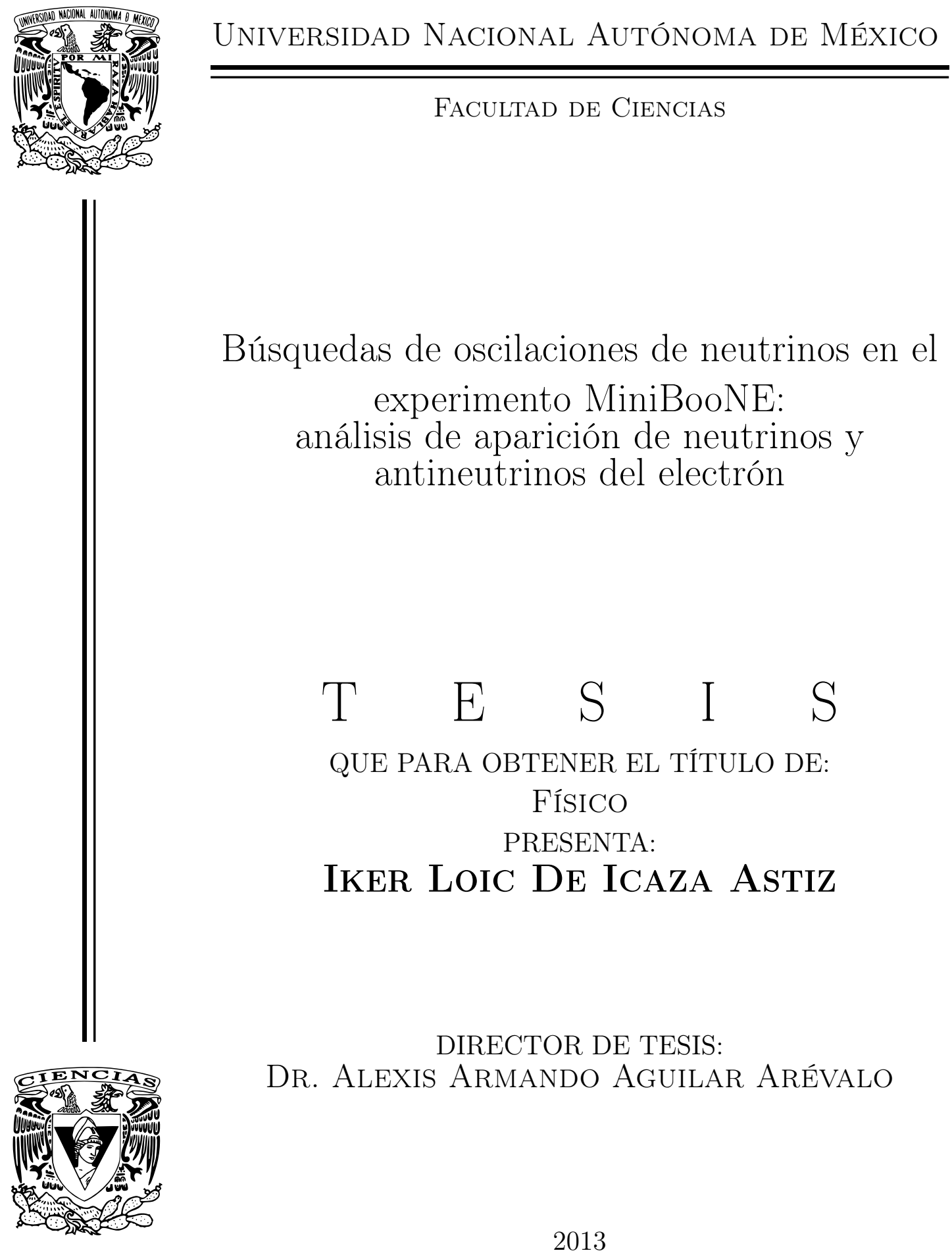


Esta página ha sido intencionalmente dejada en blanco. 


\section{Hoja de Datos del Jurado}

Datos del alumno: De Icaza

Astiz

Iker Loic

56055909

Universidad Nacional Autónoma de México

Facultad de Ciencias

Física

408023917

Datos del tutor: Dr.

Alexis Armando

Aguilar

Arévalo

Datos del sinodal 1: Dr.

Juan Carlos

D'Olivo

Saez

Datos del sinodal 2: Dra.

Myriam

Mondragón

Ceballos

Datos del sinodal 3: Dra.

Gabriela

Murguía

Romero

Datos del sinodal 4: Dr.

Víctor Manuel

Romero

Rochín

Trabajo escrito: Búsquedas de oscilaciones de neutrinos en el experimento MiniBooNE: análisis de aparición de neutrinos y antineutrinos del electrón $194 \mathrm{pp}$.

2013 
Esta página ha sido intencionalmente dejada en blanco. 


\section{Agradecimientos}

Antes que nada quiero agradecer a mi asesor el Dr. Alexis Aguilar Arévalo, con quien he estado trabajando desde mi servicio social, hace varios años ya. Con él he aprendido una gran variedad de temas: mis primeros programas serios fueron con su guía; principalmente de él adapté mi estilo de redacción de código, me enseñó más física experimental y estadística que lo que aprendí en toda la carrera; formando en mi lo que significa ser un buen experimentalista, mi perspectiva de la física de partículas elementales no podría ser tan amplia como lo es si no fuera por él; enseñándome la historia de los descubrimientos, así como las técnicas experimentales para hacerlos y la teoría que los explica. Mucho trabajo me costó, pero el conocimiento tan amplio que tengo sobre los neutrinos, experimental y teórico, se debe a la enorme paciencia de mi asesor. Mucho le agradezco haberme mandado a Fermilab, en donde fui parte de un activo y amable grupo de trabajo, en donde aprendí una forma distinta de hacer ciencia: mucho más colaborativa. Le agradezco su perfeccionismo y apoyo constante en todos estos años, sin los cuales no hubiera logrado enorgullecerme de esta tesis tanto como lo estoy ahora. Para mi es un rol a seguir, mostrándome la clase de físico en que me quiero convertir. Sobre todo le agradezco su amistad, gracias Alexis.

A mis sinodales les agradezco su expedita respuesta y asistencia.

Si vas a hacer una carrera en física más te vale hacerte de amigos como los míos; Citlali, Luigi, David, Corro, MaFer, Javier, Corinto, Laura, Susana, Ana Lucía y Martha. Amigos con los que he trasnochado haciendo tareas, recorrido la ciudad de noche saltando entre fiestas, con quienes he viajado, con quienes se me han pasado los tragos, agradezco que mi carrera haya sido junto con ellos. A ellos hace falta agregar a mis cuates activistas de conCiencias; Manuel, Carlos, Lalo y especialmente a mis amigas del Cotorreo Científico Cintli y Zitlali. Por si no fueran suficientes todavía hace falta agregar a más, en la facultad se conoce a mucha gente: Ely, Diana, Eulogio, Marce, Abryl, Ollin, Xitzel, Adela, Bermúdez, Bruno y otros tantos de los que no me acuerdo. También están mis amigos de antaño, los del pueblo o de otros pueblos; Andrea, Merlín, Raúl, Fidel, Bryan, Saraí y Villeda.

Definitivamente no hubiera decidido tomar el camino de partículas elementales si no fuera por el apoyo del Dr. Guy Paic para que hiciera mi estancia en DESY, Alemania. Así como a todos los amigos que hice allá: Bonnie, Gedas, Matteo, Benjamin, Francesco, Raquel y Castellar. Amigos que seguiré encontrando.

En Fermilab me la pasé de maravilla, agradezco muchísimo a Žarko Pavlović por orientarme en mis análisis así como por resolver mis dudas o ayudarme cada vez que me trababa, aún después de haber regresado a México. Richard Van de Water fue un gran tutor, el implacable portavoz del experimento MiniBooNE que mantiene el ánimo de la colaboración y la causa en alto, sin su ayuda mi trabajo 
por allá no hubiera llegado muy lejos. Una estancia científica no estaría completa sin las profundas y variadas pláticas (sobre ciencia u otros temas) que sostienes con personas como Ranjan, Christina, Ben, Alexander, Jason, Thomas o Francesco (el mismo del DESY). Estando fuera de los trópicos, mucho conviene la cercanía de un cálido hogar de italianos y un ruso aficionados al buen té y café, tuve ratos memorables en casa de Vito y Evgeny.

A mis hermanos; Miguel, Pilar y sobre todo a los de mi camada Yannick, Isaac, Aitor y Aramis. Mis mejores amigos, mis cómplices en tantas aventuras, son el apoyo con el podré contar toda mi vida. A mi tía Luciana que tanto nos ha ayudado y en cuya casa me he sentido tan bienvenido, por tratarme como a uno de sus hijos, siempre alentando a que siga mis sueños. A mi papá y mamá, Miguel y Mariblanca, por todo su cariño y amor, por permitirme ser como soy, distinto a mis hermanos. Por educarme como lo hicieron, tan maravillado de la vida y tan cercano a mis hermanos.

Agradezco a MiniBooNE en Fermiab por permitirme usar su cluster administrado por el software Condor y al IF-UNAM por permitirme usar las tarjetas Tesla de Nvidia, para ejecutar mis análisis. Quiero expresar mi profundo agradecimiento a la Facultad de Ciencias y la Red FAE por el apoyo que recibí para ir a Fermilab. Al ICN-UNAM, en donde trabajé la mayor parte del tiempo, por todo el café que aportó. A la facultad de Ciencias de la UNAM, el mejor lugar para estudiar física en el país. 


\section{Índice general}

$\begin{array}{ll}\text { Agradecimientos } & \text { V }\end{array}$

$\begin{array}{lc}\text { Índice general } & \text { IX }\end{array}$

Índices de figuras, tablas, algoritmos y código $\quad$ XI

Figuras . . . . . . . . . . . . . . . . . . . . . . X XI

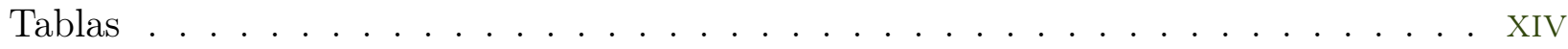

Algoritmos y código . . . . . . . . . . . . . . . . . . . XIV

Resumen / Abstract $\quad$ XV

Introducción $\quad$ XVII

1. Neutrinos 1

1.1. Acontecimientos históricos experimentales . . . . . . . . . . . . . . . . 1

1.2. El modelo estándar de partículas elementales . . . . . . . . . . . . . . . . . . . 8

1.2.1. Interacciones fundamentales . . . . . . . . . . . . . . . . . . . 10

1.2.2. Propiedades de los neutrinos . . . . . . . . . . . . . . . . . . . . 12

1.3. Fuentes de neutrinos . . . . . . . . . . . . . . . . . . . . . . . 15

1.3.1. Reactores nucleares . . . . . . . . . . . . . . . . . . 15

1.3.2. Aceleradores de partículas . . . . . . . . . . . . . . . . . . . 16

1.3.3. Manto y núcleo terrestre . . . . . . . . . . . . . . . . . . . . . . . 17

1.3.4. Atmósfera . . . . . . . . . . . . . . . . . . . 17

1.3.5. Sol .............................. . . 18

1.3.6. Supernovas y cataclismos estelares . . . . . . . . . . . . . . . . . . . . . . . . . . . . . . . . . . .

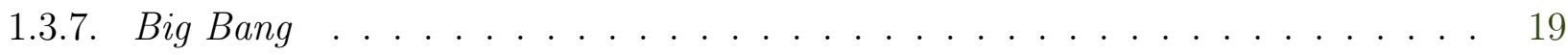

1.4. Oscilación de neutrinos . . . . . . . . . . . . . . . . . . . . . . . . . . 19

1.4.1. Oscilación de $2 \nu$ en el vacío . . . . . . . . . . . . . . . . . . . . . . 20

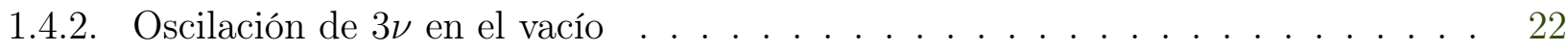

1.4.3. Efecto MSW . . . . . . . . . . . . . . . . . . . . 24

1.4.4. Consideraciones experimentales . . . . . . . . . . . . . . . 24

1.4.5. Mediciones directas de la masa de los neutrinos . . . . . . . . . . . . . . . . . 28

1.4.6. Decaimiento beta doble sin neutrinos . . . . . . . . . . . . . . . . . . . 29

1.4.7. Estatus experimental de oscilaciones de neutrinos . . . . . . . . . . . . 30

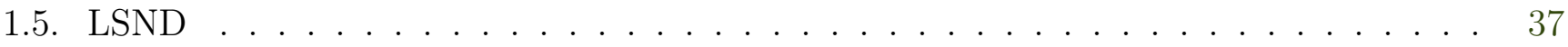


2. Experimento MiniBooNE 39

2.1. El haz de neutrinos del Booster . . . . . . . . . . . . . . . . . . . . . 40

2.1.1. El haz primario de protones . . . . . . . . . . . . . . . . . . . 4 40

2.1.2. El blanco de protones . . . . . . . . . . . . . . . . . . . . . . . . 41

2.1.3. Enfocado de mesones secundarios . . . . . . . . . . . . . . . . . 42

2.1.4. El conducto de decaimiento de mesones secundarios . . . . . . . . . . . . . . 43

2.2. El detector . . . . . . . . . . . . . . . . . . . . . . . . . 44

2.2.1. Electrónica y sistemas de adquisición . . . . . . . . . . . . . . . 45

2.2.2. Sistema de triggers . . . . . . . . . . . . . . . . . . . . . . . 4 47

2.2.3. Sistemas de calibración del detector . . . . . . . . . . . . . . . . . 48

2.3. Simulación Monte Carlo del haz . . . . . . . . . . . . . . . . . . . 50

2.3.1. Predicción del flujo de neutrinos en MiniBoonE . . . . . . . . . . . . . . 53

2.4. Simulación Monte Carlo del detector . . . . . . . . . . . . . . . . . . 56

2.4.1. Interacción cuasielástica de corriente cargada (CCQE) _ . . . . . . . . . 57

2.4.2. Producción de $\pi^{0}$ por interacciones de corriente neutra $(\mathrm{NC}) \ldots \ldots \ldots$

2.4.3. El modelo óptico de MiniBooNE . . . . . . . . . . . . . . . . . . . . 60

2.5. Reconstrucción de eventos e identificación de partículas . . . . . . . . . . . . . 61

2.5.1. Identificación de partículas . . . . . . . . . . . . . . . . . . . . 63

2.6. Resultados publicados por MiniBooNE . . . . . . . . . . . . . . 65

3. Análisis de oscilaciones $\quad 69$

3.1. Nociones de probabilidad y estadística . . . . . . . . . . . . . . . . . . 69

3.1.1. Estadística a nivel práctico . . . . . . . . . . . . . . . . . . . 71

3.2. Formato y procesamiento de datos liberados por MiniBooNE . . . . . . . . . . . . 81

3.2.1. Generalidades de los análisis realizados . . . . . . . . . . . . . . . 85

3.3. Método de búsqueda por barrido unidimensional . . . . . . . . . . . . . . . . 86

3.4. Método de búsqueda por barrido bidimensional . . . . . . . . . . . . . . . . . . 89

3.5. Método de máxima verosimilitud . . . . . . . . . . . . . . . . . . . . . . . 92

3.6. Método frecuentista Feldman-Cousins . . . . . . . . . . . . . . . . . . . 94

3.6.1. Implementación del método Feldman-Cousins . . . . . . . . . . . . . . . 96

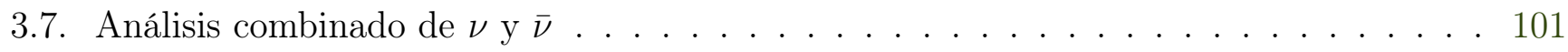

4. Resultados 103

4.1. Búsqueda de aparición de $\nu_{e}, 2007 \ldots \ldots \ldots \ldots \ldots$. . . . . . . . . . . . 105

4.2. Búsqueda de aparición de $\nu_{e}, 2009 \ldots \ldots \ldots$. . . . . . . . . . . 106

4.3. Búsqueda de aparición de $\bar{\nu}_{e}, 2010 \ldots \ldots$. . . . . . . . . . . . . 107

4.4. Búsqueda de aparición de $\nu_{e}, 2012-2013 \ldots \ldots$. . . . . . . . . . . . 109

4.5. Búsqueda de aparición de $\bar{\nu}_{e}, 2012-2013 \ldots \ldots$. . . . . . . . . . . . 110

4.6. Búsqueda combinada de aparición de $\nu_{e}$ y $\bar{\nu}_{e}, 2012 \ldots \ldots \ldots \ldots$. . . . . . . 112

5. Conclusiones 123

$\begin{array}{ll}\text { Apéndices } & 127\end{array}$

$\begin{array}{ll}\text { A. El código } & 127\end{array}$

A.1. Código serial en $\mathrm{C}++\ldots \ldots \ldots \ldots$

A.2. Código heterogéneo en CUDA . . . . . . . . . . . . . . . . . 137 
$\begin{array}{lr}\text { B. Condor } & 147\end{array}$

B.1. Uso de Condor en el pool de MiniBoonE . . . . . . . . . . . . . . . . . . . . . . 147

C. Implementación en un esquema de procesamiento en paralelo con CUDA 151

C.1. Descripción de la paralelización . . . . . . . . . . . . . . . . . . . . . . 152

D. Pruebas misceláneas del método Feldman-Cousins 155

D.1. Posición de los mínimos en los experimentos ficticios . . . . . . . . . . . . . . . . . 155

D.2. Generación de matrices de covarianza con los vectores de experimentos falsos . . . . . 156

D.3. Contornos obtenidos con Condor y CUDA . . . . . . . . . . . . . . . . 156

D.4. Diferencias con los contornos oficiales . . . . . . . . . . . . . . . . 157

D.4.1. PRNG alternativo . . . . . . . . . . . . . . . . . 157

D.4.2. Baja y alta señal . . . . . . . . . . . . . . . . . . . 157

E. Constricción de los errores $r$

E.1. Construcción de la constricción de errores . . . . . . . . . . . . . . . . . . . . 159

F. Fibra óptica en el RWM 163

$\begin{array}{lr}\text { Bibliografía } & 167\end{array}$ 


\section{Índices de figuras, tablas, algoritmos y código}

\section{Figuras}

1.1. Sección eficaz como función de $\sqrt{s}$ para la reacción $e^{+} e^{-} \rightarrow h$ del experimento ALEPH

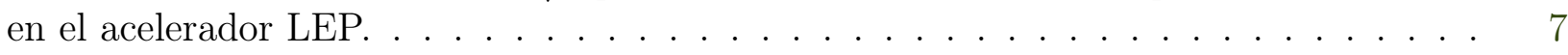

1.2. Modelo estándar de partículas elementales. . . . . . . . . . . . . . . . . . 8

1.3. Escala de masas en el modelo estándar. . . . . . . . . . . . . . . . . . . . . . . . . . 10

1.4. Compendio del conocimiento actual de las secciones eficaces de CC $\nu_{\mu}\left(\bar{\nu}_{\mu}\right)$. . . . . . . 13

1.5. Ejemplo representativo de varias fuentes de neutrinos a lo largo de varios ordenes de magnitud de energía. . . . . . . . . . . . . . . . . . 16

1.6. Análogo clásico de la oscilación de neutrinos. . . . . . . . . . . . . . . . . . . . . . . 22

a. Péndulos acoplados con resorte. . . . . . . . . . . . . . . . . . 22

b. Evolución temporal de los péndulos. . . . . . . . . . . . . . . . . . . 22

c. Modo normal de baja frecuencia. . . . . . . . . . . . . . . . . . . . 22

d. Modo normal de alta frecuencia. . . . . . . . . . . . . . . . . . . . . 22

1.7. Mezcla de neutrinos. . . . . . . . . . . . . . . . . . . . . . 23

a. Mezcla de los eigenestados de sabor y masa. . . . . . . . . . . . . . . . . . . . 23

b. Jerarquía de masa para tres neutrinos. . . . . . . . . . . . . . . . . . 23

1.8. Gráfica de la probabilidad de oscilación $P\left(\nu_{\alpha} \rightarrow \nu_{\alpha}\right)$. . . . . . . . . . . . . . . 25

1.9. Histogramas típicos de los resultados esperados. . . . . . . . . . . . . . . . . . . . . . . . . . . . . . . . . . . . . .

a. Experimento de aparición . . . . . . . . . . . . . . . . 26

b. Experimento de desaparición. . . . . . . . . . . . . . . 26

1.10. Gráfica estándar log-log de $\Delta m^{2}$ vs. $\sin ^{2} 2 \theta$ de contornos de confianza. . . . . . . . . . 28

1.11. Punto final del espectro beta. . . . . . . . . . . . . . . . . . . . . . . . . 29

1.12. Resultados de experimentos de neutrinos solares. . . . . . . . . . . . . . . . . . . . 31

a. Proporción de datos observados y esperados por el SSM. . . . . . . . . . . . . 31

b. Probabilidad de supervivencia de $\nu_{e}$ solares. . . . . . . . . . . . . . . . 31

1.13. Resultados de experimentos en el régimen atmosférico. . . . . . . . . . . . . . . . . . . . . . . . . . . . . . . .

a. Neutrinos atmosféricos de Super-Kamiokande. . . . . . . . . . . . . . . 32

b. Eventos de MINOS. . . . . . . . . . . . . . . . . . . . . 32

1.14. Resultados de experimentos de reactores. . . . . . . . . . . . . . . . . . . . . 34

a. Proporción de $L / E$ observado en KamLAND. . . . . . . . . . . . . . . . . . . 34

b. Espectro observado en Daya Bay. . . . . . . . . . . . . . . . . . . . . 34 
1.15. Varios resultados de experimentos de oscilación de neutrinos. . . . . . . . . . . . . . 36

a. Compilación de la mayoría de los resultados de oscilación de neutrinos. . . . . 36

b. Neutrinos solares y KamLAND. . . . . . . . . . . . . . . . . . . 36

c. Neutrinos atmosféricos, MINOS y T2K. . . . . . . . . . . . . 36

1.16. Anomalía de los antineutrinos de reactores nucleares de corta distancia. . . . . . . . 37

1.17. Resultados del experimento LSND. . . . . . . . . . . . . . . . . . . . . . . . . . . . . . . . . . . . . . . .

a. Exceso de eventos como función de $L / E_{\nu}$. . . . . . . . . . . . . . . . . . . . . 38

b. Ajuste de oscilaciones sobre $\left(\sin ^{2} 2 \vartheta, \Delta m^{2}\right) \ldots \ldots \ldots$. . . . . . . . 38

2.1. Vista esquemática del experimento MiniBooNE. . . . . . . . . . . . . . . . . . . . . 39

2.2. Vista aérea de la línea del haz del experimento MiniBooNE. . . . . . . . . . . . . . . 41

2.3. Estructura del haz de protones entregados por el Booster. . . . . . . . . . . . . . . . 42

2.4. Corno magnético. . . . . . . . . . . . . . . . . . . . . . . . . . . . . . . . . . 42

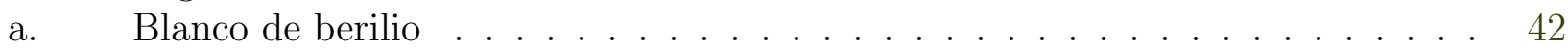

b. Fotografía del corno . . . . . . . . . . . . . . . . . . . 42

2.5. El detector MiniBooNE. . . . . . . . . . . . . . . . . . . . . . . . . . 44

a. Fotografía de la región interior y exterior del detector. . . . . . . . . . . . . 44

b. Dibujo del detector MiniBooNE. . . . . . . . . . . . . . . . . . . 44

c. Fotografía de la región de señal. . . . . . . . . . . . . . . . . . . . . 44

2.6. Dibujo esquemático del recinto del detector MiniBooNE. . . . . . . . . . . . . . . . 45

2.7. Captura de carga y tiempo para un pulso de PMT. . . . . . . . . . . . . . . . . . 46

2.8. Fotografías del interior del edificio del detector. . . . . . . . . . . . . . . . . . . . 47

a. Paneles de la electrónica involucrada en la adquisición de datos. . . . . . . . . 47

b. Rastreador de muones y fuentes de alto voltaje. . . . . . . . . . . . . . . . 47

2.9. Diagrama que resume la calibración de muones cósmicos. . . . . . . . . . . . . . . . 49

2.10. Distintas calibraciones de energía. . . . . . . . . . . . . . . . . . 50

a. Distribución de energía observada de electrones Michel. . . . . . . . . . . . 50

b. Distribución de la masa invariante de $\pi^{0} \ldots \ldots \ldots$. . . . . . . . . . 50

2.11. Flujo total predicho de cada especie de neutrinos en el detector MiniBooNE. . . . . . 54

a. Modo de neutrinos. . . . . . . . . . . . . . . . . . 54

b. Modo de antineutrinos. . . . . . . . . . . . . . . . . . . 54

2.12. Diagramas de Feynman de interacciones relevantes. . . . . . . . . . . . . . . . 59

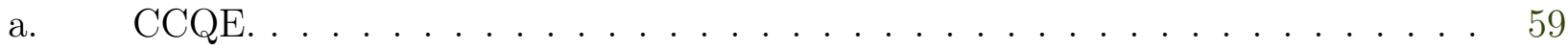

b. $\mathrm{NC} \pi^{0} \ldots \ldots \ldots \ldots \ldots \ldots \ldots$

c. $\quad \mathrm{CC} \pi^{ \pm} \ldots \ldots \ldots \ldots \ldots \ldots \ldots$

2.13. Identificación de partículas en MiniBooNE. . . . . . . . . . . . . . . . . . . 62

2.14. Parámetros internos para la reconstrucción de trazas. . . . . . . . . . . . . . . 63

a. Traza sencilla . . . . . . . . . . . . . . . . . 63

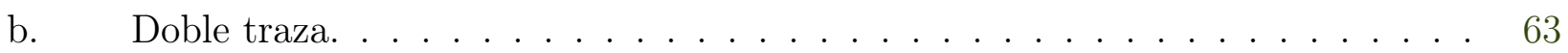

2.15. Resultados de MiniBooNE en el modo de neutrinos de 2013. . . . . . . . . . . . . . . 66

a. Distribuciones para los datos $\nu_{e}$ CCQE y exceso de eventos $\nu_{e} \ldots \ldots 6$

b. Regiones permitidas por MiniBooNE. . . . . . . . . . . . . . 66

2.16. Resultados de MiniBooNE en el modo de antineutrinos de 2013. . . . . . . . . . . . . 67

a. Distribuciones para los datos $\bar{\nu}_{e}$ CCQE y exceso de eventos $\bar{\nu}_{e} \ldots \ldots 7$

b. Regiones permitidas por MiniBooNE. . . . . . . . . . . . . . 67

3.1. Distribución normal bivariada. . . . . . . . . . . . . . . . . . . . 74

a. Muestreo de distribución bivariada. . . . . . . . . . . . . . . 74 
b. Elipses para varios valores de $\rho \ldots \ldots \ldots \ldots \ldots$. . . . . . . . . . 74

3.2. Construcción de una banda de confianza genérica y su uso. . . . . . . . . . . . . . 77

3.3. Bandas de confianza de $90 \%$ N.C. para la media de una gaussiana en unidades de la desviación media cuadrática. . . . . . . . . . . . . . . . . . . . . 78

a. Bandas de confianza implícitamente usadas. . . . . . . . . . . . . . . 78

b. Bandas de confianza del método F-C. . . . . . . . . . . . . . . . 78

3.4. Función de verosimilitud para los parámetros vida media y tasa de descomposición en escala logarítmica. . . . . . . . . . . . . . . . . . . . 80

3.5. Función densidad de probabilidad de $\chi_{k}^{2}$. . . . . . . . . . . . . . . . 80

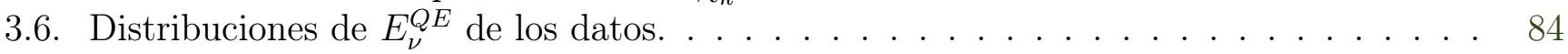

3.7. Matriz de covarianza total y su colapso. . . . . . . . . . . . . . . . . . 84

3.8. Gráfica de $\chi_{j}^{2}\left(\sin ^{2} 2 \theta\right)$ para un valor fijo de $\left(\Delta m^{2}\right)_{j} \ldots \ldots \ldots \ldots \ldots \ldots$

3.9. Superficie $\chi^{2}\left(\sin ^{2} 2 \theta, \Delta m^{2}\right)$ con cortes de nivel. . . . . . . . . . . . . . . 90

3.10. Superficie $\mathscr{L}\left(\sin ^{2} 2 \theta, \Delta m^{2}\right)$ con cortes de nivel para el método de máxima verosimilitud. 93

3.11. Malla reducida. . . . . . . . . . . . . . . . . . . . . . . . . 98

3.12. Construcción de los intervalos con el método F-C. . . . . . . . . . . . . . . . 99

a. Distribución de diferencias $\Delta \mathscr{L}_{\mathbf{f}}^{\left\{\boldsymbol{p}_{0}\right\}} \ldots \ldots \ldots \ldots \ldots \ldots$

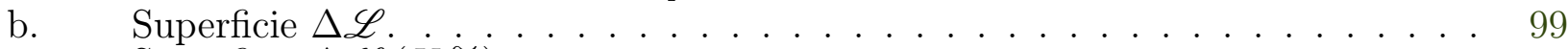

c. $\quad$ Superficie $\Delta \mathscr{L}_{c}(X \%) . \ldots \ldots \ldots \ldots$

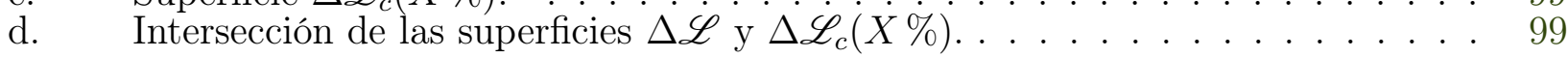

4.1. Resultados en modo de neutrinos (475-3000 MeV) datos liberados en 2007. . . . . . . 114

4.2. Resultados en modo de neutrinos (475-3000 MeV) datos liberados en 2009 . . . . . . 115

4.3. Contornos en modo de neutrinos $(200-3000 \mathrm{MeV})$ datos liberados en 2009 . . . . . . . 116

4.4. Resultados en modo de antineutrinos (200-3000 MeV) datos liberados en 2010 . . . . 117

4.5. Contornos en modo de neutrino y antineutrinos (200-3000 MeV) datos liberados en 2009 y 2010, respectivamente. . . . . . . . . . . . . . . . . . . . . 118

4.6. Contornos en modo de neutrinos (200-3000 MeV) datos liberados en 2012. . . . . . 119

4.7. Contornos en modo de antineutrinos (200-3000 MeV) datos liberados en 2012 . . . . 120

4.8. Histogramas del modo de neutrinos y antineutrinos (200-3000 MeV) datos liberados en 2012 .

4.9. Contornos para el análisis combinado de neutrinos y antineutrinos (200-3000 MeV) datos liberados en 2012 . . . . . . . . . . . . . . . . . . . . . 122

D.1. Posiciones de los mínimos de los experimentos ficticios. . . . . . . . . . . . . . . 155

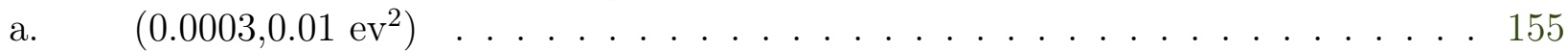

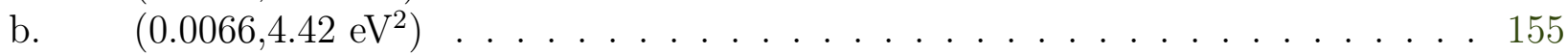

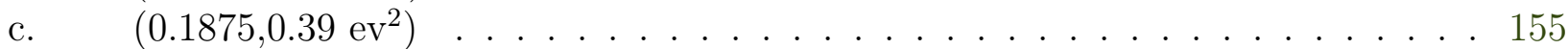

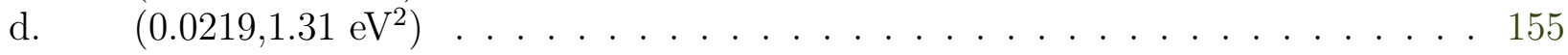

D.2. Comparación entre Condor y CUDA. . . . . . . . . . . . . . . . 156

D.3. Comparación con los contornos oficiales. . . . . . . . . . . . . . . . 158

a. Antineutrinos 2010 . . . . . . . . . . . . . . . . . . 158

b. Combinado 2012. . . . . . . . . . . . . . . . . . . 158

c. Neutrinos $2012 \ldots \ldots \ldots \ldots$. . . . . . . . . . . . . . . . 158

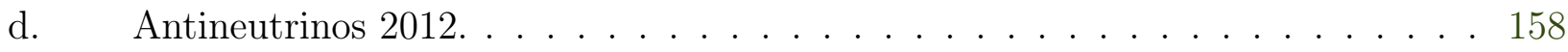

E.1. Comparación de histogramas con errores sin constreñir y constreñidos. . . . . . . . . 161

F.1. Esquema de operación del RWM. . . . . . . . . . . . . . . . . . . . . . . . 164 
F.2. Gráficas en línea del RWM. . . . . . . . . . . . . . . . . . . . . . . . . . 165

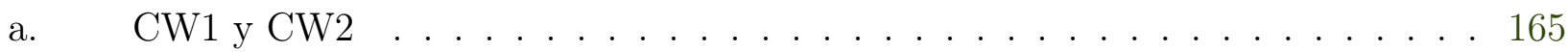

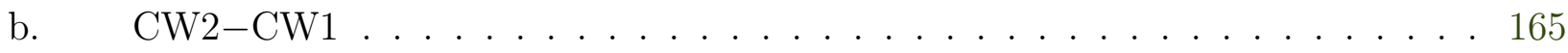

F.3. Defectos en el sistema con fibra óptica. . . . . . . . . . . . . . . . . 166

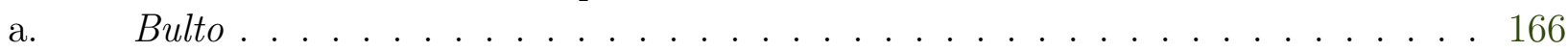

b. Corrimientos de tiempo . . . . . . . . . . . . . . . . . . 166

c. Colas amplias . . . . . . . . . . . . . . . . . 166

\section{Tablas}

2.1. Tiempos de vida y tasas de ramificación de los modos de decaimiento que producen neutrinos considerados en la simulación. . . . . . . . . . . . . . . . . . 52

2.2. Flujos predichos de $\nu_{\mu} / \bar{\nu}_{\mu}$ y $\nu_{e} / \bar{\nu}_{e}$ en el detector MiniBooNE. . . . . . . . . . . . 55

a. Flujo en modo de neutrinos. . . . . . . . . . . . . . . . . . 55

b. Flujo en modo de antineutrinos. . . . . . . . . . . . . . . . 55

2.3. Variaciones en el flujo total para cada especie de neutrino en modo de neutrinos y antineutrinos debido a incertidumbres sistemáticas. . . . . . . . . . . . . . 57

3.1. Transmutación permitida en las simulaciones para los distintos análisis. . . . . . . . . 82

3.2. Valores de $\Delta \chi^{2}$ para determinar regiones de confianza. . . . . . . . . . . . . . 87

a. Valores correspondientes a una distribución $\chi^{2}$ de un solo lado y un grado de libertad. . . . . . . . . . . . . . . . . . . . 87

b. $\quad$ Valores correspondientes a una distribución $\chi^{2}$ de dos grados de libertad. . . . 87

A.1. Funciones usadas en los programas. . . . . . . . . . . . . . . . . . . 128

A.2. Archivos que toman los programas a la entrada. . . . . . . . . . . . . . . . . 129

A.3. Especificaciones de la tarjeta NVIDIA Tesla C2075 . . . . . . . . . . . . . . . . 137

\section{Algoritmos y código}

3.1. Algoritmo del método de la búsqueda por barrido unidimensional. . . . . . . . . . . . . 89

3.2. Algoritmo del método de la búsqueda por barrido bidimensional. . . . . . . . . . . . . 91

3.3. Algoritmo del método de máxima verosimilitud. . . . . . . . . . . . . . . . . . . . . 94

3.4. Algoritmo para generar datos ficticios $\boldsymbol{D}_{\mathrm{f}}^{\left\{\boldsymbol{p}_{0}\right\}}$ en el punto $\boldsymbol{p}_{0} \in \sin ^{2} 2 \theta-\Delta m^{2} \ldots \ldots \ldots . \quad 97$

3.5. Algoritmo del método Feldman-Cousins. . . . . . . . . . . . . . . . . . . . . . . . . 101

A.1. Código serial en $\mathrm{C}++$ frequentistFC_NuBar.cpp para llevar a cabo las correcciones frecuentistas en el modo de antineutrinos. . . . . . . . . . . . . . . . . 137

A.2. Código en CUDA frequentistFC_NuBar. cu para llevar a cabo las correcciones frecuentistas en el modo de antineutrinos. . . . . . . . . . . . . . . . . . . . . 146

B.1. Bash script "submitting.sh" para mandar tareas al pool. . . . . . . . . . . . . . . . 149 


\title{
Búsquedas de oscilaciones de neutrinos en el experimento MiniBooNE: análisis de aparición de neutrinos y antineutrinos del electrón
}

\author{
por \\ Iker Loic de Icaza Astiz \\ Tesis de Licenciatura. Facultad de Ciencias, UNAM
}

\begin{abstract}
Resumen
En este trabajo se implementaron los análisis de oscilaciones por aparición de neutrinos y antineutrinos del electrón del experimento MiniBooNE empleando los datos liberados públicamente por la colaboración. Los cálculos se hicieron con algoritmos en $\mathrm{C}++$, así como en procesadores gráficos en el lenguaje CUDA. Se realizaron los análisis correspondientes a las publicaciones de 2007 y 2009 en modo de neutrinos, 2010 en modo de antineutrinos, y 2013 en modo de neutrinos y antineutrinos, construyendo los intervalos de confianza en el espacio de parámetros de oscilaciones en cada caso. Los contornos calculados de este trabajo son indistinguibles de los reportados por la colaboración MiniBooNE si no se toman en cuenta las correcciones frecuentistas de Feldman y Cousins. Al implementar estas correcciones aparecen pequeñas diferencias con los contornos oficiales en la región de bajas señales, las cuales se adscriben al método de generación de experimentos ficticios empleado aquí, y que requieren más investigación. Las probabilidades del mejor ajuste y punto nulo, así como los excesos de eventos observados, presentan pequeñas variaciones respecto a los reportados en las publicaciones, las cuales provienen de una diferencia en la definición de la región de la señal usada en este trabajo, forzada por el formato de los datos liberados, y son consistentes dentro del margen de error. Este trabajo fue realizado de manera independiente a la colaboración MiniBooNE y sus resultados no son oficiales.
\end{abstract}

Director: Dr. Alexis A. Aguilar Arévalo, Instituto de Ciencias Nucleares, UNAM. 


\title{
Neutrino Oscillations Searches in the MiniBooNE Experiment: electron neutrino and antineutrino appearance analyses
}

\author{
by
}

Iker Loic de Icaza Astiz

Bachelor Thesis. Facultad de Ciencias, UNAM.

\begin{abstract}
In this work we implemented the electron neutrino and antineutrino appearance oscillation analyses of the MiniBooNE experiment using the data publicly released by the Collaboration. The calculations were made with algorithms in $\mathrm{C}++$ as well as in graphic processors using CUDA. We performed the analyses corresponding to the publications of 2007 and 2009 in neutrino mode, 2010 in antineutrino mode, and 2013 in neutrino and antineutrino modes, and constructed the confidence intervals in the oscillations parameter space for each case. The contours calculated in this work are indistinguishable from those reported by the MiniBooNE Collaboration when the frequentist corrections of Feldman and Cousins are not taken into account. After implementing these corrections small differences with respect to the official contours appear in the low signal regions, which are ascribed to the fake experiment generation method employed here, and that require further investigation. The best fit probabilities and null point, as the observed event excesses show small variations with respect to those reported in the publications, arising from a difference in the definition of the signal region employed here and forced by the format of the released data, and are consistent within errors. This work was carried out independently to the MiniBooNE Collaboration, and its results are not official.
\end{abstract}

Thesis Supervisor: Dr. Alexis Aguilar-Arevalo, Instituto de Ciencias Nucleares, UNAM. 


\section{Introducción}

La historia de los neutrinos comienza con la investigación del decaimiento beta: después de que se hubieran observado lineas discretas en el espectro de energía del decaimiento alfa y gamma, fue una sorpresa para James Chadwick en 1914 que el espectro de energía del decaimiento beta fuera continuo. Surgieron dos posibles explicaciones: la conservación de la energía era solamente estadísticamente valida en tales procesos (preferida por Niels Bohr) o se emitía una nueva partícula sin detectar, la que se llevaba la energía y espín adicional (preferida por Wolfgang Pauli). En una carta fechada el 4 de diciembre de 1930 Pauli propone su solución al problema; una nueva partícula de espín 1/2 producida junto con el electrón pero que escapaba la detección. De esta forma el espectro continuo puede ser comprendido: tanto el electrón como esta otra partícula comparten la energía de transición, de forma que siempre sumen la energía total. Tal partícula, posteriormente bautizada por Enrico Fermi, era el neutrino. Los neutrinos fueron descubiertos hasta 1956 por Frederick Reines y Clyde Cowan.

La idea de las oscilaciones de neutrinos fue propuesta por primera por Bruno Pontecorvo en 1957, sugiriendo transiciones entre neutrinos y antineutrinos en analogía a lo que sucedía con los mesones $K^{0} / \bar{K}^{0}$. La oscilación de neutrinos entre distintos sabores fue sugerida por los japoneses Ziro Maki, Masami Nakagawa y Shoichi Sakata en 1962, después del descubrimiento de los neutrinos del muón, y requería que los neutrinos tuvieran masa. Sin embargo, esa idea no estaba apoyada por la evidencia proveniente de experimentos de reactores nucleares, a pesar de que el déficit de los neutrinos solares, observado por Ray Davis en los sesentas y setentas, admitía tal solución.

A mediados de los noventa el experimento LSND en Los Alamos encontró una señal positiva para la oscilación de neutrinos generados en un acelerador y que viajaban distancias cortas. En 1998 el experimento Super-Kamiokande en Japón presentó, por primera vez, fuerte evidencia de oscilación de neutrinos producidos en la atmósfera terrestre. Fue hasta 2001 que el experimento SNO en Canadá resolvió el problema de los neutrinos solares con evidencia irrefutable de la desaparición de neutrinos provenientes del Sol que se podía explicar en términos de oscilaciones de sabores. Algunos años después las observaciones de los neutrinos atmosféricos y solares fueron verificadas por experimentos de reactores y aceleradores, sin embargo la señal observada por LSND era inconsistente con un experimento contemporáneo, KARMEN.

Las observaciones experimentales de oscilación de neutrinos han establecido contundentemente que los neutrinos no tienen masa nula. En su gran mayoría, el fenómeno de oscilaciones se explica como la mezcla de tres eigenestados de masa con tres eigenestados de sabor. De forma independiente, es un hecho experimental que hay tres sabores activos de neutrinos. Así pues, la observación hecha por el experimento LSND implica la existencia de por lo menos un neutrino estéril, un neutrino que no forma parte de las interacciones débiles pero sí de las mezclas. El experimento MiniBooNE en Fermilab fue desarrollado para corroborar o refutar el resultado de LSND, diseñado para ser sensible a la misma región del espacio de parámetros que explicaría la observación de LSND, pero con errores 
sistemáticos diferentes y técnicas experimentales distintas.

En el primer resultado, buscando oscilaciones de neutrinos, MiniBooNE desfavorecía por completo la región de LSND, pero posteriormente al buscar oscilaciones antineutrinos, como las que investigó LSND, encontró una señal de oscilaciones semejante a la de LSND. Con la mejoría de las técnicas experimentales, la disminución de errores, el aumento de la estadística, mejorías en los análisis de datos y otros cambios; MiniBooNE encontró una señal de oscilación consistente con la de LSND en el modo de antineutrinos y en modo de neutrinos encontró una señal consistente ligeramente con oscilaciones. Así pues, MiniBooNE corrobora el resultado de LSND.

En este trabajo de tesis se utilizaron los datos publicados por el experimento MiniBooNE para realizar análisis de oscilaciones de aparición de neutrinos y antineutrinos del electrón. Se obtuvieron intervalos de confianza y límites de exclusión sobre el espacio de parámetros del modelo de oscilaciones con distintos métodos: búsqueda por barrido unidimensional, búsqueda por barrido bidimensional, máxima verosimilitud y con el método frecuentista de Feldman-Cousins. Se lograron reproducir, de forma independiente, la gran mayoría de los resultados de la búsqueda de oscilación de neutrinos, tanto en el modo de neutrinos como en el modo de antineutrinos, así como los resultados del análisis combinado de neutrinos y antineutrinos. Este ejercicio de repetir los resultados oficiales, sirvió a la colaboración como verificación de su trabajo y, en dos ocasiones, para hallar errores en sus algoritmos, ambos fueron reportados y corregidos. A pesar de que existen ciertas diferencias en los contornos obtenidos con el método frecuentista de Feldman-Cousins, los contornos obtenidos son estadísticamente equivalentes a los oficiales.

Se escribieron programas ex profeso para llevar a cabo los análisis, cada uno de ellos, así como para graficar los resultados. Los programas para el método de máxima verosimilitud y el método de Feldman-Cousins, los más robustos, han sido especialmente diseñados para ser sumamente eficientes y explotar las características peculiares del problema que puedan mejorar el rendimiento. Los análisis para el sumamente demandante método de Feldman-Cousins fueron ejecutados en un cúmulo de computadoras administradas con Condor en Fermilab y en un esquema en paralelo con tarjetas de vídeo NVIDIA del IF-UNAM, solamente se presentan los resultados obtenidos con las tarjetas de vídeo, pero los dos son congruentes. El rendimiento obtenido con las tarjetas de vídeo es un par de decenas de veces mayor que lo obtenido con un procesador serial común, en total el rendimiento obtenido en este trabajo es un par de centenas mayor que con los algoritmos oficiales. Lo cual significa una importante disminución en el tiempo de computo y de los recursos necesarios.

La estructura de esta tesis es la siguiente: el capítulo 1 tiene un breve resumen sobre neutrinos, haciendo énfasis teórico y experimental en las oscilaciones de neutrinos, se habla del estado experimental actual y se contextualiza el papel de los resultados de LSND y MiniBooNE. El capítulo 2 tiene la descripción del experimento MiniBooNE: la forma de generar el haz, detectar las partículas, las simulaciones usadas, los principales errores involucrados y los resultados obtenidos. El capítulo 3 contiene un apartado general sobre estadística y describe en detalle los distintos métodos usados para llevar a cabo los análisis, contiene varias imágenes originales. El capítulo 4 contiene los resultados de esta tesis, primero se hacen anotaciones sobre lo obtenido y al final del capítulo se presenta el conjunto de gráficas que se hicieron. El capítulo 5 contiene las conclusiones y comenta sobre la perspectiva del futuro de la física experimental de neutrinos. Los distintos apéndices contienen información adicional del trabajo de esta tesis, cabe resaltar que como complemento el último apéndice contiene una descripción sobre el trabajo que el autor hizo en una estancia en el Fermilab para contribuir al remplazo de un sistema de sincronización de tiempo.

La versión electrónica de este trabajo contiene más de setenta figuras a color y vínculos para moverse entre páginas: al presionar sobre los números de los índices, las referencias o el símbolo $\S$. 


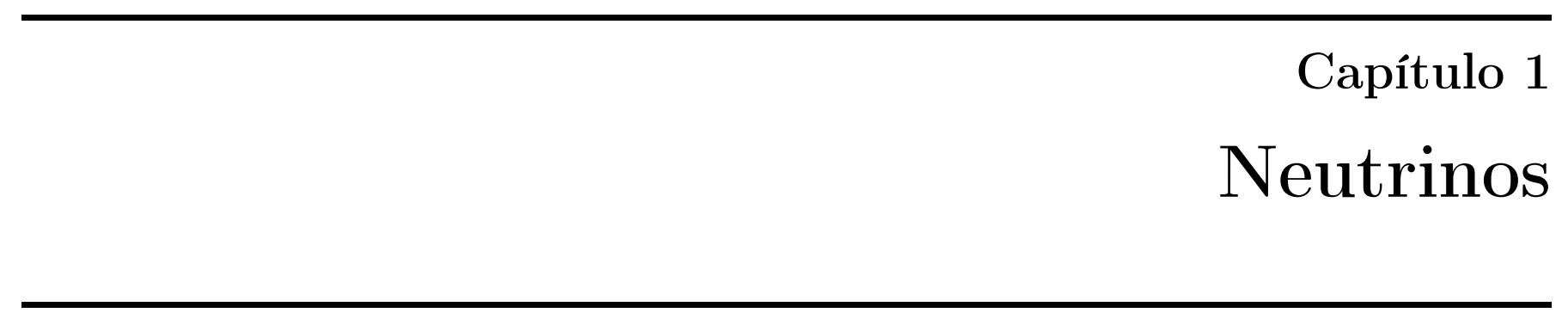

It doesn't matter how beautiful your theory is, it doesn't matter how smart you are. If it doesn't agree with experiment, it's wrong.

Richard P. Feynman

Los neutrinos son lo más cercano a la nada como ninguna otra cosa que conozcamos, además son tan elusivos que son prácticamente invisibles. Tienen un rol fundamental en la física nuclear y de partículas elementales, así como en astrofísica y cosmología. Debido a que interactúan tan débilmente con la materia, algunas de sus propiedades más básicas, como su masa y simetría de conjugación de carga, son, en buena medida, desconocidas.

\subsection{Acontecimientos históricos experimentales}

El descubrimiento del electrón en 1897 por Joseph John Thomson empezó una nueva era en la física, la física de partículas elementales. Al quitarle a los átomos su carácter de "bloque constitutivo fundamental" surgió la interrogante sobre las otras partículas que podrían estar dentro del átomo. Se descubrieron una gran variedad de partículas yendo a escalas de longitud más y más pequeñas; se logró esto con la ayuda de aceleradores de partículas, equivalentemente, yendo a energías más y más altas ${ }^{1}$ Eventualmente estos descubrimientos llevaron al actualmente aceptado modelo estándar de la física de particulas elementales en el cual los bloques constitutivos de la materia consisten de seis quarks y seis leptones, todos fermiones con espín 1/2. Interactúan entre si a través de cuatro fuerzas fundamentales: gravitación, electromagnetismo (EM), la interacción fuerte y la interacción débil. En teoría cuántica de campos estas fuerzas están descritas por el intercambio de bosones, estos junto con las partículas elementales se muestran en la figura 1.2. De entre los fermiones hay unas partículas para las cuales nuestro conocimiento sigue siendo muy limitado. Al ser leptones no participan en la interacción fuerte y como no tienen carga eléctrica tampoco participan en las interacciones EM, solo participan en la interacción débil ${ }^{2}$, lo que hace que sea muy difícil cualquier investigación experimental.

En lo que llamó un "remedio desesperado para salvar ... la ley de conservación de la energía" Wolfgang Pauli escribió una carta en 1930 a la sociedad de física de Tubinga, en la que propuso la existencia de

\footnotetext{
${ }^{1} \mathrm{El}$ otro nombre con el que se conoce a esta área, física de altas energías, enfatiza el carácter experimental.

${ }^{2} \mathrm{Si}$ no tienen una masa nula es posible que interactúen EM y gravitacionalmente.
} 
partículas neutras en el núcleo [1, 2], en aquella carta las nombró neutrones. La carta explica que de esa forma el espectro continuo de energía en el decaimiento beta, observado por James Chadwick em 1914 [3], tendría sentido asumiendo que junto con un electrón se emite un neutrón de tal forma que la suma de energías de los tres cuerpos emitidos (nucleón, electrón y neutrón) se mantiene constante. Sin embargo, en la misma carta menciona que no se atreve a publicar tal idea y que prefiere consultarlo con ellos antes; preguntando sobre que tan probable es encontrar evidencia experimental de tal partícula, más aún admite que el remedio puede parecer bastante improbable por que de existir esas partículas, ya debieron haber sido observadas hace mucho tiempo. Acaba la carta pidiendo a la sociedad de Tubinga que juzguen y escruten la propuesta y se disculpa por no poder asistir ya que debe asistir a un baile en Zúrich. Pauli determinó que además de ser neutra la nueva partícula debía tener espín $1 / 2$ y una masa muy pequeña (a lo más $10^{-2}$ veces la del protón).

La idea de Pauli ganó aceptación con el descubrimiento del neutrón como constituyente de los núcleos por James Chadwick en 1932 [4] quien uso el mismo nombre. Luego en 1934 Enrico Fermi usó la idea de Pauli en su teoría del decaimiento beta [5] y acuñó el término neutrino que significa "pequeño neutral" para diferenciarlo de los neutrones de Chadwick. El artículo de Fermi unía el neutrino de Pauli, el positrón de Dirac y el modelo de neutrones y protones de Werner Heisenberg además de que puso una base teórica sólida para el futuro trabajo experimental. En 1942 el científico chino Wang Ganchang propuso el método detectar experimentalmente a los neutrinos [6], es también Wang quien propone el método de detección de neutrones por el cual Chadwick hizo su descubrimiento.

\section{Experimento de retroceso nuclear de Rodeback y Allen}

Podría considerarse que la primera evidencia experimental de los neutrinos [7] fue encontrada en la captura del electrón de ${ }^{37} \mathrm{Ar}$ :

$$
{ }^{37} \mathrm{Ar}+e^{-} \rightarrow{ }^{37} \mathrm{Cl}+\nu_{e},
$$

la cual tiene un valor $Q$ de $816 \mathrm{keV}$. Debido a que el proceso solo tiene dos partículas en el estado final la energía de retroceso del núcleo esta fija. Usando conservación de energía y momento, debido a que la masa en reposo de ${ }^{37} \mathrm{Cl}$ es mucho mayor que $Q \approx E_{\nu}$, la energía de retroceso $T_{\mathrm{Cl}}$ esta dada por

$$
T_{\mathrm{Cl}}=\frac{E_{\nu}^{2}}{2 m_{\mathrm{Cl}}} \approx \frac{Q^{2}}{2 m_{C l}}=9.67 \mathrm{eV} .
$$

Esta energía corresponde a una velocidad de $0.71 \mathrm{~cm} / \mu$ s para el núcleo ${ }^{37} \mathrm{Cl}$. Por lo tanto la velocidad de retroceso podía ser medida por una observación retrasada en coincidencia, empezada por los electrones Auger emitidos después de la captura del electrón y detenida al medir el núcleo en retroceso [8], si el tiempo entre tales observaciones coincidía con el tiempo de vuelo de los iones en retroceso. Con una longitud de vuelo de $8 \mathrm{~cm}$, se esperaba un retraso de $8.5 \mu \mathrm{s}$. Se observó la señal alrededor de los $7 \mu \mathrm{s}$, con mejoras posteriores al hacer correcciones experimentales. El experimento de George Rodeback y James Allen en 1952 solamente fue una indicación de detección de neutrinos, sin embargo no fue capaz de asegurar la existencia de los neutrinos.

\section{Descubrimiento del neutrino por Cowan y Reines}

En 1956 Clyde Cowan, Frederick Reines et al. publicaron resultados de la detección del neutrino. El experimento de neutrinos de Cowan-Reines se vale del decaimiento beta inverso en el cual un 
antineutrino interactúa con un protón para generar un neutrón y un positrón:

$$
\bar{\nu}_{e}+\mathrm{p} \rightarrow \mathrm{n}+e^{+} .
$$

Se tienen neutrinos provenientes de un reactor nuclear los cuales interactúan con los protones de un tanque de agua con cloruro de cadmio, el positrón resultante de la interacción se aniquila inmediatamente con algún electrón del medio liberando dos rayos gamma $(\gamma)$ los cuales pueden ser detectados. Por su parte el neutrón se encuentra con el cadmio, un elemento muy efectivo para capturar neutrones, llevando a cabo la siguiente reacción:

$$
\mathrm{n}+{ }^{108} \mathrm{Cd} \rightarrow{ }^{109} \mathrm{Cd}^{*} \rightarrow{ }^{109} \mathrm{Cd}+\gamma
$$

el arreglo era tal que el fotón del decaimiento del ${ }^{109} \mathrm{Cd}$ era detectado unos $5 \mu$ s después del primer par de fotones si en verdad se trataba de la interacción de un antineutrino. Es decir la coincidencia de este par de eventos es una marca característica de la interacción de un antineutrino. Casi cuarenta años después de haber publicado sus resultados se les otorgó el premio Nobel de física en 1995.

\section{Diferencia entre $\nu_{e} \mathbf{y} \bar{\nu}_{e} \mathbf{y}$ detección de neutrinos solares}

El propósito del experimento era encontrar si los neutrinos y antineutrinos eran partículas idénticas. De ser así las reacciones

$$
\begin{aligned}
& \nu_{e}+\mathrm{p} \rightarrow e^{-}+\mathrm{n} \\
& \bar{\nu}_{e}+\overline{\mathrm{p}} \rightarrow e^{-}+\mathrm{n}
\end{aligned}
$$

debían tener la misma sección eficaz. En el experimento real Ray Davis buscaba

$$
\bar{\nu}_{e}+{ }^{37} \mathrm{Cl} \rightarrow e^{-}+{ }^{37} \mathrm{Ar}
$$

usando el reactor de Brookhaven en Estados Unidos. Usaba $4000 \mathrm{l}$ de $\mathrm{CCl}_{4}$ líquido. Los átomos producidos de Ar eran extraídos burbujeando con He en el líquido y luego congelando los átomos en una trampa de carbón [9]. Como no observó el proceso que involucraba antineutrinos logró poner el límite superior

$$
\bar{\sigma}\left(\bar{\nu}_{e}+{ }^{37} \mathrm{Cl} \rightarrow e^{-}+{ }^{37} \mathrm{Ar}\right)<0.9 \times 10^{-45} \mathrm{~cm}^{2},
$$

cuando la predicción teórica era $\bar{\sigma} \approx 2.6 \times 10^{-45} \mathrm{~cm}^{2}$.

\section{Descubrimiento de la violación de paridad en las interacciones débiles}

La paridad como una transformación de simetría se define como una inversión en el origen que resulta en $x \rightarrow-x$, significa que la probabilidad de cualquier proceso y del reflejado por un espejo tienen la misma probabilidad. Bajo transformaciones de paridad el ángulo de emisión cambia de acuerdo a $\theta \rightarrow \pi-\theta$, lo que lleva a $\Delta \theta \rightarrow-\Delta \theta$. En el clásico de experimento de Chien-Shiung $\mathrm{Wu}^{3}$ et al., se usaron átomos polarizados de ${ }^{60} \mathrm{Co}$, implementados en una sal paramagnética a una temperatura de $0.1 \mathrm{~K}$, para observar la distribución angular de emisión de electrones [10]. El decaimiento del ${ }^{60} \mathrm{Co}$ esta dado por:

$$
{ }^{60} \mathrm{Co} \rightarrow{ }^{60} \mathrm{Ni}^{*}+e^{-}+\bar{\nu}_{e}
$$

\footnotetext{
${ }^{3}$ Conocida como madame Wu.
} 
La configuración de espejo fue creado revirtiendo el campo magnético aplicado. Este experimento mostró que los electrones se emiten preferentemente en la dirección opuesta de espín del núcleo padre. Una muestra clara de que $\Delta \theta \neq 0$ y que el decaimiento beta, en efecto, viola la paridad.

Otro ejemplo de violación de paridad es el del decaimiento del pión en reposo de Richard Garwin et al. [11]. El pión positivo decae a través de la reacción

$$
\pi^{+} \rightarrow \mu^{+}+\nu_{\mu}
$$

en donde el pión tiene espín 0 y decae en reposo, lo que implica que los espines del muón y el neutrino son opuestos entre sí. Si se define la helicidad como

$$
h=\frac{\boldsymbol{\sigma} \cdot \boldsymbol{p}}{|\boldsymbol{p}|},
$$

resulta que $h\left(\mu^{+}\right)=h\left(\nu_{\mu}\right)=-1$. Al aplicar una transformación de paridad se tiene que la helicidad se transforma en +1 . La invariancia de paridad implica que las dos helicidades deben tener la misma probabilidad y que no se debe observar una polarización longitudinal del muón. La violación hubiera quedado establecida si se hubiera observado una polarización, sin embargo resultó que era máximamente violada cuando únicamente se observó $h\left(\mu^{+}\right)=+1$.

\section{Medida directa de la helicidad del neutrino}

En circunstancias especiales, medir la polarización de fotones en reacciones de captura de electrones permite medir la helicidad de los neutrinos. En el experimento de Maurice Goldhaber et al. se usó la captura del electrón de ${ }^{152} \mathrm{Eu}$ [12]. El decaimiento está dado por

$$
{ }^{152} \mathrm{Eu}+e^{-} \rightarrow \nu_{e}+{ }^{152} \mathrm{Sm}^{*} \rightarrow{ }^{152} \mathrm{Sm}+\gamma .
$$

Por conservación de momento, con el decaimiento en reposo de ${ }^{152} \mathrm{Eu}$, se tiene $\boldsymbol{p}_{{ }^{152} \mathrm{Sm}}=-\boldsymbol{p}_{\nu}$. La emisión de fotones frontales (961 keV) detienen al núcleo $\mathrm{Sm}$, implicando $\boldsymbol{p}_{\gamma}=-\boldsymbol{p}_{\nu}$. Tales fotones también llevan el pequeño retroceso de ${ }^{152} \mathrm{Sm}^{*}$, esencial para absorción resonante (para tener en cuenta el efecto Doppler) que se usa para la detección. Se puede ver que el espín del neutrino y el fotón son opuestos; lo cual, al combinar con el arreglo de los momentos, implica que la helicidad del neutrino y del fotón son la misma: $h(\nu)=h(\gamma)$. La helicidad del fotón no es más que su polarización circular, medida a través de dispersión Compton en un bloque de hierro antes del proceso de absorción. Resultó que, en efecto, los neutrinos tienen una helicidad $h(\nu)=-1$, es decir los neutrinos son izquierdos.

\section{Prueba experimental de que $\nu_{\mu}$ y $\nu_{e}$ son diferentes}

Si $\nu_{e}$ y $\nu_{\mu}$ fueran partículas idénticas entonces las reacciones $\nu_{\mu}+\mathrm{n} \rightarrow \mu^{-}+\mathrm{p}, \bar{\nu}_{\mu}+\mathrm{p} \rightarrow \mu^{+}+\mathrm{n} ; \mathrm{y}$ $\nu_{\mu}+\mathrm{n} \rightarrow e^{-}+\mathrm{p}, \bar{\nu}_{\mu}+\mathrm{p} \rightarrow e^{+}+\mathrm{n}$; debían ocurrir con la misma frecuencia, debido a que las últimas las pueden hacer $\nu_{e}$ y $\bar{\nu}_{e}$; de otra forma las últimas dos no debían ser observadas en lo más mínimo.

En 1962 el grupo de la universidad de Columbia dirigido por Leon Lederman, Melvin Schwartz y Jack Steinberger descubrió el neutrino del muón $\left(\nu_{\mu}\right)$, mostrando así que existía más de una clase de neutrinos y que junto con los $\mu$ formaban la segunda generación de leptones. Tal trabajo les hizo acreedores del premio Nobel de física en 1988. El experimento se realizó en el AGS de Brookhaven 
usando protones de $15 \mathrm{GeV}$ que impactaban un blanco de berilio [13]. Los piones y kaones secundarios creados formaban un haz casi puro de $\nu_{\mu}$. Detrás de un blindaje de $13.4 \mathrm{~m}$ de acero, que absorbía todos los hadrones y la mayoría de los muones, se encontraban 10 módulos de cámaras de chispas de una tonelada cada uno. Se discriminaba entre muones y electrones por sus trazas características, los primeros dejan trazas rectas mientras que los otros hacen cascadas electromagnéticas. En total se observaron 29 eventos de tipo muón y 6 eventos de tipo electrón ${ }^{4}$, mostrando claramente que $\nu_{\mu} \neq \nu_{e}$.

\section{Descubrimiento de la corriente neutra débil}

El desarrollo de la teoría electrodébil de Sheldon Glashow, Steven Weinberg y Abdus Salam [14]; predijo la existencia del bosón $Z$ junto con la corriente neutra ( $\mathrm{NC}$ en inglés), y los bosones $W^{ \pm}$. El descubrimiento se hizo en la cámara de burbujas Gargamelle en CERN [15, 16]. Se uso el sincrotrón de protones $P S$ con el cual se obtenía un haz de $\nu_{\mu} / \bar{\nu}_{\mu}$ que se dirigía a una cámara de burbujas de $14 \mathrm{~m}^{3}$ con gas freón. La búsqueda confiaba en eventos puramente hadrónicos sin leptones cargados en el estado final (eventos de corriente neutra), descritos por las reacciones

$$
\begin{aligned}
& \nu_{\mu}+\mathrm{N} \rightarrow \nu_{\mu}+\mathrm{X} \\
& \bar{\nu}_{\mu}+\mathrm{N} \rightarrow \bar{\nu}_{\mu}+\mathrm{X}
\end{aligned}
$$

$X$ denota el estado hadrónico final. Además se detectaban las interacciones de corriente cargada (CC)

$$
\begin{aligned}
& \nu_{\mu}+\mathrm{N} \rightarrow \mu^{-}+\mathrm{X} \\
& \bar{\nu}_{\mu}+\mathrm{N} \rightarrow \mu^{+}+\mathrm{X} .
\end{aligned}
$$

En total se detectaron 102 eventos NC y 428 eventos CC en el haz de $\nu_{\mu}$ y 64 NC y 148 CC para el haz de $\bar{\nu}_{\mu}$. Se tomaron del orden de 83000 y 207000 fotografías, para el haz de $\nu_{\mu} \mathrm{y} \bar{\nu}_{\mu}$, respectivamente. También se descubrieron eventos NC puramente leptónicos [17] provenientes de interacciones $\bar{\nu}_{\mu}+e \rightarrow$ $\bar{\nu}_{\mu}+e$. Estas observaciones fueron confirmadas por otros experimentos.

\section{Descubrimiento de los bosones de norma $W$ y $Z$}

Los bosones predichos por la teoría electrodébil finalmente fueron descubiertos en el CERN en 1983 por los experimentos $U A 1$ y $U A 2$ [18, 19, 20, 21]. Los procesos de producción eran de corriente débil y neutra dado a nivel de quarks

$$
\begin{aligned}
& \bar{d}+u \rightarrow W^{+} \rightarrow e^{+}+\nu_{e}\left(\mu^{+}+\nu_{\mu}\right) \\
& \bar{u}+d \rightarrow W^{-} \rightarrow e^{-}+\bar{\nu}_{e}\left(\mu^{-}+\bar{\nu}_{\mu}\right) \\
& \bar{d}+d \rightarrow Z^{0} \rightarrow e^{+}+e^{-}\left(\mu^{+}+\mu^{-}\right) \\
& \bar{u}+u \rightarrow Z^{0} \rightarrow e^{+}+e^{-}\left(\mu^{+}+\mu^{-}\right) .
\end{aligned}
$$

Fueron experimentos complicados, ya que la sección eficaz de producción de los bosones débiles a esas energías es muy pequeña, varios ordenes de magnitud menor que las interacciones $p \bar{p}$. Los dos experimentos observaron del orden de 25 eventos $Z, W$, que fueron incrementados posteriormente. Con el inicio del colisionador LEP de $e^{+} e^{-}$en 1989 y el SLC en SLAC el número de bosones $Z$ aumento a varios millones y sus propiedades pudieron ser bien estudiadas. Las propiedades del $W$ fueron investigadas en Fermilab.

\footnotetext{
${ }^{4}$ Se esperaban ciertos eventos de tipo electrón debido a la contaminación proveniente del decaimiento de kaones, por ejemplo $K^{+} \rightarrow e^{+}+\nu_{e}+\pi^{0}$.
} 


\section{Observación de neutrinos de la supernova 1987A}

En 1987 había tres experimentos con la capacidad de detectar neutrinos provenientes de fuentes astrofísicas Kamiokande II en Japón, IMB en Estados Unidos y el experimento Baksan en la Unión Soviética. Los tres reportan la observación de neutrinos provenientes de la explosión de una súper nova tipo II por detectores subterráneos de neutrinos fue uno de los grandes logros de la astrofísica del siglo pasado. Cerca de 25 eventos de neutrinos fueron observados en un intervalo de $12 \mathrm{~s}$. Fue la primera observación de neutrinos de una fuente astrofísica además del Sol. La supernova 1987A ocurrió en la gran nube de Magallanes, a una distancia de cerca de $50 \mathrm{kpc}$.

\section{Número de sabores de neutrinos a partir del ancho de $Z^{0}$}

El descubrimiento de la partícula $\tau$ por Martin Lewis Perl en 1975 [22] sugirió la existencia de un $\nu_{\tau}$, apuntando al esquema de tres generaciones de partículas en el modelo estándar completado en 1995 con el descubrimiento del quark top en Fermilab [23, 24] y con el descubrimiento del neutrino del tau por la colaboración DONUT en el 2000 también en Fermilab [25].

El número $N_{\nu}$ de neutrinos ligeros ${ }^{5}$ fue determinado en LEP al medir el ancho total de decaimiento $\Gamma_{Z}$ de la resonancia $Z^{0}$. Experimentalmente, la resonancia $Z^{0}$ se ajusta con cuatro parámetros diferentes que tienen pequeñas correlaciones: $m_{Z}, \Gamma_{Z}, \sigma_{\text {had }}^{0}=\frac{12 \pi}{m_{Z}^{2}} \frac{\Gamma_{e} \Gamma_{\text {had }}}{\Gamma_{Z}^{2}}$ y $R_{l}=\frac{\Gamma_{\text {had }}}{\Gamma_{l}}$.

Usando el ajuste más reciente a los cuatros experimentos de LEP [26] se deduce que el número de neutrinos es:

$$
N_{\nu}=2.9841 \pm 0.0083
$$

lo cual concuerda excelentemente con la expectativa teórica de 3 neutrinos. En la figura 1.1 se muestra la sección eficaz hadrónica como función de la energía para los datos del experimento ALEPH, del LEP. También se muestran las curvas de la predicción del modelo estándar para otros números de neutrinos.

\section{Mezcla de neutrinos}

El italiano Bruno Pontecorvo trabajando en la unión soviética sugirió por primera vez la idea de las oscilaciones de neutrinos en 1957 [28] sugiriendo la posibilidad de transiciones $\nu \leftrightarrow \bar{\nu}$ en analogía con las oscilaciones $K_{0} \bar{K}^{0}[29,30]$. En 1962 Ziro Maki, Masami Nakagawa y Shoichi Sakata sugieren que las oscilaciones entre neutrinos de distintos sabores pueden ocurrir si la masa de los neutrinos no fuera nula. Si los estados de sabor definido y los de masa definida estuvieran relacionados a través de una transformación lineal similar a un cambio de base [31]. El formalismo matemático y la formulación de las oscilaciones en el vacío fue desarrollada por Pontecorvo [32] y un año después, en 1968, es observado el "déficit de los neutrinos solares" por Raymond Davis, Don Harmer y Kenneth Hoffman [33]. En ese mismo año Vladimir Gribov y Pontecorvo sacan un artículo en el que se adjudica el déficit de neutrinos solares al fenómeno de oscilación [34].

Varios experimentos de neutrinos solares empezaron a observar un déficit de neutrinos con respecto a lo esperado, lo que fue bautizado como "el problema de los neutrinos solares". Además, también se observó un déficit de los neutrinos atmosféricos del muón que viajan hacia arriba, es decir de aquellos

${ }^{5}$ Por "ligeros" se entiende que $m_{\nu}<m_{Z / 2}$. 


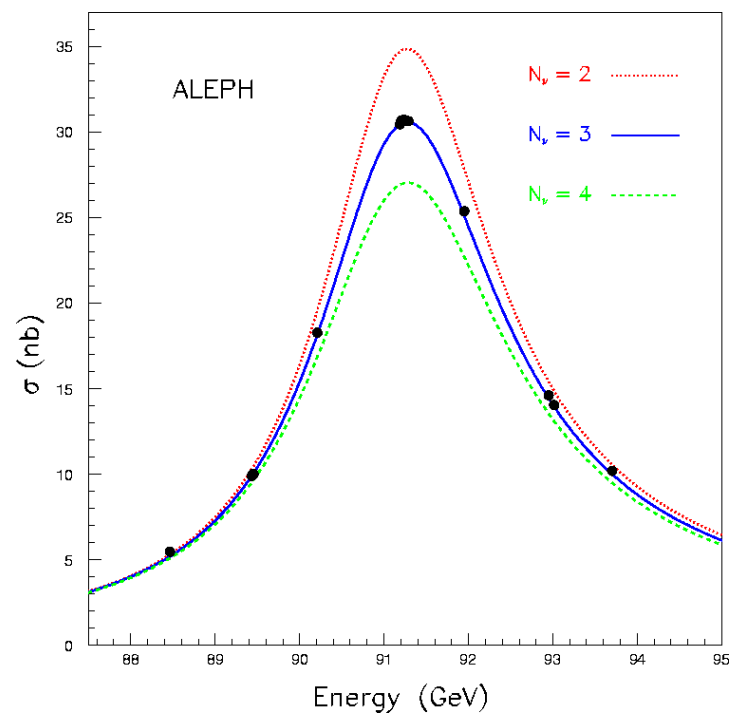

Figura 1.1: Sección eficaz como función de $\sqrt{s}$ para la reacción $e^{+} e^{-} \rightarrow h$ del experimento ALEPH en el acelerador LEP. Las diferentes curvas muestran la predicción del modelo estándar para dos, tres y cuatro sabores de neutrinos ligeros [27].

que atraviesan la Tierra. En 1998 el experimento Super-Kamiokande en Japón dio una medida muy precisa de la oscilación de neutrinos con un rango de energía de cientos de MeV hasta algunos TeV y viajando distancias comparables al diámetro terrestre $(\sim 12700 \mathrm{~km})$ [35]. Después de las observaciones de Davis en el experimento de Homestake, hubo varios experimentos radioquímicos y de radiación cherenkov subsecuentes que confirmaron el déficit, pero no fue sino hasta el 2001 que el Sudbury Neutrino Observatory (SNO) en Canadá tuvo suficientes datos para concluir que el déficit se debía a la oscilación de neutrinos [36, 37]. Adelante se hablará más al respecto, en $\S 1.3$ se describen las fuente más comunes de generación de neutrinos y en $\S 1.4 .7$ se habla más sobre los experimentos de neutrinos solares, atmosféricos y otros. En $\S 1.4$ se describe la teoría que explica la oscilación de neutrinos.

En 1985 Stanislav Mikheyev y Alexei Smirnov, profundizando en el trabajo que hizo Lincoln Wolfenstein en 1975, notaron que la materia modifica el fenómeno de oscilación. El llamado efecto MikheyevSmirnov-Wolfenstein [38, 39] (efecto MSW) es de particular relevancia en el Sol en donde los neutrinos generados por fusión en el núcleo tienen que atravesar la alta concentración de electrones para salir. Los neutrinos solares tienen energías por debajo de los $20 \mathrm{MeV}$ y viajan distancias del orden de $1.50 \times 10^{8} \mathrm{~km}$.

A mediados de los noventas el experimento LSND en el laboratorio de Los Alamos buscó oscilaciones de neutrinos del tipo $\bar{\nu}_{\mu} \rightarrow \bar{\nu}_{e}$ con un haz de neutrinos que viajaba una distancia corta $(\sim 30 \mathrm{~m})$ de la fuente al detector, encontrando una señal que favorecía este proceso. Sin embargo las distintas observaciones de oscilaciones implicaban tres regímenes muy distintos de oscilaciones, requiriendo la existencia de por lo menos un neutrino más, un serio conflicto con el modelo estándar. Las oscilaciones para neutrinos atmosféricos y solares fueron confirmadas, por su parte la observación de LSND permanece sin ser verificada o refutada de forma decisiva hasta la fecha. En $\S 1.5$ se habla más sobre LSND y en el capítulo 2 se describe el experimento MiniBooNE, construido con el objetivo de verificar o refutar el resultado de LSND. 


\subsection{El modelo estándar de partículas elementales}

A partir de un conjunto de leyes, teorías y observaciones empíricas, algunas hechas desde mediados del siglo XIX, surgió en los setenta el modelo estándar de partículas elementales (ME). Desde su concepción ha sido escudriñado por cientos de experimentos, la gran mayoría de los cuales ha dado fe de sus predicciones y su exactitud con gran precisión. Es verdaderamente inspirador que un conjunto de fuerzas, simetrías y bloques constitutivos básicos sean suficientes para describir la física detrás de fenómenos tan variados como las reacciones termonucleares y la combustión nuclear en el centro del Sol, así como el denso y caliente universo temprano momentos después del Big Bang, las reacciones que se llevan en lo alto de la atmósfera ocasionadas por partículas cósmicas, o aquellos fenómenos que se encuentran a una escala tan diminuta que hacen falta grandes acelerados para poder acceder a esos confines.

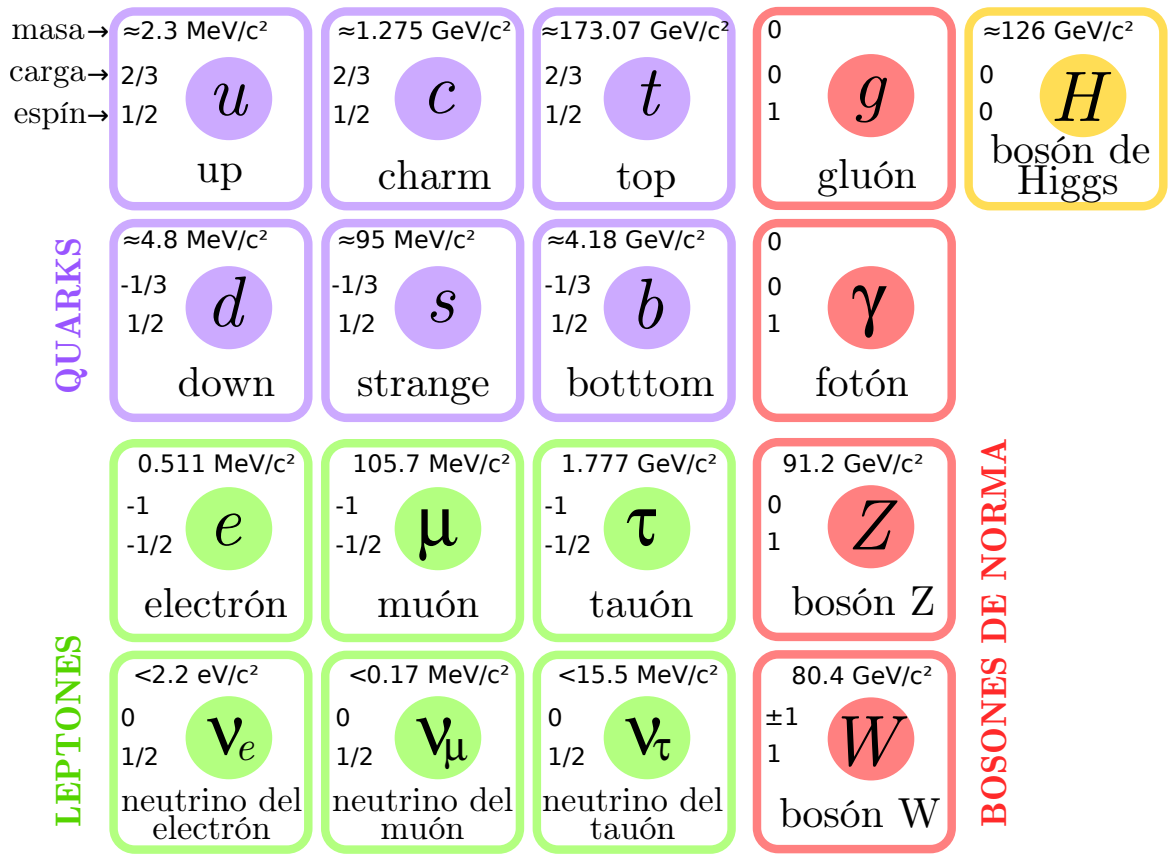

Figura 1.2: Modelo estándar de partículas elementales. Las tres primeras columnas ocupan las tres generaciones de materia; con los quarks en la parte superior y los leptones en la inferior, la cuarta columna tiene los bosones de norma y la quinta el bosón de Higgs. Ajustando los valores de las masas según el Particle Data Group [40] y parcialmente actualizado para la edición de 2014.

El modelo estándar de partículas elementales es una teoría sobre las interacciones electromagnéticas, la nuclear débil y la nuclear fuerte; que median la dinámica de las partículas subatómicas conocidas. Este modelo no incluye la interacción gravitacional. Las partículas elementales se dividen en quarks y leptones, y las interacciones son medidas por bosones. Los primeros no existen libres en la naturaleza, sino que están confinados en dobletes de quark-antiquark, formando partículas llamadas mesones (por ejemplo los piones y kaones) y en tripletes de quarks, formando bariones (los neutrones y protones por ejemplo), a los mesones y bariones en conjunto se les llama hadrones. Los quarks experimentan las cuatro interacciones fundamentales de la naturaleza, los leptones por su parte se encuentran exentos de la interacción nuclear fuerte.

A los distintos tipos de quarks se les dice sabores y hay seis distintos: up $(u)$, down $(d)$, charm $(c)$, strange $(s)$, top $(t)$ y bottom $(b)$. Por su parte hay seis leptones. Todos estos con su respectiva 
antipartícula asociada, una partícula con la misma masa pero carga opuesta. Todas estas partículas se agrupan en tres familias o generaciones, cada generación tiene una pareja de quarks (uno con carga $+2 / 3$ e y el otro con carga -1/3 e) y una pareja de leptones; uno con la carga del electrón y el otro con carga neutra. Precisamente, los leptones neutros del doblete de leptones son los neutrinos.

Los leptones se agrupan en dobletes de una partícula de carga negativa y un neutrino, a cada tipo de neutrino se le da el nombre del leptón cargado asociado con él; electrón $(e)$ y neutrino del electrón $\left(\nu_{e}\right)$, muón $(\mu)$ y neutrino del muón $\left(\nu_{\mu}\right)$; tauón $(\tau)$ y neutrino del tauón $\left(\nu_{\tau}\right)$. Al igual que los quarks, a cada tipo de neutrino de le da el nombre de sabor. Las partículas del ME y su organización en generaciones se pueden apreciar en la figura 1.2.

Además de la carga eléctrica, los quarks tienen una propiedad llamada carga de color, responsable de que respondan a la interacción nuclear fuerte. Existen tres diferentes cargas (anticargas) de color: rojo (antirojo), verde (antiverde) y azul (antiazul); los hadrones se forman con quarks de tres colores distintos y los mesones con una pareja de color-anticolor. En vista de esto se justifica la elección de la arbitraria palabra "color" para describir tal propiedad, a saber: los hadrones solo pueden ocurrir en combinaciones incoloras, de color-anticolor o de "todos" los colores (en analogía con la luz). Un antiquark tiene tanto carga eléctrica como carga de color opuesta.

La primera generación del ME, formada por $u, d$, e y $\nu_{e}$; con sus respectivas antipartículas, forman prácticamente toda la materia en la Tierra y a través de (casi) todo el universo inmediato. En particular, los protones están formados por el triplete uud y los neutrones por $u d d$, que, conjuntamente con los electrones, forman los átomos. Los neutrinos y antineutrinos del electrón son comúnmente emitidos en reacciones nucleares de isótopos radioactivos y solares, por ejemplo.

A pesar de su enorme éxito para describir los datos experimentales existentes con alta precisión, el ME generalmente no se considera como etapa definitiva en la unificación. Además de la inconsistencia con la teoría de la gravedad, nuevas observaciones y una variedad de resultados experimentales han mostrado que el cuadro que presenta el ME esta incompleto. En particular, hay varios parámetros que, a pesar de lo que se podría esperar, no están explicados por la teoría y que se tienen que determinar experimentalmente:

- Las constantes de acoplamiento $e, \alpha_{S}, \sin ^{2} \theta_{W}$.

- Las masas de los bosones $m_{W}, m_{H}$.

- Las masas de los leptones $m_{e}, m_{\mu}, m_{\tau}$.

- Las masas de los quarks $m_{u}, m_{d}, m_{s}, m_{c}, m_{b}, m_{t}$.

- Los parámetros de la matriz CKM: tres ángulos y una fase $\delta$.

Además, la jerarquía de masa permanece sin ser explicada, figura 1.3, las partículas izquierdas y derechas se tratan de forma muy distinta, la cuantización de la carga y la igualdad del valor absoluto de la carga del electrón y el protón a un nivel mejor que $10^{-21}$ no esta predicho. Como se mencionó, en el ME las masas de los neutrinos son nulas, por lo tanto cualquier evidencia de una masa no nula indicaría física "más allá del modelo estándar"6. Incluir neutrinos masivos añadiría aún más parámetros.

\footnotetext{
${ }^{6}$ Es cosa de gustos lo que significa exactamente "más allá del modelo estándar". Las masas de los neutrinos pueden ser generadas dentro de la estructura de norma de $\mathrm{SU}(3) \otimes \mathrm{SU}(2) \otimes \mathrm{U}(1)$ agrandando el contenido de partículas o añadiendo interacciones no renormalizables. A veces, aún al añadir nuevas partículas se le sigue llamando "modelo estándar", por que la estructura de norma permanece inalterada [7].
} 


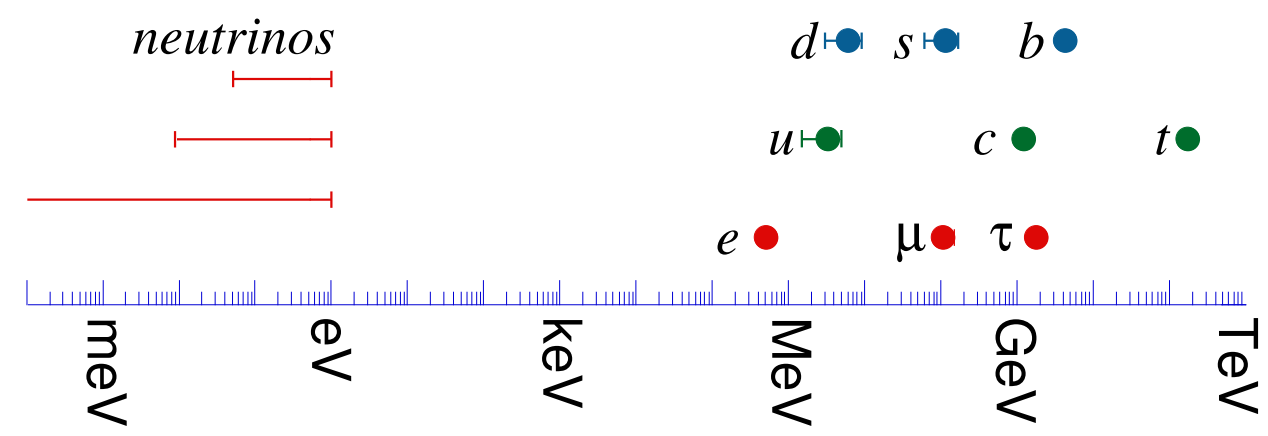

Figura 1.3: Escala de masas en el modelo estándar. La masa de las partículas de las distintas generaciones abarcan varios ordenes de magnitud, este comportamiento jerárquico sigue siendo una de las grandes interrogantes por resolver. A la izquierda se muestran las masas de los neutrinos, las partículas más ligeras, acotadas superiormente por $\sim 1 \mathrm{eV}$.

\subsubsection{Interacciones fundamentales}

El ME está construido en base a principios de simetría, es decir, la invariancia de las leyes de la física ante un conjunto determinado de transformaciones que pueden ser efectuadas sobre un sistema físico. La existencia de simetrías en un sistema, implica la conservación de una cantidad física que describe parte del sistema. Así por ejemplo, rotar el sistema de referencia en el que se describe un sistema no invalida los resultados de uno u otro sistema, se dice que hay simetría rotacional y esta implica la conservación del momento angular; la simetría traslacional, trasladar el sistema de referencia, implica la conservación del momento lineal; la simetría temporal, invariancia ante desplazamientos temporales, implica la conservación de energía del sistema.

Además de estas simetrías y sus respectivas propiedades conservadas, bien conocidas en la física clásica, el ME posee otras: simetrías locales de norma. Estas definen las cargas de las partículas y las propiedades de las fuerzas que actúan sobre ellas de manera análoga a aquella en la que la simetría cúbica de un objeto implica la existencia de cuatro vértices, seis caras y un grupo de rotaciones que intercambia las posiciones de las caras y vértices entre sí. De esta forma, las simetrías internas del ME implican que:

- Los quarks y leptones se pueden agrupar en multipletes de partículas.

- Las cargas de las partículas en cada multiplete están relacionadas de una manera definida.

- Existe un grupo de transformaciones internas que convierte a cada miembro de un multiplete en cualquier otro miembro del mismo multiplete.

Una importante implicación de estas teorías, llamadas teorías de norma, ${ }^{7}$ es que deben existir partículas mediadoras de las interacciones entre quarks y leptones. Estas partículas se conocen como bosones intermediarios o bosones de norma. Un quark o leptón emite un bosón intermediario y otro quark o leptón lo absorbe, y es mediante este intercambio de partículas que cada una siente la presencia o interacción de la otra. La interacción de un intermediario con un quark o un leptón perteneciente a un multiplete determinado, causa una de las transformaciones definidas por la simetría local de norma de la interacción asociada al intermediario, esto es, la emisión o absorción de un bosón de

\footnotetext{
${ }^{7}$ Ocasionalmente llamadas "Teorías de gauge".
} 
norma causa que un quark o leptón se transforme en otro miembro del multiplete al que pertenece e intercambie energía y momento en el proceso.

En el ME, cada fuerza (fuerte, débil y electromagnética), esta asociada con su propia simetría local de norma, la que asu vez determina un conjunto de cargas y un conjunto de bosones de norma que son los mediadores de la fuerza entre las partículas con cada tipo de carga.

\section{La interacción electromagnética}

Existe una sola simetría de norma asociada al electromagnetismo, y por lo tanto, un único tipo de carga eléctrica. Esta simetría de norma está relacionada con la invariancia de las propiedades observables de una partícula ante el cambio de fase de su función de onda (campo cuántico). Esta simetría es válida solo si la partícula tiene masa cero. Una consecuencia de que la masa del intermediario de la interacción electromagnética sea cero es que el alcance de la misma es infinito, puesta esta propiedad de la interacción es inversamente proporcional a la masa de su bosón intermediario. El bosón de norma de la interacción electromagnética es el fotón $(\gamma)$ y tanto los leptones cargados como los quarks experimentan las interacciones electromagnéticas.

\section{La interacción fuerte}

La simetría de norma de la interacción nuclear fuerte se conoce como simetría de color, y da origen a un tipo de carga llamada de color: rojo, verde o azul. Los quarks pueden tener cualquiera de los tres colores, y dos quarks con color interactúan entre sí mediante el intercambio de uno de los bosones de norma de la interacción fuerte, los ocho gluones $(g)$. De nuevo, la simetría asociada a la interacción de color implica que los gluones tienen masa cero y que la carga de color debe conservarse en una interacción de este tipo. Las propiedades de los quarks y las partículas compuestas que forman se derivan de las características de la simetría de color. Solo los quarks y los gluones experimentan las interacciones fuertes.

\section{La interacción débil}

Existen dos simetrías asociadas a la interacción débil,y por lo tanto dos tipos de carga débil: la carga isotópica débil y la hipercarga débil. Así mismo existen dos tipos de bosones intermediarios, el $W$ y el $Z$, que trasmiten la interacción débil entre partículas con carga débil. El neutrino, aunque es eléctricamente neutro, posee los dos tipos de carga débil, y por lo tanto puede interactuar con la materia mediante el intercambio ya sea de un $W$ o de un $Z$. El bosón $W$ existe en dos formas: $W^{+}$ y $W^{-}$, cada uno posee una unidad de carga eléctrica (+1e o $-1 e$ respectivamente), y una unidad de carga isotópica débil. Cuando una partícula con carga isotópica débil absorbe o emite un $W$, gana o pierde una unidad de carga eléctrica y una unidad de carga isotópica débil, cambiando su identidad.

Los proceso de decaimiento beta del neutrón, decaimiento del muón, el decaimiento beta inverso y la captura electrónica se pueden explicar mediante la emisión de bosones $W^{ \pm}$por parte de quarks o leptones que luego de la emisión se transforman en otras partículas (en la otra pareja del doblete).

En la actualidad las descripciones de las fuerzas electromagnética y débil se han fusionado en la llamada teoría electrodébil, desarrollada en los años sesenta por Weinberg, Salam y Glashow. En esta teoría ambas interacciones se presentan como dos aspectos diferentes de un mismo fenómeno. 


\subsubsection{Propiedades de los neutrinos}

Los neutrinos son partículas elementales eléctricamente neutras que interactúan débilmente y con espín semi-entero, es decir son fermiones. Son creados como resultado de ciertos tipos de decaimiento radioactivo o reacciones nucleares como aquellas que ocurren en el Sol, en reactores nucleares o cuando rayos cósmicos colisionan con los átomos. Casi todas las medidas que hay sobre neutrinos se refieren a superposiciones de eigenestados de masa, que a su vez son combinaciones de eigenestados de sabor, al respecto se habla con gran detalle en $\S 1.4$. Estas mezclas son análogas a las ya conocidas en el sector de quarks a través de la matriz CKM, sin embargo los resultados actuales de búsquedas de oscilaciones indican que no existen "eigenestados dominantes" como se presenta en el sector de quarks. Por lo tanto, no se puede asociar ningún eigenestado particular de masa con ninguna etiqueta leptónica. No obstante, los neutrinos se producen en decaimientos débiles con un sabor leptónico especifico y se detectan con un sabor leptónico especifico.

\section{Momento magnético}

El descubrimiento de las oscilaciones de neutrinos implica la existencia de, por lo menos, un eigenestado de masa distinto de cero. Debido a las fluctuaciones cuánticas, la existencia de una masa en los neutrinos sugiere fuertemente la presencia de un pequeño momento magnético, en la teoría electrodébil estándar extendida para incluir masas en los neutrinos

$$
\mu<3 e G_{F} m_{\nu} /\left(8 \pi^{2} \sqrt{2}\right)=3.2 \times 10^{-19} \mu_{B}
$$

es decir, es inobservablemente pequeña dadas las pequeñas masas conocidas [40]. Intuitivamente, en una escala de tiempo y distancia suficientemente chica en donde la incertidumbre de la energía sea muy grande, el neutrino puede ser una mezcla de $W^{+}$y $e^{-}$, y el campo magnético se acopla a ellos, después se combinan de vuelta en un neutrino. Se han obtenido varios límites a la fecha, proveniente de observaciones solares y a $90 \%$ N.C. se tiene

$$
\mu<0.32 \times 10^{-10} \mu_{B}
$$

\section{Sección eficaz de interacción}

Una forma sencilla de calcular la sección eficaz de interacción de los neutrinos se basa en la Regla de Oro de Fermi, si se puede encontrar un elemento de matriz para la interacción débil en una de estas reacciones debería ser comparable en otra. Aplicando la teoría de Fermi del decaimiento beta en ciertos casos especiales permite estimar la fuerza del acoplamiento débil. Se estima que para el rango de energía del decaimiento beta la sección eficaz es $\sim 10^{-43} \mathrm{~cm}^{2}=10^{-47} \mathrm{~m}^{2}$. ¡Aproximadamente veinte ordenes de magnitud menos que la sección eficaz de dispersión de dos nucleones con baja energía! Con este valor nominal se puede estimar la tasa de interacciones. Multiplicando la sección eficaz por la densidad de nucleones da un número de interacciones por metro y el inverso de eso es un estimado del camino libre medio. Para el agua y el plomo, con densidades de $1000 \mathrm{~kg} / \mathrm{m}^{3} \mathrm{y}$ $11400 \mathrm{~kg} / \mathrm{m}^{3}$, respectivamente; el camino libre medio de un neutrino es alrededor de:

$$
d_{\text {agua }} \approx \frac{u}{\sigma \rho}=\frac{1.66 \times 10^{-27} \mathrm{~kg}}{10^{-47} \mathrm{~m}^{2} \cdot 1000 \mathrm{~kg} / \mathrm{m}^{3}} \approx 1.7 \times 10^{17} \mathrm{~m}
$$




$$
d_{\text {plomo }} \approx \frac{u}{\sigma \rho}=\frac{1.66 \times 10^{-27} \mathrm{~kg}}{10^{-47} \mathrm{~m}^{2} \cdot 11400 \mathrm{~kg} / \mathrm{m}^{3}} \approx 1.5 \times 10^{16} \mathrm{~m} .
$$

Para tener una perspectiva de estas distancias sirve compararlo con un año luz, 1 año luz $=9.46 \times$ $10^{15}$. Así pues, jel camino libre medio de un neutrino en plomo es más de un año luz! Más aún, un neutrino con energía moderada podría atravesar más de mil años luz de plomo. Como comparación, es útil tener en cuenta que el camino libre medio en plomo de los rayos x usados para aplicaciones médicas es menor a $0.01 \mathrm{~mm}$. Esta sección eficaz también puede ser usada para evaluar el número de eventos que se esperan en un detector de cierto tamaño.

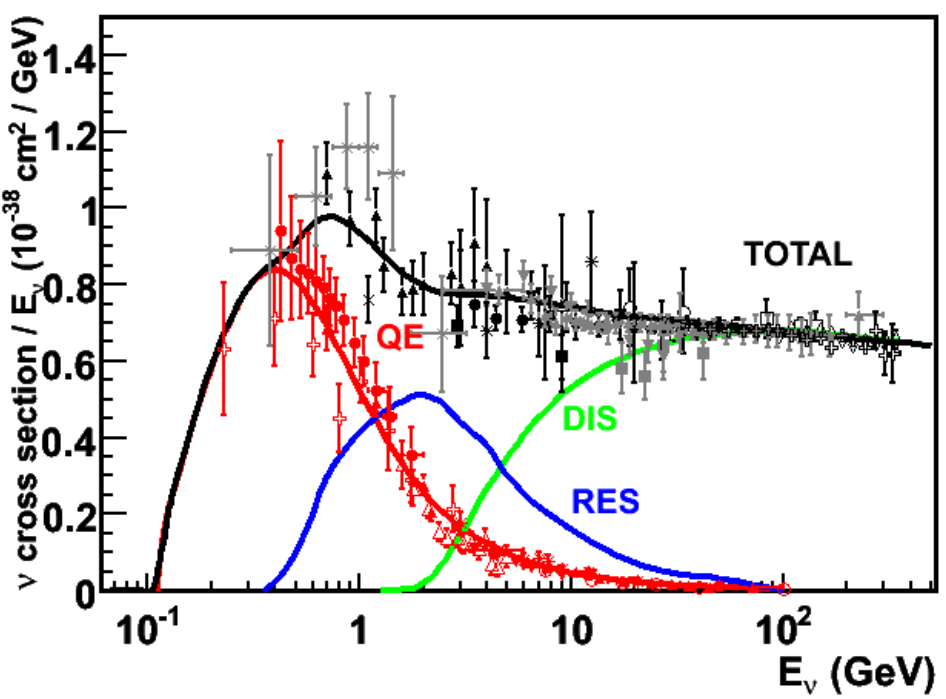

Figura 1.4: Secciones eficaces totales de CC de neutrinos y antineutrinos por nucleón dividida por la energía del neutrino y graficada como función de la energía. Estas contribuciones incluyen dispersión cuasielástica (QE), producción de resonancia (RES) y dispersión inelástica profunda (DIS). Los datos y la predicción de la dispersión cuasielástica han sido promediados sobre blancos de protones y neutrones, por lo tanto han sido divididos por un factor de dos. Tomada de [41].

Las interacciones de los neutrinos son descritas con gran precisión por el ME, las interacciones más sencillas son las que tienen con los electrones. De este tipo hay dispersión elástica $\nu_{\alpha}+e \rightarrow \nu_{\alpha}+e$, cuasielástica $\nu_{\mu}+e \rightarrow \nu_{e}+\mu$ y la producción de pares neutrino-antineutrino $e^{+}+e^{-} \rightarrow \nu+\bar{\nu}$. Por otra parte, los neutrinos también interactúan con los nucleones de varias formas, por ejemplo la dispersión cuasielástica de corriente cargada, en que a través del intercambio de un bosón $W$ un neutrino interactúa con un protón o un neutrón y surge un neutrón o un protón, respectivamente, y la pareja leptónica correspondiente. También para los nucleones se tiene dispersión elástica $\nu_{\alpha}+N \rightarrow+N$ y además se tiene la dispersión inelástica profunda (cargada o neutra) en que a través del intercambio de un bosón ( $W$ para las de corriente cargada y $Z$ para la de corriente neutra) con el nucleón se forman varios hadrones.

\section{Quiralidad}

Resultados experimentales muestran que casi todos los neutrinos producidos y observados tienen quiralidad izquierda (espín antiparalelo a su momento) y que los antineutrinos tienen quiralidad derecha, dentro del margen de error. En el ME la masa de los neutrinos es nula y solo la componente 
de quiralidad izquierda de la función de onda de la partícula $\psi_{\mathrm{L}}$, participa de las interacciones; es decir, la componente derecha $\psi_{\mathrm{R}}$ no está involucrada. Las componentes de quiralidad se definen como

$$
\psi_{\mathrm{L}, \mathrm{R}} \equiv \mathrm{P}_{\mathrm{L}, \mathrm{R}} \psi,
$$

donde los proyectores izquierdo y derechos se definen como $\mathrm{P}_{\mathrm{L}, \mathrm{R}} \equiv \frac{1}{2}\left(1 \mp \gamma^{5}\right)$, de la matriz $\gamma^{5}$ se habla más adelante.

En este contexto, si se quieren tener neutrinos masivos, estos deben satisfacer la ecuación de Dirac para fermiones en el vacío. En 1928 Paul Dirac formuló una ecuación de onda relativista que describe los campos correspondientes a partículas elementales de espín $1 / 2$ (como el electrón y los neutrinos) y sus antipartículas. Esta es una ecuación vectorial con cuatro números complejos, en contraste con la ecuación de Schrödinger que describe un campo con solo un valor complejo o escalar. La ecuación de Dirac es consistente tanto con la mecánica cuántica como con la relatividad especial. Implicó la existencia de un nuevo tipo de materia, la antimateria, que fue observada hasta después y lograba explicar la necesidad del espín como consecuencia de la unión de la mecánica cuántica y la relatividad. En su forma covariante es

$$
i \hbar \gamma^{\mu} \partial_{\mu} \psi-m c \psi=0, \quad(\mu=0,1,2,3) .
$$

En la representación de Dirac, las matrices gamma se escriben en términos de sub-matrices $2 \times 2$ de Pauli y la matriz de identidad de $2 \times 2 I_{2}$. La masa de la partícula $m, c$ es la velocidad de la luz. La notación estándar explicita es

$$
\gamma^{0}=\left(\begin{array}{cc}
I_{2} & 0 \\
0 & -I_{2}
\end{array}\right), \quad \gamma^{1}=\left(\begin{array}{cc}
0 & \sigma_{1} \\
-\sigma_{1} & 0
\end{array}\right), \quad \gamma^{2}=\left(\begin{array}{cc}
0 & \sigma_{2} \\
-\sigma_{2} & 0
\end{array}\right), \quad \gamma^{3}=\left(\begin{array}{cc}
0 & \sigma_{3} \\
-\sigma_{3} & 0
\end{array}\right) ;
$$

donde las matrices de Pauli son, usando la definición generalizada con la delta de Kronecker ${ }^{8}$

$$
\sigma_{j}=\left(\begin{array}{cc}
\delta_{j 3} & \delta_{j 1}-i \delta_{j 2} \\
\delta_{j 1}+i \delta_{j 2} & -\delta_{j 3}
\end{array}\right)
$$

Además es útil definir la matriz $\gamma^{5}=i \gamma^{0} \gamma^{1} \gamma^{2} \gamma^{3}$, que, como se vio antes, sirve para definir a los proyectores de quiralidad. Por comodidad se presenta la ecuación de Dirac, (1.1), usando unidades naturales $(\hbar=c=1)$ y el operador $\not \partial=\gamma^{\mu} \partial_{\mu}=\gamma^{0} \partial_{0}+\gamma^{1} \partial_{1}+\gamma^{2} \partial_{2}+\gamma^{3} \partial_{3}$ de la notación de Feynman:

$$
(i \not \supset-m) \psi=0 .
$$

Esta ecuación se obtiene al extremar la acción de la lagrangiana

$$
\mathcal{L}_{\mathcal{D}}=\bar{\psi}(i \not \partial-m) \psi,
$$

en donde el segundo término de la derecha es el término de masa $\mathcal{L}_{\mathcal{D}}^{\text {masa }}=m \bar{\psi} \psi$ y requiere que el campo tenga una componente izquierda y derecha $\psi=\psi_{\mathrm{L}}+\psi_{\mathrm{R}}$, porque debido a las propiedades de los proyectores de quiralidad

$$
\mathcal{L}_{\mathcal{D}}^{\text {masa }}=m\left(\bar{\psi}_{\mathrm{R}} \psi_{\mathrm{L}}+\bar{\psi}_{\mathrm{L}} \psi_{\mathrm{R}}\right)
$$

\footnotetext{
${ }^{8}$ La delta de Kronecker, $\delta_{i j}=1$ si $i=j$, y $\delta_{i j}=0$ en caso contrario.
} 
Una manera de extender el ME para incluir masas de neutrinos, es incluir campos derechos $\psi_{\mathrm{R}}$, pero al imponer las simetrías de la teoría electrodébil, exigir que los campos derechos no participen en las interacciones, es decir que sean estériles (ver §1.4.7). La razón por la cual se exige que los neutrinos sean izquierdos es por las observaciones experimentales como las de madame Wu y Goldhaber, de las que se habló en $\S 1.1$.

Sin embargo, existe otra manera para incluir la masa de los neutrinos en el ME. El físico italiano Ettore Majorana, propuso un termino de masa de la forma

$$
\mathcal{L}_{\mathcal{M}}^{\text {masa }}=m \overline{\left(\psi_{\mathrm{L}}\right)^{c}} \psi_{\mathrm{L}}
$$

que incluye el conjugado de carga $\psi^{c}$ de un espinor $\psi$, definido en la base de Majorana como $\psi^{c} \equiv i \psi^{*}$. Majorana se dio cuenta de que $\left(\psi_{\mathrm{L}}\right)^{c} \equiv i\left(\psi_{\mathrm{L}}\right)^{*}$ tiene quiralidad derecha, con lo cual el espinor queda como $\psi=\psi_{\mathrm{L}}+\left(\psi_{\mathrm{L}}\right)^{c}$ el cual satisface la llamada condición de Majorana $\psi^{c}=\psi$. En el caso de Majorana, el campo $\psi$ no puede ser acoplado a un campo electromagnético sin violar la conservación de carga, así que $\psi$ deber tener carga neutra.

Para Dirac las proyecciones quirales izquierda y derecha son independientes, mientras que para Majorana están relacionadas. Por lo mismo, en el esquema de Majorana los neutrinos tienen la mitad de grados libertad que en el esquema de Dirac. A las partículas que siguen un comportamiento descrito por la ecuación de Majorana se les llama partículas de Majorana, es decir, que son idénticas a sus antipartículas. Hasta ahora, ninguno de los fermiones del ME cumple tal propiedad. Sin embargo existe la posibilidad de que los neutrinos sean partículas de Majorana. Es importante aclarar que ninguno de los dos esquemas puede distinguirse con experimentos de oscilación de neutrinos, pero si en efecto los neutrinos fueran su propia antipartícula el decaimiento beta doble sin neutrinos podría ocurrir, §1.4.6, así como una variedad de procesos que violan número leptónico.

\subsection{Fuentes de neutrinos}

Los neutrinos son creados en ciertas reacciones nucleares, como en el Sol, el interior de la Tierra o en reactores nucleares; como subproducto de la interacción de rayos cósmicos con la atmósfera; en eventos cósmicos masivos, como el Big Bang o colapsos de super novas; o artificialmente con aceleradores de partículas.

\subsubsection{Reactores nucleares}

Los reactores nucleares son la fuente principal de neutrinos generados por los seres humanos. Se generan $\bar{\nu}_{e}$ en el decaimiento beta de los núcleos que se fragmentan en la fisión. Generalmente, los cuatro principales isótopos que contribuyen al flujo de antineutrinos son ${ }^{235} \mathrm{U},{ }^{238} \mathrm{U},{ }^{239} \mathrm{Pu}$ y ${ }^{241} \mathrm{Pu}$; a través de los antineutrinos emitidos en el decaimiento beta negativo de los respectivos fragmentos de fisión. En promedio, se producen unos $6 \bar{\nu}_{e}$ por fisión. La densidad de flujo esta dada por

$$
\Phi_{\nu}=1.5 \times 10^{12} \frac{P / \mathrm{MW}}{L^{2} / \mathrm{m}^{2}} \mathrm{~cm}^{-2} \mathrm{~s}^{-1}
$$

donde $P$ la potencia termal del reactor en MW y L es la distancia al reactor en m. Así pues, el flujo isotrópico total de los $\bar{\nu}_{e}$ emitidos es 


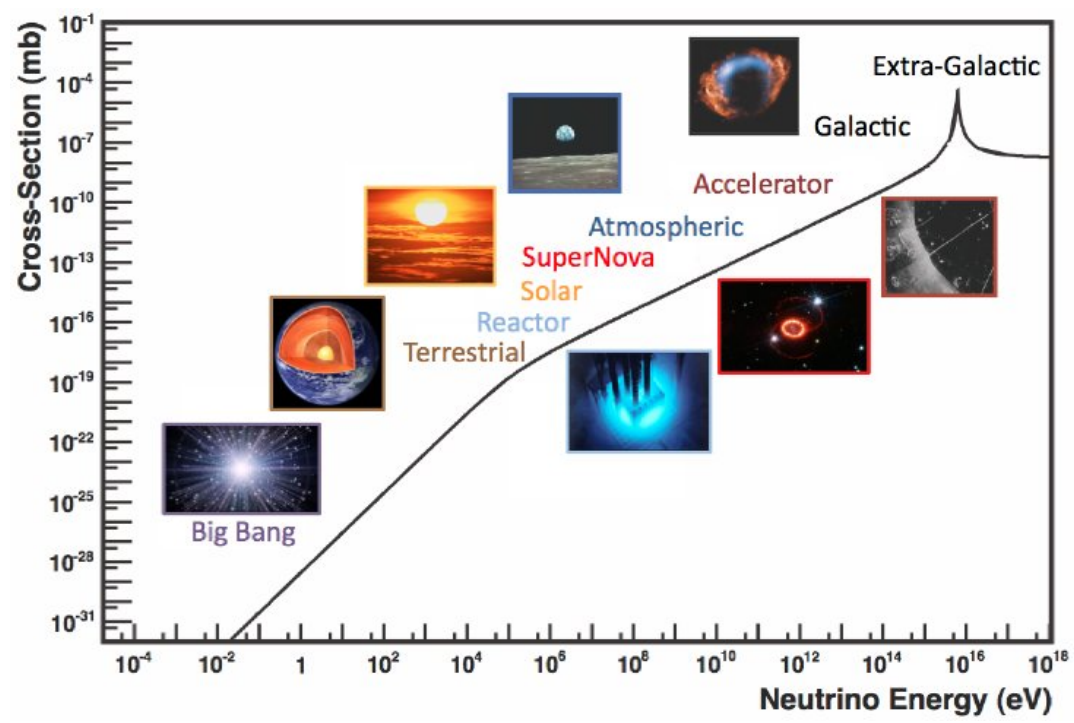

Figura 1.5: Ejemplo representativo de varias fuentes de neutrinos a lo largo de varios ordenes de magnitud de energía. Para comparar, se muestra la sección eficaz electrodébil para dispersión de electrones libres $\bar{\nu}_{e} e^{-} \rightarrow$ $\bar{\nu}_{e} e^{-}$, como función de $E_{\nu}$ (para neutrinos masivos). El pico en $10^{16} \mathrm{eV}$ se debe a la resonancia $W^{-}$. Tomado de [41].

$$
F \Phi_{\nu}=1.9 \times 10^{17} \frac{P}{\mathrm{MW}} s^{-1}
$$

Se describe brevemente el estado experimental actual de los experimentos de neutrinos de reactores en $\S 1.4 .7$.

Las bombas nucleares producen una gran cantidad de neutrinos. Inclusive, Reines y Cowan antes de buscar neutrinos de reactores consideraron la detección de neutrinos a partir de una explosión nuclear, proceso abandonado, entre otras cosas, por la dificultad de repetir el experimento.

\subsubsection{Aceleradores de partículas}

Se han usado aceleradores de partículas para generar haces de neutrinos, la técnica consiste en estrellar protones en un blanco fijo con lo cual se producen partículas inestables que decaen, entre otras cosas, a neutrinos. Con el decaimiento de muones positivos en reposo se obtiene un haz de antineutrinos del muón con energías del orden de decenas de MeV. Mucho más comunes son los haces que se obtienen del decaimiento de piones en vuelo, los piones generados con los protones del acelerador son enfocados con un campo magnético, los piones positivos (negativos) generan un haz de neutrinos (antineutrinos) del muón. Otro tipo de haz se obtiene al impactar protones con muy alta energía en blancos muy gruesos, efectivamente deteniendo a los protones, de esa forma se producen hadrones encantados pesados que decaen inmediatamente en neutrinos del electrón y muón casi en la misma proporción. Los neutrinos que se obtienen de los aceleradores son $\nu_{e}, \bar{\nu}_{e}, \nu_{\mu}$ y $\bar{\nu}_{\mu}$. Hay esfuerzos por construir complejos en donde los neutrinos se produzcan por medio del decaimiento de muones en anillos de almacenamiento de muones y " $\beta$-beams", llamados "fabricas de neutrinos". La ventaja de estas fabricas es que la composición del haz se conocería con mucha precisión y la intensidad del haz 
sería mucho mayor a lo que actualmente se tiene. En $\S 1.4 .7$ se encuentra un breve resumen del estado actual de los experimentos de aceleradores.

\subsubsection{Manto y núcleo terrestre}

Los llamados geoneutrinos provienen del decaimiento beta de isótopos radioactivos que se encuentran en el interior de la Tierra. Colectivamente, los geoneutrinos llevan información integrada sobre la abundancia de sus fuentes radioactivas dentro de la Tierra. Uno de los principales objetivos del campo emergente de geofísica de neutrinos involucra extraer información geológica valiosa de las medidas de geoneutrinos.

La mayoría de los geoneutrinos se originan en las cadenas de decaimiento $\beta^{-}$de ${ }^{40} \mathrm{~K},{ }^{232} \mathrm{Th}$ y ${ }^{238} \mathrm{U}$, de estas solo los geoneutrinos originados en las dos últimas cadenas tienen energía suficiente $(>1.8 \mathrm{MeV})$ para ser detectados por decaimiento beta inverso. Juntas, estas cadenas de decaimiento son responsables de más de $99 \%$ del calor radioactivo generado dentro de la Tierra. Los experimentos Borexino [42, 43] en Gran Sasso, Italia; y KamLAND [44, 45] en Kamioka, Japón; han observado geoneutrinos y puesto constricciones en la cantidad de calor de origen radioactivo. Estos hallazgos marcan el inicio de la geofísica de neutrinos. Se espera que el experimento $\mathrm{SNO}+$ empiece a recolectar datos en 2013 y hay una variedad de otros experimentos en planificación.

Como dato curioso, el potasio es un metal alcalino, los iones disueltos de potasio tienen un rol importante en diferentes procesos bioquímicos. En la naturaleza se encuentra formado de tres tipos distintos de isótopos de los cuales $0.0117 \%$ es potasio $40,{ }^{40} \mathrm{~K}$, el cual es inestable con una vida media de $1.25 \times 10^{9}$ años. En promedio un plátano tiene $\sim 0.5 \mathrm{~g}$ de potasio, $7.7 \times 10^{21}$ átomos de potasio, de los cuales $9.0 \times 10^{17}$ son ${ }^{40} \mathrm{~K}$. Así pues en un plátano habrá del orden de 23 decaimientos por segundo. De esos decaimientos $89.28 \%$ son a través de $\beta^{-}$y un antineutrino a ${ }^{40} \mathrm{Ca}, 10.72 \%$ de las veces habrá captura de electrón resultando ${ }^{40} \mathrm{Ar}$, un rayo gamma y un neutrino; y 0.001 de las veces decaerá a ${ }^{40} \mathrm{Ar}$ emitiendo un $\beta^{+}$y un neutrino. Un plátano contiene un positrón, por un periodo breve de tiempo, cada 73 minutos. Notemos que de esos 23 decaimientos por segundo 21 ocasiones obtendremos antineutrinos y 2 serán neutrinos. Los plátanos son una comida radioactiva: comer 600 plátanos es aproximadamente equivalente a una placa de rayos $\mathrm{X}$ en el pecho, siempre y cuando puedan ser ingeridos todos antes de que el excedente de potasio sea desechado por los riñones.

\subsubsection{Atmósfera}

Los neutrinos atmosféricos son creados por las interacciones de rayos cósmicos primarios, protones y núcleos pesados, con los núcleos en la atmósfera. La interacción de estos rayos primarios genera rayos cósmicos secundarios, que incluye todos los hadrones y sus decaimientos, con un espectro de energía que tiene un pico en el rango de $\mathrm{GeV}$ y se extiende a energías más altas aproximadamente como una ley de potencias. En particular, se producen muchos piones, los cuales decaen a neutrinos del muón y muones: $\pi^{+} \rightarrow \mu^{+}+\nu_{\mu}, \pi^{-} \rightarrow \mu^{-}+\bar{\nu}_{\mu}$. A altas energías, también los kaones contribuyen a la producciones de neutrinos y muones. Los muones que decaen antes de llegar a la superficie generan electrones y neutrinos del electrón: $\mu^{+} \rightarrow e^{+}+\nu_{e}+\bar{\nu}_{\mu}, \mu^{-} \rightarrow e^{-}+\bar{\nu}_{e}+\nu_{\mu}$. A bajas energías $(E \lesssim 1 \mathrm{GeV})$ la mayoría de los muones decaen antes de llegar a la superficie, se cumplen las siguientes 
relaciones de los distintos flujos

$$
\frac{\Phi_{\nu_{\mu}}+\Phi_{\bar{\nu}_{\mu}}}{\Phi_{\nu_{e}}+\Phi_{\bar{\nu}_{e}}} \simeq 2, \quad \frac{\Phi_{\nu_{\mu}}}{\Phi_{\bar{\nu}_{\mu}}} \simeq 1, \quad \frac{\Phi_{\nu_{e}}}{\Phi_{\bar{\nu}_{e}}} \simeq \frac{\Phi_{\mu^{+}}}{\Phi_{\mu^{-}}} .
$$

Para energías mayores a $1 \mathrm{GeV}$ aumenta la cantidad de muones que llegan a la superficie antes de decaer, lo que lleva a un aumento en la fracción del flujo de neutrino del muón sobre neutrinos del electrón. Se pueden detectar a los neutrinos con energías entre $100 \mathrm{MeV}$ y $100 \mathrm{GeV}$, en laboratorios subterráneos dispersándose en núcleos. Es un requisito que los detectores sean subterráneos ya que si no las frecuentes interacciones de los muones secundarios abrumarían al detector. El estado actual de los experimentos con neutrinos atmosféricos se resume en $\S 1.4 .7$.

\subsubsection{Sol}

De las reacciones nucleares de fusión que alimentan al Sol y otras estrellas se liberan neutrinos. Los detalles de operación del Sol se explican en el modelo estándar solar, pero puesto de forma sencilla: cuando cuatro protones se fusionan para convertirse en un núcleo de helio, dos de ellos tienen que convertirse en neutrones y cada una de esas conversiones libera un $\nu_{e}$. La energía liberada por cada fusión de ${ }^{4} \mathrm{He}$ es $Q=2 m_{e}+4 m_{\mathrm{p}}-m_{\mathrm{He}}=26.73 \mathrm{MeV}$, usando la constante solar en la Tierra $S=8.5 \times 10^{11} \mathrm{~cm}^{-2} \mathrm{~S}^{-1}$, se puede obtener una aproximación para el flujo de neutrinos en la Tierra:

$$
\Phi_{\nu} \approx \frac{S}{13 \mathrm{MeV} \text { por cada } \nu_{e}}=6.5 \times 10^{10} \mathrm{~cm}^{-2} \mathrm{~s}^{-1}
$$

Los neutrinos solares son importantes para la física de partículas: con una distancia promedio SolTierra de $1.496 \times 10^{8} \mathrm{~km}$ y neutrinos con energías de $1 \mathrm{MeV}$ se pueden probar parámetros de oscilaciones del orden de $\Delta m^{2} \sim 10^{-10} \mathrm{eV}^{2}$ lo cual no se puede probar con medios terrestres. Pero también lo son para la astrofísica: en promedio un fotón creado en el núcleo del Sol tarda varias decenas de miles de años en salir al exterior, sin embargo los neutrinos al interactuar tan débilmente logran llegar a la Tierra en un tiempo aproximado de 8 minutos. Lo que los hace magnificas herramientas para observar el interior del Sol en tiempo real ${ }^{9}$. Los principales resultados obtenidos hasta ahora de los experimentos de neutrinos solares se sintetiza en $\S 1.4 .7$.

\subsubsection{Supernovas y cataclismos estelares}

Los neutrinos se llevan la mayor parte de la energía gravitacional liberada por el colapso de estrellas masivas, eventos categorizados como supernovas tipo Ib, Ic y II. Cuando tales estrellas colapsan, la densidad de materia en el núcleo se vuelve tan grande que los protones y electrones se combinan para formar neutrones y neutrinos del electrón. La segunda fuente más importante es la energía termal del recién formado núcleo de neutrones, que se disipa a través de la formación de pares neutrinoantineutrino de todos los sabores.

Los detectores Kamiokande II e IMB detectaron 11 y 8 antineutrinos de origen termal, respectivamente; mientras que el detector Baksan encontró 5 neutrinos de origen termal o de captura del electrón, provenientes de la supernova 1987A. Todos estos, en un estallido que duró menos de 13 segundos.

${ }^{9} \mathrm{El}$ único otro proceso que da información sobre el interior son las oscilaciones del Sol, la llamada heliosismología. 
Como se esperaba, los neutrinos provenientes de la supernova llegaron a la Tierra varias horas antes que la radiación electromagnética. Los neutrinos logran atravesar la materia de la explosión rápidamente, debido a su excepcionalmente escasa interacción con la materia, mientras que los fotones son retrasados. Por la misma razón, se cree que los neutrinos llevan información de la región central de la explosión, la radiación electromagnética se dispersa o proviene de decaimientos de elementos radioactivos producidos en la onda de choque. El sistema SNEWS (Supernova Early Warning System), una red de detectores de neutrinos, vela el cielo para reportar candidatos a eventos de supernova.

La energía de los neutrinos de supernovas va de unos cuantos MeV a decenas de MeV. Sin embargo, se cree que los sitios en los que se aceleran los rayos cósmicos producen neutrinos que son, por lo menos, un millón de veces más energéticos. Estos lugares son los remanentes de supernova, ambientes con turbulentos gases. También surgen neutrinos de los brotes de rayos gamma (GRB por gammaray burst), resplandores de rayos gamma asociados con explosiones extremadamente energéticas. Se cree que la mayoría de los GRB consisten de un haz estrecho de intensa radiación liberada durante una supernova o hypernova cuando una estrella muy masiva que rota rápidamente colapsa. Los núcleos activos de galaxia (AGN por active galactic nucleus), regiones compactas en el centro de una galaxia que tienen una luminosidad mucho más alta de lo normal, también liberan neutrinos muy energéticos. Los principales experimentos que están en condiciones de observar neutrinos tan energéticos son: Baikal, AMANDA, IceCube, ANTARES, NEMO y Nestor. De hecho en abril de 2013, el experimento IceCube [46] reportó la observación de dos neutrinos con energías del orden de $10^{15} \mathrm{eV}=\mathrm{PeV}$. Neutrinos aún más energéticos, resultantes en las interacciones de rayos cósmicos extragalácticos, esperan ser observados en el observatorio Pierre Auger, en Argentina o el experimento ANITA, en la Antártica.

\subsubsection{Big Bang}

Junto con la radiación cósmica de microondas (CMB por Cosmic Microwave Background) producida por Big Bang, existe un fondo de neutrinos de muy baja energía en el universo. A estos se les llama neutrinos reliquia y se predice que tienen una temperatura ${ }^{10}$ de $1.95 \mathrm{~K}$. Varias propiedades de los neutrinos pueden ser deducidas desde la cosmología ya que afectan, por ejemplo, el fondo de radiación cósmica y la estructura a gran escala del universo y los procesos de nucleosíntesis que siguieron del Big Bang. Distintas medidas astrofísicas han determinado que el número de neutrinos es compatible con 3 y aunque aún no se descarta la posibilidad de más neutrinos ${ }^{11}$ A pesar de que hay muchísimos neutrinos del Big Bang (411 $\mathrm{cm}^{-3}$ para cada eigenestado de masa), debido a la ínfima sección eficaz no han podido ser observados directamente sin embargo su existencia ha sido corroborada a través de observaciones cosmológicas recientes [47, 48, 49].

\subsection{Oscilación de neutrinos}

En el caso de una masa no nula para el neutrino, los eigenestados débiles y de masa no son necesariamente idénticos, un hecho bien conocido en el sector de quarks donde los dos tipos de estados están conectados por la matriz CKM, introducida por Nicola Cabibbo, Makoto Kobayashi y Toshihide Maskawa [50, 51]. Esto permite el fenómeno de oscilación de neutrinos, un tipo de oscilación de sabor

\footnotetext{
${ }^{10}$ Considerablemente más fríos si su masa excede $0.001 \mathrm{eV}$.

${ }^{11}$ Depende mucho que datos se tomen en cuenta, algunos favorecen más neutrinos que otros.
} 
que ya es conocido en otros sistemas de partículas. Las oscilaciones son observables siempre y cuando los paquetes de ondas de neutrinos formen una superposición coherente de estados. Tales oscilaciones entre los diferentes sabores de neutrinos no conservan números leptónicos de sabor individuales, solo número leptónico total. Puede ser descrito por teoría cuántica de campos, sin embargo aquí se desarrolla con argumentos simplificados y aproximaciones razonables que permiten simplificar la teoría; se puede consultar una derivación sofisticada con teoría cuántica de campos en [52].

\subsubsection{Oscilación de $2 \nu$ en el vacío}

Considerando solamente dos sabores de neutrinos. Los neutrinos vienen dados por las combinaciones de eigenestados de masa $\left|\nu_{1}\right\rangle$ y $\left|\nu_{2}\right\rangle$, con masas $m_{1}$ y $m_{2}$.

Por ejemplo el estado de un neutrino del muón creado en $t=0$ está expresado por una mezcla de los eigenestados de masa.

$$
|\nu(0)\rangle=\left|\nu_{\mu}\right\rangle=-\sin \theta\left|\nu_{1}\right\rangle+\cos \theta\left|\nu_{2}\right\rangle,
$$

de forma semejante el neutrino del electrón:

$$
\left|\nu_{e}\right\rangle=\cos \theta\left|\nu_{1}\right\rangle+\sin \theta\left|\nu_{2}\right\rangle
$$

El ángulo $\theta$ caracteriza el grado de mezcla de los eigenestados de masa, es simplemente llamado ángulo de mezcla. Un tiempo $t$ después la función de es:

$$
\left|\nu_{\mu}(t)\right\rangle=-\sin \theta \mathrm{e}^{-i E_{1} t}\left|\nu_{1}\right\rangle+\cos \theta \mathrm{e}^{-i E_{2} t}\left|\nu_{2}\right\rangle
$$

donde los eigenestados se propagan como partículas libres y $E_{1}$ y $E_{2}$ son las energías de los estados $\left|\nu_{1}\right\rangle$ y $\left|\nu_{2}\right\rangle$ respectivamente. En unidades de $\hbar=c=1$. Para neutrinos relativistas $\left(E_{\nu} \gg m\right)$ podemos aproximar $E_{1}$ y $E_{2}$ por:

$$
E_{j}=\left(p^{2}+m_{j}^{2}\right)^{1 / 2} \simeq p+\frac{m_{j}^{2}}{2 p},
$$

se asume que los dos estados de masa tienen el mismo momento y además como la masa de los neutrinos es muy chica: $E_{\nu} \simeq p$. Sustituyendo estas energías, la función de onda al tiempo $t$ se convierte en:

$$
\begin{aligned}
\left|\nu_{\mu}(t)\right\rangle & =-\sin \theta \mathrm{e}^{-i t\left(p+m_{1}^{2} / 2 E_{\nu}\right)}\left|\nu_{1}\right\rangle+\cos \theta \mathrm{e}^{-i t\left(p+m_{2}^{2} / 2 E_{\nu}\right)}\left|\nu_{2}\right\rangle \\
& =\mathrm{e}^{-i t\left(p+m_{1}^{2} / 2 E_{\nu}\right)}\left[-\sin \theta\left|\nu_{1}\right\rangle+\cos \theta \mathrm{e}^{-i t\left(p+m_{2}^{2} / 2 E_{\nu}-p-m_{1}^{2} / 2 E_{\nu}\right)}\left|\nu_{2}\right\rangle\right] \\
& =\mathrm{e}^{-i t\left(p+m_{1}^{2} / 2 E_{\nu}\right)}\left[-\sin \theta\left|\nu_{1}\right\rangle+\cos \theta\left|\nu_{2}\right\rangle \mathrm{e}^{i \Delta m^{2} t / 2 E_{\nu}}\right]
\end{aligned}
$$

donde $\Delta m^{2}=m_{1}^{2}-m_{2}^{2}$. Como van casi a la velocidad de la luz podemos hacer $t \approx x / c=L$; siendo $L$ la distancia a la fuente de neutrinos de muones.

Calculemos la probabilidad $P\left(\nu_{\mu} \rightarrow \nu_{e}\right)$, de encontrar un $\nu_{e}$ en $L$, dado que $\nu_{\mu}$ partió del origen $L=0$. La probabilidad es el cuadrado de la amplitud $\left\langle\nu_{e} \mid \nu_{\mu}\right\rangle$. Usando la relación de ortonormalidad $\left\langle\nu_{i} \mid \nu_{j}\right\rangle=\delta_{i j}$ podemos calcular la probabilidad:

$$
P\left(\nu_{\mu} \rightarrow \nu_{e}\right)=\left\langle\nu_{e} \mid \nu_{\mu}\right\rangle^{2}=\left|\cos \theta \sin \theta\left(1-\mathrm{e}^{i \Delta m^{2} t / 2 E_{\nu}}\right)\right|^{2}
$$


Usando que $\cos \theta \sin \theta=\frac{1}{2} \sin 2 \theta$ se obtiene

$$
P\left(\nu_{\mu} \rightarrow \nu_{e}\right)=\sin ^{2} 2 \theta \sin ^{2}\left(\frac{\Delta m^{2} L}{4 E_{\nu}}\right)
$$

al trabajar con las unidades $\left[\Delta m^{2}\right]=\mathrm{eV}^{2},[L]=\mathrm{m}$ y $\left[E_{\nu}\right]=\mathrm{MeV}$ queda un factor de 1.27 y obtenemos la forma comúnmente usada de la fórmula de la probabilidad de oscilación de dos neutrinos:

$$
P\left(\nu_{\mu} \rightarrow \nu_{e}\right)=\sin ^{2} 2 \theta \sin ^{2}\left(\frac{1.27 \Delta m^{2} L}{E_{\nu}}\right) .
$$

El ángulo de mezcla $\sin ^{2} 2 \theta$ determina la amplitud de la oscilación, mientras que $\Delta m^{2}$ influye en la longitud de oscilación. De la fórmula (1.6) queda claro que las oscilaciones solo son posibles si $\theta$ y $\Delta m^{2}$ no son nulos, es decir que exista mezcla leptónica y exista por lo menos una masa distinta de cero. Todas las oscilaciones de dos neutrinos pueden ser caracterizadas por estas dos cantidades, porque $P\left(\nu_{\alpha} \rightarrow \nu_{\beta}\right)=P\left(\nu_{\beta} \rightarrow \nu_{\alpha}\right)$.

$P\left(\nu_{\mu} \rightarrow \nu_{\mu}\right)$ es la probabilidad de encontrar a $\nu_{\mu}$ en $L$ dado que $\nu_{\mu}$ fue producido en el inicialmente. Es el complemento de la probabilidad, es decir:

$$
P\left(\nu_{\mu} \rightarrow \nu_{\mu}\right)=1-\sin ^{2} 2 \theta \sin ^{2}\left(\frac{1.27 \Delta m^{2} L}{E_{\nu}}\right)
$$

\section{Analogía en la música y equivalente en la física clásica}

Las ondas describen algunos de los más extraordinarios fenómenos en el mundo. Pueden ser simples, como la nota de una flauta; o pueden ser mezclas complejas, como un acorde. Combinar dos ondas de tonos similares produce un efecto de "compás". Al escuchar dos flautas tocando la misma nota, una de las dos ligeramente fuera de tono, se puede oír un efecto "wah-wah-wah" conforme el sonido viene y va; ya que el sonido es una mezcla de ondas ligeramente diferentes que interfieren entre sí.

Por su parte, los neutrinos también se encuentran gobernados por un fenómeno ondulatorio conforme viajan por el espacio. La interferencia entre las ondas también produce compases regulares, el efecto wah-wah-wah resultante se detecta en ciertas propiedades del neutrino que aparecen y desaparecen. Cuando los neutrinos interactúan con la materia producen otros tipos específicos de partículas. En un momento puede producir un electrón, pero en otro bien podría producir un tauón o un muón [53].

La física básica detrás de la oscilación de neutrinos puede encontrarse en cualquier sistema de osciladores armónicos acoplados, por ejemplo un par de péndulos conectados por un resorte con pequeño coeficiente de elasticidad. Se pone en movimiento a uno de los péndulos, mientras el otro permanece en reposo. Con el tiempo, el otro péndulo se mece debido al pequeño resorte que los conecta, la amplitud del primer péndulo disminuye conforme este cede energía al segundo. Eventualmente toda la energía del sistema se transfiere al segundo péndulo y el primero se queda en reposo, de ahí el proceso se revierte y se repite hasta que se pierde la energía por la fricción.

Se puede entender el comportamiento de este sistema a través de sus modos normales de oscilación. Si los dos péndulos son idénticos, un modo es cuando los dos péndulos se mecen en la misma dirección con distancia constante entre ellos, el otro modo es cuando los péndulos se mecen en direcciones 


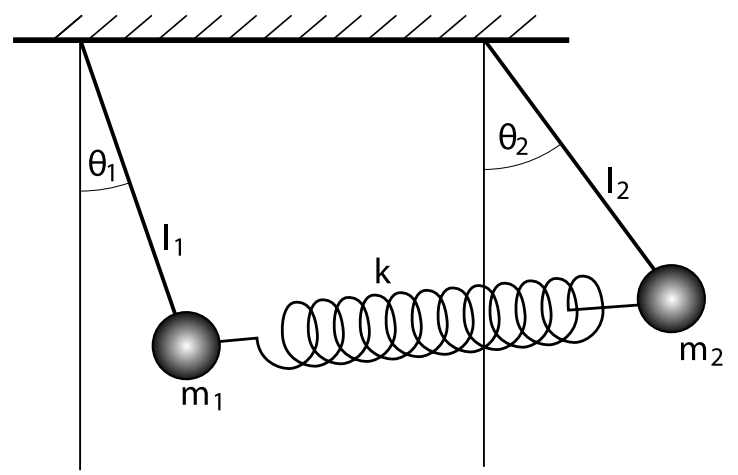

(a) Péndulos acoplados con resorte.

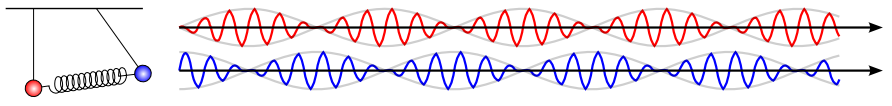

(b) Evolución temporal de los péndulos.

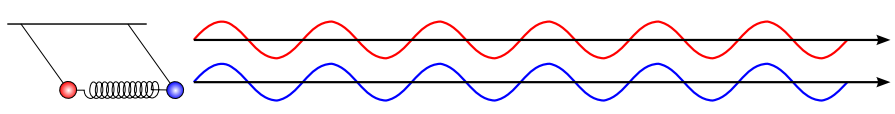

(c) Modo normal de baja frecuencia.

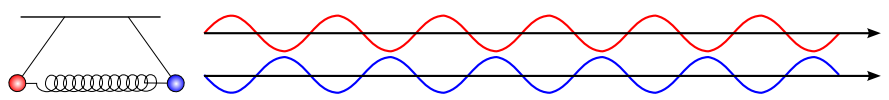

(d) Modo normal de alta frecuencia.

Figura 1.6: Análogo clásico de la oscilación de neutrinos: péndulos acoplados con un pequeño resorte. (a) Se tienen dos péndulos unidos con un pequeño resorte. (b) Al mover a uno de los dos, transfiere su movimiento al otro. Con el tiempo el sistema de los dos péndulos tendrá a los dos oscilando en la misma dirección, (c), o a los dos oscilando en direcciones opuestas, (d). Se puede describir cualquier instante del sistema como una combinación de estos dos modos normales de oscilación.

opuestas. Estos modos tienen frecuencias ligeramente distintas ya que el segundo modo involucra el ligero resorte y el primero no. El estado inicial del sistema de dos péndulos es una combinación de los dos modos, con el tiempo estos modos se desfasan, lo que se ve como una trasferencia de movimiento entre los péndulos. Si los péndulos no son iguales el análisis es ligeramente más complicado, pero el comportamiento es similar.

La descripción del sistema en términos de los dos péndulos es análogo a la base de sabor de los neutrinos. Estos son los parámetros que son fácilmente producidos y detectados, a través de interacciones que involucran el bosón $W$ en el caso de los neutrinos. La descripción en términos de los modos normales de oscilación es análoga a la base de masa de los neutrinos.

\subsubsection{Oscilación de $3 \nu$ en el vacío}

Un escenario más realista es aquel de tres sabores de neutrinos. Una vez más, los eigenestados de sabor $\nu_{\alpha}$ no necesitan ser idénticos a los eigenestados de masa $\nu_{i} \mathrm{y}$, como en el sector de quarks, están conectados por una matriz unitaria $U$, llamada matriz PMNS (Pontecorvo-Maki-Nakagawa-Sakata)

$$
\left|\nu_{\alpha}\right\rangle=U\left|\nu_{i}\right\rangle, \quad \alpha=e, \mu, \tau ; \quad i=1,2,3
$$

Para tres neutrinos de $\operatorname{Dirac}^{12} U$ esta dada como

$$
\begin{aligned}
U & =\left(\begin{array}{lll}
U_{e 1} & U_{e 2} & U_{e 3} \\
U_{\mu 1} & U_{\mu 2} & U_{\mu 3} \\
U_{\tau 1} & U_{\tau 2} & U_{\tau 3}
\end{array}\right) \\
& =\left(\begin{array}{ccc}
c_{12} c_{13} & s_{12} c_{13} & s_{13} \mathrm{e}^{-i \delta} \\
-s_{12} c_{23}-c_{12} s_{23} s_{13} \mathrm{e}^{i \delta} & c_{12} c_{23}-s_{12} s_{23} s_{13} \mathrm{e}^{i \delta} & s_{23} c_{13} \\
s_{12} s_{23}-c_{12} c_{23} s_{13} \mathrm{e}^{i \delta} & -c_{12} s_{23}-s_{12} c_{23} s_{13} \mathrm{e}^{i \delta} & c_{23} c_{13}
\end{array}\right)
\end{aligned}
$$

\footnotetext{
${ }^{12} \mathrm{Si}$ los neutrinos fueran partículas de Majorana entonces aparecería una fase de violación $C P$ con dos neutrinos, para tres neutrinos se tendrían que añadir dos fases más.
} 
donde $s_{i j}=\sin \theta_{i j}, c_{i j}=\cos \theta_{i j}(i, j=1,2,3)$. Se muestra una ilustración gráfica de los elementos de mezcla de la matriz, ignorando la fase $C P$, en la figura 1.7a. Para el caso de tres sabores de neutrinos, existen varios esquemas posibles de masa, en particular el esquema de masas jerárquico normal e invertido que se muestran en la figura 1.7b. Nótese que con tres masas distintas existen dos diferencias de masas al cuadrado linealmente independientes, es decir habiendo medido dos la tercera esta determinada, por ejemplo $\Delta m_{31}^{2}=\Delta m_{32}^{2}+\Delta m_{21}^{2}$.

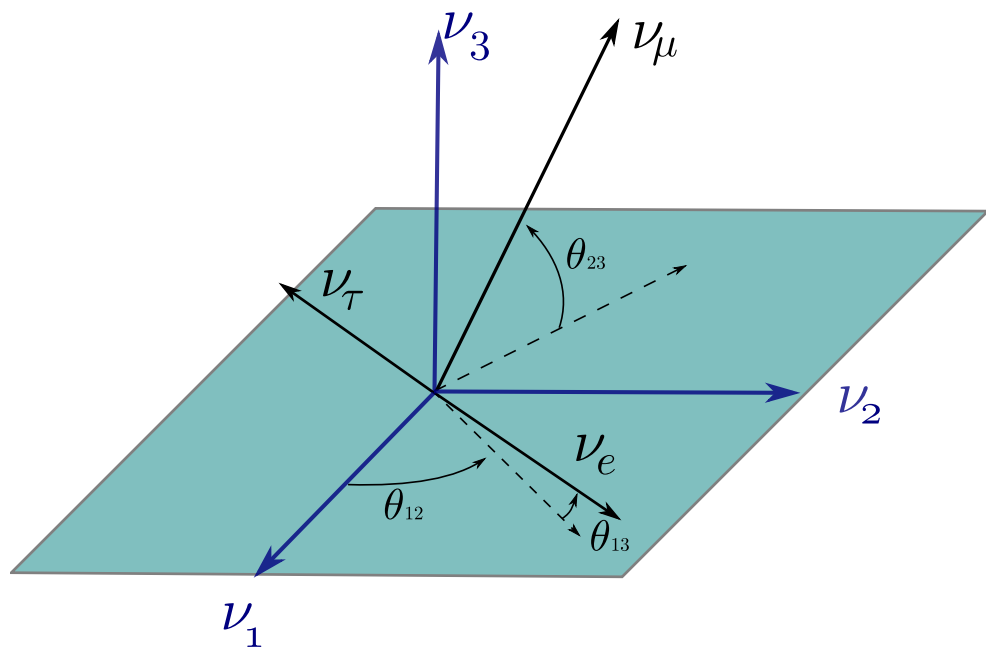

(a) Mezcla de los eigenestados de sabor y masa.

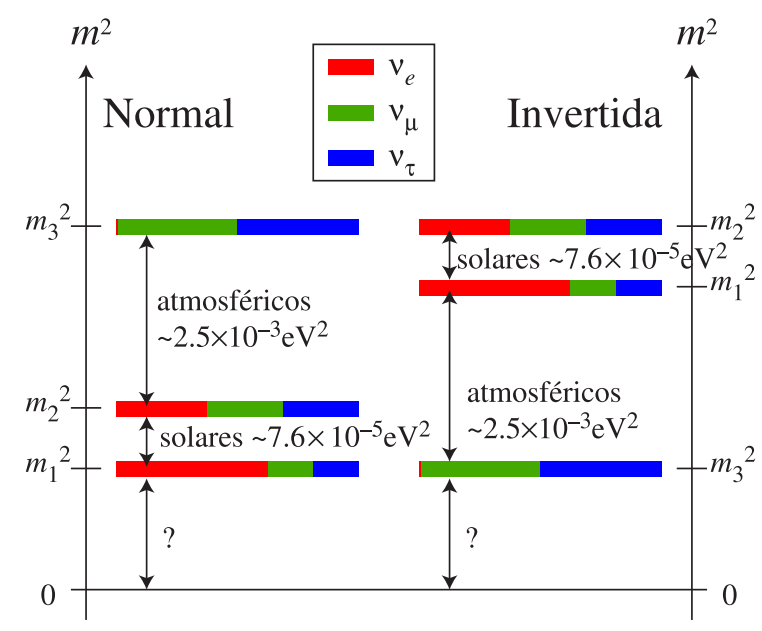

(b) Jerarquía de masa para tres neutrinos.

Figura 1.7: Representación gráfica de la mezcla de neutrinos. (a) La matriz de mezcla entre los eigenestados de masa y sabor puede ser vistos como una matriz de rotación de la base de vectores $\nu_{e}, \nu_{\mu}, \nu_{\tau}$ a la base $\nu_{1}, \nu_{2}, \nu_{3}$, se ignora la fase de violación de $C P$. (b) La jerarquía normal e invertida (caracterizada por $\Delta m_{23}^{2}=m_{3}^{2}-m_{2}^{2}<0$.) de masas para tres neutrinos, cada eigenestado de masa es una mezcla de los tres eigenestados de sabor. Las diferencias de masa de los neutrinos atmosféricos y solares difieren por dos ordenes de magnitud. Se desconoce la masa absoluta de los neutrinos.

Una forma conveniente de presentar la matriz PMNS es

$$
U=\left(\begin{array}{ccc}
1 & 0 & 0 \\
0 & c_{23} & s_{23} \\
0 & -s_{23} & c_{23}
\end{array}\right)\left(\begin{array}{ccc}
c_{13} & 0 & s_{13} \mathrm{e}^{-i \delta} \\
0 & 1 & 0 \\
-s_{13} \mathrm{e}^{+i \delta} & 0 & c_{13}
\end{array}\right)\left(\begin{array}{ccc}
c_{12} & s_{12} & 0 \\
-s_{12} & c_{12} & 0 \\
0 & 0 & 1
\end{array}\right)
$$

esta forma factorizada de la matriz resulta muy útil para interpretar datos ya que la primera matriz (extrema derecha) contiene datos relevantes para oscilaciones de neutrinos atmosféricos y de aceleradores. La segunda (en medio) contiene los parámetros accesibles para experimentos de reactores de corta distancia y la fase de violación de $C P \delta$, mientras que la tercera depende de los parámetros involucrados en oscilaciones de neutrinos solares.

Ahora se tienen más cantidades involucradas tanto en magnitud como en signo: para el caso de oscilación de dos neutrinos en el vacío el signo es irrelevante. Para neutrinos ultrarelativistas, en la ausencia de cualquier efecto de materia, la probabilidad esta dada por

$$
\begin{aligned}
P\left(\nu_{\alpha} \rightarrow \nu_{\beta}\right)= & \Delta_{\alpha \beta}-4 \sum_{i>j=1}^{3} \operatorname{Re}\left(U_{\alpha i}^{*} U_{\beta i} U_{\alpha j} U_{\beta j}^{*}\right) \sin ^{2}\left(\frac{\Delta m_{i j}^{2} L}{4 E}\right)+ \\
& +4 \sum_{i>j=1}^{3} \operatorname{Im}\left(U_{\alpha i}^{*} U_{\beta i} U_{\alpha j} U_{\beta j}^{*}\right) \sin \left(\frac{\Delta m_{i j}^{2} L}{4 E}\right) \cos \left(\frac{\Delta m_{i j}^{2} L}{4 E}\right) .
\end{aligned}
$$


Las fórmulas generales para el escenario de tres sabores son bastante complejas; sin embargo, asumiendo el dominio de masas observado (ver el estatus experimental §1.4.7):

$$
\Delta m_{21}^{2}=\Delta m_{\text {Sol }}^{2} \ll \Delta m_{31}^{2} \approx \Delta m_{32}^{2}=\Delta m_{\text {atm }}^{2} .
$$

se obtiene que para cada caso solo una escala de masas es relevante y la mayoría de los términos de la ecuación (1.11) se vuelven despreciables si $\Delta m^{2} L / 4 E \approx 1$, como es el caso en experimentos atmosféricos y de aceleradores con largas distancias, para los cuales las expresiones para las oscilaciones especificas son

$$
\begin{aligned}
& P\left(\nu_{\mu} \rightarrow \nu_{\tau}\right)=4\left|U_{\tau 3}\right|^{2}\left|U_{\mu 3}\right|^{2} \sin ^{2}\left(\frac{\Delta m_{\mathrm{atm}}^{2} L}{4 E}\right)=\sin ^{2}\left(2 \theta_{23}\right) \cos ^{4}\left(\theta_{13}\right) \sin ^{2}\left(\frac{\Delta m_{\mathrm{atm}}^{2} L}{4 E}\right) \\
& P\left(\nu_{e} \rightarrow \nu_{\mu}\right)=4\left|U_{e 3}\right|^{2}\left|U_{\mu 3}\right|^{2} \sin ^{2}\left(\frac{\Delta m_{\mathrm{atm}}^{2} L}{4 E}\right)=\sin ^{2}\left(2 \theta_{13}\right) \sin ^{2}\left(\theta_{23}\right) \sin ^{2}\left(\frac{\Delta m_{\mathrm{atm}}^{2} L}{4 E}\right) \\
& P\left(\nu_{e} \rightarrow \nu_{\tau}\right)=4\left|U_{\tau 3}\right|^{2}\left|U_{e 3}\right|^{2} \sin ^{2}\left(\frac{\Delta m_{\mathrm{atm}}^{2} L}{4 E}\right)=\sin ^{2}\left(2 \theta_{13}\right) \cos ^{2}\left(\theta_{23}\right) \sin ^{2}\left(\frac{\Delta m_{\mathrm{atm}}^{2} L}{4 E}\right) .
\end{aligned}
$$

Es importante mencionar que el futuro experimental de la física de neutrinos requiere medir los términos que no dominan la probabilidad de oscilación, lo cual significará un gran reto tecnológico y científico.

\subsubsection{Efecto MSW}

El llamado efecto MSW (o efecto de materia), descrito por Mikheyev,Smirnov y Wolfenstein [38, 39], actúa para modificar las oscilaciones de neutrinos en la materia con un perfil de densidad variable. La presencia de electrones en la materia cambia los niveles de energía de los eigenestados de masa de los neutrinos, debido a la dispersión coherente hacia adelante de corriente cargada de los neutrinos del electrón. La dispersión coherente hacia adelante es análoga al proceso electromagnético que lleva al índice de refracción de la luz en un medio. Esto significa que los neutrinos en la materia tienen una masa efectiva distinta a la que tienen en el vacío, y como la oscilación de neutrinos depende de la masa de estos entonces el efecto de oscilación es diferente. Cuando el perfil de densidad es variable se puede tener un efecto resonante que amplifica la probabilidad de transición de sabor de los neutrinos cuando estos pasan por una zona de densidad adecuada. Con los antineutrinos, el punto conceptual es el mismo pero la carga efectiva a la cual se acopla la interacción débil tiene un signo opuesto. El efecto MSW es importante para los neutrinos del electrón del Sol, donde hay una gran densidad de electrones.

Los efectos de materia para neutrinos de $\sim 1 \mathrm{GeV}$ viajando distancias cortas $(\sim 1 \mathrm{~km})$ en la relativamente baja densidad de la roca son despreciables. Por lo cual, para los fines de esta tesis, la aproximación a oscilaciones en el vacío es más que suficiente.

\subsubsection{Consideraciones experimentales}

Como se vio en $\S 1.3$ existen muchas fuentes de neutrinos, de esas las más importantes fuentes para buscar oscilaciones son: plantas nucleares $\left(\bar{\nu}_{e}\right)$, aceleradores $\left(\nu_{e}, \bar{\nu}_{e}, \nu_{\mu}, \bar{\nu}_{\mu}\right)$, la atmósfera $\left(\nu_{e}, \bar{\nu}_{e}, \nu_{\mu}, \bar{\nu}_{\mu}\right)$ y el Sol $\left(\nu_{e}\right)$. Lo que muestra que las distintas fuentes no siempre pueden investigar los mismos valores 
de los parámetros $\Delta m^{2}$ y $\sin ^{2} 2 \theta$, por ejemplo los acelerados de altas energías $(E \approx 1-100 \mathrm{GeV}$, $L \approx 1 \mathrm{~km})$ no son capaces de inspeccionar los datos de neutrinos solares $\left(E \approx 1 \mathrm{MeV}, L \approx 10^{8} \mathrm{~km}\right)$. Reexpresemos el término oscilante de la ecuación (1.5) como

$$
\sin ^{2}\left(\frac{\Delta m^{2}}{4} \frac{L}{E}\right)=\sin ^{2}\left(\pi \frac{L}{\lambda_{\mathrm{osc}}}\right)
$$

en términos de la longitud de oscilación de un periodo completo $\lambda_{\text {osc }} \equiv 4 \pi E / \Delta m^{2} \approx 2.5 \frac{E_{\nu} / \mathrm{MeV}}{\Delta m^{2} / \mathrm{eV}^{2}} \mathrm{~m}$, esta distancia crece para energías más altas o valores de $\Delta m^{2}$ más pequeños. La figura 1.8 muestra los tres casos que deben ser considerados con respecto a la posible observación de oscilaciones:

- $L \ll \lambda_{\text {osc }}$. El experimento se encuentra muy cerca de la fuente y las oscilaciones no tienen tiempo para desarrollarse.

- $L \sim \lambda_{\text {osc }}$. Una condición necesaria para observar oscilaciones y resulta ser la región más sensible.

- $L \gg \lambda_{\text {osc }}$. Varias oscilaciones han ocurrido entre la fuente y el detector. En tales circunstancias los experimentos solo miden la probabilidad promedio de transición.

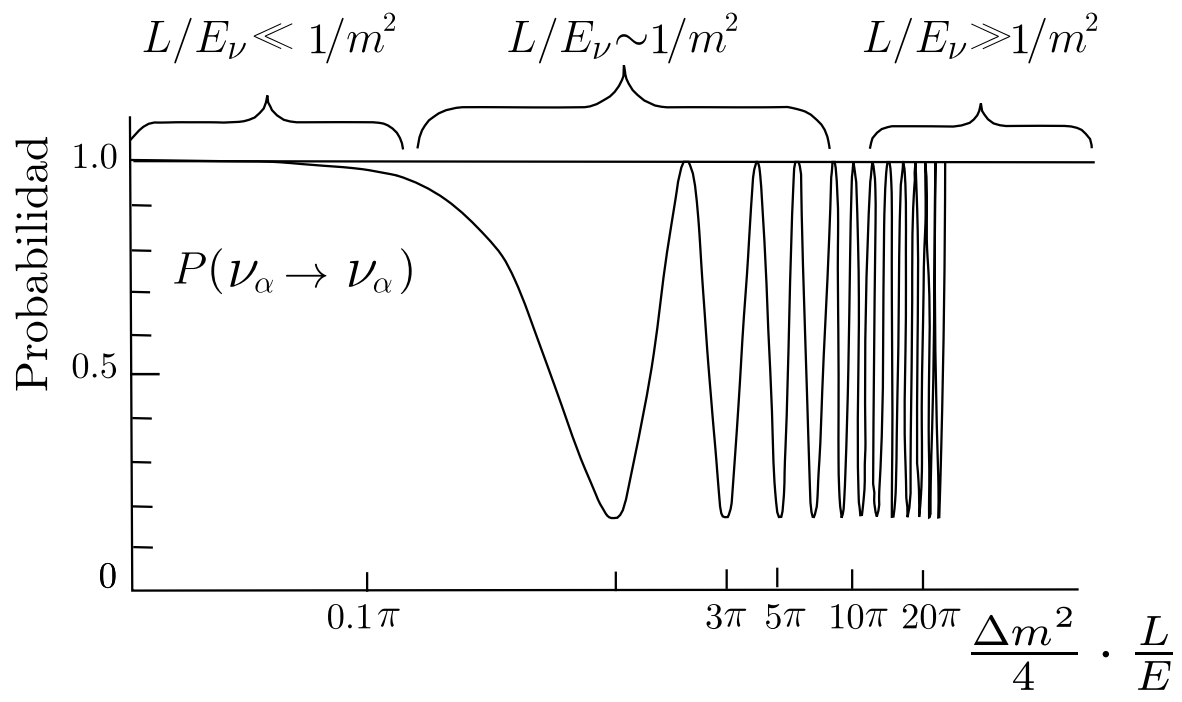

Figura 1.8: Gráfica logarítmica de la probabilidad de oscilación $P\left(\nu_{\alpha} \rightarrow \nu_{\alpha}\right)$ como función de $L / E_{\nu}$, con $\sin ^{2} 2 \theta=0.8$. Las llaves muestran tres casos posibles: no hay oscilaciones, cuando $L / E_{\nu} \ll 1 / m^{2}$; sensibilidad máxima a oscilaciones, cuando $L / E_{\nu} \sim 1 / \mathrm{m}^{2}$; solo se pueden hacer mediciones del promedio de oscilaciones debido a la resolución finita, cuando $L / E_{\nu} \gg 1 / m^{2}$.

Como ya se mencionó, usualmente se puede considerar que las mezclas de neutrinos ocurren solamente entre dos especies de neutrinos, como $\nu_{\mu} \leftrightarrow \nu_{\tau}$. Lo cual es una suposición razonable cuando: todos los ángulos de mezcla son pequeños o cuando la jerarquía de masas es tal que una diferencia de masas $\Delta m_{i j}^{2}$, por ejemplo $\Delta m_{21}^{2}$, es pequeña comparada con las otras; de tal forma hay una región en $L / E_{\nu}$ donde $\Delta m_{21}^{2} \cdot L / E_{\nu}$ es despreciable, pero $\Delta m_{32}^{2} \cdot L / E_{\nu}$ no lo es. En estos casos los resultados (o límites de exclusión) pueden ser mostrados como regiones permitidas en una gráfica de $\Delta m^{2}$ vs. $\sin ^{2} 2 \theta$, donde $\sin ^{2} 2 \theta$ es una combinación de los términos de la matriz de mezcla. Hay dos variedades de experimentos de oscilación de neutrinos: experimentos de aparición y de desaparición. Los primeros son aquellos en los que se buscan interacciones de neutrinos no esperados en el haz, (en la práctica, como nunca se tiene un haz completamente puro de un sabor dado, lo que se busca es un exceso de 
interacciones de los neutrinos no esperados). Por ejemplo la búsqueda de un exceso de interacciones de $\nu_{e}$ en un detector, para un haz primordialmente formado de $\nu_{\mu}$. Se muestra un histograma típico de los eventos que vería un experimento de aparición en la figura 1.9a.

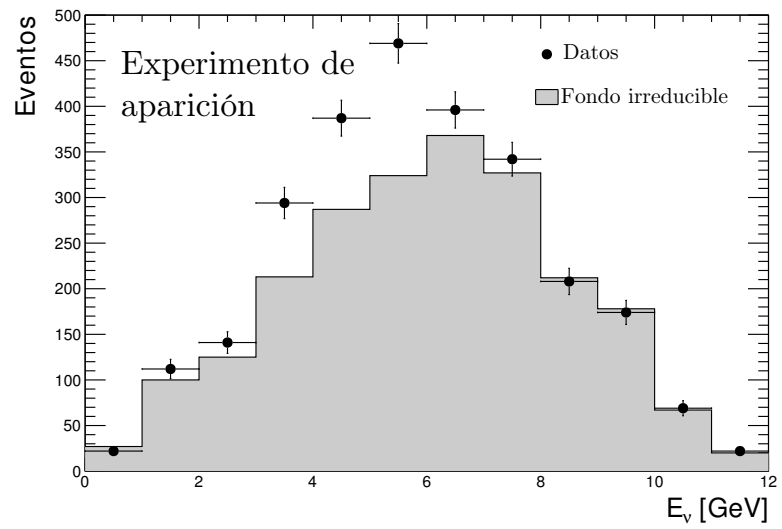

(a) Experimento de aparición

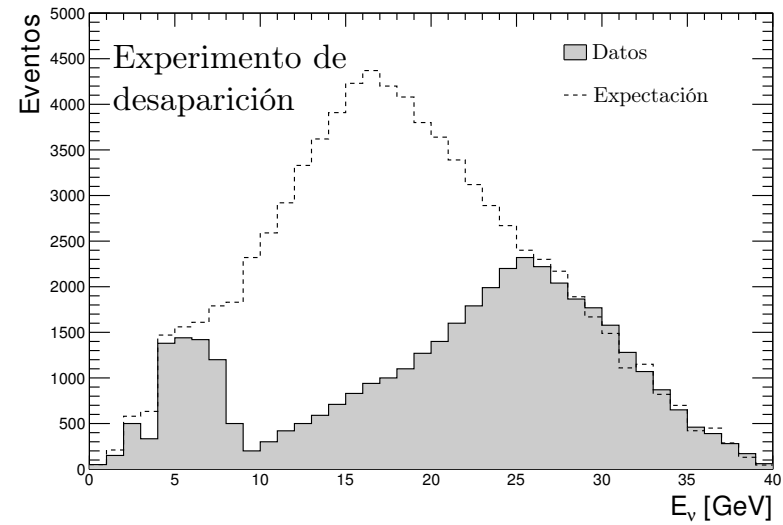

(b) Experimento de desaparición.

Figura 1.9: Histogramas típicos de los resultados esperados. (a) En un experimento de aparición se espera ver un exceso en cierta región de energía sobre el fondo irreducible. (b) En los experimentos de desaparición se observan menos eventos de los esperados en cierta región de energías.

En un experimento real la distancia que viajan los neutrinos $L$, y su energía $E_{\nu}$, tienen ciertas distribuciones, así que se debe promediar la probabilidad $P$ sobre una distribución de $L / E_{\nu}$. A manera de ejemplo, hagamos la suposición poco realista de que $b \equiv 1.27 \cdot L / E_{\nu}$ tiene una distribución gaussiana con desviación estándar $\sigma_{b}$ alrededor de un valor central $b_{0}$. Entonces el promedio de la ecuación (1.6) es:

$$
\langle P\rangle=\frac{1}{2} \sin ^{2} 2 \theta\left[1-\cos \left(2 b_{0} \Delta m^{2}\right) \exp \left(-2 \sigma_{b}^{2}\left(\Delta m^{2}\right)^{2}\right)\right]
$$

El valor de $\langle P\rangle$ esta dado por el experimento. Luego se resuelve la expresión (1.16) para $\sin ^{2} 2 \theta$ como función de $\Delta m^{2}$. Notemos distintos casos, ilustrados en la figura 1.10:

- Como las oscilaciones son completamente estropeadas por la resolución para valores grandes de $\Delta m^{2}, \sin ^{2} 2 \theta=2\langle P\rangle$ en esta región.

- La excursión máxima de la curva hacia la izquierda es para $\sin ^{2} 2 \theta=\langle P\rangle$ con buena resolución. Esta protuberancia ocurre en $\Delta m^{2}=\pi / 2 b_{0} \mathrm{eV}^{2}$.

- Para valores grandes de $\sin ^{2} 2 \theta, \Delta m^{2} \approx \frac{1}{b_{0}}\left(\langle P\rangle / \sin ^{2} 2 \theta\right)^{1 / 2}$.

- Cuando $\sin ^{2} 2 \theta=1$, se tiene $\Delta m^{2}=\sqrt{\langle P\rangle} / b_{0}$.

Para valores grandes de $\Delta m^{2}$ se pierde información del espectro y solamente se tiene una medida de la magnitud del fondo. El contoneo que se puede observar en los contornos depende de las características del experimento; como el tamaño de la fuente, la distribución de energía de los neutrinos y las peculiaridades del detector y el análisis.

Luego, en un experimento de desaparición se busca la atenuación del haz de neutrinos, digamos $\nu_{e}$, a cierta distancia de la fuente, al mezclarse con, por lo menos, otro eigenestado de sabor. La 
notación para tales experimentos es: $\nu_{e} \not \rightarrow \nu_{e}$. En contraste con la detección de algunos neutrinos del "sabor incorrecto" que establecen la mezcla en un experimento de aparición, la desaparición de algunos neutrinos de "sabor correcto" en un experimento de desaparición pasa inadvertida debido a la fluctuaciones estadísticas. Por esta razón, los experimentos de desaparición usualmente no pueden probar mezcla de poca probabilidad, es decir, de valores pequeños de $\sin ^{2} 2 \theta$. Se muestra un histograma típico de los eventos que vería un experimento de desaparición en la figura 1.9b. Los experimentos de desaparición caen en dos categorías:

- I. El flujo del haz se conoce, ya sea de la teoría o de otras medidas, por ejemplo los experimentos de reactores y algunos de aceleradores. No pueden probar mezclas con valores muy pequeños de $\sin ^{2} 2 \theta$, pero sí pueden establecer límites pequeños en $\Delta m^{2}$ para valores grandes de $\sin ^{2} 2 \theta$ debido a que $L / E_{\nu}$ puede ser muy grande. Un ejemplo de ese tipo de experimentos se muestra en la figura 1.10.

- II. La atenuación u oscilación del flujo de neutrinos del haz se mide en el mismo aparato (dos detectores ya que es impracticable en uno solo). Por encima de cierto mínimo $\Delta m^{2}$ el equilibrio es establecido en cierto punto del detector y la intensidad no cambia a lo largo del aparato. Como resultado de eso, la sensibilidad se pierde para $\Delta m^{2}$ grandes, como puede apreciarse en la figura 1.10. Hay varios experimentos que usan la estrategia de tener un detector "cercano" y uno "lejano" de forma exitosa.

Debido a su pequeña sección eficaz de interacción, para tener una tasa de eventos razonable $R$ (eventos por segundo), la masa del detector (expresada en número de nucleones $N_{T}$ ) tiene que ser muy grande y la intensidad $I\left(\nu \mathrm{cm}^{-2} \mathrm{~s}^{-1}\right)$ del haz o la fuente tiene que ser tan alta como sea posible. Así pues, para la sección eficaz $\sigma$ correspondiente $\left(\mathrm{en}^{\mathrm{cm}}{ }^{2}\right)$, un estimado de la tasa de eventos esta dado por

$$
R=N_{T} \sigma I
$$

Además, los detectores deben satisfacer varios requisitos:

- Identificación de leptones cargados para distinguir eventos de corriente cargada y corriente neutra.

- Medidas de la energía y del ángulo de dispersión del leptón cargado para determinar las variables cinemáticas del evento.

- Medidas de la energía hadrónica total, por ejemplo para reconstruir $E_{\nu}$.

- Identificación de hadrones secundarios individuales y su momento para investigar con detalle el estado hadrónico final.

- Detección de partículas de vida corta.

- Uso de distintos materiales como blanco en el detector.

Algunos de estos requisitos son excluyentes entre sí y no hay un solo detector que los cumpla todos, el diseño en cuestión y los requisitos que satisfagan los detectores dependen de las preguntas físicas que se quieran estudiar. 


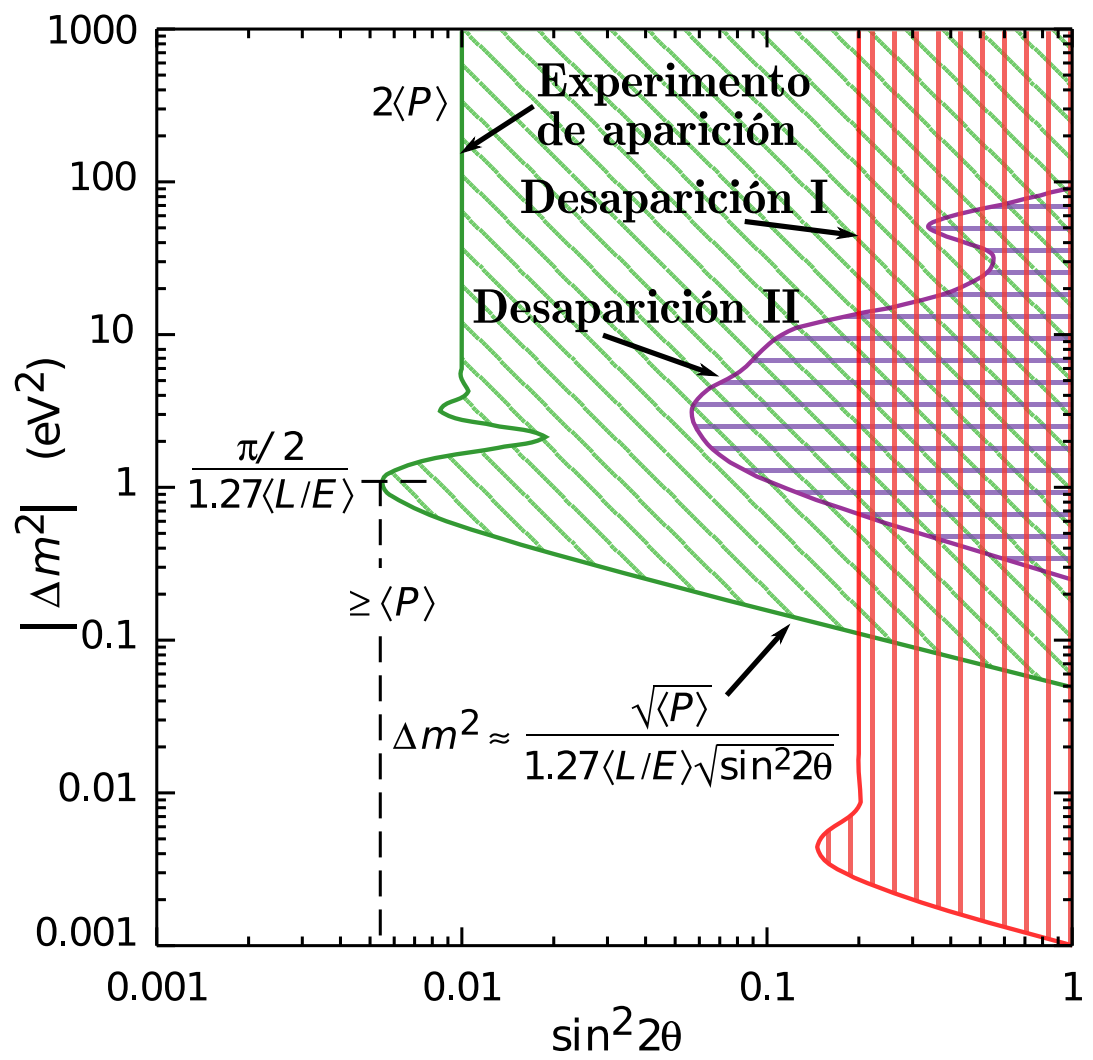

Figura 1.10: Gráfica estándar log-log de $\Delta m^{2}$ vs. $\sin ^{2} 2 \theta$ de contornos de confianza. Se muestran las regiones excluidas de los parámetros de experimentos hipotéticos de aparición y desaparición de neutrinos. Para $\Delta m^{2}$ pequeño el experimento pierde sensibilidad al estar muy cerca de la fuente, así que las oscilaciones apenas se desarrollan. Esto implica una pendiente de -2 hasta que llega a la sensibilidad máxima en el primer máximo de oscilación. Para $\Delta m^{2}$ grande las oscilaciones ya no pueden ser observadas, solo una probabilidad promedio de transición. Más detalles en el texto. Tomado de [54].

\subsubsection{Mediciones directas de la masa de los neutrinos}

Hay varias métodos directos de investigar la masa de los neutrinos ${ }^{13}$, la forma clásica para determinar la masa de $\bar{\nu}_{e}$ es el decaimiento beta. El decaimiento beta es una transición nuclear, donde el número de protones del núcleo $Z$ cambia en una unidad, mientras que el número de nucleones $A$ permanece igual. Hay tres formas de decaimiento beta: $(Z, A) \rightarrow(Z+1, A)+e^{-}+\bar{\nu}_{e}\left(\beta^{-}\right), \quad(Z, A) \rightarrow(Z-$ $1, A)+e^{+}+\nu_{e}\left(\beta^{+}\right)$, y $e^{-}+(Z, A) \rightarrow(Z-1, A)+\nu_{e}$ (captura de electrón).

La forma del espectro de energía del electrón liberado en el decaimiento beta es distinta a la forma que tendría si el antineutrino tuviera una masa nula, a saber, dada la diminuta masa de los neutrinos ${ }^{14}$, el punto final del espectro es ligeramente más pequeño, como se observa en la figura 1.11. Observar esta variación en el espectro es lo que permite poner un límite a la masa de los neutrinos. El isótopo favorito es el tritio, a través de la reacción ${ }^{3} \mathrm{H} \rightarrow{ }^{3} \mathrm{He}^{+}+e^{-}+\bar{\nu}_{e}$, para el cual el experimento Troitsk [55] tiene el mejor límite: $m_{\bar{\nu}_{e}}<2.05 \mathrm{eV}$, (95\% N.C.) Próximamente el experimento KATRIN [56] en Alemania prevé mejorar la sensibilidad a $m_{\bar{\nu}_{e}} \simeq 0.2 \mathrm{eV}$.

\footnotetext{
${ }^{13}$ Estas dos secciones no pertenecen a la búsqueda experimental de oscilaciones, sin embargo sus resultados son relevantes para la oscilación de neutrinos y la física de neutrinos en general. Por eso es que se mencionan aquí.

${ }^{14} \mathrm{La}$ invariancia en $C P T$ asegura que $m_{\bar{\nu}_{e}}=m_{\nu_{e}}$
} 


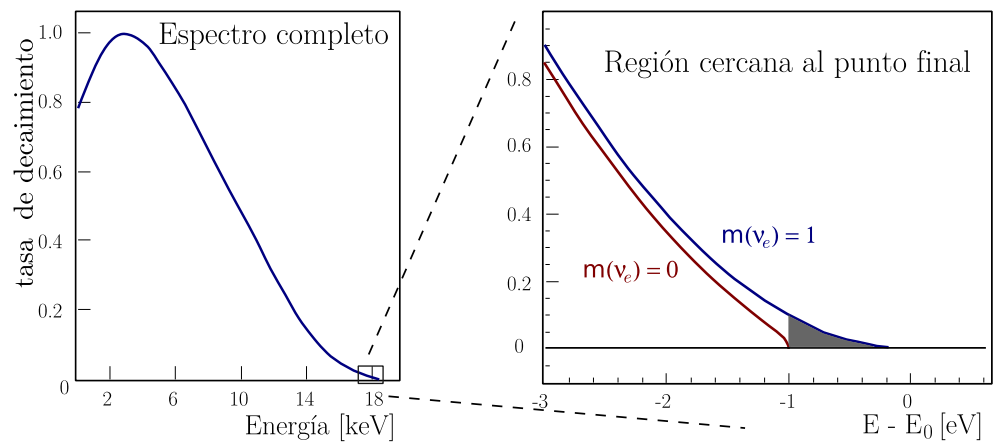

Figura 1.11: Punto final del espectro beta. El efecto de una masa finita del neutrino es un punto final reducido en $E_{0}-m_{\nu} c^{2}$. Solo $2 \times 10^{-13}$ se encuentran en la parte final de $1 \mathrm{eV}$.

A través del decaimiento en reposo del pión se puede poner un límite en la masa del neutrino del muón $\nu_{\mu}$, teniendo un conocimiento preciso del momento del muón y las masas del pión y el muón. El mejor límite obtenido a la fecha es $m_{\nu_{\mu}}<0.17 \mathrm{MeV},(90 \%$ N.C.) [57]. De forma similar, se puede obtener un límite a la masa de $\nu_{\tau}$ a través del decaimiento del leptón $\tau$, el más riguroso es $m_{\tau}<18.2 \mathrm{MeV}$, (95\% N.C.) dado por el experimento ALEPH [58].

Adicionalmente, las observaciones cosmológicas del CMB, la estructura a gran escala, las supernovas tipo Ia y otras; proveen un límite superior a la suma de la masa de los neutrinos $\sum_{k} \nu_{k}$ que varía entre una fracción de $\mathrm{eV}$ a unos cuantos $\mathrm{eV}$, estos límites dependen fuertemente de los modelos y los conjuntos de datos usados [47, 59].

\subsubsection{Decaimiento beta doble sin neutrinos}

El decaimiento beta doble $(2 \nu \beta \beta)$ es un proceso radioactivo en el cual un núcleo libera dos rayos gamma simultáneamente, caracterizado por que la carga del núcleo $Z$ cambia en dos unidades mientras que la masa atómica $A$ permanece sin alterar. Adicionalmente, un decaimiento nuclear de extrema importancia para la física de neutrinos es el decaimiento beta doble sin neutrinos ${ }^{13}(0 \nu \beta \beta)$. Este proceso requiere que los neutrinos tengan una masa diferente de cero y que sean partículas de Majorana, por lo cual se considera el proceso "dorado" para probar el carácter fundamental de los neutrinos.

Un requisito necesario para que ocurra el decaimiento $2 \nu \beta \beta$ es que el núcleo decaiga a una configuración menos energética. Además, por razones prácticas, se requiere que el decaimiento $\beta$ sencillo esté prohibido por consideraciones energéticas. La reacción que sigue este decaimiento es:

$$
(Z, A) \rightarrow(Z+2, A)+2 e^{-}+2 \bar{\nu}_{e}
$$

para el caso sin neutrinos se puede considerar como si este par de neutrinos se anularan entre sí, o equivalentemente, como si el neutrino emitido por un nucleón fuera absorbido por el otro. Así pues, solo los electrones resultan en el estado final (el par de protones quedan ligados en el núcleo) y su energía cinética es aproximadamente la diferencia en la energía de amarre, con muy buena aproximación los electrones emitidos salen en direcciones opuestas.

Medir la tasa de decaimiento del decaimiento $0 \nu \beta \beta$, además de confirmar la naturaleza de Majorana de los neutrinos, daría información en la escala de masa absoluta de los neutrinos y potencialmente en la jerarquía de masas y las fases de Majorana que aparecen en la matriz $\mathrm{PMNS}^{12}$. Es esencial insistir 
que las oscilaciones de neutrinos no pueden determinar el carácter fundamental de los neutrinos, es decir si son partículas de Dirac o Majorana, ni siquiera las fases de Majorana de la matriz de mezcla tienen algún efecto en las oscilaciones [60].

Actualmente el decaimiento $2 \nu \beta \beta$ ha sido confirmado experimentalmente, visto en 35 isótopos distintos con vida media del orden de $\sim 10^{21}$ años. Sin embargo, solo existen límites para el decaimiento sin neutrinos ${ }^{15}$ Hay cerca de una docena de experimentos corriendo y propuestos que investigarán este fenómeno.

\subsubsection{Estatus experimental de oscilaciones de neutrinos}

Se pueden buscar oscilaciones de neutrinos en una variedad de experimentos, en los cuales los mecanismos de producción de neutrinos son muy diferentes. Se han encontrado dos escalas de masas muy diferentes, la de los neutrinos solares y la de los neutrinos atmosféricos, verificadas por experimentos de reactores nucleares y de aceleradores, respectivamente. Las oscilaciones de neutrinos son un hecho experimental que se encuentra muy bien explicado en el marco de mezclas de tres neutrinos. A continuación se describe un poco sobre los distintos experimentos y los resultados que han obtenido. Se muestra el estatus, relativamente actual, de los resultados de oscilaciones en la figura 1.15a, nótese que se gráfica $\Delta m^{2} v s \tan ^{2} \theta$, razón por la cual algunos contornos son simétricos. Del lado derecho se ponen los contornos obtenidos por los experimentos de neutrinos solares (figura 1.15b) y de neutrinos atmosféricos (figura 1.15c).

\section{Experimentos con neutrinos solares}

La primera indicación de un déficit de neutrinos provino del experimento radioquímico de cloro Homestake a finales de los sesenta. Que observó una fracción de los esperados por el modelo solar estándar [61] (SSM por Standar Solar Model)de $R_{\mathrm{Cl}} / R_{\mathrm{SSM}}=0.30 \pm 0.03$, con $R_{\mathrm{Cl}}=2.56 \pm 0.16 \pm$ 0.16 SNU [33, 62 $]^{16}$ La fracción promedio de experimentos subsecuentes que usaban un blanco con Galio, SAGE [63] y GALLEX [64] (sucedido por GNO [65]), fue $R_{\mathrm{Ga}} / R_{\mathrm{SSM}}=0.52 \pm 0.03$, con $R_{\mathrm{Ga}}=68.1 \pm 3.75 \mathrm{SNU}[65]$. Además, los detectores cherenkov de agua Kamiokande [66] y SuperKamiokande [67] en Japón obtuvieron una fracción del flujo esperado de $\Phi_{\mathrm{SK}} / \Phi_{\mathrm{SSM}}=0.413 \pm 0.014$.. El déficit en los distintos flujos observados por estos experimentos sugería un efecto dependiente de la energía, que se conoció como "el problema de los neutrinos solares" [68, 69], y que fue resuelto por el detector de agua pesada SNO [36, 37] en la mina de Sudbury Canadá. Esta es la solución con ángulo de mezcla grande (large mixing angle, LMA) y surge del esquema de conversión de sabores descrito por el efecto MSW [38, 39] en el perfil de densidad exponencial del Sol. SNO presenta un resultado combinando sus datos y los de otros experimentos en un análisis de tres neutrinos de $\Delta m_{21}^{2}=\left(7.41_{-0.19}^{+0.21}\right) \times 10^{-5} \mathrm{eV}^{2} \mathrm{y} \tan ^{2} \theta_{12}=0.446_{-0.029}^{+0.030}[70]$, por su parte Super-Kamiokande presenta un resultado similar: $\sin ^{2} \theta_{12}=0.304 \pm 0.013, \Delta m_{21}^{2}=7.45_{-0.19}^{+0.20} \times 10^{-5} \mathrm{eV}^{2}$ [71], para ambos: $\theta_{12} \simeq 33^{\circ}$. Cabe resaltar que si SNO y Super-Kamiokande, experimentos de la misma naturaleza, se abstienen de combinar sus datos con los de KamLAND ${ }^{17}$ (de hecho tampoco se combinan entre sí) obtienen

\footnotetext{
${ }^{15}$ En 2001 parte de la colaboración Heidelberg-Moscow afirmó haber visto el proceso, con una vida media del orden de $\sim 10^{25}$ años, sin embargo es un resultado controversial que no ha sido aclarado.

${ }^{16} 1$ Solar Neutrino Unit, SNU $\equiv 10^{-36}$ capturas / átomo / segundo.

${ }^{17}$ Un experimento que es sensible a una región similar pero de distinta naturaleza, en $\S 1.4 .7$ se habla más de este experimento.
} 
un ángulo de mezcla similar, pero la diferencia de masa cambia $\Delta m_{21}^{2}=\left(5.6_{-1.4}^{+1.9}\right) \times 10^{-5} \mathrm{eV}^{2} \mathrm{y}$ $\Delta m_{21}^{2}=4.8_{-0.9}^{+1.8} \times 10^{-5}$, respectivamente.

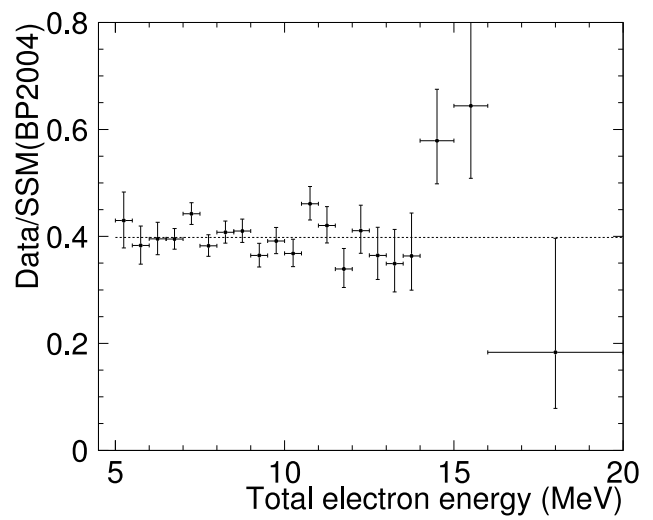

(a) Proporción de datos observados y esperados por el SSM.

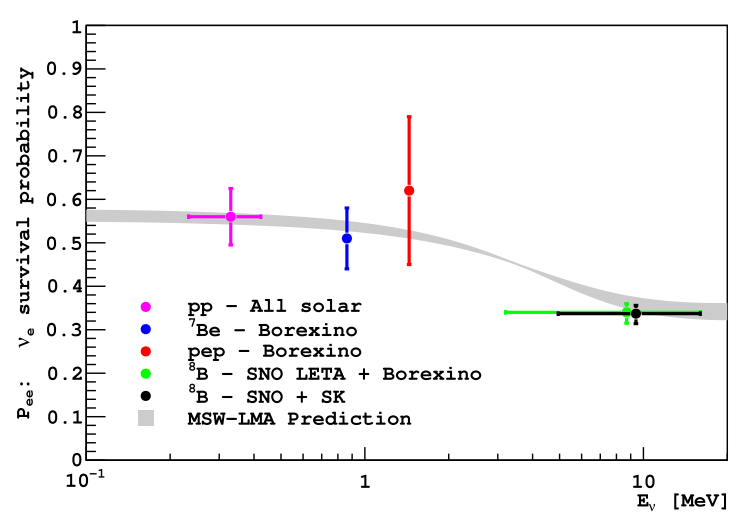

(b) Probabilidad de supervivencia de $\nu_{e}$ solares.

Figura 1.12: Resultados de experimentos de neutrinos solares. (a) Proporción de datos observados por SuperKamiokande y eventos esperados según el SSM, la línea punteada representa el promedio de los datos. Gráfica de [72] (b) Probabilidad de supervivencia de distintos neutrinos solares, con datos de Kamiokande, SuperKamiokande, SNO y Borexino, se muestra la banda a $1 \sigma$ de la predicción MSW-LMA, imagen tomada de [73].

El actual experimento Borexino [73] observó los neutrinos pep (figura 1.12b) que provienen de la inusual reacción de captura del electrón con dos protones y un electrón, formando un núcleo de deuterio y un $\nu_{e}$. Así pues, se han visto la mayoría de las componentes del flujo solar de $\nu_{e}$, excepto la componente del ciclo de carbón-nitrógeno-oxigeno (CNO). Además, Super-Kamiokande observa una asimetría día/noche con $2.7 \sigma$ de nivel de confianza, indicación de la regeneración de $\nu_{e}$ conforme atraviesa la masa terrestre.

Uno de los grandes hitos en la historia actual es la solución al problema de los neutrinos solares ${ }^{18}$, un logro de la creciente rama de la astrofísica de neutrinos. Nuevas observaciones lograran tener un mejor idea de la composición y estructura del Sol, como la distribución homogénea de elementos en el Sol. Hay nuevos experimentos en rumbo, actualmente ya casi acaban las remodelaciones a SNO para hacer SNO+ [74], además de mejorar la electrónica se cambió el agua pesada por centellador, y se espera que sea operacional el próximo año. $\mathrm{SNO}+$ buscará los neutrinos CNO y mejorará las medidas de los neutrinos pep y del ${ }^{8} \mathrm{~B}$, buscará geoneutrinos, neutrinos de supernovas e investigará el doble decaimiento beta. Se investiga la posibilidad de construir un nuevo detector de 50 kilotoneladas, LENA [75], con una gran variedad de objetivos: neutrinos solares, geoneutrinos, supernovas, oscilaciones de corta distancia con fuentes radioactivas.

\section{Experimentos con neutrinos atmosféricos}

Estos neutrinos son producidos por el decaimiento de mesones que se originan de la interacción de rayos cósmicos con el oxigeno y nitrógeno de la atmósfera terrestre. En 1965, las primeras observaciones de estos neutrinos en experimentos en Sudáfrica [76] e India [77] mostraban incompatibilidad con la expectativa con poca significación estadística, mientras que dos experimentos de calorímetros,

\footnotetext{
${ }^{18}$ Todavía hay cosas por resolver: la probabilidad de supervivencia en la región de transición vacío/materia y una parte plana del espectro que observa Super-Kamiokande que no se puede explicar con LMA.
} 
NUSEX [78] en 1988 y Frejus [79] en 1994, no observaron discrepancia. Observaciones posteriores con detectores cherenkov de agua, Kamiokande [80] e IMB [81] obtuvieron una proporción de eventos inducidos por muón y eventos inducidos por electrón $\sim 0.6$ más pequeña que el valor esperado. Estas discrepancias se conocieron como "la anomalía de neutrinos atmosféricos". La dependencia en el ángulo cenital de las mediciones de Kamiokande indicaron que el déficit era causado principalmente por neutrinos que venían por debajo del horizonte y viajaban los $\sim 10^{4} \mathrm{~km}$ del diámetro terrestre, mientras que aquellos que venían de arriba del horizonte solo viajaban $\sim 15 \mathrm{~km}$. Esto se ve apoyado por las observaciones de que el déficit crece con la distancia viajada por el neutrino desde su punto de producción. Para eventos de varios GeV Super-Kamiokande encontró en 1998 una asimetría ascendente-descendente en los eventos observados desviándose del valor esperado por $\sim 10$ desviaciones estándar $A_{\mu}=\frac{U-D}{U+D}=-0.29 \pm 0.03$, en donde $U(D)$ ocurren en el intervalo de ángulo cenital $-1<\cos \theta_{z}<-0.2\left(0.2<\cos \theta_{z}<1\right)$.

La alta precisión y estadística del conjunto de datos atmosféricos de Super-Kamiokande [35, 82, 83] junto con la confirmación de los experimentos de calorímetro metálico, Soudan2 [84] y MACRO [85], resultó en evidencia definitiva de la desaparición de neutrinos del muón producidos en la atmósfera. La mejor interpretación de estos resultados es la oscilación de $\nu_{\mu}$ hacia $\nu_{\tau}$, actualmente los mejores valores que se tienen para los parámetros de oscilación vienen del experimento de acelerador MINOS [86] y son $\left|\Delta m_{23}^{2}\right|=\left(2.41_{-0.10}^{+0.09}\right) \times 10^{-3} \mathrm{eV}^{2}$ para la diferencia de masas y $\sin ^{2} 2 \theta_{23}=0.950_{-0.036}^{+0.035}$ para el ángulo de mezcla, de forma similar a como los experimentos de reactores pueden probar los parámetros de oscilación de los neutrinos solares, es posible configurar un experimento de acelerador para que busque oscilaciones en el régimen atmosférico. La explicación en términos de oscilación $\nu_{\mu} \rightarrow \nu_{e}$ esta excluida con alto nivel de confianza ya que los eventos $\nu_{e}$ concuerdan bien con la expectación y hubieran producido un déficit en el experimento de reactor nuclear CHOOZ que no fue observado. La oscilación en neutrinos estériles también está descartada para neutrinos atmosféricos ya que esto implicaría una supresión en la señal de corriente neutra que no fue observada [87]. La interpretación en términos de no aparición de $\nu_{\tau}$ esta desfavorecida a $3.8 \sigma$ [88] de acuerdo con un análisis reciente de los efectos de esta hipótesis.

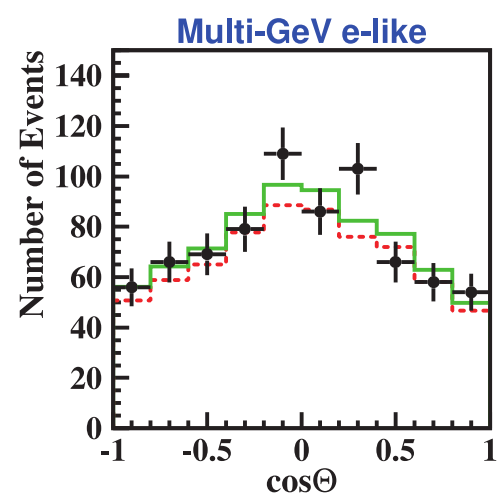

(a) Neutrinos atmosféricos de Super-Kamiokande.

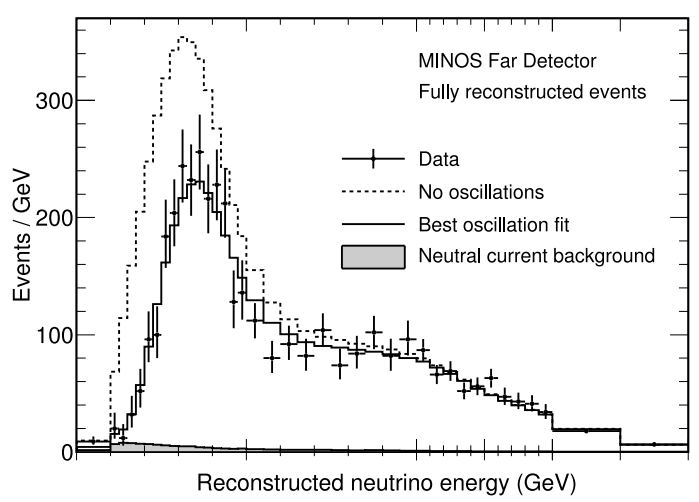

(b) Eventos de MINOS.

Figura 1.13: Resultados de experimentos en el régimen atmosférico. (a) Distribuciones de ángulo cenital del experimento Super-Kamiokande, en linea punteada se muestra el MC sin oscilar y la línea continua muestra la expectación del mejor ajuste para oscilaciones $\nu_{\mu} \leftrightarrow \nu_{\tau}$ [89]. (b) Muestras de datos del detector lejano de MINOS comparadas a la predicción con y sin oscilaciones [90].

Todavía quedan varias cosas por determinar: el octante del ángulo $\theta_{23}$, la jerarquía de masas y la fase de violación $C P$. Además del experimento Super-Kamiokande, el experimento IceCube [91] en el polo 
sur es capaz de ver oscilaciones de neutrinos atmosféricos ${ }^{19}$ y a mediados de 2013 reportó resultados significativos que son consistentes con los obtenidos por los otros experimentos. Con el objetivo de estudiar las interrogantes restantes ya se empezó la construcción del experimento INO [92] en la India, el cual se espera que entré en funcionamiento en 2018. Se han propuesto los experimentos HyperKamiokande [93] y PINGU [94] como las siguientes fases, mucho más grandes, de los experimentos Super-Kamiokande y IceCube, respectivamente.

\section{Experimentos de reactores nucleares}

Los reactores nucleares son fuentes de $\bar{\nu}_{e}$ exclusivamente, con energías de $2-10 \mathrm{MeV}$, con energías tan pequeñas un $\bar{\nu}_{e}$ que oscila a otro sabor se vuelve imposible de detectar con detectores convencionales. No hay suficiente energía para producir un $\mu$ o $\tau$, requisito para saber que la transición tomo lugar, es por eso que los experimentos de reactor típicamente buscan una señal de desaparición.

Los experimentos Gosgen [95] ( $L \sim 38-65$ m)en Suiza y Krasnoyarsk [96] $(L \sim 57-231 \mathrm{~m})$ en Rusia, Bugey [97] $(L \sim 50-94 \mathrm{~m})$ y CHOOZ [98, 99] $(L \sim 1 \mathrm{~km})$ en Francia, y Palo Verde [100] $(L \sim 750$ $890 \mathrm{~m}$ ) en Estados Unidos, no encontraron evidencia de oscilaciones, poniendo límites de exclusión en el espacio de parámetros por encima de $\sin ^{2} 2 \theta \gtrsim 10^{-1}$ y $\Delta m^{2} \gtrsim 10^{-3} \mathrm{eV}^{2}$. La región de exclusión de CHOOZ se extiende hasta valores de $\Delta m^{2}$ que son relevantes a la interpretación de datos de neutrinos atmosféricos y tiene importantes implicaciones en análisis globales que invocan el esquema de tres neutrinos.

El experimento en curso $^{20}$ KamLAND [101] detecta $\bar{\nu}_{e}$, con un blanco de centellador líquido, provenientes de varios reactores nucleares alrededor de la mina de Kamioka en Japón, que se localizan a distancias entre 150 y $210 \mathrm{~km}$. Con estas distancias superiores, el experimento es sensible a oscilaciones de neutrinos con $\Delta m^{2} \gtrsim 10^{-5} \mathrm{eV}^{2}$, similar a aquellas que influyen en neutrinos solares y observa un déficit de neutrinos consistente con los parámetros de oscilación de experimentos de solares. Enfatizando, los $\bar{\nu}_{e}$ de los reactores oscilan con parámetros consistentes con aquellos de los $\nu_{e}$ provenientes del Sol, precisamente el comportamiento esperado si la simetría CPT rige el comportamiento de los procesos leptónicos. Con el cierre a largo plazo de las centrales nucleares en Japón, KamLAND ha tenido una maravillosa oportunidad para hacer estudios del ruido y ha publicado un análisis combinando sus datos con los solares (figuras 1.14a y 1.15b), el cual otorga parámetros de oscilación: $\tan ^{2} \theta_{12}=0.436_{-0.025}^{+0.029}, \Delta m_{21}^{2}=7.53_{-0.18}^{+0.18} \times 10^{-5} \mathrm{eV}^{2}$, and $\sin ^{2} \theta_{13}=0.023_{-0.002}^{+0.002}$ [45]. Esta es la solución LMA al problema de los neutrinos solares, en contraste a otras soluciones que tenían ángulos de mezcla más pequeños. Con los datos del experimento KamLAND en los análisis globales de los datos de neutrinos solares, la solución LMA emergió con claridad como la solución favorecida para el problema de los neutrinos solares.

Actualmente hay tres experimentos de reactores que se encargan de medir el ángulo de mezcla $\theta_{13}$, a saber: Double Chooz en Francia [103], RENO en Corea [104] y Daya Bay en China [105]. Los tres reportan valores congruentes del orden de $\sin ^{2} 2 \theta_{13} \sim 0.1$, y están trabajando para presentar un resultado conjunto ${ }^{21}$, pero aquí nos limitaremos a los resultados de Daya Bay, a la fecha los más precisos. Se tiene un conjunto de detectores cercanos (cientos de metros) a los reactores y un cumulo de detectores a una distancia de $\sim 2 \mathrm{~km}$, con este arreglo se logra una mejor determinación de la tasa

\footnotetext{
${ }^{19}$ Aunque ese no es su objetivo principal.

${ }^{20}$ Ya cesó su investigación de oscilación de neutrinos, sin embargo esta en el proceso de cambiar de giro hacia la investigación de decaimiento $0 \nu \beta \beta$.

${ }^{21}$ Se debe prestar atención a los errores correlacionados, además de que deben ser evaluados de forma unificada.
} 


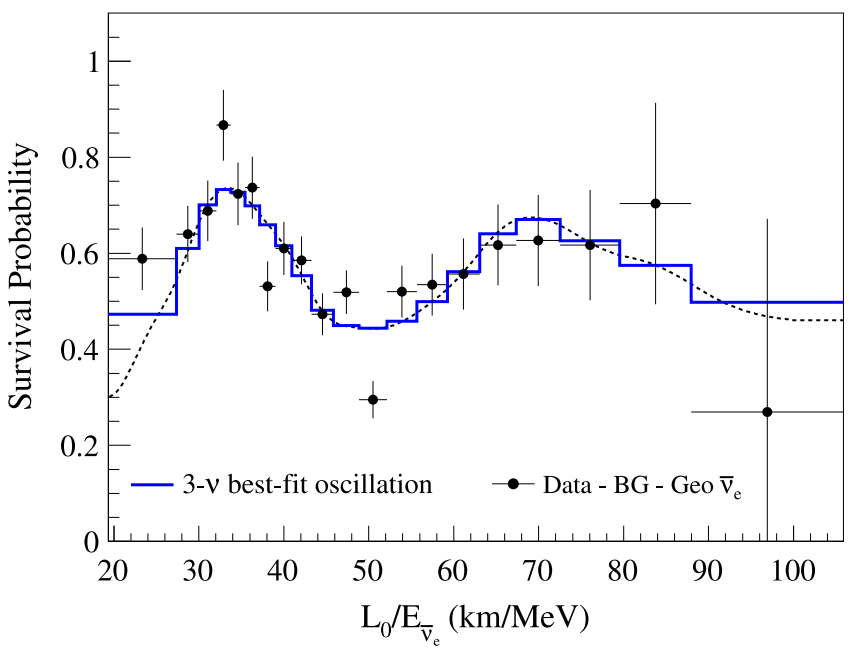

(a) Proporción de $L / E$ observado en KamLAND.

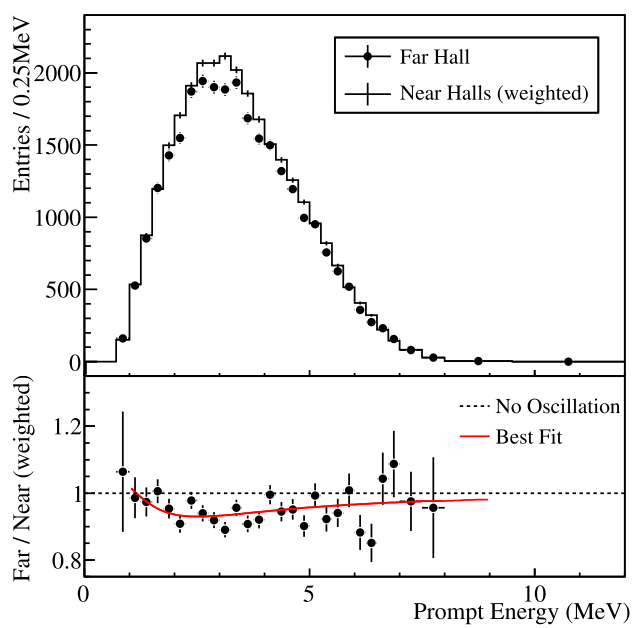

(b) Espectro observado en Daya Bay.

Figura 1.14: Resultados de experimentos de reactores. (a) Proporción del espectro observado de $\bar{\nu}_{e}$ entre lo esperado sin oscilaciones en KamLAND, tomada de [45]. (b) Espectro de energía medido en los detectores lejanos comparado con la predicción sin oscilaciones basada en lo medido en los detectores cercanos de Daya Bay, debajo se muestra la proporción de las dos muestras, tomada de [102]

de desaparición, la detección de $\bar{\nu}_{e}$ se hace con centellador dopado con Gd a través de decaimiento beta inverso y una señal retrasada de la captura del neutrón. La fracción de datos observados entre los esperados de no haber oscilación (figura 1.14b) es de $R=0.944 \pm 0.007$ (stat) \pm 0.003 (sist), así pues se tiene una incontrovertible observación $(7.7 \sigma)$ de un déficit de $\bar{\nu}_{e}$, con lo cual se obtiene $\sin ^{2} 2 \theta_{13}=$ $0.089 \pm 0.010$ (stat) \pm 0.005 (sist); que equivale a $\theta_{13} \simeq 8.7^{\circ}$ [102], el ángulo de mezcla leptónico más pequeño es comparable al ángulo de mezcla más grande de quarks. Ya se esta considerando la siguiente etapa de Daya Bay, el experimento de 20 kilotoneladas JUNO, que se encontrará a una distancia de $\sim 60 \mathrm{~km}$ de los reactores nucleares. También se considera la construcción de RENO-50 un detector de 18 kilotoneladas localizado a $50 \mathrm{~km}$ de la fuente de neutrinos. Estos nuevos experimentos pretenden hacer medidas de precisión de los parámetros de mezcla, detectar geoneutrinos, neutrinos de supernovas, investigar neutrinos estériles y determinar la jerarquía de masas.

\section{Experimentos con neutrinos de acelerador}

Como se mencionó en $\S 1.3 .2$ se pueden producir haces de neutrinos en los aceleradores de partículas, a través de varios mecanismos, con el decaimiento de mesones. El primer experimento de oscilación de neutrinos de larga distancia fue K2K [106, 107] que producía neutrinos en el laboratorio KEK de un haz de protones con momento $12 \mathrm{GeV} / c$ que impactaban un blanco de aluminio y viajaban una distancia de $L \sim 235 \mathrm{~km}$ al detector Super-Kamiokande. El segundo fue el experimento en curso MINOS [108]: protones con momento de $120 \mathrm{GeV} / c$ del Main Injector de Fermilab impactan en un blanco de grafito para generar un haz de neutrinos que viajan una distancia de $L \sim 730 \mathrm{~km}$ hasta el calorímetro de hierro en Soudan, Minnesota en Estados Unidos. Además del resultado ya mencionado de neutrinos atmosféricos, MINOS encuentra valores consistentes para los parámetros de oscilaciones de neutrinos y antineutrinos en la región a la cual es sensible [86]. Más aún, con la gran estadística que cuenta y la capacidad de diferenciar entre neutrinos y antineutrinos pueden desfavorecer $31 \%$ $(5 \%)$ del espacio de tres parámetros definido por la fase de violación de $C P \delta$, el octante de $\theta_{23}$ y la jerarquía de masa a $68 \%$ (90\%) nivel de confianza [109]. 
Además de MINOS, operan actualmente los experimentos OPERA [110] e ICARUS [111] en Gran Sasso Italia, con neutrinos producidos a $732 \mathrm{~km}$ de distancia en CERN Suiza; T2K [112] con neutrinos que viajan $295 \mathrm{~km}$ de Tokai a Kamioka en Japón y recientemente se acabó la construcción del experimento NOnuA [113], el sucesor de MINOS, con neutrinos que viajan $810 \mathrm{~km}$ desde Fermilab hasta el norte de Minnesota. OPERA se dedica a la búsqueda de oscilaciones $\nu_{\mu} \rightarrow \nu_{\tau}$ por búsqueda directa de aparición, a la fecha ha reportado 3 eventos $\nu_{\tau}$ a $3.2 \sigma$ de confianza [114]. Recientemente el experimento T2K reporta [115] medidas precisas de los parámetros de oscilación similares a aquellos encontrados por MINOS. En el futuro próximo T2K y NO $\nu$ A llevaran a importantes constricciones en la determinación de la fase de violación de $C P$, la jerarquía de masas y el octante de $\theta_{23}$. Tanto Europa como Estados Unidos investigan la posibilidad de un programa de neutrinos a gran distancia con LBNO (2 $300 \mathrm{~km})$ y LBNE $(1300 \mathrm{~km})$

Ejemplos de experimentos de oscilación de neutrinos de corta distancia son LSND, KARMEN; los cuales se resumen al final de este capítulo, y MiniBooNE, del cual se hablará con detalle en el próximo capítulo.

\section{Neutrinos estériles y oscilación de $3+n$ neutrinos}

Los experimentos de oscilación de neutrinos atmosféricos, solares, de aceleradores y de reactores se describen de forma consiste usando tres sabores activos de neutrinos, es decir con dos diferencias de masas al cuadrado y tres ángulos de mezcla. Sin embargo, varios experimentos con fuentes radiactivas, reactores y aceleradores sugieren la posible existencia de una o más especies de neutrinos que no forman parte de las interacciones débiles, es decir de neutrinos estériles.

La primera evidencia de neutrinos estériles vino en los noventa con el experimento LSND, con un haz de $\bar{\nu}_{\mu}$, generados con un acelerador, detectó un exceso de $\bar{\nu}_{e}$ en la escala de $\Delta m^{2} \sim 1 \mathrm{eV}^{2}$ significante a $3.8 \sigma$. Lo cual no concordaba con la imagen que se había formado de las oscilaciones de neutrinos. Con el objetivo de corroborar o refutar a LSND se construye el experimento MiniBooNE en Fermilab, el cual también observa un exceso de eventos a la misma escala. Debido a la gran certeza que se tiene de los resultados con neutrinos atmosféricos y al hecho de que el nivel de confianza de los resultados obtenidos por MiniBooNE y LSND es menor a 4 $\sigma$, vuelve a estas observaciones "anómalas" 22 .

Al revaluar el flujo de neutrinos emitido por reactores nucleares, se obtuvo un incrementó de $\sim 3 \%$ después de haber reconsiderando mejorías en el espectro de los neutrinos de reactores, revaluar secciones eficaces y considerando los isótopos de vida larga que se acumulan en los reactores. Basándose en el nuevo cálculo de flujo, los resultados de corta distancia $(L \leq 100 \mathrm{~m})$ muestran un déficit de $\sim 6 \%$ (98.6\% N.C.) en el flujo medido de $\bar{\nu}_{e}$, a este déficit se le llama "la anomalía de reactores nucleares" [118]. Un déficit que puede ser explicado por la desaparición de $\bar{\nu}_{e}$ debido a oscilaciones con $\Delta m^{2} \sim 1 \mathrm{eV}^{2}$.

La "anomalía del galio" surgió en experimentos de fuente radioactiva en SAGE [120] y GALLEX [121], experimentos de galio de neutrinos solares. Obtuvieron una tasa de eventos inducidos por flujos de $\nu_{e}$ producidos por intensas fuentes de ${ }^{51} \mathrm{Cr}$ y ${ }^{37} \mathrm{Ar} 15 \%$ más baja de lo esperado. Este efecto puede ser explicado por la hipótesis de desaparición de $\nu_{e}$ debido a oscilaciones con $\Delta m^{2} \gtrsim 1 \mathrm{eV}^{2}$ [122, 123].

Más aún, el análisis de datos cosmológicos como el CMB, nucleosíntesis en el Big Bang y la abundancia relativa de elementos aún admiten un neutrino extra [47, 48, 49].

\footnotetext{
${ }^{22}$ Es común escuchar sobre "la anomalía LSND", y en fechas recientes también se ha escuchado hablar sobre "la anomalía MiniBooNE".
} 


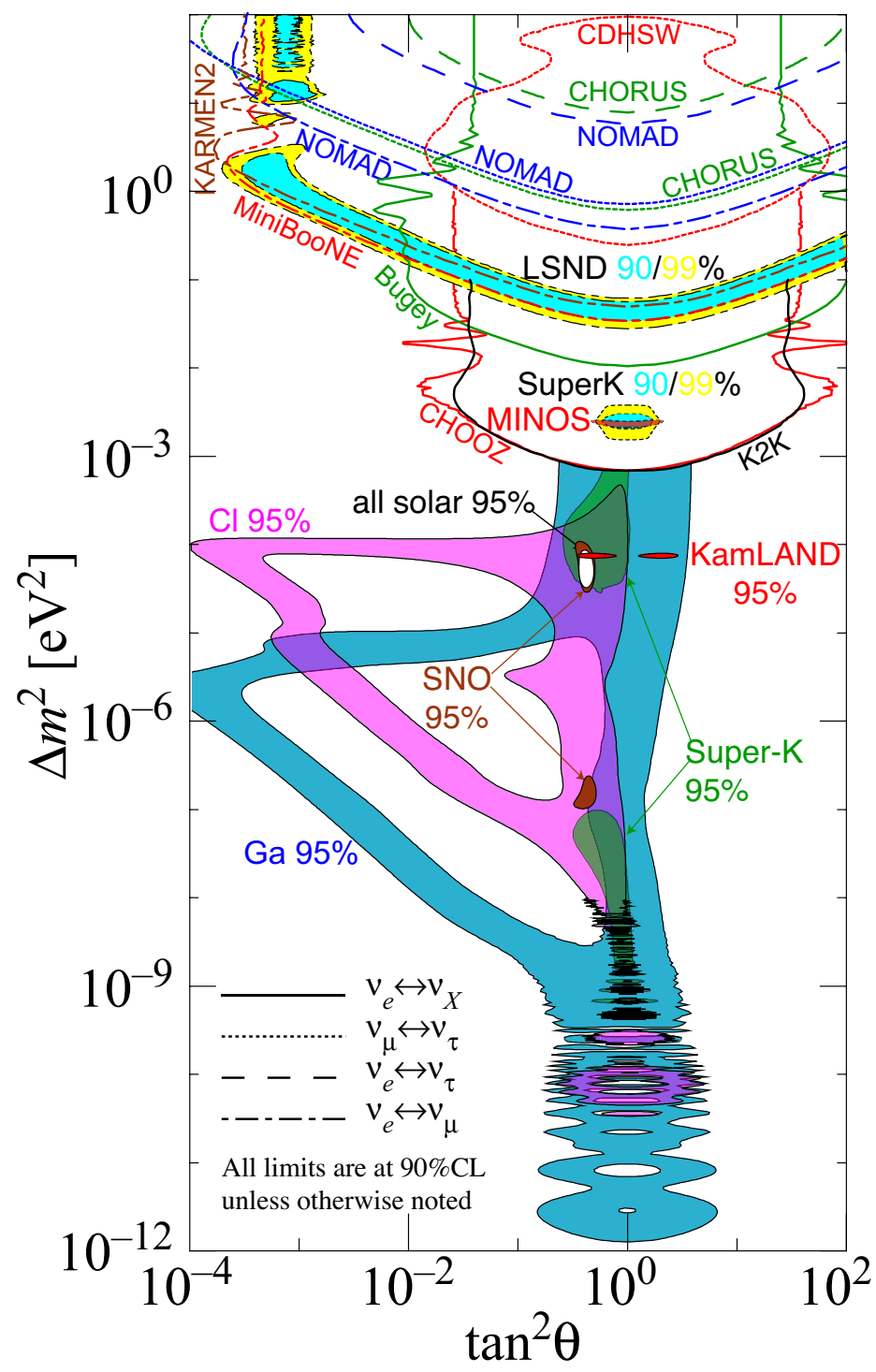

(a) Compilación de la mayoría de los resultados de oscilación de neutrinos.

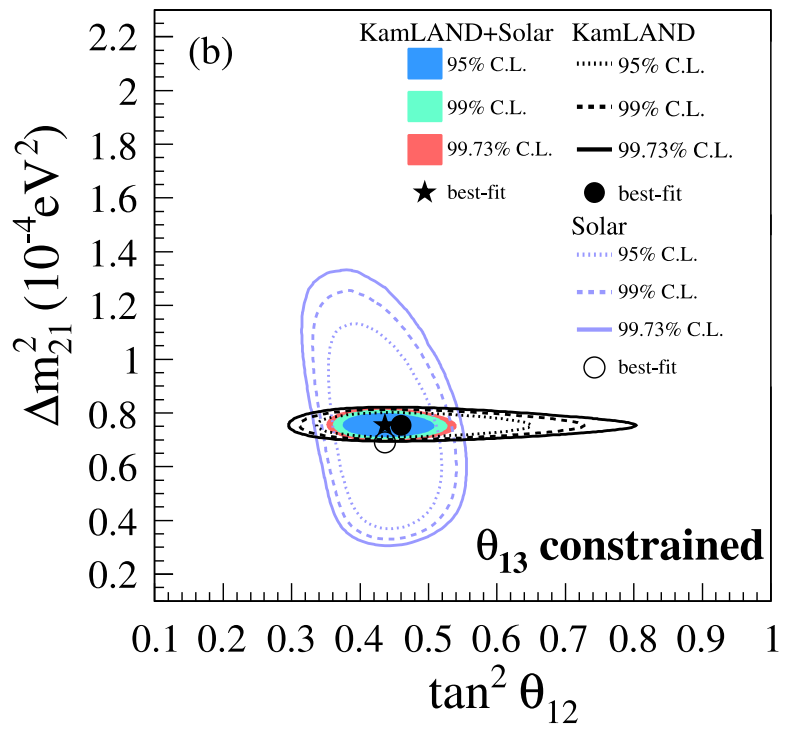

(b) Neutrinos solares y KamLAND.

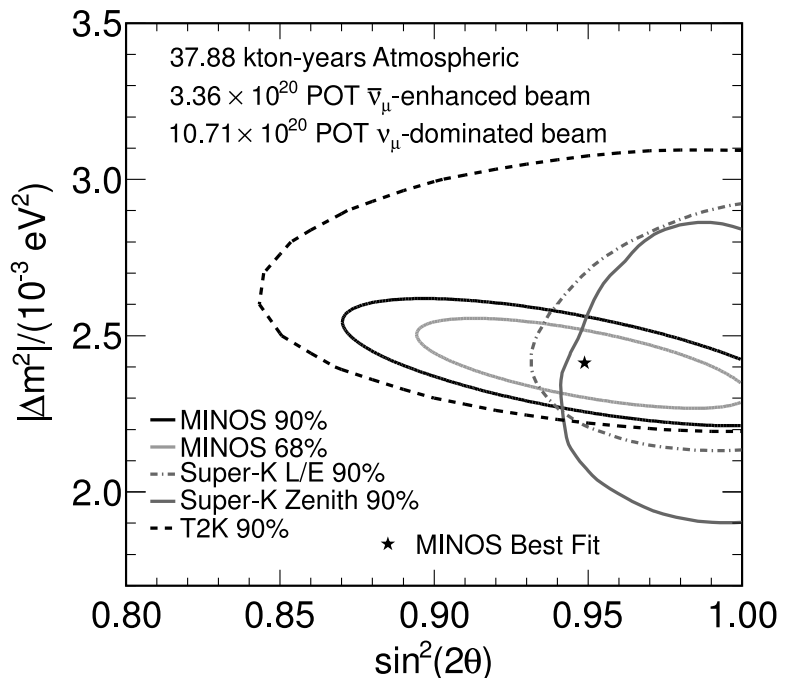

(c) Neutrinos atmosféricos, MINOS y T2K.

Figura 1.15: (a) Regiones favorecidas o excluidas de $\Delta m^{2} v s \cdot \sin ^{2} 2 \theta$ por varios experimentos basadas en análisis de oscilación de dos neutrinos. [40]. Referencias a los datos usados para esta figura pueden ser encontrados en http://hitoshi.berkeley.edu/neutrino. (b) Regiones permitidas sobre el plano $\tan ^{2} \theta_{12}-\Delta m_{21}^{2}$ para datos solares y de KamLAND para un análisis con tres neutrinos y $\theta_{13}$ constreñida por experimentos. Tomada de [45] (c) Regiones permitidas de $\left|\Delta m^{2}\right|$ vs. $\sin ^{2} 2 \theta$, asumiendo oscilaciones idénticas de neutrinos y antineutrinos. El resultado de MINOS [86] se compara al de Super-Kamiokande [116] y T2K [117].

Sin embargo, numerosos experimentos han obtenido límites de exclusión inconsistentes con lo observado por MiniBooNE y LSND. En particular el experimento con antineutrinos KARMEN [124], contemporáneo a LSND, pone un límite que excluye gran parte de las regiones permitidas por LSND y MiniBooNE, recientemente los experimentos OPERA [125] e ICARUS [126] (oscilación de neutrinos) pusieron límites que excluyen la región con $\sin ^{2} 2 \theta>10^{-2}$. Por otra parte, las distintas anomalías no parecen tener una explicación común: MiniBooNE favorece la existencia de por lo menos dos neutrinos estériles pero tal escenario sigue mostrando mucha tensión para los experimentos de desaparición.

En vista de toda esta evidencia un gran número de experimentos surgirán con el objetivo de investigar 


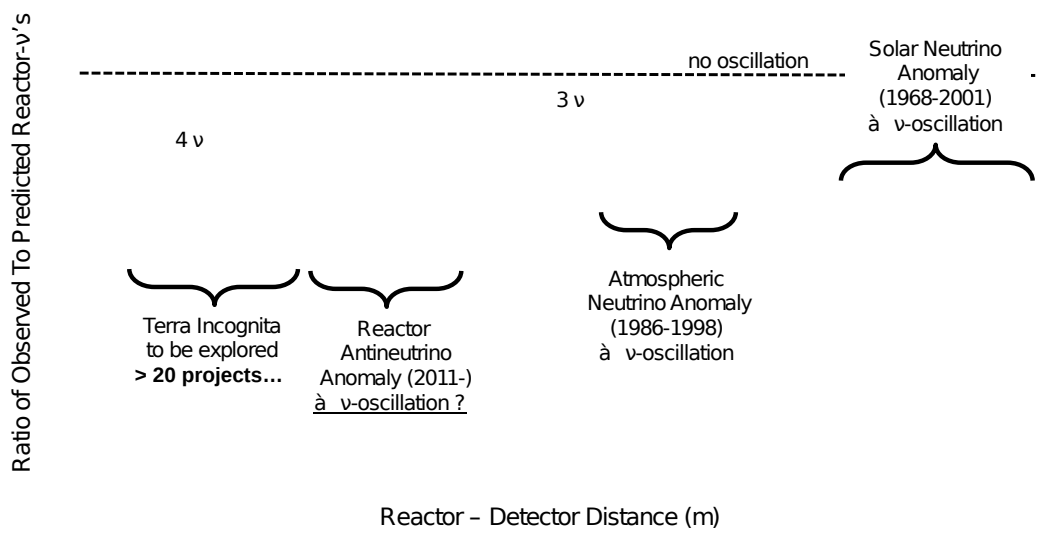

Figura 1.16: Anomalía de los antineutrinos de reactores nucleares de corta distancia. Los resultados experimentales son comparados a la predicción sin oscilación. El promedio de la tasa media, incluyendo correlaciones, es $0.943 \pm 0.023$. La línea punteada muestra una posible solución con tres neutrinos activos, mientras que la línea continua incluye un nuevo estado de masa. Estas líneas no son ajustes a los datos, solo se ponen como ilustración. Tomado de la presentación [119] sobre el artículo [118].

la posible presencia de los neutrinos estériles con una variedad de métodos y acercamientos [127]. Entre los cuales se encuentran los de fuente radioactiva SOX [128] y CeLand [129]; los de haces de aceleradores NESSiE [130], IsoDAR [131], nuSTORM [132] y MINOS+ [133]; y los de reactores Stereo, Solid, SCARR y Nucifer [134].

\subsection{LSND}

El experimento LSND (Liquid Scintillator Neutrino Detector) fue diseñado para buscar oscilaciones $\bar{\nu}_{\mu} \rightarrow \bar{\nu}_{e}$ con alta sensibilidad y para medir secciones eficaces $\nu \mathrm{C}$ [136]. Tenía la ventaja de tener una muy alta intensidad de protones, un detector de gran masa y buen sistema de identificación de partículas. El experimento KARMEN (KArlsruhe Rutherford Medium Energy Neutrino) fue contemporáneo a LSND y también presentó resultados en la búsqueda de oscilaciones. KARMEN tenía una excelente resolución de energía pero un factor de trabajo menor al de LSND. Los dos experimentos partían de un haz de alta intensidad de protones de $800 \mathrm{MeV}$ que interactuaba en un amortiguador para producir una gran cantidad de piones, la mayoría $\pi^{+}$de los cuales casi todos decaían. Los $\pi^{-}$son absorbidos y solo una pequeña fracción decae a $\mu^{-}$, que por su parte son capturados en su mayoría. Por lo tanto casi todos los neutrinos producidos provienen de decaimientos $\pi^{+} \rightarrow \nu^{+} \nu_{\mu} \mathrm{y}$ $\mu^{+} \rightarrow e^{+} \bar{\nu}_{\mu} \nu_{e}$, de los cuales la mayoría de los decaimientos (>95\%) son en reposo (decay-at-rest, DAR) y solo una pequeña fracción $(<5 \%)$ son en vuelo (decay-in-flight, DIF). Después de seis años de recolectar datos el experimento LSND obtuvo evidencia de oscilaciones de neutrinos [137] en el rango de masa $\Delta m^{2}>0.2 \mathrm{eV}^{2}$, como se muestra en la figura 1.17b. Sin embargo ese resultado no fue confirmado por el experimento KARMEN [124]. A pesar de eso se hizo un análisis conjunto de los dos experimentos [138] que revela una región de compatibilidad en una banda desde 0.2 a $1 \mathrm{eV}^{2}$ y en una región alrededor de $7 \mathrm{eV}^{2}$.

El experimento LSND observó un exceso de eventos sobre el fondo fuera del haz y el fondo de neutrinos que es consistente con oscilaciones de neutrinos. Se tiene un exceso dentro y fuera del 


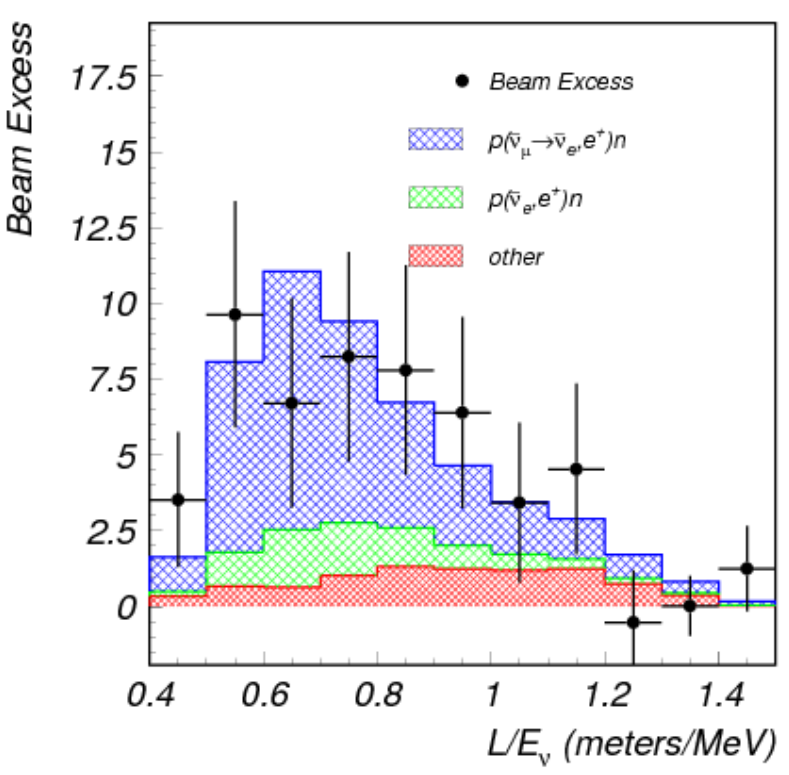

(a) Exceso de eventos como función de $L / E_{\nu}$.

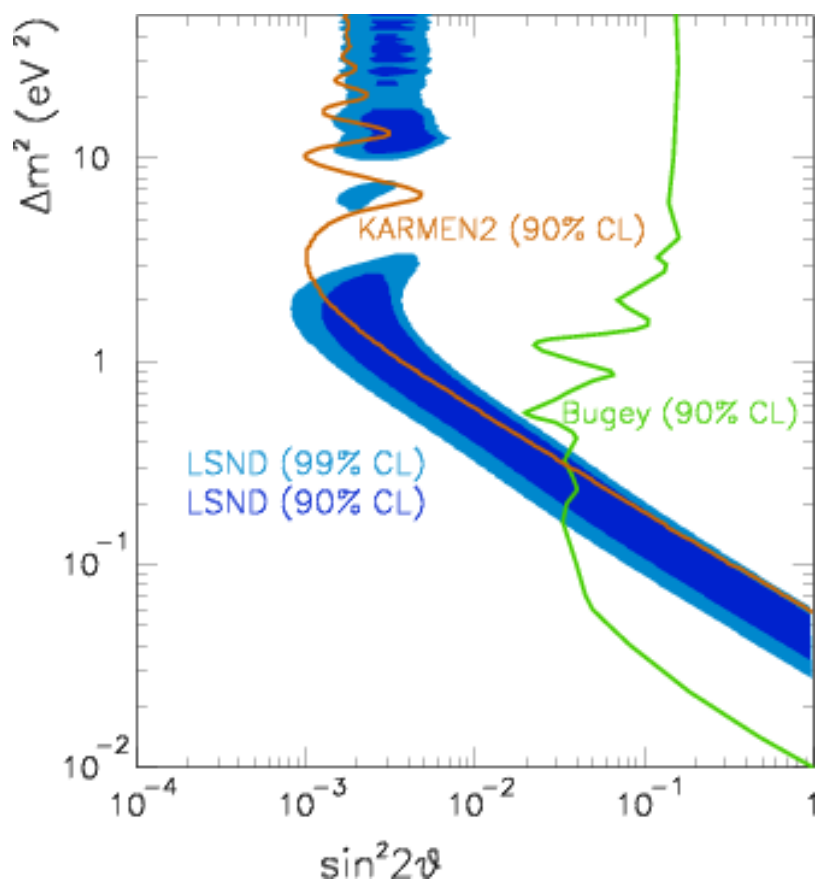

(b) Ajuste de oscilaciones sobre $\left(\sin ^{2} 2 \vartheta, \Delta m^{2}\right)$.

Figura 1.17: Resultados del experimento LSND: (a) Exceso de eventos como función de $L / E_{\nu}$ en el rango de energía $20<E_{e}<60 \mathrm{MeV}, L$ es la distancia que viajaron los $\nu$ en metros y $E_{\nu}$ es la energía de los $\nu$ en $\mathrm{MeV}$. Los datos concuerdan bien con el fondo esperado y oscilaciones de neutrinos para $\Delta m^{2}$ baja. (b) Ajuste de oscilaciones en el espacio de parámetros $\left(\sin ^{2} 2 \vartheta, \Delta m^{2}\right)$ para la muestra completa de datos de LSND de $20<E<200 \mathrm{MeV}$. Las regiones sólidas corresponden a las regiones permitidas de LSND, mientras que las curvas son los límites de exclusión de $90 \%$ N.C. de los experimentos Bugey y KARMEN en ISIS.

haz de $117.9 \pm 22.4$ con neutrones en coincidencia. Substrayendo el fondo de neutrinos provenientes de $\mu^{-}$DAR $(19.5 \pm 3.9$ eventos $)$ y el de $\pi^{-}$DIF $(10.5 \pm 4.6 \text { eventos })^{23}$ lleva a un exceso total de $87.9 \pm 22.4 \pm 6.0$ eventos significativos a $3.8 \sigma$. Este exceso corresponde a una probabilidad de oscilación de $0.264 \pm 0.067 \pm 0.045 \%$, donde el primer error es estadístico y el otro es sistemático derivado de incertidumbres en los fondos, flujo de neutrinos $(7 \%)$, eficiencia de $e^{+}(7 \%)$ y eficiencia de $\gamma(7 \%)$.

A través de un análisis estadístico, sobre el espacio de parámetros $\left(\sin ^{2} 2 \vartheta, \Delta m^{2}\right)$, a los eventos en el haz de la muestra final de oscilación, se obtienen los parámetros favorecidos para la oscilación de neutrinos. El análisis permite obtener las regiones que permiten oscilación de neutrinos por LSND. Los fondos relacionados al haz se determinan con muestras Monte Carlo (MC) para cada contribución individual al fondo. El ajuste de oscilaciones sobre el espacio $\left(\sin ^{2} 2 \vartheta, \Delta m^{2}\right)$ para toda la muestra de datos de $20<E_{e}<200 \mathrm{MeV}$ se muestra en la figura 1.17b. El ajuste incluye oscilación $\bar{\nu}_{\mu} \rightarrow \bar{\nu}_{e} \mathrm{y}$ $\nu_{\mu} \rightarrow \nu_{e}$, así como todos los fondos conocidos de neutrinos. La región sólida interna corresponde a la región permitida de $90 \%$ N.C. mientras que la externa corresponde a la de $99 \%$ N.C. Las curvas son los límites de exclusión de $90 \%$ N.C. del experimento de reactor Bugey [97] y el experimento KARMEN en ISIS [124]. La región más favorecida es la banda 0.2 a $1.0 \mathrm{eV}^{2}$, aunque una región alrededor de $7 \mathrm{eV}^{2}$ también es posible.

El experimento LSND es el precursor de MiniBooNE, de hecho el objetivo principal de MiniBooNE era corroborar o refutar el resultado obtenido por LSND, el cual, apunta a la existencia de más sabores de neutrinos; llamados neutrinos estériles.

\footnotetext{
${ }^{23}$ También incluye el fondo de interacciones $\nu_{\mu}\left(\bar{\nu}_{\mu}\right)+\mathrm{C} \rightarrow \mu^{-}\left(\mu^{+}\right)+n \mathrm{X}$.
} 


\section{Capítulo 2}

\section{Experimento MiniBooNE}

MiniBooNE (Mini ${ }^{1}$ Booster Neutrino Experiment) es un experimento de oscilación de neutrinos de corta distancia. Surgió con el objetivo de comprobar o refutar los resultados presentados por el experimento LSND [141] (ver §1.5). MiniBooNE recolectó datos de 2002 a 2012. Se encuentra en Fermilab (Fermi National Accelerator Laboratory), un laboratorio mundialmente reconocido dedicado a la física de altas energías ubicado en el estado de Illinois en Estados Unidos.

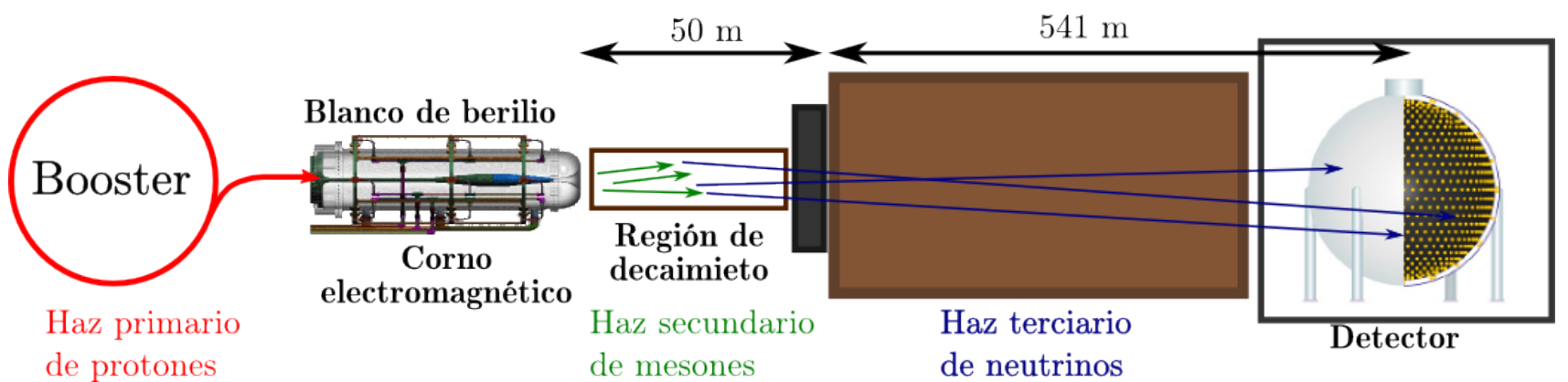

Figura 2.1: Vista esquemática del experimento MiniBooNE. El acelerador Booster manda protones de 8 GeV hacia el blanco de MiniBooNE, el cual se encuentra dentro del corno magnético. Los mesones creados de la interacción del haz primario de protones son enfocados/desenfocados en el corno magnético, formando el haz secundario. Estos mesones decaerán más adelante para formar el haz terciario de neutrinos. La componente hadrónica será detenida en el tope del haz, pero los neutrinos seguirán viajando por la tierra hasta llegar al detector, donde algunos serán detectados.

Una vista esquemática del experimento MiniBooNE se muestra en la figura 2.1. Los protones de $8 \mathrm{GeV}$ de energía provenientes del sincrotrón Booster inciden sobre un blanco de berilio (Be), el cual se encuentra dentro de un corno magnético. De la interacción de los protones con el berilio se generan mesones que son enfocados y direccionados con el corno; estos mesones decaerán en neutrinos los cuales viajaran una distancia aproximada de $541 \mathrm{~m}$ hasta el detector. La energía media de los neutrinos obtenidos, en el marco de referencia del detector, es de cientos de $\mathrm{MeV}$. Al cambiar la polaridad del corno se puede elegir entre un haz de alta intensidad primordialmente formado por neutrinos del muón $\nu_{\mu}$ o uno formado de antineutrinos del muón $\nu_{\mu}$. MiniBooNE hizo búsquedas de

\footnotetext{
${ }^{1}$ Se llama "Mini" porque inicialmente era la primera fase de un experimento con dos detectores, BooNE. Sin embargo nunca se aprobó la construcción del segundo detector.
} 
oscilación de neutrinos y antineutrinos por aparición: $\nu_{\mu} \rightarrow \nu_{e}$ y $\bar{\nu}_{\mu} \rightarrow \bar{\nu}_{e}$. Es decir de un haz de $\nu_{\mu}$ $\left(\bar{\nu}_{\mu}\right)$ se busca un exceso en el fondo intrínseco esperado de $\nu_{e}\left(\bar{\nu}_{e}\right)$.

Usando una proporción similar de distancia entre energía, $L[\mathrm{~m}] / E[\mathrm{MeV}]$, a aquella que uso LSND, se tiene sensibilidad a la misma región de oscilaciones de neutrinos de $\Delta m^{2} \sim 1 \mathrm{eV}^{2}$. De hecho la energía y distancia características hacen al experimento más sensible a oscilaciones de dos neutrinos con una diferencia de masa $>0.01 \mathrm{eV}^{2}$ [141]. Es fundamental disponer tales parámetros de la forma que se hizo ya que la probabilidad de oscilación es muy pequeña, según los resultados sugeridos por LSND, $\sim 0.25 \%$. Es importante notar que a pesar de que la proporción se mantuvo similar la distancia y la energía se elevaron dos ordenes de magnitud a como eran en LSND. Consiguiendo de esa forma cambiar el método de detección y los errores sistemáticos asociados.

Típicamente las incertidumbres en el flujo de haces de neutrinos generados con aceleradores son más de un orden de magnitud mayores a cualquier efecto esperado por fenómenos de oscilación. Esto hace que una búsqueda de oscilación por desaparición de $\nu_{\mu}\left(\bar{\nu}_{\mu}\right)$ sea sumamente desafiante para solamente un detector. Sin embargo para el caso de una búsqueda de oscilación por aparición en la cual la contaminación intrínseca de $\nu_{e}\left(\bar{\nu}_{e}\right)$ es $<1 \%$, junto con una medición de la componente rica en estadística de $\nu_{\mu}\left(\bar{\nu}_{\mu}\right)$ del haz, usada para constreñir el error sistemático del flujo y la sección eficaz, se puede lograr suficiente sensibilidad al proceso de baja amplitud $\nu_{\mu} \rightarrow \nu_{e}\left(\bar{\nu}_{\mu} \rightarrow \bar{\nu}_{e}\right)$. Más aún se requiere un haz de neutrinos (antineutrinos) de alta intensidad para generar suficiente estadística de eventos $\nu_{\mu} \rightarrow \nu_{e}\left(\bar{\nu}_{\mu} \rightarrow \bar{\nu}_{e}\right)$ y un detector con la capacidad para detectar eficazmente eventos $\nu_{e}\left(\bar{\nu}_{e}\right)$ sin confundirlos con $>99 \%$ de $\nu_{\mu}\left(\bar{\nu}_{\mu}\right)$ para que la tarea de buscar oscilaciones de neutrinos en la escala de $1 \mathrm{eV}^{2}$ sea factible.

\subsection{El haz de neutrinos del Booster}

Como se puede apreciar en la figura 2.1 la línea del haz del Booster (BNB por sus siglas en inglés: Booster Neutrino Beamline) está divida en tres etapas:

- Un haz primario de protones extraídos del Booster que inciden en un blanco de berilio.

- Un haz secundario de mesones formados por las colisiones de los protones del haz primario con los núcleos del berilio del blanco.

- Un haz terciario compuesto principalmente de neutrinos, originados del decaimiento de los mesones del haz secundario a lo largo de un túnel.

\subsubsection{El haz primario de protones}

La disposición esquemática del BNB se discute con gran detalle en el reporte técnico del diseño [142] y en la figura 2.2 se muestra una foto aérea con los puntos clave. Para generar los neutrinos que detecta MiniBooNE se parte de un haz de protones los cuales surgen de una botella con hidrógeno que pasan por un acelerador Cockcroft-Walton ${ }^{2}$, estos protones son acelerados en un acelerador lineal (linac) de $150 \mathrm{~m}$ de largo y de ahí se meten al Booster, un sincrotrón de $468 \mathrm{~m}$ de circunferencia, saliendo del Booster se dirigen al Main Injector, otro sincrotrón de protones, antes de ser acelerados los protones

${ }^{2}$ En 2012 se desmantelo este acelerador para poner uno más moderno de mayor intensidad. 
son "pateados" hacia el edificio MI-12 (Main Injector 12). El Booster entrega protones con energía cinética de $8 \mathrm{GeV}$ (momento de $8.9 \mathrm{GeV} / \mathrm{c}$ ) agrupados con la siguiente estructura: $6 \times 10^{10}$ protones forman un bunch, cada bunch tiene un ancho de 2 ns y la separación entre cada bunch es de 18 ns, se tienen aproximadamente 82 bunchs en un spill. Un spill dura $1.6 \mu$ s y tiene $4.9 \times 10^{12}$ protones. En 1 s se tienen de 4 a 10 spills, si los demás experimentos que operan en Fermilab están apagados se suele tener mayor intensidad. En la figura 2.3 se muestra un dibujo con esta estructura.

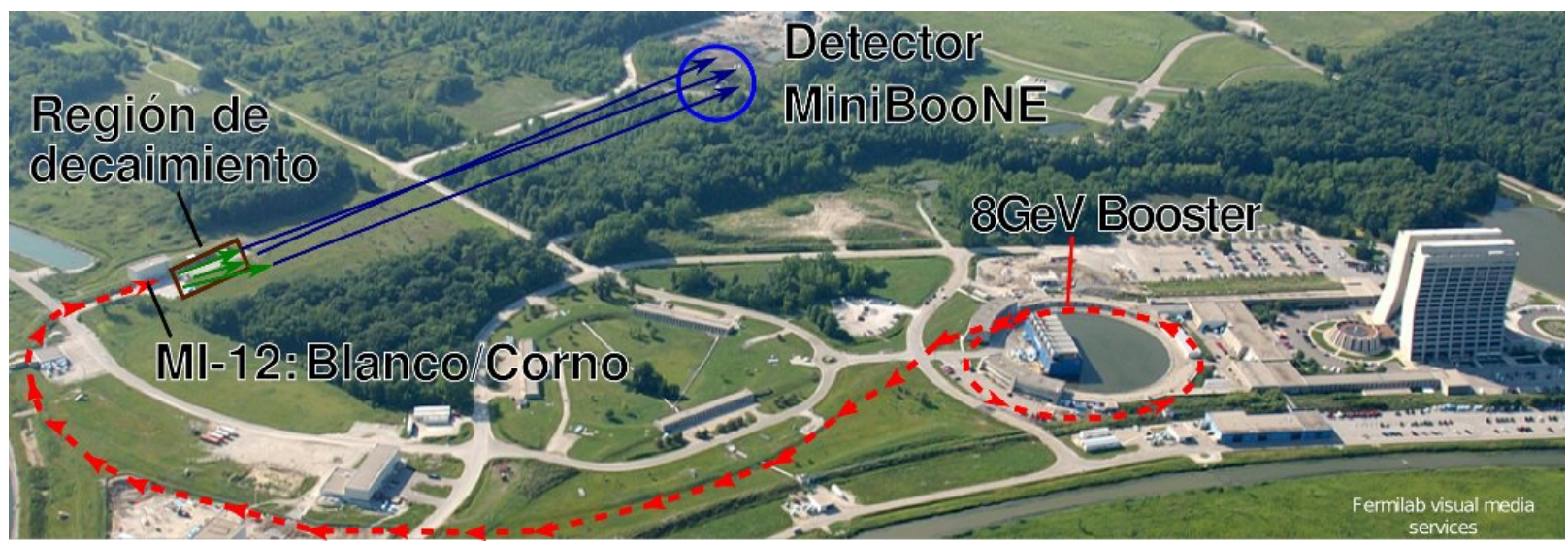

Figura 2.2: Vista aérea de la línea del haz del del experimento MiniBooNE. Protones con 8 GeV salen del sincrotrón Booster hacia el edificio MI-12 en donde se encuentra el corno magnético y dentro el blanco de berilio. Los mesones que surgen de la interacción con los protones decaen más adelante en neutrinos que llegan al detector.

La cantidad absoluta de protones en el blanco (POT: Protons On Target), su posición, perfil y tiempo son constantemente monitoreados con toroides, monitores de posición de haz, y cámaras multialámbricas, respectivamente. Para evitar que el haz se mueva demasiado de su posición nominal y se estrelle contra el material del corno, un colimador con forma de dona y un monitor de pérdida de haz están instalados en el extremo del blanco para tirar el haz si es necesario. Un programa de autoenfoque [143] la posición y ángulo del haz en tiempo real y minimiza cualquier desalineado.

\subsubsection{El blanco de protones}

Localizado en la parte subterránea de MI-12 y dentro de una caja de acero, para aislar la radiación, se encuentra el corno magnético y a lo largo del eje de este, el blanco de berilio; los cuales se muestran en la figura 2.4a. Las especificaciones del blanco se encuentran en el correspondiente reporte técnico del detector y el artículo sobre el funcionamiento del detector [144, 145] . El blanco de berilio mide $71.1 \mathrm{~cm}$ de largo (la longitud equivalente a 1.7 interacciones de protón) y $0.96 \mathrm{~cm}$ de diámetro. Compuesto de 7 pastillas de berilio (cada una de $\sim 10 \mathrm{~cm}$ de largo) y rodeado por una "manga" de berilio de $0.9 \mathrm{~cm}$ de espesor. Alrededor de las mangas se colocan tres aletas radiales dispuestas de forma simétrica, i.e. separadas a $120^{\circ}$ (no se muestran en la figura), para soportar el blanco a la vez que permiten que se enfríe el mismo al circular aire. El haz de protones impacta en la cara frontal del blanco en un área de $\sigma_{x}=1.51 \mathrm{~mm}$ y $\sigma_{y}=0.75 \mathrm{~mm}$; y se encuentra alineado con el eje del corno y el del blanco.

El berilio fue específicamente escogido como el blanco de los protones debido a su bajo número atómico $(Z=4)$, ya que minimiza las pérdidas de energía por radiación que sufren los protones incidentes, 


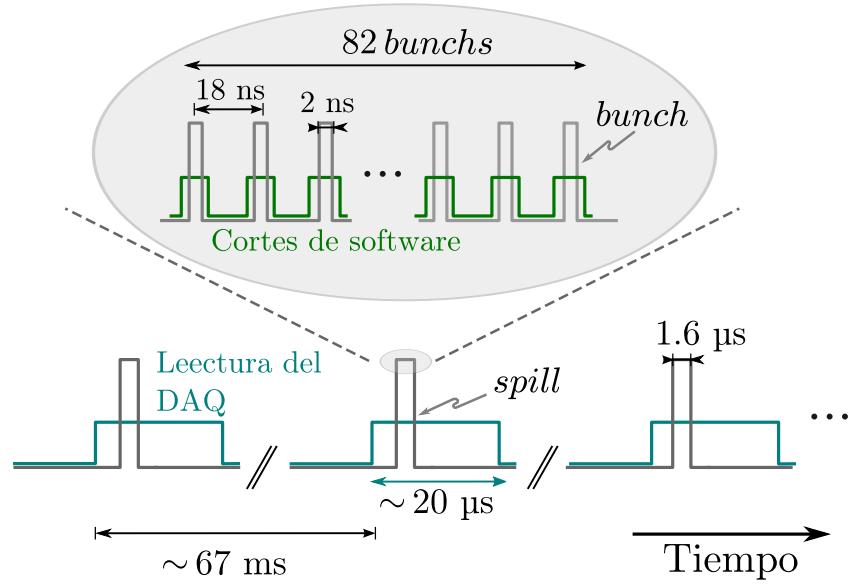

Figura 2.3: Estructura del haz de protones entregados por el Booster. $6 \times 10^{10}$ protones de 8 Gev forman un bunch con ancho de 2 ns, los cuales están separados 18 ns. 82 bunchs forman un spill, el cual dura $1.6 \mu$ s y tiene $4.9 \times 10^{12}$ protones. En 1 s se tienen de 4 a 10 spills. También se muestran las ventanas de adquisición de datos y los cortes de datos que hace el software.

antes de que interactúen con el blanco y la absorción de los mesones secundarios $\pi \pm, K \pm, K_{0}$.
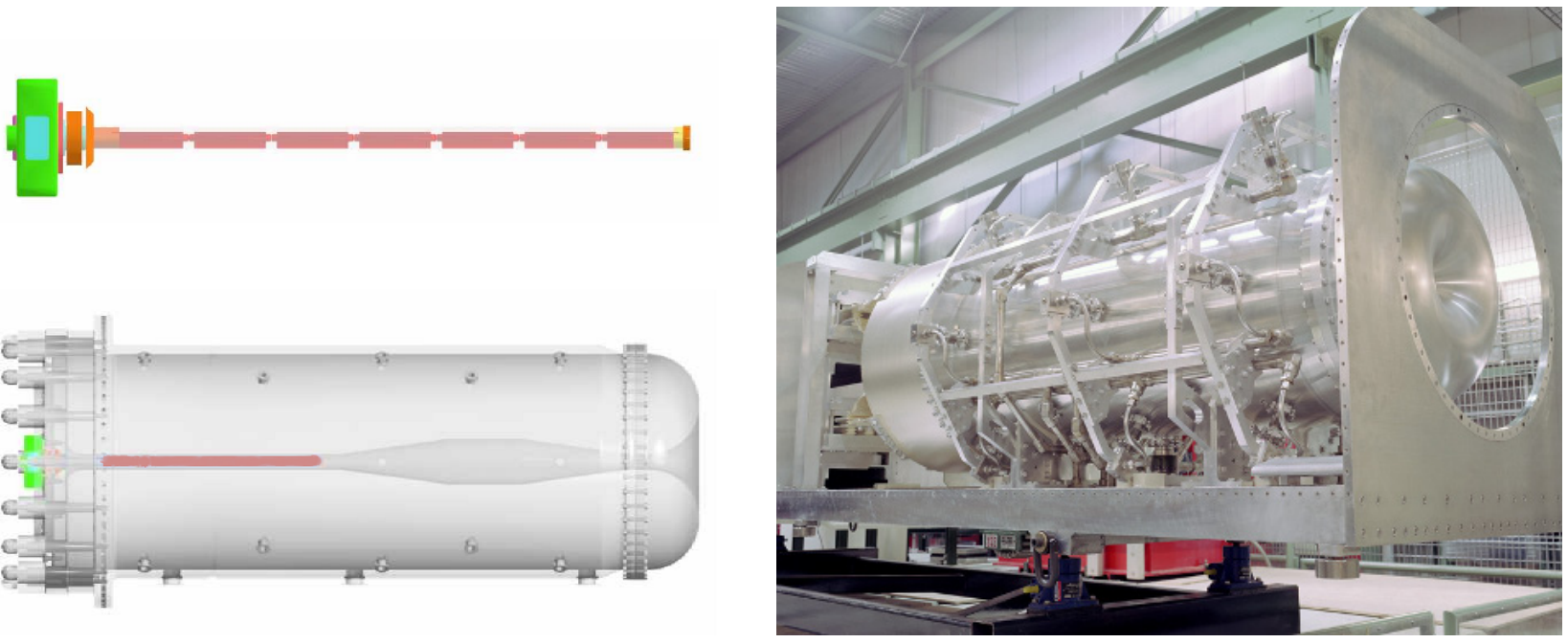

(a) Blanco de berilio (arriba) y su posición dentro del corno (abajo).

(b) Fotografía del corno magnético.

Figura 2.4: Corno magnético. Con el campo magnético toroidal generado en este se enfocan los mesones originados de las colisiones de los protones con el blanco, gracias a ello se pueden dirigir los neutrinos hacia el detector.

\subsubsection{Enfocado de mesones secundarios}

El corno magnético ${ }^{3}$ esta formado por dos corazas concéntricas de aluminio conectadas en el fondo con una tapa con forma de medio toro. Se pulsa la corriente del corno para coincidir con las colisiones

\footnotetext{
${ }^{3}$ Diseñado por Bartozsek Engineering. [146]
} 
del los protones con el blanco, produciendo un campo magnético que enfoca los mesones de corta vida que son creados de la interacción del haz primario con el blanco.

En modo de neutrinos la corriente operacional del corno tiene un pico de $174 \pm 1 \mathrm{kA}(-174 \pm 1 \mathrm{kA}$ en modo de antineutrinos) y fluye a través de la coraza interna y de vuelta por la coraza exterior produciendo de esa forma un campo magnético toroidal en el volumen entre los dos conductores coaxiales, con intensidad $B[\mathrm{kG}]=I[\mathrm{kA}] / 5 R[\mathrm{~cm}]$ con incertidumbre de $5 \%$ y dirección azimutal $[147]^{4}$. La corriente y forma del corno fueron optimizadas usando GEANT [148] para maximizar el flujo de $\nu_{\mu}$ entre $0.5-1 \mathrm{GeV}$ en el detector y minimizar el flujo por encima de $1 \mathrm{GeV}$; además de maximizar la pureza en la selección de signo del haz de neutrinos; i.e. minimiza la cantidad de antineutrinos en el modo de neutrinos y viceversa. Es importante aclarar que el campo magnético dentro de la coraza interior y fuera de la coraza exterior es despreciable. La figura $2.4 \mathrm{~b}$ muestra una foto del corno magnético, en la cual se puede apreciar el orificio por el cual desalojan los protones y mangueras con las que se regula su temperatura.

MiniBooNE corrió en modo de neutrinos desde septiembre de 2002 hasta diciembre de 2006, luego de octubre de 2007 hasta abril de 2008. En Enero de 2006 y hasta julio de 2007 empezó a correr en modo de antineutrinos; i.e. entre las corridas de neutrinos, reasumiendo en abril de 2008 y hasta abril de 2012. En total se cuenta se cuenta con datos correspondientes a $6.46 \times 10^{20}$ POT en el modo de neutrinos y con $11.27 \times 10^{20}$ en el modo de antineutrinos.

\subsubsection{El conducto de decaimiento de mesones secundarios}

Delante del blanco se encuentra un colimador de concreto de $214 \mathrm{~cm}$ de largo, con una apertura de $30 \mathrm{~cm}$ (en la cara posterior) a $35.5 \mathrm{~cm}$ (en la cara anterior) de radio. El colimador absorbe partículas secundarias que salen con ángulos grandes con respecto a la dirección del haz, de cualquier forma estás partículas no contribuirían de forma significativa al haz de neutrinos observado en el detector. Inmediatamente después del colimador, los mesones secundarios entran al conducto de decaimiento, de $50 \mathrm{~m}$ de largo y rellena con aire a presión y temperatura ambiente. Los mesones cargados decaen en vuelo produciendo neutrinos y otras partículas terciarias. Un conjunto de 10 placas retráctiles de acero y concreto se encuentran suspendidas por encima del conducto de decaimiento. Cuyo propósito es estudiar los errores sistemáticos del haz, mediante las modificaciones en el flujo de neutrinos proveniente de partículas de vida larga. Debido a la corrosión ocasionada por la exposición a la radiación dos placas se cayeron accidentalmente en diciembre de 2006 y permanecieron en el conducto de decaimiento durante parte de la corrida de antineutrinos.

Al final de la región de decaimiento se encuentra el vertedero de haz, $50 \mathrm{~m}$ después del blanco una pared de acero y concreto absorbe la componente hadrónica del haz; primordialmente los protones del haz primario que no interactuaron y los mesones que no han decaído, los neutrinos por su parte continúan su recorrido hacia el detector. La distancia de $50 \mathrm{~m}$ fue elegida para reducir los decaimientos de muones de larga vida, que de otra forma contribuirían al error sistemático de $\nu_{e}$ en la búsqueda. Dentro del conducto de decaimiento se encuentra el Little Muon Counter (LMC) un espectrómetro fuera de eje que mide la tasa y espectro de los muones producidos a un ángulo de $7^{\circ}$ del eje del haz. Más detalles del LMC se pueden consultar en [149].

\footnotetext{
${ }^{4}$ las líneas de campo son toroidales rodeando a la coraza interna
} 


\section{2. $\quad$ El detector}

El detector es un tanque esférico de $6.1 \mathrm{~m}$ de radio relleno con $\sim 950000$ litros de aceite mineral ultra puro Exxon/Mobil Marcol $7\left(\mathrm{CH}_{2}\right)$, con una densidad de $0.845 \mathrm{~g} / \mathrm{cm}^{3}$ y un índice de refracción de 1.47 el cual actúa tanto como blanco para los neutrinos, así como medio de propagación de las partículas resultantes de la interacción de los neutrinos con el aceite. Se encuentra a $541 \mathrm{~m}$ del centro del blanco y su centro esta desplazado verticalmente $1.9 \mathrm{~m}$ del eje del haz, definido por el centro del conducto de decaimiento. Esta dividido en dos regiones concéntricas: una interior de $5.5 \mathrm{~m}$ de radio y una capa exterior de $6.1 \mathrm{~m}$ de radio. La región interior es observada por 1280 tubos fotomultiplicadores (PMT por sus siglas en inglés) que observan hacia el centro del tanque, es en esta región que se observan las interacciones buscadas, por lo mismo se le llama región de señal. Las paredes de la región de señal están pintadas de negro para evitar que la luz se refleje lo que dificultaría la reconstrucción de eventos. Por su parte la región exterior cuenta con 240 PMT's apuntando en dirección tangencial a la pared del tanque, estos detectan la luz producida por partículas cargadas que penetran desde el exterior; e.g. rayos cósmicos, proveen un mecanismo extra para la detección de ruido: por eso se le llama región de veto esta pintada de blanco para maximizar la detección de partículas que la atraviesan. Hay una discriminación de > 99.99\% de eventos cósmicos de fondo [145]. En la figura 2.5 se pueden observar fotos de la región de veto y señal, así como un dibujo a escala del tanque comparado con una persona. La cobertura que tienen los PMT's para un detector con interior líquido es proporcional a la superficie del detector, se escogió un tanque esférico por que es el que maximiza la proporción entre volumen y área.

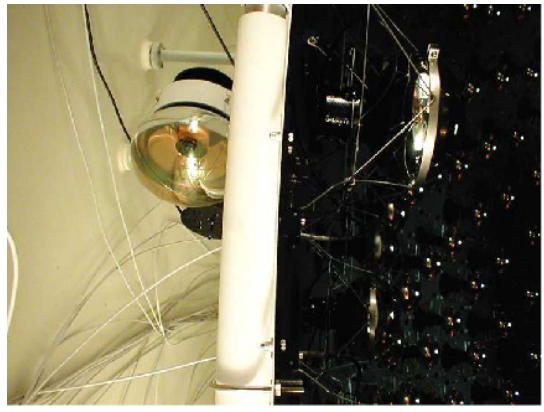

(a) Fotografía de la región interior (blanco) y exterior (negro) del detector.

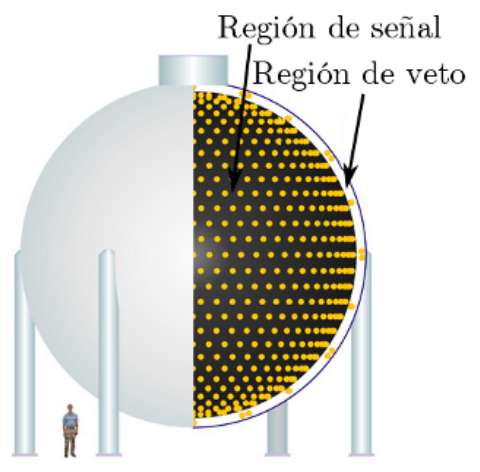

(b) Dibujo del detector MiniBooNE, notar la comparación con una persona.

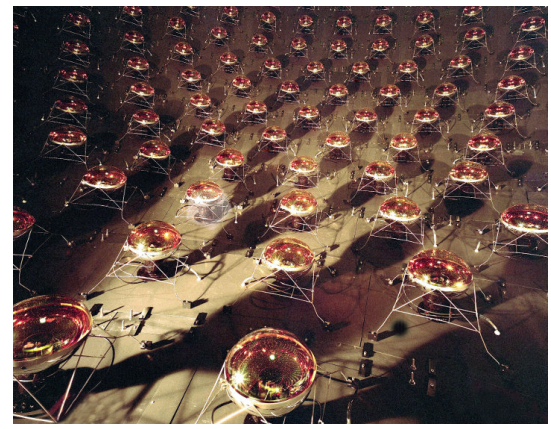

(c) Fotografía de la región de señal.

Figura 2.5: El detector MiniBooNE es un tanque relleno de aceite mineral de $6.1 \mathrm{~m}$ de radio que cuenta con una región interior de señal pintada de negro con un radio interior de $5.5 \mathrm{~m}$ y una región exterior de veto pintada de blanco. Los fotomultiplicadores detectan radiación de cherenkov y centelleo producida por las partículas cargadas que pasan por el aceite, en la región de veto se detectan las partículas cargadas que entran al tanque y en la parte interior es donde se detectan las interacciones buscadas.

El detector esta especialmente diseñado para detectar partículas cargadas relativistas que emiten radiación cherenkov [150]. Una partícula cargada que viaja a una velocidad mayor a la que lo haría la luz $\left(\sim 1.93 \times 10^{8} \mathrm{~m} / \mathrm{s}\right)$ en el aceite emite radiación cherenkov en forma de conos de luz. Los PMT's también son sensibles a la luz isotrópica emitida a través de procesos de centelleo en el aceite, es decir a la luz emitida en los procesos de fluorescencia.

Notemos que el índice de refracción del aceite es más alto que el del agua (1.33) y que su densidad es 
menor, por lo cual los electrones emiten mucha más radiación cherenkov en el aceite que en el agua. Más aún, la baja velocidad de la luz en el aceite mejora la reconstrucción de la posición de los eventos. El aceite permite la detección de muones, piones y protones de baja energía usados para rechazar eventos espurios; ya que tiene un menor umbral cherenkov y por la presencia de luz de centelleo. El aceite mineral tiene menor dispersión múltiple que el agua y una menor tasa de captura de $\mu, 8 \%$ para el aceite y $20 \%$ para el agua. La menor tasa de captura incrementa la eficiencia de la identificación de reacciones que involucran partículas cargadas. Además en el aceite se pueden sumergir componentes eléctricas sin peligro. La desventaja del aceite es que el modelo óptico que requiere para describir la generación y transmisión de la luz es mucho más complicado (§2.4.3).

El edificio que alberga al detector y la electrónica asociada, figura 2.6, se localiza debajo de un montículo de $3 \mathrm{~m}$ de tierra que sirve como blindaje de rayos cósmicos y mantiene la tasa de rayos cósmicos que pasan por el tanque por debajo de los $10 \mathrm{kHz}$, también sirve como aislante térmico para ayudar al aire acondicionado a mantener una temperatura constante en todo el edificio.

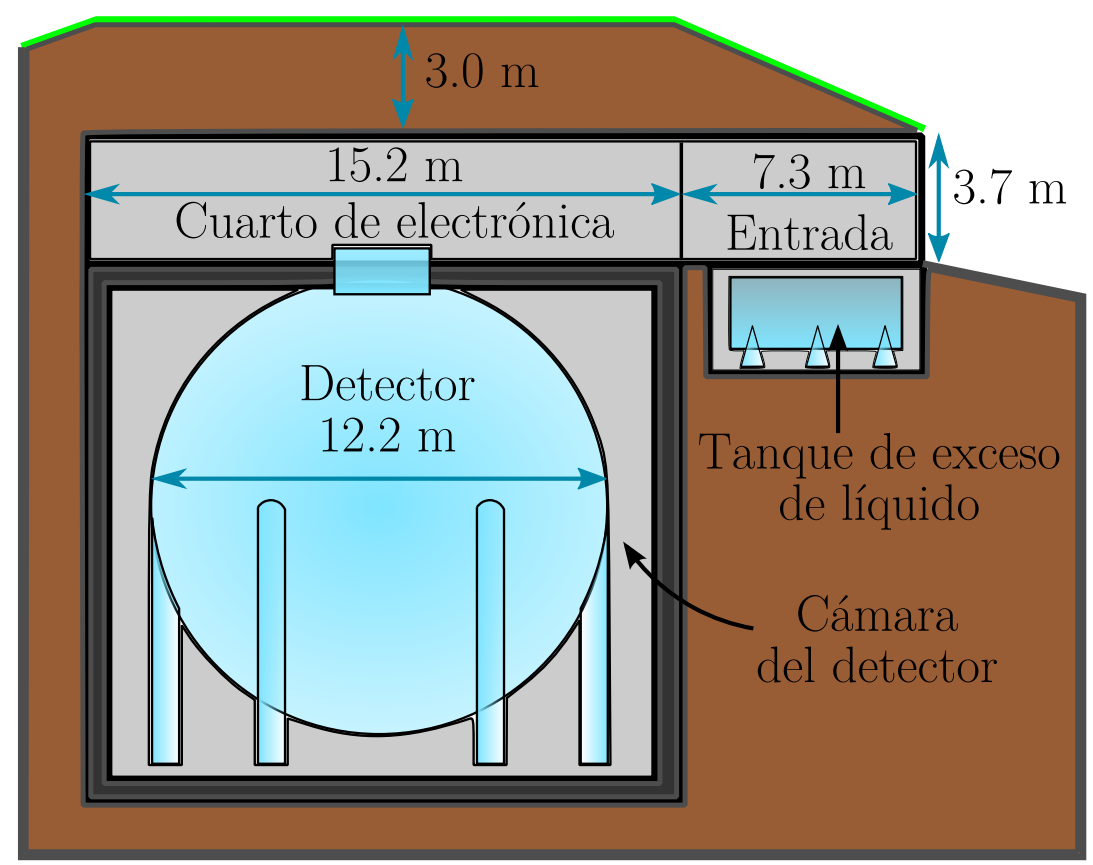

Figura 2.6: Dibujo esquemático del recinto del detector MiniBooNE. El detector esta localizado dentro de una cámara cilíndrica subterranea de $13.7 \mathrm{~m}$ de diámetro. La electrónica del detector se almacena en un cuarto superior a la cámara del tanque y por debajo de un montículo de tierra de $3 \mathrm{~m}$, que blinda el detector de muones cósmicos y lo aisla de variaciones de temperatura. También se muestra el tanque de exceso de líquido que permite la expansión térmica y recirculación del aceite en el tanque de MiniBooNE. El acceso al cuarto de electrónica es a través de una puerta que se muestra a la derecha y de ahí se puede bajar a la cámara del detector.

\subsubsection{Electrónica y sistemas de adquisición}

El experimento usa 1197 PMT's Hamamatsu R1408 usados previamente en el experimento LSND además de 323 nuevos Hamamatsu R5912. Los dos tipos de PMT's miden 8 pulgadas de diámetro y están fabricados con vidrio de baja radiactividad, la principal diferencia es que el modelo R5912 tiene una cadena de dinodos de 10 etapas mientras que el R1408 solo tiene 9 etapas [151]. El voltaje de operación de cada PMT's se ajusta con una serie de resistencias en las entradas de los preamplificadores, 
permitiendo el uso de suministros fijos de voltaje para operar todos los tubos.

Cuando un fotón llega a un tubo se genera un fotoelectrón por efecto fotoeléctrico el cual es multiplicado sucesivamente por los dinodos de los fotomultiplicadores. Cada tubo entrega dos voltajes análogos, uno que es proporcional a la carga del pulso $(V q)$ y el otro proporcional al tiempo $(V t)$ por separado. Se lee información cuando la carga almacenada es superior a lo equivalente a 0.1 fotoelectrones. Se usan tarjetas para llevar a cabo la conversión de carga-tiempo (en inglés llamadas QT board) y convertir los pulsos analógicos en digitales (conversión analógica-digital CAD) a $10 \mathrm{MHz}$ (ver figura 2.7), los cuales son almacenados en intervalos de $200 \mu$ s posteriores a una señal disparador trigger. Una sola QT board se encarga de procesar la información de 8 PMT's. En la figura 2.8a se pueden observar las QT boards.

El flujo de datos para el detector consiste de la siguiente información para cada impacto de los PMT's:

1. El número del canal del PMT.

2. El tick del reloj, contado desde el principio del evento que precede el disparador del discriminador.

3. Cuatro valores almacenados de $V q$ en conteo de CAD: charge quad.

4. Cuatro valores almacenados de $V t$ en conteo de CAD: time quad.

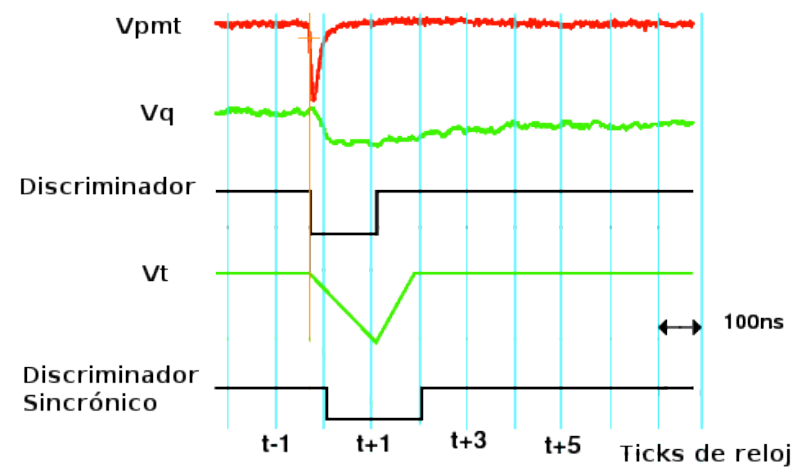

Figura 2.7: Captura de carga y tiempo para un pulso de PMT. $V p m t$ es el pulso del ánodo y $V q$ la carga integrada. La señal digital del discriminador es creada cuando la señal del ánodo atraviesa cierto umbral predeterminado. $V t$ es una señal analógica que empieza con el discriminador. La traza del discriminador sincrónico representa una señal que puede ser usada por la electrónica de disparo. $V q$ y $V t$ son señales analógicas que son digitalizadas por convertidores a los tiempos de muestreo representados por las líneas verticales, etiquetados por los ticks de reloj [144].

Cuando un impacto en un PMT satura el rango CAD (teniendo más de veinte fotoelectrones) el sistema de adquisición de datos (DAQ por sus siglas en inglés) escribe charge quads adicionales hasta que $V q$ es suficientemente chico para entrar en el rango del CAD. Los quads de carga y tiempo son usados para extraer el tiempo relativo en el cual cada impacto ocurrió con respecto a los otros impactos de un evento dado y el número de fotoelectrones que corresponden a cada impacto. El tiempo y carga de un impacto son calculados de la siguiente forma:

$$
\begin{aligned}
t & =t_{0}+(100 n s) \times N_{\text {tick }}+\Delta t_{\text {off }}+\Delta t_{q}\left(Q_{0}\right) \\
q & =Q_{0} / \text { Ganancia }
\end{aligned}
$$

donde $t_{0}$ es el tiempo del impacto relativo al tick del reloj anterior, el cual se obtiene de la pendiente de la rampa de la señal $V t$ y su intersección con la línea de base. $N_{\text {tick }}$ es el número de ticks de reloj que 
preceden el trigger del discriminador y es la medida gruesa del tiempo del trigger. El término $\Delta t_{\text {off }}$ es una calibración constante independiente del canal que quita diferencias de tiempo provenientes de diferencias en las longitudes de los cables y la cantidad de dinodos. El término $\Delta t_{q}\left(Q_{0}\right)$ es una corrección que depende de la carga en el ánodo del PMT y se encuentra en tablas. La ganancia de los PMT's y la corrección del tiempo dependiente de la carga se determinan con el sistema de calibración del láser.

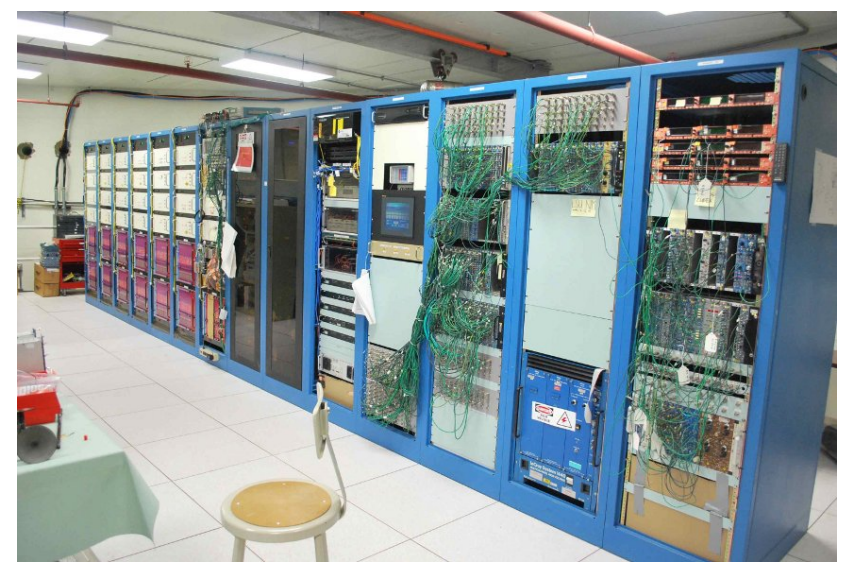

(a) Paneles de la electrónica involucrada en la adquisición de datos.

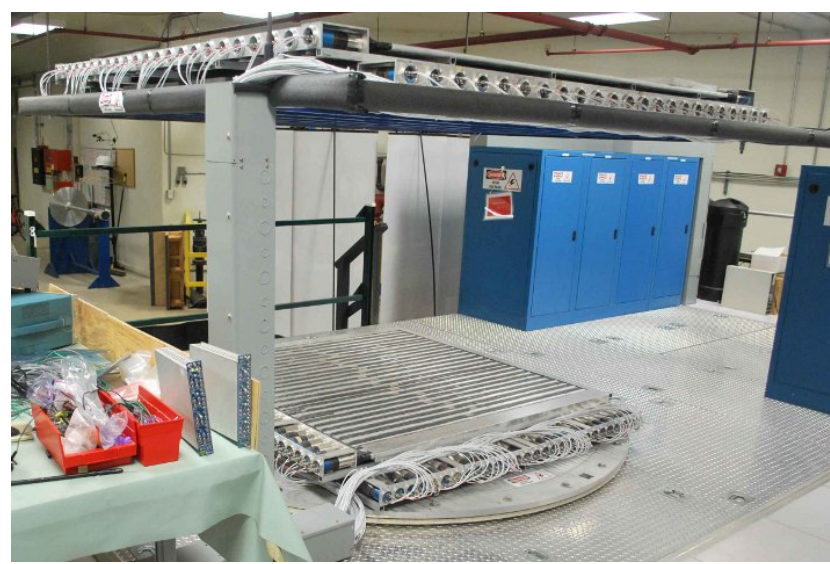

(b) Rastreador de muones y fuentes de alto voltaje.

Figura 2.8: (a) Del lado izquierdo y en rojo se pueden observar las QT boards. Del lado derecho se ven distintos NIM boards. Por la parte de atrás (no visibles) se encuentran las fuentes de poder. (b) Fotografía del rastreador de muones y fuentes de alto voltaje. Se muestra un arreglo de tiras centelladoras acopladas con PMT's usadas para determinar la dirección de muones cósmicos entrantes. A la derecha en el fondo hay armarios azules que contienen fuentes de alto voltaje.

\subsubsection{Sistema de triggers}

La lógica de los triggers de MiniBooNE se construye en el software usando información externa del acelerador y los sistemas de calibración junto con la multiplicidad de información de los PMT's.

El principal trigger físico solamente requiere que se haya mandado el haz de protones al blanco de MiniBooNE sin importar si hay o no actividad en el detector. Esta información se obtiene de los relojes de la red del acelerador (ACNET por Accelerator Network). Este trigger hace que se empiece a almacenar información $5 \mu \mathrm{s}$ antes de la llegada de los neutrinos al detector provenientes de un spill de $1.6 \mu \mathrm{s}$. De esa forma se almacena la actividad en el detector de antes y después del spill con mínimo sesgo. Un trigger del haz inicia una ventana de tiempo que inhibe la aceptación de triggers subsecuentes por $20 \mu \mathrm{s}$.

Existen otros tipos de triggers para producir conjuntos de datos con propósitos especiales. Triggers usados para recolectar eventos de neutrinos provenientes de otras fuentes en Fermilab, para buscar neutrinos provenientes de Supernovas, construir muestras para calibrar y monitorear el desempeño del detector. La frecuencia total de los triggers es de $\sim 26 \mathrm{~Hz}$, de los cuales hasta $5 \mathrm{~Hz}$ se deben a triggers del haz. En el artículo [145] se encuentra información detallada sobre todos los triggers, la forma en que operan y como se instalaron. 


\subsubsection{Sistemas de calibración del detector}

MiniBooNE emplea dos herramientas de calibración in situ: un láser pulsado y un sistema con muones cósmicos. Además, también se usan los datos del mismo experimento para calibrar la reconstrucción de ciertas cantidades físicas, como la masa de los piones neutros y el espectro de energía de los electrones Michel. Aquí se describen estos métodos con algo de detalle.

\section{El sistema de calibración del láser}

Luz emitida de un láser pulsado de diodo es enviada por fibras ópticas a cuatro frascos disipadores de luz distribuidos en varias posiciones en el interior del detector. El propósito de estos dispositivos es el de proporcionar una fuente controlada de fotones que pueda ser usada para monitorear las variaciones del tiempo y la ganancia de PMT's individuales. Los frascos tienen un diámetro de $10 \mathrm{~cm}$ y están rellenos con un medio dispersivo de nombre Ludox $\mathbb{B}$ [152].

El láser es pulsado de forma asincrónica al haz de protones a $3.3 \mathrm{~Hz}$ de forma continua durante periodos normales de toma de datos. Una caja de distribución es usada para mandar los pulsos de $\leq 100$ ns de ancho a cada uno de los cuatro frascos. Se usa la ecuación (2.1) para hacer las correcciones, se determinan por separado para los PMT R1408 y R5912. Las correcciones al tiempo se hacen con corridas que tienen distintas intensidades de luz mientras que las correcciones a la ganancia se calculan ajustando el pico del foto electrón sencillo para cada PMT en corridas de baja intensidad [153]. Las corridas con láser de baja intensidad también son usadas para determinar las tablas de eficiencia cuántica de los PMT's y verosimilitud de carga, ambas son clave para los algoritmos de reconstrucción $(\S 2.5)$. Estas calibraciones son almacenadas cada cuatro días y son principalmente realizadas usando el frasco central. Los datos de los otros tres frascos y de las muestras de electrones Michel son usadas para cotejar las calibraciones.

\section{Rastreador de muones y cubos centelladores}

Un hodoscopio se encarga de determinar el punto de entrada y la dirección de muones provenientes de rayos cósmicos que penetran el tanque. Con dos arreglos de tiras centelladoras acopladas a PMT's se miden las coordenadas $(x, y)$ de un muón cósmico entrante en dos alturas fijas $z_{b}$ y $z_{t}$ encima del tanque $\left(z_{t}-z_{b}=150 \mathrm{~cm}\right)$, ver figura $2.8 \mathrm{~b}$. Este sistema permite reconstruir trazas de muones con una resolución angular de $\sim 4^{\circ}[154]$.

Dentro del detector se encuentran siete cubos centelladores aislados ópticamente y conectados por una fibra óptica a un PMT, se usan junto con el rastreador de muones para obtener una medida precisa de la energía de muones en reposo de su rango de frenado [155].

Para un muón viajando hacia abajo que pasa por los dos arreglos de tiras centelladoras y se detiene en uno de los cubos centelladores se tienen tres puntos con los cuales se ajusta una recta, la recta ajustada se intersecta con la esfera definida por la barrera óptica del tanque para obtener el punto de entrada del muón (se escoge la solución que satisface $y>y_{\text {cubo }}$ ). El rango del muón es la distancia desde el punto de entrada al cubo. La posición de los cubos va de $15 \mathrm{~cm}$ a $400 \mathrm{~cm}$ desde la superficie del tanque, que corresponde a energías de muones en reposo desde $20 \mathrm{MeV}$ a $800 \mathrm{MeV}$. La figura 2.9 resume esta técnica. La muestra de muones en reposo es usada para la calibración de los algoritmos de reconstrucción de energía; logrando una resolución de $\pm 3 \%$ en el rango de energía relevante para 

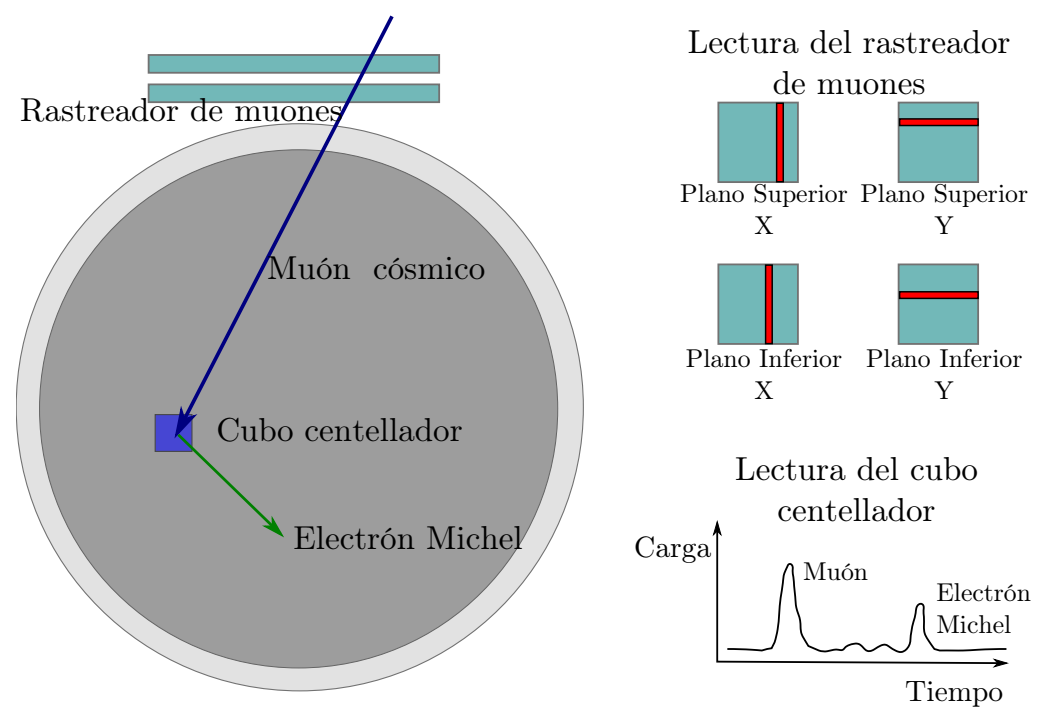

Figura 2.9: Diagrama que resume la calibración de muones cósmicos: un muón cósmico bajando atraviesa el rastreador de muones, obteniendo dos puntos de la trayectoria, y se detiene en un cubo centellador, obteniendo el tercer punto de la trayectoria del muón con los que se ajusta una recta que determina el punto de entrada del muón. Se adquiere una muestra de estos eventos que sirve para calibrar los algoritmos de reconstrucción de energía.

estos eventos. Esta se recolecta requiriendo una coincidencia retrasada entre el muón que se detiene en un cubo y su subsecuente electrón decaído.

\section{Calibración de energía con electrones Michel}

A los electrones que provienen del decaimiento de muones en reposo se les llama electrones Michel $\left(\mu^{-} \rightarrow e^{-} \bar{\nu}_{e} \nu_{\mu}\right)$. El espectro de energía de estos provee otra forma absoluta de calibrar la energía de los eventos en el tanque. La distribución de energía observada de estos electrones, mostrada en la figura 2.10a provee una medida de la resolución de energía en el punto final del espectro de los electrones Michel de $52.8 \mathrm{MeV}$.

La muestra de electrones Michel también es usada para afinar los algoritmos de reconstrucción [157] y prueba las constantes de calibración de los PMT's determinadas en las corridas con láser. Además con ella se estudian las propiedades ópticas del aceite y tiene un rol muy importante en la determinación del error sistemático del modelo óptico del detector $(\S 2.4 .3)$.

\section{Calibración de energía con $\mathrm{NC} \pi^{0}$}

Uno de los procesos frecuentes en el detector, que involucran neutrinos, es la producción de piones neutros por interacciones de corriente neutra $\nu_{\mu}-\mathrm{NC} \pi^{0}$, como se muestra en la figura 2.12b. El pión generado decae rápidamente a dos fotones, los cuales generan una cascada electromagnética que pueda ser detectada en el tanque. Cumpliendo una lista de criterios, se puede formar una muestra de eventos $\pi^{0}$, como se muestra en la figura 2.10b. Los algoritmos de reconstrucción son ajustados para que, del par de cascadas electromagnéticas, se reconstruya precisamente el pico de la masa del $\pi^{0}\left(m_{\pi^{0}}=134.9766 \mathrm{MeV}\right)$. Con esta calibración se reconstruyen fotones con energías entre $50 \mathrm{MeV}$ y $350 \mathrm{MeV}$. 


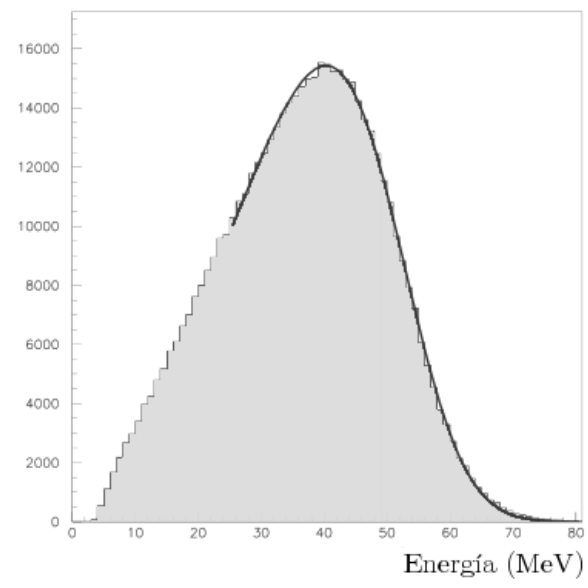

(a) Distribución de energía observada de electrones Michel.

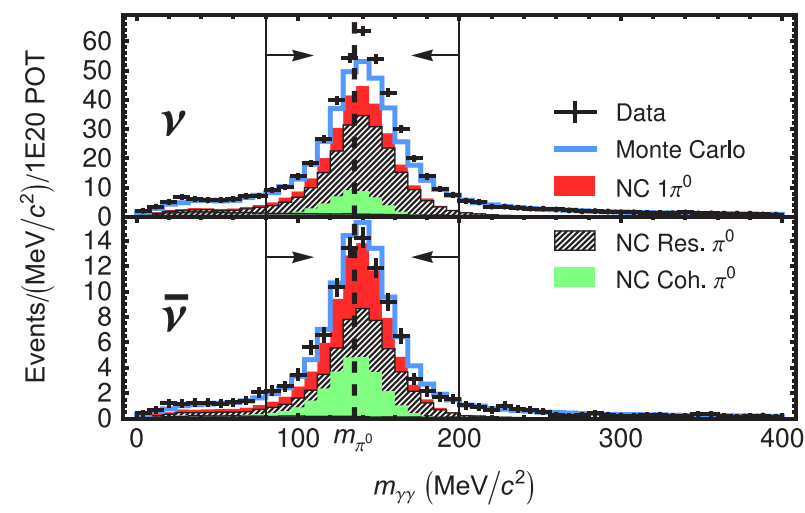

(b) Distribución de la masa invariante de $\pi^{0}$.

Figura 2.10: Distintas calibraciones de energía. (a) Distribución de energía observada de electrones Michel (histograma) y mejor ajuste (curva solida) obtenida convolucionando el espectro de energía teórico con una gausiana con ancho proporcional a $\sqrt{E}$. La resolución de energía determinada con el ajuste es de $14.8 \%$ a $52.8 \mathrm{MeV}$. (b) Distribución de la masa invariante $m_{\pi^{0}}$ para eventos que cumplen los criterios de selección, la línea punteada muestra $m_{\pi^{0}}=134.9766 \mathrm{MeV}$, tomada de [156].

\subsection{Simulación Monte Carlo del haz}

Las propiedades del flujo de neutrinos de MiniBooNE son determinadas usando una simulación Monte Carlo (MC) basada en Geant4 [158]. La simulación se puede dividir a grandes rasgos en cinco pasos:

1. Definición de la geometría de la linea del haz, especificada por la forma, localización y composición de las componentes del BNB, a partir de esta todas las otras partículas se propagan.

2. La generación de los protones primarios antes del blanco de acuerdo a las propiedades ópticas del haz.

3. La simulación de las partículas generadas en la interacción primaria p-Be. Se desarrollaron tablas personalizadas para la producción de protones, neutrones, $\pi^{ \pm}, K^{ \pm}$y $K^{0}$ en estas interacciones para adaptarse a datos externos.

4. La propagación de las partículas usando el sistema Geant4 en el blanco, corno magnético, túnel de decaimiento y el tope del haz, tomando en cuenta pérdida de energía y procesos electromagnéticos y hadrónicos que alteran la cinemática de las partículas. Las interacciones hadrónicas y decaimientos también pueden aniquilar las partículas en el proceso de rastreo y crear nuevas partículas que deben ser rastreadas. El efecto del campo magnético esperado del corno en la trayectoria de las partículas.

5. La identificación de los procesos de decaimiento de los cuales resultan neutrinos. Se maneja la simulación de los decaimientos por un modelo personalizado de decaimientofuera del sistema Geant4. El modelo de decaimiento refleja las últimas medidas de la tasa de ramificación y simula los efectos de polarización y distribución cinemática resultantes de los factores de forma de decaimiento. Se usan distintas técnicas para mejorar la precisión estadística de la predicción del flujo. 


\section{Geometría del BNB, campos magnéticos y protones primarios}

La geometría de la simulación MC en Geant4 consiste de los últimos $50 \mathrm{~m}$ de la línea del haz del Booster, el edificio del blanco y los $50 \mathrm{~m}$ del conducto de decaimiento. La descripción de la geometría se definió para igualar tanto como sea posible la verdadera linea del haz como fue construida. Cada sección es simulada con paredes de concreto rodeadas por una capa uniforme de dolomita. Toda la estructura esta llena con aire a temperatura y presión ambiental.

La simulación del edificio del blanco contiene el corno, el blanco y el colimador del haz secundario. Además de las paredes de concreto el edificio esta forrado con $1.28 \mathrm{~m}$ de blindaje de acero. Las siete pastillas y la cubierta del blanco, junto con las aletas que lo sostienen forman la simulación del blanco. El corno es construido usando un polígono de aluminio en GEANT que especifica el radio interno y externo en catorce puntos distintos a lo largo de la dirección del haz. Se pone un polígono de aire dentro del de aluminio para establecer el ancho de los conductores interior y exterior.

Los protones primarios son simulados individualmente, ya que no se esperan efectos correlacionados entre los protones de un bunch. Las propiedades del haz de protones, como la posición y perfil, han sido simuladas usando TRANSPORT [161] y verificadas por monitores [143]. Los protones son generados $1 \mathrm{~cm}$ antes del blanco con posiciones transversales $(x, y)$ extraídas de distribuciones aleatorias gausianas con media $0 \mathrm{~mm}$ y $1.51 \mathrm{~mm}$ y anchos de $0.75 \mathrm{~mm}$, respectivamente. Igualmente las desviaciones angulares de los protones de la dirección $z, \theta_{x}$ y $\theta_{y}$, son extraídas de gausianas con media $0 \mathrm{mrad}$ y anchos de $0.66 \mathrm{mrad}$ y $0.40 \mathrm{mrad}$, respectivamente. La cantidad de protones que experimentan interacciones inelásticas en el blanco (en contraposición a dispersarse) se estudia en §2.3.1. En particular mientras que la configuración describe un haz divergente, la simulación TRANSPORT indica que se espera que los protones sean convergentes en el blanco, con una "cintura" de divergencia nula en el centro del blanco. La simulación de la configuración del haz es tal que $99.8 \%$ de los protones se encuentran en una trayectoria para intersectar al blanco. Los estudios en $\S 2.3 .1$ indican que perturbaciones razonables al modelo, incluida la configuración de enfocado, no afectan el flujo predicho de neutrinos en más de $1 \%$.

Se simula el campo magnético esperado de $1 / R$ en el volumen que corresponde al espacio entre los conductores interno y externo del corno. La intensidad del campo corresponde a una corriente de $174 \mathrm{kA}$ a lo largo del conductor interior (invertido cuando se simula el modo de antineutrinos). La profundidad de penetración del campo magnético en el cilindro del conductor interior del corno (descrita en §2.3.1) se incluye en la simulación. La predicción de las trayectorias de las partículas cargadas en el campo magnético en la simulación Geant4 han sido revisadas en un estudio externo usando la rutina DRKNYS de CERNLIB [160], un método numérico implementado independientemente.

\section{Haz secundario (mesones) y terciario (neutrinos)}

Lo primero que encuentran la gran mayoría de los protones primarios es el blanco. Ya que las interacciones $\mathrm{p}-\mathrm{B}$ son la principal fuente de mesones secundarios se uso un modelo dedicado ajustado a datos externos para describir la producción de partículas en las pastillas, la manga y las aletas. La dispersión elástica se lleva acabo por los modelos existentes de Geant4, mientras que la dispersión cuasielástica de nucleones y piones usan un modelo dedicado basado en datos de dispersión hadrón-núcleo libre. Debido a la divergencia del haz primario y su dispersión, es posible que un protón primario interactúe fuera del blanco (usualmente en el aluminio del corno o el concreto de la región de decaimiento). Para estos casos la producción de partículas se maneja por el modelo hadrónico predeterminado del sistema 


\section{Geant4.}

Para las interacciones primarias p-Be se generan partículas secundarías de siete tipos $\left(\pi^{ \pm}, K^{ \pm}, K^{0}\right.$, $\mathrm{p}, \mathrm{n}$ ) de acuerdo a tablas que describen la doble sección eficaz diferencial para la producción de cada especie secundaria como función de $p_{z} \mathrm{y} p_{T}$, las componentes de momento a lo largo y transversalmente a la dirección de los protones primarios, respectivamente. La sección eficaz de producción total para una especie dada, obtenida al integrar la doble sección eficaz diferencial, determina la multiplicidad promedio de la especie en cada reacción primaria $\mathrm{p}-\mathrm{Be}$ (interacciones hadrónicas excluyendo dispersión elástica y cuasielástica) cuando se divide por la sección eficaz de la reacción.

Los neutrinos que llegan al detector son producidos en los decaimientos de piones cargados, kaones cargados y neutros y muones. Los tiempos de vida, los modos de decaimiento y las tasas de ramificación asociadas, así como las distribuciones cinemáticas de los neutrinos producidos en los decaimientos considerados en la simulación, afectan la predicción del flujo de neutrinos y se discuten a continuación. En la tabla 2.1 se dan los tiempos de vida y las tasas de ramificación de las partículas madre consideradas en la simulación, para los distintos modos de decaimiento. También se simulan los correspondientes decaimientos de partículas negativas.

\begin{tabular}{cccc}
\hline Partícula & Tiempo de vida [ns] & Modo de decaimiento & Tasa de ramificación [\%] \\
\hline \multirow{2}{*}{$\pi^{+}$} & \multirow{2}{*}{26.03} & $\mu^{+}+\nu_{\mu}$ & 99.9877 \\
& \multirow{2}{*}{12.385} & $e^{+}+\nu_{e}$ & 0.0123 \\
\hline \multirow{2}{*}{$K^{+}$} & & $\mu^{+}+\nu_{\mu}$ & 63.44 \\
& & $\pi^{0}+e^{+}+\nu_{e}$ & 4.98 \\
& $\pi^{0}+\mu^{+}+\nu_{\mu}$ & 3.32 \\
\hline \multirow{2}{*}{$K_{L}^{0}$} & \multirow{2}{*}{51.6} & $\pi^{-}+e^{+}+\nu_{e}$ & 20.333 \\
& & $\pi^{+}+e^{-}+\bar{\nu}_{e}$ & 20.197 \\
& & $\pi^{-}+\mu^{+}+\nu_{\mu}$ & 13.551 \\
\hline$\mu^{+}$ & 2197.03 & $\pi^{+}+\mu^{-}+\bar{\nu}_{\mu}$ & 13.469 \\
\hline
\end{tabular}

Tabla 2.1: Tiempos de vida y tasas de ramificación de los modos de decaimiento que producen neutrinos considerados en la simulación.

Simplemente ejecutar la simulación Geant4 y registrar los neutrinos resultantes protón por protón no otorgaría suficientes neutrinos en el detector MiniBooNE que permitan determinar precisamente el flujo en el espacio fase de interés. Por lo tanto se hicieron varias modificaciones para mejorar la estadística de la simulación MC.

Se consigue una gran mejora estadística al hacer "redecaer" a la partícula madre del neutrino. Para cada neutrino producido en el $\mathrm{MC}$ del haz, el decaimiento de la partícula que produjo el neutrino se realiza mil veces. Cada redecaimiento se realiza en el mismo lugar, pero la cinemática del decaimiento se designa aleatoriamente cada vez de las distribuciones de decaimiento, logrando diferentes momentos en las partículas hijas en cada elección.

Se usa una técnica similar para mejorar la estadística de neutrinos provenientes del decaimiento de muones. La mayoría de los muones producidos en el haz secundario no decaen antes de detenerse en el tope del haz o en las paredes de la región de decaimiento debido a su largo tiempo de vida. Para 
obtener una mejor estimación de la componente del flujo de neutrinos provenientes de muones DIF se hacen 19 copias idénticas de cada muón producido en la simulación, las cuales son propagadas de forma independiente en la simulación. Para tener en cuenta la sobreproducción de neutrinos el peso de cada neutrino proveniente del decaimiento de un muón es reducido por un factor de 20.

Se usa otra técnica de ponderación para determinar el flujo de neutrinos de alta energía. El espectro de energía de los neutrinos en MiniBooNE tiene su máximo entre 500 y $600 \mathrm{MeV}$ con una larga cola para la parte de alta energía, extendiéndose más allá de $6 \mathrm{GeV}$. Como se producen muy pocos neutrinos para estas altas energías, las fluctuaciones estadísticas son mucho mayores, incrementando la incertidumbre en la forma de la cola de alta energía. El procedimiento de redecaimiento descrito antes empeora el problema, ya que las partículas madre tienden a decaer a neutrinos de alta energía con dirección frontal lo cual resulta en una fracción significante de los mil redecaimientos apuntando al detector, todos con energías similares. Para reducir la incertidumbre estadística en la predicción de la cola de alta energía, las secciones eficaces de la producción de mesones en las interacciones p-Be son multiplicadas por una función exponencial del momento longitudinal de los mesones. Cada evento es "desponderado" por su correspondiente mejora en la sección eficaz para conservar la proporción correcta de protones/neutrinos. Esto provee una producción artificialmente grande de neutrinos a altas energías con pequeño peso estadístico, consiguiendo reducir la incertidumbre estadística en la cola de alta energía para la predicción del flujo de neutrinos.

El flujo de neutrinos en el detector MiniBooNE se determina al proyectar la trayectoria del neutrino al plano que contiene el centro del detector, $541 \mathrm{~m}$ de la fachada del blanco. Los neutrinos que se encuentran en una trayectoria hacia el detector ${ }^{5}$ son registrados en las distribuciones de flujo usadas para la simulación de eventos $\mathrm{MC}$ en el detector. Para la simulación de interacciones de neutrinos fuera del detector, en las paredes de concreto del edificio del detector o en la tierra que se encuentra más adelante, se usa un radio más grande de $1400 \mathrm{~cm}$ para determinar las distribuciones de flujo.

\subsubsection{Predicción del flujo de neutrinos en MiniBooNE}

Los resultados de la simulación se encuentran en las figuras 2.11a y 2.11b, que muestran el flujo total predicho de cada especie de neutrinos en el detector MiniBooNE en modo de neutrinos y antineutrinos, respectivamente. En cada caso, la contribución $\nu_{e} / \bar{\nu}_{e}$ es menor al $1 \%$ en el máximo del flujo de $\nu_{\mu} / \bar{\nu}_{\mu}$, aunque se eleva a energías más altas. Como se muestra, los flujos predichos muestran muchas características que se entienden mejor al analizar las fuentes de cada componente del flujo.

La contribución integrada de cada especie de neutrino y antineutrino, junto con sus cadenas principales de decaimiento, se muestran en la tabla 2.2a para la configuración en el modo de neutrinos, la tabla $2.2 \mathrm{~b}$ tiene lo correspondiente para la configuración en el modo de antineutrinos. La contribución dominante de las cadenas de decaimiento en las que el mesón secundario es producido por un nucleón se separa de cuando proviene de la interacción de un mesón. Esto debido a la diferencia cualitativa en el entendimiento sistemático para los dos procesos. Para el primer caso las secciones eficaces de producción se basan en experimentos de producción de partículas ${ }^{6}$, con incertidumbres sistemáticas propagadas de las incertidumbres reportadas en esos experimentos. Para el segundo caso, la simulación depende en el modelo de interacción hadrónica por defecto de Geant4 para producir las secciones eficaces de producción; la cual, afortunadamente, es una pequeña contribución al flujo para todos los casos.

\footnotetext{
${ }^{5} 610.6 \mathrm{~cm}$ desde el centro del detector en este plano, tomando en cuenta el desplazamiento vertical.

${ }^{6}$ Ver más detalles en la sección V del artículo [162].
} 


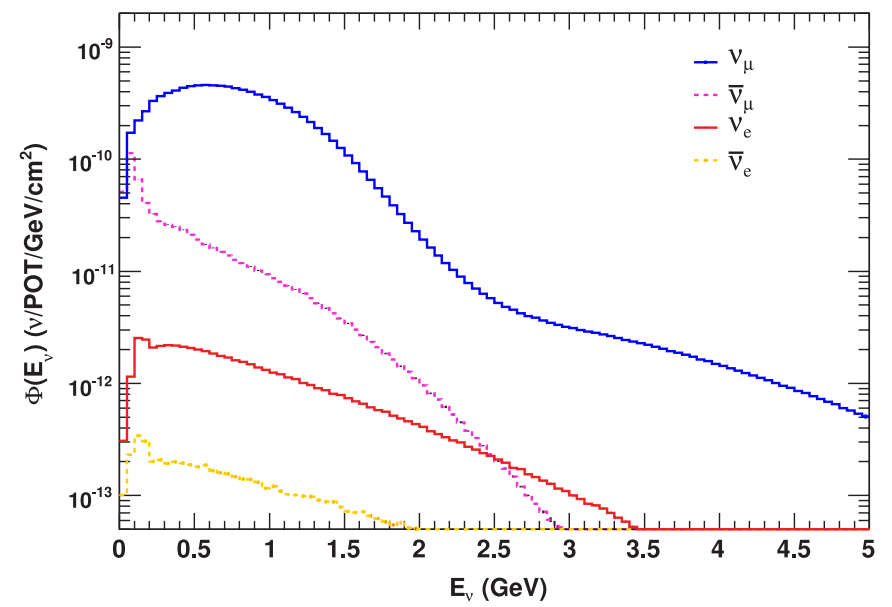

(a) Modo de neutrinos.

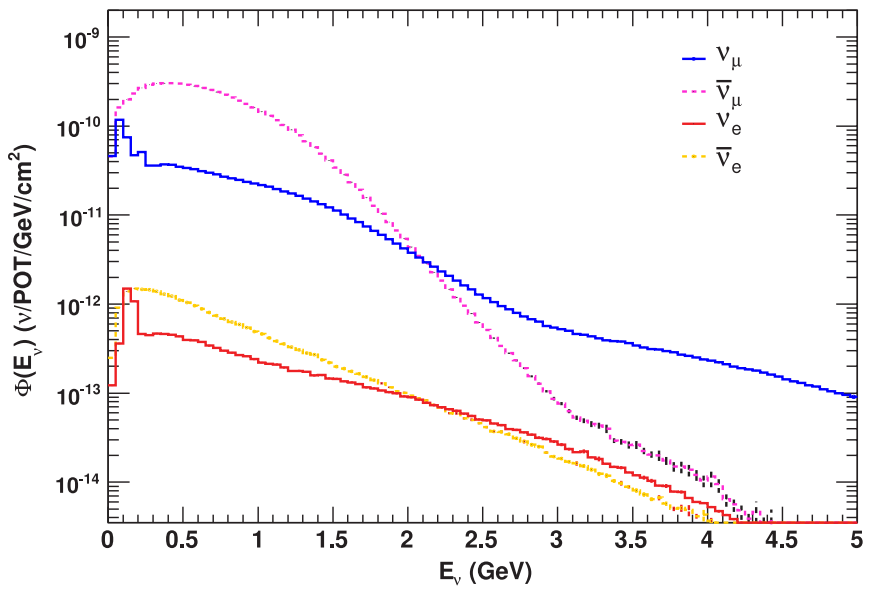

(b) Modo de antineutrinos.

Figura 2.11: Flujo total predicho de cada especie de neutrinos en el detector MiniBooNE, con el corno en modo de (a) neutrinos y (b) antineutrinos [162].

Con el corno en modo de neutrinos el flujo de $\nu_{\mu}$, es dominado por la contribución de $\pi^{+} \rightarrow \nu_{\mu}$ para energías menores a $2 \mathrm{GeV}$, y la contribución $K^{+} \rightarrow \nu_{\mu}$ se vuelve dominante para altas energías. Hay una pequeña contribución de piones producidos en el decaimiento de kaones y una contribución similar inducida por mesones terciarios. En la tabla la cadena $\pi^{-} \rightarrow \bar{\nu}_{\mu}$ se refiere a la contribución dominante al flujo de $\bar{\nu}_{\mu}$ para todas las energías. La siguiente contribución más grande viene de la cadena $\pi^{+} \rightarrow \mu^{+} \bar{\nu}_{\mu}$. Para el flujo de $\nu_{\mu}$ la contribución proveniente de la cadena análoga $\pi^{-} \rightarrow \mu^{-} \rightarrow \nu_{\mu}$ es suprimida debido al desenfoque de los $\pi^{-}$. La contribución de kaones es suprimida debido a la baja tasa de producción de $K^{-}$comparada con la producción de $K^{+}$. Aparte de las bajas energías (200 MeV) el flujo predicho de $\bar{\nu}_{\mu}$ típicamente es $\sim 6 \%$ el de $\nu_{\mu}$.

También en modo de neutrinos, las componentes dominantes para el flujo de $\nu_{e}$ son la cadena de decaimiento $\pi^{+} \rightarrow \mu^{+} \rightarrow \nu_{e}$ y el decaimiento a tres cuerpos $K^{+} \rightarrow \nu_{e}$, donde el primero domina a energías menores a $1 \mathrm{GeV}$ y el segundo a energías más altas. El máximo en el espectro $K^{+} \rightarrow \nu_{e}$ a bajas energías proviene del decaimiento $K^{+}$en reposo ${ }^{7}$. Para $\bar{\nu}_{e}$, la cadena $\pi^{-} \rightarrow \mu^{-} \rightarrow \bar{\nu}_{e}$ solo contribuye a bajas energías debido al desenfoque de $\pi^{-}$y la contribución de $K^{-} \rightarrow \bar{\nu}_{e}$ es suprimida tanto por la baja tasa de producción como por el desenfoque. El resto del espectro es dominado por el decaimiento $K_{L}^{0}$. Como en el caso $\nu_{\mu} / \bar{\nu}_{\mu}$, el flujo predicho de $\bar{\nu}_{e}$ es $\sim 10 \%$ del flujo $\nu_{e}$.

Ahora para el flujo predicho en el modo de antineutrinos. El flujo de $\bar{\nu}_{\mu}$ es dominado a todas las energías por $\pi^{-} \rightarrow \bar{\nu}_{\mu}$; la producción suprimida de $K^{-}$resulta en que la contribución $K^{-} \rightarrow \bar{\nu}_{\mu}$ es mucho más chica que la correspondiente contribución $K^{+} \rightarrow \nu_{\mu}$ en modo de neutrinos. Más aún, como los $K^{-}$en reposo son capturados, el flujo de $\bar{\nu}_{\mu}$ no muestra los picos del decaimiento en reposo a dos y tres cuerpos a bajas energías que se encuentran en $\nu_{\mu}$ proveniente del decaimiento $K^{+}$tanto en modo de neutrinos como antineutrinos. También se puede observar que el flujo a altas energías de $\nu_{\mu}$ no es substancialmente suprimido en relación con el de $\bar{\nu}_{\mu}$. De hecho, a pesar del desenfoque de $K^{+}$, el flujo de $K^{+} \rightarrow \nu_{\mu}$ es mayor que el de $K^{-} \rightarrow \bar{\nu}_{\mu}$. Esto se debe a las tasas relativas de producción y, para altas energías, el efecto de partícula dominante en donde $\pi^{+}$y $K^{+}$tienen un espectro de momentos más grande que las contrapartes negativas. El gran momento de las partículas que producen estos neutrinos, junto con su distribución angular frontal, resulta en menor desenfoque para la componente de "signo erróneo", partículas positivas para el modo de neutrinos y partículas

${ }^{7}$ El máximo del decaimiento a dos cuerpos es mucho más chico debido a la supresión por helicidad. 


\begin{tabular}{|c|c|c|c|c|}
\hline \multirow[b]{2}{*}{$\begin{array}{l}\text { Flujo }\left(\nu / \mathrm{cm}^{2} / \mathrm{POT}\right) \\
\text { Fracción del total }\end{array}$} & \multicolumn{2}{|c|}{$\nu_{\mu}$} & \multicolumn{2}{|l|}{$\bar{\nu}_{\mu}$} \\
\hline & \multicolumn{2}{|c|}{$\begin{array}{c}5.19 \times 10^{-10} \\
93.6 \%\end{array}$} & \multicolumn{2}{|c|}{$\begin{array}{c}3.26 \times 10^{-1} \\
5.86 \%\end{array}$} \\
\hline Composición & $\begin{array}{l}\pi^{+}: \\
K^{+}: \\
K^{+} \rightarrow \pi^{+}: \\
K^{0} \rightarrow \pi^{+}: \\
K^{0}: \\
\pi^{-} \rightarrow \mu^{-}: \\
\text {Otra: }\end{array}$ & $\begin{array}{c}96.72 \% \\
2.65 \% \\
0.26 \% \\
0.04 \% \\
0.03 \% \\
0.01 \% \\
0.30 \%\end{array}$ & $\begin{array}{l}\pi^{-}: \\
\pi^{+} \rightarrow \mu^{+}: \\
K^{-}: \\
K^{0}: \\
K^{0} \rightarrow \pi^{-}: \\
K^{+} \rightarrow \mu^{+}: \\
K^{-} \rightarrow \pi^{-}: \\
\text {Otra: }\end{array}$ & $\begin{array}{c}89.74 \% \\
4.54 \% \\
0.51 \% \\
0.44 \% \\
0.24 \% \\
0.06 \% \\
0.03 \% \\
4.43 \% \\
\end{array}$ \\
\hline $\begin{array}{c}\text { Flujo }\left(\nu / \mathrm{cm}^{2} / \mathrm{POT}\right) \\
\text { Fracción del total }\end{array}$ & \multicolumn{2}{|c|}{$\begin{array}{c}2.87 \times 10^{-12} \\
0.52 \%\end{array}$} & \multicolumn{2}{|c|}{$\begin{array}{c}3.00 \times 10^{-} \\
0.05 \%\end{array}$} \\
\hline Composición & $\begin{array}{l}\pi^{+} \rightarrow \mu^{+}: \\
K^{+}: \\
K_{L}^{0}: \\
\pi^{+}: \\
K^{+} \rightarrow \mu^{+}: \\
\text {Otra: }\end{array}$ & $\begin{array}{c}51.64 \% \\
37.28 \% \\
7.39 \% \\
2.16 \% \\
0.69 \% \\
0.84 \%\end{array}$ & $\begin{array}{l}K_{L}^{0}: \\
\pi^{-} \rightarrow \mu^{-} \\
K^{-}: \\
\pi^{-}: \\
K^{-} \rightarrow \mu^{-} \\
\text {Otra: }\end{array}$ & $\begin{array}{c}70.65 \% \\
19.33 \% \\
4.07 \% \\
1.26 \% \\
0.07 \% \\
4.62 \%\end{array}$ \\
\hline
\end{tabular}

(a) Flujo en modo de neutrinos.

\begin{tabular}{|c|c|c|c|c|}
\hline \multirow[b]{2}{*}{$\begin{array}{c}\text { Flujo }\left(\nu / \mathrm{cm}^{2} / \mathrm{POT}\right) \\
\text { Fracción del total }\end{array}$} & \multicolumn{2}{|c|}{$\nu_{\mu}$} & \multicolumn{2}{|c|}{$\bar{\nu}_{\mu}$} \\
\hline & \multicolumn{2}{|c|}{$\begin{array}{c}5.42 \times 10^{-11} \\
15.71 \%\end{array}$} & \multicolumn{2}{|c|}{$\begin{array}{c}2.93 \times 10^{-10} \\
83.73 \%\end{array}$} \\
\hline Composición & $\begin{array}{l}\pi^{+}: \\
K^{+}: \\
\pi^{-} \rightarrow \mu^{-}: \\
K^{0}: \\
\text { Otra: }\end{array}$ & $\begin{array}{c}88.79 \% \\
7.53 \% \\
1.77 \% \\
0.26 \% \\
2.00 \%\end{array}$ & $\begin{array}{l}\pi^{-} \\
K^{-}: \\
K^{0} \rightarrow \pi^{-}: \\
K^{0}: \\
\pi^{+} \rightarrow \mu^{+}: \\
K^{-} \rightarrow \pi^{-}: \\
\text {Otra: }\end{array}$ & $\begin{array}{l}98.4 \% \\
0.18 \% \\
0.05 \% \\
0.05 \% \\
0.03 \% \\
0.02 \% \\
1.30 \%\end{array}$ \\
\hline $\begin{array}{c}\text { Flujo }\left(\nu / \mathrm{cm}^{2} / \mathrm{POT}\right) \\
\text { Fracción del total }\end{array}$ & \multicolumn{2}{|c|}{$\begin{array}{c}6.71 \times 10^{-13} \\
0.2 \%\end{array}$} & \multicolumn{2}{|c|}{$\begin{array}{c}1.27 \times 10^{-12} \\
0.4 \%\end{array}$} \\
\hline Composición & $\begin{array}{l}K^{+}: \\
K^{0}: \\
\pi^{+} \rightarrow \mu^{+}: \\
\pi^{+}: \\
K^{+} \rightarrow \mu^{+}: \\
\text {Otra: }\end{array}$ & $\begin{array}{c}51.72 \% \\
31.56 \% \\
13.30 \% \\
0.83 \% \\
0.41 \% \\
2.17 \%\end{array}$ & $\begin{array}{l}\pi^{-} \rightarrow \mu^{-}: \\
K^{0}: \\
K^{-}: \\
\pi^{-}: \\
K^{-} \rightarrow \mu^{-}: \\
\text {Otra: }\end{array}$ & $\begin{array}{c}75.67 \% \\
16.51 \% \\
3.08 \% \\
2.58 \% \\
0.06 \% \\
2.10 \%\end{array}$ \\
\hline
\end{tabular}

(b) Flujo en modo de antineutrinos.

Tabla 2.2: Flujos predichos de $\nu_{\mu} / \bar{\nu}_{\mu}$ y $\nu_{e} / \bar{\nu}_{e}$ en el detector MiniBooNE. Contribución al flujo de decaimientos de mesones donde la partícula madre en la cadena de decaimientos es producida por interacción con un protón o un neutrón. La categoría "otra" incluye los canales con contribuciones menores a aquellas mostradas, junto con casos donde la partícula madre en la cadena de decaimiento es producida por la interacción de un mesón [162].

negativas para el modo de antineutrinos. Un efecto similar se ve para las componentes $\nu_{e} / \bar{\nu}_{e}$ en modo de antineutrinos: mientras que los $\bar{\nu}_{e}$ están dominados por decaimientos $\mu^{-}$para energías por debajo de $2 \mathrm{GeV}$, el flujo de $K^{+} \rightarrow \nu_{e}$ es mayor que el flujo de $K^{-} \rightarrow \bar{\nu}_{e}$. Una observación pertinente es que mientras la tasa absoluta de $\nu_{e} / \bar{\nu}_{e}$ proveniente de $K_{L}^{0}$ permanece igual que en modo de neutrinos, la contribución relativa es mucho mayor en modo de antineutrinos.

\section{Incertidumbres sistemáticas}

Las incertidumbres sistemáticas en la predicción del flujo de neutrinos para el modo de neutrinos y antineutrinos se encuentran resumidas en la tabla 2.3. Las distintas fuentes de incertidumbre en el haz están relacionadas, por ejemplo una variación en la cantidad de protones entregados se refleja en una variación en la cantidad de piones producidos, cada variación de un parámetro en la simulación tendrá efectos en las distribuciones de energía de los eventos empleados en los análisis. La matriz de covarianza del contenido de los bins de estas distribuciones se calcula para cada fuente de error sistemático. Así pues se construyen matrices de covarianza que se integran con otras para determinar la incertidumbre sistemática en los análisis. Estas incertidumbres provienen de distintas fuentes:

Entrega de protones: La simulación determina la tasa y espectro de los neutrinos por cada protón en el blanco. Esta información es combinada con el número de protones entregados al blanco para determinar el número de neutrinos que pasan a través del detector MiniBooNE a lo largo del periodo de captura de datos. Como resultado la predicción del número de interacciones de neutrinos en el detector varía directamente con la incertidumbre en el número de protones en el blanco. Una incertidumbre relacionada surge de la forma en como la óptica del haz de protones cambia el número 
esperado de protones interactuando en el blanco (o en otras partes), consecuentemente cambiando el flujo de neutrinos.

Como se menciona en $\S 2.1 .1$, los protones entregados son monitoreados por dos toroides antes de que estos incidan en el blanco. Se estima que la incertidumbre sistemática en las medidas es de $2 \%$, dado que el flujo total de neutrinos se escala con la cantidad de protones entregados, esta fuente de error se puede tratar como incertidumbre en la normalización del total.

Producción de partículas: Las incertidumbres en la tasa y espectro de las partículas secundarias producidas en las interacciones $\mathrm{p}$-Be afecta de forma similar la tasa y espectro de los neutrinos producidos. Esta es la incertidumbre dominante.

Interacciones hadrónicas: la tasa de las interacciones hadrónicas afecta muchos aspectos de la producción de neutrinos, incluyendo la tasa de las interacciones p-Be y la probabilidad que tienen los mesones de sobrevivir posibles interacciones hadrónicas en el blanco o corno, y decaer para producir neutrinos. Incertidumbres en la tasa de estas interacciones afectan tanto la tasa y la forma del flujo.

Campo magnético del corno: las incertidumbres en dos propiedades del campo magnético causan incertidumbres sistemáticas en el flujo de neutrinos. La primera es el efecto que tienen las variaciones de la corriente en el campo magnético, para lo cual se simulan los efectos de una variación de $174 \pm 1 \mathrm{kA}$ para obtener la incertidumbre sistemática en el flujo de neutrinos. El otro viene del modelado de la corriente dentro del cilindro interior debido al "efecto de profundidad de penetración", el cual permite que el campo magnético penetre el conductor, incrementando el campo magnético efectivo que experimentan las partículas que atraviesan el conductor interior. Es importante considerar tal efecto para partículas producidas a ángulos pequeños y con gran momento. Se simula el caso en el que este efecto existe y cuando no se encuentra, encontrando una pequeña diferencia para neutrinos con energías menores a $1 \mathrm{GeV}$ y hasta $18 \%$ cuando tienen energías de $\sim 2 \mathrm{GeV}$.

Geometría de la línea del haz: desplazamientos o desajustes de los componentes de la línea del haz pueden afectar el flujo de neutrinos en muchas formas. Por ejemplo un desajuste puede hacer que el detector se exponga a una parte distinta de la esperada del flujo de neutrinos. Un desplazamiento del blanco con respecto al corno puede resultar en una variación de las propiedades de enfoque. Estas variaciones son simuladas, sin embargo las magnitudes de las perturbaciones geométricas que son necesarias para tener un efecto substancial en el flujo $(>1 \%)$ están por encima de lo que se considera la tolerancia y precisión con la que se construyo la línea del haz. Por lo tanto, no se asigna ninguna incertidumbre sistemática significativa a la geometría del haz.

\subsection{Simulación Monte Carlo del detector}

Las tasas de interacción de neutrinos, productos y su cinemática en el detector MiniBooNE son predichas usando un generador de eventos de neutrinos basado en NUANCE v3 [163], pero adecuado al experimento MiniBooNE, como ajustes del factor de forma axial de la dispersión cuasielástica, el modelo de bloqueo de Pauli y las secciones eficaces de la producción coherente de piones neutros 


\begin{tabular}{ccccccccc}
\hline & \multicolumn{3}{c}{ Modo de neutrinos } & \multicolumn{4}{c}{ Modo de antineutrinos } \\
\cline { 2 - 9 } Fuente de incertidumbre & $\nu_{\mu}$ & $\bar{\nu}_{\mu}$ & $\nu_{e}$ & $\bar{\nu}_{e}$ & $\nu_{\mu}$ & $\bar{\nu}_{\mu}$ & $\nu_{e}$ & $\bar{\nu}_{e}$ \\
\hline Entrega de protones & $2.0 \%$ & $2.0 \%$ & $2.0 \%$ & $2.0 \%$ & $2.0 \%$ & $2.0 \%$ & $2.0 \%$ & $2.0 \%$ \\
Óptica de protones & $1.0 \%$ & $1.0 \%$ & $1.0 \%$ & $1.0 \%$ & $1.0 \%$ & $1.0 \%$ & $1.0 \%$ & $1.0 \%$ \\
Producción de $\pi^{+}$ & $14.7 \%$ & $1.0 \%$ & $9.3 \%$ & $0.9 \%$ & $13.8 \%$ & $0.1 \%$ & $2.1 \%$ & $0.1 \%$ \\
Producción de $\pi^{-}$ & $0.0 \%$ & $16.5 \%$ & $0.0 \%$ & $3.5 \%$ & $0.5 \%$ & $17.5 \%$ & $0.0 \%$ & $13.6 \%$ \\
Producción de $K^{+}$ & $0.9 \%$ & $0.2 \%$ & $11.5 \%$ & $0.3 \%$ & $3.1 \%$ & $0.0 \%$ & $22.3 \%$ & $0.4 \%$ \\
Producción de $K^{0}$ & $0.0 \%$ & $0.2 \%$ & $2.1 \%$ & $17.6 \%$ & $0.1 \%$ & $0.0 \%$ & $6.1 \%$ & $3.9 \%$ \\
Campo del corno & $2.2 \%$ & $3.3 \%$ & $0.6 \%$ & $0.8 \%$ & $1.5 \%$ & $1.0 \%$ & $3.2 \%$ & $1.5 \%$ \\
Secciones eficaces de p/n & $2.8 \%$ & $5.7 \%$ & $3.3 \%$ & $5.6 \%$ & $6.2 \%$ & $2.1 \%$ & $6.2 \%$ & $2.5 \%$ \\
Secciones eficaces de $\pi$ & $1.2 \%$ & $1.2 \%$ & $0.8 \%$ & $0.7 \%$ & $1.5 \%$ & $1.2 \%$ & $1.6 \%$ & $1.5 \%$ \\
\hline
\end{tabular}

Tabla 2.3: Variaciones en el flujo total para cada especie de neutrino en modo de neutrinos y antineutrinos debido a incertidumbres sistemáticas [162].

basados en los datos del experimento. Los varios parámetros del generador son afinados con la mejor información disponible de secciones eficaces. En la entrada se pone la predicción del flujo de neutrinos descrita en § 2.3.1, así como el material del detector (aceite mineral) y su geometría. NUANCE puede simular 99 interacciones distintas de neutrinos/antineutrinos, en un amplio rango de energías (100 MeV a $1000 \mathrm{GeV}$ ). Los procesos más importantes para este análisis son la dispersión cuasielástica de corriente cargada (CCQE por sus siglas en inglés) y la interacción por corriente neutra (NC). La figura 1.4 contiene las secciones de varios procesos relevantes en MiniBooNE. En el tiempo en que el experimento empezó a correr la mayoría de las mediciones que existían sobre secciones eficaces eran para núcleos ligeros y energías mayores, pero la mayoría de las interacciones que ocurren son para nucleones amarrados a núcleos de ${ }^{12} \mathrm{C}$, por lo tanto se modelaron efectos nucleares para incorporar el conocimiento que se tenía de las secciones eficaces de nucleones libres de blancos ligeros.

Las incertidumbres en la modelación y las secciones eficaces de los procesos que se describen más adelante se integran para formar una matriz de covarianza, la cual se usa para determinar las incertidumbres en los análisis.

\subsubsection{Interacción cuasielástica de corriente cargada (CCQE)}

Por su relativamente alta sección eficaz y la capacidad de identificar el sabor del neutrino incidente, las dispersiones cuasielástica de corriente cargada son el canal a través del cual se hace la búsqueda de oscilaciones ${ }^{8}$. Las interacciones CCQE de neutrinos ocurren con neutrones en el átomo de carbono:

$$
\nu_{\mu} \mathrm{n} \rightarrow \mu^{-} \mathrm{p}, \quad \nu_{e} \mathrm{n} \rightarrow e^{-} \mathrm{p}
$$

por lo cual se utiliza el modelo del gas relativista de Fermi de Smith-Monitz [164]. Por su parte las interacciones CCQE de antineutrinos se dan con protones:

$$
\bar{\nu}_{\mu} \mathrm{p} \rightarrow \mu^{+} n, \quad \bar{\nu}_{e} \mathrm{p} \rightarrow e^{+} n
$$

${ }^{8}$ Son responsables de $\sim 40 \%$ de todas las interacciones en el detector. 
los cuales se encuentran libres (átomos H) y amarrados (átomos C), así que se usa una combinación del formalismo Llewellyn-Smith [165] y del Smith-Moniz. El diagrama de Feynman de esta interacción se muestra en la figura 2.12a.

La energía del neutrino incidente puede ser calculada en la cinemática del estado final si se determina el ángulo de deflexión y la energía de las dos partículas salientes. En general, como es el caso en MiniBooNE, la traza del nucleón saliente es difícil de medir, pero la energía y ángulo del leptón saliente están bien determinados. En estos casos se puede aproximar la energía del neutrino asumiendo que el nucleón esta en reposo.

En una interacción cuasielástica con un nucleón libre en reposo, la energía del neutrino incidente esta completamente determinada por la energía del leptón saliente $E_{l}$ y su ángulo de deflexión $\theta_{l}$ con respecto a la dirección del neutrino:

$$
E_{\nu}^{Q E}\left(\boldsymbol{p}_{n}=0\right)=\frac{1}{2} \frac{2 M\left(E_{l}-m_{l}^{2}\right)}{M-E_{l}+\sqrt{E_{l}^{2}-m_{l}^{2}} \cos \theta_{l}}
$$

El cuadrimomento transferido $Q^{2}=-q^{2}=-\left(p_{\nu}-p_{l}\right)^{2}$ también esta dado en términos de estas cantidades:

$$
Q^{2}=2 E_{\nu} E_{l}\left(1-\beta_{l} \cos \theta_{l}\right)-m_{l}^{2},
$$

donde $\beta_{l}$ es la velocidad del leptón saliente, pero esta expresión es general y no asume nada para el nucleón blanco. El modelo de gas de Fermi en la simulación de NUANCE tiene a los neutrones en un pozo de potencial con energía de amarre $B=34 \mathrm{MeV}$ y satisfaciendo la relación de dispersión $\left(E_{n}-B\right)^{2}=\left|\boldsymbol{p}_{n}\right|^{2}-M_{n}^{2}$. El momento máximo de un neutrón dentro del núcleo de amarre (el momento de Fermi) se fija a $p_{F}=220 \mathrm{MeV} / c$. La energía de amarre y momento de Fermi se fijan a través de datos de dispersión de electrones. Se implementó un proceso de calibración para reducir los efectos de ignorar los efectos del movimiento de Fermi en el cálculo de la energía de los neutrinos en interacciones CCQE (tanto para $\nu_{e}$ como $\bar{\nu}_{\mu}$ ), este procedimiento de calibración mejora la resolución de energía a $1 \mathrm{GeV}$ de la energía del muón de $\sim 15 \%$ a $\sim 10 \%$ al reducir las colas no gaussianas en la distribución de residuos fraccionales $\left(E_{\nu}^{\text {rec }}-E_{\nu}^{\text {true }}\right) / E_{\nu}^{\text {true }}$.

En modo de neutrinos la tasa de producción de los eventos CCQE en la simulación MC fue corregida a través de un incremento de $\times \sim 1.20$ en la tasa de producción de $\pi^{+}$para ajustar el número de eventos observados $\nu_{\mu}$, resultando en un incremento de $\sim 20$ en la normalización. Por su parte en modo de antineutrinos, en donde la contribución de signo incorrecto es mayor, la predicción MC de los eventos observados requirió un incremento de 1.20 a la tasa de decaimientos de $\pi^{-}$para ajustar los datos observados de $\mu^{+}$, mientras que la producción de los $\mu^{-}$resultó ser 0.99 de la tasa predicha ${ }^{9}$. En conjunto, la normalización requirió un incremento de $13 \%$. Estas normalizaciones son compatibles con la incertidumbre combinada del flujo de neutrinos y las secciones eficaces.

\subsubsection{Producción de $\pi^{0}$ por interacciones de corriente neutra (NC)}

El fondo inducido por $\nu_{\mu}$ más grande a la búsqueda de aparición de $\nu_{e}$ en MiniBooNE es la producción de $\pi^{0}$ por interacciones de corriente neutra. El modo dominante de producción $\nu_{\mu}-\mathrm{NC} \pi^{0}$ es

\footnotetext{
${ }^{9}$ Se hizo un estudio dedicado a este efecto, ver la sección V.C de la referencia [166]
} 
la excitación de nucleones en resonancias bariónicas como $\Delta(1232)$ y su subsecuente decaimiento en dos fotones, un proceso ilustrado en la figura 2.12b. Las predicciones en la simulación del detector de este proceso toman en cuenta el formalismo de Rein y Sehgal [167]. Las predicciones que existían de las secciones eficaces de este proceso tenían una gran incertidumbre, especialmente para el rango de energías de interés. Los piones neutros también pueden producirse en el proceso de dispersión coherente de un neutrino con un núcleo como conjunto: ${ }^{10} \nu_{\mu} \mathrm{A} \rightarrow \nu_{\mu} A \pi^{0}$, el cual también se simula. La colaboración encontró que la producción coherente de $\pi^{0}$ no es despreciable, lo cual exigió una medida directa de la tasa de eventos $\mathrm{NC} \pi^{0}$ para hacer correcciones a la predicción de la simulación y para constreñir las incertidumbres asociadas con tal predicción.

Medir el proceso de producción de piones neutros por interacción de corriente neutra consistió en juntar una muestra de interacciones $\mathrm{NC} \pi^{0}$ de gran pureza $(96.5 \%)$ y determinar la producción que generan estos eventos en nueve bins de momento reconstruido para $\pi^{0}$. Se describe tal medición con gran detalle en [168]. Una comparación de estas distribuciones entre los datos y las simulaciones define un factor de corrección que puede ser aplicado a cada bin de momento reconstruido de $\pi^{0}$ para hacer que la distribución simulada esté en acuerdo con la de los datos. Los datos muestran un exceso de eventos $\pi^{0}$ en la región de bajo momento, en comparación con la predicción sin corregir; mientras que la región de gran momento concuerda satisfactoriamente.

$\mathrm{Al}$ repesar la simulación usando la corrección en el momento de $\pi^{0}$ y la fracción medida de la producción coherente de $\pi^{0}$ se obtiene una predicción del número de interacciones que producen piones neutros en el detector. El error en la producción general de $\pi^{0}$ (relevante para los análisis de oscilación) se reduce de $\sim 25 \%$ a $5 \%$, una importante mejoría en la sensibilidad de la búsqueda de aparición de $\nu_{e}[169]$.

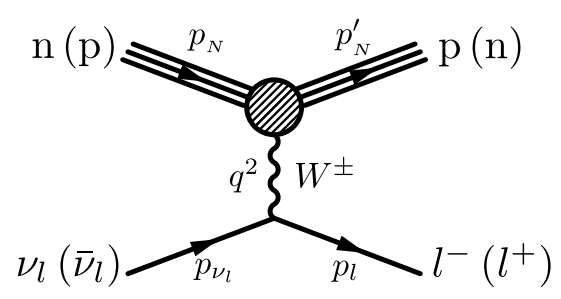

(a) CCQE.

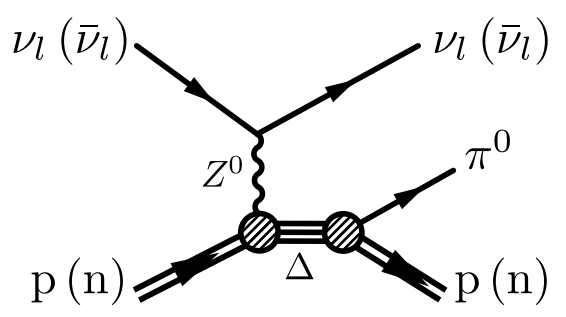

(b) $\mathrm{NC} \pi^{0}$.

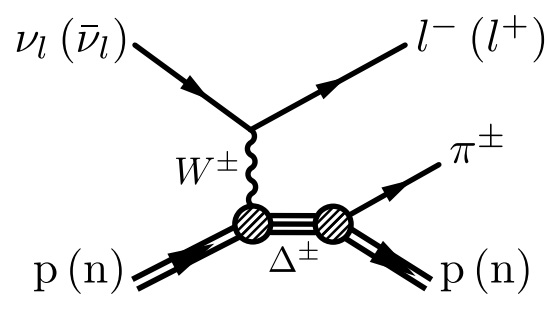

(c) $\mathrm{CC} \pi^{ \pm}$.

Figura 2.12: Diagramas de Feynman de interacciones relevantes. (a) Interacción CCQE de un $\nu_{l}$ con un nucleón para formar el correspondiente leptón cargado $l$ y el otro nucleón. (b) Producción de $\pi^{0}$ de corriente neutra $\left(\nu_{l}-\mathrm{NC} \pi^{0}\right)$ a través de la excitación de un nucleón en una resonancia $\Delta^{ \pm}$. (c) Producción de $\pi^{ \pm}$de corriente cargada $\left(\nu_{\mu}-\mathrm{CC} \pi^{ \pm}\right)$a través de la excitación de un nucleón en una resonancia $\Delta^{ \pm}$.

El generador de eventos de MiniBooNE, NUANCE, saca un conjunto de partículas y canales generados por neutrinos. Esta información es usada por la simulación del detector para modelar la propagación de partículas, la emisión y propagación de luz resultante y la respuesta de los PMT's. El experimento usa el software de simulación GEANT3 [170], en conjunto con un modelo óptico a la medida para simular las interacciones en el detector. El dominio geométrico de la simulación incluye el tanque, el suelo alrededor y la cámara del detector, incluyendo la electrónica encima. En la simulación, se asume que el aceite mineral es $\mathrm{CH}_{2}$ con una densidad de $0.845 \mathrm{~g} / \mathrm{cm}^{3}$; también se incluyen los detalles de la estructura de los PMT's, los cubos centellados y los frascos usados para la calibración con láser. Se usa el algoritmo de rastreo por defecto de GEANT3 para propagar las partículas; sin embargo se hicieron algunas modificaciones a las rutinas estándar para incluir un modelo mejorado del decaimiento Dalitz

\footnotetext{
${ }^{10}$ En oposición a la interacción de un neutrino con un solo nucleón.
} 
$\left(\pi^{0} \rightarrow e^{+} e^{-} \gamma\right)$, decaimiento del muón $(\mu \rightarrow e \gamma \gamma)$ y la posibilidad de captura del $\mu^{-}$por el carbono. El paquete por defecto de interacciones hadrónicas GFLUKA, fue sustituido por GCALOR [171].

Los eventos que provienen de interacciones fuera del tanque contribuyen significativamente al fondo de errores en la identificación de la búsqueda de oscilaciones, típicamente producen un $\mathrm{NC} \pi^{0}$ que decae en dos fotones, uno de los cuales encuentra su camino al detector. Los eventos de interacciones externas tienden a depositar toda su energía cerca de la pared del detector y sus trazas tienden a apuntar hacia el centro del detector. Se uso una muestra dedicada de eventos en la suelo para medir la tasa observada de estas interacciones, la cual demostró ser consistente con la simulación MC dentro de la incertidumbre de las medidas [172]. Las incertidumbres de estos eventos también son consideradas en los análisis.

\subsubsection{El modelo óptico de MiniBooNE}

La colaboración desarrolló un modelo óptico personalizado para caracterizar la producción y propagación de los fotones ópticos que son producidos por las partículas cargadas que atraviesan el detector.

La producción de luz ocurre a través de radiación cherenkov, centelleo y fluorescencia.

- Radiación cherenkov: cuando una partícula cargada se mueve más rápido que la luz en ese medio entonces emite radiación cherenkov, la cual es expedita y direccional. El ángulo entre la traza de la partícula y los fotones emitidos esta gobernado por la fórmula:

$$
\cos \theta_{c}=\frac{1}{\beta n}=\frac{1}{n}\left[\frac{(T+m)^{2}}{(T+m)^{2}-m^{2}}\right]^{\frac{1}{2}},
$$

donde $n$ es el índice de refracción del medio y $\beta=v / c$ es el cociente de la velocidad de la partícula y la luz. Además, del lado derecho, se ha expresado el ángulo en términos de la energía cinética $T$ y la masa de la partícula $m$. La tasa de emisión de fotones como función de la longitud de onda esta dada por:

$$
\frac{\mathrm{d}^{2} N}{\mathrm{~d} x \mathrm{~d} \omega}=\alpha q^{2}\left[1-\frac{1}{\beta^{2} n^{2}(\omega)}\right],
$$

donde, ahora, $x$ es la longitud de camino, $\omega$ la frecuencia del fotón, $\alpha$ la constante de estructura fina y $q$ la carga de la partícula. La energía cinética mínima que se necesita para producir radiación cherenkov es $T_{C h}=m\left[\left(n / \sqrt{n^{2}-1}\right)-1\right]$. El umbral de energía de los protones para producir radiación cherenkov es $T_{C h} \simeq 350 \mathrm{MeV}$.

- Centelleo y fluorescencia: las trazas de ionización de las partículas excitan los electrones en los átomos del aceite, la des-excitación subsecuente emite fotones, la cual se conoce como luz de centelleo y es por naturaleza retardada e isotrópica. Cuando los fotones ópticos son los responsables de la excitación y des-excitación de las moléculas, contrariamente a las trazas de ionización, el proceso se conoce como fluorescencia. Las características del centelleo y la fluorescencia están determinadas por la composición química del aceite. La luz de centelleo es proporcional a la pérdida de ionización de la partícula. 
La propagación o transmisión de fotones en MiniBooNE ocurre a través de procesos de dispersión, cuando un fotón óptico interactúa con una molécula y se desvía y cambia su polarización manteniendo la misma longitud de onda; fluorescencia, cuando fotones ópticos (creados por centelleo o radiación cherenkov) son absorbidos y reemitidos posteriormente con distinta longitud de onda y/o dirección ${ }^{11}$; y absorción de fotones en el aceite, la diferencia entre la tasa total de desaparición y la suma de la atenuación debido a centelleo y fluorescencia. Los estudios que se hicieron de las propiedades ópticas del aceite se encuentran en [173].

Se usa un espacio de 35 parámetros para representar todas las posibles variaciones en las propiedades ópticas del detector, se forma una matriz de covarianza que relaciona todos esos parámetros, con un procedimiento descrito en [174]. Esta matriz se usa para estimar la incertidumbre sistemática en los análisis.

\section{Simulación de la respuesta de los PMT's y adquisición digital}

El paso final de la simulación es generar la respuesta de los PMT's a los fotoelectrones de GEANT3 que llegan a los PMT's, después de eso la información se pasa a través de un código que replica el paso de los datos por la electrónica DAQ. Esto asegura que la salida final del MC es estructuralmente igual a los datos.

La respuesta de los PMT's es simulada alterando el tiempo y la carga acumulada por cada impacto de fotón, para tomar en cuenta efectos como pulsos prematuros o tardíos y comportamientos individuales de cada PMT, observados en estudios dedicados [175] y usando los datos de la calibración del láser (§ 2.2.3. También se agrega la correspondiente compensación de tiempo a cada canal. Al final la digitalización de la simulación de la respuesta de los PMT's se logra creando un pulso triangular que se usa para construir el conjunto de quads de carga y tiempo (§2.2.1).

\subsection{Reconstrucción de eventos e identificación de partículas}

Los fotones cherenkov producidos por partículas cargadas que emergen de la interacción de neutrinos son detectados por los PMT's del detector MiniBooNE. Los fotones son emitidos con un ángulo $\theta_{c}$ (ecuación (2.6)) con simetría azimutal alrededor de la traza de la partícula cargada, resultando en un patrón con forma de anillo que puede ser identificado en el arreglo de PMT's. La cantidad, distribución espacial y tiempos de arribo de estos fotones proveen información de la localización, dirección y energía de la partícula. A la extracción de tal información a partir de las medidas de la carga y tiempo de los PMT's se le llama "reconstrucción". El objetivo de esta tesis no es describir el mecanismo de reconstrucción de partículas, el cual se describe en la referencia [176].

El haz de neutrinos de $\sim 1 \mathrm{GeV}$ en MiniBooNE resulta en interacciones con relativamente poca multiplicidad de partículas salientes. En el canal más abundante, las interacciones CCQE (figura 2.12a) el nucleón suele estar debajo del umbral cherenkov y solo el leptón resultante produce significativa luz. Un muón en el detector exhibe un comportamiento mínimo de ionización a lo largo de su camino, con poca probabilidad de pérdida radiante de energía. En contraste, los electrones típicamente inducen cascadas electromagnéticas, resultando en positrones y electrones adicionales que emiten luz

\footnotetext{
${ }^{11}$ De particular importancia es la fluorescencia debido a fotones ultravioleta, los cuales no son registrados por los PMT's pero si pueden ser absorbidos y reemitidos con otra longitud de onda.
} 
cherenkov además de ser fuertemente afectados por la dispersión de Coulomb. Así pues, los muones y electrones tienen patrones de anillos cherenkov significativamente diferentes, esta diferencia es la base para distinguir la traza de un muón y de un electrón: el algoritmo de reconstrucción tiene un modelo para cada una.

El segundo proceso más abundante en MiniBooNE es la producción de $\pi^{0}$ a través interacciones de corriente neutra (figura 2.12b) en los cuales la cascada de uno de los fotones no es observada y la otra es indistinguible de la cascada de un electrón.

Como el retroceso del nucleón (núcleo para el caso de la dispersión coherente) esta debajo del umbral cherenkov, los eventos $\mathrm{NC} \pi^{0}$ están bien descritos en el algoritmo de reconstrucción por dos trazas de electrones que apuntan a un vértice común. Errores en la identificación de $\pi^{0}$ (por ejemplo, debido a una gran asimetría en la energía de los dos fotones o a un pequeño ángulo de separación entre los fotones que deja anillos cherenkov traslapados) forma la mayor parte del fondo en la búsqueda de oscilaciones. Por lo tanto, la reconstrucción e identificación de eventos $\pi^{0}$ es primordial.

Si bien se pueden adaptar otros canales en el algoritmo de reconstrucción, el análisis de aparición de $\nu_{e}\left(\bar{\nu}_{e}\right)$ solamente usa cuatro modelos de eventos: traza sencilla de electrón, traza sencilla de muón, dos trazas de fotones y dos trazas de fotones con la masa invariante de $\pi^{0}$. Estos modelos se representan en la figura 2.13 .

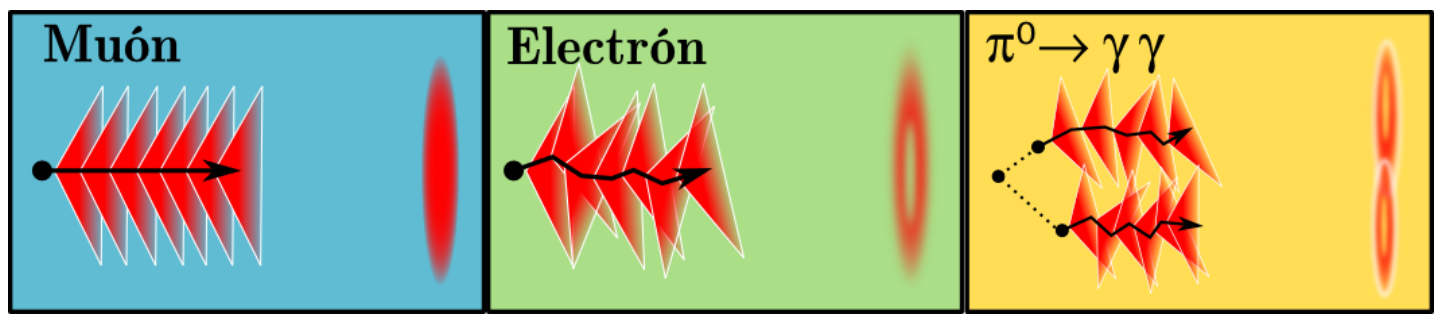

Figura 2.13: Identificación de partículas en MiniBooNE. Los eventos son reconstruidos como eventos de muón, anillo de luz bien definidos; eventos de electrón, anillos de luz difusos; o $\pi^{0}$, par de anillos difusos. MiniBooNE no puede reconocer entre el anillo dejado por un electrón y un fotón.

La reconstrucción de una traza sencilla en el detector, ya sea de electrón o de muón, se basa en un modelo con siete parámetros: las tres coordenadas del punto de inicio, $x_{0}, y_{0}, z_{0}$; el tiempo de inicio, $t_{0}$; la dirección, $\theta_{0}, \phi_{0}$; y la energía cinética, $E_{0}$. El tiempo y punto de inicio son referidos como el "vértice" del evento. La dirección esta descrita en términos del ángulo polar $\theta_{0}$ definido con respecto a la dirección del haz de neutrinos y el ángulo azimutal $\phi_{0}$ alrededor de este eje del haz. Para el modelo del electrón, las variaciones estocásticas en las cascadas electromagnéticas son modeladas en promedio, no se intenta tomar en cuenta variaciones evento por evento en el perfil de la cascada. Lo mismo va para las variaciones mucho más pequeñas de la propagación del muón. Esta simplificación permite que la traza sea completamente especificada con los siete parámetros de arriba, con los cuales se forma el vector de parámetros $\mathbf{X}=\left(x_{0}, y_{0}, z_{0}, t_{0}, \theta_{0}, \phi_{0}, E_{0}\right)$, como se muestran en figura 2.14a.

Las observables de un evento son medidas con los 1280 PMT's en la región principal del detector. Para cada PMT, se tiene: un bit indicando si el tubo registró un impacto; si el tubo registro un impacto, la carga medida del impacto y el tiempo medido del impacto.

Asumiendo que el comportamiento de los PMT's es independiente, la verosimilitud para un conjunto de mediciones en los PMT's dados los parámetros de la traza $\mathbf{X}$ se expresa como 


$$
\mathcal{L}(\mathbf{X})=\prod_{\text {Sin impacto }}(1-P(i ; \mathbf{X})) \times \prod_{\text {Con impacto }} P(i ; \mathbf{X}) f_{q}\left(q_{i} ; \mathbf{X}\right) f_{t}\left(t_{i} ; \mathbf{X}\right)
$$

en donde los productos se toman sobre todos los PMT's impactados y no impactados. Además, $P(i ; \mathbf{X})$ es la probabilidad de que el $i$-ésimo tubo sea impactado dados los parámetros $\mathbf{X} ; q_{i}, t_{i}$ son la carga y tiempo medidos en el $i$-ésimo PMT; $\left.f_{q}\left(q_{i}, \mathbf{X}\right)\right)$ es la función de densidad de probabilidad para la carga medida en el $i$-ésimo PMT, dado $\mathbf{X}$, evaluado en $q_{i}$; y $\left.f_{t}\left(t_{i}, \mathbf{X}\right)\right)$ es la función de densidad de probabilidad para el tiempo medido dado $\mathbf{X}$, evaluado en $q_{i}$.

El algoritmo de reconstrucción maximiza la verosimilitud para determinar los parámetros de las trazas bajo la hipótesis de electrón, muón o pión neutro. Subyacente a la verosimilitud hay un modelo de trazas y del detector que calcula para un parámetro dado el conjunto de funciones de distribución de probabilidad de carga y tiempo para cada PMT, tomando en cuenta producción espacialmente extendida de radiación cherenkov y de centello así como efectos de luz indirecta desde procesos ópticos subsecuentes. En particular, la reconstrucción de dos trazas requiere reconstruir dos trazas sencillas que se encuentren trasladadas distancias $s_{1}$ y $s_{2}$ de un vértice común. Adicionalmente se constriñe que este par de trazas reconstruyan la masa invariante el pión neutro. Los parámetros usados para la reconstrucción de dos trazas se pueden ver en la figura 2.14b.

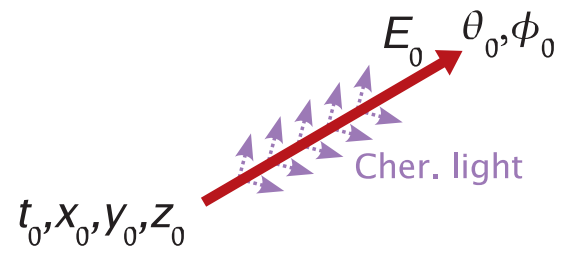

(a) Traza sencilla

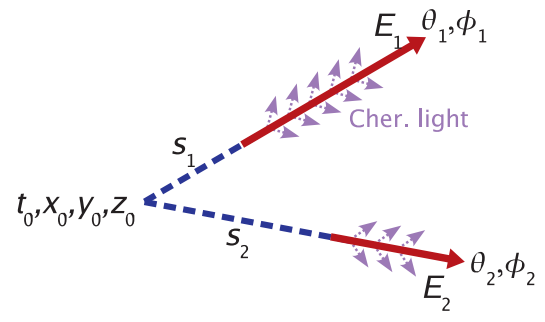

(b) Doble traza.

Figura 2.14: Parámetros internos para la reconstrucción de trazas. (a) Traza sencilla dejada por un muón o electrón y (b) traza doble dejada por dos fotones. Los parámetros de la doble traza pueden ser constreñidos para que la masa invariante de los dos fotones $m_{\gamma \gamma}$ siempre sea $m_{\pi^{0}}$. Figuras sacadas de [176].

\subsubsection{Identificación de partículas}

Además de los distintos patrones de luz que dejan los muones y electrones, se distinguen los eventos de $\nu_{\mu}\left(\bar{\nu}_{\mu}\right)$ de los $\nu_{e}\left(\bar{\nu}_{e}\right)$ en que más de $80 \%$ de las veces se observa el electrón retrasado del decaimiento de los muones. Se usa la información de la carga y el tiempo en la ventana de $19.2 \mu$ s para reconstruir las interacciones de neutrinos e identificar las partículas producidas. Se define esta ventana de tiempo como un "evento" y esta dividida en "subeventos", colecciones de impactos en los PMT's aglomerados en un tiempo de $\sim 100 \mathrm{~ns}$. Un evento CCQE $\nu_{\mu}$ puede tener dos subeventos: el primero con fotones producidos por el muón generado y el segundo del decaimiento del muón al electrón. Por su parte un evento CCQE $\nu_{e}$ solo tiene un subevento.

El máximo de la verosimilitud que arrojan los análisis del electrón, muón y el ajuste para dos trazas con masa fija pueden ser usados para probar hipótesis. Las dos cantidades 


$$
\begin{aligned}
& \mathcal{R}_{e / \mu} \equiv \log \left(\mathcal{L}_{e} / \mathcal{L}_{\mu}\right)=\log \mathcal{L}_{e}-\log \mathcal{L}_{\mu}, \\
& \mathcal{R}_{e / \pi} \equiv \log \left(\mathcal{L}_{e} / \mathcal{L}_{\pi}\right)=\log \mathcal{L}_{e}-\log \mathcal{L}_{\pi},
\end{aligned}
$$

son usadas en un evento dado para determinar si la hipótesis del electrón se prefiere sobre la hipótesis del muón o del pión neutro.

Para los eventos $\nu_{e}\left(\bar{\nu}_{e}\right) \mathrm{CCQE}$, la fracción $\mathcal{R}_{e / \mu}$ tiende a tomar valores positivos, indicando que el ajuste a la hipótesis del electrón esta favorecida sobre la hipótesis del muón. Análogamente, $\mathcal{R}_{e / \mu}$ tiende a ser negativa para los eventos $\nu_{\mu}\left(\bar{\nu}_{\mu}\right)$ CCQE, indicando que la hipótesis del muón ajusta mejor los datos que la del electrón. A grandes energías $(>1 \mathrm{GeV})$, la separación entre electrón y muón mejora gracias a que la longitud que viajan los muones crece aproximadamente linealmente con la energía, mientras que la del electrón crece más lentamente. Asimismo, la cantidad $\mathcal{R}_{e / \pi}$ prueba si un evento dado se ajusta mejor como una traza sencilla de electrón o como $\pi^{0}$. En general, la separación entre electrones y piones se vuelve más difícil a grandes energías conforme crece la asimetría en las energías de los fotones que decaen de $\pi^{0}$ y las fluctuaciones en las cascadas de un electrón sencillo se vuelven tan grandes que imitan la presencia de un segundo fotón. También, los eventos $\pi^{0}$ en que un fotón deja el tanque sin convertirse presentan un fondo irreducible a bajas energías. Evento por evento, MiniBooNE es incapaz de discernir entre neutrinos y antineutrinos, es decir entre $e^{+}$y $e^{-}$ o $\mu^{+}$y $\mu^{-}$, sin embargo acumulando muchos datos se puede construir una distribución angular que permite estimar el número de neutrinos y antineutrinos en cada modo.

\section{Criterios de selección de eventos}

El primer paso en la selección de eventos es el rechazo del ruido no relacionado al haz. Este es un fondo originado principalmente por rayos cósmicos, que pueden imitar la señal de interacción de un neutrino con la energía adecuada. Filtrar los datos de acuerdo a su sincronización con el haz provee una buena forma de rechazar eventos espurios. Característico de los eventos de neutrinos, se impone que el primer subevento tenga menos de 6 impactos en la región de veto y más de 200 impactos en los PMT's en la región de señal. Estos tres filtros rechazan > 99.99\% del fondo que no esta relacionado al haz ${ }^{12}$. Se aplica un filtro adicional que divide los eventos restantes en dos categorías: una muestra con dos subeventos (característico de CCQE $\nu_{\mu}$ ) y una muestra con un subeventos (característico de CCQE $\nu_{e}$ y su fondo respectivo). Además de estos filtros se hacen filtros cinemáticos para aumentar la pureza de cada muestra.

Muestra de $\nu_{\mu}$ CCQE: eventos con estructura de dos subeventos, el primer subevento satisface los filtros anteriores y el segundo subevento (un electrón Michel) registra un máximo de 200 y 6 impactos en la región de señal y la de veto, respectivamente. Se requiere un tiempo mínimo de 1000 ns entre el primer y segundo subevento. Tanto el vértice como el punto final de la traza del primer subevento deben ocurrir en el volumen alrededor del centro del tanque delimitado por un radio de $500 \mathrm{~cm}$. La energía reconstruida $E_{\nu}^{Q E}>150 \mathrm{MeV}$. La separación entre los vértices del muón y del electrón deben satisfacer cierta relación que depende de la energía reconstruida del muón.

\footnotetext{
${ }^{12}$ Mayor de 1000 a 1 para rayos cósmicos.
} 
Muestra de $\nu_{e}$ CCQE: identificados por tener estructura de un subevento y los filtros iniciales. El vértice reconstruido, asumiendo hipótesis de electrón, debe estar dentro de una esfera concéntrica al detector de $500 \mathrm{~cm}$ de radio. Para minimizar la cantidad de $\nu_{\mu}$ que son identificados erróneamente como $\nu_{e}$ se demanda que el punto final de la traza, asumiendo hipótesis de muón en la reconstrucción, debe estar contenido dentro de un volumen de $488 \mathrm{~cm}$ de radio. La energía visible mínima debe ser mayor a $140 \mathrm{MeV}$. Se aplica un filtro adicional basado en cinemática para rechazar eventos que provienen de interacciones exteriores a la región de interés. Hay varios filtros que vienen de los algoritmos de reconstrucción y las fracciones (2.9) y (2.10).

\subsection{Resultados publicados por MiniBooNE}

MiniBooNE tuvo diez años de corrida de 2002 a 2012, en los cuales publicó diez artículos de oscilaciones, doce artículos de secciones eficaces ${ }^{13}$ y flujos, uno sobre el detector, uno de búsqueda de supernovas, además se hicieron diecisiete tesis de doctorado. En particular los artículos sobre secciones eficaces cubren cerca del $90 \%$ de las interacciones de los neutrinos, muchas de las cuales no habían sido medidas antes, y han motivado mucho trabajo teórico sobre interacciones neutrino-núcleo. La lista completa se puede ver en: http://www-boone.fnal.gov/publications.

La tasa de neutrinos por POT se mantuvo muy estable, con variaciones menores a $<2 \%$, a lo largo de los diez años. Solamente hubo dos incidentes: se cambio un corno en 2004, pero desde entonces tanto el corno como el blanco han corrido sin problemas, imponiendo un récord; y el desplome en 2006 de unas placas de hierro en el túnel de decaimiento bajando la cantidad de antineutrinos.

De forma consistente en toda la vida del experimento los cortes del haz y el detector para los análisis de secciones eficaces y oscilación quitan solamente 2-3\%. Durante el tiempo de vida del experimento, solamente 35 PMT's y canales de electrónica fallaron $(\sim 2 \%)$.

El enfoque de esta tesis está en los artículos sobre el objetivo principal de MiniBooNE (comprobar o refutar los resultados de LSND), es decir los análisis de aparición de neutrinos (antineutrinos) del electrón para la búsqueda de oscilación de neutrinos.

- En 2007 sale el primer artículo publicado por la colaboración, intitulado "A Search for Electron Neutrino Appearance at the $\Delta m^{2} \sim 1 \mathrm{eV}^{2}$ Scale", coincidió con el primer artículo de oscilaciones [177], ahí se reporta el estado de la búsqueda de aparición de $\nu_{e}$ en un haz de $\nu_{\mu}$. Se llevaron a cabo dos análisis independientes para energías superiores a $475 \mathrm{MeV}$, ninguno de los cuales mostró un exceso significativo sobre el fondo esperado de $\nu_{e}$. Los datos encontrados resultaron inconsistentes con respecto a un modelo de oscilación de dos neutrinos. De hecho el límite obtenido a $90 \%$ N.C. excluía completamente la región permitida al mismo nivel de confianza por LSND.

- Desde la primera publicación la colaboración observa un exceso de eventos a candidatos $\nu_{e}$ en la región de energía de $200<E_{\nu}^{Q E}<475 \mathrm{MeV}$. Habiendo hecho un estudio extensivo sobre la causa de estos eventos la colaboración publicó en 2009 el artículo [178] "Unexplained Excess of Electron-Like Events From a 1-GeV Neutrino Beam", precisamente el exceso de eventos no es del

\footnotetext{
${ }^{13}$ Durante un tiempo el experimento SciBooNE se encontraba en la línea del BNB, a $\sim 100 \mathrm{~m}$ del punto de producción. Midió las secciones eficaces de los neutrinos en carbono y hierro, estas mediciones sirvieron para MiniBooNE y el experimento T2K en Japón.
} 


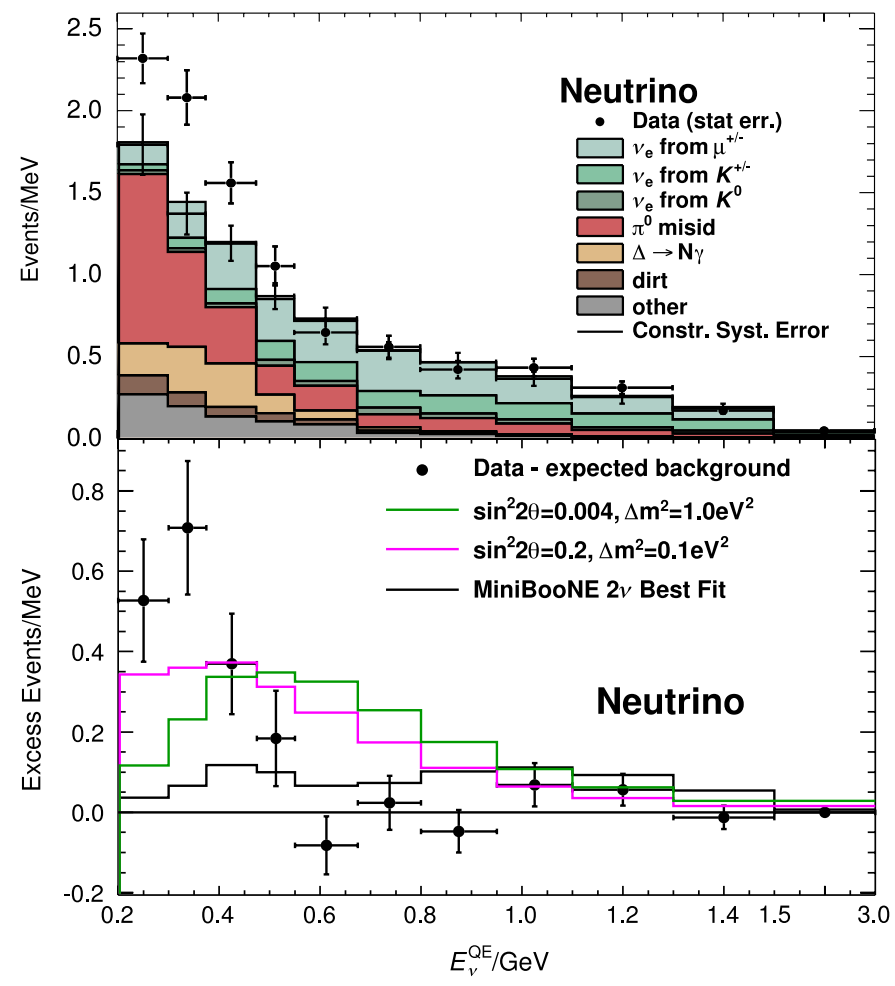

(a) Distribuciones para los datos $\nu_{e}$ CCQE como función de $E_{\nu}^{Q E}$ (arriba) y exceso de eventos $\nu_{e}$ (abajo).

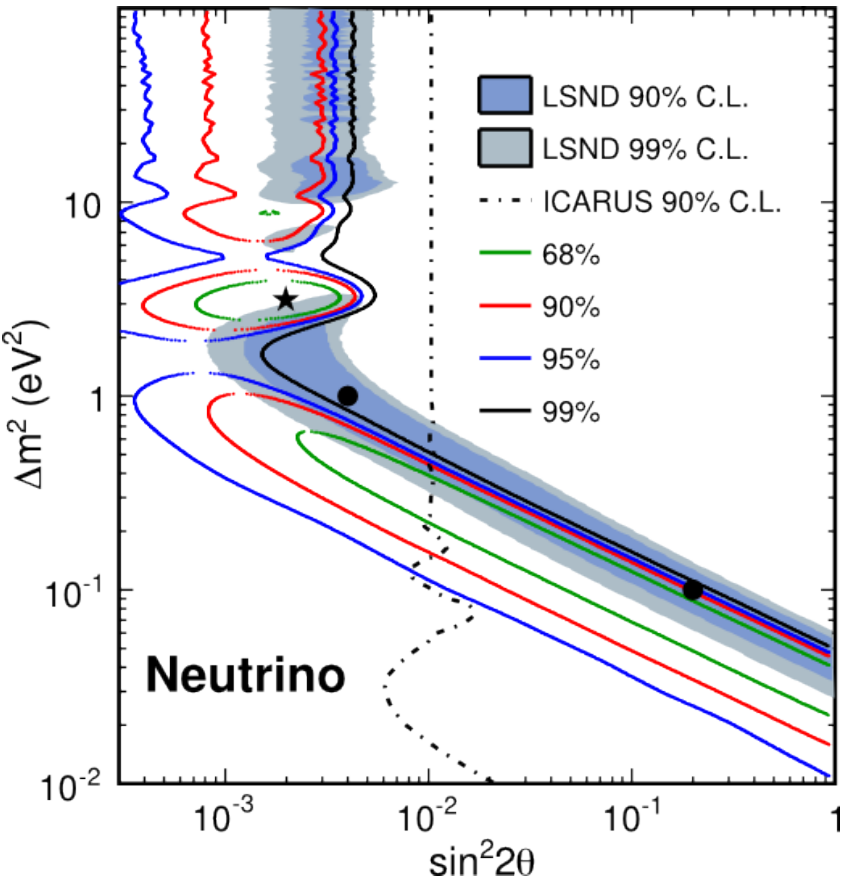

(b) Regiones permitidas para oscilaciones de dos neutrinos por MiniBooNE.

Figura 2.15: Resultados de MiniBooNE en el modo de neutrinos de 2013 [181]. (a) Distribuciones para los datos $\nu_{e}$ CCQE (puntos con error estadístico) y fondo (histograma con error sistemático), como función de $E_{\nu}^{Q E}$ (arriba); exceso de eventos $\nu_{e}$ como función de $E_{\nu}^{Q E}$, se muestran los datos (error estadístico y sistemático), la expectación del mejor ajuste y de otro par de puntos muestra (abajo). (b) Regiones permitidas por el experimento MiniBooNE para $E_{\nu}^{Q E}>200 \mathrm{MeV}$ dentro de un modelo de oscilaciones de dos neutrinos. Se muestra el límite de exclusión del experimento ICARUS [126] y las regiones permitidas de LSND. La estrella muestra el mejor ajuste de MiniBooNE y los círculos los valores de muestra de la figura de al lado.

todo consistente con el modelo de oscilación de neutrinos y su origen es desconocido, pero queda establecido que no puede adjudicarse a ninguno de los fondos conocidos. Habiendo aumentado la estadística a $6.46 \times 10^{20}$ POT el exceso en la región de bajas energías es de $128.8 \pm 20.4 \pm 38.3$ eventos, mientras que para 475-1250 MeV se observan 408 de los 385.9 \pm 35.7 eventos esperados.

- También en 2009 se reporta el estado de la búsqueda de oscilaciones en el modo de antineutrinos [179]. Con una pequeña muestra de apenas $3.39 \times 10^{20}$ POT, el experimento en su artículo "Search for Electron Antineutrino Appearance at the $\Delta m^{2} \sim 1$ eV Scale" no observa ningún exceso significativo de eventos, ni para la región de baja energía (200-475 MeV) ni para el resto. Dada la baja estadística, este resultado no es concluyente, la motivación para liberarlo yace en mostrar una comparación con el exceso a bajas energías observado en el modo de neutrinos.

- Sin embargo la situación cambia en 2010, con la publicación [180] "Event Excess in the MiniBooNE Search for $\bar{\nu}_{\mu} \rightarrow \bar{\nu}_{e}$ Oscillations" en donde con $5.66 \times 10^{20}$ POT se reporta la observación de un exceso de $43.2 \pm 22.5$ eventos para la región completa de energía (200-3000 MeV), y un exceso de $20.9 \pm 14.0$ eventos para el rango de energía 475-1250 MeV. En esta búsqueda se observa una gran compatibilidad entre las regiones permitidas de LSND y MiniBooNE.

- Habiendo aumentado la estadística del modo de antineutrinos a $11.27 \times 10^{20}$ POT se reportan 


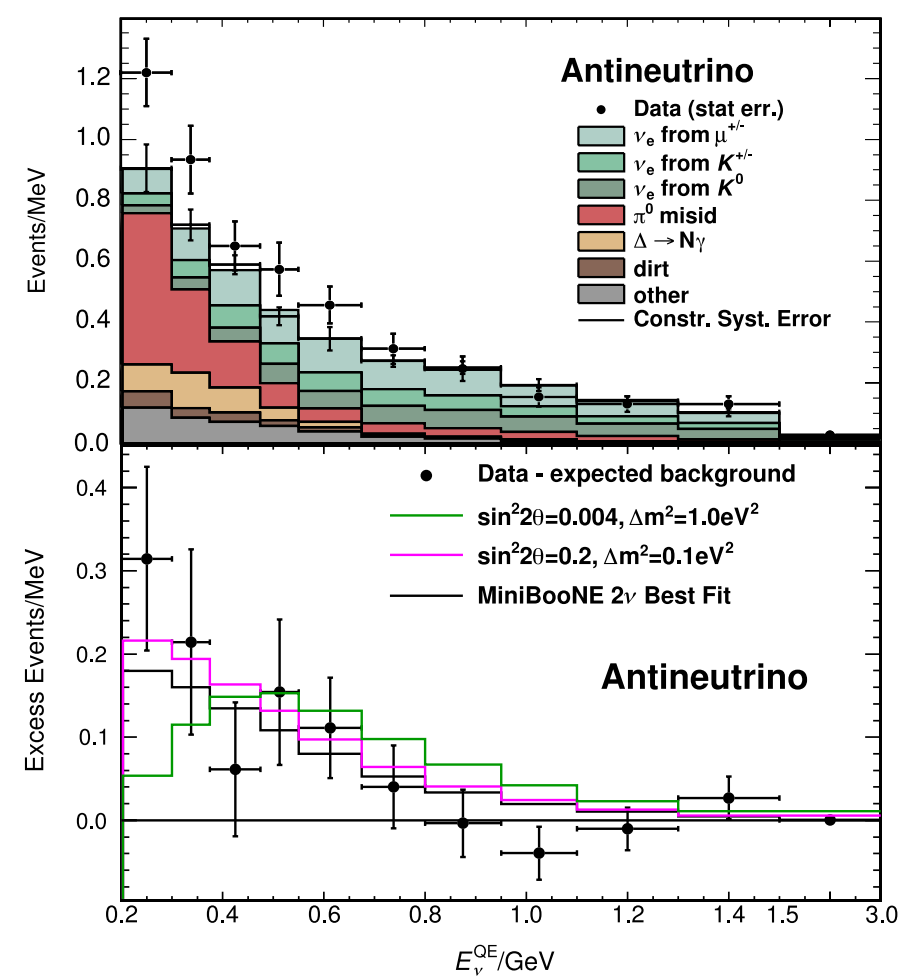

(a) Distribuciones para los datos $\bar{\nu}_{e}$ CCQE como función de $E_{\nu}^{Q E}$ (arriba) y exceso de eventos $\bar{\nu}_{e}$ (abajo).

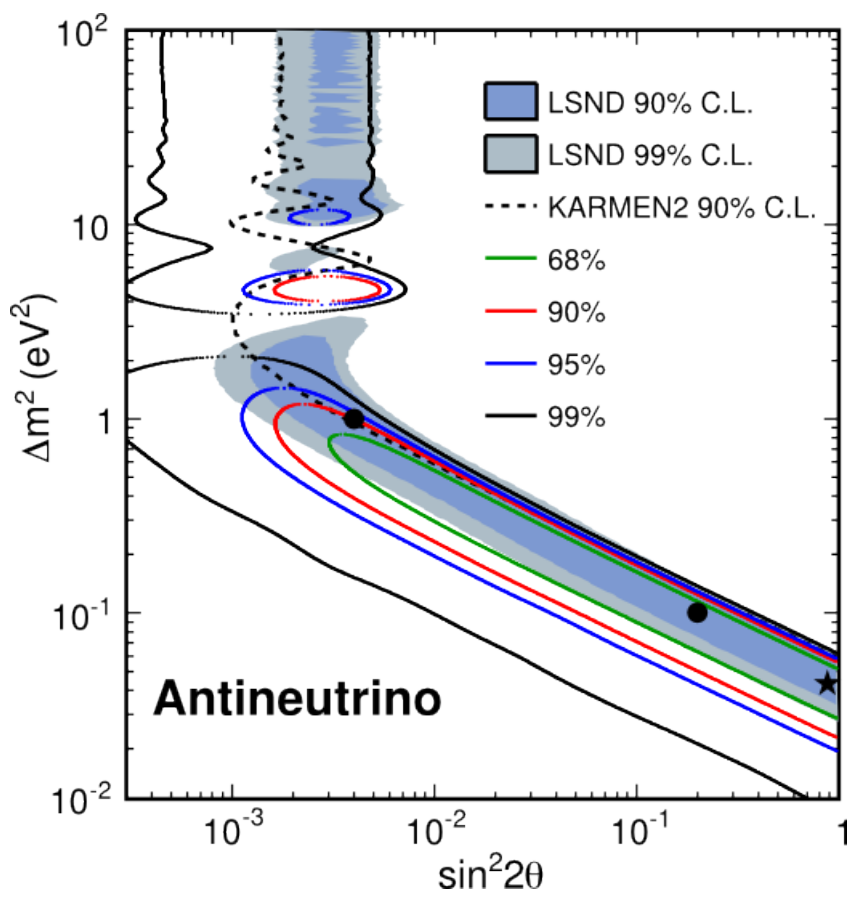

(b) Regiones permitidas para oscilaciones de dos neutrinos por MiniBooNE.

Figura 2.16: Resultados de MiniBooNE en el modo de antineutrinos de 2013 [181]. (a) Distribuciones para los datos $\bar{\nu}_{e}$ CCQE (puntos con error estadístico) y fondo (histograma con error sistemático), como función de $E_{\nu}^{Q E}$ (arriba); exceso de eventos $\bar{\nu}_{e}$ como función de $E_{\nu}^{Q E}$, se muestran los datos (error estadístico y sistemático), la expectación del mejor ajuste y de otro par de puntos muestra (abajo). (b) Regiones permitidas por el experimento MiniBooNE para $E_{\nu}^{Q E}>200 \mathrm{MeV}$ dentro de un modelo de oscilaciones de dos neutrinos. Se muestra el límite de exclusión del experimento KARMEN [124] y las regiones permitidas de LSND. La estrella muestra el mejor ajuste de MiniBooNE y los círculos los valores de muestra de la figura de al lado.

los resultados de la búsqueda de oscilaciones $\bar{\nu}_{\mu} \rightarrow \bar{\nu}_{e}$ [181], en el artículo "Improved Search for $\bar{\nu}_{\mu} \rightarrow \bar{\nu}_{e}$ Oscillations in the MiniBooNE Experiment" publicado a inicios de 2013. Además del incremento de la estadística se hicieron varias mejorías a los análisis y estimación de errores, los cuales también afectan al análisis de neutrinos. En modo de antineutrinos se reporta un exceso de $78.4 \pm 28.5(2.8 \sigma)$ eventos en el rango de energía 200-1250 MeV y en modo de neutrinos de $162.0 \pm 47.8(3.4 \sigma)$. El modo de antineutrinos es consistente con un modelo de oscilación de dos neutrinos, además de que las regiones permitidas de oscilación muestran traslape con las de LSND, sin embargo en el modo de neutrinos los datos son marginalmente compatibles con un simple modelo de oscilación de dos neutrinos y la región de compatibilidad con LSND es muy reducida. A la fecha estos son los resultados más recientes en la búsqueda de oscilaciones por aparición y se pueden ver en las figuras 2.15 y 2.16, para el modo de neutrinos y antineutrinos, respectivamente. Cabe mencionar que los datos para este análisis fueron liberados a finales de 2012 .

Actualmente MiniBooNE ha sido aprobado para hacer una corrida corta (de seis a diez semanas) para buscar materia oscura [182], en concreto buscará WIMPS (weakly interacting massive particles) de masa pequeña producidas en un haz de protones como se predicen en ciertos modelos teóricos recientes. 
En 2014 se tendrán los resultados de tal búsqueda. Actualmente la colaboración considera e investiga la posibilidad de agregar centellador orgánico al aceite del tanque, para aumentar la radiación de centelleo en el tanque [183]. Además de la inversión económica, esta nueva implementación requeriría crear un nuevo modelo óptico del detector. La generación de radiación cherenkov sería prácticamente la misma, pero la potenciación de la radiación de centelleo permitiría mejor determinación en los procesos de corriente neutra.

Con el propósito de investigar el exceso de eventos a bajas energías observado por MiniBooNE se propuso el experimento MicroBooNE [184] para medir secciones eficaces en esa región de energía. MicroBooNE construirá y operará una liquid Argon Time Projection Chamber (LArTPC) de 170 toneladas en la línea del haz Booster en el mismo Fermilab. El detector sirve como el paso necesario en un programa escalonado hacia la construcción de detectores LArTPC masivos de kilotoneladas. 


\section{Capítulo 3}

\section{Análisis de oscilaciones}

MiniBooNE es un experimento de oscilación de neutrinos, lo que hace es buscar el exceso de $\nu_{e}\left(\bar{\nu}_{e}\right)$ en un haz primordialmente de $\nu_{\mu}\left(\bar{\nu}_{\mu}\right)$, es decir, es un experimento de aparición. Sin embargo también cuenta con resultados de desaparición de neutrinos, en $\S 1.4 .7$ se explica lo que es un experimento de aparición y desaparición. Este trabajo se limita a los análisis de aparición de neutrinos y antineutrinos únicamente. En esencia, la tarea fundamental de los análisis estadísticos que se hicieron en este trabajo es determinar si la señal observada de $\nu_{e}\left(\bar{\nu}_{e}\right)$ es consistente con el modelo de oscilaciones de neutrinos en el vacío, $\nu_{\mu} \rightarrow \nu_{e}\left(\bar{\nu}_{\mu} \rightarrow \bar{\nu}_{e}\right)$.

El análisis de datos tiene varias tareas en la física como: estimar (medir) parámetros, cuantificar la incertidumbre de estas estimaciones o probar el alcance al cual las predicciones teóricas se encuentran en concordancia con los datos experimentales. Se debe reconocer el rol fundamental que juega la incertidumbre en la física de partículas elementales: la teoría no es determinista, es decir la mecánica cuántica tiene una naturaleza probabilística; hay errores aleatorios en las mediciones, presentes aún estando exentos de efectos cuánticos; hay cosas que en principio podríamos conocer pero en la práctica no las conocemos, por ejemplo por limitaciones de costo o tiempo. En estas circunstancias la estadística se vuelve una gran herramienta, ya que permite cuantificar las incertidumbres.

Nota: la sección 3.1 no es esencial para entender los resultados de esta tesis. El lector familiarizado con métodos estadísticos puede ir a $§ 3.2$ directamente.

\subsection{Nociones de probabilidad y estadística}

Desafortunadamente los estadísticos no concuerdan en los principios básicos. A grandes rasgos se puede pensar en dos escuelas: la bayesiana y la frecuentista (o clásica). Los primeros hacen uso del teorema de Bayes [185]. El enfoque bayesiano es más cercano al razonamiento cotidiano, en el cual la probabilidad es interpretada como un grado de confianza de que algo pasará o de que un parámetro tendrá un valor dado. El enfoque frecuentista es más cercano al razonamiento científico, en donde la probabilidad significa la frecuencia relativa de que algo pase. Esto la hace más objetiva, ya que puede ser determinada independientemente del observador, pero se restringe a fenómenos repetibles. En particular, se puede definir la probabilidad frecuentista de observar datos (los cuales son aleatorios), pero no para el valor verdadero de un parámetro (el cual es fijo, aún cuando sea desconocido). 
En el régimen asintótico, es decir cuando hay muchos datos y las incertidumbres estadísticas son pequeñas comparadas con la frontera física más cercana, los resultados numéricos para la estimación de parámetros y comprobación de hipótesis tienden a ser iguales para los dos enfoques. Sin embargo los resultados exactos para cada enfoque requieren información que no esta permitida por el otro enfoque:

- Los resultados frecuentistas exactos requieren como entrada las probabilidades de observar todos los datos, tanto los datos que fueron observados como los que pudieron haber sido observados (simulación).

- Los resultados bayesianos exactos requieren como entrada las creencias previas del físico haciendo el análisis. Lo cual es necesariamente subjetivo y no esta permitido en el método frecuentista.

A pesar de que se tiene cierto concepto de la probabilidad desde hace mucho, no se desarrolló una teoría matemática apropiada sino hasta 1933 cuando Andrey Kolmogorov público sus Fundamentos de la Teoría de Probabilidad [186]. En esta teoría la probabilidad es un concepto básico sin definir excepto por el hecho de que debe satisfacer los axiomas de Kolmogorov. Pensemos en esta probabilidad abstracta como la probabilidad matemática.

Probabilidad matemática. Definimos $\Omega$ como el conjunto de todos los posibles "eventos elementales" $X_{i}$ que son "exclusivos", la ocurrencia de uno de ellos implica que ninguno de los otros ocurre. Luego definimos la probabilidad de la ocurrencia de $X_{i}, P\left(X_{i}\right)$, como aquella que obedece los axiomas de Kolmogorov:

$$
\begin{aligned}
& \text { (a) } P\left(X_{i}\right) \geq 0 \text { para todo } i \\
& \text { (b) } P\left(X_{i} \cup X_{j}\right)=P\left(X_{i}\right)+P\left(X_{j}\right) \\
& \text { (c) } \sum_{\Omega} P\left(X_{i}\right)=1 .
\end{aligned}
$$

Sin embargo para la estadística necesitamos una definición operacional que permita calcular probabilidades, tal definición será más restrictiva pero siempre que satisfaga los axiomas de Kolmogorov su teoría se aplica.

Probabilidad frecuentista. Consideremos un experimento en el cual se observa una serie de eventos, algunos de los cuales son del tipo $X$. Supongamos que el número total de eventos es $N$ y que el número de eventos tipo $X$ es $n$. La probabilidad frecuentista de que cualquier evento individual sea de tipo $X$ se puede definir como el límite empírico de la proporción:

$$
P(X)=\lim _{N \rightarrow \infty} \frac{n}{N}
$$

La probabilidad frecuentista tiene las siguiente propiedades:

- No se requiere una cantidad infinita de experimentos. En principio, siempre y cuando se puedan hacer más experimentos se puede obtener la precisión requerida.

- Hay una restricción implícita: esta probabilidad solo se puede aplicar a experimentos repetibles. 
- A pesar de que ningún experimento puede ser repetido con exactamente las mismas condiciones, un físico deber ser capaz de reproducir todas las condiciones relevantes. Aún cuando eso sea imposible, debe hacer correcciones que consideren los cambios inevitables. La buena ciencia debe producir resultados reproducibles.

En este trabajo el enfoque que se sigue es el frecuentista, por lo mismo se omitirán los detalles relacionados a la escuela bayesiana. Sin embargo, por completez, se menciona el teorema de Bayes.

El teorema de Bayes: da la relación entre las probabilidades de $P(A)$ y $P(B)$, y las probabilidades condicionales de $A$ dado $B$ y $B$ dado $A, P(A \mid B)$ y $P(B \mid A)$, respectivamente. En su forma más común la relación es:

$$
P(A \mid B)=\frac{P(B \mid A) P(A)}{P(B)}
$$

donde $P(A)$ es la probabilidad a priori, el grado inicial de creencia en $A$ y $P(A \mid B)$ la probabilidad $a$ posteriori, es el grado de creencia habiendo considerado $B$.

\subsubsection{Estadística a nivel práctico}

En la teoría de la probabilidad, en general, se empieza con un problema bien definido y se calculan los posibles resultados de un experimento específico. La estadística trata de resolver el problema inverso de usar los datos para inferir cuales son las leyes relevantes para nuestro experimento. Sin embargo, debe ser claro, que la estadística depende de los resultados de la teoría de probabilidad. Un problema básico que enfrenta un experimental es el de resumir sus datos de forma eficiente, usualmente esto radica en comprobar si los datos son consistentes con una hipótesis dada y usar los datos para determinar los parámetros de un modelo.

Estimación de parámetros: se requiere determinar, de forma eficiente y sin sesgos, el valor de un parámetro y su error. Sin un estimado del error, el experimento no tiene sentido. El error permite determinar la significación o importancia del resultado obtenido. Se dice que la estimación es eficiente si se deriva tanta información como sea posible de los datos. Por su parte si carece de sesgos significa que en promedio el método que usamos nos dará el resultado correcto.

Comprobación de hipótesis: a partir de los datos se pone a prueba una hipótesis sobre el comportamiento que los describe, el resultado de tal prueba debe estar acompañado con una declaración del nivel de confianza que se tiene. Se debe considerar si los datos son compatibles con una hipótesis, no solamente si son incompatibles con la falsedad de tal hipótesis.

Estas dos tareas se encuentran íntimamente relacionadas. Para poder determinar un parámetro se asumen algunas hipótesis como verdaderas; mientras que una hipótesis específica involucre un parámetro cuyo valor requiera ser determinado como parte del procedimiento de comprobación. En lo que nos concierne la estadística es una sirviente de la ciencia, nos interesa obtener las respuestas a nuestros problemas tan pronto como sea posible. No queremos gastar mucho tiempo ${ }^{1}$ en un método muy sofisticado para resolver un problema, cuando un método más sencillo es casi tan bueno.

\footnotetext{
${ }^{1}$ Nuestro propio tiempo así como el tiempo de una computadora.
} 


\section{Tipos de errores}

Hay dos, fundamentalmente diferentes, tipos de errores asociados con cualquier procedimiento de medición, a saber: errores aleatorios (también llamados estadísticos) y errores sistemáticos. Los primeros vienen de la sencilla incapacidad que tienen los instrumentos de medición de dar mediciones infinitamente certeras, causados por fluctuaciones inherentes e impredecibles en las lecturas de los aparatos de medición. Los otros se encuentran en la índole de las equivocaciones; empujando el resultado siempre en la misma dirección. Usualmente si se identifica la causa de un error sistemático puede ser eliminada, a menos de que haya restricciones adicionales. Para el caso en que no se pueden eliminar, se deben estimar los errores sistemáticos, lo cual solo se puede hacer en el formalismo de la estadística bayesiana.

El error estadístico se reduce al tomar un gran número de mediciones ${ }^{2}$, por lo tanto es una buena práctica repetir las mediciones hasta que el error estadístico tenga un tamaño similar al sistemático. Sin embargo, es primordial saber que las mediciones adicionales no disminuirán el error total por debajo del error sistemático. Un buen físico experimental debe minimizar y estimar de forma realista los errores aleatorios de su aparato experimental, además de reducir el efecto de los errores sistemáticos lo más posible [187].

\section{Distribución normal y su utilidad}

La forma general de la distribución gaussiana (también llamada distribución normal) en una variable $x$ es:

$$
f=\frac{1}{\sigma \sqrt{2 \pi}} \mathrm{e}^{-\frac{1}{2}\left(\frac{x-\mu}{\sigma}\right)^{2}}
$$

La curva $f(x)$ es simétrica alrededor del valor $x=\mu$, conocido como la media, en donde $f$ adquiere su máximo valor. El parámetro $\sigma^{2}$, conocido como varianza ${ }^{3}$, caracteriza el ancho de la distribución y el factor $(\sigma \sqrt{2 \pi})^{-1}$ es para normalizar la distribución, es decir: $\int_{-\infty}^{\infty} f(x) \mathrm{d} x=1$. Tal factor también resulta ser el máximo valor. Así pues, conforme $\sigma$ disminuye la distribución se vuelve más angosta y aumenta su valor máximo. El área fraccionaria debajo de la curva delimitada por $\mu-\sigma \leq x \leq \mu+\sigma$ es 0.68. La función $f(x)$ representa la densidad de probabilidad, es decir, la probabilidad de que se tome un valor en el intervalo $(x, x+\mathrm{d} x)$ es $P(x) \mathrm{d} x$.

Para poder dar cierto tipo de descripción de una distribución gaussiana hace falta tener medidas del punto en el cual la distribución se centra y de su ancho. Para una muestra de $N$ medidas separadas se define la media muestral y la varianza muestral:

$$
\bar{x}=\sum_{i=1}^{N} \frac{x_{i}}{N} ; \quad s^{2}=\sum_{i=1}^{N} \frac{\left(x_{i}-\mu\right)^{2}}{N}
$$

Cabe resaltar que, en general, los valores verdaderos de la media, $\mu$, y la varianza, $\sigma^{2}$, no se conocen.

\footnotetext{
${ }^{2}$ Usualmente incrementar el número de medidas en un factor de $n$ disminuirá el error estadístico en un factor de $\sqrt{n}$.

${ }^{3} \mathrm{~A} \sigma$ se le conoce como desviación estándar.
} 
Así pues, la parte derecha de (3.5) no estima adecuadamente la varianza real, en vez se usa la fórmula:

$$
s^{2}=\frac{1}{N-1} \sum_{i=1}^{N}\left(x_{i}-\bar{x}\right)^{2}
$$

En consecuencia una medida de una cantidad no nos permite estimar la dispersión de valores, si el valor "verdadero" no es conocido. El factor $1 /(N-1)$ es necesario para que $s^{2}$ sea un estimador imparcial de la varianza de la población $\sigma^{2}$.

El teorema del límite central nos asegura que si se tiene un conjunto "grande" de $n$ variables independientes $x_{i}$ con media $\mu$ y varianza $\sigma^{2}$ entonces

$$
f=\frac{1}{n} \sum x_{i}
$$

tiende a una distribución gaussiana ${ }^{4}$ de media $\mu$ y varianza $\sigma^{2} / n$. Así pues la distribución de las variables $x_{i}$ individuales es irrelevante. La única característica importante es que $\sigma^{2}$ debe ser finito. Si las $x_{i}$ están distribuidas de forma gaussiana, entonces la distribución de la equación (3.6) también es gaussiana para todos los valores de $n \geq 1$.

Es común usar las propiedades de las distribuciones normales para interpretar la significación de resultados experimentales. El uso en tal contexto depende de los siguientes supuestos: el valor de la cantidad fue calculado correctamente, la magnitud del error fue calculada correctamente, la forma de la resolución experimental es tal que la aproximación a una gaussiana es razonable.

En una gran variedad de situaciones, el resultado de repetir un experimento muchas veces produce respuestas esparcidas, aproximadamente, a lo largo de una gaussiana; la aproximación se vuelve mejor si los errores individuales que contribuyen a la respuesta final son pequeños. Cuando esto es cierto, no tiene sentido hablar de un "máximo error posible", ya que, estrictamente, sería infinito, no se podría distinguir un experimento preciso de uno erróneo. Por eso es costumbre citar a $\sigma$ como la precisión del experimento.

\section{Correlación}

Dos variables aleatorias pueden ser tales que tengan cierta tendencia a cambiar juntas, si las variables muestran comportamiento similar se dice que están positivamente correlacionadas. Si por otra parte tienden a mostrar un comportamiento opuesto, están negativamente correlacionadas. Dos variables independientes no tienen correlación. Por comodidad se emplea la notación $\mathscr{E}(y) \equiv \bar{y}$, para el valor esperado de $y$, i.e. la media de $y$. La covarianza, es una medida de que tanto cambian dos variables juntas, para dos variables aleatorias reales $x$ y $y$, la covarianza esta dada por $^{5}$ :

$$
\langle x, y\rangle \equiv \mathscr{E}[(x-\mathscr{E}[x])(y-\mathscr{E}[y])]
$$

donde queda claro que la varianza es un caso especial de la covarianza, $\sigma_{x}^{2}=\langle x, x\rangle$. De igual forma se verifica la propiedad $\langle x, y\rangle=\langle y, x\rangle$.

\footnotetext{
${ }^{4}$ para $n$ pequeño la aproximación es mejor cerca del máximo de la distribución que en las colas.

${ }^{5}$ La notación usual para la covarianza es $\operatorname{cov}(x, y)$, sin embargo para este trabajo se prefiere algo más compacto.
} 
La forma general de la distribución gaussiana en dos variables correlacionadas esta dada por la expresión ${ }^{6}$ :

$$
f(x, y)=\frac{1}{2 \pi \sigma_{x} \sigma_{y} \sqrt{1-\rho^{2}}} \exp \left(-\frac{1}{2\left(1-\rho^{2}\right)}\left[\frac{\left(x-\mu_{x}\right)^{2}}{\sigma_{x}^{2}}+\frac{\left(y-\mu_{y}\right)^{2}}{\sigma_{y}^{2}}-\frac{2 \rho\left(x-\mu_{x}\right)\left(y-\mu_{y}\right)}{\sigma_{x} \sigma_{y}}\right]\right)
$$

donde, como se espera, $\mu_{x}, \sigma_{x}, \mu_{y}, \sigma_{y}$, son la media de $x$, la desviación estándar de $x$, la media de $y$ y la desviación estándar de $y$, respectivamente. Adicionalmente

$$
\rho=\frac{\langle x, y\rangle}{\sigma_{x} \sigma_{y}}
$$

se conoce como el coeficiente de correlación entre $x$ y $y$, el cual cumple: $\rho \leq 1$. La figura 3.1 muestra una representación gráfica de las propiedades relevantes de dos variables correlacionadas. La covarianza entre dos variables se generaliza a $n$ dimensiones con la matriz de covarianza $\mathbf{M}$, donde una componente en la posición $i, j$ contiene la covarianza entre la variable aleatoria $x_{i}$ y $x_{j}$ :

$$
\mathrm{M}_{i j} \equiv\left\langle x_{i}, x_{j}\right\rangle
$$

o de forma equivalente como el valor esperado del producto del vector aleatorio por su transpuesto:

$$
\mathbf{M}=\mathscr{E}\left(\boldsymbol{x} \boldsymbol{x}^{T}\right)
$$

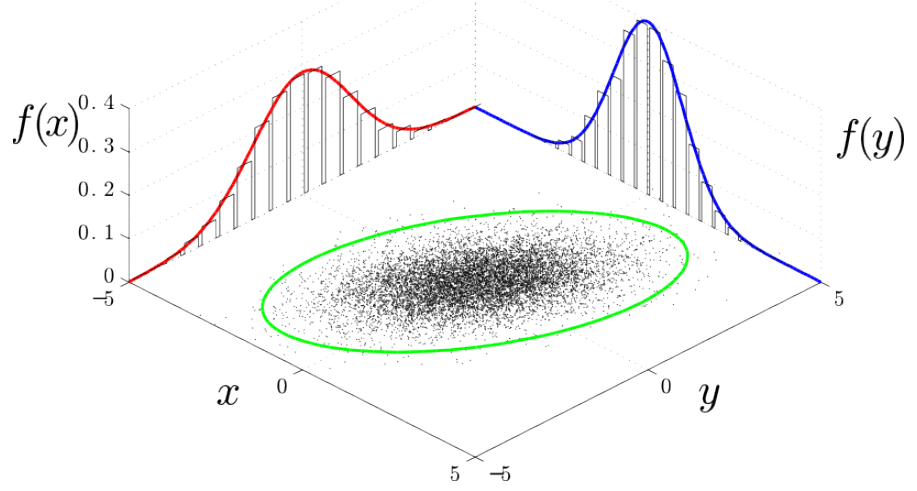

(a) Muestreo de distribución bivariada.

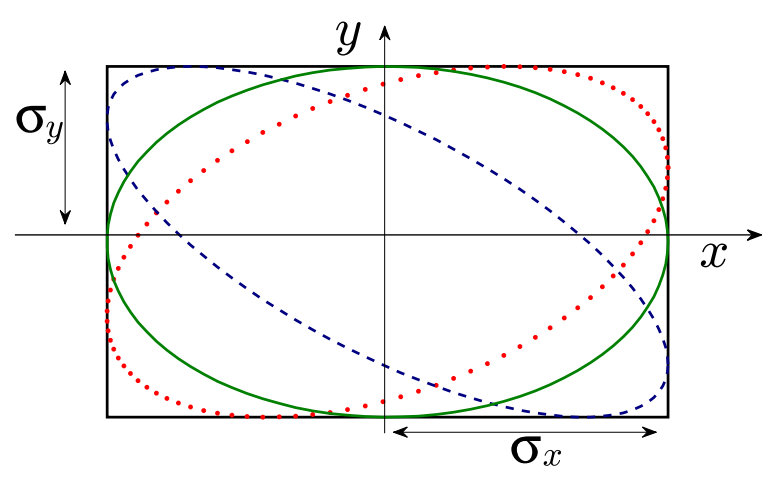

(b) Elipses para varios valores de $\rho$.

Figura 3.1: Distribución normal bivariada. (a) Se grafica una gran cantidad de muestras de una distribución normal multivariada, junto con la elipse de $3 \sigma$, las dos distribuciones marginales y los dos histogramas unidimensionales. $\left(\mu_{x}, \mu_{y}\right)$ determinan el centro de la elipse en el espacio $x-y ; \sigma_{x}$ y $\sigma_{y}$ dan una medida del ancho, la mitad de los lados de un rectángulo que circunscribe a la elipse; el coeficiente de correlación $\rho$ determina la inclinación. (b) Dejando fijo $\sigma_{x}$ y $\sigma_{y}$ se muestran varias elipses: $\rho=0$, elipse sólida; $\rho>0$, elipse punteada; $\rho<0$, elipse con guiones.

\footnotetext{
${ }^{6} f(x, y)$ es una densidad de probabilidad. La probabilidad de que $x$ y $y$ se encuentren en el intervalo $(x, x+\mathrm{d} x)$ y $(y, y+\mathrm{d} y)$ es $P(x, y) \mathrm{d} x \mathrm{~d} y$.
} 


\section{Distribución normal multivariada}

Si se tienen $n$ variables aleatorias distribuidas normalmente, $\boldsymbol{x}=\left(x_{1}, x_{2}, \ldots, x_{n}\right)$, cuya matriz de covarianza, M, es positiva definida ${ }^{7}$ la función de distribución normal multivariada no degenerada esta dada por:

$$
f(\boldsymbol{x})=\frac{1}{(2 \pi)^{n / 2} \sqrt{|\mathbf{M}|}} \exp \left(-\frac{1}{2}(\boldsymbol{x}-\boldsymbol{\mu})^{T} \mathbf{M}^{-1}(\boldsymbol{x}-\boldsymbol{\mu})\right),
$$

donde $\boldsymbol{\mu}$ es el vector de medias y $|\mathbf{M}|$ el determinante de la matriz de covarianza. Para este caso se define la función de verosimilitud por:

$$
\mathcal{L}(\overline{\boldsymbol{x}})=\frac{1}{(2 \pi)^{n / 2} \sqrt{|\mathbf{M}|}} \exp \left(-\frac{1}{2}(\boldsymbol{x}-\overline{\boldsymbol{x}})^{T} \mathbf{M}^{-1}(\boldsymbol{x}-\overline{\boldsymbol{x}})\right),
$$

ahora $\overline{\boldsymbol{x}}$ puede depender de uno o más parámetros (en el caso de esta tesis $\sin ^{2} 2 \theta$ y $\Delta m^{2}$ ) y da el valor esperado para cada $x_{i}$. Si tomamos el logaritmo natural de la ecuación (3.11) obtenemos:

$$
\ln (\mathcal{L})=-\frac{n}{2} 2 \pi-\frac{1}{2} \ln (|\mathbf{M}|)-\frac{1}{2}(\boldsymbol{x}-\overline{\boldsymbol{x}})^{T} \mathbf{M}^{-1}(\boldsymbol{x}-\overline{\boldsymbol{x}})
$$

o equivalentemente:

$$
-2 \ln (\mathcal{L})=2 n \pi+\ln (|\mathbf{M}|)+(\boldsymbol{x}-\overline{\boldsymbol{x}})^{T} \mathbf{M}^{-1}(\boldsymbol{x}-\overline{\boldsymbol{x}})
$$

En un ajuste de los parámetros se busca la máxima verosimilitud, o equivalentemente para este caso,

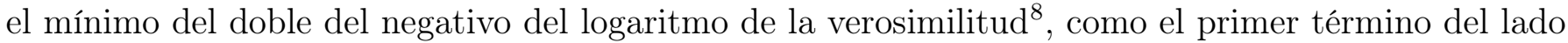
izquierdo de la ecuación pasada es constante puede ser ignorado. Se llega pues a la función que debe ser minimizada para un ajuste:

$$
\mathscr{L} \equiv-2 \ln (\mathcal{L})=\ln (|\mathbf{M}|)+(\boldsymbol{x}-\overline{\boldsymbol{x}})^{T} \mathbf{M}^{-1}(\boldsymbol{x}-\overline{\boldsymbol{x}})
$$

Veremos que $\mathscr{L}$ tiene una gran utilidad en la determinación de intervalos de confianza de los parámetros de un modelo.

\section{Intervalos y límites}

Es común llegar a ideas erróneas sobre intervalos clásicos, provenientes de mal interpretarlos como intervalos bayesianos; por lo tanto una pequeña mención a los intervalos bayesianos esta muy a la orden. Supóngase que se quiere inferir algo sobre el parámetro $\mu$, cuyo valor verdadero $\mu_{\mathbf{v}}$ es desconocido. Para eso se hace una sola medida de la observable ${ }^{9} x$, de tal forma que la función de densidad de probabilidad (f.d.p.) de obtener el valor $x$ que depende del parámetro desconocido $\mu$ es conocida: a esta f.d.p. la llamamos $P(x \mid \mu)$. Después de una sola medida se obtiene $x_{0}$. Al substituir en la f.d.p. tenemos $P\left(x_{0} \mid \mu\right)$, la cual, ahora, es una función de verosimilitud $\mathcal{L}\left(x_{0} \mid \mu\right)$. Apliquemos el teorema de Bayes, ecuación (3.3), para el valor verdadero $\mu_{\mathbf{v}}$ :

$$
P\left(\mu_{\mathbf{v}} \mid x_{0}\right)=\mathcal{L}\left(x_{0} \mid \mu_{t}\right) P\left(\mu_{\mathbf{v}}\right) / P\left(x_{0}\right)
$$

\footnotetext{
${ }^{7}$ Una matriz real simétrica $\mathbf{M}$, de $n \times n$ es positiva definida si $\boldsymbol{z}^{T} \mathbf{M} \boldsymbol{z}>0 ;\left\{\forall \boldsymbol{z} \in \mathbb{R}^{n} \mid \boldsymbol{z} \not \equiv \emptyset\right\}$. A menos de que una variable sea combinación lineal exacta de las otras, las matrices de covarianza siempre son positivas definidas.

${ }^{8}$ Se puede asegurar tal equivalencia por que el logaritmo es una función monótonamente creciente.

${ }^{9} \mathrm{~A}$ pesar de que seguido sea el caso, $x$ no necesita ser una medida de $\mu$.
} 
Un intervalo bayesiano o "intervalo creíble" $\left[\mu_{1}, \mu_{2}\right]$ correspondiente a un valor porcentual $\alpha$ de nivel de confianza puede ser construido de la f.d.p. posteriori requiriendo que

$$
\int_{\mu_{1}}^{\mu_{2}} P\left(\mu_{\mathbf{v}} \mid x_{0}\right) \mathrm{d} \mu_{\mathbf{v}}=\alpha .
$$

Nótese que existe libertad en la elección de $\mu_{1}$, dependiendo de si se quiere un límite superior, inferior o un intervalo ${ }^{10}$ central.

La construcción de intervalos frecuentistas (o clásicos) de confianza fue definida por Jerzy Neyman [188]. Esta limita a declaraciones derivadas de la f.d.p. $P(x \mid \mu)$ y provee la opción preferida para presentar resultados de un experimento de forma objetiva. Sin embargo, es crucial no interpretarlos como intervalos bayesianos, es decir como declaraciones sobre $P\left(\mu_{\mathbf{v}} \mid x_{0}\right)$. Mas bien, un intervalo de confianza $\left[\mu_{1}, \mu_{2}\right]$ es miembro de un conjunto, de tal forma que el conjunto tiene la propiedad que

$$
P\left(\mu \in\left[\mu_{1}, \mu_{2}\right]\right)=\alpha
$$

Aquí $\mu_{1}$ y $\mu_{2}$ son funciones de la observable $x$, la ecuación (3.15) se refiere a los intervalos de confianza variables $\left[\mu_{1}, \mu_{2}\right]$ de un ensamble de experimentos con $\mu$ fijo. Para un conjunto de intervalos de confianza, la ecuación (3.15) se cumple para todos los $\mu$ permitidos. En particular, los intervalos contienen a la incógnita fija $\mu_{\mathbf{v}}$, en una fracción $\alpha$ de los experimentos. Esto es completamente diferente a la afirmación bayesiana del grado de creencia de que $\mu_{\mathbf{v}}$ esta en $\left[\mu_{1}, \mu_{2}\right]$ es $\alpha$.

Si la ecuación (3.15) es satisfecha adecuadamente se dice que el intervalo "cubre" $\mu$ con la confianza establecida o, equivalentemente, que el conjunto de intervalos tienen la "cobertura" correcta. Si existe un valor de $\mu$ para el cual $P\left(\mu \in\left[\mu_{1}, \mu_{2}\right]\right)<\alpha$, entonces se dice que el intervalo "subcubre"para ese $\mu$, se tiene "subcobertura". Subcobertura significativa para cualquier $\mu$ es una seria falla. Por otra parte si hay un valor de $\mu$ para el cual $P\left(\mu \in\left[\mu_{1}, \mu_{2}\right]\right)>\alpha$, entonces se dice que el intervalo "sobrecubre" para ese $\mu$. Se dice que un intervalo es "conservador" si sobrecubre para algunos valores de $\mu$ y no subcubre ninguno. Si bien un intervalo conservador no se considera una falla tan grande como la subcobertura, tiene un gran costo: pérdida de la capacidad de rechazar hipótesis falsas.

La figura 3.2 ilustra la construcción de una "banda de confianza", en una gráfica del parámetro $\mu$ contra la cantidad medida $x$. Para cada valor de $\mu$, se examina $P(x \mid \mu)$ a lo largo de la línea horizontal a través de $\mu$. Se selecciona un intervalo $\left[x_{1}, x_{2}\right]$ que es un subconjunto de esta linea tal que

$$
P\left(x \in\left[x_{1}, x_{2}\right] \mid \mu\right)=\alpha
$$

A los intervalos $\left[x_{1}, x_{2}\right]$, se les llama "regiones de aceptación" para ese $\mu$; en la figura 3.2 se dibujan como segmentos de líneas horizontales. Para especificar de forma única las regiones de aceptación, se deben escoger criterios de selección. Se tiene la libertad de escoger el criterio, siempre y cuando la elección no esta influenciada por los datos medidos. Las elecciones más comunes son

$$
P\left(x<x_{1} \mid \mu\right)=1-\alpha
$$

que otorga "límites de confianza superiores" (que satisfacen $P\left(\mu<\mu_{2}\right)=1-\alpha$ ), y

$$
P\left(x<x_{1} \mid \mu\right)=P\left(x>x_{2} \mid \mu\right)=(1-\alpha) / 2,
$$

\footnotetext{
${ }^{10}$ Estos límites e intervalos no se limitan a una sola dimensión y en general describen un volumen $n$-dimensional.
} 


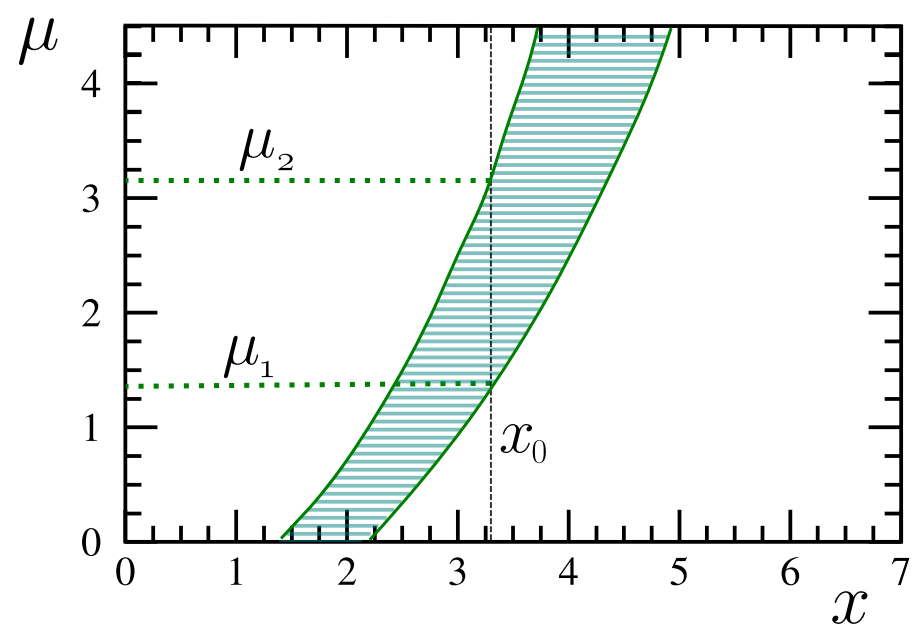

Figura 3.2: Construcción de una banda de confianza genérica y su uso. Para cada valor de $\mu$, se dibuja un intervalo horizontal de aceptación $\left[x_{1}, x_{2}\right]$ tal que $P\left(x \in\left[x_{1}, x_{2}\right] \mid \mu\right)=\alpha$. Al hacer un experimento que mide $x$ y obtiene el valor $x_{0}$, se dibuja la línea punteada vertical que pasa por $x_{0}$. El intervalo de confianza $\left[\mu_{1}, \mu_{2}\right]$ es la unión de todos los valores de $\mu$ para los cuales las correspondientes regiones de aceptación son interceptados por la línea vertical.

que otorga "intervalos de confianza centrales" (que satisfacen $P\left(\mu<\mu_{1}\right)=P\left(\mu>\mu_{2}\right)=(1-$ $\alpha) / 2$ ). Para criterios más complicados para la elección de regiones de aceptación que satisfagan la especificación más general de la ecuación (3.16), un principio de ordenamiento necesita especificar que $x$ 's incluir en la región de aceptación.

La construcción se completa cuando se dibujan regiones de aceptación para cada valor $\mu$. Al hacer un experimento que mide $x$ y obtiene el valor $x_{0}$, se dibuja una línea vertical que pasa por $x_{0}$. El intervalo de confianza es la unión de todos los valores de $\mu$ para los cuales los correspondientes intervalos horizontales son interceptados por la línea vertical; típicamente este es simplemente un intervalo conexo $\left[\mu_{1}, \mu_{2}\right]$, aunque no tiene por que ser así. Usualmente solo se presentan los extremos. Por construcción, la ecuación (3.15) es satisfecha para todos los $\mu$; por lo tanto es satisfecha por $\mu_{\mathbf{v}}$, cuyo valor es fijo pero desconocido.

Consideremos el caso de una observable $x$ que es simplemente el valor medido de $\mu$, en un experimento con una resolución gaussiana con desviación unitaria conocida y para el cual solo los valores no negativos están físicamente permitidos (por ejemplo cuando $\mu$ es una masa). La figura 3.3a muestra varias bandas de confianza de $90 \%$ N.C. para tal experimento. En línea punteada gris y con flechas en los extremos se muestra la banda que forma el límite superior ${ }^{11}$, construido usando la ecuación (3.17); con línea punteada gris y con círculos en los extremos se muestra la banda que forma el intervalo de confianza central, construido usando la ecuación (3.18). Estos límites son apropiados cuando se ha determinado, antes de realizar el experimento, que se publicará un límite superior o un intervalo de confianza central, respectivamente. Sin embargo, se puede juzgar más sensible decidir, basándose en los resultados del experimento, si se debe publicar un límite superior o intervalo de confianza central.

Supongamos que la actitud de un físico con respecto a la publicación de resultados de un experimento diseñado para medir una señal pequeña es: publicar un límite superior si el resultado tiene un nivel de confianza menor a $99 \%$ y un límite central en caso contrario. Esta clase de táctica es llamada "flip-

\footnotetext{
${ }^{11}$ La segunda línea de la banda se encuentra en $x=+\infty$.
} 


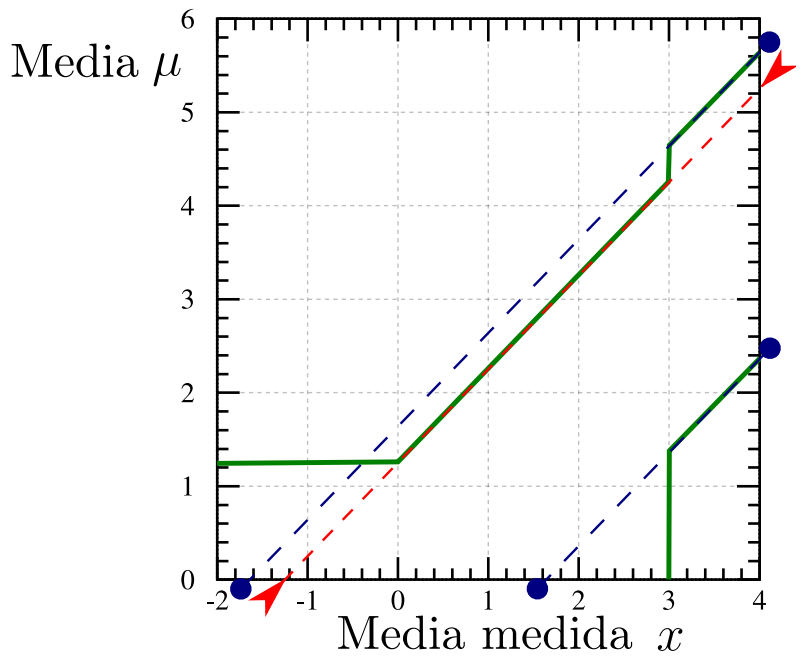

(a) Bandas de confianza implícitamente usadas.

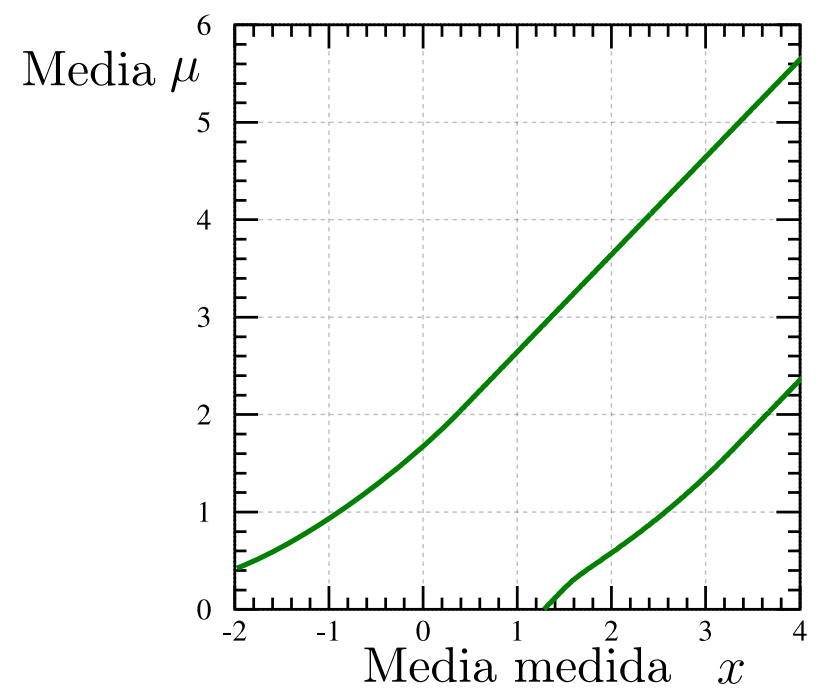

(b) Bandas de confianza del método $\mathrm{F}-\mathrm{C}$.

Figura 3.3: Bandas de confianza de $90 \%$ N.C. para la media de una gaussiana en unidades de la desviación media cuadrática. En la figura (a) se muestra la banda que forma el límite superior, en líneas punteadas y con flechas; la banda que forma el intervalo de confianza central en líneas punteadas y con círculos; y con línea sólida se ven las bandas que forman los intervalos de confianza implícitamente usados cuando la decisión está basada en los datos. En la figura (b) se aprecia la banda de confianza generada con el método de Feldman-Cousins; las cuales varían de forma "suave" entre un límite superior y un intervalo central, decidiendo por si solas que clase de límite se debe usar; las regiones de aceptación nunca son vacías, lidian de mejor forma con las regiones no físicas y otorgan límites con cobertura correcta.

flopping" 12 basándose en los datos. Además, cuando se mide un valor negativo para una cantidad físicamente positiva, se puede seguir la estrategia de pretender que se midió el valor cero cuando se cite el intervalo de confianza, lo cual introduce una tendencia conservadora.

En las curvas negras continuas de la figura 3.3a se examina el efecto que tiene tal táctica, exhibiendo las bandas de confianza. Para cada valor medido $x$ se dibuja un segmento vertical $\left[\mu_{1}, \mu_{2}\right]$ que se citará como un intervalo de confianza. Después se examinará que regiones de aceptación horizontales implican esta colección de intervalos verticales. Por ejemplo, para $\mu=2.0$, la región de aceptación tiene $x_{1}=2-1.28$ y $x_{2}=2+1.64$, solamente contiene $85 \%$ de la probabilidad $P(x \mid \mu)$. Así pues, la ecuación (3.16) no es satisfecha, el intervalo subcubre de forma significativa. Para $1.36<\mu<4.28$ la cobertura es de $85 \%$. Es importante notar que las discontinuidades que tienen las bandas generadas siguiendo la táctica de flip-flopping, son ocasionadas precisamente por elegir los intervalos basándose en los resultados.

Insistiendo en la interpretación de los intervalos de confianza pensemos en la situación en que se mide un valor negativo, sin significado físico, para tal valor su intervalo de confianza es muy angosto. Supongamos, como es común, que en tal circunstancia se cita el intervalo de $90 \%$ N.C. a partir del cero, [0., 0.01]. Esto no significa que hay una probabilidad de $90 \%$ de encontrar el valor real en el intervalo [0.,0.01], la interpretación bayesiana. Nótese que si uno mide $x<-1.8$ el resultado es insatisfactorio para las bandas en líneas punteadas, tanto para el límite superior como para el intervalo central, a pesar de que sí son intervalos de confianza con correcta interpretación estadística: al dibujar la línea vertical el intervalo de confianza es un conjunto vacío. Una forma alternativa de expresar esta

\footnotetext{
${ }^{12}$ Una frase usada para referirse a la acción de revertir una postura u opinión, en ocasiones de forma repetida.
} 
situación sería permitir valores de $\mu$ no físicos cuando se construyen las bandas de confianza y luego decir que el intervalo de confianza se encuentra completamente contenido en la región no física. Lo cual requiere saber $P(x \mid \mu)$ para valores de $\mu$ no físicos, esto puede generar dificultades conceptuales. Cuando esta situación ocurre se sabe que se esta en el $10 \%$ del ensamble "equivocado", al expresar intervalos de $90 \%$ N.C. Se puede dar el resultado incorrecto, y el ensamble de intervalos tendrá la cobertura adecuada, pero no es muy consolador.

La construcción clásica de los intervalos de confianza presenta varios problemas, a saber:

- Los intervalos no dan cobertura correcta una fracción de las veces.

- Por lo menos una fracción de las veces los intervalos de confianza pueden ser conjuntos vacíos.

- Las regiones no físicas no se manejan de forma elegante.

- Los intervalos no varían de forma continua entre límites superiores y intervalos centrales, sino que cambian de forma discontinua dependiendo en la elección.

La figura 3.3b muestra las bandas de confianza que generan los intervalos de confianza del método Feldman-Cousins, el cual lidia con los problemas de la construcción clásica de intervalos de confianza antes mencionados. Para valores grandes de $x$ los intervalos de confianza $\left[\mu_{1}, \mu_{2}\right]$ son los mismos en las dos gráficas de la figura 3.3. Por debajo de $x=1.28$ la cota inferior del nuevo intervalo de confianza es cero, así que automáticamente hay una transición de intervalos centrales y límites superiores. Por supuesto, no se esta obligado a afirmar un descubrimiento no nulo solo por que el $90 \%$ N.C. del intervalo no contiene al cero. Se debe entender que los intervalos de confianza no indican un grado de creencia.

El límite superior de $90 \%$ N.C. en $x=0$ del método $\mathrm{F}-\mathrm{C}$ es $\mu_{2}=1.64$, que es el límite superior estándar de $95 \%$ N.C. La desviación del límite estándar de $90 \%$ N.C. refleja el hecho que los intervalos proveen cobertura frecuentista solo cuando la decisión de expresar un límite superior no se basa en los datos. El método de $\mathrm{F}-\mathrm{C}$ repara la subcobertura ocasionada por el flip-flopping, tomando el compromiso necesario de aflojar los límites superiores alrededor de $x=0$. Conforme $x$ disminuye, los límites superiores decrecen de forma asintótica como $1 / x$ para valores negativos grandes de $x$. Se habla con gran detalle de este método en la sección §3.6.

\section{Aproximación parabólica}

La función de verosimilitud se acerca a una gaussiana cuando el tamaño de la muestra es grande [189]. Bajo esta condición se obtiene una f.d.p. normalmente distribuida para los parámetros, con un intervalo tipo desviación estándar dado por la disminución de $\mathrm{e}^{1 / 2}$ desde el máximo, para el caso de un solo parámetro. Tal intervalo cubre al parámetro ajustado con una probabilidad de $68 \%$. Así pues, el logaritmo de la verosimilitud es parabólico y el intervalo de confianza corresponde a la región dentro de la cual se disminuye un valor de 1/2 desde el máximo. Es una buena práctica ver cual es la forma de la función de verosimilitud, para ver hasta que punto la aproximación parabólica sigue siendo razonable. Es frecuente encontrar declaraciones como "las mediciones excluyen ... por cuatro desviaciones estándar", las cuales deben ser interpretadas con precaución. Ya que, en muchos casos, su validez depende en la suposición de que el logaritmo de la verosimilitud permanece parabólico sobre un amplio rango del espacio. Despreciar el efecto de las colas puede llevar a conclusiones erróneas. En la figura 3.4 se muestran dos funciones de verosimilitud de un experimento de decaimiento en escala logarítmica, las dos son de parámetros equivalentes: la vida media y tasa de descomposición. 
Se puede observar la semejanza que guardan con una parábola y el rango en el cual esta semejanza es razonable, también sirven para darse cuenta que verosimilitud se asimila más a una parábola.
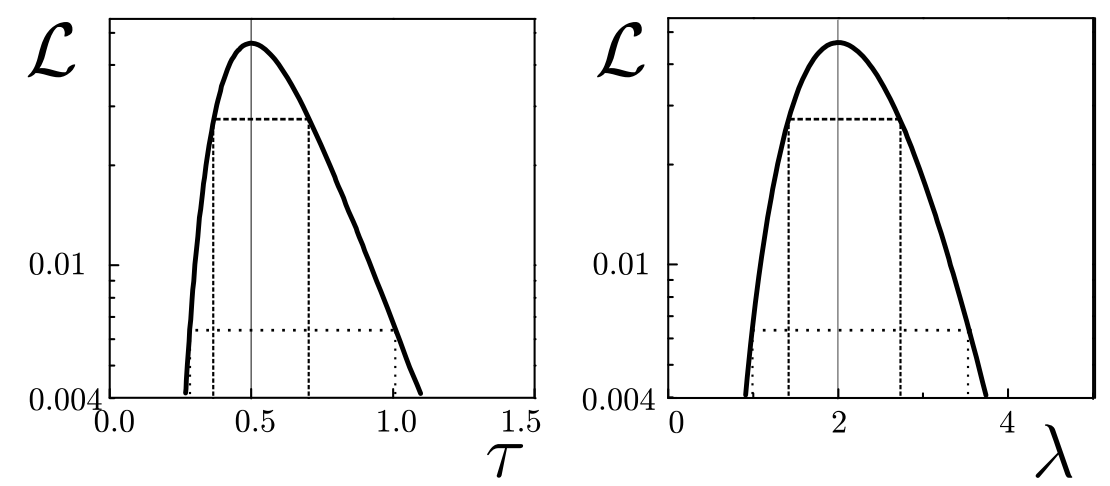

Figura 3.4: Función de verosimilitud para los parámetros vida media y tasa de descomposición en escala logarítmica. A partir del máximo se hacen cortes de $\Delta \ln \mathcal{L}=-1 / 2$ y $\Delta \ln \mathcal{L}=-2$ para determinar los intervalos de $1 \sigma$ y $2 \sigma$, respectivamente. Para ambos casos la aproximación a una parábola es razonable, sin embargo se prefiere la verosimilitud de la tasa de descomposición para determinar los intervalos ya que es más simétrica.

En general los cortes, a partir del máximo, que determinan el intervalo de confianza están dados por la distribución $\chi^{2}$. Definida como

$$
\chi_{k}^{2} \equiv \frac{x^{\frac{k}{2}-1} \mathrm{e}^{-\frac{x}{2}}}{2^{\frac{k}{2}} \Gamma\left(\frac{k}{2}\right)} .
$$

Si se tienen $k$ variables aleatorias independientes $x_{i}$, cada una distribuida de forma normal con media $\mu_{i}$ y desviación estándar $\sigma_{i}^{2}$, entonces la suma de sus cuadrados se distribuye como una $\chi^{2}$ de $k$ grados de libertad

$$
\chi_{k}^{2} \sim \sum_{i=1}^{k}\left(\frac{x_{i}-\mu_{i}}{\sigma_{i}}\right)^{2}
$$

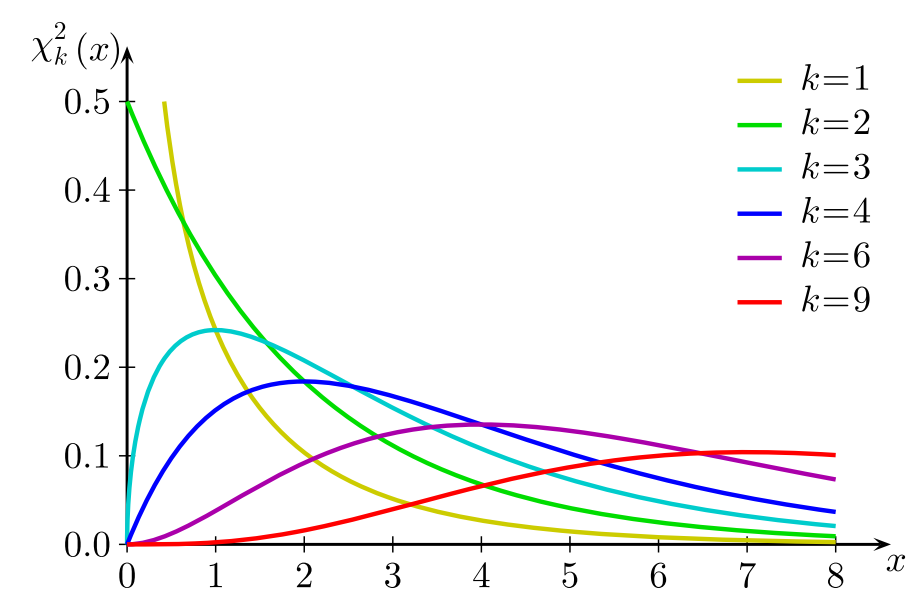

Figura 3.5: Función densidad de probabilidad de $\chi_{k}^{2}$. Curvas de distribuciones para distintos valores de grados de libertad $k$. La distancia al origen $x_{\alpha}$, que contiene una fracción $\alpha$ del área de la curva, determina el corte que se debe hacer para obtener un intervalo de $\alpha$ N.C. 
Para determinar el intervalo que cubre el mejor ajuste a $\alpha \%$ de nivel de confianza, se hace una excursión $x_{\alpha}$ desde el máximo. Donde $x_{\alpha}$ es la distancia, desde el origen, que contiene una fracción $\alpha$ del área de la curva $\chi_{k}^{2}$ correspondiente. Usualmente el número de grados de libertad $k$, es el número de parámetros que se ajustaron.

\subsection{Formato y procesamiento de datos liberados por MiniBoo- $\mathrm{NE}$}

En esta sección se menciona cuales fueron los datos utilizados para los distintos análisis y se establece una convención para su uso. Estos se encuentran en línea, en la página del experimento [190, 191, 192, 193, 194, 195] y pueden ser descargados libremente. De igual forma se explicaran los algoritmos que son comunes a todos los análisis. Los histogramas y análisis correspondientes tienen distintos rangos de energía para los eventos observados de $\nu_{e}\left(\bar{\nu}_{e}\right)$ : 475-3000 Mev y 200-3000 MeV, por lo tanto tendrán distinto número de intervalos (bins). En la tabla A.2 se presentan los archivos de los cuales provienen algunos de estos datos para el modo de antineutrinos.

- Número de bins, $n_{e}$, de energía reconstruida ${ }^{13} E_{\nu}^{Q E}$ para eventos de $\nu_{e}$ o $\bar{\nu}_{e}, n_{e}=11(8)$, para $E>200 \mathrm{MeV}(E>475 \mathrm{MeV})$.

- Número de bins, $n_{\mu}$, de energía reconstruida $E_{\nu}^{Q E}$ para eventos de $\nu_{\mu}$ o $\bar{\nu}_{\mu}, n_{\mu}=8$.

- Arreglo unidimensional $\boldsymbol{L}^{\nu_{e}}$ con los límites de los bins en $E_{\nu}^{Q E}$ (energía reconstruida del neutrino) para $\nu_{e}\left(\bar{\nu}_{e}\right.$ en modo de antineutrinos). Tiene 9 componentes cuando $E>475 \mathrm{MeV}$, es decir, define 8 bins y 12 componentes cuando $E>200 \mathrm{MeV}$, define 11 bins. Los límites usados fueron: $\boldsymbol{L}^{\nu_{e}}=(200,300,375,475,550,675,800,950,1100,1300,1500,3000)$. Las tres primeras entradas se omiten para los análisis con $E>475 \mathrm{MeV}$.

- Arreglo unidimensional $\boldsymbol{D}^{\nu_{e}}\left(\boldsymbol{D}^{\bar{\nu}_{e}}\right)$ de eventos observados $\nu_{e}\left(\bar{\nu}_{e}\right)$ por bin de $E_{\nu}^{Q E} .8 \mathrm{u} 11$ componentes. Por ejemplo, los datos liberados en 2012 en modo de antineutrinos: $\boldsymbol{D}^{\bar{\nu}_{e}}=(122,70$, $65,43,57,39,37,23,26,26,43)$.

- Arreglo unidimensional $\boldsymbol{L}^{\nu_{\mu}}$ con los límites de los bins en $0 \leq E_{\nu}^{Q E} \leq 1900 \mathrm{MeV}$ para $\nu_{\mu}\left(\bar{\nu}_{\mu}\right)$. Los límites que se usados fueron $\boldsymbol{L}^{\nu_{\mu}}=(0,500,700,900,1100,1300,1500,1700,1900)$.

- Arreglo unidimensional $\boldsymbol{D}^{\nu_{\mu}}\left(\boldsymbol{D}^{\bar{\nu}_{\mu}}\right)$ de eventos observados $\nu_{\mu}\left(\bar{\nu}_{\mu}\right)$ por bin de $E_{\nu}^{Q E}$. Correspondientemente siempre tiene 8 componentes. En 2012, modo de antineutrinos se tuvo $\boldsymbol{D}^{\bar{\nu}_{\mu}}=(9481$, 13581, 11308, 7667, 4682, 2371, 985, 380).

- Arreglo unidimensional $\boldsymbol{B}^{\nu_{e}}\left(\boldsymbol{B}^{\bar{\nu}_{e}}\right)$ del fondo estimado de candidatos a eventos $\nu_{e}\left(\bar{\nu}_{e}\right)$ por bin de enuqe. 8 u 11 números reales. En 2012 modo de antineutrinos se tuvo $\boldsymbol{B}^{\bar{\nu}_{e}}=(90.289907$, 53.077595, 57.0988, 32.937945, 43.159072, 34.174322, 36.383542, 28.737807, 28.750297, 20.098079, 42.697791).

- Arreglo unidimensional $\boldsymbol{B}^{\nu_{\mu}}\left(\boldsymbol{B}^{\bar{\nu}_{\mu}}\right)$ del estimado de eventos $\nu_{\mu}\left(\bar{\nu}_{\mu}\right)$ por bin de $E_{\nu}^{Q E}$. 8 números reales. En 2012 modo de antineutrinos se tuvo $\boldsymbol{B}^{\bar{\nu}_{\mu}}=(9998.9575,13461.302$, 11298.24, 7604.9604, 4331.8869, 2125.5371, 891.22261, 336.98711).

- Arreglo bidimensional $\mathbf{M}_{\text {frac }}^{3 \times 3}\left(\overline{\mathbf{M}}_{\text {frac }}^{3 \times 3}\right)$ de la matriz de covarianza fraccionaria con estructura de $3 \times 3$ bloques. A lo largo de la diagonal en intervalo de energía reconstruida: bloque de eventos de transmutación total, bloque del fondo estimado de eventos de $\nu_{e}\left(\bar{\nu}_{e}\right)$ y bloque del estimado

${ }^{13} Q E$ se refiere a cuasielástica. 
de eventos $\nu_{\mu}\left(\bar{\nu}_{\mu}\right)$. Los bloques fuera de la diagonal incluyen las correlaciones entre bloques. Incluye la incertidumbre sistemática para las tres muestras y la incertidumbre estadística para el fondo de $\nu_{e}\left(\bar{\nu}_{e}\right)$ y la estimación de $\nu_{\mu}\left(\bar{\nu}_{\mu}\right)$. Dependiendo del rango de energía se tiene una matriz de: $24 \times 24=(8+8+8) \times(8+8+8)$ o $30 \times 30=(11+11+8) \times(11+11+8)$ componentes.

- Tupla de eventos MC de transmutación total $\nu_{\mu} \rightarrow \nu_{e} \mathrm{y} / \mathrm{o} \bar{\nu}_{\mu} \rightarrow \bar{\nu}_{e}$. Con un renglón por evento y cuatro columnas con la siguiente información:

- Energía reconstruida $E_{\nu}^{Q E}$ del neutrino (antineutrino) en $\mathrm{MeV}$.

- Energía verdadera $E_{\nu}^{V}$ del neutrino (antineutrino) en $\mathrm{MeV}$.

- Distancia verdadera $x_{\nu}^{V}$ en cm entre el punto de producción y detección del neutrino.

- Peso w para este evento de transmutación total, sin incluir ninguna probabilidad de oscilación en la ponderación y normalizando la ponderación de tal forma que la suma de todos los pesos dividida entre el total de eventos simulados, sea igual al número esperado de eventos de transmutación total a lo largo de $E_{\nu}^{Q E}$.

Los eventos de transmutación total son obtenidos de una predicción del flujo de $\nu_{\mu}$ y $\bar{\nu}_{\mu}$ para la cual las interacciones de neutrinos son generadas asumiendo que el $100 \%$ de los eventos oscilan a $\nu_{e}$ y $\bar{\nu}_{e}$, en vez de $\nu_{\mu}$ y $\bar{\nu}_{\mu}$, y posteriormente reconstruidas. A partir de esta tupla se puede obtener la señal $\boldsymbol{S}^{\nu_{e}}\left(\boldsymbol{S}^{\bar{\nu}_{e}}\right)$ para cada bin de $E_{\nu}^{Q E}$, es decir la predicción de eventos de $\nu_{\mu} \rightarrow \nu_{e}$ $\left(\bar{\nu}_{\mu} \rightarrow \bar{\nu}_{e}\right)$.

Primera observación: en la primera publicación de datos, correspondiente al modo de neutrinos de 2007 [190], no se hacen públicos los datos correspondientes a los $\nu_{\mu}$ : eventos observados, fondo estimado, matriz de covarianza, etcétera. Estos datos son analizados usando el método de búsqueda por barrido unidimensional únicamente, en $\S 3.3$ se describe como se hace este análisis y en $\S 4.1$ se encuentran los resultados.

Segunda observación: las simulaciones para generar la matriz de covarianza y la tupla de eventos MC, con la que se genera la estimación de la señal, consideran distintos casos de transmutación total. En algunos casos la simulación contiene simultáneamente la transmutación total de neutrinos y antineutrinos mientras que en otros solo se considera un tipo de transmutación. Tal decisión esta justificada por la composición relativa de $\nu / \bar{\nu}$ para cada modo: en modo de neutrinos la componente de antineutrinos es pequeña mientras que en modo de antineutrinos la componente de neutrinos es grande. Las tablas 2.2a y 2.2b tienen los detalles de la composición del haz. La tabla 3.1 especifica el caso de transmutación total usado en las simulaciones.

\begin{tabular}{ccc}
\hline & Individual & Combinado \\
\hline \multirow{2}{*}{ Modo $\nu$} & $\nu$ oscilan & $\nu$ oscilan \\
& $\bar{\nu}$ no oscilan & $\bar{\nu}$ no oscilan \\
\cline { 2 - 3 } Modo $\bar{\nu}$ & $\bar{\nu}$ no oscilan & $\nu$ oscilan \\
& $\bar{\nu}$ oscilan & $\bar{\nu}$ oscilan \\
\hline
\end{tabular}

Tabla 3.1: Transmutación permitida en las simulaciones para los distintos análisis. 


\section{Construcción de la matriz de covarianza}

La matriz de covarianza es liberada por la colaboración lista para usarse, sin embargo conviene tener una idea de como se forma. Los errores sistemáticos asociados con la predicción del flujo de neutrinos, el modelado del detector y las secciones eficaces de los neutrinos son estimados como se describe en [177]. Las incertidumbres sistemáticas en el flujo son determinadas por las incertidumbres de las mediciones de la producción de partículas (§2.3.1), las incertidumbres de las secciones eficaces $(\S 2.4 .1$ y §2.4.2) son determinadas con datos de MiniBooNE y fuentes externas teóricas y experimentales, las incertidumbres del modelo del detector (§2.4.3) también están determinadas por datos de MiniBooNE. La matriz de covarianza es una suma de matrices de cada una de las fuentes de incertidumbre:

$$
\mathrm{M}_{i j}=\mathrm{M}_{i j}^{\Phi\left(\pi^{+}\right)}+\mathrm{M}_{i j}^{\Phi\left(\pi^{-}\right)}+\mathrm{M}_{i j}^{\Phi\left(K^{+}\right)}+\mathrm{M}_{i j}^{\Phi\left(K_{L}^{0}\right)}+\mathrm{M}_{i j}^{\mathrm{haz}}+\mathrm{M}_{i j}^{\sigma}+\mathrm{M}_{i j}^{\mathrm{NC} \pi^{0}}+\mathrm{M}_{i j}^{\text {exteriores }}+\mathrm{M}_{i j}^{\mathrm{MO}}
$$

cada componente esta asociada a la matriz de covarianza de un conjunto de parámetros de simulación subyacentes, se obtienen al muestrear el espacio de parámetros multivariado gaussiano que implica esta covarianza. Los primeros cuatro términos son de las incertidumbres que provienen del flujo de distintas partículas, luego se tiene la incertidumbre del haz, las secciones eficaces de neutrinos, los eventos de corriente neutra, los eventos que ocurren fuera del tanque y por último el modelo óptico.

Es de mucha utilidad definir al vector de estimación de eventos, $\boldsymbol{Q}^{3 \times 1}$, compuesto de tres vectores:

$$
\boldsymbol{Q}^{3 \times 1}=\left(\boldsymbol{S}^{\nu_{e}}, \boldsymbol{B}^{\nu_{e}}, \boldsymbol{B}^{\nu_{\mu}}\right)
$$

El vector de estimación de eventos para antineutrinos, $\overline{\boldsymbol{Q}}^{3 \times 1}$, se define de forma análoga.

La matriz de covarianza fraccionaria $\mathbf{M}_{\text {frac }}^{3 \times 3}$ tiene esa estructura debido a la forma en que se calculan separadamente las incertidumbres de las distintas partes que la componen. Estas partes son: la señal de eventos $\nu_{e}$, el fondo de eventos $\nu_{e}$ y la estimación de eventos $\nu_{\mu}$, por cada bin de $E_{\nu}^{Q E}$. La utilidad de la matriz fraccionaria es que se puede normalizar a cualquier cantidad de POT $^{14}$ y ajustarse para que contenga la información de cualquier punto en el espacio de parámetros $\left(\sin ^{2} 2 \theta, \Delta m^{2}\right)$. Lo primero que se tiene que hacer es construir la matriz total, es decir normalizar la matriz a la cantidad de eventos observados correspondientes al número de POT. Posteriormente se debe juntar la información de las distintas componentes, en la práctica uno no puede diferenciar entre un evento $\nu_{e}$ de señal y uno de fondo, se dice que la matriz se "colapsa". La matriz total se construye multiplicando a la matriz fraccionaria por el vector de estimación:

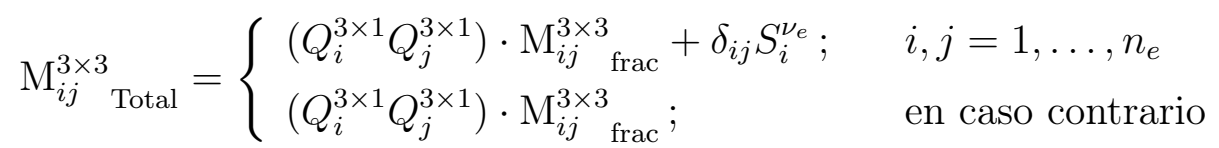

se le suma una contribución de error estadístico, correspondiente a la predicción de los eventos de señal, a los componentes de la diagonal del bloque superior izquierdo ${ }^{15}$.

A esta altura la matriz total $\mathbf{M}_{\text {Total }}^{\mathbf{3} \times \mathbf{3}}$, tiene una estructura de $3 \times 3$ bloques, de arriba a abajo los bloques de la diagonal tienen la covarianza (sistemática y estadística) de la señal de $\nu_{e}, \mathcal{S}^{\nu_{e}}$; el fondo

\footnotetext{
${ }^{14}$ Ver sección $\S 2.1 .1$.

${ }^{15}$ Ver nota 8 del capítulo 1.
} 
de $\nu_{e}, \mathcal{B}^{\nu_{e}}$; y la estimación de $\nu_{\mu}, \mathcal{B}^{\nu_{\mu}}$. Los bloques fuera de la diagonal tienen las correspondientes correlaciones: $\left\langle\mathcal{B}^{\nu_{e}}, \mathcal{S}^{\nu_{e}}\right\rangle,\left\langle\mathcal{S}^{\nu_{e}}, \mathcal{B}^{\nu_{e}}\right\rangle ;\left\langle\mathcal{B}^{\nu_{\mu}}, \mathcal{S}^{\nu_{e}}\right\rangle,\left\langle\mathcal{S}^{\nu_{e}}, \mathcal{B}^{\nu_{\mu}}\right\rangle ; \mathrm{y}\left\langle\mathcal{B}^{\nu_{\mu}}, \mathcal{B}^{\nu_{e}}\right\rangle,\left\langle\mathcal{B}^{\nu_{e}}, \mathcal{B}^{\nu_{\mu}}\right\rangle^{16}$.

Para colapsar se suman uno a uno las componentes de los bloques. Se suman las componentes de los $2 \times 2$ bloques superiores para formar el bloque superior de la matriz colapsada. Los dos bloques superiores de la última columna, así como los dos primeros bloques de la última fila, se suman para formar los bloques diagonales de la matriz colapsada. El bloque inferior izquierdo se traspasa como esta, para formar el bloque inferior izquierdo de la matriz colapsada. Logrando obtener una matriz simétrica de $2 \times 2$ bloques, $\mathbf{M}^{2 \times 2}$. Se puede ver una representación gráfica del proceso de colapso en la figura 3.7. En lo subsecuente se referirá a la matriz de covarianza total colapsada simplemente como la matriz de covarianza ${ }^{17}$ M. La matriz de covarianza para antineutrinos, $\overline{\mathbf{M}}$, se construye de forma análoga.
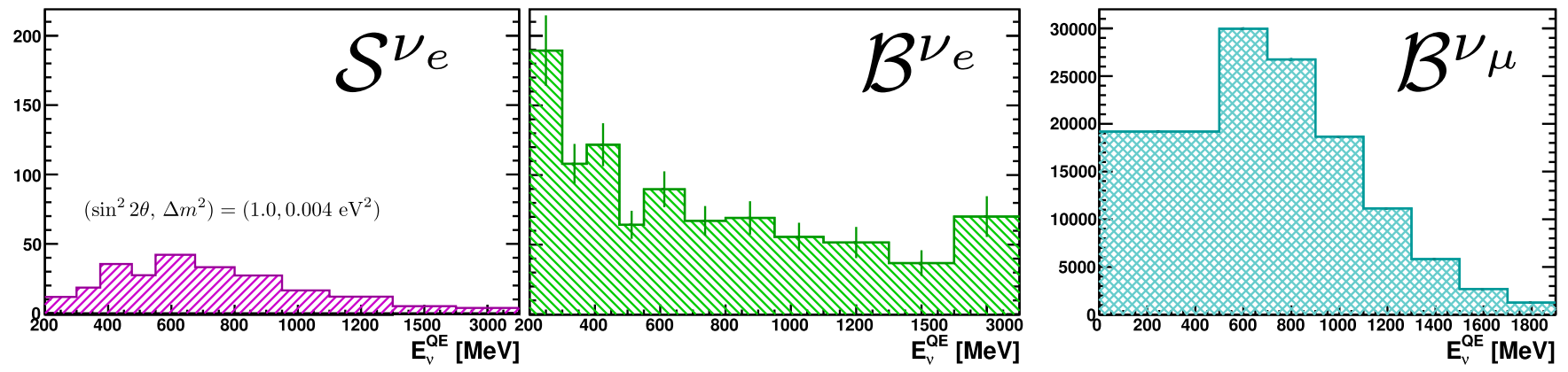

Figura 3.6: Distribuciones de $E_{\nu}^{Q E}$ de los datos de señal, fondo de $\nu_{e}$ y estimación de $\nu_{\mu}$. Notar las escalas de las distribuciones: decenas de eventos de señal, cientos de eventos de fondo $\nu_{e} \mathrm{y}$ decenas de miles de eventos $\nu_{\mu}$.

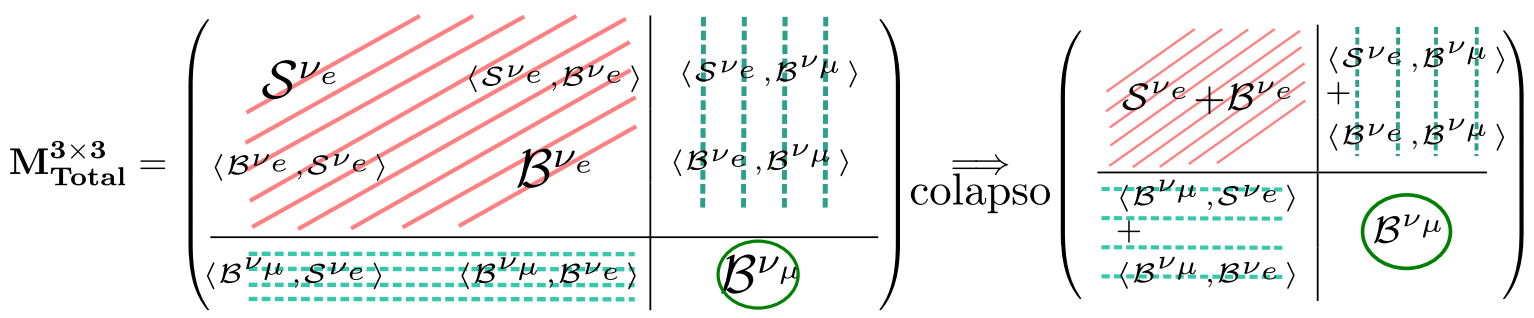

Figura 3.7: Matriz de covarianza y su colapso. Multiplicando a la matriz fraccionaria por los vectores correspondientes se obtiene la matriz total, esta tiene $3 \times 3$ bloques: los bloques diagonales tienen la incertidumbre de la señal, del fondo y estimación de neutrinos del muón; los bloques fuera de la diagonal tienen las correspondientes correlaciones. En el texto se describe el procedimiento para obtener la matriz total colapsada.

Observación: la matriz de covarianza es simétrica y positiva definida, por lo cual solo hace falta procesar los elementos correspondientes a la diagonal inferior de la misma para realizar todos los análisis que se pretenden hacer, calcular el determinante y la inversa de la matriz. La implementación computacional refleja y aprovecha esta característica. En el apéndice A se habla sobre la implementación computacional y las consideraciones que se tomaron en cuenta.

\footnotetext{
${ }^{16}$ Se reconoce el ligero abuso de notación, pero debe ser claro que $\langle\mathcal{X}, \mathcal{Y}\rangle$ define el bloque que contiene la covarianza entre la matriz de covarianza $\mathcal{X}$ y la matriz de covarianza $\mathcal{Y}$.

${ }^{17}$ Es común ver en la literatura de análisis de datos que también se le llame matriz de error.
} 
De forma similar también se colapsa el vector de estimación, en lo subsecuente $\boldsymbol{Q}$ se refiere al vector:

$$
\boldsymbol{Q}=\left(\left(\boldsymbol{S}^{\nu_{e}}+\boldsymbol{B}^{\nu_{e}}\right), \boldsymbol{B}^{\nu_{\mu}}\right)
$$

En lo subsecuente el vector de datos $\boldsymbol{D}$ es aquel formado al juntar los vectores de datos de $\nu_{e}$ y $\nu_{\mu}$ :

$$
\boldsymbol{D}=\left(\boldsymbol{D}^{\nu_{e}}, \boldsymbol{D}^{\nu_{\mu}}\right)
$$

Los vectores de estimación de eventos y datos para antineutrinos, $\overline{\boldsymbol{Q}}$ y $\overline{\boldsymbol{D}}$, respectivamente, se definen de forma análoga.

\subsubsection{Generalidades de los análisis realizados}

En este trabajo se analiza el caso de oscilación de dos neutrinos en el vacío, lo cual, como se mencionó antes, es una buena aproximación al caso general. Se transcribe aquí la fórmula de la probabilidad de oscilación para dos neutrinos en el vacío, ver $\S 1.4 .1$ :

$$
P\left(\nu_{\mu} \rightarrow \nu_{e}\right)=\sin ^{2} 2 \theta \sin ^{2}\left(\frac{1.27 \Delta m^{2} L}{E_{\nu}}\right),
$$

donde los parámetros libres que debe determinar un experimento de oscilación son: $\theta$ y $\Delta m^{2}$, sin embargo por conveniencia en los ajuste y en la forma de presentar la información el espacio de parámetros es $\left(\sin ^{2} 2 \theta, \Delta m^{2}\right)$. Por otra parte nótese la dependencia en el coeficiente $\frac{L}{E_{\nu}}$, la distancia viajada por los neutrinos entre su energía.

Para los valores de $\Delta m_{21}^{2} \sim 10^{-5} \mathrm{eV}^{2}$ y $\Delta m_{31}^{2} \sim 10^{-3} \mathrm{eV}^{2}$, medidos por experimentos solares y atmosféricos (§1.4.7), respectivamente, el cociente $L / E \sim 1$ de MiniBooNE satisface:

$$
\frac{\Delta m_{31}^{2} L}{2 E} \ll 1, \quad \frac{\Delta m_{21}^{2} L}{2 E} \ll 1 .
$$

Adicionalmente, el dominio de escalas de masa observado cumple:

$$
\Delta m_{21}^{2} \ll \Delta m_{31}^{2} \ll \Delta m_{41}^{2},
$$

donde $\Delta m_{41}^{2} \sim 1 \mathrm{eV}^{2}$ sería la diferencia que se tendría si la evidencia de LSND implicara la existencia de algún neutrino estéril. Se puede ver que, como (3.27) y (3.28) se satisfacen, la probabilidad de oscilación de $\nu_{\alpha}$ a $\nu_{\beta}$, para el caso de $3+1$ neutrinos, es:

$$
P\left(\nu_{\alpha} \rightarrow \nu_{\beta}\right)=\sin ^{2} 2 \theta \sin ^{2}\left(\frac{\Delta m_{41}^{2} L}{4 E_{\nu}}\right),
$$

es decir, es equivalente a la probabilidad de oscilación de dos neutrinos (1.5), solamente que ahora

$$
\sin ^{2} 2 \theta=4\left|U_{\alpha 4}\right|^{2}\left|U_{\beta 4}\right|^{2}
$$

con las correspondientes entradas de una matriz de mezcla de $4 \times 4, U_{e 4}$ y $U_{\mu 4}$. Es decir, el mismo proceso de ajuste para la fórmula de oscilación de dos neutrinos sirve para probar la hipótesis de la oscilación de $3+1$ neutrinos. 
Para todos los análisis que se presentan en este trabajo el espacio de parámetros $\left(\sin ^{2} 2 \theta, \Delta m^{2}\right)$ se restringe a la región $\left[\left[3 \times 10^{-4}, 1\right] \times\left[1 \times 10^{-2} \mathrm{eV}^{2}, 1 \times 10^{2} \mathrm{eV}^{2}\right]\right]$. MiniBooNE esta diseñado para buscar oscilaciones a escalas de $\sim 1 \mathrm{eV}^{2}$, si bien $\sin ^{2} 2 \theta$ esta acotado por $\sin ^{2} 2 \theta \leq 1$, puede tener valores menores, por su parte $\Delta m^{2}$, en principio, puede tener valores más grandes o chicos. Sin embargo es precisamente esta región a la cual es sensible el experimento, por eso es que para los ajustes es suficiente limitarse a esa área. Esta región ha sido dividida en una malla discreta en escala logarítmica de [190 × 190], con la excepción de los resultados de neutrinos de 2007 cuya malla es de [200 × 100].

\subsection{Método de búsqueda por barrido unidimensional}

Con este método se extrajeron los resultados de los datos del modo de neutrinos liberados en 2007, los cuales se presentan en $\S 4$.1, y los liberados en 2009, los cuales se presentan en $\S 4.2$. En el primer caso la constricción proveniente de la muestra de $\nu_{\mu}$ esta incluida en la predicción de $\nu_{e}$, mientras que en el segundo caso la constricción se realiza al incluir los datos de $\nu_{\mu}$ en el ajuste. En ambos casos el rango de energía es 475-3000 $\mathrm{MeV}$.

El estadístico usado para este método es una $\chi^{2}$, para los datos de 2007, se define como:

$$
\chi^{2}\left(\sin ^{2} 2 \theta, \Delta m^{2}\right)=\sum_{i, j=1}^{n_{e}}\left(D_{i}^{\nu_{e}}-\left(B_{i}^{\nu_{e}}+S_{i}^{\nu_{e}}\right)\right) \mathrm{M}_{i j}^{-1}\left(D_{j}^{\nu_{e}}-\left(B_{j}^{\nu_{e}}+S_{j}^{\nu_{e}}\right)\right),
$$

donde $\boldsymbol{D}^{\nu_{e}}$ es el número observado de eventos candidatos a $\nu_{e}$ solamente, $\boldsymbol{B}^{\nu_{e}}$ el número de eventos de fondo esperado según la simulación y $\boldsymbol{S}^{\nu_{e}}$ es el número de eventos de señal predicho; en bins de $E_{\nu}^{Q E}$. Todos estos tienen $n_{e}=8$ componentes. Por su parte $\mathbf{M}^{-1}$ es la inversa de la matriz de covarianza, evaluada en $\left(\sin ^{2} 2 \theta, \Delta m^{2}\right)$ y tiene $8 \times 8$ componentes. Recordemos que $\boldsymbol{S}^{\nu_{e}}$ depende de la fórmula de probabilidad de oscilación, dada por la ecuación (3.26), es decir que también es función de $\left(\sin ^{2} 2 \theta, \Delta m^{2}\right)$. Para determinar la señal en cada punto se usa la tupla de eventos MC de transmutación total descrita en $\S 3.2$, la cual cuenta con la información necesaria para generar la distribución de $E_{\nu}^{Q E}$ de la señal para cualquier punto del espacio de parámetros. El número de grados de libertad (g.l.) es igual al número de bins menos el número de parámetros libres $n_{e}-2=6$.

Por otra parte la definición que se usó para los datos de 2009, en los cuales se usa el espectro de $E_{\nu}^{Q E}$ de los $\nu_{\mu}$ como parte del ajuste es:

$$
\chi^{2}\left(\sin ^{2} 2 \theta, \Delta m^{2}\right)=\sum_{i, j=1}^{n_{e}+n_{\mu}}\left(D_{i}-Q_{i}\right) \mathrm{M}_{i j}^{-1}\left(D_{j}-Q_{j}\right)
$$

donde el vector $\boldsymbol{D}$ contiene los datos observados de $\nu_{e}$ y $\nu_{\mu}$, ecuación (3.25), formado al anexar los vectores correspondientes uno al lado del otro; $\boldsymbol{Q}$ contiene la predicción del número de eventos de fondo más la señal de $\nu_{e}$ en el primer segmento, y la predicción de $\nu_{\mu}$ en el segundo segmento, ecuación (3.24). Los vectores $\boldsymbol{D}$ y $\boldsymbol{Q}$ tienen $n_{e}+n_{\mu}=8+8=16$ componentes, por su parte se tienen $n_{e}+n_{\mu}-2-1=13$ g.l., donde al número de bins, además de restarle el número de parámetros, se le quita un grado extra debido a la normalización que da la muestra de $\nu_{\mu}$ a la simulación , §2.4.1. Ahora la matriz de $16 \times 16, \mathbf{M}$, contiene además de las correlaciones entre los bins de $\nu_{e}$, las correlaciones entre los bins de $\nu_{\mu}$, y las correlaciones entre los bins de $\nu_{e}$ y $\nu_{\mu}$. Esta matriz tiene la estructura de la matriz colapsada de la figura 3.7. 
La búsqueda por barrido unidimensional es un método iterativo. Para un valor fijo de $\left(\Delta m^{2}\right)_{j}$ en el rango de interés $\left[1 \times 10^{-2} \mathrm{eV}^{2}, 1 \times 10^{2} \mathrm{eV}^{2}\right]$ se busca el valor de $x^{\{0\}}=\left(\sin ^{2} 2 \theta\right)^{\{0\}}$ que minimiza ${ }^{18}$

$$
\chi_{j}^{2\{0\}} \equiv \chi^{2}\left(x,\left(\Delta m^{2}\right)_{j} ; \mathbf{M}^{\{0\}}\right)
$$

donde $\mathbf{M}^{\{0\}}$ representa la matriz de covarianza calculada con señal nula. La pareja $\left(x^{\{0\}},\left(\Delta m^{2}\right)_{j}\right)$ genera la señal usada para recalcular una matriz $\mathbf{M}^{\{1\}}$. A continuación se busca un nuevo valor $x^{\{1\}}=\left(\sin ^{2} 2 \theta\right)^{\{1\}}$ que minimice

$$
\chi_{j}^{2\{1\}} \equiv \chi^{2}\left(x,\left(\Delta m^{2}\right)_{j} ; \mathbf{M}^{\{1\}}\right) .
$$

Este procedimiento continúa hasta que la diferencia entre los mínimos de iteraciones sucesivas sea menor a una tolerancia de $\varepsilon \sim 1 \times 10^{-2}$, es decir:

$$
\left|\chi_{j \min }^{2\{k\}}-\chi_{j \min }^{2}\{k-1\}\right|<\varepsilon
$$

La función $\chi_{j}^{2}$, correspondiente a la última iteración, se utiliza para determinar el límite de exclusión en $\sin ^{2} 2 \theta$ para el valor $\left(\Delta m^{2}\right)_{j}$. Para cierto nivel de confianza $X \%$ se obtiene el valor $\sin ^{2} 2 \theta_{X} \%$ que satisface $^{19}$ :

$$
\chi_{j}^{2}\left(\sin ^{2} 2 \theta_{X \%}\right)=\chi_{j \min }^{2}+\Delta \chi_{X \%}^{2},
$$

donde los valores de $\Delta \chi_{X \%}^{2}$ están dados por una distribución $\chi^{2}$ de un solo lado y un grado de libertad y se muestran en la tabla 3.2a, ver la definición en (3.19). En la figura 3.8 se muestran las excursiones que se hacen desde el mínimo para obtener los límites de exclusión para un valor determinado de $\left(\Delta m^{2}\right)_{j}$. Dados los resultados consistentes con una señal nula, se decidió, a posteriori, usar tales valores de $\Delta \chi^{2}$.

\begin{tabular}{ccc}
\hline$\Delta \chi^{2}$ & \multicolumn{2}{l}{$\begin{array}{l}\text { Nivel de Confianza } \\
(\text { N.C. })\end{array}$} \\
\hline 1.64 & $90.00 \%$ & $1.28 \sigma$ \\
4.00 & $97.72 \%$ & $2.00 \sigma$ \\
9.00 & $99.86 \%$ & $3.00 \sigma$ \\
- & - & - \\
\hline
\end{tabular}

(a) Valores correspondientes a una distribución $\chi^{2}$ de un solo lado y un grado de libertad.

\begin{tabular}{lll}
\hline$\Delta \chi^{2}$ & \multicolumn{2}{l}{$\begin{array}{l}\text { Nivel de Confianza } \\
\text { (N.C.) }\end{array}$} \\
\hline 2.28 & $68.00 \%$ & $0.99 \sigma$ \\
4.60 & $90.00 \%$ & $1.64 \sigma$ \\
5.99 & $95.00 \%$ & $1.96 \sigma$ \\
9.21 & $99.00 \%$ & $2.57 \sigma$ \\
\hline
\end{tabular}

(b) Valores correspondientes a una distribución $\chi^{2}$ de dos grados de libertad.

Tabla 3.2: Valores de $\Delta \chi^{2}$ para determinar regiones de confianza. (a) Correspondientes a una distribución $\chi^{2}$ de un solo lado y un grado de libertad, (b) para una distribución $\chi^{2}$ de dos grados de libertad.

Habiendo determinado los límites de exclusión se prosigue al siguiente valor $\left(\Delta m^{2}\right)_{j+1}$, con el cual se repite el proceso antes descrito. Esto se hace para todos los valores de $\left(\Delta m^{2}\right)_{j}$ en la malla. Al tomar la unión de todas las parejas $\left(\sin ^{2} 2 \theta_{X \%},\left(\Delta m^{2}\right)_{j}\right)$ se forma el límite de exclusión de $X \%$ nivel de confianza (N.C.) en el espacio $\left(\sin ^{2} 2 \theta, \Delta m^{2}\right)$. Se construyen tantos límites de exclusión como se

\footnotetext{
${ }^{18}$ La búsqueda se hace sobre la malla predefinida de $\sin ^{2} 2 \theta, \S 3.2 .1$.

${ }^{19}$ Para obtener $\sin ^{2} 2 \theta_{X} \%$ se uso interpolación lineal, por que demostró ser suficientemente buena.
} 
estime conveniente ${ }^{20}$. El algoritmo 3.1 presenta la forma de implementar el método de la búsqueda por barrido unidimensional.

Observaciones: la iteración cero usa una matriz $\mathbf{M}^{\{0\}}$ con señal nula, es decir la matriz total se construye con un vector $\boldsymbol{Q}^{3 \times 1\{0\}}=\left(\emptyset, \boldsymbol{B}^{\nu_{e}}, \boldsymbol{B}^{\nu_{\mu}}\right)$. Por su parte la $k$-ésima iteración usa una matriz $\mathbf{M}^{\{k\}}$, la cual proviene de una matriz total construida con la señal del mejor ajuste de la iteración anterior $\boldsymbol{S}^{\{k\}}\left(x^{\{k-1\}},\left(\Delta m^{2}\right)_{j}\right)$, es decir se usa $\boldsymbol{Q}^{3 \times 1\{k\}}=\left(\boldsymbol{S}^{\{k\}}, \boldsymbol{B}^{\nu_{e}}, \boldsymbol{B}^{\nu_{\mu}}\right)$ para construir $\mathbf{M}^{\{k\}}$, ver ecuación (3.23).

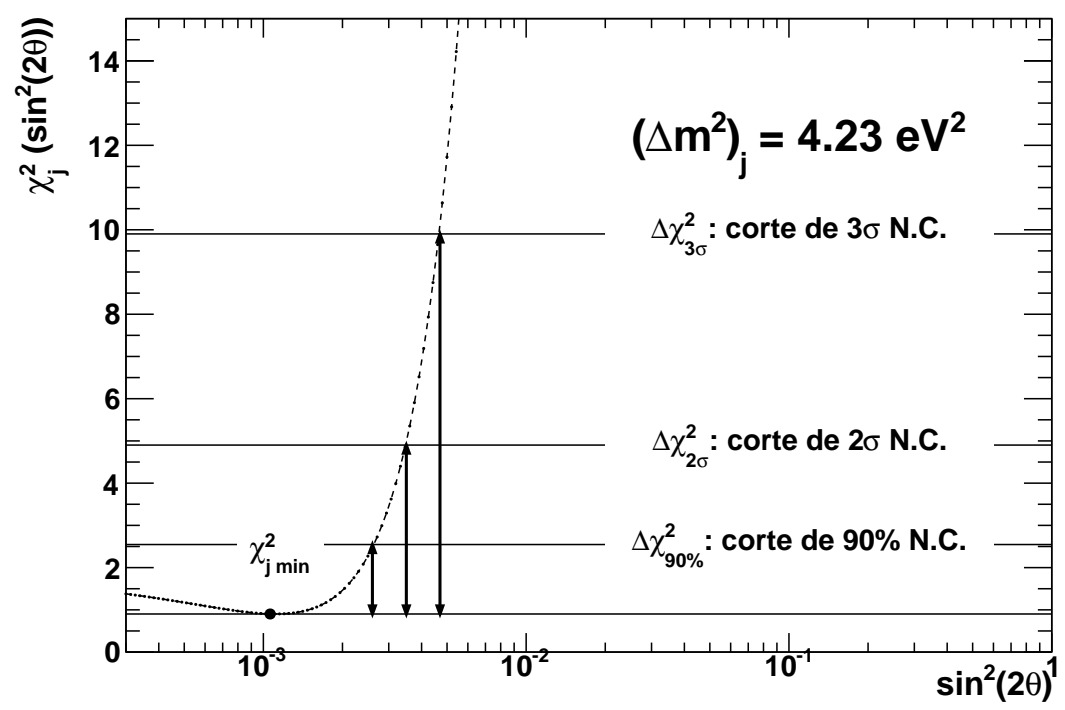

Figura 3.8: Gráfica de $\chi_{j}^{2}\left(\sin ^{2} 2 \theta\right)$ para un valor fijo de $\left(\Delta m^{2}\right)_{j}$. Excursiones desde $\chi_{j}^{2}$ min para determinar los límites de exclusión con el método de la búsqueda por barrido unidimensional. Se muestran 3 cortes, $\Delta \chi_{90 \%}^{2}, \Delta \chi_{2 \sigma}^{2}, \mathrm{y} \Delta \chi_{3 \sigma}^{2}$, con los cuales se determinan los límites de exclusión al $\sin ^{2} 2 \theta_{90 \%}, \sin ^{2} 2 \theta_{2 \sigma}, \mathrm{y} \sin ^{2} 2 \theta_{3 \sigma}$; respectivamente.

Para evaluar los estadísticos correspondientes, ecuaciones (3.30) y (3.31) para los datos de 2007 y 2009 , respectivamente, se usa la señal para cada punto $\left(\sin ^{2} 2 \theta,\left(\Delta m^{2}\right)_{j}\right) ; \sin ^{2} 2 \theta \in\left[3 \times 10^{-4}, 1\right]$, es decir, solo la matriz se mantiene fija. De esa forma la inversión de la matriz, la operación más costosa, solo se hace una vez por iteración. Por la misma razón se está exento de calcular el determinante de la matriz, término faltante en las ecuaciones (3.30) y (3.31) para ser equivalentes a la más general fórmula de verosimilitud (3.12). Usualmente se converge en la segunda o tercera iteración.

La decisión de usar un método de búsqueda por barrido unidimensional esta justificada por que el resultado obtenido es consistente con una señal nula, i.e. con la ausencia de oscilaciones de neutrinos. De forma tal que la dependencia de la $\chi^{2}$ en los parámetros de oscilación a través $\mathbf{M}$ puede despreciarse. Dada la aparente señal nula, este método permite establecer límites más restrictivos que un barrido bidimensional. El método responde a la pregunta: Si los datos observados realmente provienen de una señal nula ¿Cuál es el tamaño de la región excluida? Evidentemente en caso de una señal considerable, este método no es apropiado.

Como se mencionó en $\S 3.1 .1$, puesto que la decisión de usar un límite superior fue basada en los datos la búsqueda por barrido carece de cobertura correcta, concretamente este método otorga intervalos de

\footnotetext{
${ }^{20}$ Conforme se alejan del punto de mejor ajuste, los límites pierden veracidad.
} 
subcobertura. Más aún no es un método robusto, ya que es incapaz de distinguir entre un valor muy probable de $\Delta m^{2}$ y uno poco probable, ya que trabaja para $\Delta m^{2}$ fijos. Implícitamente se considera una aproximación gaussiana lo que permite prescindir de la construcción completa de las regiones de confianza de Neyman. En la sección §3.6 se compara este método con los demás.

Entrada: Datos y fondo de $\nu_{e}$ y $\nu_{\mu}$ (donde aplique), matriz de covarianza fraccionaria y tupla de simulación MC para la señal.

Salida : Tupla con límites de exclusión.

Declaración e inicialización de variables;

for $\left(\Delta m^{2}\right)_{j}=1 \times 10^{-2} e V^{2}$ to $1 \times 10^{2} e V^{2}$ do

$k=0$;

El siguiente loop es para encontrar la posición y el valor minimo de $\chi^{2}$, para cada $\left(\Delta m^{2}\right)_{j}$;

while $\left|\chi_{\min }^{2\{k\}}-\chi_{\min }^{2\{k-1\}}\right|>\varepsilon$ do

if $k=0$ then

$\mathbf{M}^{\{0\}} \leftarrow$ Build $\left(\mathbf{M}_{\mathrm{frac}}, \boldsymbol{Q}^{3 \times 1\{0\}}\right) ; \backslash \backslash$ matriz de covarianza con señal nula;

Invert $\left(\mathbf{M}^{\{0\}}\right)$;

for $x=3 \times 10^{-4}$ to 1 . do

FindMinimum $\left(\chi^{2\{0\}}\left(x,\left(\Delta m^{2}\right)_{j} ; \mathbf{M}^{-1}\{0\}\right)\right)$;

end

else

$\rightarrow \chi_{\min }^{2\{0\}} ; \rightarrow x^{\{0\}}$

$\mathbf{M}^{\{k\}} \leftarrow$ Build $\left(\mathbf{M}_{\text {frac }}, \boldsymbol{Q}^{3 \times 1\{k\}}\right) ; \backslash \backslash$ matriz de covarianza con señal de la iteración pasada;

Invert $\left(\mathbf{M}^{\{k\}}\right)$;

for $x=3 \times 10^{-4}$ to 1 . do

| FindMinimum $\left(\chi^{2\{k\}}\left(x,\left(\Delta m^{2}\right)_{j} ; \mathbf{M}^{-1}\{k\}\right)\right)$;

end

end

$\rightarrow \chi_{\min }^{2\{k\}} ; \rightarrow x^{\{k\}}$

$k++$;

end

$\rightarrow \chi_{j}^{2}$;

Ahora se encuentran los límites de exclusión.;

$\sin ^{2} 2 \theta_{90 \%} \leftarrow$ Interpolate $\left(\chi_{j}^{2}, \chi_{j \min }^{2}, \Delta \chi_{90 \%}^{2}\right) ; \sin ^{2} 2 \theta_{2 \sigma} \leftarrow \operatorname{Interpolate}\left(\chi_{j}^{2}, \chi_{j \min }^{2}, \Delta \chi_{2 \sigma}^{2}\right) ;$

$\sin ^{2} 2 \theta_{3 \sigma} \leftarrow$ Interpolate $\left(\chi_{j}^{2}, \chi_{j \min }^{2}, \Delta \chi_{3 \sigma}^{2}\right)$

end

$\Rightarrow$ Tupla con límites de exclusión de $90 \%, 2 \sigma, 3 \sigma$ N.C.;

Algoritmo 3.1: Algoritmo del método de la búsqueda por barrido unidimensional.

\subsection{Método de búsqueda por barrido bidimensional}

En la sección anterior se presentó el análisis de los datos de modo de neutrinos liberados en 2009, en la región de $E_{\nu}^{Q E} \geq 475 \mathrm{MeV}$ en donde no hay evidencia de un exceso de eventos. Sin embargo en la publicación [178] MiniBooNE presenta evidencia de un exceso de eventos en la región de bajas energías el cual no es compatible con la señal de oscilaciones de LSND. Si uno quisiera interpretar ese exceso en términos de oscilaciones de neutrinos un método más adecuado sería una búsqueda por barrido bidimensional usando el intervalo de $200 \leq E_{\nu}^{Q E} \leq 3000 \mathrm{MeV}$. En esta sección se describe este método y los resultados se presentan en $\S 4.2$.

El estadístico usado es una función $\chi^{2}$ para la cual la matriz de covarianza se cambia iterativamente, 
pero se permite que el número de eventos predichos para cada bin tome los valores que le corresponden para cada punto del espacio de parámetros:

$$
\chi^{2}\left(\sin ^{2} 2 \theta, \Delta m^{2}\right)=\sum_{i, j=1}^{n_{e}+n_{\mu}}\left(D_{i}-Q_{i}\right) \mathrm{M}_{i j}^{-1}\left(D_{j}-Q_{j}\right),
$$

aquí, como antes, $\boldsymbol{D}$ contiene los datos observados de $\nu_{e}$ y $\nu_{\mu}$, ecuación (3.25); $\boldsymbol{Q}$ contiene la predicción del número de eventos de $\nu_{e}$ (señal más fondo) y $\nu_{\mu}$, ecuación (3.24). Al cubrir todo el rango de energía, $\boldsymbol{D}$ y $\boldsymbol{Q}$ tienen $n_{e}+n_{\mu}=11+8=19$ componentes. De igual forma la matriz de covarianza $\mathbf{M}$, tiene $19 \times 19$ componentes. Ahora los grados de libertad son: $n_{e}+n_{\mu}-2-1=16$ g.l., una vez más el número efectivo de grados de libertad es el número de bins, menos el número de parámetros, menos uno por la normalización que se hace a la simulación con los $\nu_{\mu}$ observados.

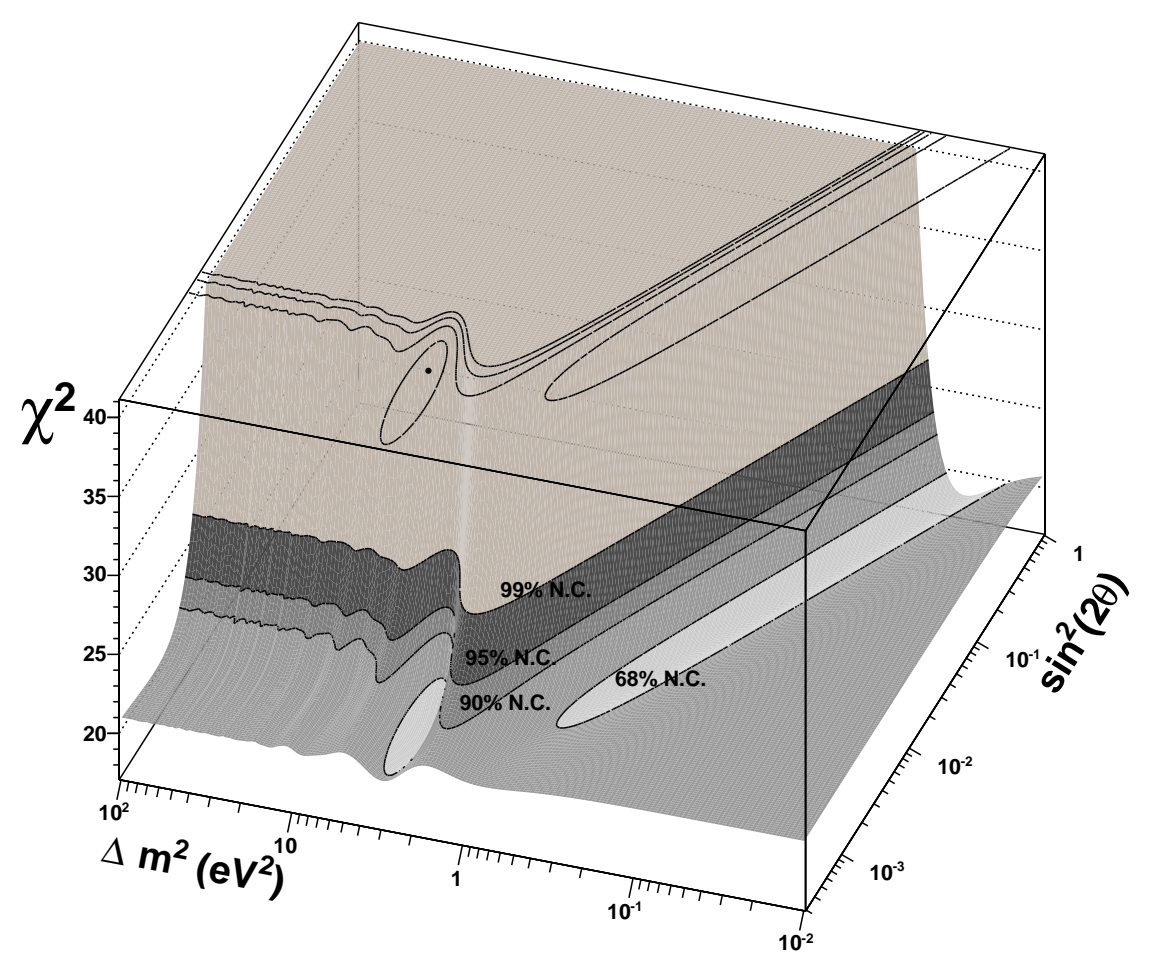

Figura 3.9: Superficie $\chi^{2}\left(\sin ^{2} 2 \theta, \Delta m^{2}\right)$ con cortes de nivel. Cortes de nivel desde $\chi_{\min }^{2}$ para determinar los contornos de confianza con el método de la búsqueda por barrido bidimensional. En la parte superior se observan las proyecciones del mínimo y de los contornos de $68 \%, 90 \%, 95 \%, 99 \%$ N.C.

Este método es muy similar al de la sección pasada, ya que es un método iterativo, solamente que ahora la búsqueda del mejor ajuste se hace en toda la malla del plano $\sin ^{2} 2 \theta-\Delta m^{2}$. En la iteración cero se empieza con una matriz con señal nula, es decir que solamente contiene información de las correlaciones del fondo de neutrinos del electrón. El método busca el valor de $\boldsymbol{x}^{\{0\}}=\left(\sin ^{2} 2 \theta, \Delta m^{2}\right)^{\{0\}}$ que minimiza

$$
\chi^{2\{0\}} \equiv \chi^{2}\left(\boldsymbol{x} ; \mathbf{M}^{\{0\}}\right)
$$

una vez más $\mathbf{M}^{\{0\}}$ representa la matriz de covarianza calculada con señal nula. Posteriormente $\boldsymbol{x}^{\{0\}}$ genera la señal para construir la matriz para la siguiente iteración, $\mathbf{M}^{\{1\}}$. El proceso se detiene cuando 
los valores del mejor ajuste de iteraciones sucesivas difieren en menos de una tolerancia de $\varepsilon \sim 1 \times 10^{-4}$, la cual se consigue a la cuarta iteración ${ }^{21}$.

De esta forma en la última iteración se obtiene una superficie $\chi^{2}$ sobre $\left(\sin ^{2} 2 \theta, \Delta m^{2}\right)$ con la cual se determinan los contornos de confianza haciendo cortes de nivel a partir del mínimo $\chi_{\text {min }}^{2}$. Estos determinan los contornos a diferentes niveles de confianza ( $X \%$ N.C.) sobre $\left(\sin ^{2} 2 \theta, \Delta m^{2}\right)$, es decir aquellos puntos que satisfacen:

$$
\chi^{2}\left(\sin ^{2} 2 \theta, \Delta m^{2}\right)=\chi_{\min }^{2}+\Delta \chi_{X \%}^{2},
$$

la distancia por encima del mínimo, $\Delta \chi_{X \%}^{2}$, a la que se hacen estos cortes esta dada por una distribución $\chi^{2}$ de dos grados de libertad, ecuación (3.19). Los valores usados se encuentran en la tabla 3.2b. En la figura 3.9 se muestran las excursiones desde el mínimo para determinar los cortes de nivel que forman los contornos. En el algoritmo 3.2 se resume la implementación del método de la búsqueda por barrido bidimensional.

Entrada: Datos y fondo de $\nu_{e}$ y $\nu_{\mu}$, matriz de covarianza fraccionaria y tupla de simulación MC para la señal. Salida : Tupla con contornos de confianza.

Declaración e inicialización de variables;

$k=0$;

while $\left|\chi_{\min }^{2\{k\}}-\chi_{\min }^{2\{k-1\}}\right|>\varepsilon$ do

if $k=0$ then

$\mathbf{M}^{\{0\}} \leftarrow$ Build $\left(\mathbf{M}_{\mathrm{frac}}, \boldsymbol{Q}^{3 \times 1\{0\}}\right) ; \backslash \backslash$ matriz de covarianza con señal nula;

Invert $\left(\mathbf{M}^{\{0\}}\right)$;

for $x_{1}=3 \times 10^{-4}$ to 1 . do

$$
\begin{aligned}
& \text { for } x_{2}=1 \times 10^{-2} e V^{2} \text { to } 1 \times 10^{2} e V^{2} \text { do } \\
& \mid \text { FindMinimum }\left(\chi^{2}\{0\}\left(x ; \mathbf{M}^{-1}\{0\}\right)\right) ;
\end{aligned}
$$

end

$\rightarrow \chi_{\min }^{2\{0\}} ; \rightarrow \boldsymbol{x}^{\{0\}}$

else

$\mathbf{M}^{\{k\}} \leftarrow \operatorname{Build}\left(\mathbf{M}_{\mathrm{frac}}, \boldsymbol{Q}^{3 \times 1\{k\}}\right) ; \backslash \backslash$ matriz de covarianza con señal de la iteración pasada; Invert $\left(\mathbf{M}^{\{k\}}\right)$;

for $x_{1}=3 \times 10^{-4}$ to 1 . do

$$
\text { for } x_{2}=1 \times 10^{-2} e V^{2} \text { to } 1 \times 10^{2} e V^{2} \text { do }
$$$$
\text { FindMinimum }\left(\chi^{2\{k\}}\left(\boldsymbol{x} ; \mathbf{M}^{-1}\{k\}\right)\right) \text {; }
$$

end

$\rightarrow \chi_{\min }^{2\{k\}} ; \rightarrow \boldsymbol{x}^{\{k\}}$

end

$k++$;

end

$\rightarrow \chi^{2}\left(\sin ^{2} 2 \theta, \Delta m^{2}\right) ; \rightarrow \chi_{\min }^{2} ;$

Ahora se encuentran los contornos de confianza.;

$\left(\sin ^{2} 2 \theta, \Delta m^{2}\right)_{68 \%} \leftarrow$ Interpolate $\left(\chi^{2}, \chi_{\min }^{2}, \Delta \chi_{68 \%}^{2}\right) ;\left(\sin ^{2} 2 \theta, \Delta m^{2}\right)_{90 \%} \leftarrow$ Interpolate $\left(\chi^{2}, \chi_{\min }^{2}, \Delta \chi_{90 \%}^{2}\right) ;$

$\left(\sin ^{2} 2 \theta, \Delta m^{2}\right)_{95 \%} \leftarrow$ Interpolate $\left(\chi^{2}, \chi_{\min }^{2}, \Delta \chi_{95 \%}^{2}\right) ;\left(\sin ^{2} 2 \theta, \Delta m^{2}\right)_{99 \%} \leftarrow$ Interpolate $\left(\chi^{2}, \chi_{\min }^{2}, \Delta \chi_{99 \%}^{2}\right) ;$

$\Rightarrow$ Tupla con contornos de confianza de $68 \%, 90 \%, 95 \%, 99 \%$ N.C.;

Algoritmo 3.2: Algoritmo del método de la búsqueda por barrido bidimensional.

Observaciones: este método es el que requiere menos operaciones, la construcción e inversión de la matriz de covarianza solamente se hace una vez por iteración, cinco veces en total.

\footnotetext{
${ }^{21} \mathrm{El}$ ajuste que se hace con la matriz nula se considera como la iteración cero.
} 
Nótese que si la matriz $\mathbf{M}$ crece arbitrariamente entonces se puede tener un valor arbitrariamente pequeño para el estadístico $\chi^{2}$ de la ecuación (3.32), creando un posible sesgo en el ajuste hacia puntos en el espacio de parámetros que carecen de significado físico, es decir deja de importar que $\boldsymbol{D}-\boldsymbol{Q} \approx 0$ ya que las componentes de la matriz inversa son muy pequeñas, $M_{i j}^{-1} \ll 1$. Sin embargo en un esquema iterativo se evita calcular la matriz en cada punto, logrando prevenir este comportamiento. Por supuesto, omitir el cálculo de la matriz de covarianza en cada punto, si bien es una aproximación razonable, impide que sea una implementación completamente robusta.

$\mathrm{Al}$ permitir que varié tanto $\sin ^{2} 2 \theta$ como $\Delta m^{2}$ la búsqueda por barrido bidimensional logra discernir entre valores poco probables de $\Delta m^{2}$ y muy probables, de hecho se consigue obtener la pareja de parámetros que mejor ajusta los datos, corrigiendo el defecto de la búsqueda unidimensional. Al considerar una aproximación gaussiana se prescinde de la necesidad de hacer la construcción completa de Neyman. Desafortunadamente los intervalos generados al cortar con un plano a partir del mínimo la superficie $\chi^{2}$ tienen regiones de subcobertura y sobrecobertura. En la sección $\S 3.6$ se discute la razón de ese comportamiento y se compara con los demás métodos.

\subsection{Método de máxima verosimilitud}

Este método es más robusto que los pasados, junto con las mejoras que fue haciendo la colaboración con los años se implementó este método para analizar los datos. La mayoría de los análisis que se presentan en el capítulo 4 se hicieron con este método, las correcciones frecuentistas (de las que se hablará en la siguiente sección) usan este método también. Se repiten los contornos de los datos liberados en 2009 del modo de neutrinos para presentar una comparación entre los distintos métodos, §4.2. Para los datos publicados del modo de antineutrinos, consistentes con una señal de oscilación, se opta por usar este método (con correcciones frecuentistas) para presentar los resultados, §4.3. Se estandariza el uso de este método para las publicaciones subsecuentes: neutrinos de 2012, §4.4; antineutrinos de 2012, §4.5; y el análisis combinado de neutrinos y antineutrinos de $2012 \S 4.6$. Todos los análisis que se hacen aquí con este método son para el rango de energías $200 \leq E_{\nu}^{Q E} \leq 3000 \mathrm{MeV}$.

El estadístico usado es el doble del negativo del logaritmo de una función de verosimilitud, $\mathscr{L} \equiv$ $-2 \ln (\mathcal{L})$ ecuación (3.12). A diferencia de los métodos pasados, ahora la matriz de covarianza se construye para cada punto en el espacio de parámetros al igual que el número de eventos predichos y se requiere el determinante de la misma:

$$
\begin{aligned}
& \mathscr{L}\left(\sin ^{2} 2 \theta, \Delta m^{2}\right)=\ln (|\mathbf{M}|)+\sum_{i, j}^{n_{e}+n_{\mu}}\left(D_{i}-Q_{i}\right) \mathrm{M}_{i j}^{-1}\left(D_{j}-Q_{j}\right) \\
& \mathscr{L}\left(\sin ^{2} 2 \theta, \Delta m^{2}\right)=\ln (|\overline{\mathbf{M}}|)+\sum_{i, j}^{n_{e}+n_{\mu}}\left(\bar{D}_{i}-\bar{Q}_{i}\right) \overline{\mathrm{M}}_{i j}^{-1}\left(\bar{D}_{j}-\bar{Q}_{j}\right),
\end{aligned}
$$

una vez más, $\boldsymbol{D}(\overline{\boldsymbol{D}})$ contiene los datos observados de $\nu_{e}\left(\bar{\nu}_{e}\right)$ y $\nu_{\mu}\left(\bar{\nu}_{\mu}\right)$, ecuación $(3.25) ; \boldsymbol{Q}(\overline{\boldsymbol{Q}})$ contiene la predicción del número de eventos de $\nu_{e}\left(\bar{\nu}_{\mu}\right)$ (señal más fondo) y $\nu_{\mu}\left(\bar{\nu}_{\mu}\right)$, ecuación (3.24); tienen $n_{e}+n_{\mu}=11+8=19$ componentes. De igual forma la matriz de covarianza $\mathbf{M}(\overline{\mathbf{M}})$, tiene $19 \times 19$ componentes. Para los grados de libertad hay que restar el número de parámetros y una unidad más ya que hizo una normalización a los datos: $n_{e}+n_{\mu}-2-1=16$ g.l. Como ya se mencionó, tanto $\boldsymbol{Q}(\overline{\boldsymbol{Q}})$ como $\mathbf{M}(\overline{\mathbf{M}})$ son funciones de $\left(\sin ^{2} 2 \theta, \Delta m^{2}\right)$. 


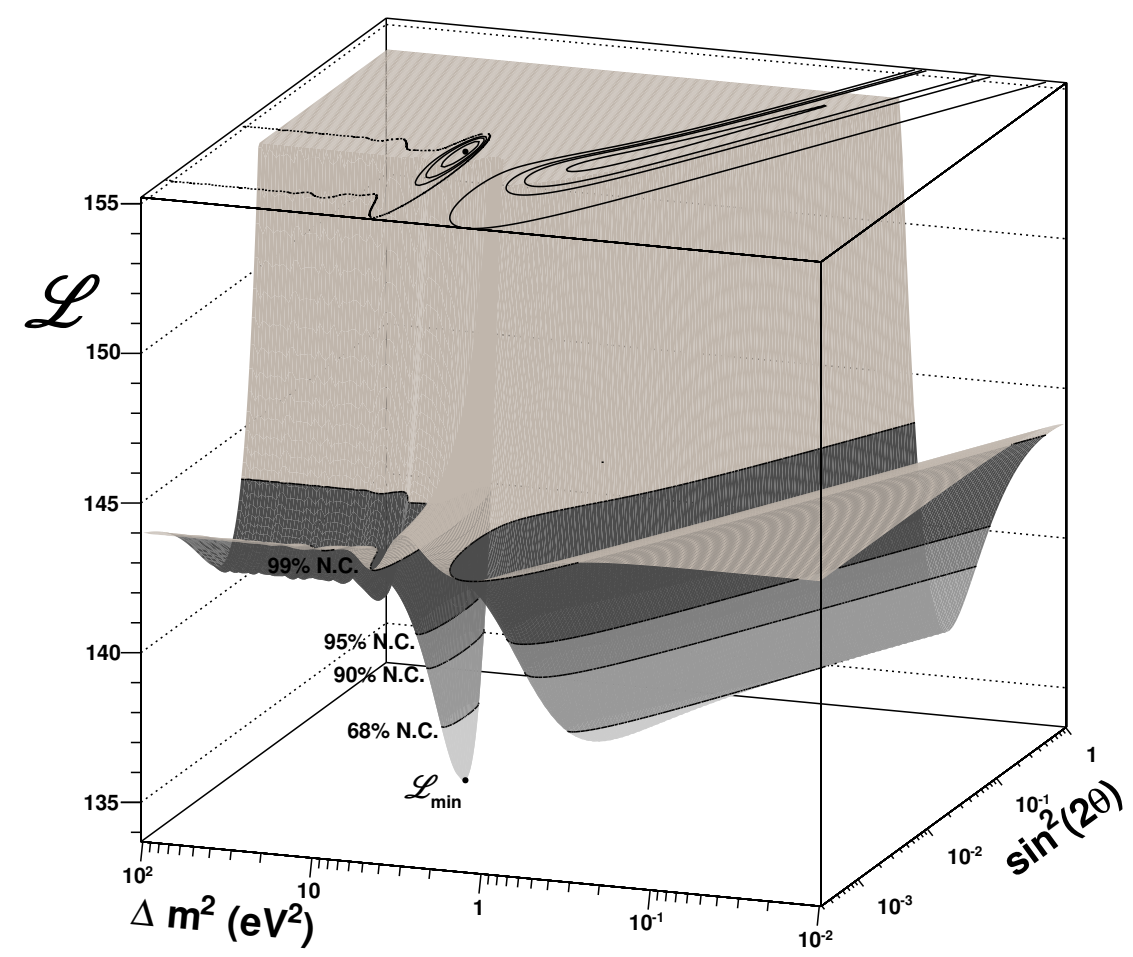

Figura 3.10: Superficie $\mathscr{L}\left(\sin ^{2} 2 \theta, \Delta m^{2}\right)$ con cortes de nivel para el método de máxima verosimilitud. Cortes de nivel desde $\mathscr{L}_{\text {min }}$ para determinar los contornos de confianza con el método de la búsqueda por barrido bidimensional. En la parte superior se observan las proyecciones del mínimo y de los contornos de $68 \%, 90 \%, 95 \%, 99 \%$ N.C.

La razón por la cual este método es más robusto que los pasados, es por que incluye la dependencia de los parámetros de oscilación en todas las partes relevantes del estadístico, evitando la necesidad de implementar un proceso iterativo. Al construir la matriz de covarianza y calcular su determinante para cada punto se tiene una mejor estimación de la semejanza de los datos con una predicción teórica. La búsqueda del mejor ajuste se hace en toda la malla del espacio $\left(\sin ^{2} 2 \theta, \Delta m^{2}\right)$, es decir se evalúa la ecuación (3.33) en cada punto de la malla y se encuentra el valor mínimo. Es más sencillo de implementar que los métodos pasados, solamente que la cantidad de operaciones es considerablemente mayor. Después de haber evaluado el estadístico en cada punto se obtiene una superficie $\mathscr{L}\left(\sin ^{2} 2 \theta, \Delta m^{2}\right)$ y el valor y posición del mejor ajuste, $\mathscr{L}_{\min }$ y $\left(\sin ^{2} 2 \theta, \Delta m^{2}\right)_{\min }$. Al igual que el método pasado, los contornos de confianza se determinan haciendo cortes de nivel a partir del mínimo; los puntos que satisfacen:

$$
\mathscr{L}\left(\sin ^{2} 2 \theta, \Delta m^{2}\right)=\mathscr{L}_{\min }+\Delta \chi_{X \%}^{2},
$$

forman el contorno de $X \%$ N.C. En la tabla $3.2 \mathrm{~b}$ se encuentran los valores de $\Delta \chi_{X \%}^{2}$ para determinar los distintos contornos. La figura 3.10 muestra las excursiones desde el mínimo para determinar los cortes de nivel que forman los contornos. El algoritmo 3.3 resume la implementación del método de máxima verosimilitud.

Observaciones: dada la importancia de este método y su aplicación para el método Feldman-Cousins de $\S 3.6$, se hizo un gran esfuerzo para optimizarlo al máximo, consiguiendo que fuera, por mucho, el método más rápido de todos. Se explota al máximo el hecho de que las matrices sean positivas 
Entrada: Datos y fondo de $\nu_{e}$ y $\nu_{\mu}\left(\bar{\nu}_{e}\right.$ y $\left.\bar{\nu}_{\mu}\right)$, matriz de covarianza fraccionaria y tupla de simulación MC para la señal.

Salida : Tupla con contornos de confianza.

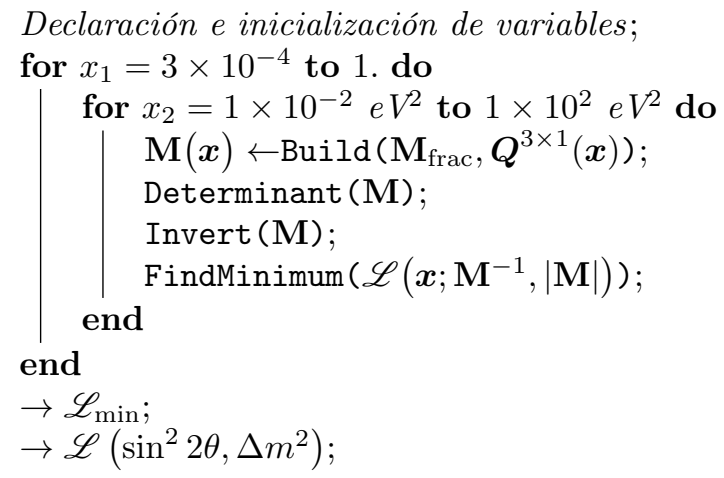

Ahora se encuentran los contornos de confianza.;

$\left(\sin ^{2} 2 \theta, \Delta m^{2}\right)_{68 \%} \leftarrow$ Interpolate $\left(\mathscr{L}, \mathscr{L}_{\min }, \Delta \chi_{68 \%}^{2}\right) ;\left(\sin ^{2} 2 \theta, \Delta m^{2}\right)_{90 \%} \leftarrow \operatorname{Interpolate}\left(\mathscr{L}, \mathscr{L}_{\min }, \Delta \chi_{90 \%}^{2}\right) ;$

$\left(\sin ^{2} 2 \theta, \Delta m^{2}\right)_{95 \%} \leftarrow$ Interpolate $\left(\mathscr{L}, \mathscr{L}_{\min }, \Delta \chi_{95 \%}^{2}\right) ;\left(\sin ^{2} 2 \theta, \Delta m^{2}\right)_{99 \%} \leftarrow \operatorname{Interpolate}\left(\mathscr{L}, \mathscr{L}_{\min }, \Delta \chi_{99 \%}^{2}\right) ;$

$\Rightarrow$ Tupla con contornos de confianza de $68 \%, 90 \%, 95 \%, 99 \%$ N.C.;

\section{Algoritmo 3.3: Algoritmo del método de máxima verosimilitud.}

definidas, consiguiendo que el cálculo del determinante sea un paso intermedio natural de la inversión de matrices.

Para este método se esta exento de la patología que surge de permitir que la matriz de covarianza tome valores arbitrariamente grandes, lo que generaría una inversa con entradas muy pequeñas, ya que al incluir el término del determinante se penaliza el uso de matrices muy grandes, consiguiendo de forma efectiva balancear la relevancia de la matriz de covarianza en la ecuación (3.33).

Hasta ahora es el método más robusto, permite discernir de forma precisa la pareja de parámetros $\left(\sin ^{2} 2 \theta, \Delta m^{2}\right)$ que mejor ajusta los datos. Como antes, se considera una aproximación gaussiana que permite prescindir de conocer las regiones de aceptación con las cuales se obtienen los intervalos de confianza, una simplificación que ayuda considerablemente a simplificar el problema. Sin embargo esta búsqueda global otorga intervalos de sobrecobertura y subcobertura, ocasionados, en esencia, por la forma no lineal de la fórmula de oscilación y la insatisfacción que provocan en las aproximaciones supuestas para la formación de los contornos. En la siguiente sección se discute con más detalle este defecto, la forma de solucionarlo y se comparan los distintos métodos.

En el apéndice A se puede ver el código que realiza el cálculo de la máxima verosimilitud.

\subsection{Método frecuentista Feldman-Cousins}

En su artículo intitulado "Unified approach to the classical statistical analysis of small signals", Gary Feldman y Robert Cousins proponen una forma de construir los límites de confianza que unifica la forma de tratar límites superiores, para resultados nulos, e intervalos de confianza de dos lados, para resultados no nulos [196]. Como se vio antes, basarse en los datos para elegir entre un límite superior o un contorno de confianza, lleva a intervalos que tienen un significado probabilístico inadecuado y que frecuentemente son incompatibles. El método Feldman-Cousins $(\mathrm{F}-\mathrm{C})$ evita límites de confianza sin sentido físico, evita tendencias conservadoras, desacopla las pruebas de bondad del ajuste de los 
intervalos de confianza, da cobertura correcta y es robusto.

Con este método se vuelven a presentar distintos resultados, desde los datos de 2009. Una vez más se presentan los resultados del modo de neutrinos de los datos publicados en $2009 \S 4.2$, aún cuando la colaboración no usa este método para el análisis. La primera ocasión que se usa este método por la colaboración, es para los datos del modo de antineutrinos de 2010, los cuales se presentan aquí en $\S$ 4.3. Siendo que este es el método más robusto hasta ahora, no se quiere dejar de analizar los datos más recientes con esta maquinaria, es decir lo datos del modo de neutrinos publicados en 2012, presentados en $\S 4.4$, y los datos del modo de antineutrinos también publicados en 2012, presentados en $§ 4.5$. A pesar del gran tiempo de procesamiento que requieren, se decidió usar el método $\mathrm{F}-\mathrm{C}$ para analizar los datos viejos para tener una comparación con los datos más nuevos. También en el análisis combinado de neutrinos y antineutrinos de los datos de 2012 se usa el método F-C, los resultados se presentan en $§ 4.5$ y en la siguiente sección se hablará sobre este análisis.

El método propuesto por Feldman y Cousins usa la libertad inherente en la construcción de intervalos de Neyman para obtener límites superiores e intervalos de dos lados. Es una elección particular de ordenamiento, basado en el coeficiente de probabilidades

$$
R=\frac{P(\boldsymbol{D} \mid \boldsymbol{Q})}{P\left(\boldsymbol{D} \mid \boldsymbol{Q}_{\min }\right)},
$$

donde como antes $\boldsymbol{D}$ son los eventos observados, $\boldsymbol{Q}$ es la predicción de eventos y cambia en cada punto $\left(\sin ^{2} 2 \theta, \Delta m^{2}\right)$ y, en particular, $\boldsymbol{Q}_{\min }$ es la predicción en el mejor ajuste.

La región de aceptación para cada punto del plano $\sin ^{2} 2 \theta-\Delta m^{2}$ es calculada realizando simulaciones de Monte Carlo de los resultados de una gran cantidad de experimentos para el conjunto de parámetros físicos desconocidos y el flujo de neutrinos del experimento. Se debe obtener $R_{c}\left(\sin ^{2} 2 \theta, \Delta m^{2}\right)$, tal que una fracción $\alpha$ de los experimentos simulados tengan $R \leq R_{c}\left(\sin ^{2} 2 \theta, \Delta m^{2}\right)$. Después de que los datos son analizados, $R$ para los datos y cada punto en el plano $\sin ^{2} 2 \theta-\Delta m^{2}$, i.e. $R\left(\boldsymbol{D} \mid\left(\sin ^{2} 2 \theta, \Delta m^{2}\right)\right)$, es comparado a $R_{c}$ y la región de aceptación son todos los puntos tales que

$$
R\left(\boldsymbol{D} \mid\left(\sin ^{2} 2 \theta, \Delta m^{2}\right)\right) \leq R_{c}\left(\sin ^{2} 2 \theta, \Delta m^{2}\right)
$$

Recuérdese que la región de aceptación es un conjunto de intervalos de la variable medida, en este caso para cada punto $\left(\sin ^{2} 2 \theta, \Delta m^{2}\right)$ se tiene un región en el espacio de datos y de la dimensión de $\boldsymbol{D}$, y el conjunto de esas regiones para cada punto en el espacio $\left(\sin ^{2} 2 \theta, \Delta m^{2}\right)$ forma la región de confianza.

En el artículo de Feldman y Cousins [196] se explica con más detalle varias de las fallas de varios de los métodos más populares para generar contornos de confianza. Reiterando, el método de $\mathrm{F}-\mathrm{C}$, a diferencia del barrido unidimensional, logra aislar efectivamente el punto de mejor ajuste; los métodos de barrido bidimensional y máxima verosimilitud logran aislar el mejor ajuste sin embargo muestran regiones considerables de subcobertura y sobrecobertura, es decir que los contornos alrededor de puntos de mejor ajuste sobre esas regiones contienen al punto en una proporción de experimentos menor o mayor que la referida. El método de $\mathrm{F}-\mathrm{C}$ cubre adecuadamente.

A pesar de lo robusto que es el método de la máxima verosimilitud, generar intervalos de confianza cortando la superficie $\mathscr{L}$ con simples planos constantes genera regiones que no tienen cobertura correcta, hay regiones de subcobertura y sobrecobertura. El método $\mathrm{F}-\mathrm{C}$ provee un mecanismo, basado en primeros principios de estadística frecuentista, para hacer las "correcciones" pertinentes 
a estos planos. Es decir los contornos se obtendrán al cortar la superficie $\mathscr{L}$ con una superficie corregida. Con el fin de hacer tales correcciones se tienen que generar distribuciones de "experimentos ficticios", que permitan estimar cual es el corte apropiado para cada punto en el plano $\sin ^{2} 2 \theta-\Delta m^{2}$. Por ejemplo, para dos grados de libertad el corte de $90 \%$ N.C. es de 4.61 unidades, el cual, para el caso gaussiano lejos de la frontera física, otorga intervalos de confianza con cobertura adecuada. Sin embargo al hacer las correcciones en los análisis hechos en este trabajo, es común observar variaciones desde 2.71 hasta 7.88 unidades para los cortes que generan intervalos con cobertura correcta. Lo que significa es que donde se requieren cortes de 2.71 se están obteniendo regiones de sobrecobertura al cortar con 4.61, y en donde se requiere cortar con 7.88 se obtienen regiones de subcobertura.

Algunos de los efectos que causan la desviación del corte de 4.61 son:

1. Naturaleza sinusoidal de la función de oscilación. La función $\mathscr{L}$ asume una f.d.p. gaussiana, pero la función de oscilación es sinusoidal. Para altos valores de $\Delta m^{2}$ las fluctuaciones pueden causar un mínimo global en una zanja equivocada de la función, incrementando el valor de $\Delta \mathscr{L}$ desde el que sería si solo hubiera una zanja.

2. Regiones unidimensionales. En algunas regiones del plano, la f.d.p. se convierte en unidimensional en vez de ser bidimensional. Por ejemplo, para valores muy bajoes de $\Delta m^{2}$ la única cantidad relevante es el número de eventos en el bin de menor energía, ya que la probabilidad de oscilación, ecuación (1.6), es proporcional a $1 / E^{2}$ para valores suficientemente pequeños de $\Delta m^{2}$. Las fluctuaciones en los bins de más alta energía no llevan a ninguna interpretación física, así pues se cancelan en el cálculo de $\Delta \mathscr{L}$. En estas regiones el corte que hace falta hacer a la superficie para obtener los contornos con correcta cobertura tiende a ser menor que lo normal.

\subsubsection{Implementación del método Feldman-Cousins}

En la implementación que se hizo del método $\mathrm{F}-\mathrm{C}$ en este trabajo, lo primero que se tiene que hacer es generar la superficie

$$
\Delta \mathscr{L}\left(\sin ^{2} 2 \theta, \Delta m^{2}\right)=\mathscr{L}\left(\sin ^{2} 2 \theta, \Delta m^{2}\right)-\mathscr{L}_{\min }
$$

la única diferencia entre esta y la que se describe en la sección anterior, ecuación (3.33), es que se le ha restado el valor constante $\mathscr{L}_{\text {min }}$. Será a esta superficie a la que se la harán correcciones. Se muestra una superficie típica en la figura 3.12b.

La generación de experimentos ficticios ${ }^{22}$ consiste en generar arreglos de datos $\boldsymbol{D}_{\mathbf{f}}^{\left\{\boldsymbol{p}_{0}\right\}}$, para cada punto $\boldsymbol{p}_{0}$ en el plano $\sin ^{2} 2 \theta-\Delta m^{2}$ que se desee evaluar. Cada uno de estos arreglos tiene la propiedad de ser una fluctuación estadística de la distribución de datos de $\nu_{e}$ y $\nu_{\mu}$ en $E_{\nu}^{Q E}$, que el experimento hubiera observado si la pareja de parámetros de oscilación reales fueran $\boldsymbol{p}_{0}$. El proceso a través del cual se generan estos datos ficticios se describe a continuación y es el mismo que se describe en el libro [197]. En esencia es una transformación lineal que genera un vector aleatorio con una estructura de dependencia dada, según lo medido por la matriz de covarianza.

Se quiere generar un vector aleatorio $\boldsymbol{y}$ de $n$ componentes, con media en cero y matriz de covarianza $\mathbf{M}=\mathscr{E}\left(\boldsymbol{y} \boldsymbol{y}^{T}\right)$, ecuación $\left(3.9^{\prime}\right)$. Para lo cual se genera un vector aleatorio $\boldsymbol{x}$ con $n$ componentes, cada

\footnotetext{
${ }^{22}$ También conocido como "Monte Carlo de juguete".
} 
una de las cuales tiene su media en cero y varianza unitaria. Luego $\boldsymbol{y}$ es simplemente

$$
\boldsymbol{y}=\mathbf{h} \boldsymbol{x}
$$

donde $\mathbf{h}$ es una matriz de $n \times n$ no singular. Nótese que

$$
\begin{gathered}
\mathscr{E}(\boldsymbol{y})=\mathbf{h} \mathscr{E}(\boldsymbol{x})=0, \\
\mathscr{E}\left(\boldsymbol{y} \boldsymbol{y}^{T}\right)=\mathbf{h} \mathscr{E}\left(\boldsymbol{x} \boldsymbol{x}^{T}\right) \mathbf{h}^{T}=\mathbf{h h}^{T}=\mathbf{M} .
\end{gathered}
$$

Como $\mathbf{M}$ es positiva definida ${ }^{7}$ siempre se puede encontrar una matriz triangular inferior no singular $\mathbf{h}$, que cumpla $\mathbf{h} \mathbf{h}^{T}=\mathbf{M}$. Ahora el problema se ha transformado en uno de descomponer una matriz simétrica positiva definida $\mathbf{M}$, en el producto de una matriz triangular inferior y su transpuesta, a esto se le llama factorización de Cholesky. Convenientemente esta matriz de covarianza se construye de igual manera que la de $\S 3.2$. El algoritmo usado para encontrar la matriz triangular inferior es el método de la raíz cuadrada ${ }^{23}$, en el cual las componentes de $\mathbf{h}$ son:

$$
\begin{aligned}
\mathrm{h}_{i 1} & =\mathrm{M}_{i 1} / \sqrt{\mathrm{M}_{11}} & & (1 \leq i \leq n) \\
\mathrm{h}_{i i} & =\sqrt{\mathrm{M}_{i i}-\sum_{j=1}^{i-1} \mathrm{~h}_{i j}^{2}} & & (1 \leq i \leq n) \\
\mathrm{h}_{i j} & =\frac{\mathrm{M}_{i j}-\sum_{k=1}^{j-1} \mathrm{~h}_{i k} \mathrm{~h}_{j k}}{\mathrm{~h}_{j j}} & & (1<j<i \leq n) \\
\mathrm{h}_{i j} & =0 & & (i<j \leq n)
\end{aligned}
$$

Por último, para formar los datos ficticios uno debe tomar el vector de estimación de eventos $\boldsymbol{Q}\left(\boldsymbol{p}_{0}\right)$, evaluado en el punto $\boldsymbol{p}_{0}$, como valor central y sumarle el vector fluctuado con las correlaciones correspondientes a ese punto, es decir:

$$
D_{\mathbf{f}}^{\left\{p_{0}\right\}}=\boldsymbol{Q}\left(\boldsymbol{p}_{0}\right)+\boldsymbol{y}
$$

Se debe generar un vector $\boldsymbol{y}$ por cada experimento ficticio que se quiera hacer, $n_{\mathbf{f}}$ experimentos ficticios para cada punto, en los análisis presentados en esta tesis $n_{\mathbf{f}}$ toma valores desde $\sim 2000$ a $\sim 6000$ experimentos ficticios. Se deben generar experimentos ficticios para tantos puntos $\boldsymbol{p}_{0}$ como se requiera. El algoritmo 3.4 resume la implementación de la generación de datos ficticios. En el apéndice A se puede ver el código que genera los experimentos ficticios.

Entrada: Matriz de covarianza M para el punto $\boldsymbol{p}_{0}=\left(\sin ^{2} 2 \theta, \Delta m^{2}\right)_{0}$ y vector de estimación de eventos $\boldsymbol{Q}\left(\boldsymbol{p}_{0}\right)$. Salida : Arreglo de vectores de datos ficticios $\boldsymbol{D}_{\mathbf{f}}^{\left\{\boldsymbol{p}_{0}\right\}}$, .

Factorización de la matriz en una triangular inferior.;

$\mathbf{h} \leftarrow$ getLowerMatrix $(\mathbf{M})$;

for $j=1$ to $n_{e}+n_{\mu}$ do

| $x_{j}=$ normalRandomGenerator $(0,1) ; \backslash \backslash$ media cero, varianza unitaria. ;

end

$\boldsymbol{y}=\mathbf{h} \boldsymbol{x} ;$

$\boldsymbol{D}_{\mathbf{f} i}^{\left\{\boldsymbol{p}_{0}\right\}}=\boldsymbol{Q}\left(\boldsymbol{p}_{0}\right)+\boldsymbol{y}$

Algoritmo 3.4: Algoritmo para generar datos ficticios $\boldsymbol{D}_{\mathbf{f}}^{\left\{\boldsymbol{p}_{0}\right\}}$ en el punto $\boldsymbol{p}_{0} \in \sin ^{2} 2 \theta-\Delta m^{2}$.

Para cada una de las $n_{\mathbf{f}}$ distribuciones de datos falsos de un punto $\boldsymbol{p}_{0} \in \sin ^{2} 2 \theta-\Delta m^{2}$ se efectúa el método de la máxima verosimilitud, es decir se evalúa el estadístico de la sección pasada, el doble del

${ }^{23}$ El método usado no es aquel descubierto por André-Louis Cholesky. 
negativo del logaritmo de una función de verosimilitud, en cada punto de la malla preestablecida de $190 \times 190=36100$. Sin embargo, ahora la ecuación (3.33) no se evalúa con los datos verdaderos sino con las distribuciones de datos falsos $\boldsymbol{D}_{\mathbf{f} i}^{\left\{\boldsymbol{p}_{0}\right\}}, i=1, \ldots, n_{\mathbf{f}}$; donde para cada $i$-ésimo experimento ficticio se busca el valor mínimo $\mathscr{L}_{\min i}^{\left\{\boldsymbol{p}_{0}\right\}}$, y el valor que adquiere la verosimilitud en el punto $\boldsymbol{p}_{0}, \mathscr{L}_{i}^{\left\{\boldsymbol{p}_{0}\right\}}\left(\boldsymbol{p}_{0}\right)$. Una aclaración sobre la notación usada en esta sección: el superíndice $\left\{\boldsymbol{p}_{0}\right\}$ significa que es con la señal de ese punto que se generaron los experimentos ficticios, por otra parte aquí en particular la notación $\left(\boldsymbol{p}_{0}\right)$ se refiere a que se evalúa la verosimilitud en el punto $\boldsymbol{p}_{0}$.

En el régimen gaussiano, se puede sustituir la probabilidad de la ecuación (3.35) por la función de verosimilitud $\mathscr{L}=-2 \ln P$, así que la ecuación (3.35) es equivalente a la diferencia que se busca de cada experimento ficticio:

$$
\Delta \mathscr{L}_{\mathbf{f} i}^{\left\{\boldsymbol{p}_{i}\right\}}=\mathscr{L}_{i}^{\left\{\boldsymbol{p}_{0}\right\}}\left(\boldsymbol{p}_{0}\right)-\mathscr{L}_{\min i}^{\left\{\boldsymbol{p}_{0}\right\}}
$$

Con los $n_{\mathbf{f}}$ experimentos ficticios se obtiene una distribución de diferencias $\Delta \mathscr{L}_{\mathbf{f} i}^{\left\{\boldsymbol{p}_{0}\right\}}, i=1, \ldots, n_{\mathbf{f}}$. Para cada punto $\boldsymbol{p}_{0}$ que se quiera corregir se debe construir una de estas distribuciones. Debido al gran tiempo de cómputo que se requiere, hacer correcciones en toda la malla de $190 \times 190=36100$ es impracticable. Por eso se hacen solamente en una "malla reducida" de $63 \times 63$ (cada tercer punto) y solamente en los puntos "cercanos" al mejor ajuste, en los distintos análisis la malla reducida tenía desde $\sim 2.2$ hasta $\sim 8.5$ miles de puntos. En la figura 3.12a se muestra una de estas distribuciones, para presentarla se ha optado por juntar los datos en bins sin embargo el algoritmo no lo requiere y de hecho es mejor no hacerlo así.

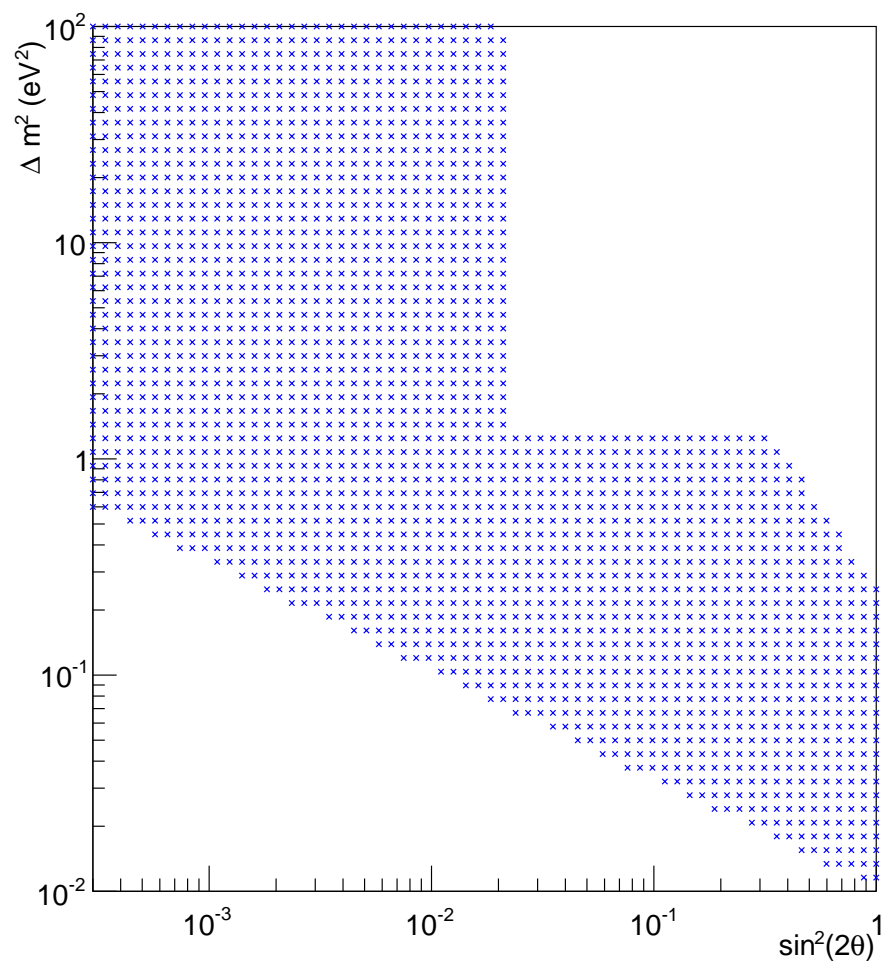

Figura 3.11: Malla reducida en donde se generan experimentos ficticios. Dependiendo de las circunstancias de cada análisis la malla aumenta hacia abajo o se triplica. La malla completa cubre prácticamente todo el espacio. 


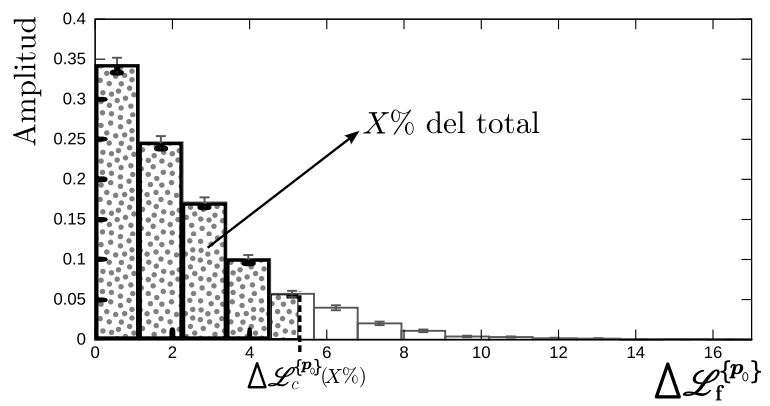

(a) Distribución de diferencias $\Delta \mathscr{L}_{\mathbf{f}}^{\left\{\boldsymbol{p}_{0}\right\}}$.
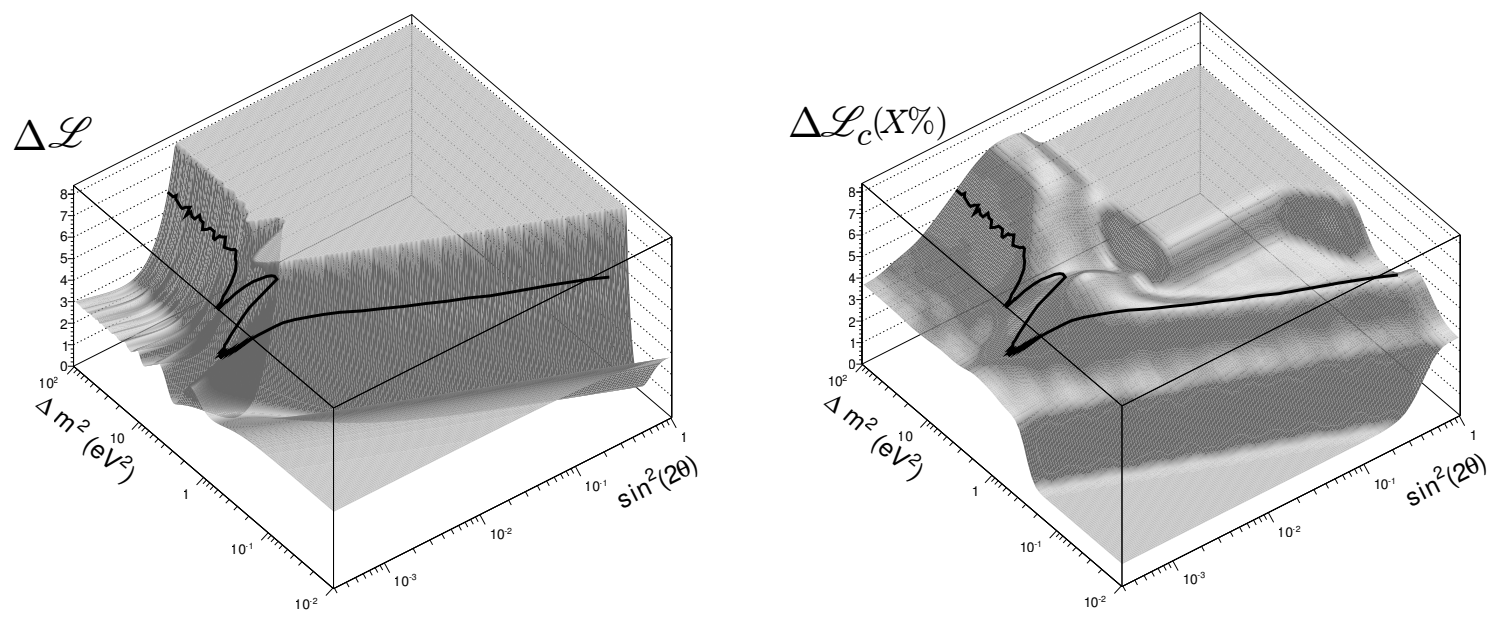

(b) Superficie $\Delta \mathscr{L}$.

(c) Superficie $\Delta \mathscr{L}_{c}(X \%)$.

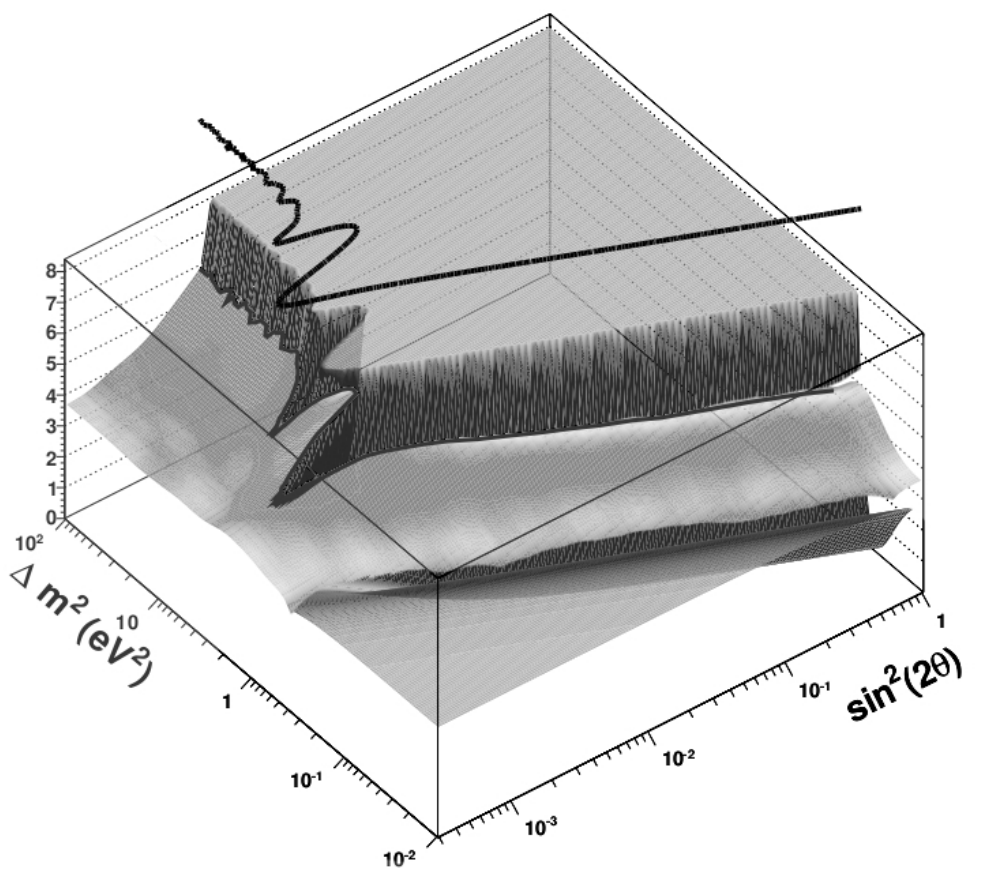

(d) Intersección de las superficies $\Delta \mathscr{L}$ y $\Delta \mathscr{L}_{c}(X \%)$.

Figura 3.12: Construcción de los intervalos con el método F-C. (a) Para cada punto $\boldsymbol{p}_{0} \in \sin ^{2} 2 \theta-\Delta m^{2}$ se construye una distribución $\Delta \mathscr{L}_{\mathbf{f}}^{\left\{\boldsymbol{p}_{0}\right\}}$ y se encuentra el valor $\Delta \mathscr{L}_{c}^{\left\{\boldsymbol{p}_{0}\right\}}(X \%)$, revisar el texto. (b) Se construye la superficie $\Delta \mathscr{L}$ en cada punto del plano $\sin ^{2} 2 \theta-\Delta m^{2}$. (c) Se construye la superficie $\Delta \mathscr{L}_{c}(X \%)$, explicación en el texto. (d) La proyección en el plano $\sin ^{2} 2 \theta-\Delta m^{2}$ de la intersección de las dos superficies, curva continua negra superior, forma el intervalo de $X \%$ N.C. Se muestra la intersección en las tres gráficas como una curva continua negra. 
Posteriormente, para generar las correcciones a cierto nivel de confianza $X \%$, se busca el valor crítico $\Delta \mathscr{L}_{c}^{\left\{\boldsymbol{p}_{0}\right\}}(X \%)$ en la distribución de diferencias para el cual $X \%$ de la distribución tenga un valor menor o igual. Se debe buscar el valor critico para cada nivel de confianza distinto. En la figura 3.12 a se puede apreciar el área que contiene el $X \%$ de los valores de $\Delta \mathscr{L}_{\mathbf{f} i}^{\left\{\boldsymbol{p}_{0}\right\}}$ y el valor que adquiere $\Delta \mathscr{L}_{c}^{\left\{\boldsymbol{p}_{0}\right\}}(X \%)$. Al observar la gráfica podría parecer que se esta integrando, por lo cual se reitera que $\Delta \mathscr{L}_{c}^{\left\{\boldsymbol{p}_{0}\right\}}(X \%)$ es el $\left(n_{\mathbf{f}} \times \frac{X}{100}\right)$-ésimo valor de la distribución de diferencias. Por ejemplo, si se hicieron 2000 experimentos ficticios, el valor crítico para la corrección de $90 \%$ N.C. sería el valor $2000 \times \frac{90}{100}=1800$-ésimo, de menor a mayor. Para cada punto $\boldsymbol{p}_{0}$ que se quiera corregir se debe encontrar $\Delta \mathscr{L}_{c}^{\left\{\boldsymbol{p}_{0}\right\}}$, obteniendo al final una superficie sobre el plano $\sin ^{2} 2 \theta-\Delta m^{2}$. En las regiones de baja y alta señal, es decir para la esquina inferior izquierda y superior derecha, se omite la generación de experimentos ficticios ya que son regiones muy lejanas del mejor ajuste o para las cuales el experimento no es muy sensible. En la figura 3.12c se muestra la superficie de valores críticos para el $X \%$ N.C., en la esquina inferior izquierda y en la superior derecha se han puesto valores arbitrarios para presentar las superficies de forma satisfactoria.

La proyección sobre el plano $\sin ^{2} 2 \theta-\Delta m^{2}$ de la intersección de las superficies $\Delta \mathscr{L}$ y $\Delta \mathscr{L}_{c}^{\left\{\boldsymbol{p}_{0}\right\}}(X \%)$ forma el intervalo de $X \%$ N.C. Con una curva negra se observa tal intersección en las figuras 3.12b y 3.12c. En la figura 3.12 d se observa la intersección de ambas superficies $\left(\Delta \mathscr{L}\right.$ en negro y $\Delta \mathscr{L}_{c}^{\left\{\boldsymbol{p}_{0}\right\}}(X \%)$ en gris) y hasta arriba la proyección de la intersección.

Existe un método alternativo teóricamente equivalente para generar los contornos a partir de las distribuciones de $\Delta \mathscr{L}_{\mathbf{f} i}^{\left\{\boldsymbol{p}_{0}\right\}}, i=1, \ldots, n_{\mathbf{f}}$; sin embargo, a pesar de que al principio se obtenían los contornos de esa forma, al final se descartó porque en la práctica presentaba varios problemas de interpolación que resultaban en contornos de peor calidad visual.

Observaciones: Es factible que el método de Feldman-Cousins sea la mejor receta para producir intervalos de confianza de Neyman. Lidia con fronteras físicas en los parámetros, nunca da intervalos de confianza vacíos y evita el problema de flip-flopping. Sin embargo hay ciertas dificultades evidentes:

1. Se quiere enfatizar que el método $\mathrm{F}-\mathrm{C}$ hace correcciones al método de máxima verosimilitud, el cual se ejecuta en aproximadamente un segundo, pero para construir los intervalos de confianza se requiere ejecutar el programa de la máxima verosimilitud $\sim 4 \times 10^{6}$ veces. ¡Aproximadamente 48 días de tiempo de cómputo! En vista de esto, hace falta recurrir a maquinaria especializada de cómputo que se encargué de hacer los cálculos lo más rápido posible. En el apéndice B se describe el sistema y uso del cluster empleado, por otra parte en el apéndice C se describe una forma novedosa de llevar a cabo los cálculos en un procesamiento paralelo con tarjetas de vídeo.

2. Los errores sistemáticos no se incorporan fácilmente en el procedimiento, básicamente se tienen que marginar con MC.

3. La "paradoja de los números pequeños". El comportamiento del método F-C para pocos eventos de señal y fondo pequeño es un poco inesperado. Para explicarlo pensemos en dos experimentos, el primero no espera eventos de fondo y el segundo espera 15 eventos; ninguno de los dos observa nada. A $90 \%$ N.C. el límite de señal es 2.44 y 0.92, respectivamente. Parecería que el segundo experimento, que anticipaba un gran fondo y no observó nada, da un mejor límite. Solo una gran fluctuación del fondo podría explicar tener 0 eventos cuando se esperaban 15, ¿cómo es que una gran fluctuación en el fondo resultó en un mejor límite a la señal? Para poder evaluar la relevancia de este resultado se recomienda acompañar los resultados con la sensibilidad ${ }^{24}$ del

\footnotetext{
${ }^{24}$ La sensibilidad de un experimento se define como el límite superior promedio que se obtendría de un ensamble de
} 
Entrada: Datos y fondo de $\nu_{e}$ y $\nu_{\mu}\left(\bar{\nu}_{e}\right.$ y $\left.\bar{\nu}_{\mu}\right)$, matriz de covarianza fraccionaria y tupla de simulación MC para la señal.

Salida : Tupla con contornos de confianza.

Declaración e inicialización de variables;

Creación de la superficie $\Delta \mathscr{L}\left(\sin ^{2} 2 \theta, \Delta m^{2}\right)$ con el método de la máxima verosimilitud;

likelihoodMethod $(\boldsymbol{D}) \Rightarrow \Delta \mathscr{L}\left(\sin ^{2} 2 \theta, \Delta m^{2}\right)$

Generación de datos ficticios y distribuciones de diferencia, en la malla reducida.;

for $x_{1}=3 \times 10^{-4}$ to 1 . do

for $x_{2}=1 \times 10^{-2} e V^{2}$ to $1 \times 10^{2} e V^{2}$ do

for $i=1$ to $n_{\mathbf{f}}$ do

$$
\mid \begin{aligned}
& \boldsymbol{D}_{\mathbf{f} i}^{\{\boldsymbol{x}\}} \leftarrow \text { fakeDataGenerator }(\boldsymbol{x}) ; \\
& \text { likelihoodMethod }\left(\boldsymbol{D}_{\mathbf{f} i}^{\{\boldsymbol{x}\}}\right) \rightarrow \Delta \mathscr{L}_{\mathbf{f} i}^{\{\boldsymbol{x}\}} ;
\end{aligned}
$$

end

Construcción de las superficies de valores críticos.;

$\operatorname{sortMinToMax}\left(\Delta \mathscr{L}_{\mathbf{f} i}^{\{\boldsymbol{x}\}}, i=1, \ldots, n_{\mathbf{f}}\right)$;

$i d 68 \leftarrow \operatorname{castInt}\left(0.68 \times n_{\mathbf{f}}\right) ; i d 90 \leftarrow \operatorname{castInt}\left(0.90 \times n_{\mathbf{f}}\right) ;$

$i d 95 \leftarrow$ castInt $\left(0.95 \times n_{\mathbf{f}}\right) ; i d 99 \leftarrow$ castInt $\left(0.99 \times n_{\mathbf{f}}\right) ;$

$\Delta \mathscr{L}_{c}^{\{\boldsymbol{x}\}}(68 \%) \leftarrow \Delta \mathscr{L}_{\mathbf{f}}^{\{\boldsymbol{x}\}} ; 0 ; \mathscr{L}_{c}^{\{\boldsymbol{x}\}}(90 \%) \leftarrow \Delta \mathscr{L}_{\mathbf{f}}^{\{\boldsymbol{x}\}} ; 90 ;$

$\left.\left.\Delta \mathscr{L}_{c}^{\{\boldsymbol{x}\}}(95 \%) \leftarrow \Delta \mathscr{L}_{\mathbf{f}}^{\{\boldsymbol{x}\}}\right\} ;, \Delta \mathscr{L}_{c}^{\{\boldsymbol{x}\}}(99 \%) \leftarrow \Delta \mathscr{L}_{\mathbf{f}}^{\{\boldsymbol{x}\}}\right\}$

end

end

Intersección de las superficies para generar los contornos.;

$\left(\sin ^{2} 2 \theta, \Delta m^{2}\right)_{68 \%} \leftarrow \operatorname{Intersect}\left(\Delta \mathscr{L}, \Delta \mathscr{L}_{c}(68 \%)\right) ;\left(\sin ^{2} 2 \theta, \Delta m^{2}\right)_{90 \%} \leftarrow \operatorname{Intersect}\left(\Delta \mathscr{L}, \Delta \mathscr{L}_{c}(90 \%)\right) ;$

$\left(\sin ^{2} 2 \theta, \Delta m^{2}\right)_{95 \%} \leftarrow \operatorname{Intersect}\left(\Delta \mathscr{L}, \Delta \mathscr{L}_{c}(95 \%)\right) ;\left(\sin ^{2} 2 \theta, \Delta m^{2}\right)_{99 \%} \leftarrow \operatorname{Intersect}\left(\Delta \mathscr{L}, \Delta \mathscr{L}_{c}(99 \%)\right) ;$

$\Rightarrow$ Tupla con contornos de confianza de $68 \%, 90 \%, 95 \%, 99 \%$ N.C.;

Algoritmo 3.5: Algoritmo del método Feldman-Cousins.

experimento, para los experimentos en cuestión es 2.44 y 4.83. En el segundo experimento el límite es mucho mejor que la sensibilidad, lo que significa que simplemente fue un resultado "afortunado" y que el límite es mucho mejor que lo que merecía ser.

\subsection{Análisis combinado de $\nu$ y $\bar{\nu}$}

Para este análisis se usan los mejores métodos, máxima verosimilitud $\S 3.5$ y las correcciones frecuentistas $\mathrm{F}-\mathrm{C} \S 3.6$, para llevar a cabo un análisis que usa todos los últimos datos disponibles, es decir combina la última publicación de datos del modo de neutrinos con la última publicación de los datos del modo de antineutrinos, a saber los datos publicados en 2012. Los contornos de confianza resultantes se pueden ver en $\S 4.6$.

Una vez más el estadístico usado es la máxima verosimilitud, ecuación (3.33), solo que ahora la suma se extiende sobre los bins de neutrinos y antineutrinos

$$
\mathscr{L}\left(\sin ^{2} 2 \theta, \Delta m^{2}\right)=\ln (|\mathbf{M}|)+\sum_{i, j}^{n_{e}+n_{\mu}+\bar{n}_{e}+\bar{n}_{\mu}}\left(D_{i}-Q_{i}\right) \mathrm{M}_{i j}^{-1}\left(D_{j}-Q_{j}\right)
$$

experimentos con el fondo esperado y sin señal verdadera, resulta útil para establecer la escala del experimento. 
por consiguiente los vectores de datos $\boldsymbol{D}$ y predicción de eventos $\boldsymbol{Q}$ tienen $19+19=38$ componentes; por su parte la matriz de covarianza $\mathbf{M}$ es de $38 \times 38$ y debe ser construida, evaluada e invertida para cada punto del espacio $\left(\sin ^{2} 2 \theta, \Delta m^{2}\right)$. En este caso el número de grados de libertad es $38-2-2=34$, ya que se le resta el par de normalizaciones que se hacen a cada muestra de datos y otro par de grados de libertad porque el ajuste, como antes, involucra dos parámetros. El aumento en el tamaño de la matriz de covarianza significa un incremento considerable en el tiempo de procesamiento y de la cantidad de operaciones necesarias para realizar la inversión, que aumenta con el cubo del número columnas. Se estima que este aumento oscila entre $4 / 6 n^{3} \sim 5 / 6 n^{3}$ [198].

Con los datos combinados se hicieron correcciones frecuentistas de Feldman-Cousins, §3.6, una vez más la diferencia es que el estadístico usado es una suma de $n_{e}+n_{\mu}+\bar{n}_{e}+\bar{n}_{\mu}=38$, de igual forma las matrices involucradas son más grandes. Pero lo demás se hace de forma análoga: la generación de datos ficticios, la búsqueda del mínimo, la construcción de las distribuciones de diferencias $\Delta \mathscr{L}_{\mathbf{f}}^{\left\{\boldsymbol{p}_{0}\right\}}, i=$ $1, \ldots, n_{\mathbf{f}}$; etcétera. Para cada experimento ficticio se busca el mínimo en la malla de $190 \times 190 \mathrm{y}$, al igual que en los otros casos, las correcciones frecuentistas solo se hacen sobre los puntos de la malla reducida de $63 \times 63$ que se encuentran en la región de interés. 


\section{Capítulo 4}

\section{Resultados}

A continuación se presentan las gráficas con los resultados obtenidos: histogramas de los eventos observados y gráficas bidimensionales de los límites impuestos o bien las regiones permitidas para los parámetros de oscilaciones de neutrinos $\left(\sin ^{2} 2 \theta, \Delta m^{2}\right)$ por MiniBooNE. Una clara señal de oscilación de neutrinos es un exceso consistente de eventos $\nu_{e}\left(\bar{\nu}_{e}\right)$, es decir que se vean más de los esperados si no hubiera oscilaciones pero que el excedente no sea tanto que no pueda ser atribuido a oscilaciones.

Uno de los objetivos de esta tesis es reproducir los resultados obtenidos por el experimento MiniBooNE empleando los datos liberados al público, es por eso que, a pesar de contar con más estadística y mejor constricción de errores, se incluyen los resultados de las primeras publicaciones. La metodología para llevar a cabo los análisis por parte de la colaboración también fue mejorando, algunos cambios se pueden percibir para aquellos datos que se analizaron con distintos métodos.

Sin embargo, estos cambios no se deben considerar mejorías por sí solos, sin tener en cuenta los problemas que solucionan. En particular, las correcciones frecuentistas de Feldman-Cousins §3.6, al método de máxima verosimilitud $\S 3.5$, otorgan regiones más estrechas. Este estrechamiento no debe verse como una mejoría por el simple hecho de que delimita una región más pequeña y, como consecuencia, se obtiene mayor certeza sobre cual es la pareja de parámetros que mejor ajusta el comportamiento observado. La función de las correcciones frecuentistas es la de otorgar cobertura adecuada, muestra la región que puede contener la pareja de parámetros al nivel de confianza estipulado. Es decir, que al repetir el experimento, el resultado obtenido caerá dentro de la región delimitada por las correcciones frecuentistas la fracción de veces correspondiente.

Con el propósito de mostrar el efecto que tiene la alta estadística de $\nu_{\mu}\left(\bar{\nu}_{\mu}\right)$ en la maquinaria del análisis, se presentan histogramas con errores constreñidos. La justificación de la constricción de los errores así como una explicación detallada del proceso se puede encontrar en el apéndice E. Para llevar acabo los ajustes y encontrar los contornos de confianza no se hizo uso de la constricción de errores, pues la constricción se aplica de manera automática a través del uso combinado de los datos de neutrinos del electrón y muón.

El formato de los datos usados se explica en $\S 3.2$. Excepto para la publicación de neutrinos de 2007 $(\S 4.1)$, en la cual se omite un histograma, los resultados presentados son:

- Histograma de eventos $\nu_{\mu}\left(\bar{\nu}_{\mu}\right)$ como función de la energía reconstruida $E_{\nu}^{Q E}$, escalados por la energía en $\mathrm{MeV}$. Los puntos con barras representan los datos observados con el correspondiente 
error estadístico, imperceptible a nivel práctico. El histograma azul (entramado cuadriculado) muestra los eventos esperados por la simulación con el correspondiente error constreñido, de igual forma que con los datos, el error es más pequeño que la escala mínima de la gráfica.

- Histograma de eventos $\nu_{e}\left(\bar{\nu}_{e}\right)$ como función de la energía reconstruida $E_{\nu}^{Q E}$, escalados por la energía en $\mathrm{MeV}$. Los puntos con barras representan los datos observados con el correspondiente error estadístico. El histograma verde (patrón punteado) muestra el fondo esperado de eventos por la simulación, con el correspondiente error constreñido. Los guiones rosa muestran la señal esperada para el punto de mejor ajuste obtenido.

- Histograma de exceso de eventos $\nu_{e}\left(\bar{\nu}_{e}\right)$ como función de la energía reconstruida $E_{\nu}^{Q E}$, escalados por la energía en $\mathrm{MeV}$. Es decir, la diferencia entre los datos (puntos con barras del histograma anterior) y el fondo esperado (histograma de patrón punteado). Los puntos con barras representan el exceso de eventos con el error total. Los guiones rosa muestran la expectativa para el punto de mejor ajuste. En la línea punteada azul se muestra la expectativa de un punto de mejor ajuste de LSND.

- Gráfica bidimensional sobre el espacio de parámetros $\left(\sin ^{2} 2 \theta, \Delta m^{2}\right)$ que delimita los límites de exclusión o los contornos de confianza. En cada gráfica se muestran tres o cuatro curvas obtenidas en este trabajo, cada uno especificando el nivel de confianza (N.C.) que denotan. Con un punto negro se muestra el mejor ajuste obtenido en cada caso, también en la gráfica se muestran las coordenadas de este. En regiones sólidas azules se muestran las regiones obtenidas por LSND que permiten la oscilación de neutrinos, la región oscura a $90 \%$ N.C. y la clara a $99 \%$ N.C. En las gráficas se transcribe la $\chi_{\min }^{2}$, obtenida para el punto de mejor ajuste, y $\chi_{\text {Nulo }}^{2}$, obtenida para el punto nulo, es decir, en ausencia de oscilación de neutrinos; así como el número de grados de libertad (g.l.) y la probabilidad para cada caso.

Todos los histogramas que se presentan a continuación tienen el último bin, correspondiente al rango de energía 1500-3000 MeV, comprimido. Esto se hace con la intención de presentar los datos de forma más estética y no tiene relevancia en la maquinaria del análisis. El resto de los bins tienen el ancho que les corresponde en la escala de energía que representan.

En todos los casos, el error estadístico mostrado en los datos se define como:

$$
\sigma_{i \text { stat }}=\sqrt{D_{i}} / \Delta E_{\nu}^{Q E}{ }_{i},
$$

donde se dividió entre $\Delta E_{\nu}^{Q E}{ }_{i} \equiv L_{i+1}^{\nu_{e}\left(\nu_{\mu}\right)}-L_{i}^{\nu_{e}\left(\nu_{\mu}\right)}$, el ancho de cada bin, para tener en cuenta el escalamiento que se hizo a los datos. Por su parte el error total se define como la suma en cuadraturas del error estadístico y el error constreñido:

$$
\sigma_{i \text { total }}=\sqrt{\sigma_{i \text { stat }}^{2}+\sigma_{i \text { const }}^{2}} \text {. }
$$

Al examinar los resultados, presentados al final de este capítulo, se debe notar: el modo que representan (neutrinos/antineutrinos), el año en que se publicaron los datos, el rango de energía que se usó, el método utilizado para obtener las regiones de confianza, la probabilidad del mejor ajuste y del punto nulo.

Los resultados obtenidos en esta tesis logran reproducir fielmente a los publicados oficialmente por la colaboración MiniBooNE, a excepción de los contornos con correcciones frecuentistas, donde se pueden observar ligeras desviaciones en algunas de las curvas, además los valores de $\chi^{2} / g . l$. y probabilidades 
de $\chi^{2}$ en la "región de señal" (200-1250 MeV) difieren de los reportados en las publicaciones puesto que en los datos liberados se usan intervalos ligeramente distintos para definir la región de señal (200$1300 \mathrm{MeV}$ ), no obstante son consistentes con las diferencias. En el caso de los contornos frecuentistas, ninguna desviación es tan dramática como para considerar que uno u otro resultado están mal, o que los resultados son inconsistentes entre sí. Existen varías sospechas sobre la causa de estas desviaciones, las cuales fueron investigadas a profundidad pero sin éxito. Las principales causas que se consideraron son: diferencias en los algoritmos para la generación de números aleatorios, diferencias en el algoritmo para crear experimentos ficticios, las diferentes resoluciones de las correcciones frecuentistas y la interpolación usada para graficar datos. En $\S$ D.4 se comparan los distintos contornos y se discute más al respecto.

La transcripción y descripción del programa que lleva a cabo las correcciones frecuentistas de FeldmanCousins al método de máxima verosimilitud se encuentra en el apéndice A. Las distintas formas en que se ejecutó, usando un cluster administrado por Condor para correr programas en $\mathrm{C}++\mathrm{y}$ con tarjetas de vídeo Nvidia para correr programas en CUDA, se encuentran en los apéndices B y C, respectivamente. Aquí se presentan únicamente los resultados obtenidos con CUDA, los resultados obtenidos con Condor son consistentes (estadísticamente equivalentes) pero no se presentan por constricciones de espacio. Todos las gráficas, histogramas e intervalos de confianza, que se presentan a continuación (además de algunas gráficas que se presentaron antes) se hicieron con el programa ROOT [199], un entorno orientado a objetos para el análisis de grandes cantidades de datos. Fue desarrollado en CERN y es el remplazo en $\mathrm{C}++$ de PAW. ROOT es muy popular en la comunidad de física de altas energías, es el caballo de batalla ${ }^{1}$ de todo físico experimental de altas energías. Sin embargo, se elaboraron programas ex profeso para el análisis de datos que establecen los límites de confianza sobre el espacio de parámetros $\left(\sin ^{2} 2 \theta, \Delta m^{2}\right)$.

\subsection{Búsqueda de aparición de $\nu_{e}, 2007$}

El primer artículo que publicó MiniBooNE corresponde al de esta búsqueda de oscilaciones [177], es decir la búsqueda de oscilaciones $\nu_{\mu} \rightarrow \nu_{e}$. Los datos así como instrucciones sobre como analizarlos se pueden obtener en línea [190] y corresponden a $(5.58 \pm 0.12) \times 10^{20}$ protones en el blanco (POT, protons on target) obtenidos de 2002 a 2004. En esta publicación, el portal no incluye explícitamente los datos de candidatos a eventos $\nu_{\mu}$, sin embargo estos han sido usados por la colaboración para constreñir el histograma de eventos $\nu_{e}$. Por tal razón no se presenta el correspondiente histograma de eventos observados y esperados de $\nu_{\mu}$. Es importante resaltar que la expectación publicada ha sido normalizada para adecuarse a los datos observados: se observan cerca de $20 \%$ más eventos que los esperados. Hay dos principales fuentes que pueden lograr ese efecto, un error en la normalización del flujo o en la normalización de la sección eficaz, principalmente de la reacción CCQE, sin embargo en este trabajo no se lidia con eso. Baste con saber que la correspondiente componente de $\nu_{e}$ es consecuentemente normalizada en el proceso de constricción de las incertidumbres.

En la figura 4.1 se muestran los resultados obtenidos para este análisis. Del lado izquierdo se muestran los histogramas de eventos candidatos a $\nu_{e} \mathrm{y}$ de exceso de eventos $\nu_{e}$, los dos normalizados por la energía. Nótese que no hay un exceso considerable de eventos: para $E_{\nu}^{Q E}>675 \mathrm{MeV}$, los puntos de los datos están esencialmente encima del fondo esperado; a pesar de que para $475<E_{\nu}^{Q E}<675 \mathrm{MeV}$ se observaron más datos que los esperados, sin embargo el error total (estadístico más sistemático) es

\footnotetext{
${ }^{1}$ En la opinión del autor, un caballo modoso, difícil de domar, terco y poco intuitivo. Pero robusto como un elefante.
} 
consistente con tal desviación. Es decir, no hay evidencia de oscilaciones de neutrinos. Del lado derecho de la figura las curvas de colores determinan los límites obtenidos con estos datos, los cuales reiteran lo antes dicho: al nivel de confianza correspondiente, se descarta la posibilidad de oscilaciones en la región superior derecha del espacio $\left(\sin ^{2} 2 \theta, \Delta m^{2}\right)$. Una interpretación alternativa sería que se permiten las oscilaciones al nivel de confianza citado para cualquier punto en la región inferior izquierda con la misma significación, así pues, el punto nulo es estadísticamente equivalente a cualquier otro punto en esa región. Se observaron un total de 388 candidatos a eventos $\nu_{e}$ y un exceso de $20.19 \pm 19.18 \pm 36.58$ eventos (error estadístico y sistemático respectivamente) en la región de $475<E_{\nu}^{Q E}<3000 \mathrm{MeV}$, es decir: no se observa un exceso significativo. El mejor ajuste se encuentra en $\left(0.0011,4.23 \mathrm{eV}^{2}\right)$, con un valor de $\chi^{2} /$ g.l. $=0.90 / 6$ lo cual da una probabilidad de $98.9 \%$ para $475<E_{\nu}^{Q E}<3000 \mathrm{MeV}$, por su parte el ajuste de oscilación para la hipótesis nula tiene un valor de $\chi^{2} / \mathrm{g}$.l. $=1.91 / 6$ con una probabilidad de $92.8 \%$ en la misma región de energía. El método usado para obtener estos límites es la búsqueda por barrido unidimensional (descrito en §3.3) estos límites no muestran ninguna diferencia perceptible a los publicados.

En dos tonos de azul se muestran las regiones que permiten oscilaciones por el experimento LSND. Los resultados obtenidos en la búsqueda de oscilaciones $\nu_{\mu} \rightarrow \nu_{e}$ por MiniBooNE son incompatibles con los de LSND. Al $90 \%$ N.C. MiniBooNE excluye la región permitida, al mismo nivel de confianza, por LSND. Concluyendo, cualitativamente, que los dos experimentos son incompatibles.

En la región de bajas energías, es decir $E_{\nu}^{Q E}<475 \mathrm{MeV}$, la colaboración observó un exceso de eventos candidatos a $\nu_{e}$, sin embargo vio que el ruido inducido por $\nu_{\mu}$, parte del cual es difícil de modelar, es grande. Había sospechas bien fundadas sobre la naturaleza de los eventos observados para el rango de bajas energías, a pesar de que no se hallaron problemas con la estimación del fondo para tal rango. Probaron que si el ajuste se hacía a partir de $475 \mathrm{MeV}$ se lograba reducir considerablemente el fondo, con poco impacto en la sensibilidad para el ajuste de oscilaciones. Así pues el ajuste se hizo en el rango $475<E_{\nu}^{Q E}<3000 \mathrm{MeV}$, sin embargo esta situación cambió con la mejora de la estimación del fondo. De lo cual se habla más en la siguiente sección.

\subsection{Búsqueda de aparición de $\nu_{e}, 2009$}

Después de haber hecho una investigación detallada sobre el exceso de eventos de electrón a bajas energías se presenta un nuevo artículo para hablar sobre un "exceso inexplicado de eventos de electrón de un haz de neutrinos de $1 \mathrm{GeV}$ " [178]. Después de varias mejoras a la forma en que se analizaban los datos, se simulaban los fondos y a la reconstrucción de los eventos; así como un incremento de la muestra de datos de $5.58 \times 10^{20}$ POT a $6.46 \times 10^{20}$ POT, el exceso de eventos de electrón perduró. En esta ocasión se incluyen los datos a eventos $\nu_{\mu}$, los cuales son usados para la maquinaria del ajuste que otorga los límites de exclusión y para constreñir los errores de los histogramas. Los datos pueden ser adquiridos por quien lo desea en la página del experimento [191].

La figura 4.2 muestra del lado izquierdo y arriba el histograma de eventos $\nu_{\mu}$, izquierda y al centro el histograma de eventos $\nu_{e}$, izquierda abajo el exceso de eventos $\nu_{e}$. Obsérvese que los eventos $\nu_{\mu}$ y la expectación son notablemente similares, en este caso la diferencia de normalización entre la predicción de $\nu_{\mu}$ y los datos corrige automáticamente la predicción de eventos de $\nu_{e}$ a través de los elementos fuera de la diagonal de la matriz de covarianza durante el ajuste.

Se muestra el histograma de eventos $\nu_{e}$ para el rango de energía $475<E_{\nu}^{Q E}<3000 \mathrm{MeV}$, más adelante se mostrará desde el mínimo de energía. Para estos datos se puede notar, a pesar de su error 
asociado, un exceso en el primer bin. El resto se encuentran por encima o debajo del fondo esperado, todos consistentes como fluctuaciones. Como antes, los resultados obtenidos son inconsistentes con la oscilación de dos neutrinos en el vacío. En total se observaron 115472 eventos $\nu_{\mu}$, en la región de señal de $475<E_{\nu}^{Q E}<1300 \mathrm{MeV}$ se observaron 420 eventos $\nu_{e}$, esperando $397.00 \pm 19.92 \pm 30.87$ lo que corresponde a un exceso de $19.92 \pm 36.74$ eventos, es decir no se observó un exceso apreciable. El mejor ajuste se encuentra en $\left(0.0009,3.46 \mathrm{eV}^{2}\right)$, con un valor de $\chi^{2} / \mathrm{g} .1 .=6.66 / 14$ lo cual da una probabilidad de $94.70 \%$ para $475<E_{\nu}^{Q E}<3000 \mathrm{MeV}$, por su parte la hipótesis nula tiene un valor de $\chi^{2}$ /g.l. $=8.80 / 16$ con una probabilidad de $92.16 \%$ en la misma región de energía. El método usado para obtener estos límites es la búsqueda por barrido unidimensional (descrito en § 3.3) para este artículo no se presentaron contornos de confianza, sin embargo al comparar los que se obtuvieron aquí con un trabajo interno [200] no se observan diferencias.

Por completez y con la intención de compararlos con los que se muestran posteriormente se agregan los contornos de confianza obtenidos usando el rango completo de energía $200<E_{\nu}^{Q E}<3000 \mathrm{MeV}$, figura 4.3. Del lado izquierdo se tienen los contornos obtenidos usando el método de búsqueda por barrido bidimensional (descrito en $\S 3.4$ ), con el cual el mejor ajuste se encuentra en $\left(0.0017,3.15 \mathrm{eV}^{2}\right)$. Por otra parte, del lado derecho se tienen los contornos obtenido con el método de máxima verosimilitud (descrita en $\S 3.5$ ), el mejor ajuste se encuentra en $\left(0.0015,3.14 \mathrm{eV}^{2}\right)$. Nótese que para este caso se tiene un contorno cerrado a $68 \%$ N.C. Adicionalmente en la parte izquierda de la figura 4.5 se muestran las correcciones frecuentistas F-C para el método de la máxima verosimilitud (§ 3.6). Por definición, el mejor ajuste y las probabilidades del mejor ajuste y el punto nulo son las mismas que para el método de máxima verosimilitud. Nótese que los límites no excluyen una región tan grande, por ejemplo el contorno de $90 \%$ N.C. excluye, aproximadamente, la región que antes excluía el contorno de $95 \%$ N.C.; más aún la región permitida de $60 \%$ N.C. incluye varias porciones de alta $\Delta m^{2}$. Se hicieron correcciones en 2285 puntos distintos, cada uno con $n_{\mathbf{f}}=2048$; en total se corrieron $2048 \times 2285=4679680$ experimentos ficticios.

El resultado obtenido en el modo de neutrinos, a pesar de las mejoras que se hicieron y del incremento de estadística, sigue siendo inconsistente con lo obtenido por el experimento LSND, las regiones que permiten oscilación por los dos experimentos son, en gran medida, disconexas y a $90 \%$ N.C. MiniBooNE sigue excluyendo la región permitida por LSND. Como se reafirmará con la última publicación de datos, el exceso observado a bajas energías no es del todo consistente con oscilaciones de neutrinos como las de LSND.

\subsection{Búsqueda de aparición de $\bar{\nu}_{e}, 2010$}

En 2009 se publica el primer artículo en que la colaboración reporta el estado de la búsqueda de oscilaciones $\bar{\nu}_{\mu} \rightarrow \bar{\nu}_{e}$ en un haz de antineutrinos [179], tal reporte no observa ningún exceso significativo de eventos $\bar{\nu}_{e}$. En esta tesis no se hizo ningún esfuerzo por reproducir los resultados de antineutrinos de este análisis, ya que no son concluyentes dada la baja estadística. Para finales de 2010, después de un incremento de $3.39 \times 10^{20}$ POT a $5.66 \times 10^{20}$ POT, MiniBooNE publicó un artículo en el que reporta un exceso de eventos $\bar{\nu}_{e}$ en la búsqueda de oscilaciones en modo de antineutrinos [180], estos datos provienen de una corrida de 2006 a 2009 y están públicamente disponibles en la página del experimento [192].

Este nuevo resultado, al igual que la versión de 2009, supone que solo hay oscilaciones $\bar{\nu}_{\mu} \rightarrow \bar{\nu}_{e}$, asume que la desaparición de $\bar{\nu}_{\mu}$ es despreciable y que los $\nu_{\mu}$ no oscilan (como lo observó el experimento en el 
modo de neutrinos). Sin embargo, en la versión estándar del análisis no se considera el exceso a bajas energías observado en el modo de neutrinos para la predicción de antineutrinos ${ }^{2}$. La colaboración determinó que estas simplificaciones podrían cambiar la probabilidad de oscilación $\bar{\nu}_{\mu} \rightarrow \bar{\nu}_{e}$ ajustada en un $10 \%$. Los resultados oficiales analizan oscilaciones en el rango completo de energía $200<$ $E_{\nu}^{Q E}<3000 \mathrm{MeV}$, oscilaciones cuando $E_{\nu}^{Q E}>475 \mathrm{MeV}$ y el caso especial de $E_{\nu}^{Q E}>200 \mathrm{MeV}$ en el que se substraen los eventos que podrían provenir del exceso a bajas energías observado en el modo de neutrinos. A pesar de que para los otros casos, $E_{\nu}^{Q E}>475 \mathrm{MeV}$ y rango total de energía con eventos substraídos, los resultados no son tan estadísticamente significativos; el exceso de eventos permanece. Para este trabajo solo se presentan los resultados para $E_{\nu}^{Q E}>200 \mathrm{MeV}$, en retrospectiva, no tiene mucho sentido desechar la región de bajas energías.

Del lado izquierdo de la figura 4.4 se aprecian los histogramas de datos. En la parte superior se observan los datos de eventos $\bar{\nu}_{\mu}$ y la expectación de eventos $\bar{\nu}_{\mu}$, en general la normalización de la estimación requirió un incremento de $13 \%$, lo cual es compatible con incertidumbre combinada del flujo de neutrinos y secciones eficaces. Los datos son una muestra de 24771 eventos que pasan los requisitos de selección CCQE de $\bar{\nu}_{\mu}$. En la parte de abajo se tiene el histograma de eventos $\bar{\nu}_{e} \mathrm{y}$ hasta abajo el exceso de eventos $\bar{\nu}_{e}$. Ahora se observa un exceso en varios bins, en tres de los cuales, es claramente más grande que lo que una fluctuación permitiría. En el rango de $200<E_{\nu}^{Q E}<3000 \mathrm{MeV}$ se observaron un total de 277 eventos $\bar{\nu}_{e}$, la expectación era de $233.74 \pm 15.29 \pm 16.51$ (error estadístico y sistemático), lo que corresponde a un exceso de $43.26 \pm 22.50$ a $1.9 \sigma$. En esta región de energía, estos números coinciden con los publicados [180] que usan la misma región de energía. Se tienen un total de 24568 eventos $\bar{\nu}_{\mu}$. Cabe notar que el exceso en $300<E_{\nu}^{Q E}<375 \mathrm{MeV}$ es mayor de lo que se esperaría para la oscilación de neutrinos, sin embargo el resto de los puntos concuerdan mejor: lo que se puede notar al compararlos con el histograma rosa de mejor ajuste. Como es de esperarse, incrementar la estadística ayuda a disminuir las fluctuaciones, lo cual se mostrará en las publicaciones más recientes. A la derecha de la figura 4.4 se encuentran las regiones que permiten oscilaciones $\bar{\nu}_{\mu} \rightarrow \bar{\nu}_{e}$ en el experimento MiniBooNE. A diferencia de los análisis pasados, ahora se tiene una mejor concordancia entre MiniBooNE y LSND, a $90 \%$ N.C. MiniBooNE y LSND tienen una amplia región de afinidad y a $99 \%$ N.C. MiniBooNE cubre casi en su totalidad a LSND. Recordemos que LSND era precisamente un experimento de oscilación de neutrinos $\bar{\nu}_{\mu} \rightarrow \bar{\nu}_{e}$. El mejor ajuste se encuentra en $\left(0.0066,4.42 \mathrm{eV}^{2}\right)$, con un valor de $\chi^{2} / \mathrm{g} .1 .=12.77 / 7$ lo cual da una probabilidad de $7.80 \%$ para $200<E_{\nu}^{Q E}<1300 \mathrm{MeV}$, por su parte la hipótesis nula tiene un valor de $\chi^{2} /$ g.l. $=25.40 / 9$ con una probabilidad de $0.26 \%$ en la misma región de energía. Estos valores son ligeramente distintos a los oficiales, en los cuales se usa la región de señal de 200-1250 MeV cuyos datos no son públicos. Estas curvas se obtienen con el método de máxima verosimilitud (descrito en § 3.5), los resultados obtenidos en esta tesis y los oficiales son idénticos, dentro del límite de precisión de cálculos de punto flotante. En la parte derecha de la figura 4.5 se encuentran los contornos obtenidos después de las correcciones frecuentistas $\mathrm{F}-\mathrm{C}$ (descrito en §3.6). Nótese que a pesar de la reducción del tamaño de los contornos, la concordancia entre MiniBooNE y LSND sigue siendo bastante buena. Estos contornos no son idénticos a los oficiales, a saber: la pequeña región de $95 \%$ N.C. alrededor de $\sim 10 \mathrm{eV}^{2}$ no aparece en los contornos oficiales y la parte izquierda del contorno de $99 \%$ N.C. es un poco más estrecha. Para cada uno de los 2199 puntos que se probaron se hicieron $n_{\mathbf{f}}=2048$ experimentos ficticios; en total se corrieron $2048 \times 2199=4503552$ experimentos ficticios.

\footnotetext{
${ }^{2}$ La colaboración también investiga el efecto que tendría el exceso a bajas energías.
} 


\subsection{Búsqueda de aparición de $\nu_{e}, 2012-2013$}

Después de la última publicación de oscilaciones, MiniBooNE continuó tomando más datos en modo de antineutrinos solamente y no hubo más datos de modo de neutrinos, los cuales eran mucho más estadísticamente significativos. Se tomaron datos en modo de neutrinos en dos periodos: 2003-2006 y 2007-2008, los dos alternando con modo de antineutrinos. Se publicaron dos resultados de la búsqueda de aparición de neutrinos, uno en $2007 \S 4.1$ y el otro en 2009 §4.2. En 2012 la colaboración vuelve a analizar los datos correspondientes a $6.46 \times 10^{20}$ POT, pero con varias mejoras. Los principales cambios son:

- Se añadió a la matriz total una matriz correspondiente a la incertidumbre de la normalización y forma del espectro del momento transferido $\left(\mathrm{Q}^{2}\right)$ para eventos $\mathrm{CC} \pi$ (producción de piones por interacciones de corriente cargada) y una matriz correspondiente a la producción de $K^{-}$. Además se usa un nuevo algoritmo para generar la matriz de covarianza asociada a la producción de piones negativos y positivos en el haz y también se añadió el error estadístico debido a la señal.

- Suavizado de las distribuciones usadas para generar algunas de las matrices parciales de covarianza.

- Nuevas constricciones a la producción de mesones $K^{+}$, debidas al experimento SciBooNE [201].

- Ajuste usando el método de máxima verosimilitud, esta es la primera vez que la colaboración publica los resultados del modo de neutrinos usando este robusto método.

Los datos se pueden descargar libremente en la página del experimento [193]. A pesar de no haber sido aceptado para publicación, el artículo [202] contiene valiosa información sobre el ajuste al modo de neutrinos. En la versión aceptada por la revista [181], el análisis al modo de neutrinos pierde cierta relevancia ${ }^{3}$.

Estos datos solamente fueron analizados usando el método de máxima verosimilitud (§ 3.5) y las correcciones frecuentistas $\mathrm{F}-\mathrm{C}$ del mismo (§3.6. La figura 4.6 presenta ambos. Del lado izquierdo se presentan los contornos que permiten oscilaciones en modo de neutrinos por el experimento MiniBooNE, se debe comparar esta figura a la equivalente del modo de neutrinos pero de 2009, el lado derecho de la figura 4.3. Después de tal comparación queda claro la importancia de las mejorías hechas a las simulaciones y estimación de errores, se pasa de tener un contorno cerrado a $68 \%$ N.C. a tener tres contornos cerrados, permitiendo oscilaciones hasta $95 \%$ N.C. El mejor ajuste se encuentra en el punto $\left(0.0020,3.14 \mathrm{eV}^{2}\right)$, en la región de $200<E_{\nu}^{Q E}<1300 \mathrm{MeV}$ tiene un valor de $\chi^{2} / \mathrm{g} .1 .=16.54 / 7$ lo cual da una probabilidad de $2.06 \%$, por su parte la hipótesis nula tiene un valor de $\chi^{2} / \mathrm{g} .1 .=22.58 / 9$ con una probabilidad de $0.72 \%$ en la misma región de energía ${ }^{4}$. Con la intención de seguir el contorno de $95 \%$ N.C. se podría extender el análisis para que abarque la región con $\sin ^{2} 2 \theta<3 \times 10^{-4}$; se omite analizar tales parámetros porque se busca ser consistente con el resto de los resultados presentados. Más aún, como se puede comprobar con las correcciones frecuentistas $\mathrm{F}-\mathrm{C}$ que aparecen del lado derecho de la figura 4.6, esa región esta sobrecubierta y en realidad el contorno de $95 \%$ N.C. es más estrecho. El lado izquierdo de la figura 4.5 tiene los contornos equivalentes de la publicación pasada

\footnotetext{
${ }^{3}$ Los datos correspondientes a este análisis fueron publicados a finales de 2012, sin embargo el artículo salió hasta 2013.

${ }^{4}$ Los resultados oficiales son: $\chi^{2} /$ g.l. $=13.2 / 6.8 \rightarrow P=6.1 \%$ para el mejor ajuste y $\chi^{2} /$ g.l. $=22.8 / 8.8 \rightarrow P=0.5 \%$ para la hipótesis nula; en la región de señal 200-1250 MeV.
} 
del modo de neutrinos, es decir los que tienen correcciones frecuentistas. También en este caso se puede notar la mejora significativa que otorgan las nuevas estimaciones de los errores.

Dentro del margen de error esperado por la incertidumbre en las operaciones de punto flotante, los contornos obtenidos con el método de máxima verosimilitud, lado izquierdo de la figura 4.6, son idénticos a los oficiales. Las correcciones frecuentistas por su parte, lado derecho de la misma figura, presentan ligeras diferencias; en los análisis que se realizaron en este trabajo el lado izquierdo del contorno de $95 \%$ N.C. es ligeramente más estrecho y la diferencia más notable es que en la publicación oficial el mismo contorno no presenta dos regiones disconexas para $\Delta m^{2}>2 \mathrm{eV}^{2}$. Para las correcciones frecuentistas de estos resultados, en buena parte motivado por la diferencias ya mencionadas, se corrieron una gran cantidad de experimentos ficticios. Como se explica en $\S 3.6$, hacer correcciones frecuentistas es muy demandante, por lo mismo solo se hace para algunos puntos, con los cuales se interpola para el resto, o se omite por completo para las regiones que corresponden a la esquina inferior izquierda y superior derecha de la gráfica. Se probaron 7232 puntos distintos en el espacio de parámetros $\left(\sin ^{2} 2 \theta, \Delta m^{2}\right)$, con distintas cantidades de experimentos ficticios, la mayoría solamente con 2048 , pero aquellos puntos que se encontraban en la región dispareja a los resultados oficiales se probaron con $2048 \times 2=4096$ y hasta con $2048 \times 3=6144$ experimentos ficticios. En total se corrieron 18616320 experimentos ficticios ${ }^{5}$.

Del lado izquierdo de la figura 4.8 se presentan los histogramas del modo de neutrinos. En la parte superior se aprecia el histograma de candidatos a eventos $\nu_{\mu}$ normalizado a la energía de cada bin, el espectro de $\nu_{\mu}$ tiene un máximo alrededor de los $600 \mathrm{MeV}$ y concuerda formidablemente con la expectación (recuérdese que esta ha sido normalizada dentro del rango de incertidumbre aceptado para adaptarse a lo observado). Debajo se encuentran los histogramas de eventos $\nu_{e}$, a diferencia de los presentados en la figura 4.2, estos incluyen el intervalo de bajas energías. En este par de histogramas se debe apreciar el exceso de eventos, más allá de lo que se esperaría para oscilación de dos neutrinos, de los tres primeros bins: ni aún considerando la incertidumbre de cada uno se asemejan al mejor ajuste de oscilaciones (histograma con guiones rosas). Además, en general lo observado por MiniBooNE no guarda semejanza con lo que se hubiera observado si el mejor ajuste fuera aquel encontrado por LSND (histograma con lineas punteadas azules). En el rango de la señal $200<E_{\nu}^{Q E}<1300 \mathrm{MeV}$, se observaron un total de 964 eventos $\nu_{e}$, cuando la expectación era de $816.81 \pm 28.58 \pm 40.74$ (error estadístico y sistemático) lo que corresponde a un exceso de $147.19 \pm 49.77$ eventos $\nu_{e}$ a $3.0 \sigma^{6}$. En el rango completo de energía, se observan un total de 115472 eventos $\nu_{\mu}$ y 1068 eventos $\nu_{e}$, formando un total de 116540 candidatos a eventos que pasan los criterios de selección. Tomando en cuenta la cantidad de protones enviados al blanco en modo de neutrinos, en promedio se requieren $5.54 \times 10^{15}$ protones en el blanco para detectar un neutrino que pase los criterios de selección, es decir, se obtiene un evento que pase todos los criterios cada minuto o par de minutos de haz corriendo.

\subsection{Búsqueda de aparición de $\bar{\nu}_{e}, 2012-2013$}

En 2012 la colaboración publica ${ }^{3}$ un artículo sobre el estatus de la búsqueda de oscilaciones [181], en este muestra los resultados de la búsqueda de modo de neutrinos con la misma estadística que se

\footnotetext{
${ }^{5}$ De hecho se corrieron muchísimos más, pero debido a algunos errores o por ser consistente con los otros resultados presentados esos experimentos no se usan.

${ }^{6}$ Los resultados oficiales son: 952 eventos $\nu_{e}$, expectación de $790.1 \pm 28.1 \pm 38.7$ y un exceso de $162.0 \pm 47.8(3.4 \sigma)$; en la región de señal 200-1250 MeV.
} 
tenía en la publicación de 2009 pero varias mejorías, los resultados de la búsqueda de antineutrinos contando casi con el doble de estadística y mejorías en la constricción de errores, simulaciones y análisis. Los datos en modo de antineutrinos corresponden a $11.27 \times 10^{20}$ POT y corresponden al rango completo de energía de $200<E_{\nu}^{Q E}<3000 \mathrm{MeV}$. Los datos pueden ser descargados de la página del experimento [194]. Los resultados que se presentan aquí corresponden a los de la primera versión del artículo [202], siendo los resultados presentados en la publicación [181] ligeramente distintos, ya que la simulación de la señal usada para esa publicación permite que tanto los neutrinos como antineutrinos oscilen, a diferencia de la simulación usada en la primera versión (y en este trabajo) que no permite que los neutrinos oscilen (ver tabla 3.1). Esos análisis también fueron reproducidos, pero se omiten por constricciones de espacio y porque presentan problemas de interpolación que resultan en contornos de peor calidad visual. Baste saber que usar la otra simulación afecta ligeramente los resultados finales, pero siguen siendo consistentes con los presentados aquí.

Entre las mejoras que se cuentan, hay una mejor determinación del fondo intrínseco de $\nu_{e}$ proveniente del decaimiento de $K^{+}$gracias a las medidas de neutrinos de gran energía hechas por el experimento SciBooNE [201], una mejor determinación de los $\pi^{0}$ de corriente neutra y fondos de eventos externos en modo de antineutrinos debidos al incremento de la estadística en la muestra de datos y el uso de un ajuste de máxima verosimilitud con correcciones frecuentistas.

Para obtener las regiones de confianza que permiten oscilaciones por el experimento MiniBooNE en modo de antineutrinos, figura 4.7, se uso el método de máxima verosimilitud (§ 3.5), parte izquierda de la figura, del cual se hicieron correcciones frecuentistas (§3.6), parte derecha de la figura. Este par de gráficas deben ser comparadas con las correspondientes de la publicación de 2010, parte derecha de la figura 4.4 y parte derecha de la figura 4.5, respectivamente. Se debe notar que los contornos actuales no son tan estrechos, en particular el de $99 \%$ N.C., sin embargo el incremento de estadística ha ayudado a disminuir las fluctuaciones, lo que queda claro al observar los contornos de $60 \%$ N.C. del método de máxima verosimilitud. En este modo se puede observar una gran concordancia entre lo obtenido por LSND y MiniBooNE. El mejor ajuste de oscilación en el rango completo de energía se encuentra se encuentra en el punto $\left(0.8423,0.05 \mathrm{eV}^{2}\right)$, en la región de $200<E_{\nu}^{Q E}<1300 \mathrm{MeV}$ tiene un valor de $\chi^{2} /$ g.l. $=5.47 / 7$ lo cual da una probabilidad de $60.30 \%$, por su parte la hipótesis nula tiene un valor de $\chi^{2} /$ g.l. $=16.78 / 9$ con una probabilidad de $5.22 \%$ en la misma región de energía ${ }^{7}$. Los contornos frecuentistas muestran ligeras diferencias con los resultados oficiales: la parte izquierda del contorno de $99 \%$ N.C. es ligeramente más estrecho en este trabajo y existen variaciones en las regiones de 95 N.C. para $\Delta m^{2}>10 \mathrm{eV}^{2}$, lo cual seguramente es un artificio del método de interpolación usado para graficar. Este fue el primer resultado frecuentista que se obtuvo en este trabajo, por la misma razón una gran cantidad de puntos fueron investigados para estudiar las diferencias que se observaron con los resultados oficiales. Se hicieron correcciones frecuentistas en 8478 puntos distintos, en la mayoría se corrieron 2048 experimentos ficticios y en las regiones que mostraban discrepancias se corrieron 4096 o 6144 ; en total se corrieron 23375872 experimentos ficticios $^{5}$.

Del lado derecho de la figura 4.8 se presentan los histogramas de datos del modo de antineutrinos para la muestra de $11.27 \times 10^{20}$ POT. Como antes, en la parte superior se encuentra el histograma de eventos $\bar{\nu}_{\mu}$, de este es interesante ver que la forma del espectro es bastante similar a su contra parte del modo de neutrinos pero aproximadamente la mitad de altura. Debajo se encuentra las histogramas de los candidatos a eventos $\bar{\nu}_{e}$, intuitivamente queda claro el efecto que tiene el incremento de la estadística al ver el comportamiento exponencial que siguen los eventos $\bar{\nu}_{e}$ (en medio), a diferencia

\footnotetext{
${ }^{7}$ Los resultados oficiales son: $\chi^{2} /$ g.l. $=5.0 / 7 \rightarrow P=66 \%$ para el mejor ajuste y $\chi^{2} /$ g.l. $=16.6 / 8.9 \rightarrow P=5.4 \%$ para la hipótesis nula; en la región de señal 200-1250 MeV.
} 
de la publicación pasada, figura 4.4, en donde algunos puntos del espectro se desvían de la tendencia global. Hasta abajo se observa el exceso de eventos $\bar{\nu}_{e}$, salvo el bin de 200-275, que tiene un exceso ligeramente mayor a lo esperado, y el bin de 950-1 100, con una ligera carencia, el comportamiento de los datos es consistente con oscilaciones (guiones rosas). La concordancia con lo que hubiera observado MiniBooNE si el fenómeno estuviera descrito por el mejor ajuste de LSND (líneas punteadas azules) no es tan bueno pero sigue siendo bastante cercano. MiniBooNE observó un total de 482 eventos, con una expectación de $400.64 \pm 20.02 \pm 20.45$ (error estadístico y sistemático) que corresponden a un exceso de $81.36 \pm 28.62$ eventos $\bar{\nu}_{e}$ a $2.8 \sigma$ en la región de señal $200<E_{\nu}^{Q E}<1300 \mathrm{MeV}^{8}$. Para el rango completo de energía, en total se observaron un total de 50455 candidatos a eventos $\bar{\nu}_{\mu} \mathrm{y}$ 551 candidatos a eventos $\bar{\nu}_{e}$, para un total de 51006 candidatos a eventos que pasan los criterios de selección. En el modo de antineutrinos se enviaron $11.27 \times 10^{20}$ protones al blanco, en promedio hacen falta $2.19 \times 10^{20}$ protones en el blanco para detectar un antineutrino que pase los criterios de selección, cuatro veces más que para el modo de neutrinos.

A esta altura del experimento aumentar la cantidad de datos no otorgaría mucho información, ya que si bien con más datos se conseguiría disminuir el error estadístico no lograría disminuir el error sistemático.

\subsection{Búsqueda combinada de aparición de $\nu_{e}$ y $\bar{\nu}_{e}, 2012$}

En agosto de 2012 la colaboración MiniBooNE presenta un análisis combinado de los modos de neutrinos y antineutrinos [202], correspondiente a $6.46 \times 10^{20}$ POT y $11.27 \times 10^{20}$ POT respectivamente. Sin embargo, el artículo es rechazado por la futilidad que significa hacer un análisis combinado de dos comportamientos que claramente no son compatibles, injustificando su compatibilidad a pesar del exceso de eventos que ambos ven. En este trabajo se reproducen esos análisis, a pesar de que en la versión final de esa publicación [181], no se hayan presentado.

Las curvas obtenidas que permiten oscilación de neutrinos combinando los datos de neutrinos y antineutrinos se presentan en la figura 4.9. Del lado izquierdo las regiones usando el método de máxima verosimilitud (§3.5) y del derecho las obtenidas al hacer correcciones frecuentistas (§ 3.6). Para este resultado la diferencia entre los dos casos es marginal, apenas aumentando ligeramente los contornos. Los datos de neutrinos trasladan las regiones hacia valores más bajos de $\Delta m^{2}$. Para generar los contornos frecuentistas se probaron 2208 puntos, la mayoría con 2048 experimentos ficticios, pero los puntos en donde se observaron diferencias menores se hicieron 4096; en total se corrieron 5130240 experimentos ficticios. El mejor ajuste de oscilación en el rango completo de energía se encuentra se encuentra en el punto $\left(1.00,0.038 \mathrm{eV}^{2}\right)$, en la región de $200<E_{\nu}^{Q E}<1300 \mathrm{MeV}$ tiene un valor de $\chi^{2}$ /g.l. $=25.42 / 16$ lo cual da una probabilidad de $6.27 \%$, por su parte la hipótesis nula tiene un valor de $\chi^{2}$ /g.l. $=48.01 / 18$ con una probabilidad de $0.01 \%$ en la misma región de energía ${ }^{9}$. Para la región de señal de 200-1 $300 \mathrm{MeV}$ se observaron un total de 1446 eventos $\nu_{e}$ y $\bar{\nu}_{e}$, cuando se esperaban $1206.85 \pm 34.74 \pm 53.41$ (error estadístico y sistemático), lo que equivale a un exceso combinado de $239.15 \pm 63.71$ eventos a $3.7 \sigma^{10}$.

\footnotetext{
${ }^{8}$ Los resultados oficiales son: 478 eventos $\bar{\nu}_{e}$, expectación de $399.6 \pm 20.0 \pm 20.3$ y un exceso de $78.4 \pm 28.5(2.8 \sigma)$; en la región de señal 200-1250 MeV.

${ }^{9}$ Los resultados oficiales son: $\chi^{2} /$ g.l. $=24.7 / 15.6 \rightarrow P=6.7 \%$ para el mejor ajuste, en la región de señal 200$1250 \mathrm{MeV}$. No se tiene información para la hipótesis nula.

${ }^{10}$ Los resultados oficiales son: 1430 eventos $\nu_{e}$ y $\bar{\nu}_{e}$, expectación de $1189.7 \pm 34.5 \pm 52.6$ y un exceso de $240.3 \pm$ $62.9(3.8 \sigma)$; en la región de señal 200-1 $250 \mathrm{MeV}$.
} 
Las diferencias que hay entre los contornos oficiales y los obtenidos en esta tesis para las correcciones frecuentistas se encuentran en la parte izquierda de la curva de $3 \sigma$ N.C.: en este trabajo la hendidura alrededor de $6 \mathrm{eV}^{2}$ es más pronunciada y por debajo de $0.5 \mathrm{eV}^{2}$ la curva es más estrecha. Para $\Delta m^{2}>8 \mathrm{MeV}$ también hay diferencias, pero seguramente se deben solamente a artificios de la interpolación.

Las marcadas diferencias en los espectros de $\nu_{e} \mathrm{y} \bar{\nu}_{e}$ no se pueden explicar satisfactoriamente con modelos de $3+1$ neutrinos, los tres parámetros de tal formalismo son incapaces de acomodar los distintos comportamientos observados (revisar § 3.2.1). Los modelos con 2 o 3 neutrinos estériles, los cuales cuentan con una fase de violación $C P$ que daría cuenta de los distintos comportamientos, parecen ajustar los datos exitosamente [203].

También se estudió el efecto de las correlaciones entre los neutrinos y antineutrinos en los análisis, anulando los términos correspondientes a esas relaciones. El nivel de confianza al cual se obtienen regiones cerradas disminuye a $\sim 90 \%$. Se omiten en la presentación de este trabajo por constricciones de espacio. 

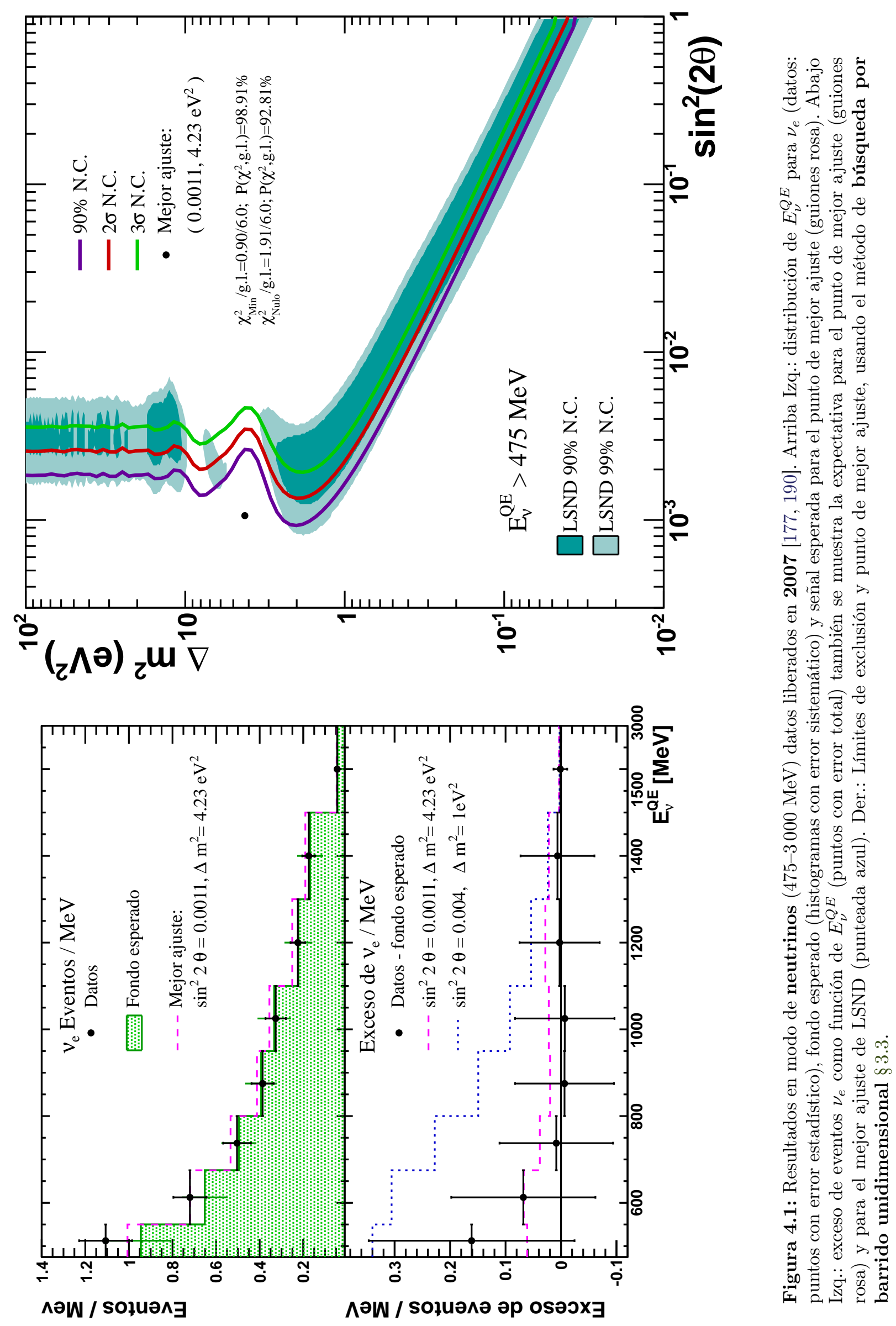


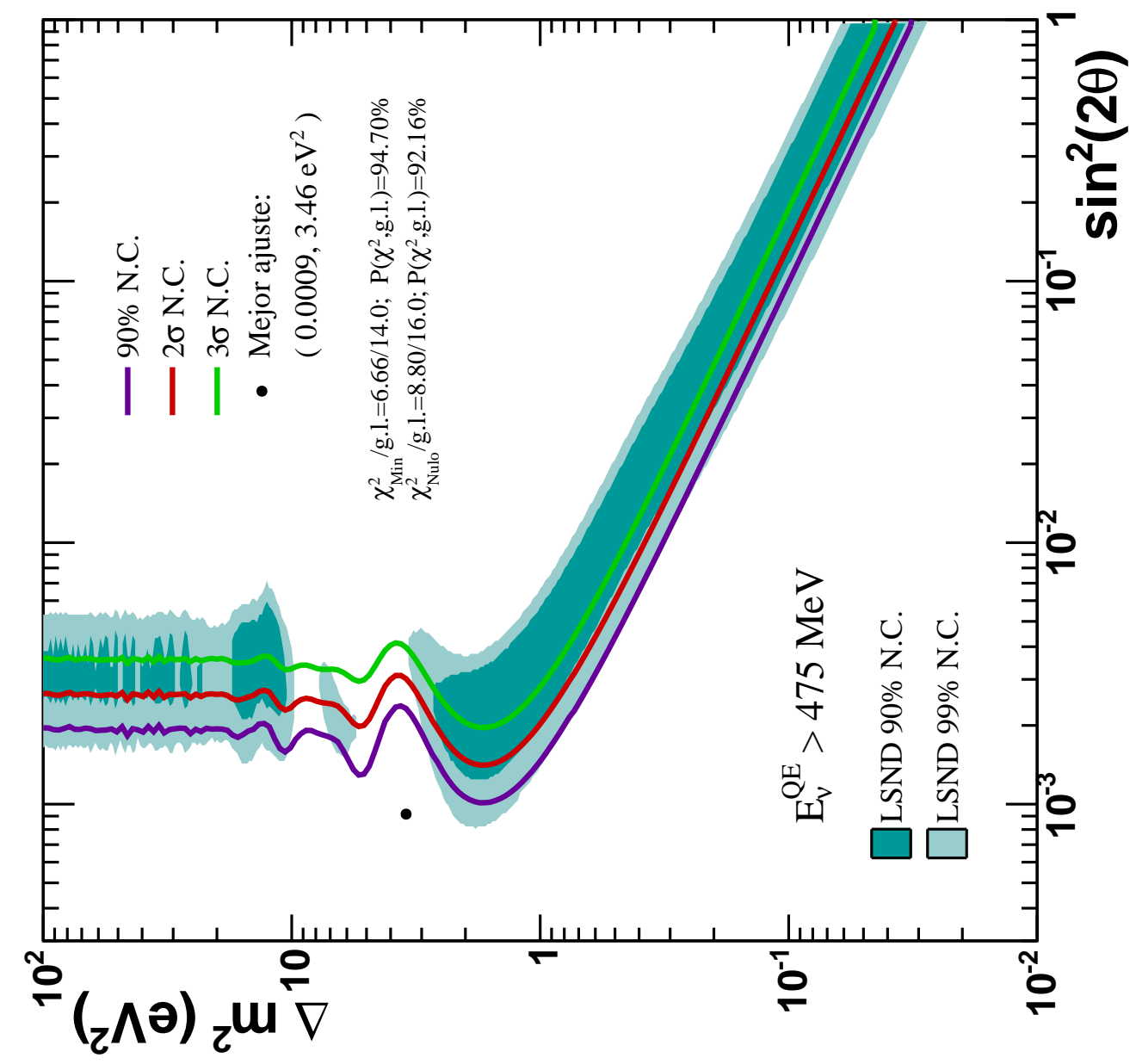

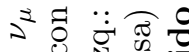

莡

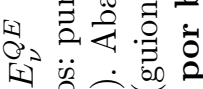

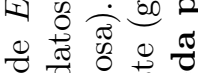

五

\%

قै

行国

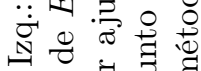

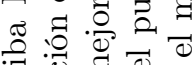

5 웅

$\dot{F}$.

बㄹ.

我灾焉

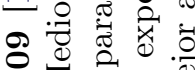

只 $\approx$

वैंक

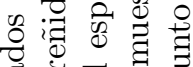

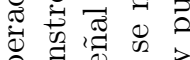

$\because 8$ क

की 矛 3

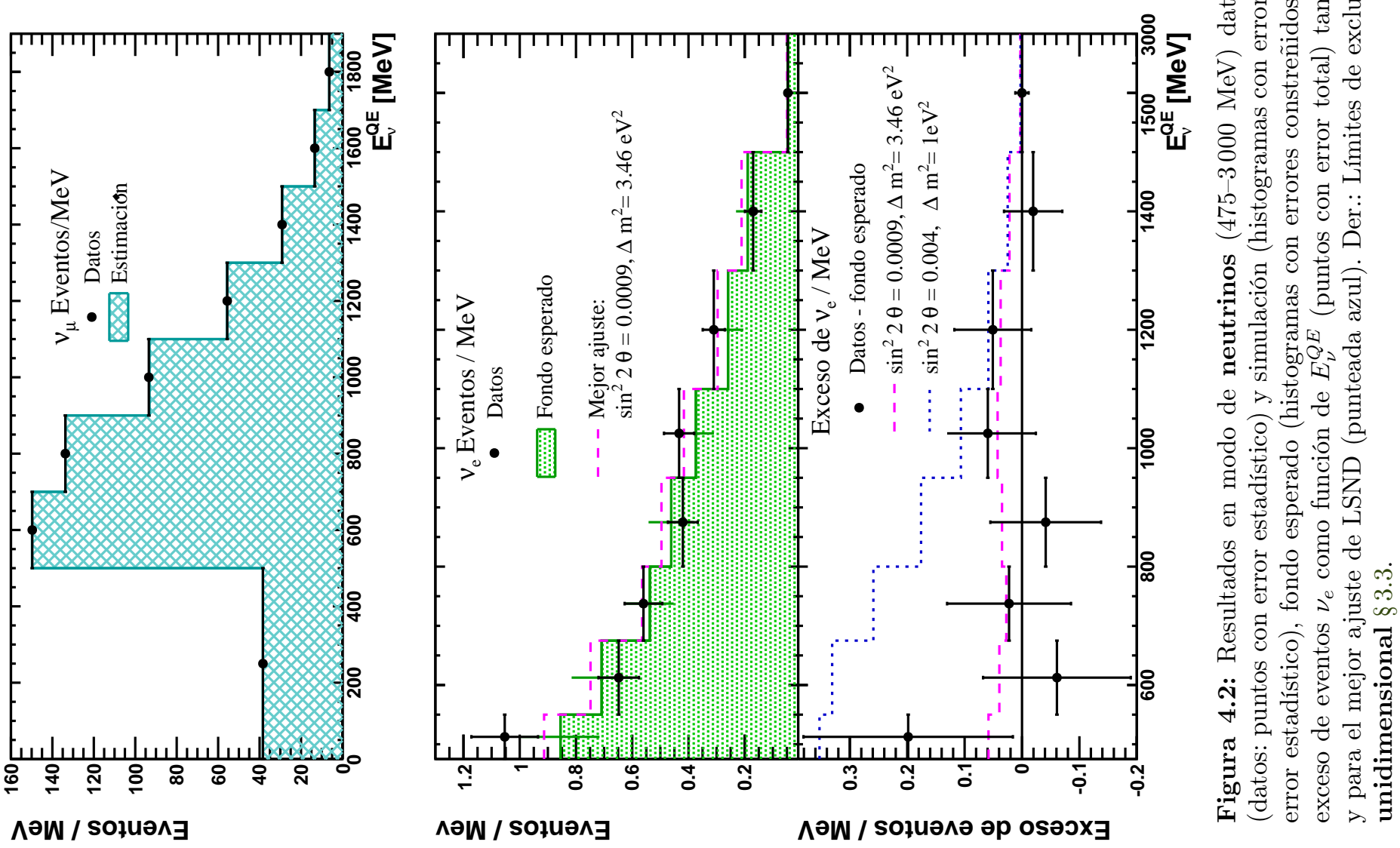



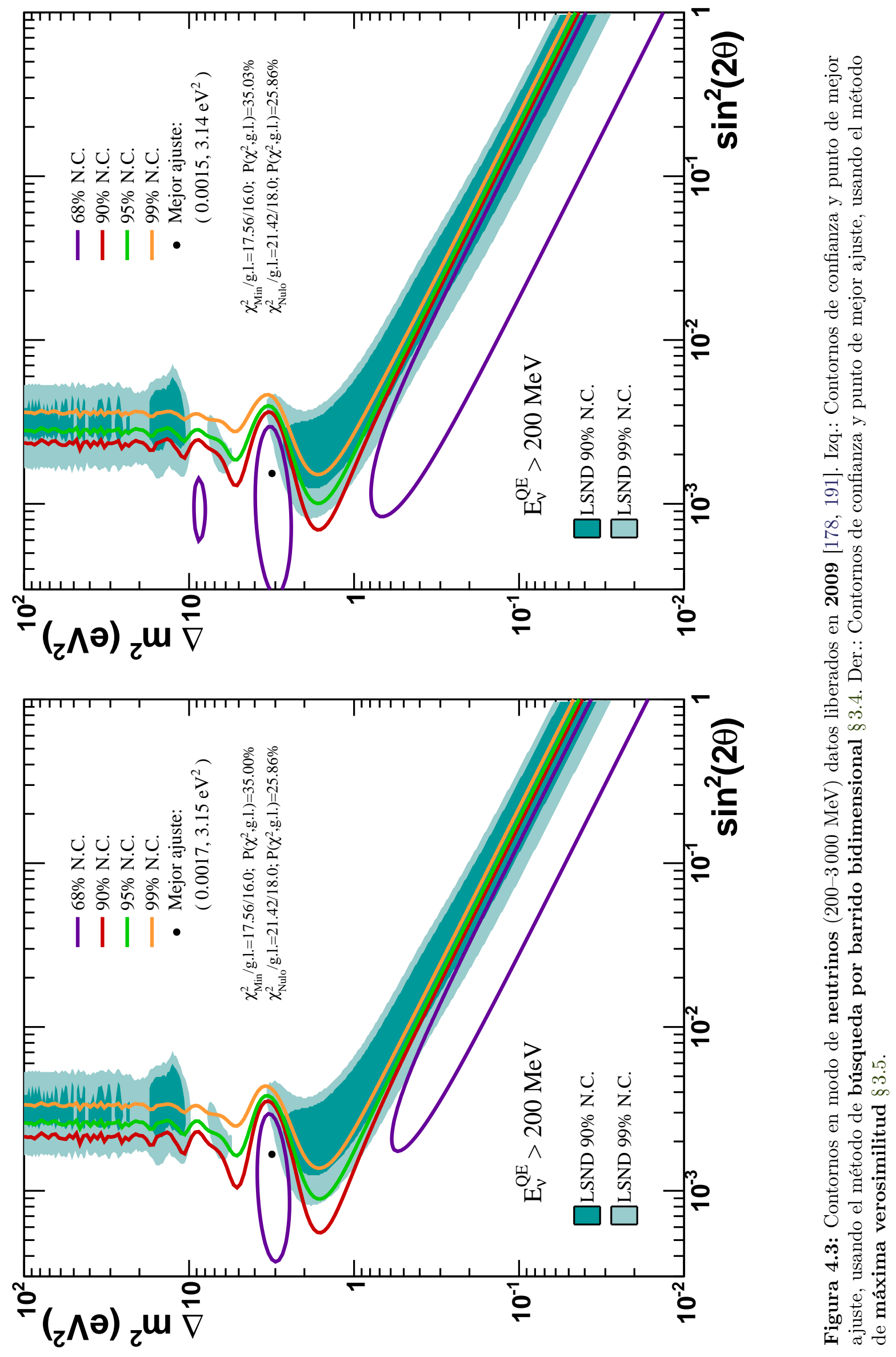


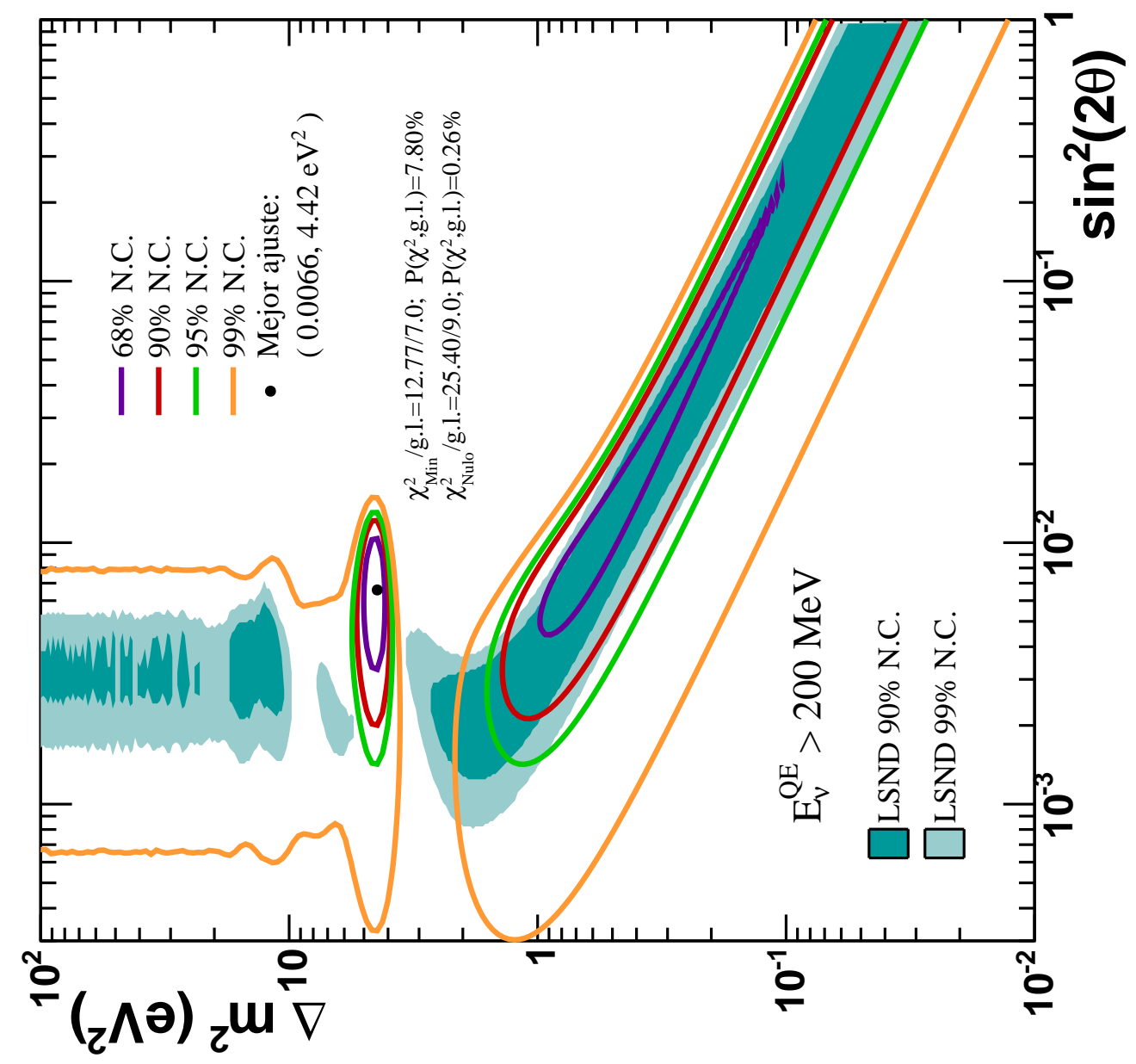

$\omega^{2}$ 。ี

ॠ

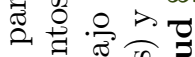

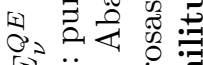

거 $\ddot{0} \dot{0} \dot{0} \tilde{\sigma}$

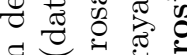

0

苛

勿

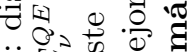

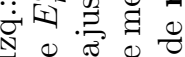

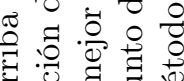

安苛范

$\therefore$ व

윽 윰 윰

ó

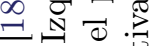

윽 워

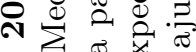

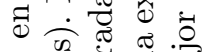

o :

त्वृ की

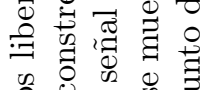

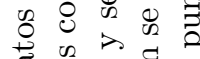

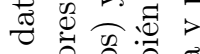

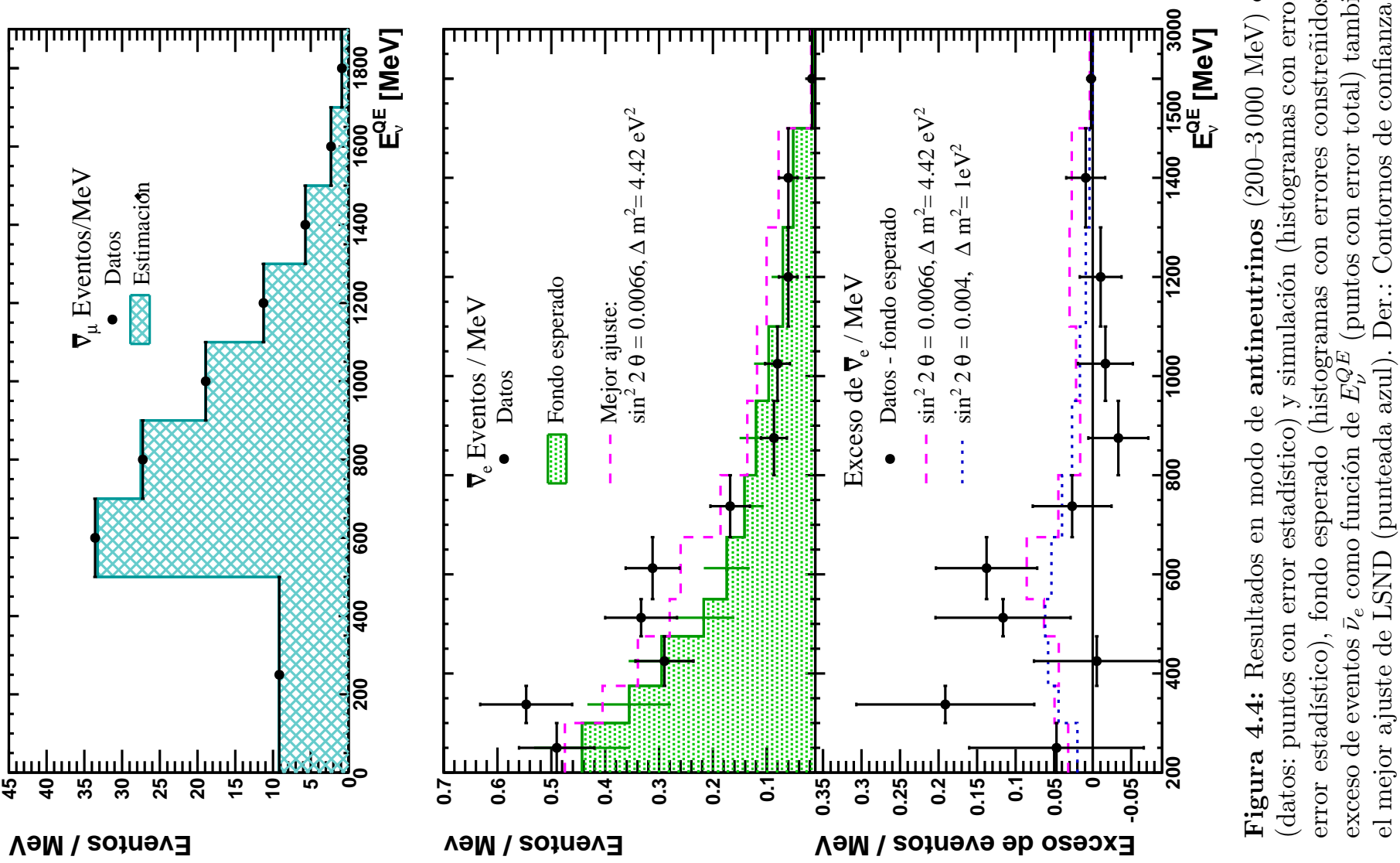



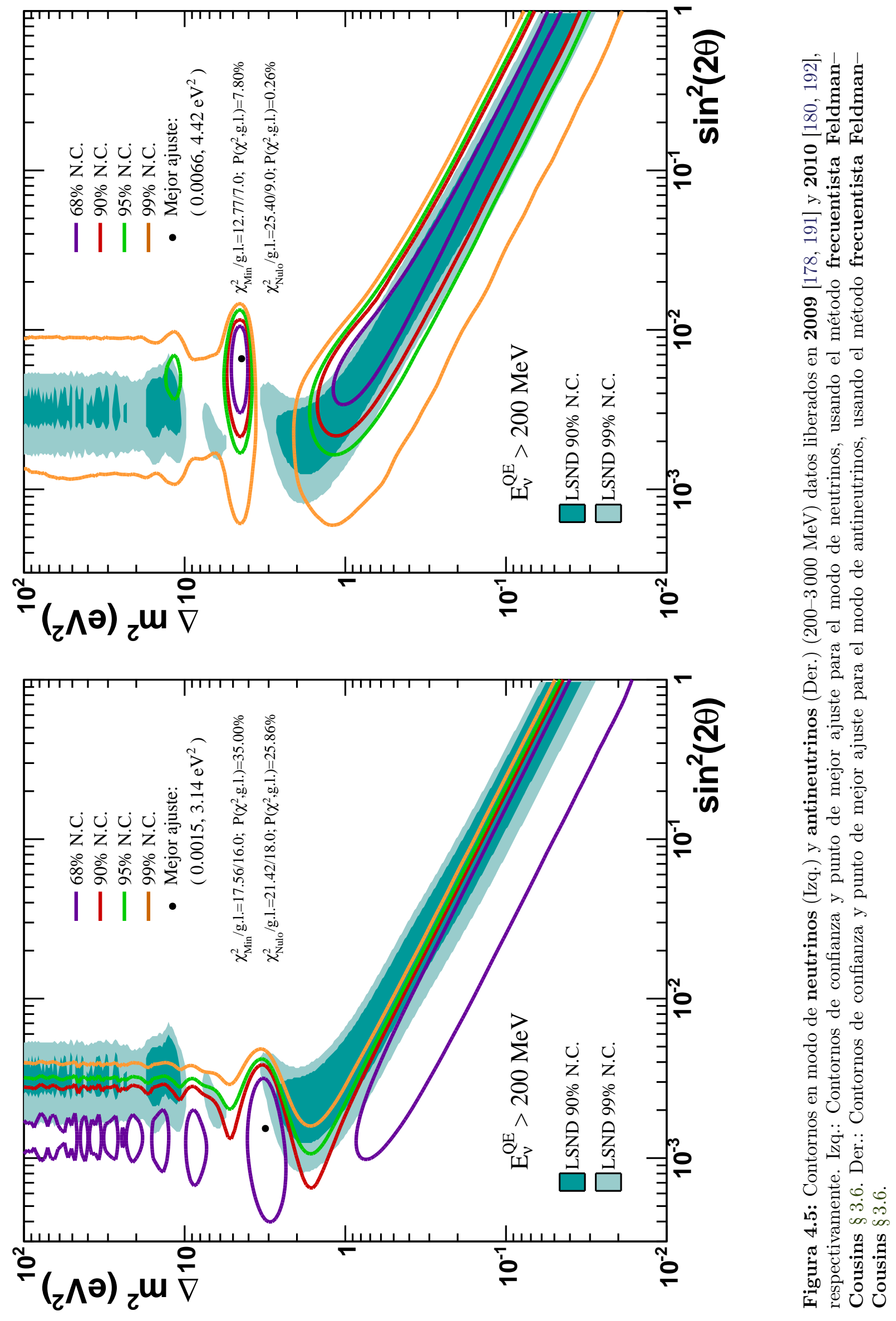


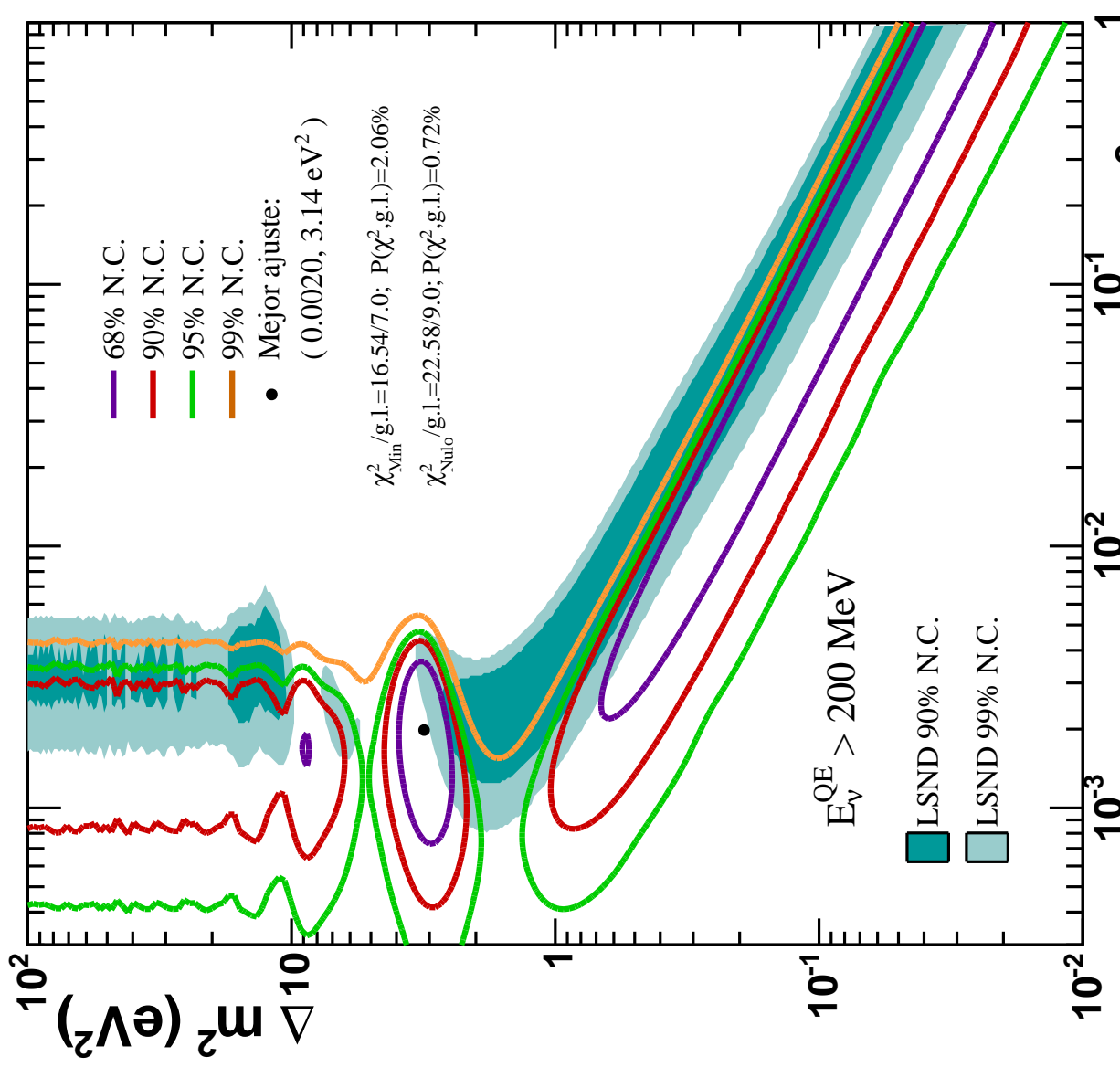

苍

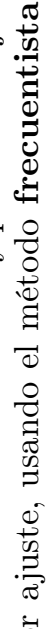

$\because \frac{\bar{\sigma}}{0}$

ஸे ช

-

N

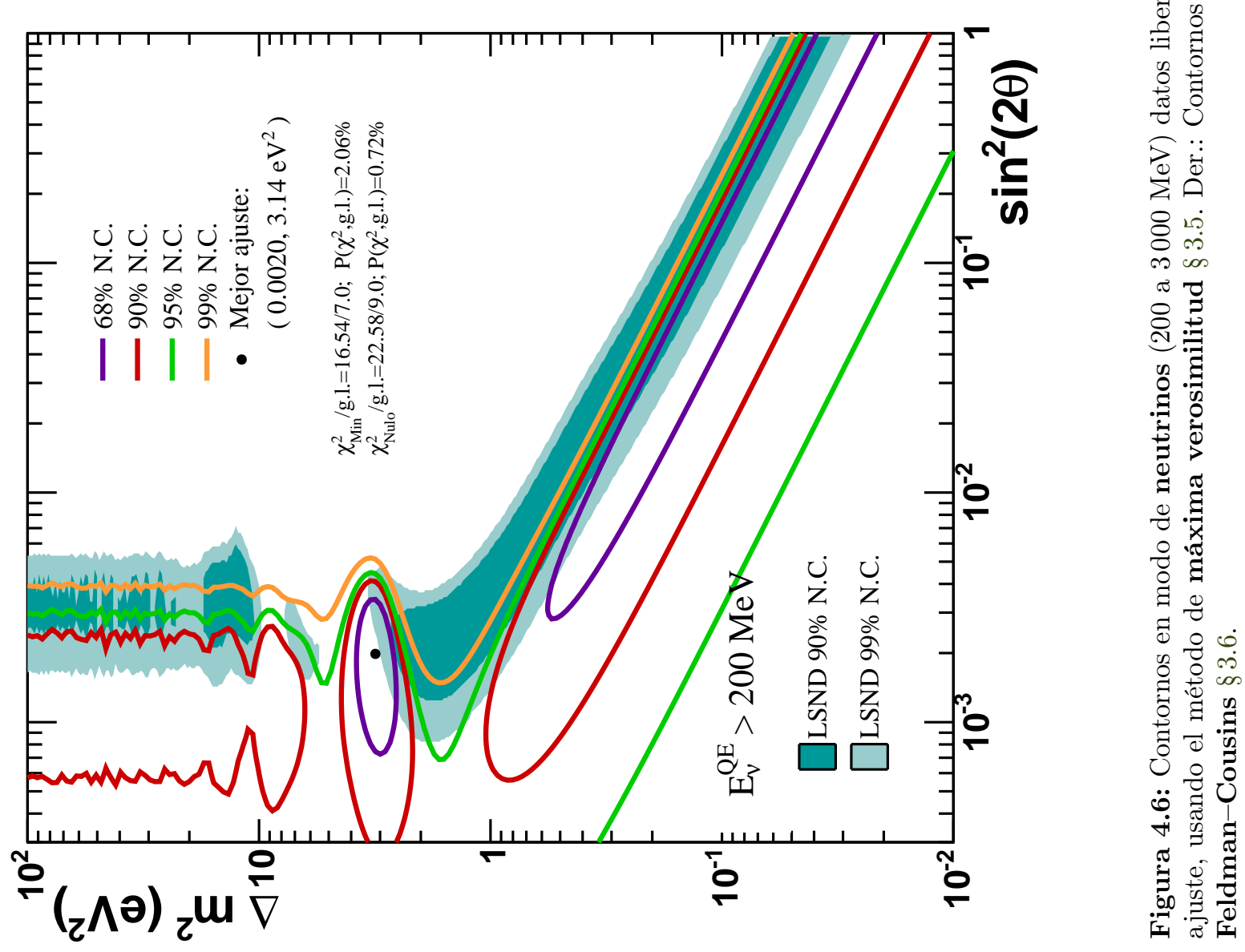



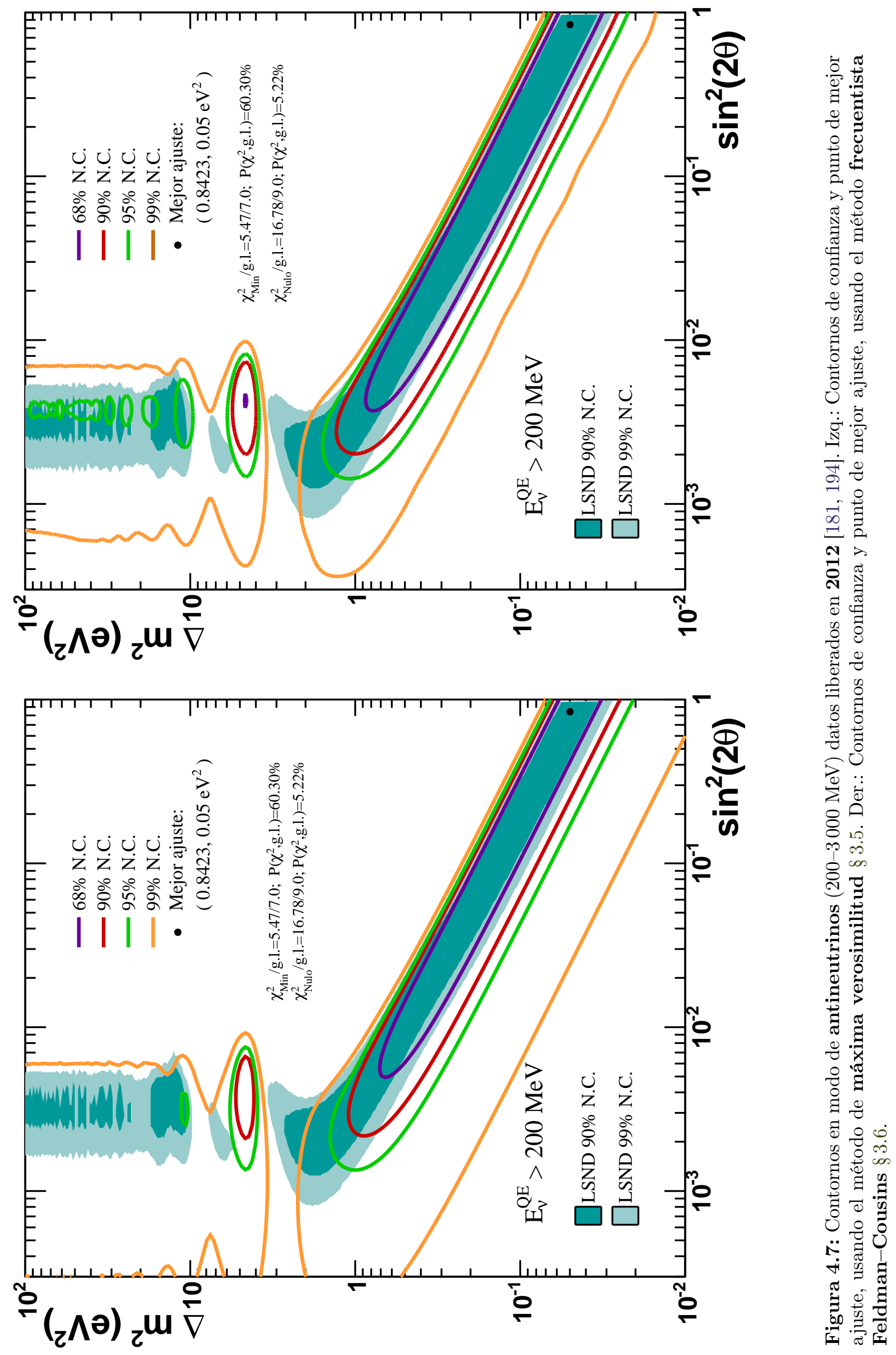

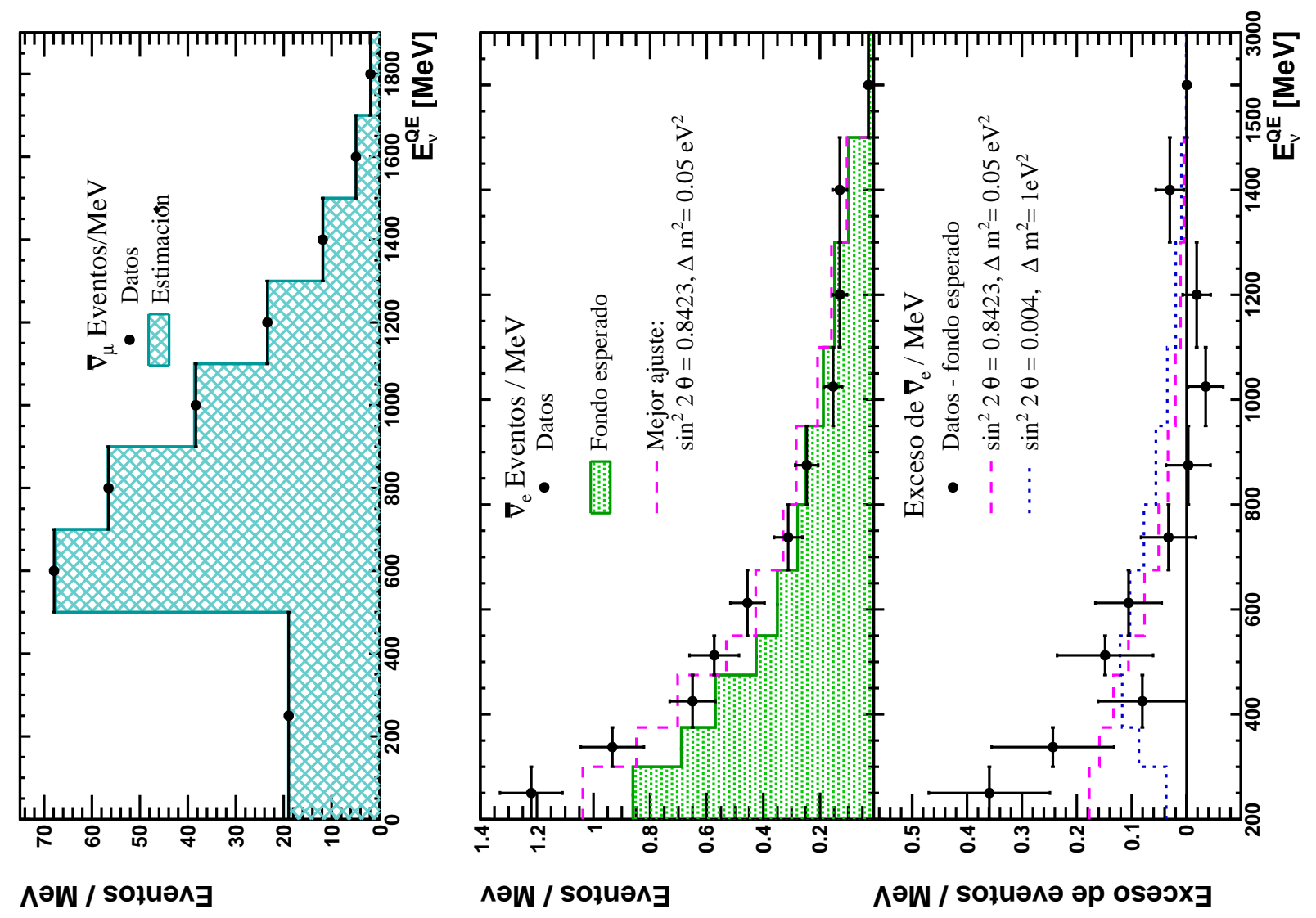

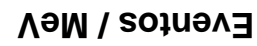

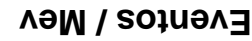

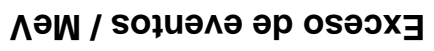

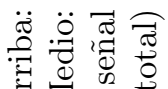

文文

स है : 0

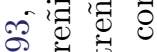

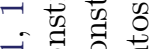

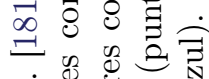

ง

穴氙

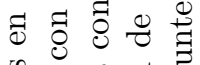

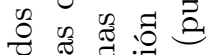

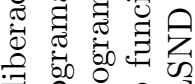

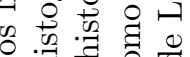

㝴

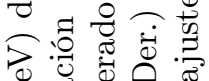

西

○ छ

ठำ

bे

过官氙芯

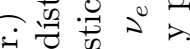

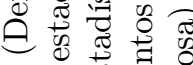

की

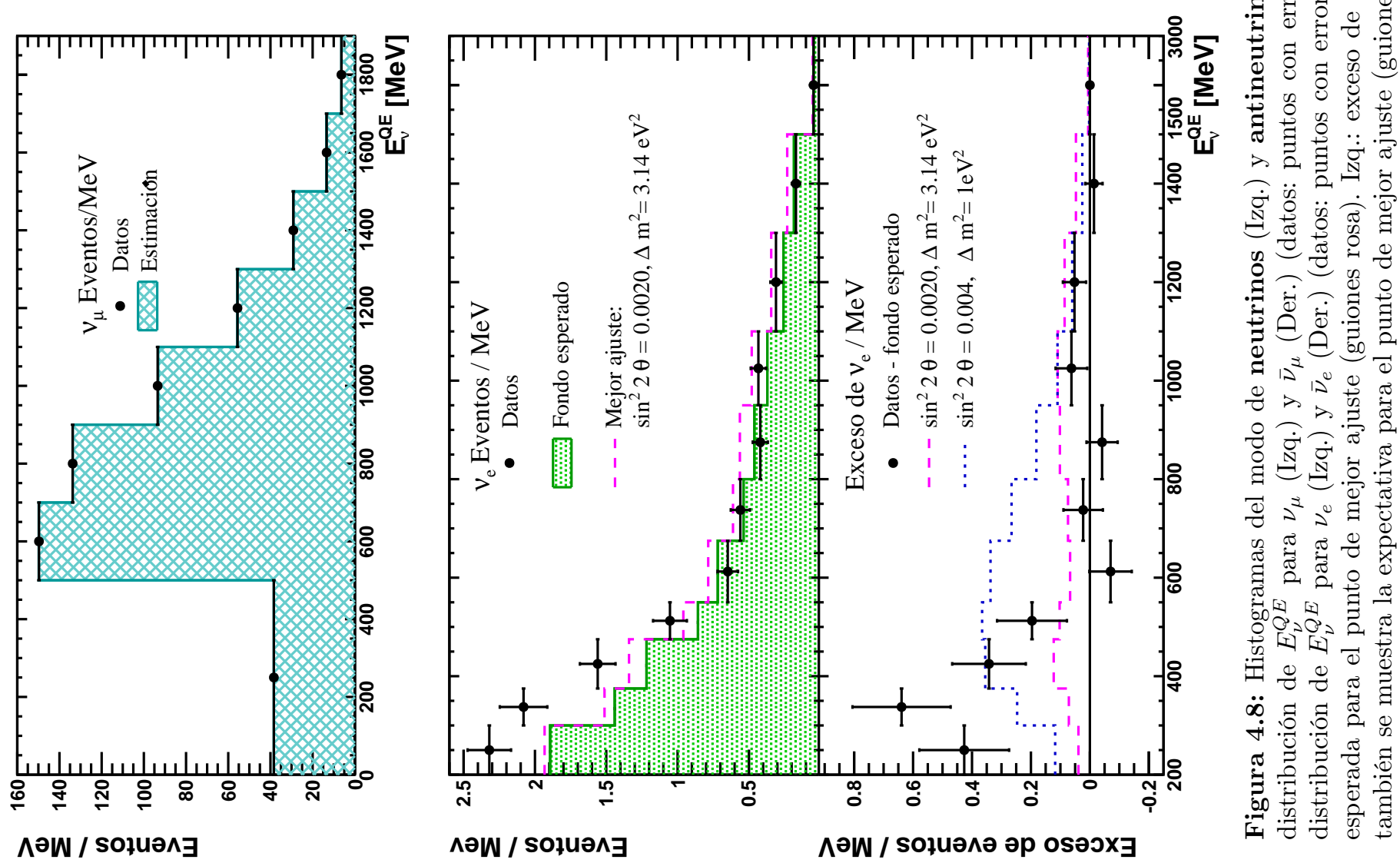



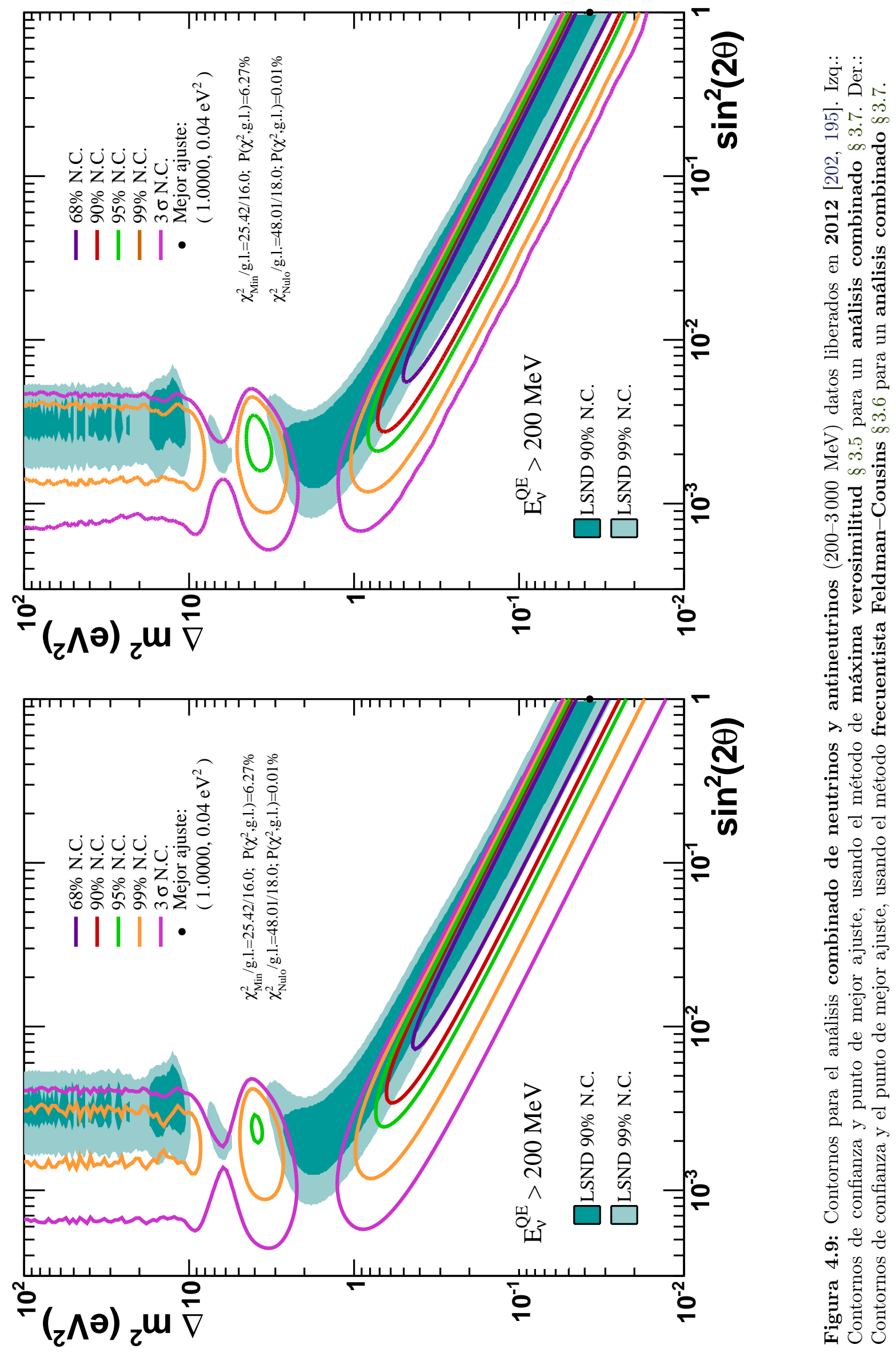


\section{Capítulo 5}

\section{Conclusiones}

Los experimentos de oscilación de neutrinos solares, atmosféricos, de reactores y de aceleradores han establecido una masa no nula para los neutrinos, así como una mezcla entre ellos. Estas observaciones parecen ser consistentes con el paradigma de tres neutrinos del modelo estándar de partículas elementales. Sin embargo, los anómalos resultados del experimento LSND de los Alamos y MiniBooNE de Fermilab, podría sugerir la existencia de por lo menos un neutrino estéril. Los experimentos de corta distancia con reactores nucleares, muestran una anomalía que también sugiere la existencia de neutrinos estériles. Además, observaciones cosmológicas aún permiten la existencia de un neutrino más. A pesar de todas esas pistas que apuntan al neutrino estéril, todavía hay serías incongruencias y sigue siendo un asunto por resolver que será atendido por varios experimentos en el futuro próximo.

Los neutrinos detectados en el experimento MiniBooNE en Fermilab son generados de la interacción de protones de $8 \mathrm{GeV}$ provenientes del acelerador Booster, estos protones forman mesones que son enfocados por un corno magnético y que decaen, principalmente, en $\nu_{\mu}$ (modo de neutrinos). Cambiando la polaridad del corno se obtiene un haz principalmente de $\bar{\nu}_{\mu}$ (modo de antineutrinos). MiniBooNE es un detector cherenkov esférico de 800 t y $12 \mathrm{~m}$ de diámetro, que puede diferenciar sabores de neutrinos (entre $\nu_{e}$ y $\nu_{\mu}$ o $\bar{\nu}_{e}$ y $\bar{\nu}_{\mu}$ ). Observar un exceso de eventos $\nu_{e}\left(\bar{\nu}_{e}\right)$ por encima de la cantidad de eventos que se esperan ver, es interpretado como una indicación de oscilación de neutrinos. MiniBooNE tomó datos de 2002 hasta 2012 en ambos modos.

El experimento MiniBooNE ha observado en la región de señal de $200<E_{\nu}^{Q E}<1250 \mathrm{MeV}$ un exceso de eventos $\nu_{e}$ en el modo de neutrinos de $162.0 \pm 47.8(3.4 \sigma)$, así como un exceso de eventos $\bar{\nu}_{e}$ en el modo de antineutrinos de $78.4 \pm 28.5(2.8 \sigma)$, pero con marcadas diferencias entre uno y otro. En el modo de antineutrinos los resultados son consistentes con la señal de oscilación de LSND, cumpliendo de esa forma su objetivo principal de mostrar evidencia a favor de la observación de LSND. Interpretar el exceso de eventos observado en modo de neutrinos como un efecto de oscilaciones es desfavorecido y, en realidad, actualmente no existe una buena explicación para tal efecto, el cual debe ser estudiado. Si se insiste en una explicación para ambos efectos, el exceso combinado es de $240.3 \pm 62.9(3.8 \sigma)$. Adjudicar el comportamiento observado a la existencia de un neutrino estéril adicional esta desfavorecido, ya que en modelos de $3+1$ neutrinos no se espera un comportamiento tan distinto entre neutrinos y antineutrinos. Ajustar los datos a un modelo de $3+2$ neutrinos resolvería las tensiones entre el comportamiento de neutrinos y antineutrinos, ya que existe una fase de violación $C P$ que permitiría tales diferencias. Como un experimento de oscilaciones con un único detector, las incertidumbres sistemáticas prácticamente dominan el error total medido, por lo cual más estadística 
no añadiría nueva información significativa a la cuestión de oscilaciones. Además de las valiosas observaciones sobre la anomalía LSND, MiniBooNE ha sido un experimento bastante exitoso, entre sus más notables logros están las mediciones de secciones eficaces, cubriendo cerca del $90 \%$ de las interacciones de los neutrinos con energías de $\sim 1 \mathrm{GeV}$.

Utilizando los datos disponibles al público se lograron reproducir la mayoría de los resultados de oscilaciones de MiniBooNE en este trabajo. Se usaron cuatro métodos distintos para reproducir los análisis de la búsqueda de aparición de neutrinos y antineutrinos del electrón. Con la búsqueda por barrido unidimensional se analizaron los datos de neutrinos de 2007 y 2009. La búsqueda por barrido bidimensional se usó solamente para analizar los datos de neutrinos de 2009. El método de máxima verosimilitud fue usado para analizar los datos de neutrinos de 2009, antineutrinos de 2010, para analizar los datos individuales de neutrinos y antineutrinos, así como para hacer el análisis combinado de neutrinos y antineutrinos de 2012. El método frecuentista de Feldman-Cousins fue usado para hacer correcciones al método de la máxima verosimilitud, es decir, se usó para los mismos datos que para tal método. Los contornos obtenidos con los tres primeros métodos no muestran ninguna diferencia perceptible con respecto a los oficiales, sin embargo los obtenidos con las correcciones F-C muestran ligeras diferencias en ciertas regiones de los contornos exteriores, muy posiblemente ocasionadas por particularidades de los algoritmos usados para generar experimentos ficticios. De cualquier forma los resultados aquí obtenidos no son contradictorios a los oficiales y de ninguna forma significan una conclusión opuesta.

En el análisis de aparición más reciente, el exceso de eventos calculado en este trabajo fue de $147.19 \pm 49.77$ eventos $\nu_{e}$ a $2.9 \sigma$ y $81.36 \pm 28.62$ eventos $\bar{\nu}_{e}$ a $2.8 \sigma$, para el modo de neutrinos y antineutrinos, respectivamente. En el análisis combinado de neutrinos y antineutrinos se obtuvo un exceso de $239.15 \pm 63.71 \nu_{e}$ y $\bar{\nu}_{e}$ a $3.7 \sigma$. En el mejor ajuste y en el punto nulo se obtuvo $\chi 2 / g . l=16.54 / 7$ con probabilidad de $2.06 \%$ y $\chi 2 / g . l=22.58 / 9$ con probabilidad de $0.72 \%$ para el modo de neutrinos, $\chi^{2} /$ g.l. $=5.47 / 7$ con probabilidad de $60.30 \%$ y $\chi 2 / g . l=16.78 / 9$ con probabilidad de $0.05 \%$ para el modo de antineutrinos, y de $\chi 2 / g . l=25.42 / 16$ con probabilidad de $6.27 \%$ y $\chi 2 / g . l=40.01 / 18$ con probabilidad de $0.01 \%$ para el análisis combinado. Las diferencias entre estos valores y los oficiales se dan porque públicamente no se tiene acceso a los datos del bin que llega a $1250 \mathrm{MeV}$, escogido con el objetivo de maximizar la señal, si no que llega hasta $1300 \mathrm{MeV}$ lo que da origen a ciertas variaciones.

Se escribieron programas ex profeso para llevar a cabo los análisis antes mencionados, así como para procesar los datos, graficarlos y presentarlos. Para ajustar los datos de oscilaciones, se usó un generador de números aleatorios basado en el método de Mersenne Twister escrito por un tercero, todo lo demás fue escrito por el autor. Pensando en maximizar la eficiencia y minimizar el tiempo de cómputo, se puso particular énfasis en el diseño de los programas para hacer el método de la máxima verosimilitud y las correcciones frecuentistas $\mathrm{F}-\mathrm{C}$. Se implementó el método frecuentista $\mathrm{F}-\mathrm{C}$ en un esquema serial en $\mathrm{C}++$, ejecutándose en un cluster administrado por el sistema Condor en Fermilab; también se implementó en un esquema de procesamiento en paralelo en CUDA, ejecutándose en tarjetas de vídeo NVIDIA en el IF-UNAM. Obteniendo en ambos casos resultados consistentes entre sí, de la misma forma ambos resultados obtienen diferencias análogas a los resultados oficiales. La implementación en CUDA permite prescindir del numeroso cluster de $60 \sim 70$ núcleos por un par de tarjetas de vídeo. La ejecución en las tarjetas de vídeo es $\sim 24$ más rápida que en un procesador estándar, y en total un par de centenas de veces más rápida que los programas oficiales. Este trabajo llena un vacío en la documentación de los análisis de oscilación, verificando que en efecto se puede llegar a los resultados oficiales de MiniBooNE usando los datos liberados, ya que los publicados fueron calculados usando el propio marco de análisis del experimento y deben esperarse algunas diferencias. 
Además, corrobora que todo se haya hecho bien: gracias a este trabajo se encontraron dos pequeños errores en los análisis oficiales, ambos fueron notificados y uno de ellos fue consecuentemente corregido por la colaboración para la publicación de 2013.

La utilidad de este trabajo radica en el mejoramiento de las técnicas computacionales, el método frecuentista $\mathrm{F}-\mathrm{C}$ se ha vuelto en la estrategia cotidiana para una gran cantidad de análisis en la física de altas energías, sobre todo para la física de neutrinos en donde las señales suelen ser pequeñas; y demanda una gran cantidad de poder de cómputo. En vista de que el futuro de la física experimental de neutrinos requerirá ajustes más completos y demandantes, es importante.

El futuro a largo plazo de MiniBooNE es incierto y es probable que pronto será definitivamente apagado. Pensando a largo plazo, la colaboración contempla la posibilidad de agregar centellador orgánico al aceite del tanque, lo cual permitiría disminuir el fondo de interacciones neutras y hacer nuevos estudios de secciones eficaces. A corto plazo, se ha aprobado que MiniBooNE haga una corrida de prueba para buscar WIMPS de masa pequeña, esta nueva búsqueda requirió mejorar el sistema de sincronización temporal entre el blanco y la electrónica del detector, el autor colaboró con miembros de la colaboración en Fermilab para implementar el nuevo sistema. El haz que suministra neutrinos a MiniBooNE se volverá a usar para el experimento MicroBooNE, el cual empezará a tomar datos en 2014 y estudiará a detalle el exceso a bajas energías observado en modo de neutrinos. Inevitablemente estos neutrinos atravesarán el detector MiniBooNE, así pues solamente sería cuestión de mantener el detector prendido para obtener más datos. A MicroBooNE le serviría tener a MiniBooNE prendido como referencia y las precisas mediciones a bajas energías de MicroBooNE ayudarían a disminuir los errores que tiene MiniBooNE.

Aún con el espectacular avance que se ha hecho en los estudios de las oscilaciones de neutrinos, se debe seguir con un extenso y desafiante programa de investigación para: obtener un mejor entendimiento del patrón de oscilaciones y las mezclas de neutrinos, de sus orígenes y del estado de la violación de la simetría $C P$ en el sector de leptones. Particularmente se debe: determinar el signo de $\Delta m_{31}^{2}$, determinar o constreñir significativamente la escala absoluta de la masa de neutrinos, medir la fase de violación de $C P \delta$, hacer medidas precisas de los ángulos de mezcla y las diferencias de masa, entender de forma fundamental el mecanismo que da masas y mezclas a los neutrinos y a la no conservación de los números leptónicos individuales. Otra de las interrogantes que queda por resolver sobre los neutrinos es si son partículas de Dirac o de Majorana, lo cual no puede ser observado en experimentos de oscilación de neutrinos, la incontrovertible observación del doble decaimiento beta sin neutrinos, establecería que los neutrinos son su propia antipartícula, o sea que son partículas de Majorana. Dependiendo del valor de la violación $C P$, es posible que los neutrinos sean, por lo menos en parte, causantes de la asimetría materia/antimateria. Además de que podrían ser la partícula (o alguna de las partículas) de la materia oscura, formando en conjunto la llamada "materia oscura caliente".

A nivel personal, la mayor relevancia que tiene esta tesis se mide en la cantidad de cosas que se aprendieron. A pesar de no ser ajeno a la programación, la gran cantidad de líneas de código que se requirieron escribir para llevar a cabo los análisis y presentarlos de la forma más adecuada (incluido el archivo fuente de este escrito) sirvieron de magnifica práctica para dominar con gran maestría tales habilidades. El tiempo necesario en hacer cualquier programa disminuyo considerablemente conforme la experiencia aumentaba, el autor sabe la importancia que tiene en la ciencia la pericia en el manejo de computadoras y también sabe que las habilidades que se aprendieron en la realización de esta tesis le acompañaran en toda su vida profesional. En particular, el uso de tarjetas gráficas y el computo en paralelo son parte de las herramientas que ofrece el futuro de la computación y tener la capacidad de 
usarlas propone nuevas técnicas para la solución de problemas. En un área tan prolífica y dinámica como la física de neutrinos existen una gran cantidad de cosas por aprender, si bien el panorama adquirido no es completo, sí es muy amplio. Mucho énfasis se ha puesto en entender cabalmente y a detalle la estadística involucrada en los análisis, mas que solamente usar las técnicas de forma mecanizada. De forma similar, se hizo un esfuerzo notable por familiarizarse con el experimento, entendiendo los compromisos ineludibles de la labor experimental y la mejor forma de lidiar con ellos, estudiando la gran cantidad de detalles que hacen falta tener presente para operar un experimento de tal magnitud, modesto para el área de altas energías pero suficientemente grande como para que su operación exitosa requiera el esfuerzo constante e incansable de decenas de científicos e ingenieros por más de diez años. Esta tesis trata de reflejar el aprendizaje del autor en todas estas áreas.

Rara vez en la ciencia el camino y los pasos que se deben seguir hacia nuevos descubrimientos es tan claro como ahora. La física de neutrinos es uno de los puentes que hay entre los fenómenos más inmensos, como los grandes eventos astronómicos y cosmológicos, con los más diminutos, como los núcleos atómicos y las partículas elementales. Vivimos en una época en que la luz que arrojan los nuevos descubrimientos rompe con la perspectiva mantenida por mucho tiempo del universo. El descubrimiento de la masa no nula de los neutrinos como consecuencia de las oscilaciones ha jugado un rol fundamental en esta revolución. Los datos recolectados en los experimentos actuales y futuros serán la punta de lanza en la verificación o rectificación de nuestras teorías, en la creación de nuevas teorías o para abrir posibilidades que no han sido imaginadas.

La odisea hacia el entendimiento de los neutrinos y del universo en su conjunto continua. Sin embargo, como la historia de este campo lo ha demostrado tantas veces, lo apasionante de los neutrinos es que se debe esperar lo inesperado.

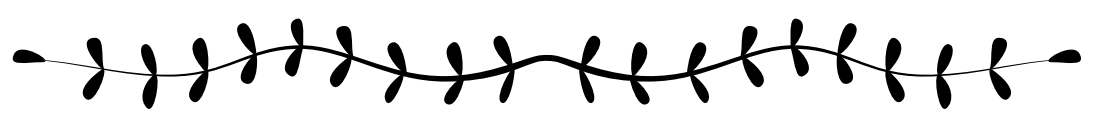




\section{Apéndice A \\ El código}

Se transcriben las últimas versiones de los códigos que se usaron para llevar a cabo las correcciones frecuentistas de Feldman-Cousins del modo de antineutrinos para los datos liberados en agosto de 2012. Para el modo de neutrinos el programa es análogo solo que usando los datos correspondientes. Para el modo combinado $\nu-\bar{\nu}$ se deben usar matrices más grandes y hay que prestar atención en la diagonalización e inversión de las mismas, pero de igual forma el programa es análogo.

En la transcripción del código se muestran explícitamente las funciones escritas ad hoc, en la tabla A.1 se resume su uso. De particular importancia son las funciones: float* choleskyFactor (float matrix[]) y float* choleskyInverse (float matrix []) que llevan a cabo el proceso de inversión de matrices. Con la primera función a partir de una matriz hermitiana y positiva definida ${ }^{1} \mathbf{M}$ se obtiene una matriz triangular inferior $\mathbf{h}$ que al multiplicarla con su transpuesta se recupera la matriz original: $\mathbf{h} \times \mathbf{h}^{\mathrm{T}}=\mathbf{M}$. Se decidió separar el proceso de inversión en dos etapas para así poder obtener el logaritmo del determinante de la matriz a invertir después de la primera etapa: la suma del logaritmo de los elementos de la traza de la matriz triangular inferior, linea 377 del código A.1. Después la segunda función toma una matriz cholesky triangular inferior y regresa la matriz inversa $\mathbf{M}^{-1}$. Dado que estas matrices son simétricas basta reservar el arreglo unidimensional h [NBC* (NBC+1)/2] para una matriz simétrica de dimensión NBC $\times$ NBC, para hacer referencia al elemento en el renglón i y la columna j de lo que sería una matriz bidimensional se escribe la dirección unidimensional $[i *(i+1) / 2+j]$. Haciendo estos cambios en todo el código se logra una ligera optimización ${ }^{2}$ de $\sim 10 \%$ al ahorrar memoria y accesos duplicados innecesarios. Los datos que toman los códigos a la entrada se muestran en la tabla A.2.

Se hizo uso de paqueterías de un tercero para el generador de números pseudo aleatorios (en inglés PRNG) la paquetería en cuestión y las instrucciones sobre su implementación se encuentran en [204]. El PRNG que usa esa paquetería es un Mersenne Twister, desarrollado en 1997 por Makoto Matsumoto y Takuji Nishimura [205], se basa en la recurrencia lineal de una matriz sobre un campo binario finito $F_{2}$. Genera rápidamente números pseudo aleatorios de muy alta calidad, diseñado específicamente para rectificar muchas de las fallas encontradas en algoritmos más viejos. La variante usada es la MT19937 cuya longitud de periodo es de $2^{19937}$ - 1; 19937 es un primo Mersenne, de donde toma el nombre el algoritmo.

\footnotetext{
${ }^{1}$ Características que por definición cumplen las matrices de covarianza.

${ }^{2}$ Importante optimización en vista de que se requieren $>10^{11}$ iteraciones.

${ }^{3}$ Esta matriz no es la misma que el bloque correspondiente de la matriz combinada. Solo los eventos $\bar{\nu}$ son usados
} 


\begin{tabular}{|c|c|}
\hline Nombre de la función & Detalles \\
\hline float dm2Tic(int index) & $\begin{array}{l}\text { Toma un entero entre } 0 \text { y } 189 \text {, regresa la coor- } \\
\text { denada de } \Delta m^{2} \text { correspondiente. }\end{array}$ \\
\hline float s2tTic(int index) & $\begin{array}{l}\text { Toma un entero entre } 0 \text { y } 189 \text {, regresa la coor- } \\
\text { denada de } \sin ^{2} 2 \theta \text { correspondiente. }\end{array}$ \\
\hline float s2tTicCU(int index) & $\begin{array}{l}\text { Igual que la pasada solo que optimizado para } \\
\text { su uso en CUDA. }\end{array}$ \\
\hline bool lowerRegion(double $\mathrm{x}$, double $\mathrm{y}$ ) & $\begin{array}{l}\text { Toma las coordenadas de los índices y regre- } \\
\text { sa verdadero si estos se encuentran en la re- } \\
\text { gión inferior exenta de calcular correcciones } \\
\text { frecuentistas. }\end{array}$ \\
\hline bool upperRegion(double $\mathrm{x}$, double $\mathrm{y}$ ) & $\begin{array}{l}\text { Toma las coordenadas de los índices y regresa } \\
\text { verdadero si estos se encuentran en la región } \\
\text { superior exenta de calcular correcciones fre- } \\
\text { cuentistas. }\end{array}$ \\
\hline bool upperSquare (double $\mathrm{x}$, double $\mathrm{y}$ ) & $\begin{array}{l}\text { Toma las coordenadas de los índices y regresa } \\
\text { verdadero si estos se encuentran en el cuadra- } \\
\text { do superior exento de calcular correcciones } \\
\text { frecuentistas. }\end{array}$ \\
\hline float fileExists (const char $*$ fileName) & $\begin{array}{l}\text { Sirve para determinar si un archivo dado ya } \\
\text { existe, con el propósito de no sobre escribir } \\
\text { archivos. }\end{array}$ \\
\hline float* choleskyFactor(float matrix []) & $\begin{array}{l}\text { Toma la dirección de una matriz hermitiana } \\
\text { y positiva definida } M \text {, regresa la dirección de } \\
\text { una matriz triangular inferior } h \text {, que satisfa- } \\
\text { ce: } \mathbf{h} \times \mathbf{h}^{\mathrm{T}}=\mathbf{M}\end{array}$ \\
\hline float* choleskyInverse (float matrix []) & $\begin{array}{l}\text { Toma la dirección de una matriz cholesky } \\
\text { triangular inferior y regresa la dirección de } \\
\text { la inversa de la matriz original } \mathbf{M}^{-1}\end{array}$ \\
\hline
\end{tabular}

Tabla A.1: Funciones usadas en los programas.

Encontrar el mejor ajuste para un experimento ficticio dado consiste en calcular el valor de $\mathscr{L}$ sobre el espacio de parámetros $\left(\sin ^{2} 2 \theta, \Delta m^{2}\right)$ de $\left[3 \times 10^{-4}, 1\right] \times\left[1 \times 10^{-2}, 1 \times 10^{2}\right]$ el cual ha sido dividido en escala logarítmica en una malla de $190 \times 190=36100$ y de esos valores encontrar el mínimo, lo cual es considerado una iteración. Hacer la corrección de un punto en el espacio de parámetros requiere, por lo menos, $n_{\mathbf{f}}=2048$ iteraciones o "experimentos ficticios". Esta búsqueda del mínimo se hace de forma exhaustiva, i.e. calculando y probando cada uno de esos valores, no se hicieron intentos por implementar un método de minimización iterativo por dos razones:

- Permitir que el espacio de parámetros tomará valores continuos representa una posible pérdida de eficiencia ya que requeriría que el histograma para la señal ${ }^{5}$ fuera calculado para cada punto. En vez de simplemente cargar el arreglo que contiene los histogramas para cada punto en el espacio de parámetros, ver tabla A.2.

aquí para la parte de la transmutación total de la matriz además de que hubo variaciones adicionales en las simulaciones MC para construir esta matriz. Ver tabla 3.1.

${ }^{4}$ Una discusión sobre el peso de cada evento en la simulación MC se encuentra en Table 2.3

${ }^{5}$ Ver capítulo 3 para más detalles sobre la señal 


\begin{tabular}{|c|c|}
\hline Nombre del archivo & Contenido y observaciones. \\
\hline $\begin{array}{l}\text { miniboone } \\
\text { nuebardata_lowe. } \\
\text { txt }\end{array}$ & Candidatos observados a eventos $\bar{\nu}_{e}$ por intervalo de energía reconstruida. \\
\hline $\begin{array}{l}\text { miniboone } \\
\text { numubardata.txt }\end{array}$ & $\begin{array}{l}\text { Candidatos observados a eventos CCQE } \bar{\nu}_{\mu} \text { como función de energía re- } \\
\text { construida (notar que no tiene los mismos intervalos que } \bar{\nu}_{e} \text { ) }\end{array}$ \\
\hline $\begin{array}{l}\text { miniboone } \\
\text { nuebarbgr_lowe.txt }\end{array}$ & $\begin{array}{l}\text { Fondo estimado de candidatos a eventos } \bar{\nu}_{e} \text { por intervalo de energía re- } \\
\text { construida. }\end{array}$ \\
\hline $\begin{array}{l}\text { miniboone_numubar. } \\
\text { txt }\end{array}$ & $\begin{array}{l}\text { Estimación de candidatos a eventos CCQE } \bar{\nu}_{\mu} \text { por intervalo de energía } \\
\text { reconstruida. }\end{array}$ \\
\hline $\begin{array}{l}\text { miniboone_full_ } \\
\text { fractcovmatrix_ } \\
\text { nubar_lowe.txt }\end{array}$ & $\begin{array}{l}\text { Arreglo bidimensional de la matriz de covarianza fraccionaria para even- } \\
\text { tos } \bar{\nu}_{\mu} \rightarrow \bar{\nu}_{e} \text { de transmutación total, fondo estimado de eventos } \bar{\nu}_{e} \text { y } \\
\text { estimado de eventos CCQE } \bar{\nu}_{\mu} \text { (tres bloques diagonales lado a lado) por } \\
\text { intervalo de energía reconstruida, incluyendo incertidumbre sistemática } \\
\text { para las tres muestras e incertidumbre estadística para la estimación de } \\
\text { los eventos de fondo de } \bar{\nu}_{e} \text { y CCQE } \bar{\nu}_{\mu} \cdot{ }^{3}\end{array}$ \\
\hline signalsbarSMALL.txt & $\begin{array}{l}\text { Arreglo que contiene los histogramas de señal para los } 190 \text { valores de } \\
\Delta m^{2} \text {. Multiplicando por el valor de } \sin ^{2} 2 \theta \text { correspondiente, el cual ha } \\
\text { sido factorizado en la generación del arreglo; ecuación }(1.6) \text {, se obtie- } \\
\text { ne la señal para cada punto. Se ha construido a partir de miniboone_ } \\
\text { numubarnuebarfullosc_ntuple.txt que contiene } 86403 \text { eventos de } \\
\text { transmutación total } \bar{\nu}_{\mu} \rightarrow \bar{\nu}_{e} \text {, con información de la energía reconstruida, } \\
\text { energía verdadera, distancia recorrida y el peso para cada evento }{ }^{4} \text {. La } \\
\text { generación del arreglo de señales permite ahorrar una cantidad enorme } \\
\text { de tiempo que se iría en generar las señales para cada punto cada vez } \\
\text { que se requieran, es fundamental en la optimización del código. }\end{array}$ \\
\hline
\end{tabular}

Tabla A.2: Archivos que toman los programas a la entrada. Los primeros cinco archivos se encuentran en línea, el último fue generado ex profeso para estos programas.

- Poner en funcionamiento un método iterativo de minimización representaba un gran reto en la implementación a CUDA.

Sin embargo el autor reconoce que el siguiente paso en la optimización del código y uno necesario en espacios de más de dos dimensiones es la implementación de un algoritmo iterativo para la búsqueda del mínimo.

\section{A.1. Código serial en $\mathrm{C}++$}

Una iteración, es decir generar $190 \times 190$ matrices, invertir cada una y para cada una calcular $\mathscr{L}$ para así poder encontrar el mejor ajuste toma 1.08 s en un procesador @ $2.53 \mathrm{GHz}$ y ocupa $\sim 500 \mathrm{KiB}$ de memoria.

El código escrito en $\mathrm{C}++$ se corrió en Condor, más detalles al respecto se encuentran en el apéndice B. En la versión final se hizo que un solo procesador se encargará del cálculo de las correcciones frecuentistas de un solo punto. Consecuentemente cada procesador imprime dos archivos: likelyEff_ NuBar2048NF_XXX-YYY.txt y fakeExperimentsDistribution_NuBar_2048NF_XXX-YYY.txt en las carpetas effectiveLikely y fakeDistributions, respectivamente; XXX y YYY son los índices de 0 a 
189 para $\sin ^{2} 2 \theta$ y $\Delta m^{2}$ respectivamente, para el punto calculado. Es importante que cada procesador escriba archivos independientes y que luego estos sean recolectados para su análisis, por que a pesar de que es posible que todos los procesadores accedan y escriban en el mismo archivo es común que haya conflictos. El primer archivo almacena la superficie efectiva ${ }^{6} \chi_{\mathbf{E f}}^{2}$. El segundo archivo tiene la distribución de experimentos ficticios $\Delta \mathscr{L}_{\mathbf{f} i}^{\left\{\boldsymbol{p}_{0}\right\}}, i=1, \ldots, n_{\mathbf{f}}$; para un punto $\boldsymbol{p}_{0}=\left(\sin ^{2} 2 \theta, \Delta m^{2}\right)_{0}$ con índices XXX y YYY. Se escribió un programa para llevar a cabo el análisis de estas distribuciones, del cual se prescinde aquí.

El código A.1 transcribe la implementación en $\mathrm{C}++$. Las primeras líneas cargan los paquetes necesarios, definen las variables globales y las funciones necesarias. La rutina principal empieza alrededor de la línea 170, en donde se verifica que los argumentos que necesita sean los adecuados, estos se pasan con el bash script B.1. Por la línea 220 se empiezan a leer los archivos de datos, a lo largo de esa sección se definen los arreglos que los almacenaran. De la línea 320 a la 435 se construye la superficie $\mathscr{L}\left(\sin ^{2} 2 \theta, \Delta m^{2}\right)$ con los datos verdaderos y se escriben al archivo likelihoodNuBars. txt, también es aquí que se construye la superficie equivalente $\Delta \mathscr{L}\left(\sin ^{2} 2 \theta, \Delta m^{2}\right)=\mathscr{L}\left(\sin ^{2} 2 \theta, \Delta m^{2}\right)-\mathscr{L}_{\min }$. Posteriormente empiezan las definiciones para generar los experimentos ficticios. En la línea 450 se corrobora que no se este trabajando con puntos, que por su lejanía del mejor ajuste, se encuentren exentos de calcular correcciones frecuentistas. De ahí hasta la línea 550 se genera el arreglo de vectores ficticios. Alrededor de la línea 560 hasta la 648 se hace la minimización de $\mathscr{L}_{\mathrm{F}}\left(\sin ^{2} 2 \theta, \Delta m^{2}\right)$ para cada experimento ficticio, la diferencia entre este bloque y el que esta entre la línea 320 y 435 es que aquí se usan datos ficticios, pero el algoritmo es el mismo. En esta parte es donde se construye la distribución de experimentos ficticios $\Delta \mathscr{L}_{\mathbf{f} i}^{\left\{\boldsymbol{p}_{0}\right\}}, i=1, \ldots, n_{\mathbf{f}}$ del punto $\boldsymbol{p}_{0}=\left(\sin ^{2} 2 \theta, \Delta m^{2}\right)_{0}$, con la cual se obtiene un punto de la superficie efectiva $\chi_{\mathbf{E f}}^{2}\left(\boldsymbol{p}_{0}\right)$.

Código A.1: Código serial en $\mathrm{C}++{ }_{21}$ frequentistFC_NuBar.cpp para llevar a cabo las correcciones frecuentistas en el modo de antineutrinos.

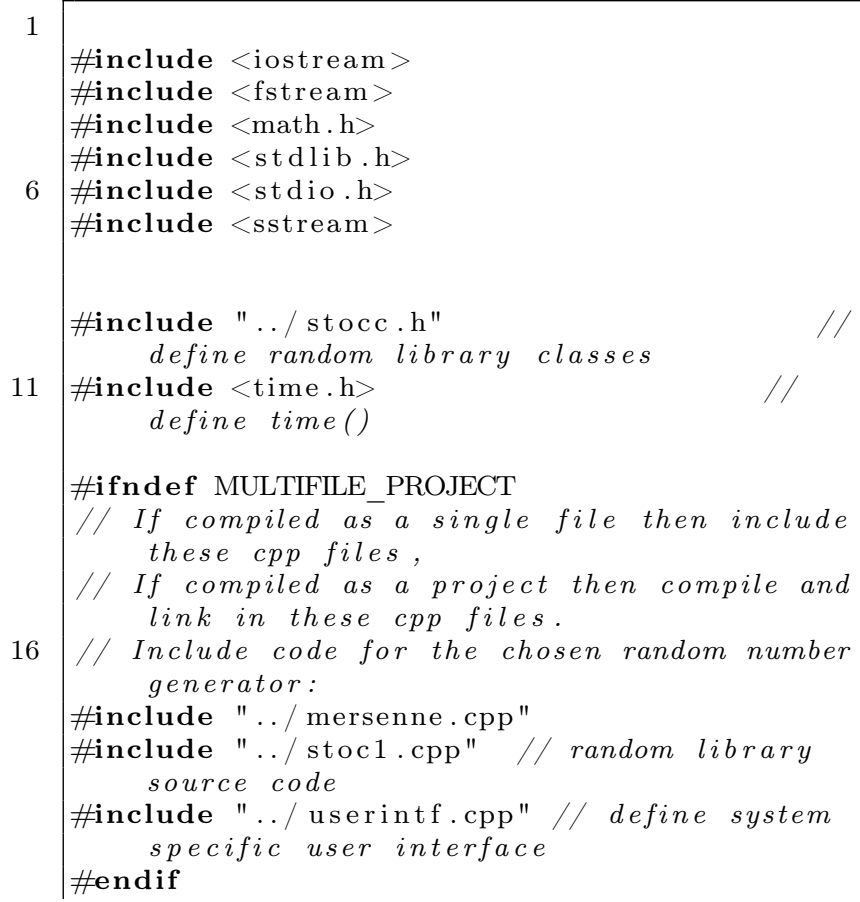

1

\#include $<$ iostream $>$

\#include $<$ fstream $>$

\#include $<$ math. $\mathrm{h}>$

\#include $<$ stdlib.h $>$

6

\#include $<$ sstream $>$

\#include "../ stocc.h"

define random library classes

11

\#include <time. $\mathrm{h}>$

define time()

\#ifndef MULTIFILE_PROJECT

// If compiled as a single file then include these cpp files,

// If compiled as a project then compile and link in these cpp files.

/ Include code for the chosen random number generator:

\#include "../ mersenne.cpp"

\#include "../stoc $1 . \mathrm{cpp} "$ // random library source code

\#include "../ userintf.cpp" // define system \#endif specific user interface

16
${ }^{6}$ Superficie teóricamente equivalente, pero que en la práctica no es tan fina debido a problemas de interpolación. \#define $\mathrm{NB} 11$

\#define $\mathrm{NBmu} 8$

\#define NBT $(2 * \mathrm{NB}+\mathrm{NBmu}) / /$ number bins total

\#define $\mathrm{NBC}(\mathrm{NB}+\mathrm{NBmu}) / /$ number of bins collapsed

26 \#define N $190 / /$ Grid

\#define NF 2048

\#define step 3

using namespace std;

31

float dm2Tic(int index)

float $\operatorname{dm} 2 \mathrm{~min}=1 . \mathrm{e}-02$;

float $\operatorname{dm} 2 \max =1 . \mathrm{e} 02$;

return pow $(10,(\log 10(\mathrm{dm} 2 \mathrm{~min})+\operatorname{index} *($ $\log 10(\mathrm{dm} 2 \max )-\log 10(\mathrm{dm} 2 \mathrm{~min})) /(\mathrm{N}-1)) \quad)$;

\}

float s2tTic(int index)\{

float $\mathrm{s} 2 \mathrm{t} \min =3 . \mathrm{e}-04$;

41

float $\mathrm{s} 2 \mathrm{t} \max =1$;

return exp10f( $\log 10(\operatorname{sitmin})+$ index $*(\log 10($

\} $\mathrm{s} 2 \mathrm{tmax})-\log 10(\mathrm{~s} 2 \mathrm{tmin})) /(\mathrm{N}-1))$;

float s2tTicCU(int index)\{

return exp10f $(-3.522878745 \mathrm{f}+$ index $*(3.522878745 \mathrm{f}) /(\mathrm{N}-1))$;

\} 
bool lowerRegion(double $\mathrm{x}$, double $\mathrm{y})\{$

double $\mathrm{x} 1=0$;

double $\mathrm{y} 1=78$;

double $\mathrm{x} 2=180$;

double $\mathrm{y} 2=0$;

if $((((\mathrm{y} 2-\mathrm{y} 1) /(\mathrm{x} 2-\mathrm{x} 1)) *(\mathrm{x}-\mathrm{x} 1)+\mathrm{y} 1)>\mathrm{y})\{$

\} return true

else

\}

return false;

61

bool upperRegion(double $\mathrm{x}$, double $\mathrm{y})\{$

double $\mathrm{x} 1=93$;

double $\mathrm{y} 1=190$;

double $\mathrm{x} 2=190$;

66

double $\mathrm{y} 2=60$

if $((((\mathrm{y} 2-\mathrm{y} 1) /(\mathrm{x} 2-\mathrm{x} 1)) *(\mathrm{x}-\mathrm{x} 1)+\mathrm{y} 1)<\mathrm{y})\{$

else

71

\}

return false;

bool upperSquare (double $\mathrm{x}$, double $\mathrm{y})\{$

if $(x>94 \& \& y>94)$

return true;

else

return false;

81

\}

float fileExists (const char $*$ fileName) \{

ifstream infile (fileName);

86

\}

return infile. $\operatorname{good}()$;

float $*$ choleskyFactor (float matrix []) \{

float epsilon $=1 \mathrm{e}-8$;

float sumh2;

float $\mathrm{h}[\mathrm{NBC} *(\mathrm{NBC}+1) / 2]$

// Calculate the lower triangular matrix such that:

96

$/ / h \quad x\left(h^{\wedge} T\right)=$ matrix

for (int $\quad \mathrm{i}=0 ; \quad \mathrm{i}<\mathrm{NBC} ; \quad \mathrm{i}++)\{$

if (fabs (matrix [0])>epsilon $)\{$ $\mathrm{h}[\mathrm{i} *(\mathrm{i}+1) / 2]=\operatorname{matrix}[\mathrm{i} *(\mathrm{i}+1) / 2+0] /$

\} sqrt (matrix [0]);

101

\}

else

$\mathrm{h}[\mathrm{i} *(\mathrm{i}+1) / 2]=0.0 ;$

for $($ int $\mathrm{j}=1 ; \mathrm{j}<\mathrm{NBC} ; \mathrm{j}++)\{$

sumh $2=0.0 ;$

for (int $\mathrm{jj}=0 ; \quad \mathrm{j}<\mathrm{j} ; \quad \mathrm{j} \mathrm{j}++)\{$

$\operatorname{sumh} 2+=h[j *(j+1) / 2+j j] * h[j *(j+1) / 2+j j$ ];

\}

$\mathrm{h}[\mathrm{j} *(\mathrm{j}+1) / 2+\mathrm{j}]=\operatorname{sqrt}(\operatorname{matrix}[\mathrm{j} *(\mathrm{j}+1) / 2+$ j] $-\operatorname{sumh} 2)$;

111

for $($ int $\quad \mathrm{i}=\mathrm{j} ; \quad \mathrm{i}<\mathrm{NBC} ; \quad \mathrm{i}++)\{$

$\operatorname{sumh} 2=0$;

for $($ int $\quad \mathrm{i} i=0 ; \quad \mathrm{ii}<\mathrm{j} ; \quad \mathrm{i} \mathrm{i}++)\{$

$\operatorname{sumh} 2+=\mathrm{h}[\mathrm{i} *(\mathrm{i}+1) / 2+\mathrm{i} i] * \mathrm{~h}[\mathrm{j} *(\mathrm{j}+1) / 2+$

i i ] ;

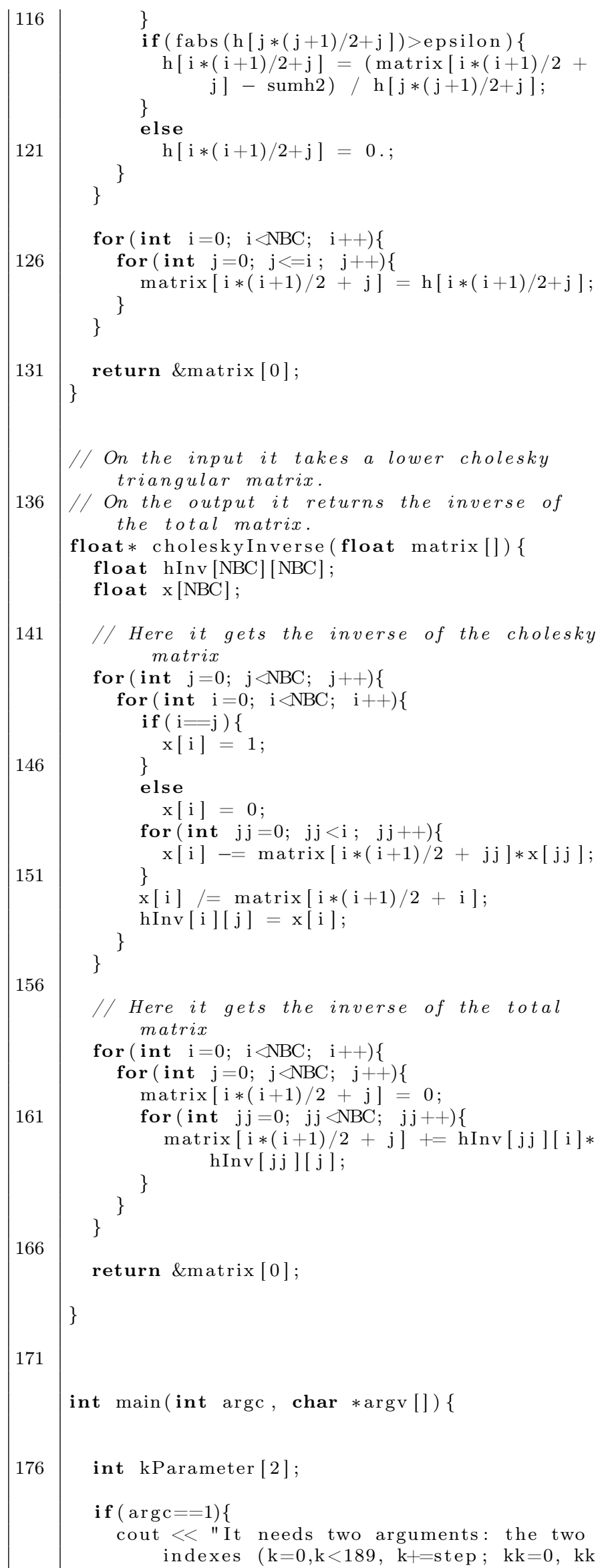


$<189$, kk=+step) for the fake

experiments, only multiples of step." $<<$ endl

else \{

$\mathrm{kParameter}[0]=\operatorname{atoi}(\operatorname{argv}[1])$

if $(\mathrm{kParameter}[0] \%$ step $!=0 \quad|| \mathrm{kParameter}$ $[0]<0 \quad||$ kParameter $[0]>189)\{$

cout $<<$ "It needs two arguments: the two indexes $(\mathrm{k}=0, \mathrm{k}<189, \mathrm{k}+=\mathrm{step} ; \mathrm{kk}$ $=0, \mathrm{kk}<189, \mathrm{kk}=+$ step ) for the fake experiments, only multiples of step . " $<$ endl;

$\mathrm{kParameter}[1]=$ atoi $(\operatorname{argv}[2])$;

if $(\mathrm{kParameter}[1] \%$ step $!=0 \quad|| \mathrm{kParameter}$

$[1]<0 \quad||$ kParameter $[1]>189)\{$

cout $<<$ "It needs two arguments: the two indexes $(\mathrm{k}=0, \mathrm{k}<189, \mathrm{k}+=\mathrm{step} ; \mathrm{kk}$ $=0, \mathrm{kk}<189$, $\mathrm{kk}=+$ step ) for the fake experiments, only multiples of step . " $<<$ endl;

cout $<<$ "Reading data files. $\backslash \mathrm{n} "<<$ endl;

float $*$ da NuebarNumubar $\mathrm{h}$;

da_NuebarNumubar_h = new float $[\mathrm{NBC}]$;

ifstream daNuebārFile ("../NuBarData/ miniboone nuebardata lowe.txt");

if (daNuebarFile e is open $(\overline{)})\{$

for (int $\mathrm{i}=0 ; \quad \mathrm{i}<\overline{\mathrm{NB}} ; \mathrm{i}++)\{$ \} daNuebarFile $\gg$ da_NuebarNumubar_h[i ];

daNuebarFile. close ();

else \{

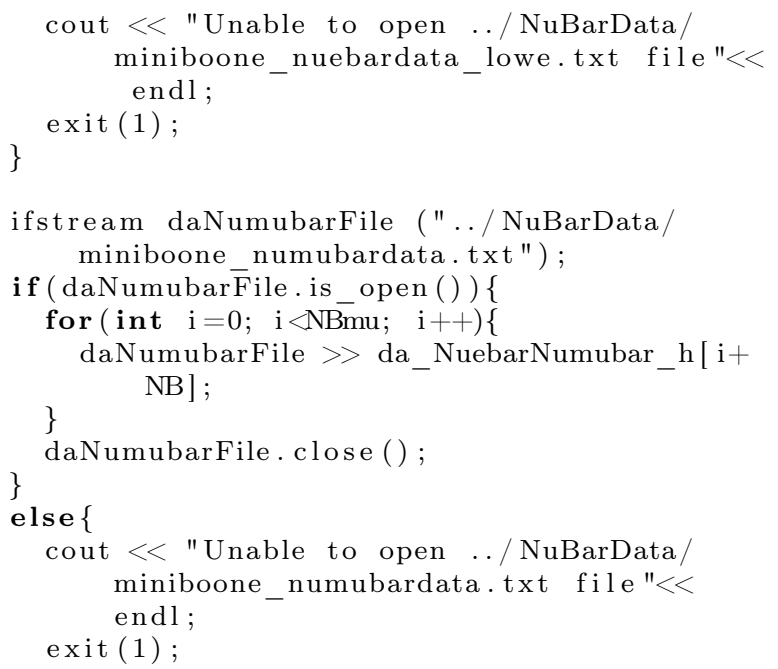

ifstream daNumubarFile ("../NuBarData/ miniboone_numubardata.txt");

if (daNumubar $\bar{F}$ ile. is open ()) \{

for (int $\mathrm{i}=0 ; \quad \mathrm{i}<\mathrm{NBmu} ; \quad \mathrm{i}++$ ) \{ daNumubarFile $\gg$ da NuebarNumubar h $[\mathrm{i}+$ $\mathrm{NB}]$; daNumubarFile. close ();

else \{

cout $<<$ "Unable to open ../NuBarData/ miniboone_numubardata.txt file $"<<$ endl ;

exit (1);

\}

\}

float *sgbgNueNumubarSMALL_h;

sgbgNueNumubarSMALL_h $=$ new float $[\mathrm{NBC}]$;

ifstream bgNuebarFile ("../NuBarData/ miniboone_nuebarbgr_lowe.txt");

if (bgNuebarFile. is_open ()) \{

for (int $\mathrm{i}=0 ; \quad \mathrm{i}<\overline{\mathrm{NB}} ; \quad \mathrm{i}++)\{$

bgNuebarFile $\gg$ sgbgNueNumubarSMALL_h[i ];

\}

bgNuebarFile.close ();

else \{

cout $<<$ "Unable to open ../NuBarData/ miniboone nuebarbgr lowe.txt file $"<<$ endl ; exit (1)

float * collapsed SgBgNueNumubarSMALL $\mathrm{h}$; collapsed_SgBgNueNumubarSMALL_h $=$ new float [NBmu ];

ifstream bgNumubarFile ("../NuBarData/ miniboone numubar.txt");

if (bgNumubarFile. is open ()) \{

for (int $\mathrm{i}=0 ; \mathrm{i}<\mathrm{NBmu} ; \mathrm{i}++)\{$ bgNumubarFile $\gg$ collapsed SgBgNueNumubarSMALL h [i ]; sgbgNueNumubarSMALL $\mathrm{h}[\mathrm{i}+\mathrm{NB}]=$ collapsed_SgBgNueNumubarSMALL_h [i ]

\} \}

bgNumubarFile. close ();

else \{

cout $<<$ "Unable to open ../NuBarData/ miniboone_numubar.txt file" $<<$ endl; \} exit (1)

float $*$ fracMat $3 \times 3$ h ; fracMat3x3_h = new float [NBT $* \mathrm{NBT}]$; ifstream fracMat3x3File ("../NuBarData/ miniboone_full_fractcovmatrix_nubar_lowe .txt"); 
301

float $* \operatorname{sgNuebar} \mathrm{h}$;

sgNuebar $\mathrm{h}=$ new float $[\mathrm{NB} * \mathrm{~N}]$;

ifstream ${ }^{-}$sgNuebarFile ("signalsbarSMALL.txt ");

if ( $\operatorname{sgNuebarFile.is\_ open~())~}\{$

for $($ int $\mathrm{kk}=0 ; \mathrm{kk}<\mathrm{N} ; \mathrm{kk}++)\{$

for (int $\quad$ i i $=0 ;$ i i $<\mathrm{NB} ; \quad$ i i ++$)\{$ sgNuebarFile $\gg$ sgNuebar_h[ii $+\mathrm{NB} * \mathrm{kk}$ ];

\}

exit (1);

cout $<<$ "Starting Real Likelihood surface calculation. $\backslash \mathrm{n} "<<$ endl ;

float deltaLikelyReal $[\mathrm{N}][\mathrm{N}]=\{0$.$\} ;$

float likelyRealBF $=1 \mathrm{e} 5$;

int min_position [2];

float collapsed SgBgNueNumubar [NBC] ;

float sgbgNueNumubar [NBT];

float totMat $2 \times 2[\mathrm{NBC} *(\mathrm{NBC}+1) / 2]$;

float detolog;

float *lowerMat $\mathrm{h}$;

lowerMat_h $=\mathbf{n e w}$ float $[\mathrm{NBC} *(\mathrm{NBC}+1) / 2]$;

float $*$ invMat $\mathrm{h}$;

invMat_h $=\mathbf{n e w}$ float $[\mathrm{NBC} *(\mathrm{NBC}+1) / 2]$;

for (int $\quad \mathrm{i}=0 ; \quad \mathrm{i}<\mathrm{NB} ; \quad \mathrm{i}++)\{$

sgbgNueNumubar $[\mathrm{i}+\mathrm{NB}]=$ sgbgNueNumubarSMALL_h [ i ];

\}

for (int $\quad \mathrm{i}=0 ; \quad \mathrm{i}<\mathrm{NBmu} ; \quad \mathrm{i}++$ )

collapsed_SgBgNueNumubar $[i+N B]=$ collapsed_SgBgNueNumubarSMALL_h [i ];

$\operatorname{sgbgNueNumubar}[\mathrm{i}+2 * \mathrm{NB}]=$ sgbgNueNumubarSMALL_h $[\mathrm{i}+\mathrm{NB}]$;

\}

for (int $\mathrm{k}=0 ; \mathrm{k}<\mathrm{N} ; \mathrm{k}++)\{/ / \mathrm{s} 2 t$

for $($ int $\mathrm{kk}=0 ; \mathrm{kk}<\mathrm{N} ; \mathrm{kk}++)\{/ / d m 2$

for (int $\mathrm{i}=0 ; \quad \mathrm{i}<\mathrm{NB} ; \quad \mathrm{i}++)\{$

collapsed_SgBgNueNumubar $[\mathrm{i}]=(\mathrm{s} 2 \mathrm{t}$ Tic $(\mathrm{k}) * \overline{\mathrm{s} g N u e b a r} \mathrm{~h}[\mathrm{i}+\mathrm{NB} * \mathrm{kk}])+$ sgbgNueNumubarSMALL_h [i ] ; $\operatorname{sgbgNueNumubar}[\mathrm{i}]=\operatorname{s} 2 \mathrm{t} \overline{\mathrm{T}} \mathrm{ic}(\mathrm{k}) *$ \} sgNuebar_h[i $+\mathrm{NB} * \mathrm{kk}]$;

/ Total matrix creation and collapsing for (int $\mathrm{i}=0 ; \quad \mathrm{i}<\mathrm{NB} ; \mathrm{i}++)\{$

for $($ int $\mathrm{j}=0 ; \mathrm{j}<\mathrm{i}+1 ; \mathrm{j}++)\{$ $\operatorname{totMat} 2 \times 2[i *(i+1) / 2+j]=$ fracMat $3 \times 3$ h $[\mathrm{i} * \mathrm{NBT}+\mathrm{j}] *$ sgbgNueNumubar [i] * sgbgNueNumubar [j] + fracMat3x3_h $[(\mathrm{i}+\mathrm{NB}) * \mathrm{NBT}+\mathrm{j}] *$ sgbgNueNumubar $[i+N B] *$ sgbgNueNumubar [j] + fracMat3x 3 h $[\mathrm{i} * \mathrm{NBT}+(\mathrm{j}+\mathrm{NB})] *$ sgbgNueNumubar [i]* $\operatorname{sgbgNueNumubar}[\mathrm{j}+\mathrm{NB}]+$ fracMat3x3_h $[(\mathrm{i}+\mathrm{NB}) * \mathrm{NBT}+(\mathrm{j}+\mathrm{NB})$ ]*sgbgNueNumubar $[\mathrm{i}+\mathrm{NB}] *$ sgbgNueNumubar $[j+\mathrm{NB}]$;

\}

totMat $2 \times 2[i *(i+1) / 2+i]+=$

\} sgbgNueNumubar [i ];

for $($ int $\mathrm{i}=2 * \mathrm{NB} ; \quad \mathrm{i}<\mathrm{NBT} ; \mathrm{i}++)\{$

for (int $\mathrm{j}=0 ; \mathrm{j}<\mathrm{NB} ; \mathrm{j}++)\{$ totMat $2 \times 2[(\mathrm{i}-\mathrm{NB}) *((\mathrm{i}-\mathrm{NB})+1) / 2+\mathrm{j}]=$ fracMat $3 \times 3$ h $[\mathrm{i} * \mathrm{NBT}+\mathrm{j}] *$ sgbgNueNumūbar [i] $*$ sgbgNueNumubar [j] + fracMat $3 \times 3$ h $[i * N B T+(j+N B)] *$ sgbgNueNumubar [i ] * \} sgbgNueNumubar $[\mathrm{j}+\mathrm{NB}]$;

\}

for (int $\mathrm{i}=2 * \mathrm{NB} ; \mathrm{i}<\mathrm{NBT} ; \mathrm{i}++)\{$

for (int $\mathrm{j}=2 * \mathrm{NB} ; \mathrm{j}<\mathrm{i}+1 ; \mathrm{j}++)\{$ totMat $2 \times 2[(\mathrm{i}-\mathrm{NB}) *((\mathrm{i}-\mathrm{NB})+1) / 2+(\mathrm{j}-$ $\mathrm{NB})]=$ fracMat $3 \times 3 \quad \mathrm{~h}[\mathrm{i} * \mathrm{NBT}+\mathrm{j}] *$ sgbgNueNumubar [i $] \bar{*}$ \} 
\}

deltaLikelyReal[k][kk] $+=($

da NuebarNumubar h[i] collapsed_SgBgNuēNumubar [i ] ) * invMat_h[ $\mathrm{i} *(\mathrm{i}+1) / 2+\mathrm{i}] *($

da_NuebarNumubar_h[i] -

\}

collapsed SgBgNueNumubar [i ]);

deltaLikelyReal[k][kk] += detolog;

if ( isnan (deltaLikelyReal[k][kk]))\{ cout $<<$ "deltaLikelyReal: " $<<$ deltaLikelyReal [k][kk] $<<"$ CHANGOS NAN! Index : $\quad "<<\mathrm{k}<<"$ " $<<\mathrm{kk}<<$ endl; exit (1);

\}

if ( deltaLikelyReal [k] [ kk]<likelyRealBF ) \{ likelyRealBF $=$ deltaLikelyReal $[\mathrm{k}][\mathrm{kk}$ ]; min position $[0]=\mathrm{k}$; \} min_position $[1]=\mathrm{kk}$

cout $<<$ fixed $<<$ "likelyRealBF $\backslash$ t" $<<$ likelyRealBF $<<$ endl;

cout $<<$ fixed $<<$ "s2t $\backslash \mathrm{t} "<<$ min position $[0]<<" \backslash \mathrm{t} "<<$ s2tTic (min_position [0]) $<<$ endl

cout $<<$ fixed $<<$ "dm2 $\backslash$ t" $<<$ min_position [1] $<<" \backslash \mathrm{t} "<<\operatorname{dm} 2 \mathrm{Tic}($ min_position $[1])<<$ endl ;

// Writing ntuple likelihood on a text file

Using the following format:

$/ /(D m 2(e V 2), \quad \sin 2(2 t h),-2 \ln (L))$

ofstream myfilentuple ("likelihoodNuBars. txt") ;

else cout $<<$ "Unable to open file "<<endl;

cout $<<$ "Printing likelihoodNuBars.txt" $<<$ endl;

cout $<<"$ $<<$ endl;

float epsilon $=1 \mathrm{e}-8$;

float $h[N B C][N B C]$;

float sumh2;

int32_t seed $=\left(\right.$ int $\left.32 \_\mathrm{t}\right)$ time $(0) ; / /$ Start random seed.

441

StochasticLib1 sto(seed);// Make instance of random library

float fakeVector $[\mathrm{NBC}]$;

float fluctuatedVector [NBC];

float gaussRand [NBC];
446 int $\mathrm{k}=\mathrm{kParameter}[0]$;

int $\mathrm{kk}=\mathrm{kParameter}[1]$;

// It will not try to find anything here. if (lowerRegion (k, kk) || upperRegion (k, kk) I| upperSquare (k, kk)) \{

cout $<<$ "I'm in forbidden region!" $<<$ endl;

\}

exit $(0)$

for (int $\quad \mathrm{i}=0 ; \quad \mathrm{i}<\mathrm{NB} ; \quad \mathrm{i}++)\{$

sgbgNueNumubar[i] = s2tTic $(\mathrm{k}) *$ sgNuebar_h[ $\mathrm{i}+\mathrm{NB} * \mathrm{kk}]$

collapsed_SgBgNueNumubar[i] = s2tTic $(\mathrm{k}) *$ sgNuebar_h[i $+\mathrm{NB} * \mathrm{kk}]+$

\} sgbgNueNümubarSMALL_h [i ];

//Total matrix creation and collapsing for (int $\mathrm{i}=0 ; \mathrm{i}<\mathrm{NB} ; \mathrm{i}++)\{$

for $($ int $\mathrm{j}=0 ; \mathrm{j}<\mathrm{i}+1 ; \mathrm{j}++)\{$ totMat $2 \times 2[i *(i+1) / 2+j]=$ fracMat $3 \times 3+h[$ $\mathrm{i} * \mathrm{NBT}+\mathrm{j}] * \operatorname{sgbgNueNumubar}[\mathrm{i}] *$ sgbgNueNumubar $[j]+$ fracMat $3 \times 3$ h $[(i$ $+\mathrm{NB}) * \mathrm{NBT}+\mathrm{j}] * \operatorname{sgbgNueNumubar}[\mathrm{i}+\mathrm{NB}] *$ sgbgNueNumubar $[j]+$ fracMat $3 \times 3$ h $[i *$ $\mathrm{NBT}+(\mathrm{j}+\mathrm{NB})] * \operatorname{sgbgNueNumubar}[\mathrm{i}] *$ sgbgNueNumubar $[j+N B]+$ fracMat $3 \times 3$ _h $[(\mathrm{i}+\mathrm{NB}) * \mathrm{NBT}+(\mathrm{j}+\mathrm{NB})] * \operatorname{sgbgNueNumubar}[$ \} $\mathrm{i}+\mathrm{NB}] * \operatorname{sgbgNueNumubar}[\mathrm{j}+\mathrm{NB}]$;

totMat $2 \times 2[i *(i+1) / 2+i]+=$ sgbgNueNumubar [i ]

\}

for (int $\mathrm{i}=2 * \mathrm{NB} ; \mathrm{i}<\mathrm{NBT} ; \mathrm{i}++)\{$

for (int $\mathrm{j}=0 ; \mathrm{j}<\mathrm{NB} ; \mathrm{j}++)$ \{ totMat $2 \times 2[(\mathrm{i}-\mathrm{NB}) *((\mathrm{i}-\mathrm{NB})+1) / 2+\mathrm{j}]=$ fracMat $3 \times 3 \_\mathrm{h}[\mathrm{i} * \mathrm{NBT}+\mathrm{j}] *$ $\operatorname{sgbgNueNumubar}[\mathrm{i}] * \operatorname{sgbgNueNumubar}[\mathrm{j}]$ + fracMat $3 \times 3$ _h $[\mathrm{i} * \mathrm{NBT}+(\mathrm{j}+\mathrm{NB})] *$ $\operatorname{sgbgNueNumubar}[\mathrm{i}] * \operatorname{sgbgNueNumubar}[\mathrm{j}+$ $\mathrm{NB}]$;

\}

for $($ int $\mathrm{i}=2 * \mathrm{NB} ; \quad \mathrm{i}<\mathrm{NBT} ; \mathrm{i}++)\{$

for $($ int $\mathrm{j}=2 * N B ; \quad \mathrm{j}<\mathrm{i}+1 ; \mathrm{j}++)\{$

totMat $2 \times 2[(\mathrm{i}-\mathrm{NB}) *((\mathrm{i}-\mathrm{NB})+1) / 2+(\mathrm{j}-\mathrm{NB})]$ $=$ fracMat $3 \times 3 \ldots h[i * N B T+j] *$ $\operatorname{sgbgNueNumubar}[\mathrm{i}] * \operatorname{sgbgNueNumubar}[\mathrm{j}$ ];

\}

]

float $*$ da_NuebarNumubarFakes_h; 
for (int $\mathrm{j}=1 ; \mathrm{j}<\mathrm{NBC} ; \mathrm{j}++)\{$

sumh2 $=0.0$

for $($ int $\quad j \mathrm{j}=0 ; \quad j \mathrm{j}<\mathrm{j} ; \quad \mathrm{j} \mathrm{j}++)\{$ \} $\operatorname{sumh} 2+=h[j][j j] * h[j][j j] ;$

$\mathrm{h}[\mathrm{j}][\mathrm{j}]=\operatorname{sqrt}(\operatorname{totMat} 2 \times 2[\mathrm{j} *(\mathrm{j}+1) / 2+\mathrm{j}]-$ sumh2);

for (int $i=j ; \quad i<N B C ; \quad i++)\{$ $\operatorname{sumh} 2=0$.;

for $($ int $\quad \mathrm{i} i=0 ; \quad \mathrm{ii}<\mathrm{j} ; \quad \mathrm{i} \mathrm{i}++)\{$ \} sumh $2+=\mathrm{h}[\mathrm{i}][\mathrm{i} i] * \mathrm{~h}[\mathrm{j}][\mathrm{i} i]$

if (fabs $(h[j][j])>\operatorname{epsilon})\{$ $\mathrm{h}[\mathrm{i}][\mathrm{j}]=(\operatorname{totMat} 2 \times 2[\mathrm{i} *(\mathrm{i}+1) / 2+\mathrm{j}]-$ sumh2) / h $[j][j]$;

\}

else

\}

$\mathrm{h}[\mathrm{i}][\mathrm{j}]=0$.;

511

\}

for (int $\mathrm{i}=0 ; \quad \mathrm{i}<\mathrm{NBC} ; \quad \mathrm{i}++)$ \{

for (int $\mathrm{j}=\mathrm{i}+1 ; \mathrm{j}<\mathrm{NBC} ; \mathrm{j}++)\{$ \}

$\mathrm{h}[\mathrm{i}][\mathrm{j}]=0$.

\}

seed $=($ int32_t $)$ time $(0)$;

// Loop to generate a collection of fake vectors.

for (int $\mathrm{kkk}=0 ; \mathrm{kkk}<\mathrm{NF} ; \mathrm{kkk}++)\{$

for (int $\mathrm{i}=0 ; \quad \mathrm{i}<\mathrm{NBC} ; \quad \mathrm{i}++)\{$ gaussRand $[\mathrm{i}]=$ sto. $\operatorname{Normal}(0,1)$;

for (int $\mathrm{i}=0 ; \quad \mathrm{i}<\mathrm{NBC} ; \quad \mathrm{i}++)\{$

fakeVector $[\mathrm{i}]=$ \} collapsed_SgBgNueNumubar [i ] ;

// Here we get the vector with fluctuations according to the

// covariance matrix. And the new fake vector.

for (int $\mathrm{i}=0 ; \mathrm{i}<\mathrm{NBC} ; \mathrm{i}++)\{$

fluctuatedVector $[i]=0$.;

for $($ int $\mathrm{j}=0 ; \mathrm{j}<\mathrm{NBC} ; \mathrm{j}++)\{$ if $(j>i)\{$

\}

break

fluctuatedVector [i] $+=h[i][j] *$ gaussRand $[\mathrm{j}]$;

541
\}

fakeVector [i] += fluctuatedVector [i ];
\}

for (int $\quad \mathrm{i}=0 ; \quad \mathrm{i}<\mathrm{NBC} ; \quad \mathrm{i}++)\{$

da_NuebarNumubarFakes_h $[\mathrm{i}+\mathrm{kkk} * \mathrm{NBC}]=$ fakeVector [i];

\}

\section{\} / for $k k k<N F$}

cout $<<$ "Fake data vector ready." $<<$ endl;

cout $<<$ "Starting effective likely array computation" $<<$ endl;

cout $<<$ "With: " $<<$ endl;

cout $<<" \mathrm{k}=$ " $<<$ kParameter $[0]<<", \quad \mathrm{kk}=$ " $<<$ kParameter $[1]<<$ endl

float deltaLikelyFakes [NF];

float ratioBiggerTemp;

float da NuebarNumubarTemp $[\mathrm{NBC}]$;

float collapsed SgBgNueNumubarTemp [NBC] ;

float sgbgNueNumubarTemp [NBT];

float totMat $2 \times 2 \mathrm{Temp}[\mathrm{NBC} *(\mathrm{NBC}+1) / 2]$;

float detologTemp;

float likelyTemp;

float likelyBFTemp $=1 \mathrm{e} 8$;

float likelyParameterTemp;

float *lowerMatTemp ;

float $*$ invMatTemp;

int $\mathrm{i}, \mathrm{j}, \mathrm{l}, \mathrm{ll}$;

for $($ int $\mathrm{kkk}=0 ; \mathrm{kkk}<\mathrm{NF} ; \mathrm{kkk}++)\{$

for $(i=0 ; \quad i<N B C ; \quad i++)\{$ da_NuebarNumubarTemp [i] = da_NuebarNumubarFakes_h $[\mathrm{i}+\mathrm{NBC} * \mathrm{kkk}]$;

\}

for $(i=0 ; \quad i<N B ; \quad i++)\{$

sgbgNueNumubarTemp $[i+N B]=$ sgbgNueNumubarSMALL_h [i] ;

\}

for $(i=0 ; \quad i<N B m u ; \quad i++)\{$

collapsed_SgBgNueNumubarTemp $[\mathrm{i}+\mathrm{NB}]=$ collapsed SgBgNueNumubarSMALL h [i]

$\operatorname{sgbgNueNumub}$ arTemp $[\mathrm{i}+2 * \mathrm{NB}]=$ sgbgNueNumubarSMALL_h $[\mathrm{i}+\mathrm{NB}]$;

likelyBFTemp $=1 \mathrm{e} 8$

for $(1=0 ; \quad l<\mathrm{N} ; \quad 1++)\{$

for $(11=0 ; \quad 11<N ; \quad 11++)\{$

for $(i=0 ; \quad i<N B ; \quad i++)\{$ collapsed_SgBgNueNumubarTemp $[\mathrm{i}]=$ ( s2tTic CU $(1) * \operatorname{sgNuebar} \mathrm{h}[\mathrm{i}+\mathrm{NB} *$ 11]) + sgbgNueNumubarSMALL_h[i ];

$\operatorname{sgbgNueNumubarTemp}[\mathrm{i}]=\operatorname{s} 2 \mathrm{tTicCU}(1)$ \} *sgNuebar_h[i $+\mathrm{NB} * 11]$;

for $(i=0 ; \quad i<N B ; \quad i++)\{$ for $(j=0 ; \quad j<i+1 ; j++)\{$ 
totMat $2 \times 2 \operatorname{Temp}[\mathrm{i} *(\mathrm{i}+1) / 2+\mathrm{j}]=$ fracMat $3 \times 3$ _h $[\mathrm{i} * \mathrm{NBT}+\mathrm{j}] *$ sgbgNueNumubarTemp [i ] * sgbgNueNumubarTemp [j] + fracMat3x3_h $[(\mathrm{i}+\mathrm{NB}) * \mathrm{NBT}+\mathrm{j}] *$ sgbgNueNumubarTemp [i+NB]* sgbgNueNumubarTemp [j] + fracMat3x3_h $[\mathrm{i} * \mathrm{NBT}+(\mathrm{j}+\mathrm{NB})] *$ sgbgNueNumubarTemp [i] * sgbgNueNumubarTemp $[\mathrm{j}+\mathrm{NB}]+$ fracMat $3 \times 3$ h $[(\mathrm{i}+\mathrm{NB}) * \mathrm{NBT}+(\mathrm{j}+\mathrm{NB}$ )] $\operatorname{sgbgNue} \bar{N}$ umubarTemp [i+NB]* sgbgNueNumubarTemp $[\mathrm{j}+\mathrm{NB}]$;

\}

totMat $2 \times 2$ Temp $[\mathrm{i} *(\mathrm{i}+1) / 2+\mathrm{i}]+=$ \} sgbgNueNumubarTemp [i ] ;

for $(i=2 * N B ; \quad i<N B T ; \quad i++)\{$

for $(\mathrm{j}=0 ; \mathrm{j}<\mathrm{NB} ; \mathrm{j}++)\{$

totMat $2 \times 2$ Temp $[(\mathrm{i}-\mathrm{NB}) *((\mathrm{i}-\mathrm{NB})+1) / 2$ $+\mathrm{j}]=$ fracMat $3 \times 3 \ldots h[\mathrm{i} * \mathrm{NBT}+\mathrm{j}] *$ sgbgNueNumubarTemp [i ]* sgbgNueNumubarTemp [j] + fracMat3x3_h [i $* \mathrm{NBT}+(\mathrm{j}+\mathrm{NB})] *$ sgbgNueNumubarTemp [i ]* sgbgNueNumubarTemp [ j+NB] ; \}

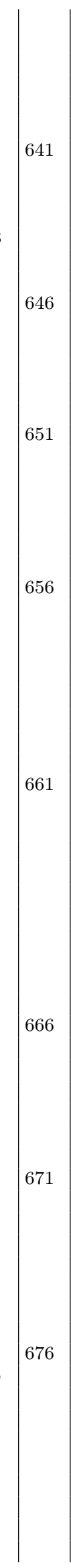

\author{
likelyTemp $+=\operatorname{detologTemp}$; \\ likelyBFTemp $=($ likelyTemp $<$ \\ likelyBFTemp) ? likelyTemp : \\ likelyBFTemp ;
likelyParameterTemp $=(\mathrm{kParameter}$ $[0]==1 \quad \& \&$ kParameter $[1]==11)$ ? likelyTemp : likelyParameterTemp ;

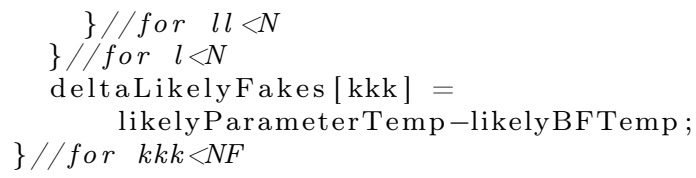

ofstream chi2FrecFCNuBar (name.c_str(), ios $:$ : app );

if (chi2FrecFCNuBar . is_open ()) \{ chi2FrecFCNuBar $<<$ fixed $<<$ dm2Tic ( kParameter $[1])<<" \backslash \mathrm{t} "<<\mathrm{s} 2 \mathrm{tTic}($ kParameter $[0])<<" \backslash \mathrm{t} "<<-2 * \log ($ ratioBiggerTemp /NF) $<<" \backslash \mathrm{t} "<<$ ratioBiggerTemp $<<" \backslash \mathrm{t} "<<\mathrm{NF}<<$ endl ; \} chi2FrecFCNuBar.close ();

else cout $<<$ "Unable to open " $<$ name. $c \operatorname{str}()<<"$ file $"<<$ endl;

cout $\overline{<}<$ "Printing " $<<$ name. $c_{-}$str ()$<<$ endl;

bool itExist $=$ fileExists (nameDistribution. c_str());

ofstream fakeDistos (nameDistribution.c str (), ios::app);

if (fakeDistos.is_open ()) \{

if (! itExist) \{

fakeDistos $<<$ "\#\#\# k" $<<" \backslash \mathrm{t} "<<$ "kk" $<<$ endl;

fakeDistos $<<$ "\#\#\# $"<<$ kParameter [0] $<<" \backslash \mathrm{t} "<<$ kParameter $[1]<<$ endl;

fakeDistos $<<$ fixed $<<$ s 2 tTic ( kParameter $[0])<<" \backslash \mathrm{t} "<<\operatorname{dm} 2$ Tic ( kParameter $[1])<<$ endl ;

fakeDistos $<<$ fixed $<<$ "deltaLikelyReal $="<<" \backslash \mathrm{t} "<<$ deltaLikelyReal [ kParameter [0]] [kParameter [1]] $<<$ \} endl ;

for $($ int $\mathrm{kkk}=0 ; \mathrm{kkk}<\mathrm{NF} ; \mathrm{kkk}++)\{$ 
681

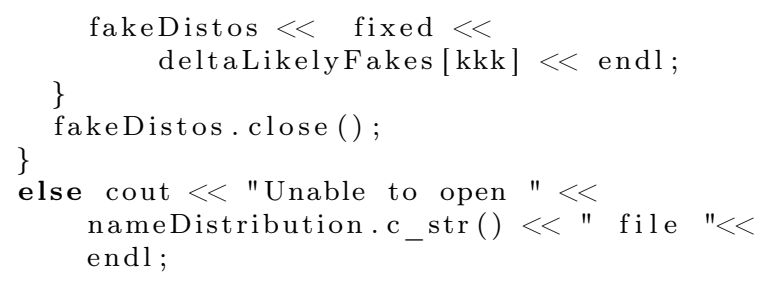

\section{A.2. Código heterogéneo en CUDA}

En $C U D A$ no se tiene información detallada sobre el tiempo que tarda una sola iteración, mas bien se cuenta con el tiempo que tarda una ejecución del kernel. Se inicializan 128 blocks con 96 threads cada uno $^{7}$ esta combinación es la que maximiza la eficiencia al maximizar la transferencia de datos. En una ejecución del kernel se calculan las correcciones frecuentistas para 6 puntos distintos, es decir se hacen $6 \times 2048$ iteraciones las cuales tardan 521.646 s o 8 min y 41 s. Logrando una mejora de $\sim 24 \times$ sobre el rendimiento obtenido en $\mathrm{C}++$. Se uso una tarjeta gráfica NVIDIA Tesla C2075, algunas características de la misma se encuentran en la tabla A.3.

\begin{tabular}{ll}
\hline CUDA Driver Version / Runtime Version & $5.0 / 5.0$ \\
CUDA Capability Major/Minor version number: & 2.0 \\
Total amount of global memory: & 5375 MBytes ( 5636292608 bytes) \\
(14) Multiprocessors $\times($ 32) CUDA Cores/MP: & $448 \mathrm{CUDA}$ Cores \\
GPU Clock rate: & $1147 \mathrm{MHz}(1.15 \mathrm{GHz})$ \\
Memory Clock rate: & $1566 \mathrm{Mhz}$ \\
Memory Bus Width: & 384 -bit \\
Total amount of constant memory: & 65536 bytes \\
Total number of registers available per block: & 32768 \\
Warp size: & 32 \\
Maximum number of threads per multiprocessor: & 1536 \\
Maximum number of threads per block: & 1024 \\
Maximum sizes of each dimension of a block: & $1024 \times 1024 \times 64$ \\
Maximum sizes of each dimension of a grid: & $65535 \times 65535 \times 65535$ \\
Maximum memory pitch: & 2147483647 bytes \\
Concurrent copy and kernel execution: & Yes with 2 copy engine $(\mathrm{s})$ \\
Run time limit on kernels: & No \\
\hline
\end{tabular}

Tabla A.3: Especificaciones de la tarjeta NVIDIA Tesla C2075.

Así como esta el código no es capaz de ejecutarse en cualquier tarjeta gráfica, ya que requiere 115 MB de memoria ${ }^{8}$. Se uso una tarjeta dedicada a hacer cálculos por lo cual ha sido liberada de procesar los gráficos de la computadora en que se encuentra conectada y no tiene un tiempo límite de ejecución. Portar el código a una tarjeta con tiempo límite de ejecución o que no disponga de tanta memoria requiere que la cantidad de operaciones que lleva a cabo el kernel sea disminuida.

\footnotetext{
${ }^{7}$ Se define manualmente la cantidad de threads, los blocks quedan entonces definidos. Ver la línea 567 del código A.2

${ }^{8}$ Memoria de la tarjeta gráfica
} 
A pesar de que el código en CUDA sigue un esquema heterogéneo, con ciertas operaciones ejecutadas de forma serial y otras de forma paralela, se reconoce que no se ha maximizado del todo esta característica y no se ha hecho ningún esfuerzo en mantener el procesador del CPU ocupado mientras ocurre la ejecución del kernel.

Para compilar se usa el compilador nvcc de CUDA con la opción de máxima optimización: -03 ${ }^{9}$. Además se usa la opción -ptxas-options=-v para obtener información sobre la cantidad de registros que usa el programa, esto con el objetivo de encontrar la combinación de threads y blocks que maximizan la ocupación: 192 y 128, respectivamente. Como ya se menciono antes se eligió maximizar la transferencia de datos, por que aún cuando el rendimiento en los dos casos (maximizando la ocupación y maximizando la transferencia de datos) es esencialmente idéntico maximizar la ocupación limita la cantidad de registros de los que se dispone.

\$ nvcc frequentistFC_NuBar.cu -o freqDev1 --ptxas-options=-v -03

Para ejecutar los programas se uso el comando nohup para que corrieran sin necesidad de mantener la conexión con la computadora anfitrion:

\$ nohup time./freqDev1 $01>$ run1.out 2> run1.err $</$ dev/null \&

La información que se imprime a la pantalla es almacenada en el archivo run1.out y la información sobre el estado final de la ejecución, por ejemplo el tiempo que tardó la ejecución o la señal con la que acabó o fue interrumpida, es almacenada en el archivo run1.err

Dado que es una tarjeta la que se encarga de hacer todos los análisis solamente se imprime un archivo de la superficie efectiva $\chi_{\mathbf{E f}}^{2}$, likelyEff_NuBar2048NF.txt. Al igual que en el programa serial se imprime un archivo para cada distribución de $\Delta \mathscr{L}_{\mathbf{f} i}^{\left\{\boldsymbol{p}_{0}\right\}}, i=1 \ldots n_{\mathbf{f}}$ y se sigue la misma notación. El programa que analiza estas distribuciones es el mismo que en el caso serial.

Código A.2: Código en CUDA frequentistFC_NuBar.cu para llevar a cabo las correcciones frecuentistas en el modo de antineutrinos. generator:

${ }^{9}$ Esta opción solo optimiza la parte serial del código.

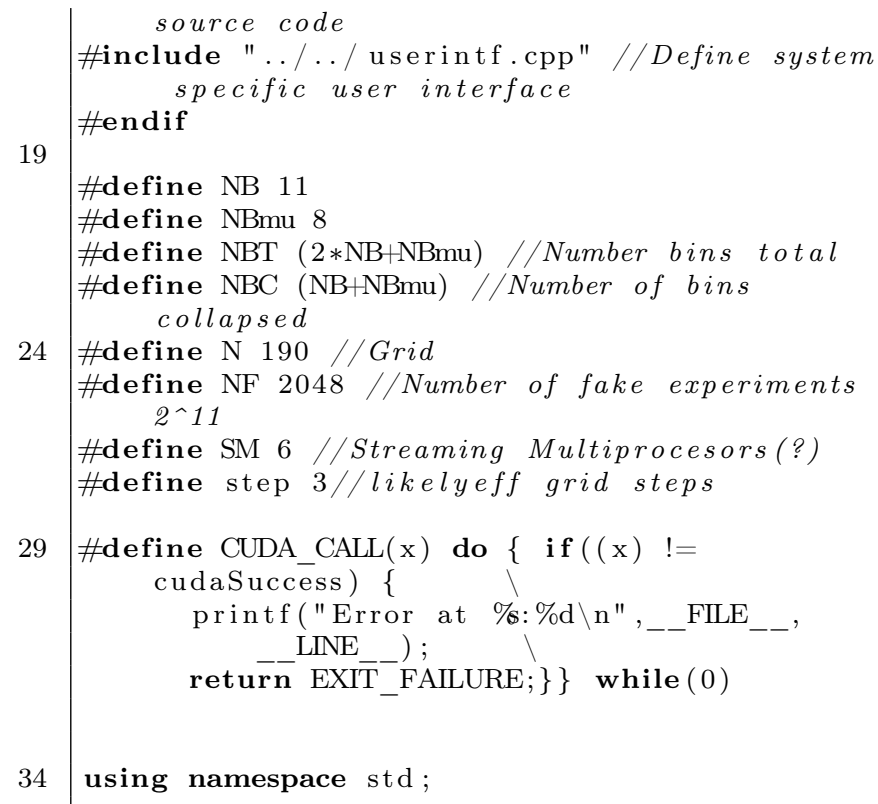


39

void cudaSafeCall ( cudaError t error, char* message $)\{$

if (error!=cudaSuccess) $\{$ fprintf(stderr," ERROR: \% : \% $\backslash \mathrm{n}^{\prime \prime}$, message, error ); exit $(-1) ; \quad\}$

44

host__ float dm2Tic(int index) \{

float $\mathrm{dm} 2 \mathrm{~min}=1 . \mathrm{e}-02$;

float $\mathrm{dm} 2 \max =1 . \mathrm{e} 02$;

return pow $(10,(\log 10(\mathrm{dm} 2 \mathrm{~min})+$ index $*($

\} $\log 10(\mathrm{dm} 2 \max )-\log 10(\mathrm{dm} 2 \min )) /(\mathrm{N}-1)) \quad)$;

_host_float s2tTic(int index) \{

float $\mathrm{s} 2 \mathrm{tmin}=3 . \mathrm{e}-04$;

float $\mathrm{s} 2 \mathrm{tmax}=1$;

return exp10f $(\log 10(\mathrm{~s} 2 \mathrm{tmin})+$ index $*(\log 10($

\} $\mathrm{s} 2 \mathrm{tmax})-\log 10(\mathrm{~s} 2 \mathrm{tmin})) /(\mathrm{N}-1))$;

54 \}

_host__ bool lowerRegion (double x, double y)

double $\mathrm{x} 1=0$;

double $\mathrm{y} 1=78$;

double $\mathrm{x} 2=180$;

double y $2=0$

if $((((\mathrm{y} 2-\mathrm{y} 1) /(\mathrm{x} 2-\mathrm{x} 1)) *(\mathrm{x}-\mathrm{x} 1)+\mathrm{y} 1)>\mathrm{y})\{$

64 \} return true

else

return false;

69

\}

__host__ bool upperRegion (double $\mathrm{x}$, double $\mathrm{y}$ ) \{

double $\mathrm{x} 1=93$;

double $\mathrm{y} 1=190$;

double $\mathrm{x} 2=190$

74

ouble $\mathrm{y} 2=60$

if $((((\mathrm{y} 2-\mathrm{y} 1) /(\mathrm{x} 2-\mathrm{x} 1)) *(\mathrm{x}-\mathrm{x} 1)+\mathrm{y} 1)<\mathrm{y})\{$

\}

return true;

else

79

\}

return false;

_host__ bool upperSquare (double $\mathrm{x}$, double $\mathrm{y}$ )

84

if $(\mathrm{x}>94 \& \& \mathrm{y}>94)$

return true;

else

return false;

89

\}

host bool fileExists (const char *fileName ) \{

ifstream infile (fileName);

94

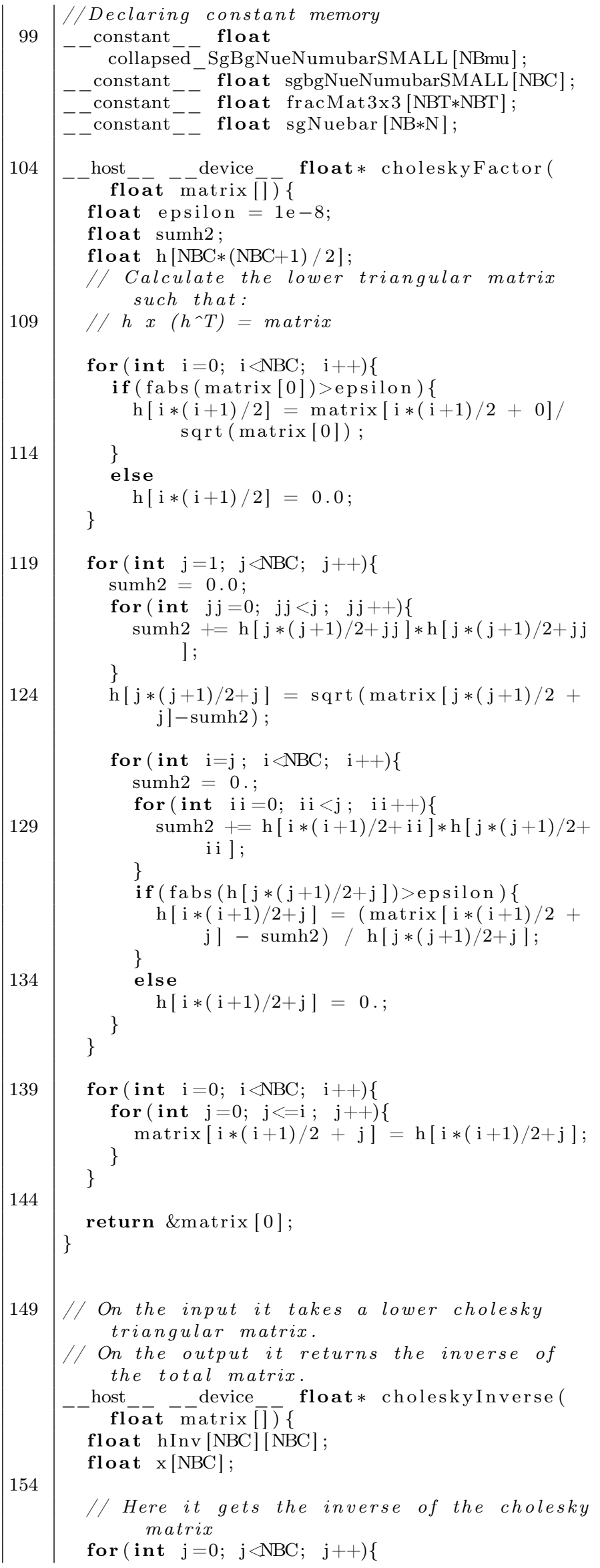

// On the input it takes a lower cholesky triangular matrix.

// On the output it returns the inverse of the total matrix.

host____ device __ float $*$ cholesky Inverse (

flōat matrix [] $)\{$

float hInv[NBC][NBC];

float $\mathrm{x}[\mathrm{NBC}]$;

// Here it gets the inverse of the cholesky matrix

for (int $\mathrm{j}=0 ; \mathrm{j}<\mathrm{NBC} ; \mathrm{j}++)\{$ 
for $($ int $\quad \mathrm{i}=0 ; \quad \mathrm{i}<\mathrm{NBC} ; \quad \mathrm{i}++)\{$

i f $(\mathrm{i}=\mathrm{j})\{$

\}

$\mathrm{x}[\mathrm{i}]=1$;

else

$\mathrm{x}[\mathrm{i}]=0$;

for (int $\mathrm{j} j=0 ; \quad \mathrm{j}<\mathrm{i} ; \mathrm{j} \mathrm{j}++)\{$

\}

$\mathrm{x}[\mathrm{i}]-=$ matrix $[\mathrm{i} *(\mathrm{i}+1) / 2+\mathrm{jj}] * \mathrm{x}[\mathrm{jj}]$;

$\mathrm{x}[\mathrm{i}] /=\operatorname{matrix}[\mathrm{i} *(\mathrm{i}+1) / 2+\mathrm{i}]$;

\}

$\mathrm{hInv}[\mathrm{i}][\mathrm{j}]=\mathrm{x}[\mathrm{i}]$

/ Here it gets the inverse of the total matrix

for (int $\mathrm{i}=0 ; \mathrm{i}<\mathrm{NBC} ; \mathrm{i}++)\{$

for (int $\mathrm{j}=0 ; \mathrm{j}<\mathrm{NBC} ; \mathrm{j}++)\{$

matrix $[\mathrm{i} *(\mathrm{i}+1) / 2+\mathrm{j}]=0$;

for (int $\mathrm{j} j=0 ; \quad \mathrm{j}<\mathrm{NBC} ; \mathrm{j} \mathrm{j}++)\{$ $\operatorname{matrix}[\mathrm{i} *(\mathrm{i}+1) / 2+\mathrm{j}]+=\operatorname{hInv}[\mathrm{jj}][\mathrm{i}] *$ $\operatorname{hInv}[\mathrm{jj}][\mathrm{j}]$;

return \&matrix [0];

184

\}

global__ void fakeExperiments (float *

da NuebarNumubarFakes, float *

deltaLikelyFakes, int *kParameter \{

int tid;

tid $=$ blockDim.$x *$ blockIdx $x+$ threadIdx.$x$

189

i f $($ t i d $<\mathrm{NF} * \mathrm{SM})\{$

float da NuebarNumubar $[\mathrm{NBC}]$;

for (int $\overline{\mathrm{i}}=0 ; \mathrm{i}<\mathrm{NBC} ; \mathrm{i}++)\{$

da NuebarNumubar [i] $=$

da_NuebarNumubarFakes [ i + NBC*tid ] ;

194

float collapsed_SgBgNueNumubar [NBC] ;

float sgbgNueNumubar [NBT] :

float totMat $2 \times 2[\mathrm{NBC} *(\mathrm{NBC}+1) / 2]$;

float detolog;

float likelyTemp;

float likelyBF $=1 \mathrm{e} 8$;

float likelyParameter ;

float *lowerMat;

float $*$ invMat;

204

int $\mathrm{i}, \mathrm{j}, \mathrm{l}, \mathrm{ll}$;

int $\mathrm{kTest}=\mathrm{kParameter}[\mathrm{tid} / \mathrm{NF}] / 1000$;

int $\mathrm{kkTest}=\mathrm{kParameter}[\mathrm{tid} / \mathrm{NF}] \% 1000$;

209

for $(\mathrm{i}=0 ; \quad \mathrm{i}<\mathrm{NB} ; \quad \mathrm{i}++)\{$

sgbgNueNumubar $[\mathrm{i}+\mathrm{NB}]=$

sgbgNueNumubarSMALL [ i ] ;

\}

for $(\mathrm{i}=0 ; \mathrm{i}<\mathrm{NBmu} ; \mathrm{i}++)\{$

collapsed SgBgNueNumubar $[\mathrm{i}+\mathrm{NB}]=$ collapsed_SgBgNueNumubarSMALL [ i ] ;

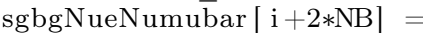

\} sgbgNueNumubarSMALL [ i+NB] ;

for $(1=0 ; \quad l<N ; \quad l++)\{$ for $(11=0 ; \quad 11<\mathrm{N} ; \quad 11++)\{$

for $(\mathrm{i}=0 ; \quad \mathrm{i}<\mathrm{NB} ; \quad \mathrm{i}++)\{$

collapsed_SgBgNueNumubar [i ] $=$ ( s2tTicCU ( l )*sgNuebar [i $+\mathrm{NB} * 11$ ]) + sgbgNueNumubarSMALL [i ] ; sgbgNueNumubar [i] = s2tTicCU (l)* \} sgNuebar [i $+\mathrm{NB} * 11]$;

for $(i=0 ; \quad i<N B ; \quad i++)\{$

for $(\mathrm{j}=0 ; \mathrm{j}<\mathrm{i}+1 ; \mathrm{j}++)\{$ totMat $2 \times 2[\mathrm{i} *(\mathrm{i}+1) / 2+\mathrm{j}]=$ fracMat $3 \times 3[\mathrm{i} * \mathrm{NBT}+\mathrm{j}] *$ sgbgNueNumubar [i ]* sgbgNueNumubar [j] + fracMat $3 \times 3[(\mathrm{i}+\mathrm{NB}) * \mathrm{NBT}+\mathrm{j}] *$ sgbgNueNumubar $[\mathrm{i}+\mathrm{NB}] *$ sgbgNueNumubar [j] + fracMat $3 \times 3[\mathrm{i} * \mathrm{NBT}+(\mathrm{j}+\mathrm{NB})] *$ sgbgNueNumubar [i ]* sgbgNueNumubar $[\mathrm{j}+\mathrm{NB}]+$ fracMat $3 \times 3[(\mathrm{i}+\mathrm{NB}) * \mathrm{NBT}+(\mathrm{j}+\mathrm{NB})$ ]*sgbgNueNumubar $[\mathrm{i}+\mathrm{NB}] *$ sgbgNueNumubar [j+NB];

\} $\operatorname{totMat} 2 \times 2[\mathrm{i} *(\mathrm{i}+1) / 2+\mathrm{i}]+=$ sgbgNueNumubar [i ] ;

\}

for $(\mathrm{i}=2 * \mathrm{NB} ; \quad \mathrm{i}<\mathrm{NBT} ; \mathrm{i}++)\{$

for $(\mathrm{j}=0 ; \mathrm{j}<\mathrm{NB} ; \mathrm{j}++)\{$

totMat $2 \times 2[(\mathrm{i}-\mathrm{NB}) *((\mathrm{i}-\mathrm{NB})+1) / 2+\mathrm{j}$ ]$=\operatorname{fracMat} 3 \times 3[\mathrm{i} * \mathrm{NBT}+\mathrm{j}] *$ sgbgNueNumubar [i ]* sgbgNueNumubar [j] + fracMat $3 \times 3[\mathrm{i} * \mathrm{NBT}+(\mathrm{j}+\mathrm{NB})] *$ sgbgNueNumubar [i ] * \} sgbgNueNumubar $[\mathrm{j}+\mathrm{NB}]$;

\}

for $(\mathrm{i}=2 * \mathrm{NB} ; \quad \mathrm{i}<\mathrm{NBT} ; \mathrm{i}++)\{$

for $(\mathrm{j}=2 * \mathrm{NB} ; \mathrm{j}<\mathrm{i}+1 ; \mathrm{j}++)\{$ totMat $2 \times 2[(\mathrm{i}-\mathrm{NB}) *((\mathrm{i}-\mathrm{NB})+1) / 2+($ $\mathrm{j}-\mathrm{NB})]=\operatorname{fracMat} 3 \times 3[\mathrm{i} * \mathrm{NBT}+\mathrm{j}] *$ sgbgNueNumubar [i ]* \} sgbgNueNumubar [j] ;

\}

lowerMat $=$ choleskyFactor $($ totMat $2 \times 2) ;$

detolog $=0 . ;$

for $(\mathrm{i}=0 ; \quad \mathrm{i}<\mathrm{NBC} ; \quad \mathrm{i}++)\{$ detolog $+=2 * \log f($ lowerMat $[\mathrm{i} *(\mathrm{i}+1)$ $/ 2+\mathrm{i}])$;

\}

invMat $=$ choleskyInverse (lowerMat $)$

likelyTemp $=0$.;

for $(\mathrm{i}=0 ; \quad \mathrm{i}<\mathrm{NBC} ; \mathrm{i}++)\{$

for $(\mathrm{j}=0 ; \mathrm{j}<\mathrm{i} ; \mathrm{j}++)\{$

likelyTemp $+=2 *(($

da_NuebarNumubar [i ] collapsed SgBgNueNumubar [i ] ) * invMat $[\mathrm{i} *(\mathrm{i}+1) / 2+\mathrm{j}] *($ da NuebarNumubar [j] collapsed_SgBgNueNumubar [j ] )) 
259

\} likelyTemp $+=$ (da_NuebarNumubar[i] - collapsed SgB̄BNueNumubar [i] ) * invMat $[\mathrm{i} *(\overline{\mathrm{i}}+1) / 2+\mathrm{i}] *($ da NuebarNumubar [i] -

\} collapsed_SgBgNueNumubar [i]) ;

likelyTemp $+=\operatorname{detolog}$;

likelyBF $=($ likelyTemp $<$ likelyBF $)$ ?

likelyTemp : likelyBF ;

likelyParameter $=(\mathrm{kTest}=\mathrm{l} \& \& \mathrm{kkTest}$ =ll)? likelyTemp :

likelyParameter ;

\}$/ /$ for $\quad l l<N$

\} / / for $l<N$

deltaLikelyFakes [tid ] = likelyParameter likelyBF ;

\}// if tid $<N F * S M$

\}//KERNEL: fakeExperiments ()

274

int main(int $\operatorname{argc}, \quad$ char $* \operatorname{argv}[])\{$

int modulusThree, index;

string strIndex;

stringstream fileIndex;

i f $(\operatorname{argc}==1)\{$

cout $<<$ "It needs two arguments modulusthree $(0,1,2)$ and the run number" $<<$ endl;

\} exit (1);

else \{

modulusThree $=$ atoi $(\operatorname{argv}[1])$;

if (modulusThree $!=0$ \&\& modulusThree $!=1 \quad \& \&$ modulusThree $!=2)\{$

cout $<<$ "modulusThree needs to be 0,1 or $2^{\prime \prime}<<$ endl

\} exit (1)

index $=$ atoi $(\operatorname{argv}[2])$;

fileIndex $<<$ index

\}

strIndex $=$ fileIndex.str ();

string strNF;

stringstream out;

out $<<\mathrm{NF}$;

strNF $=$ out.str () ;

string name $=$ "chi2Eff NuBar";

name. append ( $\operatorname{strNF})$;

name. append ("NF ");

name. append ( $\operatorname{str} \overline{I n} \operatorname{dex})$;

name. append ("Run.txt");

string nameDistribution $="$

fakeDistributions /

fakeExperimentsDistribution_NuBar _ " ; nameDistribution . append ( $\operatorname{strNF})$;

nameDistribution . append ("NF_");

string strKIndex, strKKIndex ;
// //Select best device to run on

// int num_devices, device;

// CUDA CALLL(cudaGetDeviceCount(E) num_devices));

$/ /$ if (num_devices > 1) \{

$/ / \quad$ int $m \bar{a} x_{-}$multiprocessors $=0$, max device $=0$;

$/ /$ for (device $=0 ;$ device $<$ num_devices; device ++ ) \{

cudaDeviceProp properties,

CUDA CALL(cudaGetDeviceProperties(E) properties, device));

if (max_multiprocessors < properties . multiProcessorCount) \{

max multiprocessors $=$ properties.

multiProcessorCount;

max_device $=$ device;$$
\text { \} }
$$

\}

CUDA_CALL(cudaSetDevice (max_device));

cout $<<$ "I'm running in device: " <

max_device $<<$ endl;

$1 /\}$

cout $<<$ "I'm running in device: " $<<1<<$ endl;

cudaSafeCall (cudaSetDevice (1), " cudaSetDevice" ) ;

// Capture the start time

float elapsedTime;

cudaEvent_t start, stop;

CUDA_CALL $\overline{(}$ cudaEventCreate ( \&start ) );

CUDA_CALL( cudaEventCreate ( \&stop ) );

CUDA_CALL( cudaEventRecord ( start, 0 ) );

cout $<<$ "Reading data files." $<<$ endl;

float *da_NuebarNumubar_h;

da NuebarN $\bar{N}$ mubar $h=$ new float $[\mathrm{NBC}]$;

ifstream daNuebārFile ("../NuBarData/ miniboone nuebardata lowe.txt");

if (daNuebarFile.is_open $(\overline{)})\{$

for (int $\mathrm{i}=0 ; \quad \mathrm{i}<\mathrm{NB} ; \quad \mathrm{i}++)\{$ \} daNuebarFile $\gg$ da_NuebarNumubar_h[i];

\} daNuebarFile.close ();

else \{

cout $<<$ "Unable to open .. / NuBarData/ miniboone_nuebardata_lowe.txt fil e $"<<$ endl ;

\} exit (1);

ifstream daNumubarFile (". ./NuBarData/ miniboone numubardata.txt");

if (daNumubarFile.is_open () ) \{

for (int $\mathrm{i}=0 ; \mathrm{i}<\mathrm{NBmu} ; \quad \mathrm{i}++)\{$

daNumubarFile $\gg$ da_NuebarNumubar_h[i+ $\mathrm{NB}]$;

\}

daNumubarFile. close ();

\}

else \{

cout $<<$ "Unable to open ../NuBarData/ miniboone numubardata.txt file $"<<$ endl ;

\} 
float * collapsed_SgBgNueNumubarSMALL_h; collapsed_SgBgNueNumubarSMALL_h $=$ new float [NBmu ];

ifstream bgNumubarFile (" . / NuBarData/ miniboone numubar.txt");

if ( bgNumubarFile. is open ()) \{

for (int $\mathrm{i}=0 ; \mathrm{i}<\mathrm{NBmu} ; \mathrm{i}++)\{$ bgNumubarFile $>$ collapsed_SgBgNueNumubarSMALL_h [i ] ; sgbgNueNumubarSMALL $\mathrm{h}[\mathrm{i}+\mathrm{NB}]=$ collapsed_SgBgNueNumubarSMALL_h [i ] ;

float $*$ fracMat $3 \times 3 \ldots$ h

fracMat $3 \times 3 \quad h=$ new float $[\mathrm{NBT} * \mathrm{NBT}]$;

ifstream fracMat $3 \times 3$ File ("../NuBarData/ miniboone full fractcovmatrix nubar lowe .txt");

if ( fracMat $3 \times 3$ File. is open ()) \{

for (int $\mathrm{i}=0 ; \quad \mathrm{i}<\mathrm{NBT} ; \quad \mathrm{i}++)\{$ for $($ int $\mathrm{j}=0 ; \mathrm{j}<\mathrm{NBT} ; \mathrm{j}++)\{$ fracMat $3 \times 3$ File $\gg$ fracMat $3 \times 3 \_$h $[i * N B T+$ j ] ; \} \} \}

fracMat3x3File.close ();

409

else \{

cout $<<$ "Unable to open ../ NuBarData/ miniboone full fractcovmatrix nubar lowe . txt file $\overline{\bar{N}}<<$ endl; \} exit (1);

float $*$ sgNuebar h ;

sgNuebar $\mathrm{h}=$ new float $[\mathrm{NB} * \mathrm{~N}]$;

ifstream sgNuebarFile ("signalsbarSMALL.txt $")$;

if ( $\mathrm{sgNuebarFile.is \_ open} \mathrm{())} \mathrm{\{}$

for $($ int $\mathrm{kk}=0 ; \mathrm{kk}<\mathrm{N} ; \mathrm{kk}++)\{$

for (int i i $=0 ;$ i i $<N B ; \quad$ i i ++$)\{$ sgNuebarFile $\gg$ sgNuebar h $[\mathrm{i} i+\mathrm{NB} * \mathrm{kk}$ ];

\}

else \{

cout $<<$ "Unable to open signalsbarSMALL. txt file $"<<$ endl;

\} exit (1)

cout $<<$ "Starting Real Likelihood surface calculation" $<<$ endl;

float deltaLikelyReal $[\mathrm{N}][\mathrm{N}]=\{0$.$\} ;$

float likelyRealBF $=1 \mathrm{e} 5$;

int min position [2];

float collapsed_SgBgNueNumubar $[\mathrm{NBC}]$;

float sgbgNueNumubar [NBT] ;

float totMat $2 \times 2[\mathrm{NBC} *(\mathrm{NBC}+1) / 2]$;

float detolog;

float *lowerMat_h;

lowerMat_h $=$ new float $[\mathrm{NBC} *(\mathrm{NBC}+1) / 2]$;

float $*$ invMat $\mathrm{h}$;

invMat_h $=$ new float $[\mathrm{NBC} *(\mathrm{NBC}+1) / 2]$;

444

for (int $i=0 ; \quad i<N B ; \quad i++)\{$ sgbgNueNumubar $[\mathrm{i}+\mathrm{NB}]=$ sgbgNueNumubarSMALL_h [i ];

\}

for (int $\mathrm{i}=0 ; \mathrm{i}<\mathrm{NBmu} ; \mathrm{i}++)\{$ collapsed_SgBgNueNumubar $[\mathrm{i}+\mathrm{NB}]=$ collapsed_SgBgNueNumubarSMALL_h [i ]; sgbgNueNumubar $[\mathrm{i}+2 * \mathrm{NB}]=$

\} sgbgNueNumubarSMALL_h [i+NB];

// Loop trough a variety of values of $s 2 t$ and dm2 using the real

// data and building deltaLikelyReal array

for (int $\mathrm{k}=0 ; \mathrm{k}<\mathrm{N} ; \mathrm{k}++)\{/ / s 2 t$

for $($ int $\mathrm{kk}=0 ; \mathrm{kk}<\mathrm{N} ; \mathrm{kk}++)\{/ / d m 2$

for (int $\mathrm{i}=0 ; \quad \mathrm{i}<\mathrm{NB} ; \quad \mathrm{i}++)$ \{

collapsed_SgBgNueNumubar [i] $=(\mathrm{s} 2 \mathrm{tTic}$ $(\mathrm{k}) * \overline{\mathrm{s}} g$ Nuebar $\mathrm{h}[\mathrm{i}+\mathrm{NB} * \mathrm{kk}])+$ sgbgNueNumubarSMALL_h [i];

$\operatorname{sgbgNueNumubar}[\mathrm{i}]=\operatorname{s} 2 \mathrm{t} \overline{\mathrm{T}} \mathrm{ic}(\mathrm{k}) *$ \} sgNuebar_h $[\mathrm{i}+\mathrm{NB} * \mathrm{kk}]$;

//Total matrix creation and collapsing for (int $\mathrm{i}=0 ; \mathrm{i}<\mathrm{NB} ; \mathrm{i}++)\{$

for (int $\mathrm{j}=0 ; \mathrm{j}<\mathrm{i}+1 ; \mathrm{j}++)\{$ totMat $2 \times 2[i *(i+1) / 2+j]=$ fracMat $3 \times 3$ h $[\mathrm{i} * \mathrm{NBT}+\mathrm{j}] *$ sgbgNueNumubar [i] * sgbgNueNumubar [j] + fracMat $3 \times 3$ h $[(i+N B) * N B T+j] *$ sgbgNueNumubar [ $i+\mathrm{NB}] *$ sgbgNueNumubar [j] + fracMat $3 \times 3$ h $[\mathrm{i} * \mathrm{NBT}+(\mathrm{j}+\mathrm{NB})] *$ sgbgNueNumubar [i] * sgbgNueNumubar $[\mathrm{j}+\mathrm{NB}]+$ fracMat $3 \times 3$ h $[(i+N B) * N B T+(j+N B)$ ] $* \operatorname{sgbgNueNumubar}[\mathrm{i}+\mathrm{NB}] *$ sgbgNueNumubar $[\mathrm{j}+\mathrm{NB}]$;

\}

totMat $2 \times 2[i *(i+1) / 2+i]+=$ sgbgNueNumubar [i ];

\}

for (int $\mathrm{i}=2 * \mathrm{NB} ; \quad \mathrm{i}<\mathrm{NBT} ; \quad \mathrm{i}++)\{$

for (int $\mathrm{j}=0 ; \mathrm{j}<\mathrm{NB} ; \mathrm{j}++)\{$ 
cout $<<$ fixed $<<$ "likely min " $<<$

likelyRealBF $<<$ endl;

cout $<<$ fixed $<<$ "s 2 t $"<<$ min position [0] $<<" \backslash \mathrm{t} "<<$ s2tTic (min_position $[0])<<$ endl ; cout $<<$ fixed $<<$ "dm2 $"<<$ min position [1] $<$ " $\backslash \mathrm{t} "<<$ dm2Tic (min_position [1]) $<<$ endl ;

//Constant memory allocations

CUDA_CALL(cudaMemcpyToSymbol ( collapsed_SgBgNueNumubarSMALL, collapsed_SgBgNueNumubarSMALL_h , collapsed_SgBgNueNumubarSMAL $\bar{L}$ _buffSize) ) ;

CUDA_CALL(cudaMemcpyToSymbol( 
sgbgNueNumubarSMALL,

sgbgNueNumubarSMALL_h, sgbgNueNumubarSMAL $\bar{L}$ buffSize));

CUDA_CALL(cudaMemcpyToSymbol (fracMat3x3 , fracMat $3 \times 3$ h, fracMat $3 \times 3$ buffSize)); CUDA_CALL(cudaMemcpyToSymbol(sgNuebar, sgNuebar_h, sgNuebar_buffSize));

int nThreads $=96$;

int $\mathrm{nBlocks}=(\mathrm{NF} * \mathrm{SM}+\mathrm{nThreads}-1) /$ nThreads ;

cout $<<$ "nThreads $="<<" \backslash \mathrm{t} "<<$ nThreads $<<$ endl;

cout $<<$ "nBlocks $="<<" \backslash \mathrm{t} "<<$ nBlocks $<<$ endl ;

double epsilon $=1 \mathrm{e}-8$;

double $\mathrm{h}[\mathrm{NBC}][\mathrm{NBC}]$;

double sumh2;

int32_t seed $=($ int32_t $)$ time $(0) ; / /$ Start rāndom seed.

StochasticLib1 sto(seed);// Make instance of random library

double fakeVector $[\mathrm{NBC}]$;

double fluctuatedVector [NBC];

double gaussRand $[\mathrm{NBC}]$;

float *da NuebarNumubarFakes $\mathrm{h}$;

da_NuebarN $\bar{N} u m u b a r F a k e s \_h=$ new float $[\mathrm{NBC} * \mathrm{NF} *$ $\mathrm{SM}]$

float ratioBigger;

int $\mathrm{s}$;

int $\mathrm{sOld}=0$;

int $* \mathrm{kParameter} \mathrm{h} ; / /$ Hardware dependent

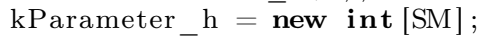

// Loop trough a variety of values of s2t and dm2 to generate the

// effective likely array.

for (int $\mathrm{k}=\operatorname{modulusThree;} \mathrm{k}<\mathrm{N} ; \mathrm{k}+=$ step $)\{$

limitExceeded :

for $($ int $\mathrm{kk}=\operatorname{modulusThree} ; \mathrm{kk}<\mathrm{N} ; \mathrm{kk}+=$ step ) \{

// It will not try to find anything here.

if (lowerRegion ( $\mathrm{k}, \mathrm{kk}) \quad$ | upperRegion (k $\mathrm{kk})||$ upperSquare $(\mathrm{k}, \mathrm{kk}))\{$

\} continue ;

604

$\mathrm{s}=\mathrm{sOld}$;

while $(\mathrm{s}<\mathrm{SM})\{$

i f $(\mathrm{k}>=\mathrm{N})\{$

$\mathrm{kk}=\mathrm{N}$;

609

\}

goto limitExceededTwice;

if $(\mathrm{kk}>=\mathrm{N})\{$

sOld $=\mathrm{s}$;

$\mathrm{k}+=$ step;

\}

goto limitExceeded;

// It will not try to find anything here.

if (lowerRegion ( $\mathrm{k}, \mathrm{kk})$ || upperRegion ( $\mathrm{k}, \mathrm{kk})||$ upperSquare $(\mathrm{k}, \mathrm{kk}))\{$ sOld $=\mathrm{s}$; $\mathrm{k}+=$ step; \}

goto limitExceeded;

for (int $\quad \mathrm{i}=0 ; \quad \mathrm{i}<\mathrm{NB} ; \quad \mathrm{i}++)\{$

$\operatorname{sgbgNueNumubar}[\mathrm{i}]=\operatorname{s} 2 \mathrm{tTic}(\mathrm{k}) *$ sgNuebar h[i $+\mathrm{NB} * \mathrm{kk}]$;

collapsed_SgB̈gNueNumubar [i] = $\mathrm{s} 2 \mathrm{t} \overline{\mathrm{Tic}}(\mathrm{k}) * \mathrm{sgNuebar} \mathrm{h}[\mathrm{i}+\mathrm{NB} * \mathrm{kk}]$ \} + sgbgNueNumubarSMALL_h[i ] ;

/ Total matrix creation and collapsing

for (int $\mathrm{i}=0 ; \quad \mathrm{i}<\mathrm{NB} ; \mathrm{i}++)\{$

for (int $\mathrm{j}=0 ; \mathrm{j}<\mathrm{i}+1 ; \mathrm{j}++)\{$ $\operatorname{totMat} 2 \times 2[\mathrm{i} *(\mathrm{i}+1) / 2+\mathrm{j}]=$ fracMat $3 \times 3$ h $[\mathrm{i} * \mathrm{NBT}+\mathrm{j}] *$ sgbgNueNumubar [i ] * sgbgNueNumubar [j] + fracMat3x3_h $[(\mathrm{i}+\mathrm{NB}) * \mathrm{NBT}+\mathrm{j}] *$ sgbgNueNumubar $[\mathrm{i}+\mathrm{NB}] *$ sgbgNueNumubar [j] + fracMat3x3_h $[\mathrm{i} * \mathrm{NBT}+(\mathrm{j}+\mathrm{NB})] *$ sgbgNueNumubar [i ] * sgbgNueNumubar [j+NB]+ fracMat $3 \times 3$ h $[(\mathrm{i}+\mathrm{NB}) * \mathrm{NBT}+(\mathrm{j}+\mathrm{NB}$ )]*sgbgNue $\bar{N} u m u b a r[\mathrm{i}+\mathrm{NB}] *$ sgbgNueNumubar $[\mathrm{j}+\mathrm{NB}]$;

\}

totMat $2 \times 2[\mathrm{i} *(\mathrm{i}+1) / 2+\mathrm{i}]+=$ \} sgbgNueNumubar [i ] ;

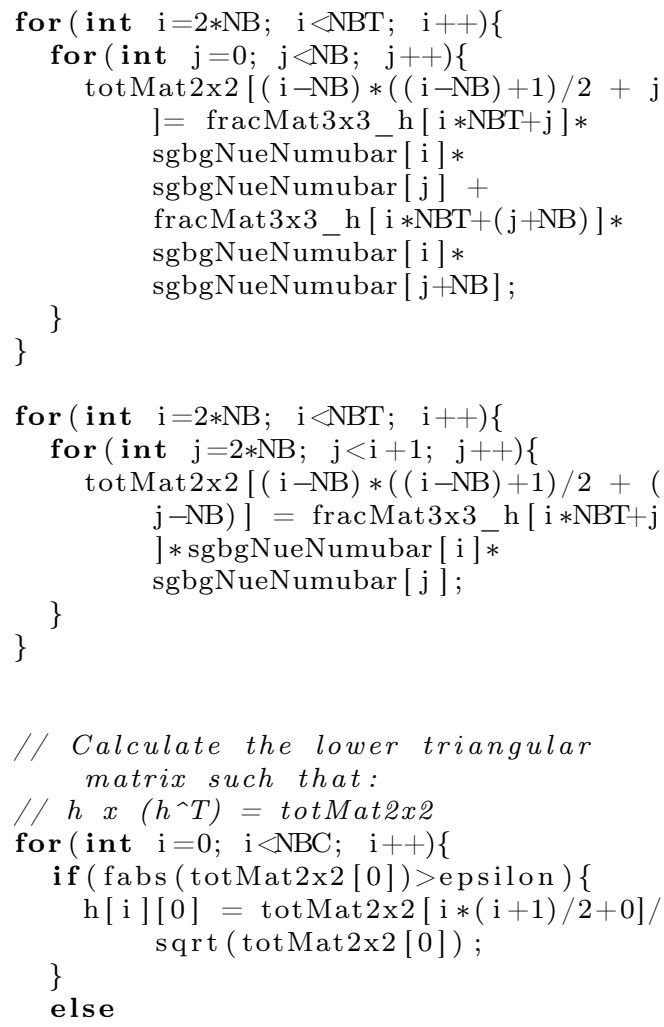


\}

for (int $\mathrm{j}=1 ; \mathrm{j}<\mathrm{NBC} ; \mathrm{j}++)\{$

$\operatorname{sumh} 2=0.0$

for (int $\mathrm{j} j=0 ; \quad j \mathrm{j}<\mathrm{j} ; \quad \mathrm{j} \mathrm{j}++)\{$ \} $\operatorname{sumh} 2+=h[j][j j] * h[j][j j] ;$

$\mathrm{h}[\mathrm{j}][\mathrm{j}]=\operatorname{sqrt}(\operatorname{totMat} 2 \times 2[\mathrm{j} *(\mathrm{j}+1) / 2+$ j]-sumh2)

669

674

679

684

689

694 \}

for $($ int $\quad \mathrm{i}=\mathrm{j} ; \quad \mathrm{i}<\mathrm{NBC} ; \quad \mathrm{i}++)\{$

sumh2 $=0$.;

for $($ int $\quad \mathrm{i} \mathrm{i}=0 ; \quad \mathrm{ii}<\mathrm{j} ; \quad \mathrm{ii}++)\{$ \} $\operatorname{sumh} 2+=h[i][i i] * h[j][i i] ;$

if $($ fabs $(h[j][j])>\operatorname{epsilon})\{$ $\mathrm{h}[\mathrm{i}][\mathrm{j}]=(\operatorname{totMat} 2 \times 2[\mathrm{i} *(\mathrm{i}+1) / 2+$

\} $\mathrm{j}]-\operatorname{sumh} 2) / \mathrm{h}[\mathrm{j}][\mathrm{j}]$;

else

$\mathrm{h}[\mathrm{i}][\mathrm{j}]=0 . ;$

$$
\}
$$

for (int $i=0 ; \quad i<N B C ; \quad i++)\{$

for (int $\mathrm{j}=\mathrm{i}+1 ; \mathrm{j}<\mathrm{NBC} ; \mathrm{j}++)\{$ \}

$\mathrm{h}[\mathrm{i}][\mathrm{j}]=0$.;

\}

seed $=($ int32_t $)$ time $(0)$;

// Loop to generate a collection of fake vectors.

for $($ int $\mathrm{kkk}=0 ; \mathrm{kkk}<\mathrm{NF} ; \mathrm{kkk}++)\{$

for (int $\mathrm{i}=0 ; \quad \mathrm{i}<\mathrm{NBC} ; \quad \mathrm{i}++$ ) \{ gaussRand $[\mathrm{i}]=$ sto. $\operatorname{Normal}(0,1)$;

for (int $\mathrm{i}=0 ; \quad \mathrm{i}<\mathrm{NBC} ; \quad \mathrm{i}++)\{$

fakeVector $[\mathrm{i}]=$ \} collapsed_SgBgNueNumubar [i ];

// Here we get the vector with fluctuations according to the

// covariance matrix. And the new fake vector.

for (int $\mathrm{i}=0 ; \quad \mathrm{i}<\mathrm{NBC} ; \quad \mathrm{i}++)\{$

fluctuatedVector $[\mathrm{i}]=0$.;

for (int $\mathrm{j}=0 ; \mathrm{j}<\mathrm{NBC} ; \mathrm{j}++)\{$ if $(\mathrm{j}>\mathrm{i})\{$

\}

break ;

fluctuatedVector [i] $+=\mathrm{h}[\mathrm{i}][\mathrm{j}] *$ gaussRand [j ] ;

\}

fakeVector $[\mathrm{i}]+=$ fluctuatedVector

\} [i ] ;

714

for (int $\quad \mathrm{i}=0 ; \quad \mathrm{i}<\mathrm{NBC} ; \quad \mathrm{i}++)\{$

da_NuebarNumubarFakes_h [i $+\mathrm{kkk} * \mathrm{NBC}$ $+\mathrm{s} * \mathrm{NBC} * \mathrm{NF}]=$ fake $\overline{\mathrm{V}}$ ector $[\mathrm{i}]$;

\}

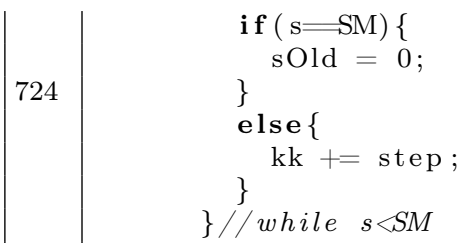

limitExceededTwice :

cudaS afeCall ( cudaMemcpy (

da_NuebarNumubarFakes_d,

da ${ }^{-}$NuebarNumubarFakes ${ }^{-} \mathrm{h}$,

da_NuebarNumubarFakes_buffSize, cudaMemcpyHostToDevice $\overline{)}$, " cudaMemcpy" ) ;

cudaSafeCall (cudaMemcpy (kParameter d , kParameter_h, kParameter_buffSize , cudaMemcpyHostToDevice), " cudaMemcpy" ) ;

734

\}

cout $<<" \backslash \mathrm{n} \backslash \mathbf{n} \backslash$ nLaunching kernel" $<<$ endl ;

cout $<<$ "With: " $<<$ endl;

for $(\mathrm{s}=0 ; \mathrm{s}<\mathrm{SM} ; \mathrm{s}++)\{$

cout $<<" \mathrm{k}="<<\mathrm{kParameter} \mathrm{h}[\mathrm{s}$

] $/ 1000<<", \quad \mathrm{kk}="<<$

kParameter h $[\mathrm{s}] \% 1000<<$ endl

fakeExperiments $<<<$ nThreads, nBlocks $>>>$ ( da NuebarNumubarFakes d,

dētaLikelyFakes_d, $\mathrm{kP}$ arameter_d);

cudaSafeCall ( cudaMemcpy (

deltaLikelyFakes_h,

deltaLikelyFakes d,

deltaLikelyFakes buffSize

cudaMemcpyDevice $\bar{T}$ oHost ), "

cudaMemcpy" ) ;

cout $<<$ "Done!" $<<$ endl

ofstream chi2FrecFCuBar (name.c_str(), ios : : app );

if ( chi2FrecFCuBar.is open ())

for (int $\mathrm{s}=0 ; \mathrm{s}<\mathrm{SM} ; \mathrm{s}++)\{$

ratioBigger $=0$;

for (int $\mathrm{i}=0 ; \quad \mathrm{i}<\mathrm{NF} ; \mathrm{i}++)\{$

if (deltaLikelyReal [kParameter h [s ]/1 10000$][$ kParameter $\mathrm{h}[\mathrm{s}$ ]\%1000] $<=$ deltaLikelyFakes h [i $+\mathrm{s} * \mathrm{NF}])\{$

ratio Bigger ++ ;

kParameter_h[s]\%1000) $<<" \backslash \mathrm{t} "$

$<$ s2tTic ( k $<<" \backslash \mathrm{t} "<<-2 * \log ($ ratioBigger

$\mathrm{NF})<<" \backslash \mathrm{t} "<<$ ratioBigger $<<"$ t" $<<\mathrm{NF}<<$ endl

chi 2 FrecFCuBar $<<$ fixed $<<$ dm2Tic ( kParameter h $[\mathrm{s}] \% 1000)<<" \backslash \mathrm{t} "$

$<<$ s 2 tTic $(\bar{k}$ Parameter h $[\mathrm{s}] / 1000)$ $<<" \backslash \mathrm{t} "<<-2 * \log$ (ratioBigger $\mathrm{NF})<<" \backslash \mathrm{t} "<<$ ratioBigger $<<"$ $\backslash \mathrm{t} "<<\mathrm{NF}<<$ endl ;

\}

\}

$\mathrm{kParameter} \_\mathrm{h}[\mathrm{s}]=\mathrm{k} * 1000+\mathrm{kk}$;

$\mathrm{s}++$; 
else cout $<<$ "Unable to open " $<$ name. c_str ()$<<"$ file " $<<$ endl;

cout $<<$ "Printing " $<$ name. $\mathrm{c}$ str ()$<<$ endl ;

for (int $\mathrm{s}=0 ; \mathrm{s}<\mathrm{SM} ; \mathrm{s}++)\{$ out.str( std:: string () );

out.clear ();

out $<<$ kParameter h $[\mathrm{s}] / 1000$;

strKIndex $=$ out.str () ;

nameDistribution . append (strKIndex);

nameDistribution . append ("-");

out.str ( std : : string () );

out. clear ();

out $<<$ kParameter_h $[\mathrm{s}] \% 1000$;

strKKIndex $=$ out. - tr () ;

nameDistribution . append (strKKIndex); nameDistribution . append (".txt");

bool itExist $=$ fileExists ( nameDistribution.c_str ());

ofstream fakeDistos (nameDistribution .c_str (), ios : : app);

if (fakeDistos.is_open ()) \{

if (! it Exist) \{

fakeDistos $<<$ "\#\#\# k" $<<" \backslash \mathrm{t} "<<$ "kk" $<<$ endl;

fakeDistos $<<$ "\#\#\# " $<<$

kParameter_h [s] $/ 1000<<" \backslash \mathrm{t} "$

$<<$ kParameter_h[s]\%1000 < endl;

fakeDistos $<<$ fixed $<<$ s2tTic ( kParameter_h [s]/1000) $<<" \backslash \mathrm{t}$ $"<<$ dm2Tic $($ kParameter_h [s 1\%1000) $<<$ endl ;

fakeDistos $<<$ "deltaLikelyReal = $"<<" \backslash \mathrm{t} "<<$ deltaLikelyReal [ kParameter_h[s]/1000][ kParameter ${ }^{-}$h $\left.[\mathrm{s}] \% 1000\right]<$ endl

\} ;

for (int $\quad \mathrm{i}=0 ; \quad \mathrm{i}<\mathrm{NF} ; \quad \mathrm{i}++$ ) \{

fakeDistos $<<$ deltaLikelyFakes_h [ $\mathrm{i}+\mathrm{s} * \mathrm{NF}]<<$ endl

\} \}

fakeDistos.close ();

794

799

809

814

819

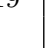

delete [] deltaLikelyFakes_h;

return 0 ;

$824\} / /$ int $\operatorname{main}()$ 


\section{Apéndice B}

\section{Condor}

Condor (actualmente conocido como HTCondor) es un sistema de software computacional de alto rendimiento para la distribución gruesa de tareas computacionales intensivas paralelas [206]. Se puede usar para administrar la carga de trabajo en un clúster dedicado de computadoras o para mandar trabajos a computadoras de escritorio ociosas. Es desarrollado por el equipo Condor en la universidad de Wisconsin-Madison y sigue la filosofía de código abierto.

Condor cuenta con un mecanismo de cola de tareas, una política de horarios, esquema de prioridades, monitoreo y administración de recursos entre otras funcionalidades. Los usuarios mandan sus tareas a Condor, estos se colocan en la cola de tareas y Condor decide cuando y donde se correrán, registra el progreso de las tareas e informa al usuario tras la finalización de la tarea. Cada estación de trabajo conectada al Condor pool corre un daemon que observa el $I / O$ de los usuarios y la carga del CPU. A una estación que se encuentra ociosa por cierto tiempo se le asigna una tarea de la cola, la cual correrá hasta que el daemon detecte un golpe de teclado, movimiento del ratón o demasiado uso del CPU ajeno a Condor (por ejemplo cuando un usuario se conecta a través de ssh a su computadora para mandar trabajos). En ese momento se genera un ticket con el estado y progreso de la tarea y esta es removida de la estación de trabajo y se manda de vuelta a la cola de tareas donde será reasignada a otra computadora.

\section{B.1. Uso de Condor en el pool de MiniBooNE}

En el desarrollo de esta tesis fue fundamental el uso de Condor, ya que permitió lograr las primeras versiones de los análisis. Inclusive con las versiones finales y más optimizadas del código el tiempo necesario de cómputo es de más de mil horas por cada análisis, muchas más para el análisis combinado. MiniBooNE cuenta con un clúster dedicado al análisis de datos de $\sim 80$ núcleos, con velocidades entre 2.3 y $2.5 \mathrm{GHz}$. La disponibilidad de los mismos es variable y, en general, para hacer los análisis no se dispuso de más de 70 de ellos simultáneamente (posiblemente por que el resto no era compatible con la arquitectura del programa).

Además de estar registrado en Fermilab como investigador visitante, se requirió una cuenta en el FNALU, el Unix cluster central para Fermilab. Así como una cuenta de kerberos del Fermilab y una 
computadora kerberizada para hacer accesos remotos al FNAL Realm. ${ }^{1}$ Una vez que el administrador del pool de MiniBooNE te da de alta como usuario de ese pool puedes mandar tareas a Condor.

Trabajando remotamente, es decir en una computadora ajena al pool de MiniBooNE, se debe conseguir un ticket de kerberos. En una terminal se pone el siguiente comando y se introduce el password solicitado:

\$ kinit -A user_name@FNAL.GOV

Password for user_name@FNAL.GOV:

Con un ticket válido (los tickets expiran después de 24 horas) se puede accesar a cualquier máquina del pool, eso se hace mediante Secure Shell (ssh)

\$ ssh -Y -1 user_name computer_name.fnal.gov

Es importante que al finalizar una sesión de trabajo se destruya el ticket para inhabilitar accesos espurios.

\section{\$ kdestroy}

Una vez conectado se mandan los datos que se analizaran así como el código fuente del programa que llevará a cabo el cómputo. Antes de mandar trabajos se realizan distintas pruebas para revisar que el programa consiga acceso a los datos que necesita y que no haya ningún problema de compatibilidad. La versión del compilador gcc disponible en el pool difiere de la versión con la cual se escribieron los programas. La versión que se uso es la 4.5.1, para cargarla se usa el comando:

\$ setup gcc v4_5_1

Posteriormente se compila usando la opción de máxima optimización: la instrucción -03. Con lo cual se logra una mejora en el rendimiento de $\sim 3 \times$. La introducción de dichas flags produjo bugs en ciertas funciones, estos se resolvieron cambiando la redacción del código. Se comprobó que el resultado obtenido con y sin instrucciones de optimización fuera estrictamente el mismo.

\$ g++ frecuentistFC_NuBar.cpp -o frecuentistFC_NuBar -03

Mandar una tarea al pool se hace con:

\$ boone-jobsub frecuentistFC_NuBar

Por cada tarea que se manda a Condor se generan cinco archivos. El nombre de estos archivos se forma anexando al nombre del ejecutable la fecha y hora en que fueron mandadas las tareas y un número único. Pensando en que se mandó la tarea frecuentistFC_NuBar el cuatro de noviembre de 1970 a las 12:34:42. Los archivos que se generan son:

- frecuentistFC_NuBar_19701104_123442_1.cmd: Archivo que describe propiedades de Condor específicas del ejecutable.

\footnotetext{
${ }^{1}$ En la red hay instrucciones sobre como kerberizar computadoras para que sean compatibles con Fermilab [207]
} 
- frecuentistFC_NuBar_19701104_123442_1.err: Contiene cualquier señal de error que mande la ejecución.

- frecuentistFC_NuBar_19701104_123442_1.1og: Bitácora que contiene información detallada sobre la ejecución; dirección IP de la máquina perteneciente al pool desde la cual se manda el ejecutable, dirección IP de la máquina que lo ejecuta, tiempo que tarda, memoria que se aloja, etcétera.

- frecuentistFC_NuBar_19701104_123442_1.out: Aquí se guarda la salida a la pantalla del ejecutable.

- frecuentistFC_NuBar_19701104_123442_1.sh: bash script ejecution wrapper.

Una vez la tarea se ha completado Condor manda un correo al correo asociado a la cuenta de FNALU informando el termino de la ejecución. Detallando la fecha y hora en que fue mandada la tarea y cuando se terminó su ejecución, el tiempo que tomó, la señal de error que se registró, el tamaño de la memoria virtual usada y otros parámetros comunes. Con el propósito de administrar y monitorear el progreso de las tareas los siguientes comandos resultan útiles:

- condor_q -global: Para revisar la lista de tareas, se obtiene información sobre todas las tareas; las que se encuentran en espera, detenidas, ejecutándose. Se obtiene el nombre del dueño de cada tarea, un número con el cual se identifica cada una, la fecha en que fue mandada y el tiempo de ejecución entre otras cosas.

- condor_q -submitter user_name: Se obtiene la lista de tareas y sus atributos que mandó el usuario user_name.

- condor_hold <ID \#>: Para detener así como evitar que empiece la ejecución de una tarea. Como argumento se debe poner el número de identidad de la tarea que se desee detener.

- condor_release <ID \#>: Permite que una tarea detenida pueda ser puesta de vuelta en la cola de espera. Como argumento se debe poner el número de identidad de la tarea que se desee liberar.

- condor_rm <ID \#>: Se usa para quitar una tarea de la cola. De igual forma se debe poner el número que identifica a la tarea en cuestión.

En el recuadro B.1 se encuentra transcrito el bash script usado para automatizar el envío de tareas. Como argumento toma un archivo en el que se encuentran los índices de las coordenadas que se requieren calcular. Para evitar conflictos en la escritura cada ejecutable escribe en su propio archivo de salida.

Código B.1: Bash script "submitting.sh" para mandar tareas al pool.

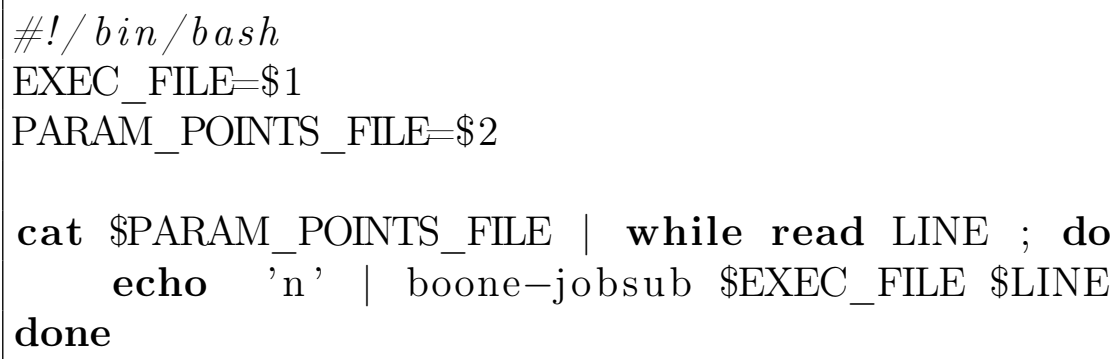




\section{Apéndice C}

\section{Implementación en un esquema de procesamiento en paralelo con CUDA}

La Arquitectura Unificada de Dispositivos de Cómputo, CUDA por sus siglas en inglés Compute Unified Device Architecture es una plataforma de computación en paralelo y un modelo de programación creado por la compañía NVIDIA e implementado por las unidades gráficas de procesamiento, GPU por sus siglas en inglés Graphics Processing Units, que producen [209].

Los microprocesadores basados en una sola unidad central de procesamiento llevaron un rápido incremento en el rendimiento y reducción de precios en los programas computacionales por más de dos décadas. Estos procesadores dieron miles de millones de operaciones de punto flotante por segundo (GFLOPS) a las computadoras de escritorio y decenas de millones de GFLOPS a los servidores clúster. Este implacable crecimiento en el rendimiento ha permitido que las aplicaciones de software den mayor funcionalidad, que tengan mejor interfaz para el usuario y que generen más resultados útiles. Los usuarios por su parte solicitan aún más mejoras una vez que se acostumbran a las ya obtenidas, generando así un ciclo positivo para la industria de computo [210].

Durante esta racha la mayoría de los desarrolladores de software contaron con las mejoras en el hardware para incrementar la velocidad de sus programas. Sin embargo esta racha se ha frenado desde 2003 debido a problemas con el consumo de energía y a la disipación de calor, limitando el incremento de la frecuencia del reloj y el nivel de actividades productivas que pueden ser realizadas en el periodo de un CPU. Los productores de microprocesadores han cambiado a modelos en los que se usan múltiples unidades de procesamiento, conocidos como procesadores multinúcleo, en cada chip para incrementar el poder de procesamiento. Este cambio ha ejercido un impacto tremendo en la comunidad de desarrolladores de software [211].

Tradicionalmente la mayoría de las aplicaciones son escritas como programas secuenciales, como las describiera John von Neumann en su reporte seminal de 1945 [212]. La ejecución de estos programas puede ser entendida por un humano pasando secuencialmente a través del código. La expectativa de que un programa corra más rápido con cada nueva generación de microprocesadores ya no es estrictamente valida. Un programa secuencial solo correrá en uno de los núcleos de un procesador, el cual no se volverá significativamente más rápido que lo que se usa hoy en día.

Las aplicaciones que continuarán gozando de mejoras de rendimiento con cada nueva generación de microprocesadores son los programas paralelos, en los cuales múltiples hilos (threads) de ejecución 
cooperan para completar el trabajo más rápidamente. Se ha referido a este nuevo, dramáticamente remontado, incentivo para el desarrollo de programas en paralelo como la "revolución de concurrencia" [211]. Programar en paralelo no es nuevo, la comunidad de computo de alto rendimiento ha estado desarrollando programas paralelos por décadas. Estos programas corrían en costosas computadoras de gran escala. Solo unas cuantas aplicaciones elite justifican el uso de estos costos aparatos, limitando la práctica computo paralelo a un reducido número de desarrolladores. Ahora que todos los microprocesadores son computadoras paralelas, el número de aplicaciones que deben ser desarrolladas como programas en paralelo ha incrementado dramáticamente. Ahora hay una gran necesidad para que los desarrolladores de software aprendan programación en paralelo.

CUDA le da a los desarrolladores acceso al conjunto de instrucciones virtuales y a la memoria de los elementos de computación en paralelo de los GPU's, usando CUDA los GPU's se vuelven accesibles para ser usados para cálculos como CPU's. A diferencia de los CPU's, los GPU's tienen una arquitectura en paralelo de la cantidad de datos que se pueden transferir que enfatiza la ejecución de muchos threads lentamente, en vez de ejecutar un solo thread muy rápidamente. Se puede acceder a la plataforma de CUDA a través de una variedad de librerías y compiladores, existen extensiones de $\mathrm{C} / \mathrm{C}++$ y Fortran que permiten hacer uso de las capacidades de las tarjetas de vídeo. Propulsado por la sumamente demandante industria de videojuegos, las tarjetas de vídeo, usadas para generar los gráficos de una computadora, han logrado salirse del mercado que las vio nacer y se han logrado usar de forma exitosa para acelerar en un orden de magnitud o más aplicaciones de biología computacional, criptografía, simulaciones de fluidos o mecánica cuántica.

\section{C.1. Descripción de la paralelización}

La paralelización de este análisis se hizo de forma "expedita", en general la forma más sencilla de paralelizar un algoritmo es forzar que la paralelización ocurra en algún bucle. Con eso en mente se reconocen tres niveles (de mayor a menor) en los cuales se puede implementar la paralelización:

- Cada thread calcula todos los experimentos ficticios para cada punto del espacio de parámetros. Para lo cual se deben de lanzar tantos threads como puntos se quieran probar y cada thread hará un total de $n_{\mathbf{f}}$ iteraciones, es decir $n_{\mathbf{f}} \times 190 \times 190$ inversiones de matrices. Haciendo 2000 experimentos ficticios, son más 72 millones de inversiones, muchísimos cálculos para un solo thread. La memoria se saturaría por completo.

- Cada thread calcula un experimento ficticio. Se deben lanzar tantos threads como experimentos ficticios. En este esquema, cada thread invierte 36100 matrices, lo cual requiere una cantidad de memoria que exige una tarjeta de vídeo muy robusta.

- Cada thread calcula un valor de $\mathscr{L}$ de un experimento ficticio, se requieren lanzar $190 \times 190=$ 36100 threads y cada uno invertirá una matriz.

Paralelizar en el nivel superior no es posible ya que requiere muchísima más memoria que la que cuenta cualquier tarjeta de vídeo actual. Paralelizar en el nivel inferior no es tan eficiente, ya que se hacen muy pocos cálculos para la cantidad de datos transferidos a la tarjeta: idealmente uno quiere reducir el número de veces que se transfieren datos a la tarjeta, ya que es un proceso lento. Es decir, en cada transmisión de datos se quieren hacer muchas cuentas. Es común que mientras menor sea el nivel en que se paralelice, mayor será el esfuerzo para pasar el código serial a código paralelo. En 
este trabajo en particular es cierto, el nivel de paralelización que se escogió es el intermedio, todos los algoritmos relevantes (colapso e inversión de matrices, generación de datos ficticios, búsqueda de mínimo) son los mismos que en el caso serial. La dificultad de hacer el programa paralelo está en la gestión de las distintas tareas, la transferencia de datos entre el CPU y el GPU y los lanzamientos de threads.

El kernel ${ }^{1}$ esta diseñado para que cada thread lleve a cabo un experimento ficticio, pero también esta diseñado para que en cada llamada al kernel se puedan correr los experimentos ficticios de más de un punto, usualmente se hacían los experimentos ficticios de 3 a 12 puntos en cada llamada al kernel. La cantidad de puntos que cada llamada efectuará es fácilmente modificable en el programa, esta puesto de tal forma que sea fácil obtener la ocupación total de todos los threads.

En esencia lo que hace el programa es: generar datos ficticios de forma serial para todos los experimentos ficticios que requiera una llamada al kernel, mandar esos datos al GPU, llamar al kernel en donde se harán todos los experimentos ficticios, transferir al CPU el resultado de cada experimento ficticio. Esto se hace para todos los puntos en la malla reducida en que se quieran correcciones frecuentistas.

La memoria que usa la tarjeta de vídeo es la global (la más lenta) y constante (rápida). Se obtendrían serías ventajas si se lograse implementar un programa que haga uso de la memoria compartida o de textura, pero si es que es posible se tendría que cambiar el modelo de paralelización radicalmente.

Hay una gran cantidad de mejoras que se pueden hacer al programa, desde implementar algoritmos de inversión más eficientes y que requieran menos memoria, hasta cambiar el nivel de paralelización y el tipo de memoria que se usa. Comentando al respecto:

- No se reconoce un esquema, sencillo o complicado de programar, en el cual se pueda hacer uso de la memoria compartida o de textura de forma útil.

- Posterior a la realización de los análisis, el autor dio con un algoritmo más eficiente para realizar la inversión de matrices [198], este otorga una mejoría de $~ 30 \%$ en cada iteración del código serial. Hace falta probar que este nuevo algoritmo sea estable y robusto, tanto de forma serial como paralela. Es una mejoría sencilla bastante prometedora para el esquema paralelo, porque reduce el uso de operaciones lentas.

- Con el objetivo de permitir que estos análisis se puedan hacer en tarjetas más modestas o tarjetas que no estén dedicadas a hacer análisis (es decir que tengan que ocuparse de los gráficos de una computadora), hace falta agregar "descansos" al kernel o que varios threads se ocupen de una iteración simultáneamente (una mezcla entre el nivel intermedio e inferior de paralelización que se mencionaron antes), la primera opción es más fácil de implementar, la segunda no tanto.

- Mantener al CPU trabajando mientras corre el GPU, actualmente el CPU lanza el kernel y espera ocioso a que este acabe, momento en el cual hace más operaciones y lanza otro kernel.

Sin embargo, actualmente ya se tiene un rendimiento de $\sim 25$ veces más rápido que el esquema completamente serial, lo que es un logro notable.

\footnotetext{
${ }^{1}$ Conjunto de operaciones que se hacen en el GPU de forma paralela.
} 
Apéndice D

\section{Pruebas misceláneas del método Feldman-Cousins}

Se hicieron varias pruebas para comprobar el funcionamiento adecuado de todos los pasos del algoritmo que se usó, así como para investigar la posibilidad de nuevas formas de hacer los análisis. A continuación se mencionan algunas.

\section{D.1. Posición de los mínimos en los experimentos ficticios}

Se hizo con el objetivo de ver si era posible limitar el espacio de la búsqueda del punto de mejor ajuste en cada experimento ficticio. De esa forma se disminuiría el tiempo de cómputo. Sin embargo se observó que a pesar de que hay ciertas tendencias estas no son generales y se concluyó que restringir la búsqueda a solo una sección del ya restringido espacio de parámetros introduciría un sesgo en el análisis.

Sin embargo estas pruebas muestran sugerencias sobre la posible selección de la posición de una adivinanza inicial para aquellos algoritmos minimizadores que requieran una. La figura D.1 muestra la posición de los mínimos en $\left(\sin ^{2} 2 \theta, \Delta m^{2}\right)$ y el punto usado para generar los experimentos ficticios.

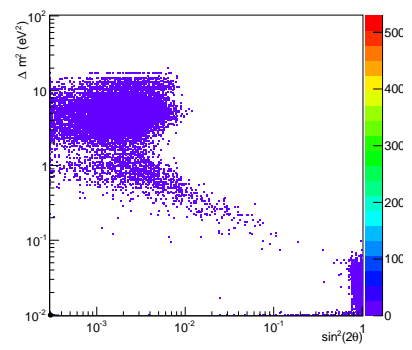

(a) $\left(0.0003,0.01 \mathrm{ev}^{2}\right)$

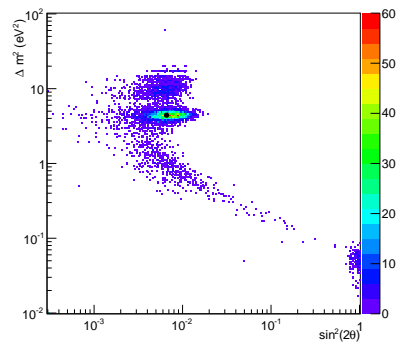

(b) $\left(0.0066,4.42 \mathrm{eV}^{2}\right)$

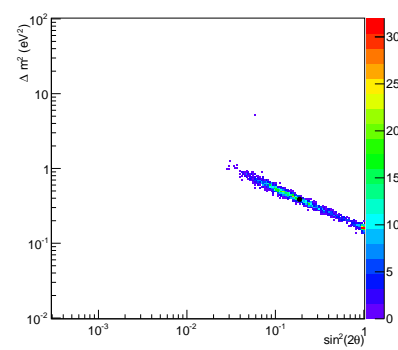

(c) $\left(0.1875,0.39 \mathrm{ev}^{2}\right)$

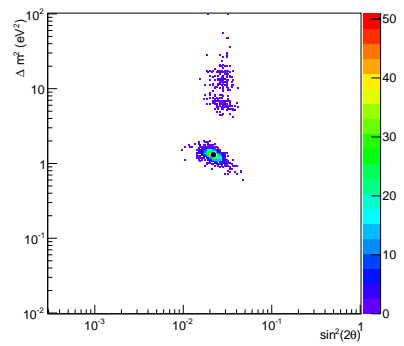

(d) $\left(0.0219,1.31 \mathrm{eV}^{2}\right)$

Figura D.1: Posiciones de los mínimos de los experimentos ficticios. Con el punto negro se generaron los experimentos ficticios (se muestra la coordenada debajo de cada figura), en escala de colores esta la cantidad de veces que un mínimo se obtuvo en cada coordenada. 


\section{D.2. Generación de matrices de covarianza con los vectores de experimentos falsos}

Una de las pruebas que se hizo para determinar si el generador de experimentos ficticios estaba funcionando fue generar matrices de covarianza con los vectores de experimentos ficticios, ecuación (3.9). Al comparar la matriz de covarianza generada con experimentos ficticios y la matriz colapsada a partir de los datos reales, ver $\S 3.2$, se requería que el error fraccionario entrada por entrada fuera del orden del error estadístico, es decir el inverso de la raíz del número de experimentos ficticios. Después de probar con varios algoritmos para generar números aleatorios se escogió el Mersenne Twister [205].

\section{D.3. Contornos obtenidos con Condor y CUDA}

Los contornos que se presentan en el capítulo 4 se hicieron analizando los datos con CUDA, sin embargo también se hicieron con Condor, de hecho las primeras versiones fueron efectuadas con Condor. En la figura D.2 se muestran los contornos del modo de antineutrinos de 2012 obtenidos tanto con Condor como con CUDA, nótese que los contornos de $68 \%$ y $90 \%$ N.C. son idénticos y que las diferencias en los contornos de $95 \%$ (en la parte superior) y $99 \%$ (del lado izquierdo) son menores.

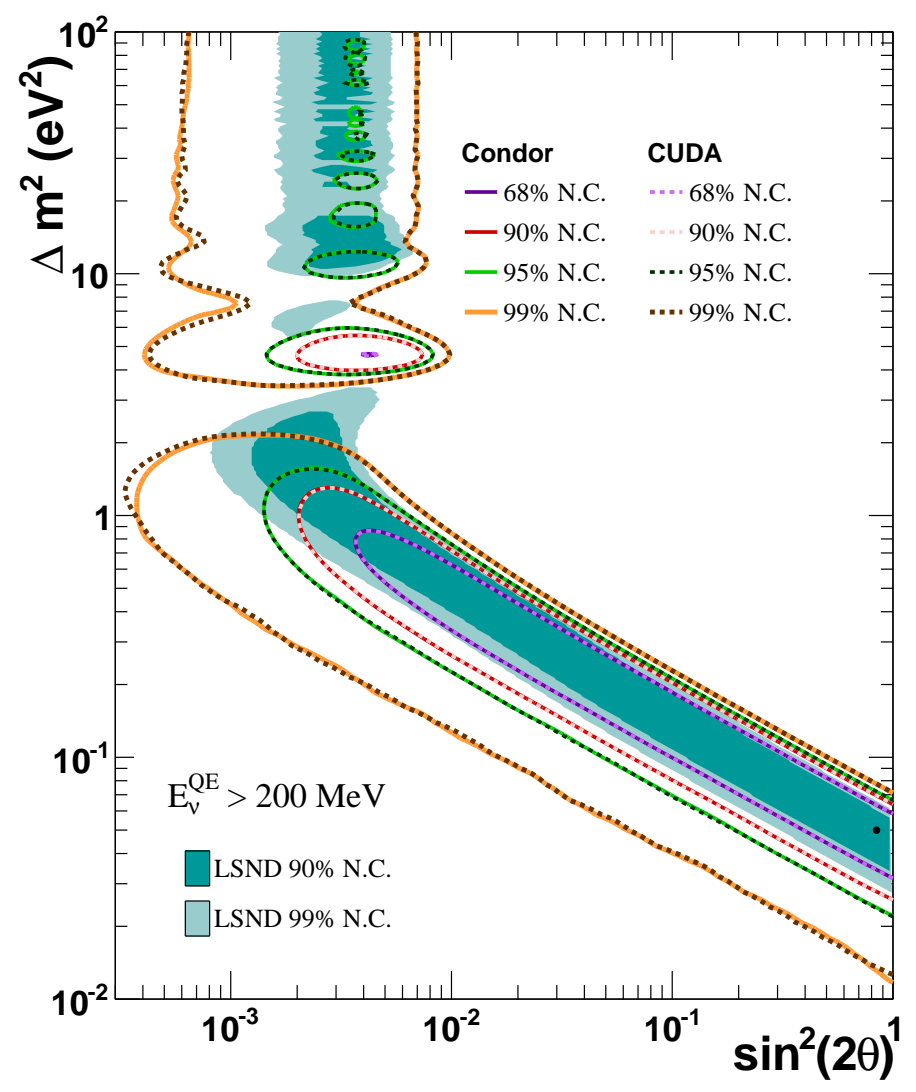

Figura D.2: Comparación entre Condor (línea sólida) y CUDA (línea punteada). Se muestran los contornos del modo de antineutrinos de 2012 solamente, pero todos muestran un comportamiento semejante. 


\section{D.4. Diferencias con los contornos oficiales}

En su mayoría, los contornos oficiales fueron reproducidos en este trabajo. Casi todos los contornos que fueron presentados son idénticos, sin embargo en todos los resultados frecuentistas se observan algunas diferencias en los contornos exteriores. En la figura D.3 se comparan los contornos.

Además de una búsqueda extensiva de errores en todo el código se hicieron varias pruebas para tratar de determinar la fuente de las diferencias.

\section{D.4.1. PRNG alternativo}

Como ya se mencionó el generador de números aleatorios (pseudo random number generator, PRNG) para generar los experimentos ficticios fue el Mersenne Twister que se puede descargar de [204]. Una de las sospechas que se tenía sobre las causas que originaban las diferencias en los contornos frecuentistas oficiales y los obtenidos en esta tesis era el PRNG. Se lanzaron nuevas corridas usando la función implementada por defecto en $\mathrm{C}$ para generar números aleatorios, rand(), y una transformación Box-Muller ${ }^{1}$. Los contornos obtenidos con este PRNG alternativo son notablemente más burdos, sin embargo son equivalentes a los obtenidos con el Mersenne Twister. A pesar de ser más burdos siguen siendo más estrechos que los oficiales.

\section{D.4.2. Baja y alta señal}

Pensando que las diferencias con los contornos oficiales se debían a una propagación sistemática del error en los cálculos de punto flotante, se investigó el efecto que tendría alterar el peso de la señal en la generación de experimentos ficticios. Así pues, se hicieron análisis en los cuales los experimentos ficticios fueron generados con un aumento de $0.1 \%$ en la señal y con una disminución de $0.001 \%$. En ambos casos los contornos obtenidos fueron sumamente distintos, muchísimo más estrechos. La prueba no logró concluir nada.

\footnotetext{
${ }^{1}$ Una combinación definitivamente inferior a la usada.
} 


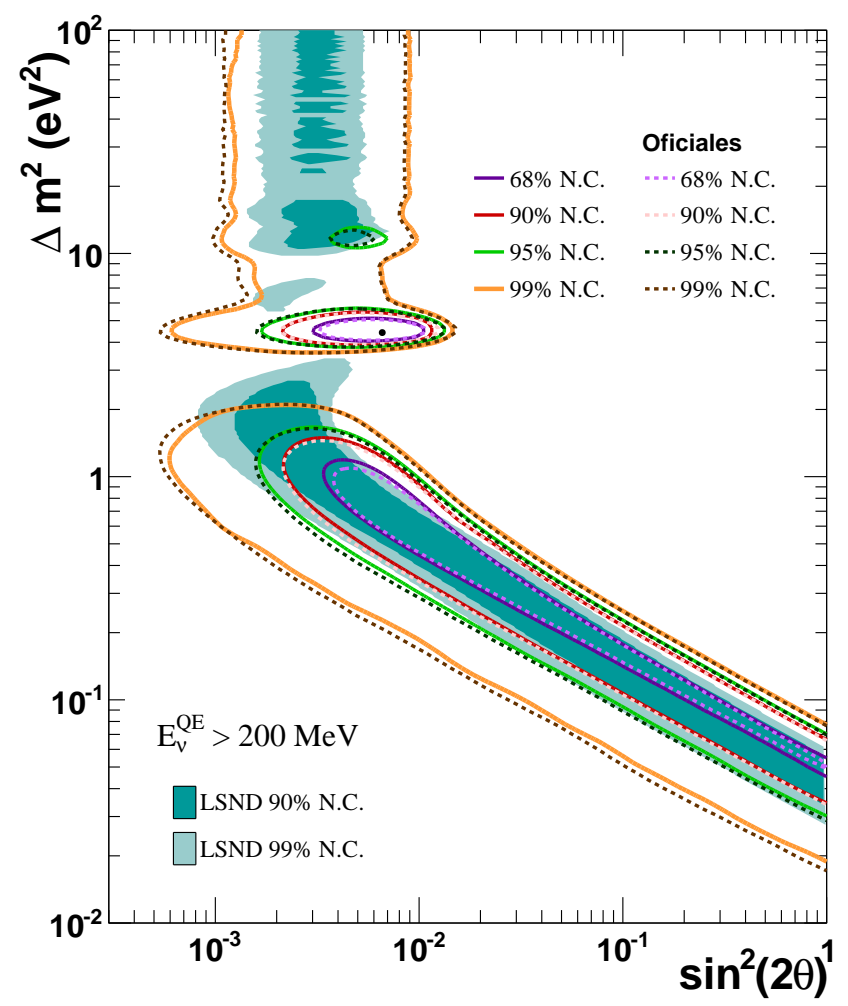

(a) Antineutrinos 2010 .

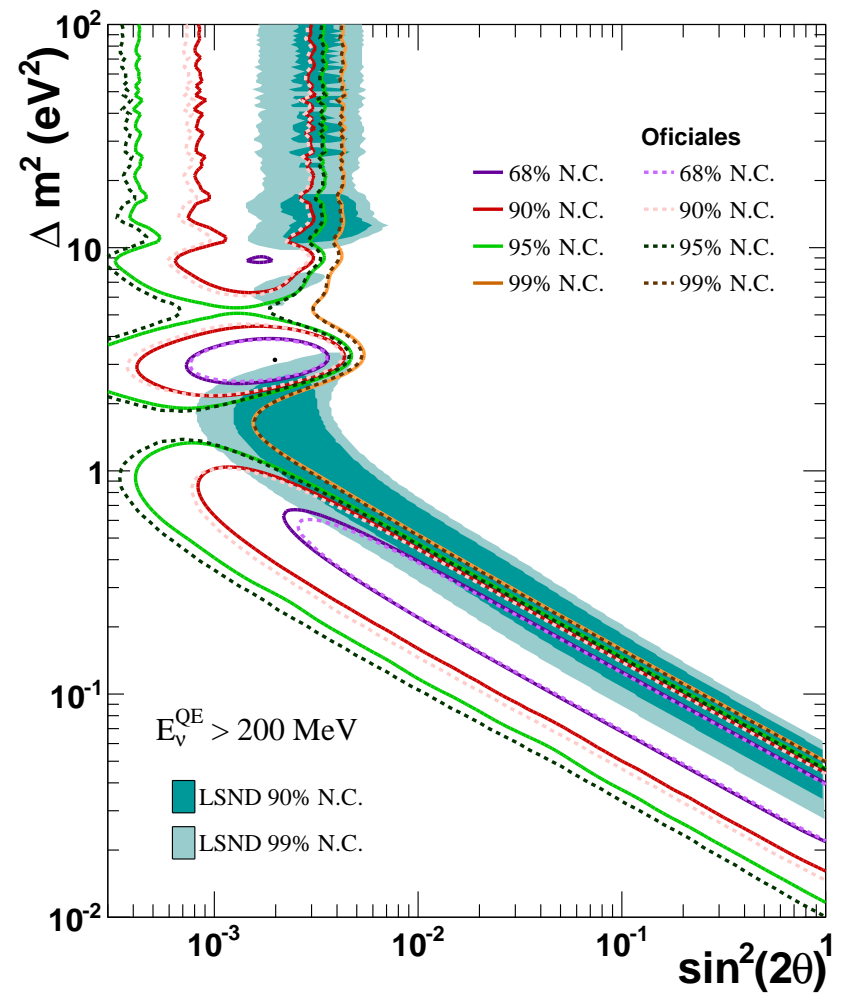

(c) Neutrinos 2012 .

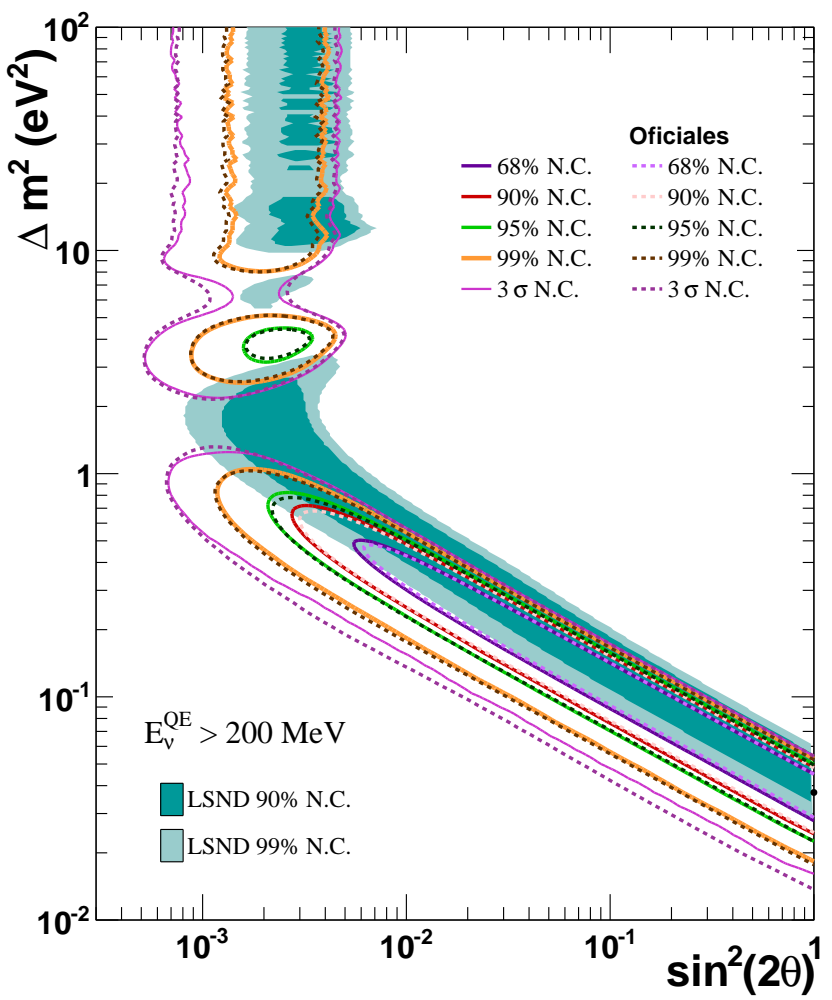

(b) Combinado 2012.

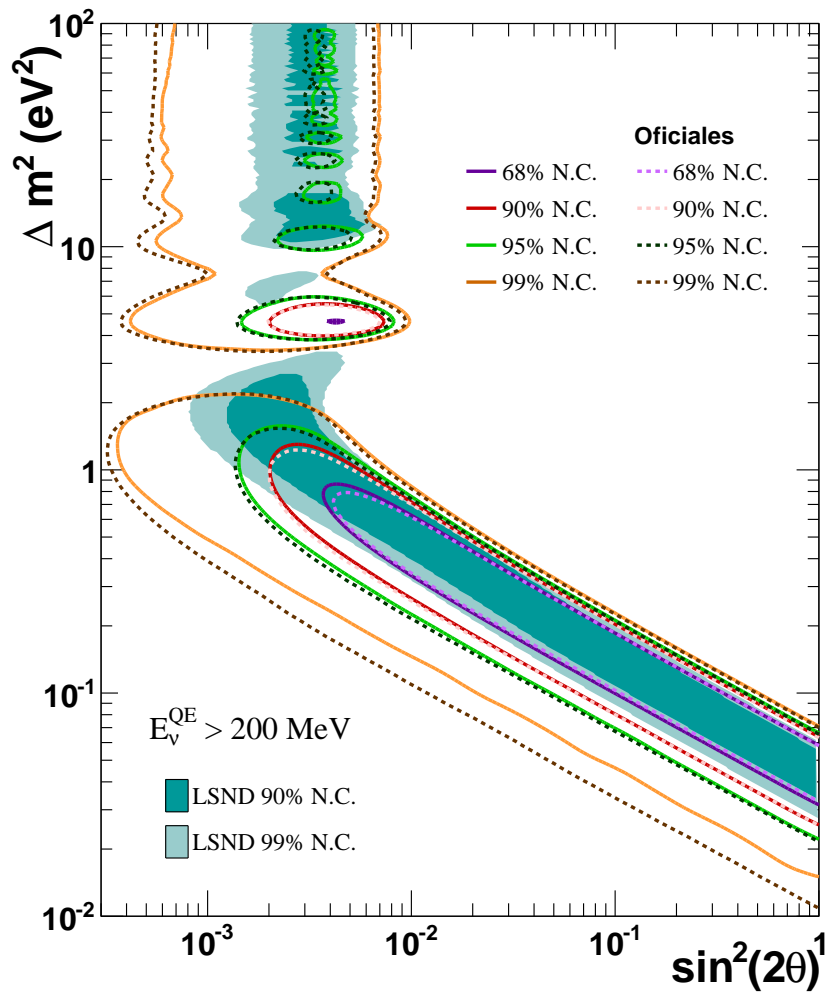

(d) Antineutrinos 2012.

Figura D.3: Comparación con los contornos oficiales. 


\section{Apéndice E}

\section{Constricción de los errores}

Además de constreñir la magnitud de las incertidumbres en el número de eventos $\nu_{e}\left(\bar{\nu}_{e}\right)$, la distribución de evento observados $\nu_{\mu}\left(\bar{\nu}_{\mu}\right)$ y las correlaciones entre las dos muestras proveen una forma de corregir la forma y normalización de la distribución predicha de $\nu_{e}\left(\bar{\nu}_{e}\right)$. A pesar de que no se aplique una corrección a la distribución de $\nu_{e}\left(\bar{\nu}_{e}\right)$, la presencia de los términos fuera de la diagonal en la verosimilitud $\mathscr{L}(3.12)$ permiten soluciones en que los bins de $\nu_{e}\left(\bar{\nu}_{e}\right)$ y $\nu_{\mu}\left(\bar{\nu}_{\mu}\right)$ muestren discrepancias similares con respecto a los datos, como podría ser una normalización residual entera más grande que las barras de error, mientras que da un valor aceptable de like. Como resultado, un exceso de eventos en la distribución de $\nu_{e}\left(\bar{\nu}_{e}\right)$ solo sera atribuido a una señal de oscilación si es más grande que las diferencias que pueden ser tomadas en cuenta a través de las correlaciones entre $\nu_{\mu} / \nu_{e}\left(\bar{\nu}_{\mu} / \bar{\nu}_{e}\right)$, es decir, un exceso de eventos $\nu_{\mu}\left(\bar{\nu}_{\mu}\right)$ se verá reflejado como un exceso $\nu_{e}\left(\bar{\nu}_{e}\right)$ y este último exceso no necesariamente significa una señal de oscilación.

\section{E.1. Construcción de la constricción de errores}

Pensemos solamente en una $\chi^{2}$, como la de la ecuación (3.31), para calcular explícitamente como sería la corrección equivalente, se puede intentar resolver para el número de eventos en la distribución $\nu_{e}$ y $\nu_{\mu}$ que minimizan la siguiente función $\chi^{2}$ :

$$
\chi^{2}=\sum_{i, j=1}^{n_{e}+n_{\mu}} \Delta_{i} \mathrm{M}_{i j}^{-1} \Delta_{j}+\sum_{k}^{n_{m u}} \frac{\left(F_{k}-D_{k}\right)^{2}}{D_{k}}, \quad \frac{\partial \chi^{2}}{\partial F_{i}}=0
$$

donde $\boldsymbol{D}$ es el vector de datos, $\boldsymbol{F}$ es el vector de datos ajustados, $\Delta_{i}=F_{i}-Q_{i}$ es la diferencia entre el número ajustado de eventos y la predicción MC y como antes $\mathbf{M}$ la matriz de covarianza. El conjunto de ecuación que se necesitan resolver son:

$$
\frac{\partial \chi^{2}}{\partial F_{i}}=2 \sum_{i, j=1}^{n_{e}+n_{\mu}} \mathrm{M}_{i j}^{-1} \Delta_{j}+2 \frac{\left(F_{i}-D_{i}\right) \delta_{i\left(\text { bin } n_{\mu}\right)}}{D_{i}}=0,
$$

donde $\delta_{i\left(\text { bin } n_{\mu}\right)}$ cuando $i$ es un bin de $\nu_{\mu}$ y cero de otra forma. Al definir la matriz $\mathrm{B}_{i j}^{-1}$ como sigue:

$$
\mathrm{B}_{i j}^{-1}= \begin{cases}\mathrm{M}_{i j}^{-1} ; & i \leq n_{e} \text { о } j \leq n_{e} \\ \mathrm{M}_{i j}^{-1}+\frac{1}{D_{i}} ; & i>n_{e} \text { y } j>n_{e}\end{cases}
$$


se puede escribir la ecuación (E.1) de la siguiente forma:

$$
\sum_{j} \mathrm{~B}_{i j}^{-1} F_{j}=\sum_{j} \mathrm{M}_{i j}^{-1} Q_{j}+\frac{D_{i} \delta_{i\left(b i n n_{\mu}\right)}}{D_{i}} .
$$

La solución de este conjunto de ecuaciones es

$$
F_{i}=\sum_{k} \mathrm{~B}_{i k}\left(\sum_{j} \mathrm{M}_{k j}^{-1} Q_{j}+\delta_{k\left(b i n n_{\mu}\right)}\right)
$$

y la matriz de covarianza para los valores $F_{i}$ esta dada por

$$
\mathrm{B}_{i j}=\left\langle\delta F_{i} \delta F_{j}\right\rangle .
$$

Los errores contenidos en la matriz $\mathrm{B}_{i j}$ han sido efectivamente constreñidos por el alto número de eventos en los bins de la distribución de $\nu_{\mu}$. Las transformaciones en las ecuaciones (E.2) y (E.4) son usadas para presentar los resultados de las distribuciones ajustadas y constreñir las barras de error, sin embargo no son parte de la maquinaría de ajuste. De forma intuitiva se puede pensar que las reducidas barras de error sobre la distribución $\nu_{\mu}$ otorgan un peso más fuerte en el ajuste, en la figura E.1 se puede observar la disminución de las barras de error en la muestra de $\bar{\nu}_{\mu}$, también hay ligeras diferencias en las posiciones de los histogramas, sin embargo son prácticamente imperceptibles.

A modo de ejemplo se muestra ahora de forma analítica como los eventos $\nu_{\mu}$ constriñen a los eventos $\nu_{e}$, con el ejemplo más sencillo posible de un bin de $\nu_{e} \mathrm{y}$ un bin de $\nu_{\mu}$. En tal circunstancia se tienen la incertidumbre estadística $\sigma_{\nu_{e}} \mathrm{y} \sigma_{\nu_{\mu}}$ en el número de eventos en la muestras de eventos $\nu_{e}$ y $\nu_{\mu}$, respectivamente; la incertidumbre sistemática $\delta_{\nu_{e}}$ y $\delta_{\nu_{\mu}}$ de cada muestra; y la correlación $\rho$ entre la incertidumbre sistemática de ambas muestras. Así la matriz de error para el sistema puede ser escrita como:

$$
\mathbf{E}_{2 \times 2}=\left(\begin{array}{cc}
\sigma_{\nu_{e}}^{2}+\delta_{\nu_{e}}^{2} & \rho \delta_{\nu_{e}} \delta_{\nu_{\mu}} \\
\rho \delta_{\nu_{e}} \delta_{\nu_{\mu}} & \sigma_{\nu_{\mu}}^{2}+\delta_{\nu_{\mu}}^{2}
\end{array}\right)
$$

Definiendo $\alpha_{\nu_{e}}=\sigma_{\nu_{e}} / \delta_{\nu_{e}}$ y $\alpha_{\nu_{\mu}}=\sigma_{\nu_{\mu}} / \delta_{\nu_{\mu}}$, la inversa de la matriz de error es

$$
\mathbf{E}_{2 \times 2}^{-1}=\frac{1}{\left(\alpha_{\nu_{e}}^{2}+1\right)\left(\alpha_{\nu_{\mu}}^{2}+1\right)-\rho^{2}}\left(\begin{array}{cc}
\frac{\alpha_{\nu_{\mu}}^{2}+1}{\delta_{\nu_{e}}^{2}} & \frac{-\rho}{\delta_{\nu} \delta_{\nu_{\mu}}} \\
\frac{-\rho}{\delta_{\nu_{e}} \delta_{\nu_{\mu}}} & \frac{\alpha_{\nu_{e}}^{2}+1}{\delta_{\nu_{\mu}}^{2}}
\end{array}\right)
$$

Por su parte para la $\chi^{2}$, si $s$ es el número de eventos de oscilación en la muestra de $\nu_{e}, \Delta_{\nu_{e}}$ y $\Delta_{\nu_{\mu}}$ son las respectivas diferencias entre los eventos observados y esperados para las distintas muestras, entonces la $\chi^{2}$ se puede escribir en forma vectorial como

$$
\chi^{2}=\left(\begin{array}{ll}
\Delta_{\nu_{e}}-s & \Delta_{\nu_{\mu}}
\end{array}\right) \mathbf{E}_{2 \times 2}^{-1}\left(\begin{array}{c}
\Delta_{\nu_{e}}-s \\
\Delta_{\nu_{\mu}}
\end{array}\right)
$$

al desarrollar se obtiene

$$
\begin{aligned}
\chi^{2} & =\frac{1}{\left(\alpha_{\nu_{e}}^{2}+1\right)\left(\alpha_{\nu_{\mu}}^{2}+1\right)-\rho^{2}} \times \\
& \times\left[\left(\alpha_{\nu_{\mu}}^{2}+1\right) \frac{\left(\Delta_{\nu_{e}}-s\right)^{2}}{\delta_{\nu_{e}}}-2 \rho \frac{\Delta_{\nu_{\mu}}\left(\Delta_{\nu_{e}}-s\right)}{\delta_{\nu_{e}} \delta_{\nu_{\mu}}}+\left(\alpha_{\nu_{e}}^{2}+1\right) \frac{\Delta_{\nu_{\mu}}^{2}}{\delta_{\nu_{\mu}}^{2}}\right]
\end{aligned}
$$


Diferenciando esta última expresión con respecto a $s$ e igualando con cero produce el mejor ajuste para los eventos de señal:

$$
s=\Delta_{\nu_{e}}\left[1-\frac{\rho}{\alpha_{\nu_{\mu}}^{2}+1} \frac{\Delta_{\nu_{\mu}} / \delta_{\nu_{\mu}}}{\Delta_{\nu_{e}} / \delta_{\nu_{e}}}\right]
$$

además, al tomar el doble de la inversa de la segunda derivada se obtiene la incertidumbre en el número de eventos de señal

$$
\delta_{s}^{2}=\sigma_{\nu_{e}}^{2}+\left[1-\frac{\rho^{2}}{\alpha_{\nu_{\mu}}^{2}+1}\right] \delta_{\nu_{e}}^{2}
$$

Hay dos importantes casos extremos que notar de las ecuaciones (E.10) y (E.11):

- Si no hay correlación entre los bins de $\nu_{e}$ y $\nu_{\mu}(\rho=0)$, o si la muestra de $\nu_{\mu}$ tiene una gran incertidumbre estadística $\left(\alpha_{\nu_{\mu}} \rightarrow \infty\right)$ entonces la señal extraída y su incertidumbre es $\Delta_{\nu_{e}} \pm \sqrt{\sigma_{\nu_{e}}^{2}+\delta_{\nu_{e}}^{2}}$.

- Si los $\nu_{e}$ y $\nu_{\mu}$ están completamente correlacionados $(\rho=1)$ y la muestra de $\nu_{e}$ tiene incertidumbre estadística despreciable $\left(\alpha_{\nu_{\mu}}=0\right)$, la señal extraída y su incertidumbre es $\Delta_{\nu_{e}}\left(1-\frac{\Delta_{\nu_{\mu}} / \delta_{\nu_{\mu}}}{\Delta_{\nu_{e}} / \delta_{\nu_{e}}}\right) \pm$ $\sigma_{\nu_{e}}$.
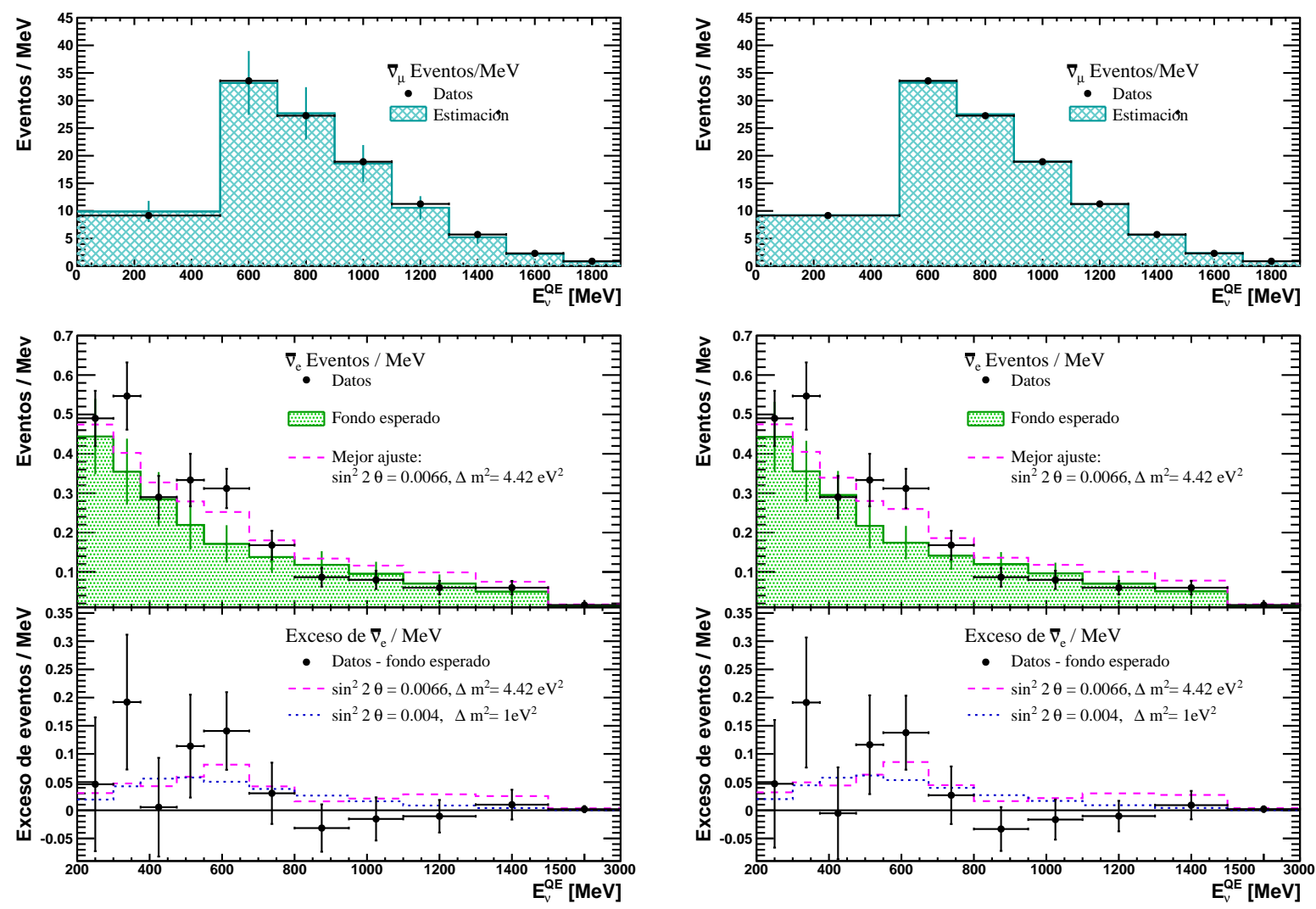

Figura E.1: Comparación de histogramas con errores sin constreñir y constreñidos. Datos de 2010 del modo de antineutrinos. A la izquierda se muestran los histogramas sin constreñir y a la derecha con el error constreñido. La constricción de los errores para los $\bar{\nu}_{\mu}$ es notable, el efecto en los otros histogramas es humilde. 


\section{Apéndice $\mathrm{F}$}

\section{Fibra óptica en el RWM}

Como una oportunidad para conocer el experimento MiniBooNE en persona, a algunos de sus colaboradores, la forma en la que se trabajaba y el laboratorio Fermilab, el autor de esta tesis viajó a Estados Unidos para hacer una corta estancia de investigación del 20 de marzo al 7 de mayo de 2012. Además de trabajar en los análisis de datos que ya se discutieron, se implementó y se empezó la caracterización de una fibra óptica que serviría para ayudar en la sincronización temporal de los eventos. Este trabajo se hizo en colaboración con los investigadores del laboratorio de Los Alamos: Dr. Žarko Pavlović y Dr. Richard Van de Water.

\section{La señal del RWM en MiniBooNE}

El Resistive Wall Monitor (RWM) es una bobina de alambre localizada en el edificio MI12, debajo de la tierra y un par de metros antes del blanco en el eje del haz. Fue instalado entre 2001 y 2002 y ha estado en operación desde entonces. Justo antes de impactar el blanco de Be, los protones inducen una señal en esta espira. El primer bunch de protones, con $6 \times 10^{10}$ protones, dispara la electrónica en MI12 y de ahí viaja un pulso cuadrado por un cable hacia el edificio del detector.

En el detector se almacena la señal cuadrada que viajo desde MI12 por el cable de cobre (copper wire first hit, CW1). Esta misma señal rebota de vuelta hacia MI12 al llegar al extremo del cable, el cual esta terminado con una resistencia ${ }^{1}$. En MI12 rebota por segunda ocasión y por segunda ocasión se dirige hacia el detector. En el detector se almacena la señal del cable de cobre que viajó por segunda vez (copper wire second hit, CW2). Nótese que esta señal viajó la distancia entre MI12 y el detector un total de tres veces. En la figura F.1 se resume el esquema de operación del RWM. Hay otros sistemas usados para monitorear la posición y el ancho del haz de protones.

Se hace esto con el objetivo de tener en cuenta los cambios en el tiempo de propagación debido a cambios en la constante dieléctrica y el tamaño del cable, como resultado de cambios en la temperatura. Al tomar la diferencia de tiempos entre la primera y segunda señal se puede calcular la velocidad de propagación en cualquier momento. Con la velocidad de propagación y la etiqueta de tiempo de la primera señal se puede calcular con precisión la hora a la cual los protones pasan por el RWM. Tener una mejor resolución de tiempo ayuda a discriminar eventos de corriente neutra, en particular $\pi^{0}$.

\footnotetext{
${ }^{1}$ Este rebote es análogo al que haría una onda mecánica al llegar a un pared.
} 


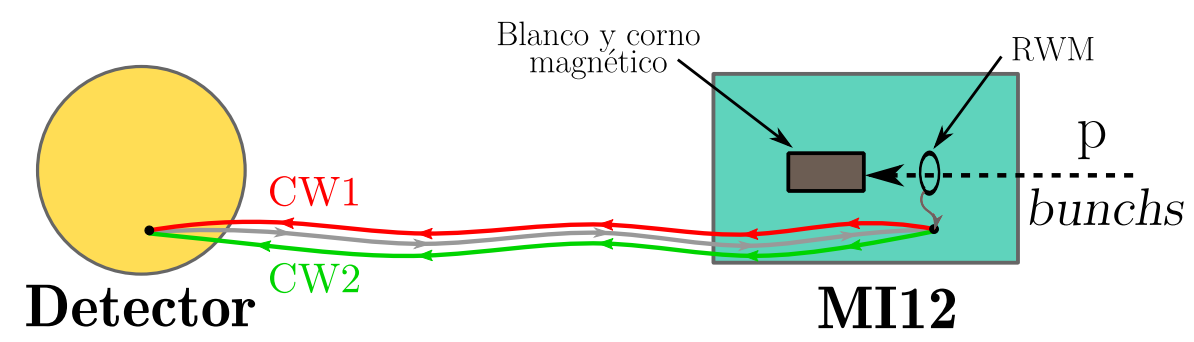

Figura F.1: Esquema de operación del RWM. Los bunchs de protones provenientes del Booster atraviesan el RWM antes de impactar el blanco de Be. Una señal viaja de MI12 al detector a través de un cable de cobre, en el detector se almacena esta señal (CW1). Rebota en el detector y otra vez en MI12 para ser almacenada por segunda ocasión en el detector (CW2). De ida y vuelta viaja a través del mismo cable.

Sean $t_{1}$ la etiqueta de tiempo para CW1, $t_{2}$ la etiqueta de tiempo para CW2, $t_{\text {viaje }}=d / v$ el tiempo que toma la señal para viajar de MI12 al detector y $t$ la etiqueta de tiempo de interés, aquella asociada al paso de los protones por el RWM. Insistiendo, $t_{\text {viaje }}$ cambia con las variaciones de temperatura.

Así pues: $t=t_{1}-t_{\text {viaje }}$; notemos que $t_{2}-t_{1}=\frac{2 d}{v}=2 t_{\text {viaje }}$. Implícitamente se acepta que la variación en la temperatura, y por lo mismo la variación en la velocidad de propagación, es despreciable en el intervalo de tiempo que le toma a la señal dar dos vueltas. Al remplazar el tiempo de viaje se obtiene:

$$
t=t_{1}-\frac{t_{2}-t_{1}}{2}
$$

El RWM es capaz de producir una señal para cada bunch de protones en el spill, pero solo se dispara en el primer bunch del spill ya que la estructura del haz de protones es muy estable y se conoce suficientemente bien como para reconstruir los otros bunchs, en $\S 2.1 .1$ se habla sobre la estructura del haz de protones. Esta señal analógica, la que inducen los protones en la espira, es discriminada y convertida en un pulso cuadrado en cajas NIM (Nuclear Instrumentation Module) en el edificio del blanco, MI12. El pulso cuadrado viaja por un cable de cobre al edificio del detector, ahí después de ser discriminado, se almacena y rebota al MI12. El umbral del discriminador en el detector es suficientemente bajo como para permitir el paso de la atenuada señal reflejada, pero suficientemente alto como para prohibir la entrada de una señal que haya rebotado más veces. A pesar de que los datos del RWM no entran directamente en los análisis, permitieron verificar el funcionamiento de muchas cosas: ver que se pueden relacionar los eventos de neutrinos en el detector a cada bunch (revisar § 2.1.1) de protones y comprobar que el pulso de corriente que fluye a través del corno magnético es esencialmente estático a la escala del tiempo del paso de protones.

Inconvenientes con el RWM: A pesar de que es un sistema robusto que permite notar pequeñas variaciones, es muy susceptible a perturbaciones externas. Mover, conectar y desconectar cables suele tener efectos inesperados en las diferencias de tiempo. Más aún, un alambre de cobre de más de $500 \mathrm{~m}$ de largo actúa como antena y durante las tormentas eléctricas suele haber variaciones en los tiempos. En el pasado se tuvieron serios problemas con esto, parte de la electrónica resulto dañada por culpa de las altas corrientes que se inducían. Para evitar más daños se instaló un circuito op-amp en cada uno de los extremos del cable para que actuaran como fusibles, una fuerte tormenta eléctrica quemaría tales circuitos pero no componentes importantes o caras. Sin embargo, la señal del RWM se pierde hasta que alguien remplace el circuito quemado. No todas las tormentas queman los circuitos, pero es común que una tormenta de menor intensidad cause desplazamientos en las etiquetas de tiempo, tal 
conducta no tiene otra explicación que un mal funcionamiento del op-amp. Si bien se sigue teniendo la señal del RWM, esta ya no es del todo confiable. La figura F.2 muestra los histogramas de los tiempos registrados por el RWM y que se muestran en línea ${ }^{2}$. En la figura F.2a se muestran los histogramas de los tiempos registrados de CW1 y CW2, su variación oscila de 30 a 40 ns, dominada por fluctuaciones del Booster. Mientras que la figura F.2b muestra la diferencia de tiempos, en esta figura se pueden notar las pequeñas variaciones que la diferencia de tiempo atraviesa, variaciones no del todo esperadas. Es importante notar que como estas variaciones son menores a 15 ns no son perceptibles en las otras dos gráficas.

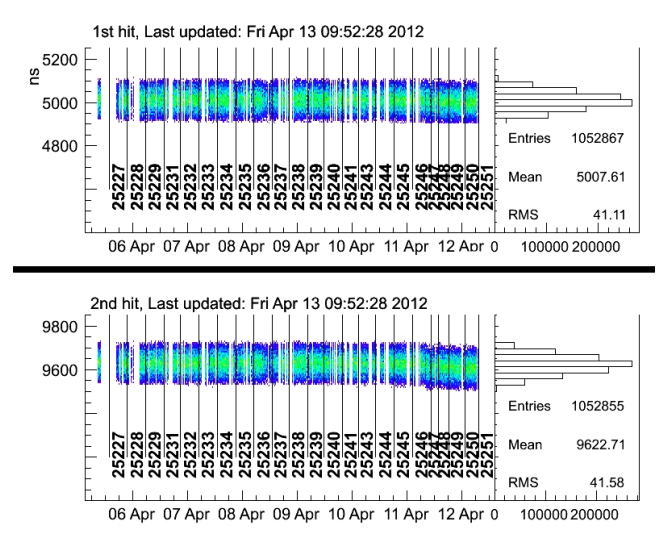

(a) CW1 y CW2

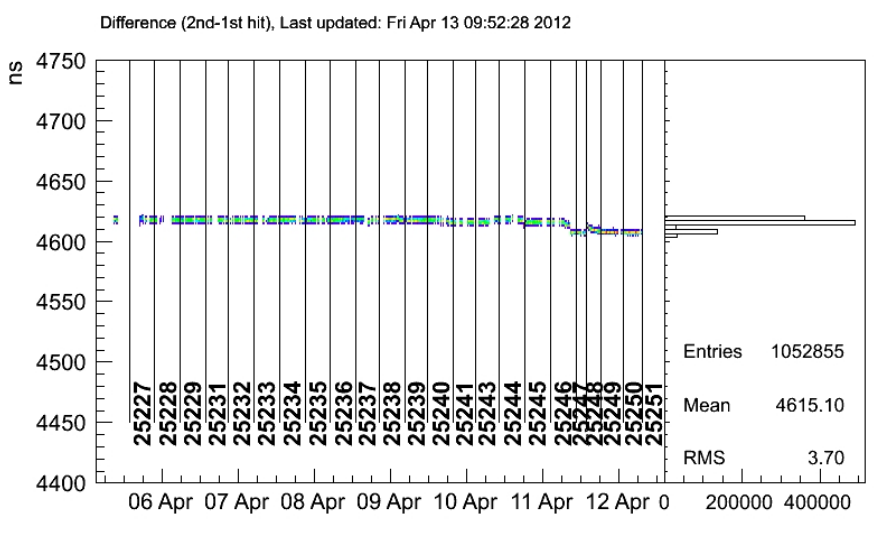

(b) CW2-CW1

Figura F.2: Gráficas en línea del RWM. En el eje horizontal se tiene la fecha y un número que identifica la corrida a la que se relacionan, en el eje vertical se gráfica la etiqueta de tiempo.

\section{Nuevo sistema con fibra óptica}

Con la intención de lidiar con tales inconvenientes se instaló un nuevo sistema para remplazar el cable de cobre con una fibra óptica. Ya que se espera que el tiempo de propagación de las señales sea más estable puesto que la fibra es menos susceptible a las variaciones de temperatura, la atenuación de la señal es despreciable y no sería afectada por los truenos ya que es un dieléctrico. Se tendió una fibra óptica que conectaba MI12 con el detector, la señal que generaban los protones al pasar por el RWM era separada en una componente que viajaba por el cable, como antes se venía haciendo, y otra componente que viajaba por la fibra hasta el detector, en donde se almacenaba (fiber optics, FO). Dada la supuesta baja susceptibilidad de la fibra a los cambios de temperatura, no se requería rebotar señales. Se omiten los detalles de tal implementación ya que a final de cuentas tuvo que cambiarse. Una vez conectada la fibra y habiendo configurado los sistemas de adquisición de datos, se procedió a probar el nuevo sistema, comparándolo con el ya existente que usaba el cable de cobre. Varios defectos surgieron.

Bulto: Se observó un ligero exceso de eventos cuando se tomaba la diferencia entre los tiempos del cable de cobre y la fibra óptica. Por ejemplo cuando se toma la diferencia FO-CW1 la mayoría de los eventos tienen una diferencia de $3.1 \mu$, sin embargo hay una cantidad considerable 15 ns después. No se puede atribuir este comportamiento a ninguna de las muestras, CW1, CW2 o FO; ya que todas tienen una dispersión mayor. Tampoco es razonable pensar que este comportamiento es

\footnotetext{
${ }^{2}$ Solo accesibles por la colaboración.
} 
una fluctuación estadística. La figura F.3a muestra un bulto estándar. Después se encontró la razón de este comportamiento: uno de los discriminadores estaba siendo disparado en la parte posterior (descendente) del pulso. Tal pulso dura unos cuantos microsegundos, la parte creciente del pulso es estable ( $\leq 1 \mathrm{~ns}$ ) y se llega al máximo en unos cuantos picosegundos, sin embargo el extremo descendiente lleva varios nanosegundos en llegar al mínimo y no siempre lo hace de la misma forma.

Corrimientos: Una lección que se aprendió es que se requiere conectar a la fibra óptica en un modulo reservado. Colaboradores del experimento SciBath trabajaban con la señal del RWM mientras se determinaba la configuración del hardware y se hacían pruebas, cada vez que empezaban a recolectar datos se conectaban a la señal del RWM. La bitácora que mantenían de sus actividades fue de gran ayuda para determinar que los corrimientos de tiempo se debían a ellos, como se muestra en la figura F.3b.

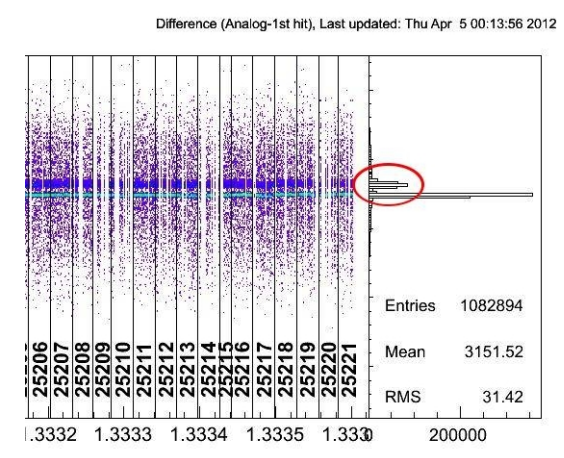

(a) Bulto

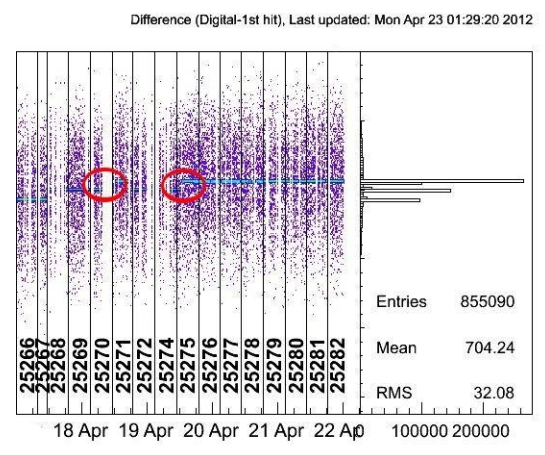

(b) Corrimientos de tiempo

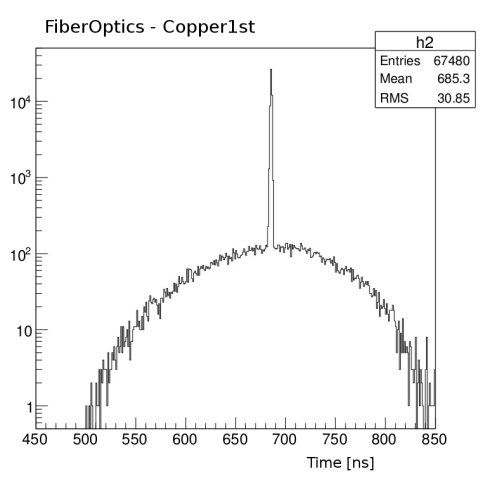

(c) Colas amplias

Figura F.3: Defectos en el sistema con fibra óptica.

Colas amplias: Al comparar los tiempos entre la fibra y el cobre, o entre los dos distintos canales en que se conectaba la fibra, se podía ver una dispersión alrededor de la media de 100 ns. Este problema es despreciable en un histograma lineal, pero al graficar en escala logarítmica se puede observar una fracción de $5 \%$ a $10 \%$ fuera del pico central, como lo muestra la figura F.3c. Este problema no se pudo resolver, pero todo parecía indicar a que tenía que ver con los módulos que almacenaban la señal de la fibra óptica.

Desafortunadamente, un par de días antes de concluida la estancia, un trueno quemó el discriminador encargado de separar la señal de la fibra y el cable de cobre. Lo que hizo que trabajar a distancia se volviera mucho más complicado. Después de que se reparó el sistema surgieron nuevos comportamientos anómalos, los cuales ya no podían ser investigados remotamente. Después de que se rechazó la primera solicitud de la colaboración para la búsqueda de materia oscura, la implementación de la fibra óptica entró en hiato. El trabajo que se llevo a cabo en Fermilab no fue suficiente para lograr implementar un sistema de sincronización de tiempo completamente funcional, pero fue útil como parte del proceso necesario de instrumentación. Al final, el mecanismo del sistema que fue instalado cambió, pero se basó en una propuesta que surgió de este trabajo.

Una componente importante del análisis es el uso del tiempo de sincronización con respecto al haz, lo cual se lograba con la alta estadística de datos $\nu_{\mu}\left(\bar{\nu}_{\mu}\right)$. Dado que el experimento planea hacer una búsqueda de materia oscura en la cual la gran mayoría de los neutrinos estarán ausentes, hace falta un mecanismo que permita sincronizar precisamente los eventos con respecto a la llegada del haz. 


\section{Bibliografía}

[1] W. Pauli, "Carta sin publicar a la sociedad de física de Tubinga," diciembre 1930.

[2] L. M. Brown, "The idea of the neutrino," Physics Today, vol. 31, no. 9, pp. 23-28, 1978.

[3] J. Chadwick, "The intensity distribution in the magnetic spectrum of beta particles from radium (B + C)," Verh.Phys.Gesell., vol. 16, pp. 383-391, 1914.

[4] J. Chadwick, "The Existence of a Neutron," Proceedings of the Royal Society of London. Series A, vol. 136, no. 830, pp. 692-708, 1932.

[5] E. Fermi, "Versuch einer Theorie der $\beta$-Strahlen. I," Zeitschrift für Physik, vol. 88, pp. 161-177, 1934.

[6] K. C. Wang, "A Suggestion on the Detection of the Neutrino," Phys. Rev., vol. 61, pp. 97-97, Jan 1942.

[7] K. Zuber, Neutrino Physics. Taylor \& Francis, second ed., 2011.

[8] G. W. Rodeback \& J. S. Allen, "Neutrino Recoils Following the Capture of Orbital Electrons in A ${ }^{37}$," Phys. Rev., vol. 86, pp. 446-450, May 1952.

[9] R. Davis, "Attempt to Detect the Antineutrinos from a Nuclear Reactor by the $\mathrm{Cl}^{37}\left(\bar{\nu}, e^{-}\right) \mathrm{A}^{37}$ Reaction," Phys. Rev., vol. 97, pp. 766-769, Feb 1955.

[10] C. S. Wu, E. Ambler, R. W. Hayward, D. D. Hoppes, \& R. P. Hudson, "Experimental Test of Parity Conservation in Beta Decay," Phys. Rev., vol. 105, pp. 1413-1415, Feb 1957.

[11] R. L. Garwin, L. M. Lederman, \& M. Weinrich, "Observations of the Failure of Conservation of Parity and Charge Conjugation in Meson Decays: the Magnetic Moment of the Free Muon," Phys. Rev., vol. 105, pp. 1415-1417, Feb 1957.

[12] M. Goldhaber, L. Grodzins, \& A. W. Sunyar, "Helicity of Neutrinos," Phys. Rev., vol. 109, pp. 10151017, Feb 1958.

[13] G. Danby et al., "Observation of High-Energy Neutrino Reactions and the Existence of Two Kinds of Neutrinos," Phys. Rev. Lett., vol. 9, pp. 36-44, Jul 1962.

[14] S. Weinberg, "A model of leptons," Phys. Rev. Lett., vol. 19, pp. 1264-1266, Nov 1967.

[15] F. Hasert et al., [Gargamelle] , "Search for elastic muon-neutrino electron scattering," Physics Letters $B$, vol. 46, no. 1 , pp. $121-124,1973$.

[16] F. Hasert et al., [Gargamelle] , "Observation of neutrino-like interactions without muon or electron in the Gargamelle neutrino experiment," Nuclear Physics B, vol. 73, no. 1, pp. 1 - 22, 1974.

[17] F. Hasert et al., [Gargamelle], "Observation of neutrino-like interactions without muon or electron in the Gargamelle neutrino experiment," Physics Letters B, vol. 46, no. 1, pp. 138 - 140, 1973.

[18] G. Arnison et al., "Experimental observation of lepton pairs of invariant mass around $95 \mathrm{GeV} / c^{2}$ at the CERN SPS collider," Physics Letters B, vol. 126, no. 5, pp. 398 - 410, 1983.

[19] G. Arnison et al., "Experimental observation of isolated large transverse energy electrons with associated missing energy at $s=540 \mathrm{GeV}$," Physics Letters B, vol. 122, no. 1, pp. $103-116,1983$.

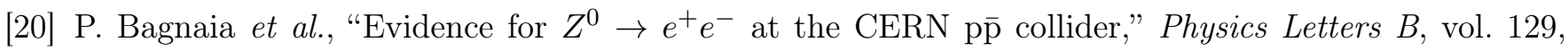


no. $1-2$, pp. $130-140,1983$.

[21] M. Banner et al., "Observation of single isolated electrons of high transverse momentum in events with

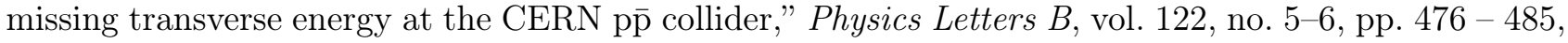
1983.

[22] M. L. Perl et al., "Evidence for Anomalous Lepton Production in $e^{+}-e^{-}$Annihilation," Phys. Rev. Lett., vol. 35, pp. 1489-1492, Dec 1975.

[23] S. Abachi et al., [D0] , Observation of the Top Quark," Phys. Rev. Lett., vol. 74, pp. 2632-2637, Apr 1995.

[24] F. Abe et al., [CDF], "Observation of Top Quark Production in $\bar{p} p$ Collisions with the Collider Detector at Fermilab," Phys. Rev. Lett., vol. 74, pp. 2626-2631, Apr 1995.

[25] K. Kodama et al., [DONUT] , "Observation of tau neutrino interactions," Physics Letters B, vol. 504, no. 3, pp. $218-224,2001$.

[26] J. A. Bagger, "Precision electroweak measurements on the $Z$ resonance," Physics Reports, vol. 427, no. 5-6, pp. $257-454,2006$.

[27] D. DeCamp et al., "Determination of the number of light neutrino species," Physics Letters B, vol. 231, no. 4, pp. 519 - 529, 1989.

[28] B. Pontecorvo, "Mesonium and anti-mesonium," Sov.Phys.JETP, vol. 6, p. 429, 1957.

[29] T. Lee, R. Oehme, \& C.-N. Yang, "Remarks on Possible Noninvariance Under Time Reversal and Charge Conjugation," Phys.Rev., vol. 106, pp. 340-345, 1957.

[30] T. Lee \& C. Wu, "Weak Interactions: Decays of neutral K mesons," Ann.Rev.Nucl.Part.Sci., vol. 16, pp. 511-590, 1966.

[31] Z. Maki, M. Nakagawa, \& S. Sakata, "Remarks on the Unified Model of Elementary Particles," Progress of Theoretical Physics, vol. 28, pp. 870-880, Nov. 1962.

[32] B. Pontecorvo, "Neutrino Experiments and the Problem of Conservation of Leptonic Charge," Soviet Journal of Experimental and Theoretical Physics, vol. 26, p. 984, May 1968.

[33] R. Davis, D. S. Harmer, \& K. C. Hoffman, "Search for Neutrinos from the Sun," Phys. Rev. Lett., vol. 20, pp. 1205-1209, May 1968.

[34] V. Gribov \& B. Pontecorvo, "Neutrino astronomy and lepton charge," Physics Letters B, vol. 28, no. 7, pp. 493 - 496, 1969.

[35] Y. Fukuda et al., [Super-Kamiokande] , "Evidence for Oscillation of Atmospheric Neutrinos," Phys. Rev. Lett., vol. 81, pp. 1562-1567, Aug 1998.

[36] Q. R. Ahmad et al., [SNO], "Measurement of the Rate of $\nu_{e}+d \rightarrow p+p+e^{-}$Interactions Produced by ${ }^{8}$ B Solar Neutrinos at the Sudbury Neutrino Observatory," Phys. Rev. Lett., vol. 87, p. 071301, Jul 2001.

[37] Q. R. Ahmad et al., [SNO], "Direct Evidence for Neutrino Flavor Transformation from NeutralCurrent Interactions in the Sudbury Neutrino Observatory," Phys. Rev. Lett., vol. 89, p. 011301, Jun 2002.

[38] L. Wolfenstein, "Neutrino oscillations in matter," Phys. Rev. D, vol. 17, pp. 2369-2374, May 1978.

[39] S. Mikheev \& A. Y. Smirnov, "Resonance Amplification of Oscillations in Matter and Spectroscopy of Solar Neutrinos," Sov.J.Nucl.Phys., vol. 42, pp. 913-917, 1985.

[40] J. Beringer et al., [Particle Data Group] , "Review of particle physics," Phys. Rev. D, vol. 86, p. 010001, Jul 2012.

[41] J. A. Formaggio \& G. P. Zeller, "From eV to EeV: Neutrino cross sections across energy scales," Rev. Mod. Phys., vol. 84, pp. 1307-1341, Sep 2012.

[42] G. Bellini et al., [Borexino] , "Observation of geo-neutrinos," Physics Letters B, vol. 687, no. 4-5, pp. 299 - 304, 2010.

[43] G. Bellini et al., [Borexino] , "Measurement of geo-neutrinos from 1353 days of Borexino," Phys.Lett., vol. B722, pp. 295-300, 2013. 
[44] Kamland Collaboration et al., [KamLAND], "Partial radiogenic heat model for Earth revealed by geoneutrino measurements," Nature Geoscience, vol. 4, pp. 647-651, Sept. 2011.

[45] A. Gando et al., [KamLAND] , "Reactor On-Off Antineutrino Measurement with KamLAND." arXiv:1303.4667, 2013.

[46] M. G. Aartsen et al., [IceCube] , "First Observation of PeV-Energy Neutrinos with IceCube," Phys. Rev. Lett., vol. 111, p. 021103, Jul 2013.

[47] P. Ade et al., [Planck] , "Planck 2013 results. XVI. Cosmological parameters." arXiv:1303.5076, 2013.

[48] E. Komatsu et al., [WMAP] , "Seven-Year Wilkinson Microwave Anisotropy Probe (WMAP) Observations: Cosmological Interpretation," Astrophys.J.Suppl., vol. 192, p. 18, 2011.

[49] G. Mangano \& P. D. Serpico, "A robust upper limit on from bbn, circa 2011," Physics Letters B, vol. 701, no. 3, pp. 296 - 299, 2011.

[50] N. Cabibbo, "Unitary Symmetry and Leptonic Decays," Phys. Rev. Lett., vol. 10, pp. 531-533, Jun 1963.

[51] M. Kobayashi \& T. Maskawa, "CP-Violation in the Renormalizable Theory of Weak Interaction," Progress of Theoretical Physics, vol. 49, pp. 652-657, Feb. 1973.

[52] E. Akhmedov \& A. Smirnov, "Paradoxes of neutrino oscillations," Physics of Atomic Nuclei, vol. 72, no. 8, pp. 1363-1381, 2009.

[53] Janet Conrad, "Explain it in 60 seconds: Neutrino mixing." http://www.symmetrymagazine.org/ article/may-2005/explain-it-in-60-seconds.

[54] D. Groom et al., [Particle Data Group] , "Review of Particle Physics," The European Physical Journal, vol. C15, pp. 1+, 2000.

[55] V. N. Aseev et al., [Troitsk], "Upper limit on the electron antineutrino mass from the Troitsk experiment," Phys. Rev. D, vol. 84, p. 112003, Dec 2011.

[56] G. Drexlin, [KATRIN] , "KATRIN: Direct measurement of a sub-eV neutrino mass," Nucl.Phys.Proc.Suppl., vol. 145, pp. 263-267, 2005.

[57] K. Assamagan et al., "Upper limit of the muon-neutrino mass and charged-pion mass from momentum analysis of a surface muon beam," Phys. Rev. D, vol. 53, pp. 6065-6077, Jun 1996.

[58] R. Barate et al., [ALEPH] , "An Upper limit on the tau-neutrino mass from three-prong and five-prong tau decays," Eur.Phys.J., vol. C2, pp. 395-406, 1998.

[59] G. L. Fogli et al., "Observables sensitive to absolute neutrino masses. ii," Phys. Rev. D, vol. 78, p. 033010, Aug 2008.

[60] C. Giunti, "No effect of Majorana phases in neutrino oscillations," Physics Letters B, vol. 686, no. 1, pp. $41-43,2010$.

[61] J. N. Bahcall, A. M. Serenelli, \& S. Basu, "New Solar Opacities, Abundances, Helioseismology, and Neutrino Fluxes," Astrophys.J., vol. 621, pp. L85-L88, Mar. 2005.

[62] B. Cleveland et al., "Measurement of the solar electron neutrino flux with the Homestake chlorine detector," Astrophys.J., vol. 496, pp. 505-526, 1998.

[63] J. Abdurashitov et al., [SAGE] , "Solar neutrino flux measurements by the Soviet-American Gallium Experiment (SAGE) for half the 22 year solar cycle," J.Exp.Theor.Phys., vol. 95, pp. 181-193, 2002.

[64] W. Hampel et al., [GALLEX] , "GALLEX solar neutrino observations: Results for GALLEX IV," Phys.Lett., vol. B447, pp. 127-133, 1999.

[65] M. Altmann et al., [GNO] , "Complete results for five years of GNO solar neutrino observations," Phys.Lett., vol. B616, pp. 174-190, 2005.

[66] Y. Fukuda et al., [Kamiokande] , "Solar neutrino data covering solar cycle 22," Phys.Rev.Lett., vol. 77, pp. 1683-1686, 1996.

[67] Y. Fukuda et al., [Super-Kamiokande], "Measurements of the solar neutrino flux from SuperKamiokande's first 300 days," Phys.Rev.Lett., vol. 81, pp. 1158-1162, 1998. 
[68] J. N. Bahcall, N. A. Bahcall, \& G. Shaviv, "Present status of the theoretical predictions for the Cl-36 solar neutrino experiment," Phys.Rev.Lett., vol. 20, pp. 1209-1212, 1968.

[69] J. N. Bahcall, "Solar neutrinos. I: Theoretical," Phys.Rev.Lett., vol. 12, pp. 300-302, 1964.

[70] B. Aharmim et al., [SNO], "Combined analysis of all three phases of solar neutrino data from the sudbury neutrino observatory," Phys. Rev. C, vol. 88, p. 025501, Aug 2013.

[71] H. Sekiya for the Super-Kamiokande Collaboration, "Solar neutrino analysis of Super-Kamiokande." arXiv:1307.3686, 2013.

[72] K. Abe et al., [Super-Kamiokande] , "Solar neutrino results in Super-Kamiokande-III," Phys.Rev., vol. D83, p. 052010, 2011.

[73] G. Bellini et al., [Borexino] ,"First evidence of pep solar neutrinos by direct detection in borexino," Phys. Rev. Lett., vol. 108, p. 051302, Feb 2012.

[74] V. Lozza, [SNO+], "Scintillator phase of the SNO+ experiment," J.Phys.Conf.Ser., vol. 375, p. 042050, 2012.

[75] M. Wurm et al., "The next-generation liquid-scintillator neutrino observatory LENA," Astroparticle Physics, vol. 35, no. 11, pp. 685 - 732, 2012.

[76] F. Reines et al., "Evidence for High-Energy Cosmic-Ray Neutrino Interactions," Phys. Rev. Lett., vol. 15, pp. 429-433, Aug 1965.

[77] C. Achar et al., "Detection of muons produced by cosmic ray neutrinos deep underground," Physics Letters, vol. 18, no. 2, pp. 196 - 199, 1965.

[78] M. Aglietta et al., [NUSEX] , "Experimental study of atmospheric neutrino flux in the NUSEX experiment," Europhys.Lett., vol. 8, pp. 611-614, 1989.

[79] K. Daum et al., [Frejus] , "Determination of the atmospheric neutrino spectra with the Frejus detector," Z.Phys., vol. C66, pp. 417-428, 1995.

[80] Y. Fukuda et al., [Kamiokande] , "Atmospheric muon-neutrino / electron-neutrino ratio in the multiGeV energy range," Phys.Lett., vol. B335, pp. 237-245, 1994.

[81] R. Becker-Szendy et al., [IMB] , "The Electron-neutrino and muon-neutrino content of the atmospheric flux," Phys.Rev., vol. D46, pp. 3720-3724, 1992.

[82] Y. Ashie et al., [Super-Kamiokande] , "A Measurement of atmospheric neutrino oscillation parameters by Super-Kamiokande I," Phys.Rev., vol. D71, p. 112005, 2005.

[83] J. Hosaka et al., [Super-Kamiokande], "Three flavor neutrino oscillation analysis of atmospheric neutrinos in Super-Kamiokande," Phys.Rev., vol. D74, p. 032002, 2006.

[84] M. C. Sanchez et al., [Soudan 2] , "Measurement of the L/E distributions of atmospheric neutrinos in Soudan 2 and their interpretation as neutrino oscillations," Phys.Rev., vol. D68, p. 113004, 2003.

[85] M. Ambrosio et al., [MACRO], "Matter effects in upward going muons and sterile neutrino oscillations," Phys.Lett., vol. B517, pp. 59-66, 2001.

[86] P. Adamson et al., [MINOS] , "Measurement of neutrino and antineutrino oscillations using beam and atmospheric data in minos," Phys. Rev. Lett., vol. 110, p. 251801, Jun 2013.

[87] S. Fukuda et al., [Super-Kamiokande] , "Tau neutrinos favored over sterile neutrinos in atmospheric muon-neutrino oscillations," Phys.Rev.Lett., vol. 85, pp. 3999-4003, 2000.

[88] K. Abe et al., [Super-Kamiokande] , "A Measurement of the Appearance of Atmospheric Tau Neutrinos by Super-Kamiokande," Phys.Rev.Lett., vol. 110, p. 181802, 2013.

[89] Y. Ashie et al., [Super-Kamiokande] , "Measurement of atmospheric neutrino oscillation parameters by Super-Kamiokande I," Phys. Rev. D, vol. 71, p. 112005, Jun 2005.

[90] P. Adamson et al., [MINOS], "Measurement of the neutrino mass splitting and flavor mixing by MINOS," Phys.Rev.Lett., vol. 106, p. 181801, 2011.

[91] M. Aartsen et al., [IceCube] , "Measurement of Atmospheric Neutrino Oscillations with IceCube," Phys. Rev. Lett. 111,, vol. 081801, 2013. 
[92] S. K. Agarwalla, S. Choubey, \& A. Raychaudhuri, "Neutrino mass hierarchy and with a magic baseline beta-beam experiment," Nuclear Physics B, vol. 771, no. 1-2, pp. 1-27, 2007.

[93] K. Abe et al., "Letter of Intent: The Hyper-Kamiokande Experiment — Detector Design and Physics Potential —." arXiv:1109.3262, 2011.

[94] W. Winter, "Neutrino mass hierarchy determination with IceCube-PINGU," Phys.Rev., vol. D88, p. 013013, 2013.

[95] G. Zacek et al., [CALTECH-SIN-TUM] , "Neutrino Oscillation Experiments at the Gosgen Nuclear Power Reactor," Phys.Rev., vol. D34, pp. 2621-2636, 1986.

[96] G. Vidyakin et al., "Limitations on the characteristics of neutrino oscillations," JETP Lett., vol. 59, pp. 390-393, 1994.

[97] B. Achkar et al., [Bugey] , "Search for neutrino oscillations at 15, 40 and 95 meters from a nuclear power reactor at Bugey," Nuclear Physics B, vol. 434, no. 3, pp. 503 - 532, 1995.

[98] M. Apollonio et al., [CHOOZ] , "Limits on neutrino oscillations from the CHOOZ experiment," Phys.Lett., vol. B466, pp. 415-430, 1999.

[99] M. Apollonio et al., [CHOOZ] , "Search for neutrino oscillations on a long baseline at the CHOOZ nuclear power station," Eur.Phys.J., vol. C27, pp. 331-374, 2003.

[100] F. Boehm et al., [Palo Verde] , "Final results from the Palo Verde neutrino oscillation experiment," Phys.Rev., vol. D64, p. 112001, 2001.

[101] A. Piepke, [KamLAND] , "KamLAND: A reactor neutrino experiment testing the solar neutrino anomaly," Nucl.Phys.Proc.Suppl., vol. 91, pp. 99-104, 2001.

[102] F. An et al., [Daya Bay] , "Improved Measurement of Electron Antineutrino Disappearance at Daya Bay," Chin. Phys., vol. C37, p. 011001, 2013.

[103] F. Ardellier et al., [Double Chooz] , "Double Chooz: A Search for the neutrino mixing angle theta(13)." arXiv:hep-ex/0405032, 2006.

[104] J. Ahn et al., [RENO] , "RENO: An Experiment for Neutrino Oscillation Parameter $\theta_{13}$ Using Reactor Neutrinos at Yonggwang." arXiv:1003.1391, 2010.

[105] X. Guo et al., [Daya-Bay], "A Precision measurement of the neutrino mixing angle $\theta_{13}$ using reactor antineutrinos at Daya-Bay." arXiv:hep-ex/0701029, 2007.

[106] S. Ahn et al., [K2K] , "Detection of accelerator produced neutrinos at a distance of 250-km," Phys.Lett., vol. B511, pp. 178-184, 2001.

[107] M. Ahn et al., [K2K] , "Measurement of Neutrino Oscillation by the K2K Experiment," Phys.Rev., vol. D74, p. 072003, 2006.

[108] E. Ables et al., [MINOS] , "P-875: A Long baseline neutrino oscillation experiment at Fermilab," tech. rep., Fermi National Accelerator Laboratory, 1995.

[109] P. Adamson et al., [MINOS] , "Electron neutrino and antineutrino appearance in the full MINOS data sample," Phys.Rev.Lett., vol. 110, p. 171801, 2013.

[110] M. Guler et al., [OPERA], "OPERA: An appearance experiment to search for $\nu_{\mu} \rightarrow \nu_{\tau}$ oscillations in the CNGS beam. Experimental proposal," tech. rep., Organisation européenne pour la recherche nucléaire, 2000.

[111] F. Arneodo et al., [ICARUS], "The ICARUS experiment: A Second generation proton decay experiment and neutrino observatory at the Gran Sasso Laboratory." arXiv:hep-ex/0103008, 2001.

[112] K. Abe et al., [T2K] , "The T2K Experiment," Nucl.Instrum.Meth., vol. A659, pp. 106-135, 2011.

[113] D. Ayres et al., [NO $\nu$ A] , "NO $\nu$ A: Proposal to build a 30 kiloton off-axis detector to study $\nu_{\mu} \rightarrow \nu_{e}$ oscillations in the NuMI beamline," tech. rep., Fermi National Accelerator Laboratory, 2004.

[114] U. Kose for the OPERA Collaboration, [OPERA] , "Study of Neutrino Oscillations in the OPERA Experiment." arXuv:1305.2513, 2013.

[115] K. Abe et al., [T2K Collaboration], "Measurement of Neutrino Oscillation Parameters from Muon Neutrino Disappearance with an Off-axis Beam." arXiv:1308.0465, 2013. 
[116] Y. Itow. Proceedings of the 25th International Conference on Neutrino Physics and Astrophysics (Neutrino 2012), Kyoto, Japan, June 2012. To be published.

[117] K. Abe et al., [T2K] , "First Muon-Neutrino Disappearance Study with an Off-Axis Beam," Phys.Rev., vol. D85, p. 031103, 2012.

[118] G. Mention et al., "Reactor antineutrino anomaly," Phys. Rev. D, vol. 83, p. 073006, Apr 2011.

[119] TPC-Paris, Sterile Neutrinos, December 2012.

[120] J. N. Abdurashitov et al., [SAGE] , "Measurement of the solar neutrino capture rate with gallium metal. iii. results for the 2002-2007 data-taking period," Phys. Rev. C, vol. 80, p. 015807, Jul 2009.

[121] W. Hampel et al., [GALLEX] , "Final results of the 51Cr neutrino source experiments in GALLEX," Physics Letters B, vol. 420, no. 1-2, pp. 114 - 126, 1998.

[122] M. A. Acero, C. Giunti, \& M. Laveder, "Limits on nu(e) and anti-nu(e) disappearance from Gallium and reactor experiments," Phys.Rev., vol. D78, p. 073009, 2008.

[123] C. Giunti \& M. Laveder, "Statistical Significance of the Gallium Anomaly," Phys.Rev., vol. C83, p. 065504, 2011.

[124] B. Armbruster et al., [KARMEN], "Upper limits for neutrino oscillations $\bar{\nu}_{\mu} \rightarrow$ nuebar from muon decay at rest," Phys. Rev. D, vol. 65, p. 112001, Jun 2002.

[125] N. Agafonova et al., [OPERA], "Search for $\nu_{\mu} \rightarrow \nu_{e}$ oscillations with the OPERA experiment in the CNGS beam," JHEP, vol. 1307, p. 004, 2013.

[126] M. Antonello et al., [ICARUS], "Experimental search for the "LSND anomaly" with the ICARUS detector in the CNGS neutrino beam," Eur.Phys.J., vol. C73, p. 2345, 2013.

[127] K. Abazajian et al., "Light Sterile Neutrinos: A White Paper." arXiv:1204.5379, 2012.

[128] Borexino Collaboration, "SOX: Short distance neutrino Oscillations with BoreXino." arXiv:1304.7721, Apr. 2013.

[129] A. Gando et al., "White paper: CeLAND - Investigation of the reactor antineutrino anomaly with an intense 144Ce-144Pr antineutrino source in KamLAND." arXiv:1309.6805, 2013.

[130] U. Kose, "NESSiE: The Experimental Sterile Neutrino Search in Short-Base-Line at CERN." arXiv:1304.7127, Apr. 2013.

[131] A. Adelmann et al., "Cost-effective Design Options for IsoDAR." arXiv:1210.4454, 2012.

[132] D. Adey et al., [nuSTORM], "nuSTORM - Neutrinos from STORed Muons: Proposal to the Fermilab PAC," tech. rep., Fermi National Accelerator Laboratory, 2013.

[133] G. Tzanankos et al., [MINOS+], "MINOS+: a Proposal to FNAL to run MINOS with the medium energy NuMI beam," tech. rep., Fermi National Accelerator Laboratory, 2011.

[134] A. Porta, [Nucifer] , "Reactor neutrino detection for non proliferation with the Nucifer experiment," J.Phys.Conf.Ser., vol. 203, p. 012092, 2010.

[135] W. C. Louis, "The LSND and KARMEN Neutrino Oscillation Experiments," in Neutrino Oscillations: Present Status and Future Plans (J. Thomas \& P. Vahle, eds.), ch. 7, World Scientific Publishing Company, 2008.

[136] C. Athanassopoulos et al., [LSND], "The liquid scintillator neutrino detector and LAMPF neutrino source," Nuclear Instruments and Methods in Physics Research Section A: Accelerators, Spectrometers, Detectors and Associated Equipment, vol. 388, no. 1-2, pp. 149 - 172, 1997.

[137] A. Aguilar et al., [LSND], "Evidence for neutrino oscillations from the observation of $\bar{\nu}_{e}$ appearance in a $\bar{\nu}_{\mu}$ beam," Phys. Rev. D, vol. 64, p. 112007, Nov 2001.

[138] E. D. Church, K. Eitel, G. B. Mills, \& M. Steidl, "Statistical analysis of different $\bar{\nu}_{\mu} \rightarrow \bar{\nu}_{e}$ searches," Phys. Rev. D, vol. 66, p. 013001, Jun 2002.

[139] A. A. Aguilar-Arevalo, An improved Neutrino Oscillations Analysis of the MiniBooNE Data. PhD thesis, Columbia University, 2008.

[140] G. S. Karagiorgi, Searches for New Physics at MiniBooNE: Sterile Neutrinos and Mixing Freedom. PhD 
thesis, Columbia University, 2010.

[141] E. Church et al., "A proposal for an experiment to measure $\nu_{\mu} \rightarrow \nu_{e}$ oscillations and $\nu_{\mu}$ disappearance at the Fermilab Booster: BooNE ," FERMILAB-PROPOSAL-0898, Fermi National Accelerator Laboratory, 1997.

[142] I. Stancu et al., [BooNE], "Technical Design Report for the 8GeV Beam." http://www-boone.fnal. gov/publicpages/8gevtdr2.0.ps.gz, 2001.

[143] T. Kobilarcik, J. Devaoy, \& C. Moore, "Automatic Beam Correction," in Particle Accelerator Conference, 2003.

[144] I. Stancu et al., [BooNE], "The MiniooNE Detector Technical Design Report." http://www-boone. fnal.gov/publicpages/detectortdr.ps.gz, 2001.

[145] A. A. Aguilar-Arevalo et al., [MiniBooNE] , "The MiniBooNE detector," Nuclear Instruments and Methods in Physics Research Section A: Accelerators, Spectrometers, Detectors and Associated Equipment, vol. 599, no. 1, pp. 28 - 46, 2009.

[146] L. Bartozsek, "Bartozsek Engineering." http://www. bartoszekeng.com.

[147] L. Bugel \& M. Sorel, "Magnetic Field Measurements for the MiniBooNE Prototype Horn," MiniBooNE Technical Note 34, Fermi National Accelerator Laboratory, 2001.

[148] CERN Program Library Long Writeup W5013, "GEANT Detector and Simulation Tool," 1993.

[149] R. Nelson, "Constraining the kaon content in the MiniBooNE secondary beam with the Little Muon Counter," AIP Conf.Proc., vol. 842, pp. 831-833, 2006.

[150] P. A. Cherenkov, "Visible emission of clean liquids by action of $\gamma$ radiation," Doklady Akademii Nauk SSSR, vol. 2, 1934.

[151] Hamamatsu, "Hamamatsu Photonics." www.hamamatsu.com.

[152] G. Davison, "Ludox ß, silica coloidal." http://www.gracedavison.com/products/ludox/overview. htm.

[153] I. Stancu, "Gain Calibration and the Speed of Light from Low Intensity Laser Runs," MiniBooNE Technical Note 63, Fermi National Accelerator Laboratory, 2002.

[154] R. Imlay, B. Metcalf, G. Schofield, M. Sung, \& W. M., "Study of the Angular Reconstruction of Muons in MiniBooNE Using the Muon Tracker," MiniBooNE Technical Note 99, Fermi National Accelerator Laboratory, 2003.

[155] R. Imlay, B. Metcalf, S. Ouedraogo, M. Sung, \& M. Wascko, "Energy Calibration of Stopping Muons in MiniBooNE Using the Muon Tracker and Cubes," MiniBooNE Technical Note 106, Fermi National Accelerator Laboratory, 2003.

[156] A. A. Aguilar-Arevalo et al., [MiniBooNE] , "Measurement of $\nu_{\mu}$ and $\bar{\nu}_{\mu}$ induced neutral current single $\pi^{0}$ production cross sections on mineral oil at $E_{\nu} \sim \mathcal{O}(1 \mathrm{GeV})$," Phys. Rev. D, vol. 81, p. 013005, Jan 2010.

[157] Y. Liu \& I. Stancu, "The MiniBooNE Charge Likelihoods and Light Scattering in Michel Electron Events," MiniBooNE Technical Note 126, Fermi National Accelerator Laboratory, 2004.

[158] S. Agostinelli et al., "Geant4-a simulation toolkit," Nuclear Instruments and Methods in Physics Research Section A: Accelerators, Spectrometers, Detectors and Associated Equipment, vol. 506, no. 3, pp. $250-303,2003$.

[159] M. Sorel, Search for Sterile Neutrinos Using the MiniBooNE Beam. PhD thesis, Columbia University, 2005.

[160] CERN, "Cern program library." http://cernlib.web.cern.ch/cernlib/.

[161] D. C. Carey, K. Brown, \& F. Rothacker, "Third order TRANSPORT with MAD input: A Computer program for designing charged particle beam transport systems," SLAC Report, SLAC, 1998.

[162] A. A. Aguilar-Arevalo et al., [MiniBooNE] "Neutrino flux prediction at MiniBooNE," Phys. Rev. D, vol. 79, p. 072002, Apr 2009.

[163] D. Casper, "The nuance neutrino physics simulation, and the future," Nuclear Physics B - Proceedings 
Supplements, vol. 112, no. 1-3, pp. 161 - 170, 2002.

[164] R. Smith \& E. Moniz, "Neutrino reactions on nuclear targets," Nuclear Physics B, vol. 101, no. 2, pp. $547-, 1972$.

[165] C. Llewellyn Smith, "Neutrino Reactions at Accelerator Energies," Phys.Rept., vol. 3, pp. 261-379, 1972.

[166] A. A. Aguilar-Arevalo et al., [MiniBooNE], "Measurement of the neutrino component of an antineutrino beam observed by a nonmagnetized detector," Phys. Rev. D, vol. 84, p. 072005, Oct 2011.

[167] D. Rein \& L. M. Sehgal, "Neutrino-excitation of baryon resonances and single pion production," Annals of Physics, vol. 133, no. 1, pp. 79 - 153, 1981.

[168] Z. Djurcic et al., "Measurement of the Rate of NC $\pi^{0}$ Production at MiniBooNE," MiniBooNE Technical Note 194, Fermi National Accelerator Laboratory, 2007.

[169] A. A. Aguilar-Arevalo et al., [MiniBooNE], "First Observation of Coherent $\pi^{0}$ Production in Neutrino Nucleus Interactions with $E_{\nu}<2 \mathrm{GeV}$," Physics Letters B, vol. 664, no. 1-2, pp. 41 - 46, 2008.

[170] R. Brun, F. Bruyant, M. Maire, A. McPherson, \& P. Zanarini, "GEANT3," 1987. Revised version.

[171] C. Zeitnitz \& T. Gabriel, "The GEANT-CALOR interface and benchmark calculations of ZEUS test calorimeters," Nuclear Instruments and Methods in Physics Research Section A: Accelerators, Spectrometers, Detectors and Associated Equipment, vol. 349, no. 1, pp. 106 - 111, 1994.

[172] H.-J. Yang, "Measurement of Dirt Events," MiniBooNE Technical Note 203, Fermi National Accelerator Laboratory, 2006.

[173] B. C. Brown, S. Maza, A. Pla-Dalmau, \& H. A. Tanaka, "Studies of the Optical Properties of Marcol 7 Mineral Oil," MiniBooNE Technical Note 145, Fermi National Accelerator Laboratory, 2004.

[174] R. B. Patterson, A Search for Muon Neutrino to Electron Neutrino Oscillations At $\Delta m^{2}>0.1$ eV $^{2}$. PhD thesis, Princeton University, Nov 2007.

[175] B. T. Fleming et al., "Photomultiplier tube testing for the MiniBooNE experiment," Nuclear Science, IEEE Transactions on, vol. 49, no. 3, pp. 984-988, 2002.

[176] R. Patterson et al., "The extended-track event reconstruction for MiniBooNE," Nuclear Instruments and Methods in Physics Research Section A: Accelerators, Spectrometers, Detectors and Associated Equipment, vol. 608, no. 1, pp. 206 - 224, 2009.

[177] A. A. Aguilar-Arevalo et al., [MiniBooNE], "Search for Electron Neutrino Appearance at the $\Delta m^{2} \sim 1$ eV Scale," Phys. Rev. Lett., vol. 98, p. 231801, Jun 2007.

[178] A. A. Aguilar-Arevalo et al., [MiniBooNE], "Unexplained Excess of Electronlike Events from a 1-GeV Neutrino Beam," Phys. Rev. Lett., vol. 102, p. 101802, Mar 2009.

[179] A. A. Aguilar-Arevalo et al., [MiniBooNE], "Search for Electron Antineutrino Appearance at the $\Delta m^{2} \sim 1 \mathrm{eV}^{2}$ Scale," Phys. Rev. Lett., vol. 103, p. 111801, Sep 2009.

[180] A. A. Aguilar-Arevalo et al., [MiniBooNE], "Event Excess in the MiniBooNE Search for $\bar{\nu}_{\mu} \rightarrow \bar{\nu}_{e}$ Oscillations," Phys. Rev. Lett., vol. 105, p. 181801, Oct 2010.

[181] A. A. Aguilar-Arevalo et al., [MiniBooNE] , "Improved Search for $\bar{\nu}_{\mu} \rightarrow \bar{\nu}_{e}$ Oscillations in the MiniBooNE Experiment," Phys. Rev. Lett., vol. 110, p. 161801, Apr 2013.

[182] A. A. Aguilar-Arevalo et al., [MiniBooNE] , "Low Mass WIMP Searches with a Neutrino Experiment: A Proposal for Further MiniBooNE Running." arXiv:1211.2258, 2012.

[183] A. A. Aguilar-Arevalo et al., [MiniBooNE] , "Letter of Intent: A new investigation of $\nu_{\mu} \rightarrow \nu_{e}$ oscillations with improved sensitivity in an enhanced MiniBooNE experiment," tech. rep., Fermi National Accelerator Laboratory, 2012.

[184] H. Chen et al., [MicroBooNE], "Proposal for a New Experiment Using the Booster and NuMI Neutrino Beamlines: MicroBooNE," fermilab-proposal-0974, Fermi National Accelerator Laboratory, 2007.

[185] F. James, Statistical methods in experimental physics. World Scientific, second ed., 2006.

[186] A. N. Kolmogorov, Foundations of the Theory of Probability. Chelsea Publishing Company, second 
english ed., 1956.

[187] L. Lyons, Statistics for nuclear and particle physicist. Cambridge University Press, first ed., 1986.

[188] J. Neyman, "Outline of a theory of statistical estimation based on the classical theory of probability," Philosophical Transactions of the Royal Society of London. Series A, Mathematical and Physical Sciences, vol. 236, no. 767, pp. 333-380, 1937.

[189] G. Bohm \& G. Zech, Introduction to Statistics and Data Analysis for Physicists. DESY, first ed., 2010.

[190] MiniBooNE, "Data Release for A. A. Aguilar-Arevalo et al., "A Search for Electron Neutrino Appearance at the $\Delta m^{2} \sim 1 \mathrm{eV}^{2}$ Scale"." http://www-boone.fnal.gov/for_physicists/data_release/nue/.

[191] MiniBooNE, "Data Release for A. A. Aguilar-Arevalo et al., "Unexplained Excess of ElectronLike Events From a $1 \mathrm{GeV}$ Neutrino Beam"." http://www-boone.fnal.gov/for_physicists/data_ release/lowe/.

[192] MiniBooNE, "Data Release for A. A. Aguilar-Arevalo et al., "Event Excess in the MiniBooNE Search for $\bar{\nu}_{\mu} \rightarrow \bar{\nu}_{e}$ Oscillations"." http://www-boone.fnal.gov/for_physicists/data_release/nuebar2010/.

[193] MiniBooNE, "Data Release for A. A. Aguilar-Arevalo et al., "A Combined $\nu_{\mu} \rightarrow \nu_{e}$ and $\bar{\nu}_{\mu} \rightarrow \bar{\nu}_{e}$ Oscillation Analysis of the MiniBooNE Excesses"." http://www-boone.fnal.gov/for_physicists/ data_release/nue_nuebar_2012/numode.html\#fit200.

[194] MiniBooNE, "Data Release for A. A. Aguilar-Arevalo et al., "A Combined $\nu_{\mu} \rightarrow \nu_{e}$ and $\bar{\nu}_{\mu} \rightarrow \bar{\nu}_{e}$ Oscillation Analysis of the MiniBooNE Excesses"." http://www-boone.fnal.gov/for_physicists/ data_release/nue_nuebar_2012/nubarmode.html\#fit200.

[195] MiniBooNE, "Data Release for A. A. Aguilar-Arevalo et al., "A Combined $\nu_{\mu} \rightarrow \nu_{e}$ and $\bar{\nu}_{\mu} \rightarrow \bar{\nu}_{e}$ Oscillation Analysis of the MiniBooNE Excesses"." http://www-boone.fnal.gov/for_physicists/ data_release/nue_nuebar_2012/combined.html\#fit200.

[196] G. J. Feldman \& R. D. Cousins, "Unified approach to the classical statistical analysis of small signals," Phys. Rev. D, vol. 57, pp. 3873-3889, Apr 1998.

[197] L. Devroye, Non-Uniform Random Variate Generation. Springer-Verlag, first ed., 1986.

[198] A. Krishnamoorthy \& D. Menon, "Matrix inversion using cholesky decomposition," CoRR, vol. ab$\mathrm{s} / 1111.4144,2011$.

[199] R. Brun \& F. Rademakers, "ROOT - An object oriented data analysis framework," Nuclear Instruments and Methods in Physics Research Section A: Accelerators, Spectrometers, Detectors and Associated Equipment, vol. 389, no. 1-2, pp. 81 - 86, 1997. New Computing Techniques in Physics Research.

[200] Low E. Group, "Studies and Oscillation Fits with the New All-Hadronic Monte Carlo," MiniBooNE Technical Note 254, Fermi National Accelerator Laboratory, 2008.

[201] G. Cheng et al., [SciBooNE] , "Measurement of $K^{+}$production cross section by 8 gev protons using high-energy neutrino interactions in the sciboone detector," Phys. Rev. D, vol. 84, p. 012009, Jul 2011.

[202] A. A. Aguilar-Arevalo et al., [MiniBooNE] , "A Combined $\nu_{\mu} \rightarrow \nu_{e}$ and $\bar{\nu}_{\mu} \rightarrow \bar{\nu}_{e}$ Oscillation Analysis of the MiniBooNE Excesses." arXiv:1207.4809, 2012.

[203] J. M. Conrad, W. C. Louis, \& M. H. Shaevitz, "The LSND and MiniBooNE Oscillation Searches at High $\Delta m^{2}, "$ Ann.Rev.Nucl.Part.Sci., vol. 63, p. 45, 2013.

[204] F. Agner, "Pseudo random number generators." http://www. agner.org/random/.

[205] M. Matsumoto \& T. Nishimura, "Mersenne twister: a 623-dimensionally equidistributed uniform pseudorandom number generator," ACM Trans. Model. Comput. Simul., vol. 8, pp. 3-30, Jan. 1998.

[206] D. Thain, T. Tannenbaum, \& M. Livny, "Distributed computing in practice: the Condor experience," Concurrency and Computation: Practice and Experience, vol. 17, no. 2-4, pp. 323-356, 2005.

[207] Fermilab, "Configuring kerberos on newer Linux distributions." http://fermilinux.fnal.gov/ documentation/security/kerberos-newer-linux/.

[208] J. Sanders \& E. Kandrot, CUDA by Example: An Introduction to General-Purpose GPU Programming . Addison-Wesley Professional, first ed., 2010.

[209] NVIDIA, "Nvidia corporation." http://www.nvidia.com/object/cuda_home_new.html. 
[210] D. B. Kirk \& W. W. Hwu, Programming Massively Parallel Processors: A Hands-on Approach. Morgan Kaufmann, first ed., 2010.

[211] H. Sutter \& J. Larus, "Software and the Concurrency Revolution," Queue, vol. 3, pp. 54-62, Sept. 2005.

[212] J. Neumann, "First draft of a report on the EDVAC," tech. rep., Moore School of Electrical Engineering University of Pennsylvania, 1945.

[213] A. A. Aguilar-Arévalo \& V. Sandberg, "Event Logging of Beam Timing and Cable Propagation Delay Measurements with the BooNE Data Acquisition System," MiniBooNE Technical Note 4, Fermi National Accelerator Laboratory, 1998. 